\begin{abstract}
s
12th European Congress of Trauma and Emergency Surgery
\end{abstract}

April 27-30, 2011

Milan, Italy

Congress President

Zago Mauro

Milan, Italy 
European Journal of Trauma and Emergency Surgery

Official Publication of the European Society for Trauma and Emergency Surgery

Supplement

Vol. 37, 2011

Abstracts of the 12th European Congress of Trauma and Emergency Surgery

April 27-30, 2011, Milan/Italy

Organized by

European Society for Trauma \& Emergency Surgery

Italian Trauma and Emergency Surgery Society

Contents

S4 Oral Presentations

S82 Video Presentations

S89 Posters

S182 Case Reports

S207 Index of Authors 
Dear Colleagues,

Milan is ready to host the 12th European Congress on Trauma and Emergency Surgery. We are proud and honored to welcome all European and Oversea participants with all their accompanying persons.

We worked hard to try to make your stay in Italy unforgettable, both from scientific and social sides.

We are sure the Congress and the reported experiences of many distinguished colleagues will increase our knowledge allowing us to learn more and more, nourishing our scientific curiosity, getting in touch with new ideas, improving to work in multidisciplinary teams, never ending our thirst of training.

These were some of the meanings behind the motto we have chosen for our Meeting: Feeding Brains, training for Life.

Trauma and Emergency Surgery, both in dedicated Centers and in General Hospitals, is a matter of Team-working and a continuing challenge in organisation, technical skills, educational planning, decision-making and, most important, patient outcome. For this reason, ESTES is working for a European specific curriculum and expertise in Acute Care Surgery.

The scientific programme and the precongress Courses of the 2011 ESTES Congress offer a cutting edge update both for those who work primarily in an emergency setting and for those who take emergency call on a less frequent basis.

It is now time to enjoy Milan and the ESTES Congress!

Welcome!

Mauro Zago, Congress President

Hayato Kurihara, Congress General Secretary 


\section{2th European Congress of Trauma and Emergency Surgery}

\section{Oral Presentations \\ EMERGENCY SURGERY 1}

\section{1}

\section{EMERGENCY COLORECTAL SURGERY IN OCTOGENARIANS: IS IT ACTUALLY AN ISSUE?}

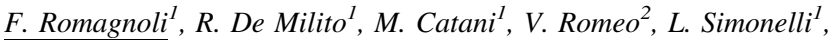 \\ F. Guerra ${ }^{3}$, C. Modini ${ }^{1}$
}

\begin{abstract}
${ }^{1}$ Dea-emergency Department, Policlinico Umberto I, Rome, Italy, ${ }^{2}$ Dea-emergency Department, Sapienza Università di Roma, Rome, Italy, ${ }^{3}$ Dea, Policlinico Umberto I, Rome, Italy
\end{abstract}

Introduction: Elderly individuals represents one of the most rapidly growing segments of the world's population, hence surgeons will carry out surgical procedures on more and more elderly patients. Emergency surgery often represents an unfavorable condition and is associated with higher mortality rates and thus, the elderly patient presenting with emergent colorectal disease may be an increased risk for postoperative complications and mortality. Previous studies have addressed the outcomes of elderly patients undergoing a variety of elective surgical procedures, including colorectal resection. The aim of this study was to determine the short-time outcomes of octogenarians following emergency colorectal resection, predictive factors impacting the surgical outcome.

Materials and methods: We evaluated 355 patients underwent surgery in our tertiary level hospital Emergency Department for complication of colorectal disease from January 2007 to December 2009. The results were then analyzed in terms of morbidity and mortality in 30 days, stratified on the basis of patient's characteristics and pathology, timing and type of treatment

Results: Ninety-three patients on 355 were over 80 years of age. In the octogenarians the associated pathologies with a higher incidence were cardiac (40\%), respiratory (11\%) and diabetes (8\%). The global morbidity and mortality were 14 and $30.1 \%$ respectively. Although oldness represent significant risk factor for mortality, the difference between octogenarians and others were not statistically significant in terms of morbidity.

Conclusion: Our experience represents one of the largest retrospective series in nonelective setting for a variety of colorectal diseases in octogenarians patients. The results of the present study, both in terms of mortality and morbidity, are absolutely competitive with those published. The analysis of the results has shown outcomes perfectly in line with the best that was found in literature, also among the non elderly patients, demonstrating that the training of the emergency surgery team to treat patients at risk is just as effective by itself. References: 1. McGillicuddy EA, Schuster KM, Davis KA, Longo WE (2009) Factors predicting morbidity and mortality in emergency colorectal procedures in elderly patients. Arch Surg 144(12):1157-1162. 2. Person B, Cera SM, Sands DR, et al (2008) Do elderly patients benefit from laparoscopic colorectal surgery? Surg Endosc 22(2):401-405. 3. Leong QM, Aung MO, Ho CK, Sim R (2009) Emergency colorectal resections in asian octogenarius: factors impacting surgical outcome. Surg Today 39:575-579. 4. Mulcahy HE, Patchett SE, Daly L, O'Donoghue DR (1994) Prognosis of elderly patients with large bowel cancer. $\mathrm{Br}$ J Surg 81:419-421. 5. Morel PH, Egeli RA, Wachtl S, Rohner A (1989) Results of operative treatment of gastrointestinal tract tumors in patients over 80 years of age. Arch Surg 124:662-664. 6. Richardson JD, Cocanour CS, Kern JA, et al (2004) Perioperative risk assessment in elderly and high-risk patients. J Am Coll Surg 199:133-146. 7. Colorectal Cancer Collaborative Group (2000) Surgery for colorectal cancer in elderly patients. A systematic review. Lancet 356:968-974. 8. Ozturk E, Yilmazlar T (2007) Factors affecting the mortality risk in elderly patients undergoing surgery. NZ J Surg 77:156-159. 9. Pavlidis TE, Marakis G, Ballas K, Rafailidis S, Psarras K, Pissas D, Papanicolaou K, Sakantamis A (2006) Safety of bowel resection for colorectal surgical emergency in the elderly. Colorectal Dis 8:657-662.

Disclosure: No significant relationships.

\section{2}

\section{PROGNOSTIC FACTORS IN PATIENTS WITH COLORECTAL PERFORATION}

G. Muraoka ${ }^{1}$, M. Hamada ${ }^{2}$, Y. Saisaka ${ }^{2}$, J. Ishihara ${ }^{2}$, K. Tanaka $^{2}$,

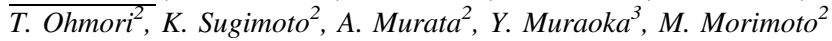

${ }^{1}$ Surgery, Kochi Health Sciences Center, Kochi, Japan, ${ }^{2}$ Emergency Department, Kochi Health Sciences Center, Kochi, Japan, ${ }^{3}$ Mathematics, Hyogo College of Medicine, Nishinomiya, Japan 
Introduction: Colorectal perforation is an abdominal emergency with high mortality. The aim of this study is to assess the prognostic factors for survival in patients suffering from colorectal perforation.

Materials and methods: We analyzed 59 patients underwent urgent surgery for colorectal perforation between March 2005 and December 2009. We examined demographics, pre- and post-operative clinical variables and blood chemicals, APACHE II score on admission and after surgery treatment.

Results: Eleven patients died and the overall mortality was $18 \%$. APACHE II scores on admission were a significantly higher in nonsuvivors than in survivors $(14.7 \pm 7.1$ vs. $21.9 \pm 9.0, \mathrm{p}=0.013)$. Compared with survivors, non-survivors displayed significantly low $\mathrm{PaCO}_{2}$, low white blood cell count, high PT-INR, low base excess and high lactate preoperatively, and low white blood cell count, low platelet count and high base excess postoperatively. There was significantly great difference of platelet count between pre- and postoperative data in two groups $(\mathrm{p}=0.01)$. Multiple logistic regression analysis yielded a model containing three significant variables, postoperative platelet count (a.Plt), postoperative base excess (a.BE) and the difference of platelet count before and after surgery (d.Plt). The regression model to predict $\mathrm{p}$ is $\operatorname{logit}(\mathrm{p})=$ $1.4252+0.1286[$ a.Plt $]+0.3076[$ a.BE $]+0.1515[$ d.Plt $]$. This model gives correct prediction for survival, i.e. positive predictive value $41 / 47(=0.872)$, negative predictive value $5 / 5(=1.000)$, Accuracy $46 / 52(=0.885)$, showing good match in our clinical cases.

Conclusion: The regression model including three explanatory variables, postoperative platelet count, postoperative base excess and the difference of platelet count between pre- and postoperation, could accurately predict the outcome of the patients with colorectal perforation. Further prospective examination should be required to get the conclusion.

Disclosure: No significant relationships.

\section{3}

\section{INFLUENCE OF DETERSION IN LEG ULCERS MANAGEMENT}

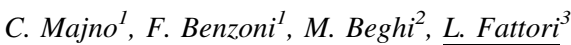

${ }^{1}$ Osp San Gerardo, Monza, Italy, ${ }^{2}$ Surgery- Chirurgia Iii, Osp San Gerardo, Monza, Italy, ${ }^{3}$ Surgery Chirurgia Iii, OSP San Gerardo, Monza, Italy

Introduction: Current treatment modalities for chronic leg ulcers are time consuming, expensive and only moderately successful. Treatment options include compression bandages, dressings and surgery but modalities are not completely coded. The aim was to evaluate prospectively the evolution of leg ulcers with different ways of detersion. Endpoints of the study were changes in wound healing, wound pain intensity, and comfort.

Materials and methods: This prospective case series examined 58 patients with painful chronic venous leg ulcers who were treated for 6 weeks with proper detersion and advanced dressing associated with appropriate compression bandaging. Patients were randomly assigned to two groups, for which cleaning was performed with either tap water (Group-A) or tap water plus soap (Group-B). Patients were enrolled according to the following criteria: age (60-85), leg ulcer set in by no more than 6 months and not less than 2 weeks, maximal extension $<100 \mathrm{~cm}^{2}$ and ABPI $>0.8$. Exclusion criteria were infection, antibiotic treatment ended by less than 2 weeks, insulin-dependent diabetes, immune suppressive therapy and immobilization. Ulcers were monitored at regular intervals using the PUSH tool. Pain was determined by VAS scale. Microbiological sampling was performed. Results: Healing was detected in $8 / 29$ patients in Gr.A and in $15 / 29$ in Gr.B; evidence of infection was found in 2 patients in Gr.A and 1 in Gr.B ( $p<0.01)$ The PUSH tool was statistically $(\mathrm{p}<0.05)$ different in the two groups for exudate amount and tissue type. Concerning the wound pain, no difference was found at the beginning of the study, than a decrease was detected, probably due to the healing process. A remarkable $(\mathrm{p}<0.5)$ reduction of bacteria and infection was detected in Gr.B vs Gr.A. Comfort was better in Gr.B than Gr.A.

Conclusion: The findings demonstrate that the process of healing is shortened if detersion is performed by tap water and soap, and that the comfort of patients is improved.

Disclosure: No significant relationships.

\section{O3A}

\section{ANY ADVANTAGE OF LAPAROSCOPIC APPENDECTOMY IN ACUTE APPENDICITIS?}

$\underline{\text { D.E. Böler }}^{1}$, N. Cabioglu, A.K. Belli ${ }^{3}$, B. Akce ${ }^{1}$, C. Uras $^{4}$

${ }^{1}$ Surgery, Bakirkoy Acibadem Hospital, Istanbul, Turkey, ${ }^{2}$ Surgery, Haseki Research Hospital, Istanbul, Turkey, ${ }^{3}$ Surgery, Bakırkoy Acıbadem Hospital, Istanbul, Turkey, ${ }^{4}$ Surgery, Acibadem Bakirkoy Hospital, Istanbul, Turkey

Introduction: Laparoscopic appendectomy versus open appendectomy has been a matter of debate. The goal of this study was to investigate the advantages or disadvantages of laparoscopic appendectomy (LA) in acute appendicitis compared to the open technique. Materials and methods: Between August 2000 to September 2010, 572 patients were operated with a diagnosis of acute appendicitis by an experienced surgical team. Of 572, 2 patients were excluded from the study because they were converted from laparoscopic technique to open appendectomy (OA). Of 570 cases, 429 patients $(75 \%)$ diagnosed with acute appendicitis were included into the study, whereas patients with perforated or other complicated appendicitis were excluded. The demographic data, histopathological diagnosis of the removed appendix, the operating room occupation time, length of hospitalization and postoperative complications were analysed.

Results: Of 429 patients, $386(90 \%)$ underwent LA, whereas 43 $(10 \%)$ had an OA. Age, gender, and body mass index were similarly distributed in both open and laparoscopic groups. The mean operating room occupation time and the hospital stay time were also found to be similar between $\mathrm{OA}^{-}$and LA-groups (OA, $50.1 \pm 18.3 \mathrm{~min}$ vs. LA, $52.6 \pm 15 \mathrm{~min}, \mathrm{p}=0.34$; and $\mathrm{OA}$, $1.8 \pm 0.7$ days vs. LA, $1.67 \pm 0.6$ days, $\mathrm{p}=0.29$; respectively). However, patients who underwent LA had a lower overall postoperative complication rate (OA, 9.3\% vs LA, 3.1\%, p = 0.042), and a lower wound infection rate compared to the OA-group (OA, $9.5 \%$ vs LA, $1.6 \%, p=0.001$ ).

Conclusion: Laparoscopic appendectomy in acute appendicitis seems to be comparable with the open technique regarding the duration of the operation and length of the hospital stay. However, laparoscopic approach could be considered superior over the open appendectomy due to a lower postoperative complication rate.

Disclosure: No significant relationships. 
04

\section{NEW CLINICAL INDEX FOR INFLUENCING THE DECISION FOR RELAPAROTOMY IN PATIENTION WITH PURULENT PERITONITIS}

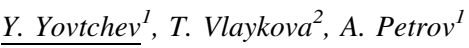 \\ ${ }^{1}$ Surgical Disease, University Hospital, Stara Zagora, Bulgaria, \\ ${ }^{2}$ Biochemistry, Medcal Faculty, Stara Zagora, Bulgaria
}

Introduction: The purpose of the study was to analyze clinical parameters related to the development of postoperative complication and to the indications for relaparotomy in patients operated for purulent peritonitis and a concomitant oncological disease of the colon or rectum.

Materials and methods: For a 14-year period (March 1995-March 2009) 58 emergency operations of severe purulent peritonitis secondary to perforated colon carcinoma have been performed. A retrospective study on medical records of this group of patients was performed, taking consideration of all clinical parameters that were supposed to influence the decision making for relaparotomy and to be related to disease outcome. Studied parameters included body temperature, general or local peritoneal reaction, leukocytosis, paresis of gastrointestinal organs, ileus and/or presence of intestinal content in peritoneal drain fluid. A new quantitative index was introduced to evaluate the necessity for relaparotomy.

Results: The onset of the first clinical signs associated with this complication and the evaluation of the need for reoperation were determined on the basis of criteria introduced by us, that consequently turned out to be statistically significant in decision making for relaparotomy $(\mathrm{p}=0.022)$. In this retrospective study, no relationship between empirical antibiotic therapy and either the decision for relaparotomy $(\mathrm{p}=0.655)$ or clinical outcome $(\mathrm{p}=0.431)$ was established. There was no statistically significant association between patients with one surgical intervention and those with relaparotomy due to anastomosis leakage $(\mathrm{p}=0.34)$.

Conclusion: Complementary diagnostic methods could assist in decision making for reoperation but could also provide falsely negative information and therefore, to result in considerably delayed relaparotomy.

Disclosure: The purpose of the study was to analyze clinical parameters related to the development of postoperative complication and to the indications for relaparotomy in patients operated for purulent peritonitis and a concomitant oncological disease of the colon.

\section{O4A}

\section{A OPEN ABDOMEN FOR MANAGING NON TRAUMA PATIENTS: 5-YEARS EXPERIENCE AT A LEVEL II EMERGENCY HOSPITAL}

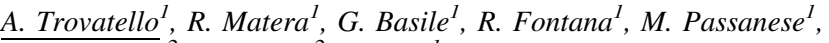 \\ M. Carpinteri $^{2}$, A. Bucolo ${ }^{2}$, P. Tine ${ }^{I}$
}

${ }^{1}$ Surgery, Hospital, Siracusa, Italy, ${ }^{2}$ Anesthesiology And Intensive Care Unit, Hospital, Siracusa, Italy

Introduction: Damage control surgery and the open abdomen technique, widely used in trauma, are now being utilized more often in non-trauma patients.

Materials and methods: The 5-years experience at a level II emergency hospital of non trauma patients managed with the open abdomen technique is reported. The number of operations, definitive fascial closure, fistula rate, complications and length of stay are discussed
Results: Thirty-nine patients were managed with an open abdomen technique over a period of 5 years. The most common indications for the open abdomen technique were intraabdominal hypertension, severe intraabdominal infection and damage control surgery in trauma group Conclusion: The use of this approach in selected urgent situations may reduce complications in these surgical critical patients.

Disclosure: No significant relationships.

\section{5}

\section{ROLE OF SIRS SCORE ON ADMISSION AND INITIAL CONCENTRATION OF IL-6 IN PREDICTION OF OUTCOME IN SEVERE ACUTE PANCREATITIS}

\author{
P.D. Gregoric ${ }^{1}$, N. Ivancevic ${ }^{2}$, D. Radenkovic ${ }^{3}$, A. Sijacki ${ }^{2}$, \\ B. Karadzic ${ }^{2}$, I. Pejovic ${ }^{3}$, D. Bajec ${ }^{2}$
}

${ }^{1}$ Iii, Clinic of Emergency Surgery, Clinical Center of Serbia and School of Medicine, University of Belgade, Serbia, Belgrade, Serbia, ${ }^{2}$ Clinic of Emergency Surgery, Clinical Center of Serbia and School of Medicine, University of Belgade, Serbia, Belgrade, Serbia, ${ }^{3} 1 \mathrm{st}$ Surgery Clinic, Clinical Center of Serbia and School of Medicine, University of Belgade, Serbia, Belgrade, Serbia

Introduction: Background: Early recognition of severe form of acute pancreatitis is important because these patients need more aggressive diagnostic and therapeutical approach and can develop systemic complications such as: sepsis, coagulopathy, acute lung injury (ALI), acute respiratory distress syndrome (ARDS), multiple organ dysfunction syndrome (MODS), multiple organ failure (MOF).

Aims: To determine role of the combination of systemic inflammatory response syndrome (SIRS) score and serum Interleukin-6 (IL-6) level on admission as predictor of illness severity and outcome of Severe Acute Pancreatitis (SAP).

Materials and methods: Methods: We evaluated 234 patients with first onset of SAP appears in last $24 \mathrm{~h}$. A total of 77(33\%) patients died. SIRS score and serum IL-6 concentration were measured in first hour after admission

Results: In 105 patients with SIRS score 3 and higher, initial measured IL-6 levels were significantly higher than in the group of remaining 129 patients $(72 \pm 67$ vs. $18 \pm 15 \mathrm{pg} / \mathrm{mL})$. All nonsurvivals were in the first group, with SIRS score 3 and 4 and initial IL-6 concentration $113 \pm 27 \mathrm{pg} / \mathrm{mL}$. The values of $\mathrm{C}$-reactive protein (CRP) measured after 48 h, Acute Physiology and Chronic Health Evaluation (APACHE II) score on admission and Ranson score showed the similar correlation, but serum amylase level did not correlate significantly with Ranson score, IL-6 concentration and APACHE II score

Conclusion: The combination of SIRS score on admission and IL-6 serum concentration can be early, predictor of illness severity and outcome in SAP.

Disclosure: No significant relationships.

\section{ELBOW FRACTURE DISLOCATION}

\section{O6}

\section{COVERAGE OF DORSAL ASPECT OF THE HAND IN EMERGENCY}

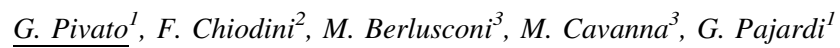

${ }^{1}$ Hand Surgery, Multimedica Hospital, Milan, Italy, ${ }^{2}$ Traumatology Ii, Instituto Clinico Humanitas, Rozzano, Italy, ${ }^{3}$ Trauma Surgery Ii, Istituto Clinico Humanitas, Rozzano, Italy 
Introduction: Dorsum of the hand is one the most exposed body region during social and working activities making trauma at this level very frequent. The dorsal aspect of the hand, from a functional point of view, allows proper gliding of extensor tendons, but also must be remember that our hands are part of our appearance. The aspect of the hand must, at least, be sufficiently normal as to pass unnoticed: if not, the patients will hide their hand in its pocket for the rest of its life. The authors present their indications for reconstruction of the dorsum of the hand after complex trauma.

Materials and methods: Twenty-five consecutive patients, mean age 30 years, with complex trauma of the dorsum of the hand, underwent surgical reconstruction between 2005 and 2009. In 16 patients, where the donor site could be closed primarily, an interosseous posterior flap (IOP) was used, while in 9 patients, with a major loss of substance, a free gracilis flap (GF) covered by thin split graft was preferred. All Patients were reviewed with a minimum of 1 year follow-up.

Results: All cases eventually healed, with complete survival of the flaps, without major complications. All the patients were satisfied both from a cosmetical and functional point of view. Also scars, at donor site level, were well accepted.

Conclusion: IOP and GF are two reliable coverage solutions. Both are versatile and well adaptable on the dorsum of the hand. A proper indication, a careful harvesting and a precise post-operative follow up and protocol lead to a good both cosmetical and functional results.

References: IOP and GF are two reliable coverage solutions. Both are versatile and well adaptable on the dorsum of the hand. A proper indication, a careful harvesting and a precise post-operative follow up and protocol lead to a good both cosmetical and functional results.

Disclosure: No significant relationships.

\section{O7}

\section{MANAGEMENT OF EXTENSIVE VOLAR WRIST WOUNDS: "SPAGHETTI WRIST". OUR EXPERIENCE}

\section{Smarrelli}

Hand Surgery, IRCCS Humanitas; Humanitas-Gavazzeni, MilanoBergamo, Italy

Introduction: The extensive volar injuries occurring between the distal crease of the wrist and the flexor musculotendinous junctions at the volar forearm, have been defined in several ways: full-house syndrome; suicide wrist and spaghetti wrist that remains the most common definition, involving at least three completely transected structures, including at least one nerve and one artery.

Materials and methods: On between January 2003 and January 2008, we treated 22 cases. We reviewed the patients reporting the cause of injury, the number of anatomical structures involved, the side, further complications and results. The outcome was tested by clinical examination and interview to each patient, based on the assessment of five criteria: tendon function, opposition, intrinsic function, deformity and sensation. Besides, every 6 months within the first 2 years, the patients underwent electromyographies; then they submit a final evaluation with DASH Score.

Results: We report our results for each criteria and the mean DASH score was 11.6; among several prognostic factors, we observed that ulnar nerve involvement strongly determine the prognosis. Generally we had good results about flexor tendon function; mechanism of trauma, number of structures involved, age and early mobilization are important prognostic factors too.

Conclusion: The management of these wounds is challenging since from their clinical presentation and requires a systematically approach respecting well defined procedures a good knowledge of the crosssectional anatomy, a check-list of all the structures and some tips and tricks in order to avoid mistakes and induce in underestimating.
References: Results of treatment of extensive volar wrist lacerations: Spaghetti Wrist. Puckett CL.; Meyer VH. Plastic and Reconstructive Surgery. Vol.75.714-719.1985 Spaghetti wrist: management and results. Chin G, Weinzweig N, Mead M, Gonzalez M. Plastic and Reconstructive Surgery. 1998;102(1):96-102. Results of treatment of the extensive volar wrist lacerations: "spaghetti wrist". Kabak S, Halici M., Baktir A., Turk C.Y., Avsarogullari L. Eur J Emerg Med.9.71-71. 2002 Management and functional outcomes of combined injuries of flexor tendons, nerves and vessels at the wrist. Noaman H. Microsurgery 27: 536-543. 2007 Microsurgical treatment of injury to peripheral nerves in uper and lower limb: a critical review of the last 8 years. Portincasa A, Gozzo G, Parisi D, Annacontini L, Campanale A, Basso G, Maiorella A. Microsurgery 27: 455-462. 2007. Disclosure: No significant relationships.

08

\section{EVALUATION OF A VARIABLE-ANGLE ANGLE-STABLE VOLAR PLATE IN A DISTAL RADIUS C-FRACTURE MODEL-A BIOMECHANICAL STUDY}

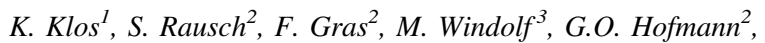 \\ T. Mückley ${ }^{2}$
}

${ }^{1}$ Friedrich-schiller-universität Jena, Klinik für Unfall-, Hand- und Wiederherstellungschirurgie, FSU Jena, Jena, Germany, ${ }^{2}$ Klinik Für Unfall- Hand- Und Wiederherstellungschirurgie, Friedrich-SchillerUniversität Jena, Jena, Germany, ${ }^{3}$ Biomedical Services, AO Research Institute Davos, Davos Platz, Switzerland

Introduction: The present study was performed to investigate whether variable-angle angle-stable plating provides comparable stability to conventional (fixed-angle) angle-stable constructs in the management of complex intra-articular fractures of the distal radius.

Materials and methods: An AO 23 C2.1 intra-articular fracture was created in seven pairs of human cadaver radii, One radius of each pair received a juxta-articular 2.4-mm LCP volar distal radius plate, while the contralateral one received a $2.4 \mathrm{~mm}$ variable angle LCP twocolumn volar distal radius plate (both plates: Synthes, Oberdorf, Switzerland). Parameters tested were construct stiffness (static axial loading with $150 \mathrm{~N}$ ), range of motion, and secondary loss of reduction (dynamic 150-N axial loading over 5,000 cycles). Stiffness and range of motion were measured both pre- and post-cycling.

Results: The polyaxial group showed significantly greater initial and final stiffness than did the fixed-angle group. The range of motion was significantly greater, both initially $(\mathrm{P}=0.002)$ and at the end of cyclic testing, in the fixed-angle constructs. The conventional-plate constructs showed a mean loss of reduction of $0.9 \mathrm{~mm}$, which was significantly greater than the mean loss of the variable-angle plate constructs, of $0.3 \mathrm{~mm}$.

Conclusion: The polyaxial radius plate provides a sound construct and seems to be a reliable device for the management of intra-articular distal radius fractures.

Disclosure: No significant relationships.

\section{9}

\section{HINGED ELBOW FIXATION IN TRAUMA OF THE ELBOW}

\section{T. Kozák}

Ortopedie A Traumatologie, Nemocnice Znojmo, Znojmo, Czech Republic 
Introduction: The elbow joint has a tendency to early post- traumatic stiffness. That why the early rehabilitation after surgery is always needed. The aim of this study was to evaluate the results of use of an unilateral hinge elbow fixator in acute and chronic trauma situations. Materials and methods: We treated 38 patients, 27 patients for acute trauma fracture or dislocation, 11 patients for posttraumatic stiffness. In acute cases there where 7 patients operated and the fixator was used to shield ligament suture and/or osteosynthesis. In nine patients was the fixator applied without open surgery. In chronic cases with posttraumatic stiffness there were eight elbows released during open surgery and the fixator served for shielding of interposition arthroplasty and/or ligament reconstruction. In seven patients the elbow was distracted and released by closed technique.

Results: In all we achieved healing of fractures and ligaments with the preservation of range of movement. The mean MEPS in acute cases at the time of last control was 76 points. In chronic cases the MEPS improved from 62 to 78 points. The final range of motion was $105^{\circ}$ in acute and $100^{\circ}$ in chronic cases on average.

Conclusion: In cases of fracture and acute instability, the application of the hinge external fixator provides an alternative how to achieve osseous and ligamentous healing. In chronic situations, the use of the elbow fixator results in most patients in regaining very of good functional results. Application of the elbow external fixator is relative delicate method which requires an experienced.

Disclosure: No significant relationships.

\section{0}

PULSED ELECTROMAGNETIC FIELDS AS AN ADJUNCT IN THE TREATMENT OF FRESH SCAPHOID FRACTURES. A RANDOMIZED DOUBLE-BLIND PLACEBO CONTROLLED MULTI-CENTRE TRIAL

\author{
P. Hannemann ${ }^{1}$, K. Göttgens ${ }^{1}$, P.R.G. Brink ${ }^{1}$, B. van Wely ${ }^{2}$, \\ K. Kolkman ${ }^{3}$ A. Werre
}

${ }^{1}$ Trauma Surgery, Maastricht University Medical Centre, Maastricht, Netherlands, ${ }^{2}$ Surgery, Radbound University Nijmegen Medical Centre, Nijmegen, Netherlands, ${ }^{3}$ Surgery, Rijnstate Hospital, Arnhem, Netherlands, ${ }^{4}$ Surgery, Canisius Wilhelmina Hospital, Nijmegen, Netherlands

Introduction: Failure of treatment of scaphoid fractures can result in longer immobilization as well as avascular necrosis, non-union, early osteo-arthritis and can seriously impair wrist function with significant psychosocial and financial consequences. Previous data indicate that bone healing may be improved using physical forces. The influence of pulsed electromagnetic fields (PEMF) on healing of fresh scaphoid fractures was studied.

Materials and methods: A multi-centre, prospective, double-blind, placebo-controlled, randomized trial was conducted on the effects of PEMF on fresh scaphoid fractures. Half of all applied devices were disabled at random. Clinical and radiological (X-ray) follow up occurred at 4, 6, 9, 12, 24 and 52 weeks.

Results: 24 patients (45.3\%) were included in group A (PEMF/ intervention) and $29(54.7 \%)$ in group B (placebo). Baseline characteristics were comparable.

At 6 weeks follow-up four patients (16.7\%) in group A and $5(17.2 \%)$ in group B showed radiologic consolidation, $\mathrm{p}>0.05$.

In group $B$ pressure pain at the anatomic snuff box and longitudinal compression pain was less, respectively in $5(17.2 \%)$ and 2 patients (6.9\%) compared to $11(45.8 \%)$ and 9 patients $(37.5 \%)$ in group A, $\mathrm{p}=0.03$, resp 0.008 Time interval until clinical and radiological consolidation was respectively 10.5 [Standard deviation (SD) 13.3] and 21.7 (SD 28.5) weeks in group A and 6.6 (SD 5.3) and 15.6 (SD 12.5) weeks in group $B$, although not statistical significantly different.
Conclusion: Early bone growth stimulation by PEMF seems to have no statistically significant advantage in the treatment of fresh scaphoid fractures and more research is needed tot study the clinical effects of early bone growth stimulation by physical forces.

Disclosure: No significant relationships.

\section{1}

\section{RADIUS FIXATOR: IMPROVED TECHNIQUE OF NON- BRIDGING FIXATION OF DISTAL RADIUS FRACTURES}

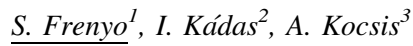

${ }^{1}$ Department 1, Trauma Center, Budapest, Hungary, ${ }^{2} 3$, National Institute of Traumatolgy, Budapest, Hungary, ${ }^{3}$ Iii. Trauma Department, Trauma Center, Péterfy S. Hospital, Budapest, Hungary

Introduction: The use of non-bridging external fixator in unstable distal radius fractures has been reported ever since the end of 90's. There has been certain skepticism, whether it is suitable in cases, where the bone stock is compromised e.g. by osteoporosis or bone loss. We report on a series, where a special non-bridging fixator provided good results.

Materials and methods: Between 01.02. 2004-31.12.2008 67 patients with AO type A3-B2-C1 fractures were treated with the above technique, and followed prospectively. Technique: fracture is reduced under regional anaesthesia using traction. Two $2.2 \mathrm{~mm}$ threaded $\mathrm{K}$-wires are introduced in the distal fragment, at an angle of $30^{\circ}-40^{\circ}$. If necessary, additional $\mathrm{K}$-wires are used to fix the fragments. Two $3 \mathrm{~mm}$ Schanz-screws are inserted into the radius shaft at an angle of $60^{\circ}$. The K-wires and Schanz-screws are connected by two $4 \mathrm{~mm}$ rods in crossed positions, providing rotational and angular stability of the construct. Mobilization of the wrist begins immediately. Metal work is removed at 6 weeks In the last 11 cases the combination of two K-wires attached to "headless" screws (similar to Herbert-screws) were used, to increase the stability of fixator, even in porotic bones. The threads of these screws fixed the pins in both cortices of the distal fragment. Follow-up was 12 months.

Results: Radiological results: good $67 \%$, fair $22 \%$ poor $11 \%$ Functional outcome (modified Gartland-Werley score): excellent or good $75 \%$, fair $19 \%$, poor $6 \%$. Complications: pin site infection 2 , deep infection 0 , aseptic loosening 2

Conclusion: Non-bridging "radius fixator" provided reliable fixation of fracture while allowing immediate carpal function. We recommend its use in selected cases of distal radius fracture. Adherence to precise technique is essential.

Reference: Hayes AJ, Duffy PJ, McQueen MM. Bridging and nonbridging external fixation in the treatment of unstable fractures of the distal radius. Acta Orthopaedica 2008;79(4):540-547.

Disclosure: No significant relationships.

\section{2}

\section{ANGLE STABLE PLATE OR ANGLE STABLE LOCKED NAIL FIXATION IN DISTAL RADIUS FRACTURES. AN EXPERIMENTAL EXAMINATION AND PHASE I CLINICAL STUDY}

\section{W. Friedl, M. Rinner}

Orthopädie Unfallchirurgieund Handchirurgie, Klinikum Aschaffenburg, Aschaffenburg, Germany 
Introduction: Distal radius fractures are typical and frequent fracture of elderly woman with reduced bone density. The angle stable plate, often also multidirectional is today the most common stabilisation device. We tested experimental and clinical as alternative the XS radius nail witch is a 4.5 or $3.5 \mathrm{~mm}$ straight nail and witch is introduced after guide wire placement and over drilling with a canulated drill of the same diameter. It is locked parallel to the joint in three different directions with angular stability with threaded wires.

Materials and methods: 16 radius sawbones were osteotomised corresponding to a A3 Fracture and stabilised with a angle stable plate (8) and XS nail (8). 1,000 alternating load cycles from 20 to $200 \mathrm{~N}$ were performed and the deformation was registered. Also a FE analysis with the MSC Patran/Marc software were performed.

Also the calculated deformation in the FE study was $20 \%$ lower. Also deformation amplitude was lower with $0.31 \mathrm{~mm}$ compared to $0.42 \mathrm{~mm}$ in the plate group. The differences however were not significant.

Results: Both devices show good biomechanical results. The XS nail has the advantage of mainly intraosseus position, simple operation technique with introduction over a guide wire from the proc. Styloideus radii and over drilling with a canulated drill of the same size. The exposure of the $\mathrm{N}$ rad.superf. must be performed. First clinical evaluation is presented.

Due to the results we developed a anatomically adapted XS radius nail. The results of the first 100 patients are presented.

Conclusion: Both angular stable plate and XSR nail can be used in distal radius fracture.

Disclosure: No significant relationships.

\section{HIP FRACTURE-DISLOCATION}

\section{3}

\section{A COMPARISON OF FIXATION TECHNIQUES FOR DISTAL FEMORAL VALGUS DEFORMITY CORRECTION: FIXATOR ASSISTED NAILING VERSUS FIXATOR ASSISTED PLATING}

\section{F.M. Kovar}

Department Of Trauma Surgery, Medical University Vienna, Vienna, Austria

Introduction: Fixator assisted nailing (FAN) and fixator assisted plating (FAP) are two techniques that can be used to correct femoral valgus deformities. Although FAN violates the knee joint, FAP requires a large exposure. The aim of the present study is to investigate if there is a difference in accuracy of correction when comparing FAN and FAP techniques for the treatment of femoral valgus deformity.

Materials and methods: We reviewed the medical records and radiographs of all patients who underwent surgery for correction of femoral valgus deformities between 2002 and 2009. If the medical records contained incomplete data or the follow-up was limited, patients were excluded from the study. Radiographs were measured by a single investigator. Data of surgery and latest date of follow-up were recorded.

Results: FAN: Twenty extremities (18 subjects) were treated using FAN. Seven male and 11 female subjects with an average age of 36 years (range 14-68 years) were included in the study. Average pre- and postoperative mechanical lateral distal femoral angle (mLDFA) was $81 \AA$ (range $67-86 \AA$ ) and $89 \AA$ (range $80-100 \AA$ ), respectively $(\mathrm{p}=0.009)$. Average pre-and postoperative medial proximal tibial angle (MPTA) was $88 \AA$ (range $62-100 \AA$ ) and $88 \AA$ (range 78-96 ̊), respectively. Average follow-up was 17 months (range 1-68 months). FAP: Seven extremities (six subjects) were treated with FAP. Two male and four female subjects with an average age of 16 years (range 15-19 years) were included in the study. Average pre- and postoperative mechanical lateral distal femoral angle (mLDFA) was $80 \AA$ (range 71-87 $\AA$ ) and $88 \AA$ (range 81-94 ̊).

Conclusion: Both methods for femoral deformity correction are safe and effective surgical techniques. Based on our results, FAP may be a more accurate technique for deformity correction in the distal femur. Disclosure: No significant relationships.

\section{4}

\section{ARTHROPLASTY OR FEMORAL NECK FRACTURES- HEMI OR TOTAL REPLACEMENT}

\section{$\underline{\text { J. Waddell }}$}

Division of Orthopaedic Surgery, St. Michael's Hospital, Toronto, ON, Canada

Introduction: There is increasing interest in the use of arthroplasty as a treatment for displaced femoral neck fractures in patients over 65 years of age.

Materials and methods: All publications in the English literature over the past 10 years regarding the treatment of displaced femoral neck fractures have been reviewed. Over 500 publications were available for review and from these a series of recommendations formulated.

Results: Fixation with either multiple screws or a sliding hip screw is suitable for valgus impacted or undisplaced femoral neck fractures (Garden I and II) and is generally indicated for reduction and fixation of displaced fractures (Garden III and IV) in patients under 60 years of age. In patients over 60 years of age or patients at any age with poor bone quality, significant comorbidity or pre-existing hip arthritis arthroplasty is the appropriate treatment. Non-cemented monopolar arthroplasties should be reserved for patients with minimal functional activity in supported living (nursing home). Cemented modular monopolar or bipolar arthroplasties should be used in patients in assisted living situations (retirement homes). Total hip replacements should be reserved for patients capable of independent living and self-care.

Conclusion: The need for guidelines regarding the use of arthroplasty in displaced femoral neck fractures is necessary in order to ensure appropriate surgery for these patients which will allow them early immediate weight bearing and a relatively low complication rate. Selecting the appropriate implant should be determined by the patient's functional level rather than patient age.

Disclosure: No significant relationships.

\section{5}

\section{CUT THROUGH OF THE HELICAL BLADE OF THE PROXIMAL FEMORAL NAIL ANTIROTATION}

\author{
H.Frei ${ }^{1}$, T. Hotz ${ }^{2}$, K.P. Käch ${ }^{3}$
}

${ }^{1}$ Chirurgie, Kantonsspital Winterthur, Winterthur, Switzerland, ${ }^{2}$ Chirurgie, Kantonsspital Winterhur, Winterthur, Switzerland,

${ }^{3}$ Trauma, Surgical Clinic, Winterthur, Switzerland

Introduction: For the treatment of unstable trochanteric femoral fractures the proximal femoral nail antirotation (PFNA) was designed 
with a helical blade rather than a screw for better purchase in the femoral head due to compression of spongious bone.

Materials and methods: During a period of 24 months we treated fractures of the proximal femur with the PFNA. The patients were analyzed regarding to a "cut through" of the helical blade into the hip joint. The perioperative data were analyzed as well as the technical performance of the operation and implant positioning.

Results: In the analyzed period 210 Patients were treated with the PFNA. 16 patients died in the first four postoperative weeks. 26 patients with unplanned reoperations were recorded: there occurred 2 infections, 5 reinventions due to technical problem, 6 because of nonunion of the fracture, 8 because of excessive lateral sliding of the blade. Seven patients showed acetabular penetrations of the helical blade ("cut throughs"). Analyzing the technical performance, all patients with a "cut through" showed a correct implant position and good reduction of the fractures.

Conclusion: The PFNA is an advanced implant for the treatment of challenging unstable proximal femoral fractures. Although the helical blade offers new technical advantages as compression of the spongious bone in the femoral head and rotational stability, there seems to be a tendency of possible migration of the helical blade into the femoral head and even cutting through into the Acetabulum by repeated physiological forces only. The classic cut out is missing. Disclosure: No significant relationships.

\section{6}

\section{PERIPROSTHETIC FRACTURES AROUND HIP REPLACEMENTS TREATED BY OSTEOSYNTHESIS: FOLLOW UP ON 66 CONSECUTIVE VANCOUVER TYPE B1 AND C FRACTURES}

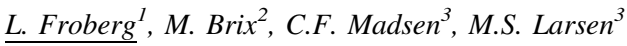

${ }^{1}$ Orthopaedic, Odense University Hospital, Odense, Denmark, ${ }^{2}$ Orthopaedics And Trauma, Odense University Hospital, Odense, Denmark, ${ }^{3}$ Orthopaedics, Odense University Hospital, Odense, Denmark

Introduction: Periprosthetic femoral fracture (PFF) is a severe complication to hip joint replacement. Treatment of such fractures is technically demanding, with a frequency of reoperations up to $30 \%$. The purpose of this study was to describe the outcome of PFF around hip implants in a consecutive cohort of patients treated by locking plate osteosynthesis. The primary outcome was reoperation.

Materials and methods: From May 2002 to October 2010, 61 consecutive patients (63 fractures) with Vancouver type B1 or C PFF around hip joint replacement were osteosynthesised at Odense University Hospital, Denmark.

Results: Twenty-five fractures were classified as type B1 and 38 as type C. The median age at PFF was 82 years (range 49-97). In addition to locking plate osteosynthesis locking attachment device $(n=4)$ and cables $(n=5)$ were used. At a mean follow up 28 months after PFF (range 0-99 months) 31 patients (33 fractures) had diseased and 30 patients (30 fractures) were still alive. At follow up seven fractures $(11 \%)$ had a reoperation due to: failure of fixation $(n=4)$, deep infection $(n=2)$ or loosening of prosthesis $(n=1)$. In addition three had their plate removed due to local irritation at the insertion place. All patients reoperated due to failure of fixation had a new low energy fall, whereby a fracture occurred at the stress rising area where the plate overlapped the prosthesis. In those patients, the plate overlapped half the length of the prosthesis.
Conclusion: A low re-operation rate can be obtained in patients suffering from type B1 and C PFF treated by locking plate osteosynthesis. In concordance to other studies we recommend: (1) Careful preoperative evaluation of the fracture type according to the Vancouver classification system (2) spanning of the prosthesis all the way to avoid stress rising areas.

Disclosure: No significant relationships.

\section{7}

\section{RETROSPECTIVE STUDY OF PERIPROSTHETIC FEMORAL FRACTURES TREATED BY POLYAXIAL LOCKING IMPLANTS}

\author{
E. Wilde ${ }^{1}$, G. Heinrichs ${ }^{1}$, R. Thietje ${ }^{2}$, A.P. Schulz ${ }^{1}$, A. Paech ${ }^{1}$
}

${ }^{1}$ Trauma + Orthopaedics, University Lübeck, Lübeck, Germany, ${ }^{2}$ Department of Trauma and Orthopaedic Surgery, BG Trauma Hospital Hamburg, Hamburg, Germany

Introduction: Following the increasing number of geriatric patients and their higher level of activity the periprosthetic fracture especially of the femur needs a sufficient surgical treatment as a basis to regain their mobility. Polyaxial implants imposes good possibilities for this surgical problem.

Materials and methods: In our retrospective study we treated 58 consecutive patients with periprosthetic femoral fractures type Vancouver $\mathrm{B} 1$ and $\mathrm{C}$ with an angular stable plate as index procedure during a 5 year period in a trauma hospital (study centre). The outcome was measured using radiographics, Barthel-Index and timed up\&go test as well as a questionnaire.

Results: In $96.5 \%$ (56 patients) primary bony healing occurred after the index procedure. 55 Patients regained full weight bearing, in average this was reached after 8.6 weeks. Three patients stayed bedridden as they did before the injury. Early implant failure occurred in two cases and was due to proximal screw dislocation (3.5\% revision rate). At follow up $89 \%$ regained the mobility status as before injury and $78 \%$ had reached the same social status. The Barthel-Index was at 35 points at the beginning of rehabilitation and increased up to 85 points $(70-100)$ of possible 100 at the follow up. Compared with the preoperative level this means a decrease of only 10 points. The mean up\&go time was measured as $21.4 \mathrm{~s}$.

Conclusion: A periprosthetic femoral fracture means a major deterioration of the lower limb function and therefore a risc of loss for the self independence and social status in the geriatric patient. In our retrospective study we could show that polyaxial locking plates ca achieve excellent surgical results.

Disclosure: No significant relationships.

\section{8}

\section{TREATMENT OF TRAUMATIC HIP DISCLOCATIONS ASSOCIATED WITH ACETABULAR FRACTURES}

\author{
F. Castelli ${ }^{1}$, G. Pesenti ${ }^{1}$, D. Capitani ${ }^{2}$
}

${ }^{1}$ Dea, Niguarda Ca Granda, Milan, Italy, ${ }^{2}$ Department Of Orthopedic Surgery And Traumatology, Niguarda Ca Granda Hospital, Milano, Italy 
Introduction: Backgruond Traumatic dislocation of the hip is an extremely severe injury. Although previously considered an uncommon lesion, it now is seen more often as result of MVA. In most cases this injury can result in a high incidence of complications. Early diagnosis, in politrauma patient too, and a early closed reduction constitute the gold standard of a proper treatment of this injury.

Materials and methods: $369 \mathrm{pz}$ with surgical acetabular fracture have been selected in 70 months period 209 (67.4\%) were male and $101(32.6 \%)$ female. Average age is 38.2 yo. $(80.6 \%>20$ yo $<60$ yo). In the present study $126 \mathrm{pz}(34.14 \%)$ with hip dislocation associated with acetabuar fracture were included. $71 \mathrm{pz}(56.34 \%)$ were posterior wall fxt, $2(1.59 \%)$ posterior column fxt, $3(2.38 \%)$ posterior wall + posterior column, $1(0.79 \%)$ pure transverse fxt, $24(19.05 \%)$ transverse + pw fxt. $101 \mathrm{pz}(32.6 \%)$ have a posterior fracture-dislocation, $21(6.7 \%)$ central and 4 (1.2\%) anterior. Average follow-up was 62 months (min $18 € \max$ 114). Every $\mathrm{pz}$ received an early closed reduction ( $>6 \mathrm{~h}$ from trauma) when directly admitted. Once the dislocation is reduced when surgical reconstruction is indicated it is done best in the first 10 days after injury. $10 \mathrm{pz}(3.2 \%)$ that reported a irreducible fracture-dislocation had required an early open reduction and internal fixation.

Results: Results In 79 pz (62.69\%) we obtain a stable and concentric closed reduction. In 2 cases of uncommon pattern of postero-superior wall fracture $(1.59 \%)$ occur a secondary dislocation, in $17 \mathrm{pz}$ $(13.49 \%)$ the hip remains unstable, $1 \mathrm{pz}(0.79 \%)$ have an associated femoral neck fracture, $13 \mathrm{pz}$ of posterior column plus posterior wall and transverse plus posterior wall fractures $(10.31 \%)$ after closed reduction of the posterior dislocation we obtain a central dislocation. $21 \mathrm{pz}(16.6 \%)$ received closed reduction $>6$ h. $6 \mathrm{pz}(28.5 \%)$ on the whole $21 \mathrm{pz}$ that received a delayed reduction have developed AVN. $105 \mathrm{pz}(83.4 . \%)$ received closed reduction $<6$ h. $2 \mathrm{pz}(1.9 \%)$ on the whole $105 \mathrm{pz}$ developed AVN. The average AVN rate in our study was $6.3 \%$ with 8 cases of AVN on 126 acetabular fracturedislocation.

Conclusion: Conclusion In summary, this study suggest: (a) There are two different mechanism of intra-articular incarceration of bone fragments: (1) Primary incarceration with one or several fragments, perhaps pedunculated to the capsule that enter the acetabulum at the moment of injury. (2) Secondary incarceration with fragments maintaining a capsular flap pedicle that is drawn into the joint during the closed reduction; (b) Early closed or open reduction $<6 \mathrm{~h}$ constitute the gold standard of a proper treatment of this injury reducing the AVN rate of approximately 15 times.

References: 1. Letournal E, Judet R Fractures of the Acetabulum. Berlin: Springer; 1993. 2. Matta JM Fractures of the Acetabulum: accuracy of reduction and clinical results in patient managed operatively within three weeks after injury. J Bone Jt Surg. 1996;78A:1632-45. 3. Tile M Fractures of the pelvis and acetabulum. Baltimore: Williams and Wilkins;1995. 4. Leunig M, Sledge JB, Gill TJ, Ganz R. Traumatic labral avulsion from the stable rim: a constant pathology in displaced transverse acetabular fractures Arch Orthop Trauma Surg 2003;123:392-5. 5. Ganz R, Gill TJ, Gautier E, Ganz K, Krugel N, Berlemann U. Surgical dislocation of the adult hip. A technique with full acess to femoral head and acetabulum without the risk of avascular necrosis. J Bone Jt Surg 2001;83B;1119-24. 6. Mast J, Jakob R, Ganz R. Planning and reduction technique in fracture surgery. New York: Springer; 1989. 7. Rashleigh-Belcher HJC, Cannon SR. Recurrent dislocation of hip joint with a Bankart-type lesion. J Bone Jt Surg. 1986;68B:398-9. 8. Richter H, Huston JJ, Zych G. The use of spring plates in the internal fixation of Acetabular fractures. J Orthop Trauma. 2004;18:179-181.

Disclosure: No significant relationships.
019

\section{V-SHAPED CEMENT AUGMENTATION OF THE PROXIMAL FEMUR TO PREVENT SECONDARY HIP FRACTURES}

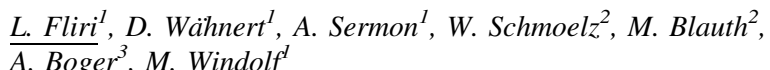

${ }^{1}$ Biomedical Services, AO Research Institute Davos, Davos Platz, Switzerland, ${ }^{2}$ Trauma Surgery \& Sports Medicine, Medical University Innsbruck, Innsbruck, Austria, ${ }^{3}$ Biomaterials, Synthes $\mathrm{GmbH}$, Oberdorf, Switzerland

Introduction: Hip fractures due to osteoporosis are associated with a high risk of secondary fractures on the contralateral side. In case of poor bone quality, a prophylactic mechanical reinforcement of the contralateral side during operation of the initial fracture could be of interest. This biomechanical in vitro study investigates the potential of a prophylactic V-shaped bone cement augmentation of the proximal femur in terms of increased energy absorption until fracture.

Materials and methods: Five pairs of human cadaveric proximal femurs were tested in a configuration simulating a fall on the greater trochanter. The femoral neck of one specimen was augmented with $10-13 \mathrm{ml}$ polymethylmethacrylate (PMMA) from the lateral cortex towards inferior and superior, spanning a V-shaped cement pattern. The contralateral femur served as a non-augmented control. Clinical relevant proximal femoral fractures were generated with a $45 \mathrm{~kg}$ mass in free fall. Displacement and loading progression were recorded during fracturing. Energy absorption until fracture, fracture load and stiffness were statistically evaluated.

Results: Augmented samples showed significantly increased energy absorption until failure compared to their control $(p=0.043)$. Mean energy absorption was $20.3 \mathrm{~J}$ (SD 14.1) for the augmented and $10.0 \mathrm{~J}$ (SD 5.8) for the non-augmented group, respectively. No significant differences were found between the two groups for fracture load $(\mathrm{p}=0.5)$ and stiffness $(\mathrm{p}=0.5)$.

Conclusion: Biomechanically, a V-shaped prophylactic cement augmentation carries potential to prevent secondary hip fractures indicated by increased energy absorption until fracture. Further investigations are necessary to minimize interference with the biology and to maximize the mechanical benefit of prophylactic augmentation. Reference: 1. PMID:7094454 2. PMID:19257816.

Disclosure: No significant relationships.

\section{O20}

\section{FLOATING HIP. IPSILATERAL PELVIC AND FEMORAL FRACTURES} V. Guimerá García, P. Caba, C. Olaya-Gonzalez, M. Aroca Peinado,
J.L. León Baltasar, C. Resines Erasun

Orthopaedic \& Trauma Surgery, Hospital Universitario 12 de Octubre, Madrid, Spain

Introduction: The combination of ipsilateral pelvic and femoral fractures is a surgical challenge. Few studies have discussed this complex injury pattern. The aim of this paper is to describe our experience in this type of injury.

Materials and methods: Data were obtained from our Hospital Trauma registry. We measured demographics, ISS, NISS, mechanism of injury, length of hospitalization, surgical timing and methods of fixation and complications. Acetabular fractures were classified according to Letournel and Judet. Pelvic and femoral fractures were 
classified using Tile and AO/OTA system (Tile A fractures not considered). Following Muller et al., floating hips were described as types $\mathrm{A}, \mathrm{B}$ or $\mathrm{C}$.

Results: Between May 1995 and May 2010, 36 patients were identified. 22 males and 14 females with an average age of 33.5 years. The average ISS was 31.9 and NISS 42.6. Motor vehicle collision was involved in the majority of patients followed by high falls. The most common injury pattern was pelvic fracture with ipsilateral femoral fracture (Muller type B) and both pelvic and acetabular fracture (Muller type C), respectively. Patients were treated following DCO and staged treatments. The femur fracture was commonly fixed before acetabulum with separated approaches (ilioinguinal and lateral or retrograde).

Conclusion: Floating hip injury is a surgical challenge because of fracture fixation sequence and methods. Most patients can be treated in supine position using both percutaneous and anterior approaches for pelvis/acetabulum and lateral or retrograde for the femur.

References: Liebergall M, et al. The floating hip. Ipsilateral pelvic and femoral fractures. J Bone Jt Surg Br. 1992;74:93-100. Muller EJ, et al. Ipsilateral fractures of the pelvis and the femur-floating hip? A restrospective analysis of 42 cases. Arch Orthop Trauma Surg. 1999;119:179-182. Liebergall M, et al. The floating hip injury: patterns of injury. Injury. 2002;33:717-722. Burd TA, et al. The floating hip: complications and outcomes. J Trauma. 2008;64:442-8.

Disclosure: No significant relationships.

\section{EMERGENCY SURGERY 2}

\section{1}

\section{LAPAROSCOPIC SURGERY FOR THE ACUTE ABDOMEN: OUR EXPERIENCE}

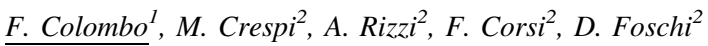

${ }^{1}$ General Surgery Department, Luigi Sacco University Hospital, Milan, Italy, ${ }^{2}$ Generale Surgery, L.Sacco Hospital, Milan, Italy

Introduction: The progressive improvement of the laparoscopic techniques and the technological instruments have allowed to extend the indications of minimally invasive surgery also to the treatment of the acute abdominal pathologies. The timing of the surgery is a crucial factor that allows a safe use of the laparoscopic technique. A correct indication must also be matched by an adequate skill and proper training in laparoscopic surgery, allowing the surgeon to deal with more complex cases and self manage the problems or the possible intra-operative conversion to laparotomy.

Materials and methods: We selected patients undergoing emergency surgery in our Surgical Division, in the period between January 2007 and August 2010. In the present study we included 320 cases of the most acute abdominal diseases occured in the Emergency Department: acute appendicitis, acute cholecystitis, perforation of the intestinal tract, bowel obstruction, acute colo-rectal diseases.

Results: Our laparoscopic rate for the abdominal acute surgery was around $40 \%$. Excluding the surgery for the acute colo-rectal diseases the mini-invasive approach reach the $75 \%$ value. In particular we observed a very high rate of the laparoscopic technique for the treatment of the appendicitis and cholecystitis (90\%). We evaluated the conversion and the morbidity rate of our experience.

Conclusion: We believe that laparoscopic surgery currently plays a key role in the management and treatment of the acute abdominal diseases, both as a diagnostic approach and as therapeutic procedure. Our data, according to the literature and international guidelines, authorize us to continue in this direction.

Disclosure: No significant relationships. $\mathbf{0 2 2}$

\section{A DISTINCT LACK OF CONTRAST IN CONTRAST-INDUCED NEPHROPATHY}

\author{
B. Bruns ${ }^{l}$, D.N. Holena ${ }^{1}$, N. Tolstoi ${ }^{1}$, H. BaL ${ }^{2}$, K. Holtz $^{3}$, \\ P.M. Reilly ${ }^{I}$, C.W. Schwab
}

${ }^{1}$ Division Of Traumatology, Surgical Critical Care, And Emergency Surgery, University of Pennsylvania School of Medicine, Philadelphia, PA, United States of America, ${ }^{2}$ School Of Medicine, University of Pennsylvania, Philadelphia, United States of America, ${ }^{3}$ School Of Arts And Sciences, University of Pennsylvania, Philadelphia, United States of America

Introduction: Iodinated contrast is a known nephrotoxin but is widely used in the workup of traumatic injury. CIN is defined as an increase in serum creatinine by $\geq 0.5 \mathrm{mg} / \mathrm{dl}$ or a creatinine increase by $\geq 25 \%$. RIFLE, a consensus method of describing acute renal injury, defines three classes of severity ('Risk', 'Injury', 'Failure') and two outcomes ('Loss', 'End stage renal disease'). We sought to determine the incidence of patients meeting criteria for contrast-induced nephropathy (CIN) in a cohort of trauma patients who did or did not receive iodinated contrast and to evaluate the utility of RIFLE criteria in classification of CIN cases.

Materials and methods: Prospectively collected data from 2009 to 2010 were analyzed retrospectively. Inclusion criteria were age $\geq 18$ years of age and a traumatic mechanism of injury.

Results: 256 patients were analyzed. Median age was 46 years (interquartile range (IQR) 27-66), 61\% were male, and $49 \%$ were AfricanAmerican. Blunt trauma was the mechanism of injury in $85 \%$ of patients. Median injury severity score (ISS) was 10 (IQR 5-18). There was no difference in rates of diabetes, shock on admission, or preexisting kidney disease between patients that got iodinated contrast (IC, $\mathrm{n}=137)$ and those who did not $(\mathrm{NC}, \mathrm{n}=119)$. Median ISS and \% penetrating mechanism were higher in the $\mathrm{CG}$ group. The overall rate of CIN was $5.2 \%$ (5.9\% in IC vs. $4.3 \%$ in NC, $\mathrm{p}=0.38)$. When stratified by RIFLE criteria, 4 (3.4\%) patients in NC and $6(4.4 \%)$ patients in IC achieved the 'risk' category. One $(0.8 \%)$ patient in the NC and $2(1.5 \%)$ patients in the IC group achieved the 'injury' classification.

Conclusion: The risk of meeting creatinine criteria for CIN is $\sim 5 \%$ regardless of whether or not contrast is administered. When stratified by RIFLE criteria, most patients fell into the 'risk' and 'injury' categories Disclosure: No significant relationships.

\section{$\mathbf{0 2 3}$}

\section{SURGEON-PERFORMED ULTRASOUND IN MANAGEMENT OF ACUTE SURGICAL PATIENTS}

M.L. Boella, P.M. Gini, A. D'Elia, I. Righi, G. Ferrari

Chirurgia Generale, Ospedale civile di legnano, legnano, Italy

Introduction: Aim of our study is to evaluate the importance and reliability of surgeon-performed ultrasound in managing acute surgical patients.

Materials and methods: 137 consecutive abdominal and/or thoracic US were performed by surgeons, $90.5 \%$ in acute and $9.5 \%$ in emergency setting at patient's bed. A specific surgical question to be answered or a collection to be drained were the goals of exams. The results of procedures were checked according to CT scan or surgical findings. The surgeons were asked if any advantage were found in self-performing exams.

Results: 83 exams (60.6\%) were diagnostic: 23 for shock, 17 for sepsis and 43 for acute abdominal pain. The questions asked were about: 
presence of free fluid or collections (55), presence of cholecystitis (22), other topics (6); the fluid were sampled in 11 cases under US.

54 exams, $(39.4 \%)$ were therapeutics: a percutaneous drain was required in 38 patients and a skin-marker for further drain in 16.

The goal was achieved in 125 exams (91.2\%), whilst 7 exams gave no answer, 1 was wrong and 4 collections were not drained.

In 97 cases $(70.8 \%)$ one or more advantages were found as saving time in 74 cases, avoiding transfer of critical patients in 23 , expanding drain indications in 19, correcting diagnosis in 10, easing surgery in 5 and allowing reevaluation in 3 .

Conclusion: Self-performed ultrasound is a useful tool for the acute care surgeon in managing a critical patient. Clinical ultrasound should be considered a reliable extension of physical exam at patient's bed and could be a matter of acute care surgeon training.

Disclosure: No significant relationships.

\section{4}

\section{ENDOSCOPIC CYSTOGASTROSTOMY- CYSTODUODENOSTOMY IN PATIENTS WITH PANCREATIC PSEUDOCYSTS: A 3 YEARS EXPERIENCE}

\author{
H. Yanar, E. Sivrikoz, S. Sarici, K. Günay, C. Ertekin, R. Güloğu
}

General Surgery, Istanbul Faculty of Medicine, Istanbul, Turkey

Introduction: Pancreatic pseudocysts are frequent sequelae of acute pancreatitis and endoscopic drainage of these collections has gained acceptance as an alternative to surgical drainage but it is associated with variable success rates.

Materials and methods: Symptomatic, $>5 \mathrm{~cm}$ diameter pancreatic pseudocysts were evaluated with CT-scan and gastroscopy. If the cysts were related with gastric or duodenal lumen and a bulging was seen on endoscopy, an endoscopic cystogastrostomy or cystoduodenostomy was performed. The drainage applied transmural technique in all cases. We have reviewed our experience with endoscopic drainage of pancreatic pseudocysts.

Results: Between January 2007 to September 2010, 580 patients admitted to Emergency Service with a diagnosis of acute pancreatitis. Among these pancreatic pseudocysts developed in 38 patients. In 21 patients endoscopic drainage was performed. With pure pseudocyst, successful resolution of symptoms and collection occurred in all patients $(9 / 9,100 \%)$. However, drainage of pseudocysts with organized necrosis was associated with a higher failure rate $(6 / 12,50 \%)$ than other collections. In one patient laparotomy was performed due to active bleeding. Two patient died in the ICU because of sepsis.

Conclusion: Endoscopic drainage seems to be successful in the management of pancreatic pseudocysts with low morbidity and mortality. Re-intervention rates are acceptable in regard of preventing surgical intervention and adequate resolution of symptoms and collections seems to obtained.

Disclosure: No significant relationships.

\section{$\mathbf{O 2 5}$}

\section{RELEVANCE OF PRIMARY TREATMENT OF MAXILLO- FACIAL INJURIES CAUSED BY DOG BITE IN CHILDREN}

\author{
A. Szentirmai ${ }^{1}$, T. Kassai ${ }^{2}$, A.T. Renner ${ }^{3}$
}

${ }^{1}$ Maxillo-facial Dept, Trauma Center, Budapest, Hungary, ${ }^{2}$ Pediatric Dept, Trauma Center, Budapest, Hungary, ${ }^{3}$ Hand And Microsurgery, Trauma Center, Budapest, Hungary
Introduction: Animal bites on the face may result in severe deformities and distorting soft tissue cicatrices, which cause a constantly visible, not-maskable, and permanent skewness of the face for a life. Our objectives are: to reduce the extent of these deformities and to optimally restore the function and the aesthetics, preferably during the course of the primary treatment.

Materials and methods: A retrospective review was performed on 2,694 patients, who suffered a dog bite to the maxillo-facial region from 2003 to 2007. 2,371 (358 children, 2,013 adults) were outpatients, 323 ( 72 children, 251 adults) required hospitalization. Amongst 72 children, the face injury was predominant. In $84 \%$ of the cases a renowned dog caused the injury. All dogs underwent the obligatory vaccinations.

Results: All patients underwent primary, definitive treatment, including osteosynthesis and soft tissue reconstruction. Treatment at the ICU was required in case of one adult patient; 8 from the 72 children required ICU-treatment. Antibiotics were applied by all patients. Septic complications requiring repeated surgery occurred by one adult and two children. Minor secondary soft tissue corrections were necessary in case of two children. The titanium plates have been removed form each children. No disturbed growth has been observed in any of the cases. All patients and parents were satisfied with the functional and aesthetical result.

Conclusion: In our opinion, primary, definitive treatment of potentially infected, destructed complex face injuries is crucial, because, based on our experience, this way of treatment provides with best clinical outcome and shortest hospitalization period. The preconditions of this conception should be established in the everyday treatment of injured people. Face deformities, acquired in the childhood, result in lifelong disorders of personality development, thus we consider the preventive reference extremely important. It would be necessary to propagate the applicable preventive guidelines of the American Society of Plastic Surgery.

Disclosure: No significant relationships.

\section{6}

\section{ANTIBIOTICS VS. SURGERY IN ACUTE APPENDICITIS: AN INTENTION TO TREAT PROSPECTIVE RANDOMISED CONTROLLED STUDY}

\section{Pisano $^{1}$, L. Campanati ${ }^{1}$, N. Colaianni ${ }^{1}$, F. Gazzotti ${ }^{1}$, M. Lotti $^{l}$,}

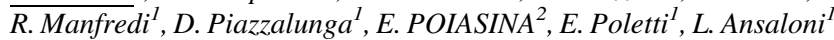

${ }^{1}$ St General Surgical Unit Department Of Surgery, Aziendda Ospedaliera Ospedali Riuniti Bergamo, Bergamo, Italy, ${ }^{2}$ General Surgery, Ospedali Riuniti di Bergamo, Bergamo, Italy

Introduction: The standard of care for acute appendicitis still remains surgery. Although antibiotics are somewhat proposed as an alternative, the power of studies, even prospective, that challenged the gold standard is poor. The aim of this work is to present the project of an ongoing prospective randomized controlled study comparing antibiotics to surgery in acute appendicitis.

Materials and methods: Preliminary agreement has been reached over Andersson's score as the most comprehensive diagnostic tool for patients suspected to suffer of acute appendicitis. According to the Andersson's score 3 groups are individuate; class 1 patients have very low probability and at the opposite the class 3 patients have very high probability to have acute appendicitis. Class 2 patients have intermediate probability: we add ultrasound to better discern acute appendicitis patients. Consequently patients requiring immediate surgery, class 1 and class 2 with negative ultrasound and/or positive gynaecological consultation are excluded. Remaining patients, meeting the inclusion criteria, are randomized. In order to perform a no inferiority analysis between antibiotics and surgery the population size was calculated: 110 
patients in each arm were obtained. Patients in the antibiotics group receive Ertapenem e.v. infusion $1 \mathrm{~g}$ for day for 3 consecutive days followed by Amoxicillina plus Clavulanic per os $1 \mathrm{gr} 3$ tid for 7 days. Patients in the surgery group receive Amoxicillina plus Clavulanic e.v. $2 \mathrm{gr}$ followed by surgery. Discharge criteria and follow up are the same. Results: The primary outcome was the same for both arms: absence of symptoms and normalization of laboratory test after 2 weeks, no major complications or recurrence into 1 year.

The primary outcome was the same for both arms: absence of symptoms and normalization of laboratory test after 2 weeks, no major complications or recurrence into 1 year.

Conclusion: with this study we hope to scientifically stress the historical paradigm of acute appendicitis and surgery.

Disclosure: No significant relationships.

\section{ANKLE FRACTURE-DISLOCATION}

\section{$\mathbf{O 2 7}$}

\section{COMPARISONS OF MEDICINAL LEECH THERAPY WITH VENOUS CATHETERIZATION IN THE TREATMENT OF VENOUS CONGESTION OF THE SURAL FLAP}

\section{N. Mozafari ${ }^{1}$, S.N. Hosseini ${ }^{2}$, M.R. Ghazisaidi ${ }^{l}$, M. Abdolzadeh $^{l}$}

${ }^{1}$ Plastic and Reconstructive Surgery, 15 Khordad Hospital, Tehran, Iran, ${ }^{2}$ Burns Department, Zanjan University of Medical Sciences, Zanjan, Iran

Introduction: The originally described distally based sural flap technique has a risk of partial or total flap necrosis as high as $25 \%$. The purpose of this study was to compare the medicinal leech therapy (MLT) with venous catheterization (VC) for blood volume removal, infection, wound dehiscence, and flap necrosis in the distally based sural flap with venous congestion.

Materials and methods: Fifty-six conventional distally based sural flaps with venous congestion during reconstructive surgeries were randomly divided into two groups, MLT group and VC group. The results of comparisons were analyzed using SPSS software (SPSS for Windows Ver.11.5).

Results: There were significant differences in terms of the average volume of removed blood (53.6 vs. $172.2 \mathrm{cc}$ ), infection (10.7 vs. $34.6 \%$ ), wound dehiscence (10.7 vs. $42.3 \%$ ), flap necrosis (3.6 vs. $19.2 \%)$ and nursing (7.8 vs. 5.19) and patient's satisfaction (8.03 vs. 5.6) in the VC group and MLT group respectively. Although local heparin irrigation was performed in the VC group, the catheter was exchanged in 10 patients due to obstruction by clot.

Conclusion: It is recommended that the $\mathrm{VC}$ be used for congested pedicled flaps instead of leech therapy, since VC is more effective, easy and safe in blood removal, and it has less complication.

Reference: There are 30 references for this study.

Disclosure: No significant relationships.

\section{8}

\section{COMPLEMENT ACTIVATION IS INHIBITED BY MAGGOT EXCRETIONS}

\section{G. Cazander ${ }^{1}$, M.W.J. Schreurs ${ }^{2}$, G.N. Jukema ${ }^{3}$}

${ }^{1}$ Surgery, Bronovo Hospital, Den Haag, Netherlands, ${ }^{2}$ Immunology, Erasmus Medical Center, Rotterdam, Netherlands, ${ }^{3}$ Department of
Trauma Surgery, VU University Medical Centre, Amsterdam, Netherlands

Introduction: Traumatic injury in humans causes activation of the innate immune system which results in an inflammatory response, that is essential for tissue repair. However, excessive complement activation can lead to tissue damage. Maggot therapy is successfully used to treat infected wounds, although the mechanism of action is unknown. In this research, we hypothesized that maggot excretions/ secretions (ES) affect complement activation in order to modulate the host's inflammatory response.

Materials and methods: Therefore, the effect of ES on complement activation was investigated in healthy sera and in pre- and postoperatively gained sera from trauma patients. Different immunoassays, that are also clinically used to determine complement deficiencies in patients, were performed in absence or presence of ES.

Results: Results show that ES reduced complement activation in healthy and immune-activated sera up to $99.9 \%$ ( $p<0.0001$ ), via all pathways of complement activation. ES proved to be temperaturetolerant and boiled ES showed the best complement-inhibiting properties. Protease treatment of ES decreased complement-inhibiting effects markedly, indicating the involvement of an ES derived protein constituent in complement inhibition.

Conclusion: This research shows the first pathway independent complement-inhibitor, probably a boiled stable protein, that already is successfully used in clinical practice. The immunosuppressive effect of ES may explain part of the improved wound healing caused by maggot therapy. If ES inhibit the inflammatory response, the healing process can progress. Furthermore, the complement-inhibitor(s) present in maggot ES could provide a novel treatment modality for diseases, resulting from an (over)active complement system, e.g. infections in trauma patients, ischemic-reperfusion injury and severe inflammatory response syndrome (SIRS).

Disclosure: No significant relationships.

\section{9}

\section{COMPARISONS OF EXTRA-ARTICULAR DISTAL TIBIA FRACTURES: PERCUTANEOUS LOCKED PLATING VERSUS INTRAMEDULLARY NAILING}

\section{F. Bilgili ${ }^{1}$, S. Sökücü ${ }^{2}$, S. Kllıç ${ }^{2}$, A.S. Parmaksızoglu ${ }^{2}$, K.S. çepni ${ }^{2}$, Y.S. Kabukçuogไu}

${ }^{1}$ Orthopaedic and Traumatology Department, Taksim Training and Research Hospital, Istanbul, Turkey, ${ }^{2}$ Taksim Training and Research Hospital, Istanbul, Turkey

Introduction: The purposes of this study were to compare and evaluate the clinical and radiographic results, as well as post-operative complication rates, of treatment of extra-articular distal third tibial fractures with intramedullary nailing (IMN) or percutaneous locked plating (PLP).

Materials and methods: A retrospective review identified 46 patients with surgically treated distal extra-articular tibial fractures of between 3 and $11 \mathrm{~cm}$ proximal to the plafond. Eighteen patients were treated with closed IMN (Group I) and 28 patients were treated with PLP fixation (Group II). Patients with at least 1-year follow-up were included. The mean age was 40.5 (range 16-70) years; $78 \%$ of the patients were men. The rates of union, malunion, malalignment (defined as $>5^{\circ}$ of angulation in any plane), infection, and removal of implants were compared according to final outcomes. The average followup period was 28 months. Twenty-one fractures (27\%) were open. 
Results: The age and gender were similar in both groups. None of the patients had infection. Two patients (7\%) had nonunion in Group II. Two patients treated with IMN, compared with three patients treated with PLP, had a malalignment. Three patients had significant change in alignment between the immediate postoperative and the final radiographic evaluation in Group II. Significant differences were found with regard to union time between the two groups $(\mathrm{p}<0.05)$. The average union times were 16 weeks in Group I and 19 weeks in Group II.

Conclusion: For earlier union time compared to PLP, we advise considering the use of IMN for extra-articular fractures in the distal third of the tibia.

References: 1. Ozkaya U, Parmaksizoglu AS, Gul M, Sokucu S, Kabukcuoglu Y. Minimally invasive treatment of distal tibial fractures with locking and non-locking plates. Foot Ankle Int. 2009 Dec;30(12):1161-7. 2. Vallier HA, Le TT, Bedi A. Radiographic and clinical comparisons of distal tibia shaft fractures $(4$ to $11 \mathrm{~cm}$ proximal to the plafond): plating versus intramedullary nailing. Orthop Trauma. 2008;22(5):307-11.

Disclosure: No significant relationships.

\section{O30}

\section{INTRA-ARTICULAR FRACTURES ON DISTAL TIBIA- SHALL WE DELAY THE OPERATION?}

\author{
B. Bonchev ${ }^{I}$, R. Kastelov ${ }^{2}$, N. Vodenicharski ${ }^{I}$, D. Valchev ${ }^{I}$
}

${ }^{1}$ Orthopaedic And Trauma Clinic, Saint Anna University Hospital, Sofia, Bulgaria, ${ }^{2}$ Orthopaedic And Trauma Clinic, Saint Anna University Hospial, Sofia, Bulgaria

Introduction: The purpose of present study is to represent our experience in emergency operative treatment-ORIF of second and third type tibial pylon fractures from Rüedi and Allgöwer classification. To discuss advantages and challenges, which it gives to us in compared to delay of operation more then $48 \mathrm{~h}$ (damage control) and non-operative treatment.

Case report: Results: The results were report according to FFI (Foot Function Index). Very good with FFI under 30 were report in 42 patients $(77.7 \%)$. Good with FFI from 31 to 70,9 patients $(16.6 \%)$ Bad with FFI above 71 in 3 patients $(6.7 \%)$ treated non-operative. Complications: Superfitial infection of operative wound in $6(11.1 \%)$ patients. Valgus deformity-AP view of x-ray in $5(9.25 \%)$ patients. Varus deformity in $2(3.7 \%)$ patients.

Materials and methods: For the period of August 1998 until March 2010 in University hospitals "Pirogov" and "Saint Anna" Sofia, Bulgaria were treated 54 patients with pylon fractures. $45(83.3 \%)$ male and $9(16.7 \%)$ female. The middle age is 43.5 years. $9(16.6 \%)$ were open fractures. Classification A type-3(5.55\%), B type-13(24\%) and C type-28(60.45\%). 49 patients were operated with ORIF. 22 patients operated until $24 \mathrm{~h}$ and 27 patients until $48 \mathrm{~h}$ from the trauma. Non-operative five patients.

Discussion: Emergency operative treatment of the tibial pylon fractures before soft tissue damage (until $48 \mathrm{~h}$ of trauma) is more successful to achieve anatomic reposition of the fracture. Early operation and early mobilization is connected with recovery of soft tissues trofic. We report good recovery of limb function only after surgical treatment.

Results: The results we report according to FFI (Foot Function Index). Very good FFI under 30 in 42 patients $(77.7 \%)$. Good FFI from 31 to 70,9 patients $(16,6 \%)$. Bad FFI above 71 in 3 patients $(6.7 \%)$ treated non-operative. Complications: Superficial infection of operative wound in six patients $(11.1 \%)$. Valgus deformity-AP view of x-ray in $5(9.25 \%)$ patients. Varus deformity in $2(3.7 \%)$ patients. There was no need of artrodesis or allopastic.

Conclusion: Emergency operative treatment of the tibial pylon fractures before soft tissue damage (until $48 \mathrm{~h}$ of trauma) is more successful to achieve anatomic reposition. Early operation and early mobilization is connected with recovery of soft tissues trofic. We report good recovery of limb function only after surgical treatment. Disclosure: No significant relationships.

\section{1}

\section{LOCKED PLATING OF DISTAL TIBIA FRACTURES-A BIOMECHANICAL COMPARISON OF STEEL VERSUS TITANIUM IMPLANTS}

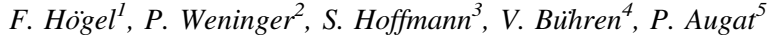

${ }^{1}$ Trauma, Berufsgenossenschaftliche Unfllklinik Murnau e.V., Murnau, Germany, ${ }^{2}$ Trauma, Lorenz Böhler Krankenhaus, Vienna, Austria, ${ }^{3}$ Biomechanics, Berufsgenossenschaftliche Unfllklinik Murnau e.V., Murnau, Germany, ${ }^{4}$ Trauma Surgery, Trauma Center Murnau, Murnau, Germany, ${ }^{5}$ Biomechanical Research Laboratory, Traumacenter Murnau, Murnau, Germany

Introduction: Distal tibia fractures are a challenge to stabilize and often gain a relative instability which leads to non unions. Therefore it is of interest if locked plating with a steel plate is of higher stiffness and less interfragmentary movement than osteosynthesis with a locked titanium plate.

Materials and methods: In each group five tibiae were used (medium Tibia, Sawbone 4th generation, Schweden). A locked steel plate (AxSOS, Stryker, USA) and a locked titanium plate (LCP, Synthes, USA) were used at the distal tibia. An osteotomy was performed at the distal shaft intersection with a gap of $10 \mathrm{~mm}$. Axial load of $350 \mathrm{~N}$ was applied through the physiological load axis and torsional load was applied with a torque of 0 to $+10 \mathrm{Nm}$ and back to $0 \mathrm{Nm}$. The stiffness was calculated from the machine datas and the interfragmentary movement was measured by an optoelectronic measurement system (Pontos, GOM, Germany).

Results: Under axial load no significant differences regarding the stiffness was found between the implants, but interfragmentary movement was significantly lower using a steel plate than a titanium plate (titanium plate $1.03 \mathrm{~mm}$, steel plate $0.6 \mathrm{~mm}$ ) in mean. Torsional stiffness of steel plates was significantly higher than with titanium plates but interfragmentary movement showed no significant differences.

Conclusion: Treatment of distal tibia fractures using angle stable steel plates showed significantly higher stiffness under torsional load and significantly lower interfragmentary movement under axial load compared to titanium plates. It is of benefit to treat those fractures with a steel plate concerning the biomechanical properties.

Disclosure: No significant relationships.

\section{$\mathbf{0 3 2}$}

\section{POTENTIAL APPLICATION OF THE HLS-FAMILY IN THE FIXATION OF THE CALCANEUS FRACTURE}

\author{
I. Kádas ${ }^{1}, \underline{\text { Z. Magyari }}{ }^{2}$, Z. Biró ${ }^{2}$, A. Kocsis $^{3}$ \\ ${ }^{1} 3$, National Institute of Traumatology, Budapest, Hungary, \\ ${ }^{2}$ Department 3, National Institute of Traumatology, Budapest, \\ Hungary, ${ }^{3} 3$, National Institute of Traumatology, Budapest, Hungary
}


Introduction: Calcaneus fractures with depression of the articular surface treated according to Zadravecz's technique suggested a new solution, comprising the achievement of reduction via ligamentotaxis and the use of a traditional screw with head and thread, exerting a compressive effect. It results in distraction and compression at the same time. We tried to resolve this contradiction with designing of an implant, which is suitable for both distraction or keeping the distance between the fragments as well.

Materials and methods: Usually, there are threads at both ends of the HLS screw (HeadLess Screw). There is a thread on the end close to the head as well. Thread diameter and inclination are either larger, the same or smaller, when compared to the thread at the tip of the screw. Both threads are rotating into the same direction. Distractive, neutralizing and compressive effects are results of the different thread inclinations. After fracture reduction, these screws may be applied for fractures requiring ligamentotaxis of the external malleolus. We decided to use an intramedullary distraction screw. A compressive screw is applied for fractures of the internal malleolus.

Results: We treated 81 patients with the modified Zadravecz's technique from the year 2005 on, using the headless screws in a distractive manner. There were two deep infection, one superficial wound healing disturbances. Good reposition 56, acceptable 20 poor 5. All the patients was mobilized at the end of the therapy without walking aids. 65 returned to the original profession.

Conclusion: Based of our preliminary results, the so called "reduction screw-family" may be used for achieving compressive (approximating), neutralizing (distance keeping) and distractive (distraction) effects as well.

Disclosure: No significant relationships.

\section{$\mathbf{0 3 3}$}

\section{SURGICAL TREATMENT OF LISFRANC INJURIES; CLINICAL OUTCOME AND PATIENT SATISFACTION}

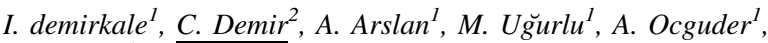 M. Dogan ${ }^{I}$}

${ }^{1}$ Orthopaedics and Travmatology, ataturk education and research hospital, ankara, Turkey, ${ }^{2}$ Orthopaedics and Travmatology, ataturk education and research hospital, Ankara, Turkey

Introduction: Lisfranc fracture-dislocations can cause severe tarsometatarsal malalignment if have not treated. In this study potential prognostic factors for functional outcome and individual perceptions of disability were retrospectively analyzed and compared with radiological findings.

Materials and methods: This study consisted of 15 patients who were treated between January 2004 and January 2010. The mean duration of follow-up was 26.6 months (range 6-60 months). There were 13 men and 2 women with a mean age of 33.9 years (range 19-55 years). Two patient had sustained from polytrauma whereas 13 patient had only foot injury. Within open reduction treated patients, stabilization was managed by Kirschner wires at five patients and cannulated screws at nine patients. Patients were retrospectively reviewed by clinically, radiologically, with American Orthopaedic Foot and Ankle Society (AOFAS) Midfoot Scale and the Foot and Ankle Disability Index (FADI) scoring systems. To evaluate radiological findings we have used a new classification system which assesses tarsometatarsal and intertarsal alignment.

Results: The AOFAS midfoot scale values ranged from 9 to 100 points with an average of 65 . The functional results on the FADI score at the follow-up examination varied from 38.5 to 100 points (mean 70). Consequently, satisfactory $\mathrm{x}$-rays (types A and C) were found in only five patients, whereas ten patient showed unsatisfactory radiologic results.

Conclusion: Although radiological results do not necessarily correlate with our functional outcomes; high patient satisfaction was observed in this study. It should be emphasized that further studies with larger groups are needed to corroborate the discordance between higher clinical results and worse anatomic alignment.

References: 1. Calder JD, Whitehouse SL, Saxby TS. Results of isolated Lisfranc injuries and the effect of compensation claims. J Bone Jt Surg Br. 2004 May;86(4):527-30. 2. Mulier T, Reynders $\mathrm{P}$, Sioen W, et al. The treatment of Lisfranc injuries. Acta Orthop Belg. 1997;63(2):82-90.

Disclosure: No significant relationships.

\section{4}

\section{MINI-INVASIVE TECHNIQUE FOR THE INDIRECT REDUCTION AND TREATMENT OF CALCANEAL FRACTURES}

\section{K. Memisoglu, H. Atmaca, C.C. Kesemenli, H. Sarman, H. Ceylan}

Orthopaedics and Traumatology, Kocaeli University School of Medicine, Kocaeli, Turkey

Introduction: Calcaneal fractures are the most commonly seen tarsal bone fractures, constituting $2 \%$ of all fractures, and $65 \%$ of all tarsal fractures. $70 \%$ of these fractures are intraarticular fractures. Closed reduction-percutaneous pinning, open reduction-internal fixation, and primary arthrodesis are procedures used in the surgical treatment. The aim of surgical treatment is to restore the normal biomechanics of the Achilles heel and rear of the foot by anatomical reduction of the articular surface. Minimization of soft tissue complications seen in the treatment of calcaneal fractures may be achieved by utilizing indirect closed reduction and $\mathrm{K}$ wire or screw assisted minimally invasive percutaneous osteosynthesis methods.

Materials and methods: In this study, a new technique for the treatment of calcaneal fractures with associated soft tissue problems is described by presenting three patients treated by indirect reduction of comminuted calcaneal fractures and Endobutton ${ }^{\circledR}$ (Smith \& Nephew) assisted minimally invasive fixation techniques.

Results: No complications occured during or after any of the operations, and no infection or wound problems were seen in any of the patients. All three patients, who worked as heavy manual laborers, returned to work in less than 1 year following treatment, and none of the patients complained of foot expansion or any step or walking disorders.

Conclusion: Endobutton ${ }^{\circledR}$ assisted minimally invasive fixation surgery, which has a short learning curve and a low risk of complications or infection, may be an alternative method to be applied especially in cases of calcaneal fractures where there is a split parallel to the long axis of the calcaneus or where there is additional soft tissue pathology or a high risk of soft tissue complications.

\section{Reference:}

1. Eastwood DM, Langkamer VG, Atkins RM. Intra-articular fractures of the calcaneum. Part I. Open reduction and internal fixation by extended lateral transcalcaneal approach. J Bone Jt Surg Br. 1993;75:189-95.

Disclosure: No significant relationships. 


\section{BASIC AND CLINICAL RESEARCH 1}

\section{$\mathbf{O 3 5}$}

\section{BLOOD LOSS DURING EXTENSIVE EXCISION AND MICROGRAFT IN LARGE SIZED BURN P}

\author{
J.Wu, G. Luo, Y. Peng
}

Institute Of Burn Research, State Key Lab of Trauma, Burn and Combined Injury, Third Military Medical University, Southwest Hospital, Chongqing, China

Introduction: Precise estimation of blood loss before and during operation is essential to ensure completing a major surgery. The blood loss for microskin grafting after extensive eschar excision in major burn patients.

Materials and methods: The quantity of blood loss was calculated prospective by the change of the levels of hemoglobin in peripheral blood and analyzed with the clinical data.

Results: There were 71 patients in the study. The average total burn area was $74.63 \%$ TBSA. The operations of extensive excision and microskin graft in these patients were carried out on day 4.63 post burn injury. The average excised burn area was $31.68 \%$ TBSA (from 10 to $50 \%$ TBSA), and the average operation time last $2.70 \mathrm{~h}$. It was found that the blood loss per operation was $2,055.96,875.90 \mathrm{ml}$, which meant that 77.93 blood was averagely lost when $1 \%$ TBSA burn escar was excised and micrografted, or $0.42 \mathrm{ml} / \mathrm{cm}^{2}$.

Conclusion: It is necessary to improve the accuracy of the estimation of blood loss during the operation of extensive excision and microskin graft in burn patients.

Disclosure: No significant relationships.

\section{6}

\section{PANTON-VALENTINE LEUKOCIDIN ASSOCIATED STAPHLOCOCCAL INFECTION (PVL-SA)-THE MORBIDITY, RESOURCE BURDEN, AND POTENTIALLY FATAL CONSEQUENCES OF MISSED DIAGNOSES}

\section{J.H. Sarginson ${ }^{1}$, M. Morgan ${ }^{2}$}

${ }^{1}$ Department Of Plastic And Reconstructive Surgery, Royal Devon and Exeter Hospital, Exeter, United Kingdom, ${ }^{2}$ Department Of Microbiology, Royal Devon and Exeter Hopsital, Exeter, United Kingdom

Introduction: Panton-Valentine Leukocin is a leucocidal toxin secreted by particularly virulent strains of S. Aureus responsible for aggressive soft-tissue infection, most often presenting as recurrent cutaneous abscesses requiring serial surgical drainage. Though largely a community-based pathogen, over recent years, PVL-SA has become an increasing concern in the hospitals of Europe, as it is difficult to eradicate, highly contagious, and can progress to a haemorrhagic pneumonia which carries a mortality of $75 \%$.

Materials and methods: We present a series of missed diagnoses of PVL-SA, which presented to general and specialist surgery at a South West England hospital over a 3 year period.

Results: The missed diagnoses resulted in unnecessarily high morbidity for the sufferers-requiring serial surgical drainage with the concurrent anaesthetic risks, unchecked spread of infection through households and communities, and avoidable transmission of infection to health care workers and clinicians.
Conclusion: Despite the aggressive nature of the toxin, PVL-SA remains a little-known and poorly understood organism amongst surgeons. The resource burden on treating centres, the morbidity suffered by the individual and the community at large, and the potentially fatal consequences of respiratory infection with PVL-SA necessitate a low threshold of suspicion, and prompt diagnosis by the treating clinician. We present our series, along with a synopsis of the diagnostic characteristics of the pathogen, in order that it might be better recognised more appropriately managed.

Disclosure: No significant relationships.

\section{7}

\section{THE EFFECTS OF LOCALLY APPLIED SIMVASTATIN ON THE EXPERIMENTAL RAT FEMUR NON-UNION MODEL}

\section{Oner, I.H. Kafadar, A. Kocer, M. Halici, A. Guney}

Orthopaedics and Traumatology, ERCIYES University Medical Faculty, Talas, Turkey

Introduction: The aim of this study was to assess the effects of locally applied simvastatin on non-union that was performed experimentally on rat femur.

Materials and methods: Thirtytwo male Wistar-Albino rats were included in this study. Rats were randomly divided into four equal groups as follows: Group $\mathrm{K}_{4}$, Group $\mathrm{K}_{8}$ (control groups), Group $\mathrm{S}_{4}$ and Group $\mathrm{S}_{8}$ (study groups). Right femurs of rats were osteotomized after anaesthesia was given. Osteotomized femurs of rats were fixed by Kirschner $(\mathrm{Kr})$ wires and staple nail after $1.8 \mathrm{~mm}$ gap was performed. At the end of 10th week, non-union of each femur was confirmed radiographically. $1 \mathrm{~mL} /$ day of physiological serum was locally applied of control groups and $10 \mathrm{mg} / \mathrm{kg} /$ day of simvastatin was locally applied of study groups for a week. $\mathrm{K}_{4}$ and $\mathrm{S}_{4}$ groups were sacrificed 4 week after the end of the local treatment. $\mathrm{K}_{8}$ and $\mathrm{S}_{8}$ groups were sacrificed 8 week after the end of the local treatment. Before the sacrification, intracardiac blood samples were taken for biochemical analysis, and radiography were taken. After the sacrification, right femurs of rats were taken for histopathological evaluation.

Results: There were significant differences between the control and study groups when evaluated radiologically. Significantly higher level of bone specific alkaline phosphatase (BAP) and osteocalcine values were found in study groups than those of controls. There was significant healing in study groups when compared with the controls histopathologically.

Conclusion: These results show that local application of simvastatin produces beneficial effects biochemical, radiological and histopathologically on rat femur non-union model.

Disclosure: No significant relationships.

\section{8}

\section{CHANGES OF COAGULATION FACTOR PATTERNS IN THE EARLY COURSE AFTER MULTIPLE INJURY}

\section{Burggraf, B. Hussmann, S. Lendemans}

Department Of Trauma Surgery, University Hospital of Essen, Essen, Germany

Introduction: In the initial phase after multiple injury coagulopathy is frequently seen and patients are still at high risk for death due to 
uncontrolled haemorrhage. Consumption and transfusion induced dilution of coagulation factors are major causes of coagulopathy. Today, a vast amount of haemostatic active agents are routinely available. While present research is focusing on optimal transfusion protocols and ,point of care" strategies, little is known about the underlying changes of coagulation factor patterns. The purpose of our study was therefore to evaluate possible alterations of clotting factors in the early post injury phase.

Materials and methods: Patients admitted directly from the scene of accident, with an ISS $\geq 16$ points were included. Ten healthy volunteers formed the control group. Besides routine tests of coagulation, activities of clotting factors were determined.

Results: Study group consists of 32 patients. Mean age was 49 years, ISS was 24 points in average. Our data shows a significant reduction in mean activity of factors II (77\%), V (75\%), VII (86\%), X (81\%), XII $(76 \%)$ und XIII $(81 \%)$, as well as serum calcium levels $(2.06 \mathrm{mmol} / \mathrm{l})$ and prothrombin time $(86 \%)$, following severe trauma. In contrast, activity of factor VIII (202\%) is significantly increased. The remaining factors and fibrinogen show a tendency towards reduced activity and levels, respectively.

Conclusion: Present study describes the changes of coagulation factors in the initial course after severe injury. As supposed previously by other authors, we see a widespread reduction of clotting factor activity. Furthermore, we found a clear increase of factor VIII activity. The underlying mechanisms have yet to be determined. Further investigation, especially increasing the study group population, is necessary. This will allow subgroup analysis with respect to trauma mechanism and severity and potentially divergent clotting factor constellations. Long-term objective should aim on a patient focused coagulation therapy with subsequent reduction in mortality after severe trauma.

Disclosure: No significant relationships.

\section{O39}

\section{CRITICAL BLEEDING AND DAMAGE CONTROL RESUSCITATION IN TRAUMA SETTING AND MOREOVER. A POINT OF CARE APPROACH WITH THROMBOELASTOGRAPHY: WHAT ELSE?}

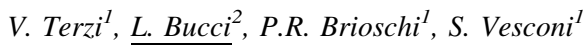 \\ ${ }^{1}$ I.c.u. Bozza, A.O. Niguarda, Milan, Italy, ${ }^{2}$ I.c.u. Bozza, A.O. \\ Niguarda, Milan, Italy
}

Introduction: Hemorrhage is considered the most preventable cause of in-hospital deaths, especially in acute trauma setting. The damage control approach with surgery or interventional radiological techniques has the goal to stop the bleeding as soon as possible. Damage control resuscitation has been proposed with two fundamental targets: to restore intravascular volume and to correct the imbalanced coagulation system [1]. As a part of primary care of the hemorrhagic patient, point-of-care (POC) diagnostic tool such thromboelastography/metry $\left(\mathrm{TEG}^{\mathrm{TM}} / \mathrm{TEM}^{\mathrm{TM}}\right.$,) have become employed by anesthesiologists, intensivists, blood-bank specialists and trauma surgeons [2, 3] In critical bleeding settings such as trauma, major surgery, GI tract hemorrhage, aortic aneurismal rupture, post-partum hemorrhage $(\mathrm{PPH})$ the rapid, near-patient visco-elastic assay of the entire coagulation system (i.e. factors, fibrinogen, platelets and the fibrinolysis enhancement, if any) can direct targeted therapeutical approaches (FFP, fibrinogen sources, platelets, antifibrinolytic agents, adjunctive agents such rFVIIa, PCC, desmopressin) [4, 5].

Materials and methods: Recently in our institution we retrospectively evaluated the use of POC-TEG in critical bleeding setting
(2007-2009 years; 32 total patients: trauma, major surgery, burns, sepsis) with massive transfusion protocol (i.e. $>10 \mathrm{RBC} / 24 \mathrm{~h}$ ), using blood components or blood derivatives (i.e. rFVIIa, tranexamic acid) according to TEG traces/values [6].

Results: The 28-days survival was evaluate, according for trauma patients to TRISS method and a VLAD (Variable Life-Adjusted Display) analysis. An improvement in survival with TEG-guided approach was observed.

Conclusion: To restore physiology and homeostasis represent the primary care project of all the involved specialists managing a critical bleeding patient (trauma surgeons and the other specialists always at work in the ED) with ematologist and blood-banker [7], in order to ameliorate the trauma team as well and to manage such a precious resource as blood and blood components.

References: 1. 2009;96:111-18. 2. 2008;106:1366-75. 3. 2009;40: 119-123. 4. 2010;14:R52. 5. 2010;14:R55. 6. AttiCongressoSIAARTI2009:Firenze, Italy. 7. 2007;47(2 Suppl): 176S-181S

Disclosure: No significant relationships.

\section{0}

\section{GROUPVIB PHOSHOLIPASEA2 IS ASSOCIATED WITH ACUTE LUNG INJURY FOLLOWING TRAUMA AND HEMORRHAGIC SHOCK}

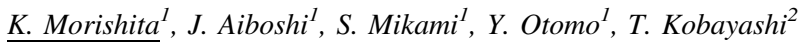

${ }^{1}$ Acute Critical Care and Disaster Medicine, Tokyo Medical and Dental University, Tokyo, Japan, ${ }^{2}$ Ochanomizu University, Tokyo, Japan

Introduction: A variety of inflammatory mediators enter into the systemic circulation through the mesenteric lymph duct after hemorrhagic shock, thus leading to acute lung injury (ALI)/MODS. Our studies have already demonstrated that the post hemorrhagic shock mesenteric lymph contains biologically active lipids, such as lyso-phosphatidylcholine and lyso-phosphatidylethanolamine with unsaturated fatty acid). We hypothesize that GroupVIB phosholipa$\mathrm{seA}_{2}\left(\mathrm{iPLA}_{2} \gamma\right)$ may be the enzyme involved in the production of these lipids. The purpose of our study was to determine the role of $\mathrm{iPLA}_{2} \gamma$ in ALI following trauma and hemorrhagic shock(T/HS) using (E)6-(bromomethylene)-3-(1-naphthalenyl)-2H-tetrahydropyran-2-one (BEL), an $\mathrm{iPLA}_{2} \gamma$ specific inhibitor.

Materials and methods: Male SD rats were anesthetized and femoral artery and jugular vein were cannulated. The blood was withdrawn from the T/HS group until the mean arterial pressure was reduced to $40 \mathrm{mmHg}$ and maintained for $30 \mathrm{~min}$. The animals were resuscitated over $2 \mathrm{~h}$ (shed blood + normal saline). Evans blue dye (EBD) was injected and then the lungs were harvested. The concentration of EBD was measured by the EBD method. The trauma and sham shock (T/SS) group underwent the same procedures without shock and resuscitation. Rats were randomly allocated into three groups: T/SS + dimethyl superoxide (DMSO), T/HS + DMSO, T/HS + BEL (final concentration $10 \mu \mathrm{M})$. BEL or DMSO was injected $30 \mathrm{~min}$ before $\mathrm{T} / \mathrm{HS}$ or T/SS. The animals were exposed to T/HS and T/SS as described above. Results: Lung permeability of T/HS + BEL $(0.024 \pm 0.0041 \mu \mathrm{g} / \mathrm{g}$ of wt) was significantly decreased in comparison to that of T/HS + DMSO $(0.044 \pm 0.014 \mu \mathrm{g} / \mathrm{g}$ of $\mathrm{wt} ; \mathrm{p}<0.01)$. Furthermore the lung permeability of T/HS + BEL was not significantly different from that of T/SS + DMSO $(0.019 \pm 0.014 \mu \mathrm{g} / \mathrm{g}$ of $\mathrm{wt})$.

Conclusion: BEL attenuates pulmonary permeability following T/HS. $\mathrm{iPLA}_{2} \gamma$ may be the key enzyme involved in the pathogenesis of ALI/ MODS.

Disclosure: No significant relationships. 


\section{1}

\section{INVESTIGATING THE ROLE OF NFאB IN SYSTEMIC INFLAMMATION RESPONSE FOLLOWING HEMORRHAGE/RESUSCITATION IN COMBINATION WITH CHRONIC ALCOHOLIC LIVER DISEASE-A NEW ANIMAL MODEL}

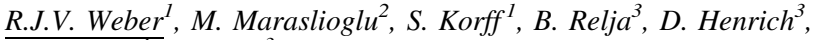 \\ M. Lehnert ${ }^{4}$ I. Marzi $^{3}$
}

${ }^{1}$ Trauma-, Hand- And Reconstructive Surgery, J.W. Goethe University Hospital, Frankfurt, Germany, ${ }^{2}$ University of Frankfurt, Frankfurt, Germany, ${ }^{3}$ Department Of Trauma Surgery, University of Frankfurt, Frankfurt, Germany, ${ }^{4}$ Department Of Trauma, Hand And Reconstructive Surgery, Goethe University Frankfurt, Frankfurt, Germany

Introduction: Chronic alcohol abuse is associated after multiple trauma with a longer ICU stay and a higher 30 day-mortality. This may be due to an impaired immune response associated with chronic alcohol abuse. Aim of our project was to create a reliable animal model to analyse the immune response after hemorrhage/resuscitation in mice chronically fed ethanol.

Materials and methods: Male transgenic mice (cis-NFkB-EGFPreporter gen mouse) were pair fed with an ethanol-diet for 4 weeks $(6.3 \% \mathrm{vol} / \mathrm{Vol})$. Hepatic damage and systemic inflammation was evaluated by transaminase release, serum IL-6, liver-body-weight ratio, histology and epifluorescence-microscopy. Hepatic fat content was quantitated by Soxleth-analysis. After ethanol feeding a hemorrhagic shock $(90 \mathrm{~min})$ followed by resuscitation was performed. $2 \mathrm{~h}$ after resuscitation animals were sacrificed and tissues were harvested. Results: Ethanol feeding induced a significant elevation of serumAST-levels: $\quad 129.08 \pm 16.0 \mathrm{IU} / \mathrm{L} \quad$ (alcohol) $\quad$ (mean \pm SEM) vs. $69.47 \pm 7.83 \mathrm{U} / \mathrm{L}$ (control) and IL6-levels: $450.87 \pm 52.8 \mathrm{IU} / \mathrm{L}$ (alcohol) versus $189.39 \pm 7.52 \mathrm{IU} / \mathrm{L}$ (control). Liver-/Body-weightratio increased to $5.85 \pm 0.18 \%$ (alcohol) versus $4.30 \pm 0.09 \%$ (control). Red oil $\mathrm{O}$ staining and Soxleth extraction $(26.25 \pm 0.52$ versus $4.61 \pm 0.09 \%, \mathrm{p}<0.05)$ showed strong fat accumulation in livers fed ethanol. Ethanol fed mice display enhanced hepatic $\mathrm{NF} \kappa \mathrm{B}$ related EGPF-expression in epifluorescence-microscopy. In a pilot study, hepatic AST release after $\mathrm{H} / \mathrm{R}$ in ethanol fed mice showed a trend towards an increased liver damage $(3,398 \pm 1,118 \mathrm{IU} / \mathrm{L}$ (alcohol) vs. $956 \pm 400 \mathrm{IU} / \mathrm{L}$ (control), $\mathrm{p}=0.1$ )

Conclusion: Using transgenic mice our model is a valuable instrument for detailed investigation of the reactions following hemorrhage-resuscitation combined with chronic alcohol abuse that are partially associated with $\mathrm{NF} \kappa \mathrm{B}$-activation.

Disclosure: No significant relationships.

\section{2}

\section{TRAUMA BAY PREDICTORS OF MORTALITY AFTER MASSIVE TRANSFUSION PROTOCOL ACTIVATION: A TEN YEAR EXPERIENCE}

D.N. Holena, C.A. Sims, P.K. Kim, B. Sarani, J.L. Pascual, B.M. Braslow, P.M. Reilly, C.W. Schwab

Division Of Traumatology, Surgical Critical Care, And Emergency Surgery, University of Pennsylvania School of Medicine, Philadelphia, PA, USA
Introduction: Massive transfusion protocols (MTP) offer an efficient mechanism to deliver large volumes of blood to exsanguinating trauma patients, but many will not survive despite this mobilization of resources. We sought to identify physiologic and resuscitation variables associated with mortality after activation of the MTP.

Materials and methods: Data were collected prospectively from 2000 to 2009. Inclusion criteria were age $\geq 18$ years and MTP activation. Patient demographics, mechanism of injury, and admission vital signs (heart rate (HR), systolic blood pressure (SBP), Glascow coma scale (GCS), shock index (SI-calculated as HR/SBP), and temperature) were collected. Predictor variables found to be significantly associated with mortality on univariate analysis were entered into a multiple logistic regression model.

Results: A total of 374 patients met inclusion criteria. Median age was 30 years [inter-quartile range (IQR) 21], 85\% were male, $74 \%$ were African American, and $69 \%$ had a penetrating mechanism. Injury severity score (ISS) and SI were high (median ISS 25, IQR 18; median SI 0.98, IQR 0.58). Overall mortality was $47 \%$. Patients who died were more likely to have a blunt mechanism injury, lower admission SBP, higher HR, higher SI, lower temperature, and to receive more packed red blood cell (PRBC) units during trauma bay resuscitation (TBR). On univariate analysis, blunt mechanism, SBP, SI, GCS, temperature, and number of PRBC units during TBR were associated with mortality. After multiple logistic regression analysis, blunt mechanism (OR 2.70, 95\% CI 1.40-5.19), SI (OR 1.19 per 0.2 units, 95\% CI 1.03-1.37), and PRBCs during TBR (OR 1.34 per unit, 95\% CI 1.13-1.57) remained significantly associated with mortality. Conclusion: Blunt mechanism of injury, elevated SI, and number of PRBCs during TBR are significantly associated with mortality after MTP activation. Further efforts towards risk stratification may allow identification of patients who will not benefit from MTP activation. Disclosure: No significant relationships.

\section{3}

\section{LEUKOTRIENE B4: PROGNOSTIC FACTOR FOR PULMONARY COMPLICATIONS FOLLOWING MULTIPLE TRAUMA?}

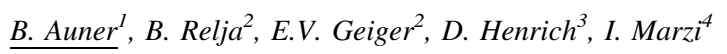

${ }^{1}$ Department Of Trauma, Hand And Reconstructive Surgery, University of Frankfurt, Frankfurt, Germany, ${ }^{2}$ Department Of Trauma, Hand And Reconstructive Surgery, Goethe University Frankfurt, Frankfurt, Germany, ${ }^{3}$ Department of Trauma Surgery, University of Frankfurt, Frankfurt, Germany, ${ }^{4}$ Unfallchirurgie, Univ. Klinikum Frankfurt am Main, Frankfurt, Germany

Introduction: An overwhelming inflammatory response after multiple trauma leads to organ dysfunction and increased susceptibility to infections and septic complications. The lung is regularly involved in posttraumatic complications such as pneumonia, acute lung injury (ALI), acute respiratory distress syndrome (ARDS) and multiple organ failure. Lung infiltration with neutrophils represents a key event in these processes. Leukotriene B4 (LTB4) is one of the most potent chemoattractants and activators of neutrophils. Aim of the present study was to evaluate the significance of LTB4 as an indicator for pulmonary complications (PC) in the posttraumatic course.

Materials and methods: This prospective study included 100 multiply traumatized patients (ISS $\geq 16$ ), divided into a group without 
PC $(n=70)$ and with PC $(n=30$; pneumonia, respiratory failure, ALI, ARDS, pulmonary embolism). Ten healthy volunteers served as control group. Plasma LTB4 levels were measured in the emergency room (ER) and the following 5 days (1-5) using ELISA. Statistics: Kruskal-Wallis; $\mathrm{p}<0.05$ was considered significant.

Results: LTB4 levels were significantly higher in patients who developed PC as compared to patients without PC or controls (127.8, 95.57 and $59.08 \mathrm{pg} / \mathrm{mL}$, respectively), immediately after the admission. PC were associated with significantly longer duration of mechanical ventilation, ICU and total hospital stay. The cutoff level for LTB4 to detect risk for PC was $109.6 \mathrm{pg} / \mathrm{mL}$ ( $72 \%$ specificity and $67 \%$ sensitivity, ER).

Conclusion: LTB4 might be a suitable prognostic factor for early detection of patients at risk for pulmonary complications after multiple trauma. Furthermore, the high levels of LTB4 indicate the strong pro-inflammatory stimulus after severe trauma.

Disclosure: No significant relationships.

\section{4 \\ ENDOSCOPIC STENTING FOR COLON OBSTRUCTION. A SUITABLE APPROACH AS BRIDGE TO ELECTIVE SURGERY AND PALLIATION}

N. Tamini ${ }^{1}$, L. Nespoli ${ }^{1}$, L. Gianotti ${ }^{2}$, M. Dinelli $^{3}$, A. Ardito $^{2}$, R. Frego ${ }^{3}$, A. Redaelli, E. Bolzonaro ${ }^{2}$, A. Nespoli ${ }^{2}$

${ }^{1}$ Department of Surgery, Milano-Bicocca University, Monza, Italy, ${ }^{2}$ Surgery, Milano-Bicocca University, monza, Italy, ${ }^{3}$ Endoscopy, San Gerardo Hospital, Monza, Italy

Introduction: Colonic obstruction often requires an emergency treatment. Surgery in such setting may be demanding for the surgeon, at high risk for the patient and often is a temporary or palliative therapeutic option. The role of self-expandable metallic stent (SEMS) is debated but it may be an alternative as bridge to elective operation or definitive palliation avoiding surgery.

Materials and methods: We admitted 117 patients with large bowel obstruction over a period of 6 years. Sixty-seven $(57.2 \%)$ of the these 117 were treated by endoscopic stenting. Forty-two of $67(62.7 \%)$ patients with the intent of bridge to elective surgery (SEMS group) and $25(37.3 \%)$ for definitive palliation. Fifty of $117(42.8 \%)$ patients underwent emergency surgery (ES) without stenting.

Results: Obstruction was successfully treated by SEMS in $96.7 \%$ of the cases $(65 / 67)$ within a median of $24 \mathrm{~h}$ from admission. We observed three $(4.5 \%)$ self-limiting bleeding and three stent migration with necessity of further endoscopy for replacement. No perforation was recorded. In two patients $(3.3 \%)$ the endoscopic procedure failed for technical reasons. Moreover, we compared the SEMS group to the ES group. The median time from SEMS positioning to elective operation was 5 days. The two groups were well matched for baseline characteristics. The rate of total complications was respectively $45.2 \%(19 / 42)$ versus $72.0 \%(36 / 50)(p=0.009)$, surgical site infections $26.2 \%(11 / 42)$ versus $54.0 \%(27 / 50)(\mathrm{p}=0.006)$, anastomotic leak $12.5 \%$ versus $20.0 \%(\mathrm{p}=\mathrm{NS})$, need of intensive care $7.1 \%(3 / 42)$ versus $24.0 \%(12 / 50)(\mathrm{p}=0.02)$, Hartmann procedure $4.8 \%(2 / 42)$ versus $12.0 \%(6 / 50)(\mathrm{p}=\mathrm{NS})$, stoma formation $11.9 \%$ $(5 / 42)$ versus $20.0 \%(10 / 50)(\mathrm{p}=\mathrm{NS})$, mean length of hospitalization 13.9 (6.3) versus $22.5(20.2)$ days $(\mathrm{p}=0.01)$.

Conclusion: Endoscopic colon stenting is safe and feasible to manage patients with acute obstruction. Preoperative SEMS as bridge to elective surgery is associated with a significant better outcome and a shorter hospitalization when compared with emergency surgery.

Disclosure: No significant relationships.
045

\section{IMPACT OF MODERN HOSPITAL MANAGEMENT AND SPECIALIZED LAPAROSCOPIC TRAINING ON THE QUALITY OF EMERGENCY MINIMALLY INVASIVE SURGERY: EXPERIENCE IN A GOVERNMENTAL HOSPITAL IN A GULF COUNTRY}

\author{
N. Balalaa ${ }^{I}$, R. selli Kanth ${ }^{2}$, N. Balalaa ${ }^{1}$
}

${ }^{1}$ General Surgery, Alain hospital, Alain, United Arab Emirates, ${ }^{2}$ Surgery, Alain hospital, Alain, United Arab Emirates

Introduction: to assess the impact on the quality of emergency minimally invasive surgery in a governmental hospital in a Gulf country (Alain|IUAE) of a modern hospital management and specialized laparoscopic training.

Materials and methods: Study concerned all laparoscopic procedure $\mathrm{s}$ for emergency done 1 year before (01-11॥2009) and 1 year after (11\2009-10\\2010) introduction of a specialized training program. Number of laparoscopic procedure, complexity of cases, conversions rate and complications for period before the program were assessed and compared to the period after the program.

Results: Before starting the specialized program a total of 97 lap. Procedures was done compared to 186 after the program $(\mathrm{p}=0.001)$. The conversion rate has decreased from $13.6 \%$ before to $2,1 \%$ after the program $(p=0.0001)$, while complexity of case $(14$ vs. $26 \%$, $p=0.025)$ and needs for post operative ICU treatment ( 1 vs. $4.8 \%$, $\mathrm{p}=0.035$ ) was increased. There was no mortality or major complications required reoperation in both periods, but the overall complication rate was decreased from $19.5 \%$ before to $10.2 \%$ $(\mathrm{p}=0.032)$ after the training program. For the entire study there was only 2 out of 58 cholecystectomy had postoperative cystic duct leakage treated with ERCP and stent and 2 out 160 appendicectomy had postoperative intraabdominal abscess treated with PAD. The overall port side infection rate was $1.4 \%$.

Conclusion: The improvement of laparoscopic surgery for emergency resulting from modern hospital management and standardized training through specialized leadership was obvious at Alain-hospital and reached the required international standard. However, training needs to be continued and practices homogenized.

Reference: Allemann P, Probst H, Demartines N, Schäfer M. Prevention of infectious complications after laparoscopic appendectomy for complicated acute appendicitis-the role of routine abdominal drainage. Langenbecks Arch Surg. 2010. Disclosure: No significant relationships.

\section{6}

\section{LAPAROSCOPIC COLORECTAL SURGERY IN EMERGENCY}

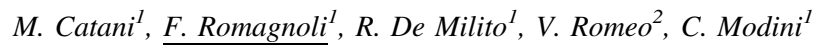

${ }^{1}$ Dea-emergency Department, Policlinico Umberto I, Rome, Italy, ${ }^{2}$ Dea-emergency Department, Sapienza Università di Roma, Rome, Italy

Introduction: Laparoscopic colectomy (LC) is slowly becoming the standard of care for elective resections. However, the use of LC in the emergency setting is relatively unstudied. The authors describe their experience with a series of emergent and urgent LC cases for a variety of benign and neoplastic colorectal diseases.

Materials and methods: This study reviewed 34 patients referred to the Emergency Department of a tertiary level hospital over a 3-years 
period and underwent laparoscopic colectomy performed by the same surgeon, skilled in advanced laparoscopic technique. Patient demographics, indication for surgery, operative details and postoperative complications were examined. Laparoscopic group was compared with case-matched open group for complication and length of hospital stay.

Results: Twenty-one LC were performed for benign complicated disease, 12 for malignant disease and one for iatrogenic perforation during colonoscopy. Two cases were converted to open procedure $(5.8 \%)$, the mean operative time was $188 \mathrm{~min}$ (range 75-350 min). The average postoperative length of hospital stay was 6.57 days (median 6 days) versus 11, 12 days for the open group (p 0.019), with no post operative mortality. No specific complications or complications requiring other surgical procedure were observed, three patients had minor complication (wound infection).

Conclusion: With increasing experience, $\mathrm{LC}$ is a feasible option in nonelective situations lowering complication rate and length of hospital stay. It should be stressed that the steep learning curve for elective LC must be overcome before these procedures are attempted in emergent or urgent setting. Because this is a retrospective series, further comparative studies are needed

References: 1. Champagne B, Stulberg JJ, Fan Z, Delaney CP. The feasibility of laparoscopic colectomy in urgent and emergent settings. Surg Endosc 2009;23:1791-96. 2. Biondo S, Kreisler E, Millan M, Fraccalvieri D, Golda T, Frago R, Miguel B. Impact of surgical specialization on emergency colorectal surgery outcomes. Arch Surg 2010;145(1):79-86. 3. Skala K, Gervaz P, Buchs N, Inan I, Secic M, Mugnier-Konrad B, Morel P. Risk factors for mortality-morbidity after emergency-urgent colorectal surgery. Int $\mathrm{J}$ Colorectal Dis. 2009;24:311-6. 4. Ingraham AM, Cohen ME, Bilimoria KY, Feinglass JM, Richards KE, Hall BL, Ko CY. Comparison of hospital performance in noemergency versus emergency colorectal operations at 142 hospitals. J Am Coll Surg 2010;210:155-165.

Disclosure: No significant relationships.

\section{7}

\section{OUR EARLY EXPERIENCES WITH SINGLE-INCISION LAPAROSCOPIC SURGERY: THE FIRST 12 PATIENTS}

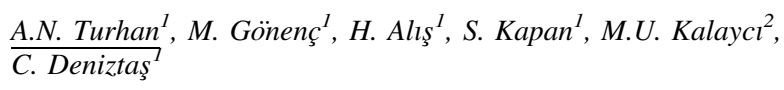

${ }^{1}$ General Surgery, Bakirkoy Dr. Sadi Konuk EAH, İstanbul, Turkey, ${ }^{2} 4$ th General Surgery, Okmeydani Research and Training Hospital, Istanbul, Turkey

Introduction: Single-incision laparoscopic surgery (SILS) is a new laparoscopic procedure. Herein we present our experience of this novel technique: single incision laparoscopic (SIL) appendectomy.

Materials and methods: Appendectomy was performed to 12 patients using conventional laparoscopic instruments through a single small umbilical incision (length $\sim 3 \mathrm{~cm}$ ). The patients received standard postoperative care for acute appendicitis.

Results: The study consisted of 12 patients ( 3 women and 9 men) with an average age of 33 (16-58) years. The mean BMI was 24 (21-27). Mean operative time was $42.7 \mathrm{~min}$ (15-90) min. Loop was used for ligating appendix radix. In one patient conversion to laparoscopic appendectomy was performed due to technical problems. No operative and post operative complications occurred. The average postoperative hospital stay was 1 day.
Conclusion: These promising results suggested that laparoscopic appendectomy can be effectively and safely achieved through a single umbilical incision using conventional instruments.

Disclosure: No significant relationships.

048

\section{TREATMENT OF ACUTE APPENDICITIS WITH LAPAROSCOPIC APPENDECTOMY: A TWO-YEAR, SINGLE-CENTER EXPERIENCE}

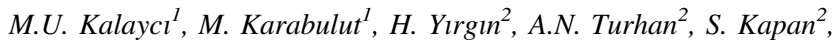 \\ S. BUYUKASIK ${ }^{1}$ H. Allş ${ }^{2}$
}

${ }^{1}$ General Surgery, Bakirkoy DR Sadi Konuk Research And Teaching Hospital, Istanbul, Turkey, ${ }^{2}$ General Surgery, Bakirkoy Dr. Sadi Konuk EAH, İstanbul, Turkey

Introduction: Aim: Laparoscopic appendectomy (LA) has been gaining acceptance in treatment of acute appendicitis in recent years. In this study the efficiency and safety of LA has been investigated. Materials and methods: 565 patients with acute appendicitis who underwent laparoscopic appendectomy at General Surgery Clinic of Bakirkoy Dr Sadi Konuk Training and Research Hospital from February 2008 to November 2010, were evaluated retrospectively regarding socio-demographic data, operation findings and surgical outcomes.

Results: Male to female ratio was $269 / 296$ with a mean age of 23.3 (13-83) years. Mean operative time was $55(13-180)$ min. Perforated appendicitis was seen in 90 patients Abdominal cavity was drained in $53(9.3 \%)$ patients. No complications occurred intraoperatively. Postoperative complication occurred in $60(10.6 \%)$ patients: localized abscess in $33(5.84 \%)$. Mean hospital stay was $1.2(1-15)$ days. No laparoscopy related complication and mortality was observed.

Conclusion: Laparoscopic appendectomy is a safe and effective method as well as open appendectomy in acute appendicitis in experienced centers.

Disclosure: No significant relationships.

\section{9}

\section{PHYSIOLOGY IS MORE IMPORTANT THAN ETIOLOGY WHEN CONSIDERING A PATIENT FOR RELAPAROTOMY. ANALYSIS OF 473 RELAPAROTOMIES}

I. Martínez-Casas
I, J. Sancho
P. Sánchez-Velazquez

${ }^{1}$ Servicio De Cirugía General Y Digestiva, Hospital del Universitari Mar, Barcelona, Spain, ${ }^{2}$ Servicio De Cirugía General Y Digestiva, Hospital del Mar, Barcelona, Spain

Introduction: Slow decision making and poor selection of patients can be deleterious in relaparotomies. Our aim was to identify preoperative risk factors for mortality after relaparotomy

Materials and methods: 473 relaparotomies on 380 patients were prospectively analysed. Past medical history, initial surgery and period between surgeries were recorded.

Results: There was a majority of males $(63 \%)$. Mean age was $62.5 \pm 17$ years. Initial ASA scores were mainly II (41\%) and III (44\%). 69 patients (18\%) were reoperated on more than once $(\mathrm{n}=93)$ and suffered higher mortality (26 vs.13\%; $\mathrm{P}=0.008)$. 
Overall mortality was $20.3 \%(\mathrm{~N}=77)$ and was associated to age $(73 \pm 11$ vs. $61 \pm 17$ years; $\mathrm{P}<0.001)$, initial ASA score $(\mathrm{P}<0.001)$, but not to the urgency of initial surgery ( 23 vs. $18 \%$; $\mathrm{P}=0.2)$. Factors associated with mortality were cardiac disease $(21$ vs. $13 \% ; \mathrm{P}=0.01$ ), chronic renal failure (30 vs. $15 \% ; \mathrm{P}=0.045$ ), diabetes mellitus ( 23 vs. $14 \% ; \mathrm{P}=0.046$ ), anti-aggregant therapy (31 vs. $15 \% ; \mathrm{P}=0.013)$ and malignancy $(21 \mathrm{vs} .13 \% ; \mathrm{P}=0.02)$. Relaparotomies were mainly on demand $(94.5 \%)$ and were categorized by aetiology in peritonitis (40\%), abscess (19\%), haemorrhage (19\%), abdominal wall (11\%) and exploratory laparotomy (11\%). Peritonitis and exploratory laparotomies had higher mortality $(\mathrm{P}=0.006)$. Mortality was associated with mechanical ventilation $(\mathrm{P}<0.001)$, vasoactive drugs $(\mathrm{P}<0.001)$, parenteral nutrition $(\mathrm{P}<0.001)$, blood transfusion $(\mathrm{P}<0.001)$ or haemodialysis $(\mathrm{P}=0.04)$ in the period between laparotomies. The presence of tachycardia $(\mathrm{P}=0.02)$, hypotension $(\mathrm{P}=0.001)$, SIRS score $\geq 2(\mathrm{P}=0.006)$, leukocytosis $(\mathrm{P}=0.01)$, s-creatinine $>2 \mathrm{mg} / \mathrm{dL}(\mathrm{P}<0.001)$, thrombopenia $(\mathrm{P}=$ 0.005), hyperbilirubinemia $(\mathrm{P}=0.02)$, hypoalbuminemia $(\mathrm{P}<0.001)$, hypoprothrombinemia $(\mathrm{P}<0.001)$ and lactic acidosis $(\mathrm{P}=0.01)$ were associated with higher mortality rates. Multivariate analysis isolated lactic acidosis and hypoalbuminemia before relaparotomy as independent risk factors for mortality.

Conclusion: Mortality after relaparotomy is aggravated by a lower physiological reserve and severity of the patient before surgery. Lactic acidosis and hypoalbuminemia are independent risk factors for mortality after relaparotomy.

Disclosure: No significant relationships.

\section{EMERGENCY SURGERY 4}

\section{0}

\section{ULTRASONOGRAPHIC FINDINGS IN SMALL BOWEL OBSTRUCTION: A RETROSPECTIVE STUDY}

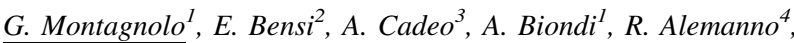 E. Contessini Avesani ${ }^{1}$}

${ }^{1}$ Surgery, Fondazione IRCCS "Ca' Granda” Policlinico Maggiore, Milano, Italy, ${ }^{2}$ IRCCS, Ca' Granda, Policlinico Maggiore Milano, Milano, Italy, ${ }^{3}$ Università di Milano, Milan, Italy, ${ }^{4}$ Surgery, Fondazione IRCCS, Milano, Italy

Introduction: The clinical-radiographic evaluation of small-bowel obstruction (SBO) is critical in discriminating patients who need surgery from those who do not. Abdominal sonography has been advocated by some authors as a valuable diagnostic tool for the diagnosis of bowel obstruction and for early recognition of strangulation. Aim of this study is to evaluate the role of ultrasonography in the management of SBO.

Materials and methods: 334 patients whose clinical or plain x-ray findings suggested small bowel obstruction who underwent abdominal ultrasonography, were collected and subgrouped according to the treatment outcome (surgery vs conservative treatment). Sonographic findings associated with surgery and failure of conservative treatment were reviewed.

Results: Six sonographic findings were considered: bowel loops diameter $>35 \mathrm{~mm}$, bowel wall thickness, absence of mucosal folds, absence of peristaltic movements, sonographic features of bowel content, free peritoneal fluid. At $\chi^{2}$-square test, sonographic findings significantly associated with SBO needing surgery were: bowel loops diameter $>35 \mathrm{~mm}$, absence of mucosal folds, free peritoneal fluid and absence of peristaltic movements.

Conclusion: Ultrasonography should be considered a useful diagnostic tool in the decision-making algorithm of SBO. In this study, four findings-bowel loop diameter $>35 \mathrm{~mm}$, absence of mucosal folds, free peritoneal fluids and absence of peristalsis-were shown to be predictive of SBO needing surgery.

References: Ogata M, Mateer JR, Condon RE. Prospective evaluation of abdominal sonography for the diagnosis of bowel obstruction. Ann Surg. 1996;223(3):237-41. Jang TB, Schindler D, Kaji AH. Bedside ultrasonography for the detection of small bowel obstruction in the emergency department. Emerg Med J. 2010. Maglinte DD, Howard TJ, Lillemoe KD, Sandrasegaran K, Rex DK. Small-bowel obstruction: state-of-the-art imaging and its role in clinical management. Clin Gastroenterol Hepatol. 2008;6(2):130-9.

Disclosure: No significant relationships.

\section{1}

US Driven Protocol For Right Lower Quadrant Abdominal Pain: Cost-Effective And Faster

\author{
M. Zago, D. Mariani, H. Kurihara, A. Casamassima, F. Luzzana, \\ M. Ripamonti
}

General And Emergency Surgery, Istituto Clinico Città Studi, Milano, Italy

Introduction: In the era of defensive medicine and advanced imaging the abdominal pain algorithm is often time-consuming and expensive. There is a trend to confirm suspected diagnosis always with the most sensitive study. We prospectively analyzed the impact of systematic use of surgeon-performed US in managing lower right quadrant (RLQ) pain in ED.

Materials and methods: Patients (pts) admitted with RLQ pain to our ED from February to September 2010 were included. US was performed immediately after the clinical exam (before lab results) if the surgeon in shift was trained. Non US-trained surgeons applied a traditional work-up (Lab, plain films, US and/or CT requested to imaging specialists). The following items were analyzed: time to surgical diagnosis (visit-to-indication-for-surgery), rates of CT exams, non-surgical admissions, readmissions for surgery within a week and costs for the two algorithms. X-square test was used for statistical analysis.

Results: We analyzed 135 pts admitted in ED for RLQ pain. Eightyeight pts were included in the US-driven protocol (group A), 47 in the traditional one (group B). Forty-eight pts were hospitalized (43 of whom submitted to operation, 5 discharged the day after), 87 discharged from the ED. Results were compared for group A and group $\mathrm{B}$ respectively. Mean time to surgical diagnosis was 12 versus $106 \min (\mathrm{p}<0.001)$, rate of CT exams 12 versus $62 \%(\mathrm{p}<0.03)$, non-surgical hospitalizations 0 versus $10.6 \%$ ( $p<0.05$ ), readmissions for surgery 1 versus $6 \%(\mathrm{p}=\mathrm{NS})$, mean cost for the two algorithms 118 vs. $350 €$ (according to the current regional reimbursement system, excluding ED extra-time and inappropriate hospitalizations).

Conclusion: US-driven protocol for RLQ pain was safer, cheaper, faster and more cost-effective than the traditional one. Modern health systems needs a proper training programs on clinical US to increase accuracy and cost containment.

References: 1. Binnebsel M, et al. Chirurg 2009;80(7):579-87. 2. Jo YH, et al. Am J Emerg Med. 2010;28(7):766-70.

Disclosure: No significant relationships. 


\section{2}

\section{CONSERVATIVE MANAGEMENT OF PERIAMPULLARY PERFORATION DUE TO ENDOSCOPIC SFINCTEROTOMY AT ERCP: EXPERIENCE IN RECENT 4 YEARS}

\section{H. Yanar, S. Sarici, E. Sivrikoz, C. Ertekin, K. Günay, R. Güloglu}

General Surgery, Istanbul Faculty of Medicine, Istanbul, Turkey

Introduction: Due to high rate of therapeutic ERCP recently, the incidence of iatrogenic intestinal perforation due to papillotomy is increased. If perforations are detected early, conservative management is $80-90 \%$ successful. We hereby report a series of cases by whom ERCP and endoscopic sfincterotomy (EST) resulted in perforation and were managed conservatively.

Materials and methods: Patients who presented to our emergency service with suspicion of perforation after ERCP were treated according to their physical exam, hemogram, biochemistry results, plain radiograms and computerized tomography scans. Retroperitoneal contrast leakage and peritoneal sign were considered indications for emergent surgical intervention. Patients without peritoneal signs routinely underwent radiologic studies to confirm or exclude the suspicion of retroperitoneal duodenal perforation. Subcutaneous emphysema, retroperitoneal air, pneumothorax, and pneumomediastinum resulted in conservative management. During the conservative management patients were placed on nasogastric tube drainage, parenteral fluid replacement and wide-spectrum antibiotic therapy.

Results: Between February 2007 and October 2010, 967 ERCP were performed at our institution and in 20 patients retroperitoneal perforation was diagnosed after EST. All patients had extensive retroperitoneal air, but in patients eight subcutaneous emphysema and in three patients pneumomediastinum were detected at presentation. Co-existent morbidities were pneumothorax in two patients, pancreatitis in five patient, retroperitoneal contrast leakage with hard abdominal signs in one patient. One patient with pneumothorax was placed on closed tube drainage, the other was managed conservatively due to $<20 \%$ pneumothorax. Five patients with abdominal hard signs were operated. In three patients no perforation was found. After Kocher maneuver retroduodenal perforation was found in two patients and duodenorrhaphy, stone extraction from common bile duct and t-tube drainage was performed. Other 15 patients were treated conservatively. Among these two patient operated on diagnosis of retroperitoneal abscess, one of them died because of septic shock. Conclusion: Traditionally, duodenal perforations after ERCP have been managed surgically. Selected cases can be successfully treated conservatively.

Disclosure: No significant relationships.

\section{3}

\section{LEARNING CURVE FOR SINGLE-PORT LAPARASCOPIC APPENDECTOMY: A CASE-CONTROL STUDY IN A TAIWAN MEDICAL CENTER}

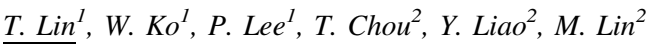

${ }^{1}$ Department Of Traumatology, National Taiwan University Hospital, Taipei, Taiwan, ${ }^{2}$ Surgery, National Taiwan University Hospital, Taipei, Taiwan

Introduction: An attempt to search for less invasive procedure of laparoscopic appendectomy for acute appendicitis has undertaken. Single-port laparoscopic appendectomy (SIPLA) using umbilicus as a port entry has been proposed effective and efficient in managing acute appendicitis. The study was intended to delineate the learning curve of SIPLA for non-complicated appendicitis.

Materials and methods: From July 2009 to June 2010, a total of thirty patients underwent SIPLA for non-complicated appendicitis was enrolled in the study. Another 30 patients of conventional threeport laparoscopic appendectomy in the same time were collected as a control group. Two separate surgeons performed all the operation procedures in each group. The demography of the patients such as body mass index (BMI), intra-operative parameters including operating time (OpT) and all the instruments used, were recorded. The patients in SIPLA group were sequentially sub-grouped into three groups. OpT was used as an indicator for analyzing learning curve.

Results: The patient characteristics were similar among all comparative groups. Using OpT as an indicator, we found after ten patients, a significant decrease in OpT was achieved (first 10 cases: $102.6 \mathrm{~min}$, second: $79.5 \mathrm{~min}$, third: $75.3 \mathrm{~min}$, control group: $72.0 \mathrm{~min}, \mathrm{p}<0.01$ by one way ANOVA). Using multivariate regression model, the OpT of first ten SIPLA cases was significantly longer $(28 \mathrm{~min})$ than the other group of patients, after adjusted for BMI $>21$, pathology of suppurative appendicitis, and endoloop use $(\mathrm{p}<0.001)$.

Conclusion: The learning curve of single-port laparoscopic appendectomy is most critical in first ten cases. A minimum of ten cases is required to overcome the learning difficulty.

References: Lin, YY, Shabbir A, So JB. Laparoscopic appendectomy by residents: evaluating outcomes and learning curve. Surg Endosc, 2010;24(1):125-30. Chouillard E, et al. Single-incision laparoscopic appendectomy for acute appendicitis: a preliminary experience. Surg Endosc. 2010.

Disclosure: No significant relationships.

\section{4}

\section{LOW-PRESSURE LAPAROSCOPIC CHOLECYSTECTOMY FOR ACUTE CHOLECYSTITIS IN “HIGH RISK” PATIENTS}

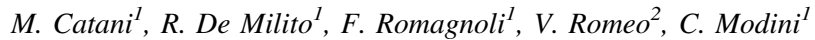

${ }^{1}$ Dea-emergency Department, Policlinico Umberto I, Rome, Italy, ${ }^{2}$ Dea-emergency Department, Sapienza Università di Roma, Rome, Italy

Introduction: The intra-abdominal pressure used for laparoscopic cholecystectomy (LC) is usually $12-15 \mathrm{mmHg}$, the pneumoperitoneum with carbon dioxide has a significant effect on both cardiovascular and respiratory function. These effects are transient in young healthy patients but it may be dangerous in "high risk" patients with poor reserve. The latter is true especially in those patients treated in emergency, where the post operative pain control represents one of the major goal to achieve. Previous studies have demonstrated that physiologic effects of pneumoperitoneum at $6-8 \mathrm{mmHg}$ permit to perform the laparoscopic procedure also in these patients in elective setting. The aim of this study is to assess the feasibility of performing a low-pressure $\mathrm{LC}$ at $6-8 \mathrm{mmHg}$ in patients treated in emergency setting for acute cholecystitis, where the available space for dissection is required for a safe procedure.

Materials and methods: From January 2006 to October 2010, 130 patients admitted to the Emergency Department DEA II of Policlinico Umberto I-Rome for acute cholecystitis and treated with early LC have retrospectively reviewed, 23 were classified as "high risk" and have been operated with low-pressure partially downwards LC at 6-8 $\mathrm{mmHg}$. Duration of procedure, conversion rate, complication and post operative length of stay were analyzed.

Results: All of 23 procedure were completed laparoscopically with no conversion. The mean duration of procedure was $79 \min (60-150)$, comparable with what observed in same procedure at normal pneumoperitoneum. $1(4.3 \%)$ post operative complication consisting in 
rectal muscle haematoma was reported, the mean post operative hospital stay was 3.2 days (3-8).

Conclusion: $\mathrm{LC}$ at $6-8 \mathrm{mmHg}$ is safe and effective also in high risk patients treated for acute cholecystitis with no increase of conversion rate and worsening of other clinical outcomes, in the hand of experienced surgeon. Although the increase in technical difficulty because of smaller space for dissection, the appropriate technique and low-pressure pneumoperitoneum allow to treat also patients at "high risk".

References: 1. Sandhu T, Yamada S, Ko-iam W. Low-pressure pneumoperitoneum versus standard pneumoperitoneum in laparoscopic cholecystectomy, a prosecutive randomized trial. Surg Endosc. 2009;23:1044-47. 2. Ekici Y, Bozbas H, Haberal M. Effect of different intra-abdominal pressure levels on QT dispersion in patients undergoing laparoscopic cholecystectomy. Surg Endosc. 2009;23:2543-49. 3. Catani M, Guerricchio R, De Milito R, Simi M. "Low pressure" laparoscopic cholecystectomy in high risk patients (ASA III and IV): our experience. Chirurgia Italiana. 2004;56:71-80. 4. Joshipura VP, Thakker R et al. A prospective randomized controlled study comparing low pressure versus high pressure pneumoperitoneum during laparoscopic cholecystectomy. Surg Laparosc Endosc Percutan Tech;19:234-40. 5. Catani M, Modini C. Laparoscopic cholecystectomy in acute cholecystitis a proposal of safe and effective technique. Hepato-Gastroenterology. 2007;54:2186-91.

Disclosure: No significant relationships.

\section{5}

\section{CAUSES OF THERAPEUTIC FAILURE IN SECONDARY PERITONITIS IN PATIENTS WITH ADEQUATE EMPIRIC ANTIBIOTIC TREATMENT AND CORRECT SOURCE CONTROL}

\section{E. Membrilla Fernández ${ }^{1}$, J.J. Sancho ${ }^{2}$, M. Girvent Montllor ${ }^{2}$, G. Adrio $^{2}$, D. Fraccalvieri ${ }^{2}$, J.J. Inigo Noain ${ }^{2}$, A. Monzón ${ }^{2}$, A. Luna Aufroy ${ }^{2}$, A. Sitges Serra ${ }^{2}$}

${ }^{1}$ General Surgery And Digestive, Hospital Universitario Del Mar, Barcelona, Spain, ${ }^{2}$ Hospital del Mar, Barcelona, Spain

Introduction: Effective source control and adequate empiric therapy are critical in secondary peritonitis. A significant proportion of patients with source control and adequate empiric therapy suffer a therapeutic failure (TF) defined as surgical site infection (SSI) or exitus. This study was designed to analyze its causes.

Materials and methods: An open observational prospective multicentric study at 24 Spanish Hospitals (04/2005-08/2007). There were 362 patients with secondary peritonitis; 262 (72.4\%) with communityacquired peritonitis and $100(27.6 \%)$ with postoperative peritonitis (POP). Patients were only included if at least two quadrants of the abdomen were affected and with a positive peritoneal culture. Severity scores, origin of peritonitis, surgical technique, genus, species and microbial sensitivity to antibiotics, (SSI) and mortality were recorded. Results: There were 204 patients (56\% of the total), with adequate empiric antibiotic therapy and effective source control (98 women) with a mean age of 60 years $\mathrm{IC}_{95 \%}=[57.8-63.1]$. TF was higher in POP (58 vs. $37 \%$; $\mathrm{P}=0.017$ ). The TF group had more patients with ASA III/IV (45 vs. $28 \%$; $\mathrm{P}=0.0039$ ), a higher prevalence of cardiac insufficiency ( 13 vs. $5 \%$; $\mathrm{P}=0.03$ ) and a higher Mannheim Peritonitis Index $(27 \pm 7$ vs. $22 \pm 7 ; \mathrm{P}=0.001)$. There were not significant differences in the APACHE II or Charlson scores. The TF group had more patients with preoperative peritonitis lasting over 24 h $(77$ vs. $63 \% ; \mathrm{P}=0.043)$ and more organic failure (53 vs. $15 \%$; $\mathrm{P}=0.001)$, more tachycardia $(99 \pm 19$ vs. $93 \pm 17 ; \mathrm{P}=0.019)$ and taquipnea ( $22 \pm 7$ vs. $19 \pm 7 ; \mathrm{P}=0.038$ ), higher C-reactive protein levels $(18 \pm 25$ vs. $39 \pm 28 \mathrm{mg} / \mathrm{L} ; \mathrm{P}=0.003)$ and deeper hypoalbuminemia $(2.68 \pm 0.9$ vs. $3.10 \pm 0.8 \mathrm{~g} / \mathrm{dL} ; \mathrm{P}=0.021)$ than the group with no complications. Patients with therapeutic failure had higher prevalence of anaerobic bacteria (44 vs. $32 \% ; \mathrm{P}=0.049)$ and Enterococcus spp. (24 vs. $12 \% ; \mathrm{P}=0.018)$.

Conclusion: Cardiac insufficiency and hypoalbuminemia were associated to postoperative septic complications. The elevated Mannheim Peritonitis Index is dependant of the existence of organ failure. Disclosure: No significant relationships.

\section{2}

\section{EARLY RECOGNITION OF VITALLY THREATENED PATIENTS: EVALUATION OF THE PERFORMANCE OF A NEW EARLY WARNING SCORE}

D. Den Hartog
E.M.M. Van Lieshout
,

${ }^{1}$ Surgery-traumatology, Erasmus MC, Rotterdam, Netherlands,

${ }^{2}$ Surgery, Erasmusmc Rotterdam, Rotterdam, Netherlands

Introduction: Early warning scores may aid prediction of major adverse events in hospitalized patients. Recently, a new early warning score (the 'SIT score') was introduced in the Netherlands. The aim of the present study was to assess the relationship between the SIT score and the occurrence of major adverse clinical events during hospitalization of patients admitted to a general and trauma surgery ward.

Materials and methods: Prospective cohort study. All 572 consecutive patients admitted between March 2009 and September 2009 to the general and trauma surgery ward of our university medical center were included. Follow-up was limited to the time the patient was hospitalized. Logistic regression analysis was used to assess the relationship between the SIT score and the occurrence of the combined endpoint consisting of death, reanimation, ICU admission, emergency surgery, and severe complications. The performance of the SIT score was analyzed using sensitivity, specificity, positive and negative predictive values, and receiver-operator characteristics (ROC) curves.

Results: During a median follow-up of 4 days, 46 (8\%) patients reached the combined endpoint. Logistic regression analysis showed that a SIT score $\geq 3$, adjusted for baseline American Society of Anesthesiologists classification, was associated with a significantly higher risk of reaching the combined endpoint (odds ratio 11.3, 95\%CI 5.5-22.9). When considering a SIT score $\geq 3$ a positive test result, the area under the ROC curve was $85 \%$, sensitivity was $74 \%$, and specificity was $82 \%$.

Conclusion: A SIT score $\geq 3$ is an independent predictor of major adverse events in patients admitted to a general and trauma surgery ward. Disclosure: No significant relationships.

\section{SHOULDER FRACTURE-DISLOCATION}

\section{6}

\section{VAC INSTILL ${ }^{\circledR}$ THERAPY IN THE MANAGEMENT OF OSTEOMYELITIS}

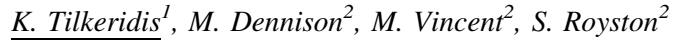

${ }^{1}$ Orthopaedic, Sheffield Teaching Hospitals, Northern General Hospital, Sheffield, United Kingdom, ${ }^{2}$ Orthopaedics, northern general hospital, sheffield, United Kingdom 
Introduction: V.A.C. Instill ${ }^{\odot}$ is an innovative system that combines the benefits of V.A.C. ${ }^{\odot}$ Therapy with Instillation therapy with antibiotics or antiseptics. Our aim is to show that using this system following a surgical debridement, the combination of irrigation with antibiotics and removal of exudates, eradicates the infection effectively and prepares the cavity for bone grafting.

Materials and methods: From April 2007 up to date 17 patients treated with this method. Full data for ten patients is available. All were males with a mean age of 39 years (range 20-56). Six were smokers and the duration of the symptoms ranged from 5 months to 10 years. Seven patients suffered from tibial osteomyelitis, one had femoral stumb osteomyelitis, one a distal radius osteomyelitis and one had calcaneal osteomyelitis. All patients had bone exposed after debridement. Treatment protocol included instillation of antibiotics, locally as indicated per sensitivity test, regularly evaluation of the inflammatory markers and cultures from the deep sponge.

Results: The average duration of the VAC Instill therapy was 32 days (range 20-71 days), and the average time to wound closure was 39 days (22-90). One patient developed local Vancomycin sensitivity. All patients but two, treated successfully with the method. From the two patients who failed, one eventually treated with the Lautenbach procedure and one sustained a bellow knee amputation.

Conclusion: VAC Instill therapy is a promising method in the treatment of chronic osteomyelitis ( $80 \%$ success). Our results are comparable to other techniques, like the Lautenbach procedure 1 . These are only early results; further studies are needed to back these findings.

Reference: 1. Lautenbach E. Proceedings: Chronic osteomyelitis: irrigation and suction after surgery. The Journal of Bone and Joint Surgery. Br. Vol. 1975;57(2):259.

Disclosure: No significant relationships.

\section{7}

\section{CLINICAL OUTCOME AFTER MINIMAL INVASIVE PLATE OSTEOSYNTHESIS FOR PROXIMAL HUMERUS FRACTURES}

\author{
Y.P. Acklin ${ }^{1}$, K. Stoffel ${ }^{1}$, C. Sommer ${ }^{2}$
}

${ }^{1}$ Surgical Department/trauma Unit, Kantonsspital Graubünden, Chur, Switzerland, ${ }^{2}$ Chirurgie, Kantonsspital Graubuenden, Chur, Switzerland

Introduction: Plate osteosynthesis of proximal humerus fractures is a well established treating option. The standard approach is the deltapectoral, but few data using the minimal invasive antero-lateral delta split approach exists. Our goal was to evaluate the clinical outcome of minimal invasive plate osteosynthesis for proximal humerus fractures. Materials and methods: From 12.2007 until 04.2009, 121 patients with 122 acute proximal humerus fractures were managed with a minimal invasive locking plate osteosynthesis and a minimal followup of 1 year. Two patients died, 5 were in a nursing home, and 49 were lost to follow-up due to foreign residency or change of address. Complete data was available for 65 patients (mean age 61 years) with 66 fractures operated by 15 different surgeons. Outcomes were assessed with radiography and functional outcome scores.

Results: At follow-up the median Constant score was 81 (range from 38 to 91 ) corresponding to $95 \%$ of the opposite arm. The results were excellent in 52 patients $(79 \%)$, good in 8 patients $(12 \%)$, fair in 4 patients $(6 \%)$ and poor in 2 patients $(3 \%)$. No significant differences in the ANOVA calculation were stated comparing the Constant-Murley scores achieved in AO-type $\mathrm{A}, \mathrm{B}$ or $\mathrm{C}$ fractures and no significant correlation between patient age and adjusted outcome score could be found. Implant related complications e.g. screw perforation occurred in five $(8 \%)$ patients, avascular necrosis was observed in five patients (8\%), in two patients $(3 \%)$ the ventral branch of the axillary nerve were damaged without any clinical consequents. One deep infection was treated with debridement and antibiotics.

Conclusion: Minimal invasive plating of the proximal Humerus allows good reduction and is a feasible approach in a teaching hospital. Complications rates and functional outcome are in some aspects superior to comparable published data using the delta-pectoral approach.

Disclosure: No significant relationships.

\section{8}

\section{INTRAMEDULLARY NAILING OF PROXIMAL HUMERUS} FRACTURES-PROSPECTIVE STUDY

\section{Kloub, P. Kopacka, K. Holub}

Traumatology, Hospital Ceske Budejovice, Ceske Budejovice, Czech Republic

Introduction: Stable osteosynthesis of proximal humerus fractures with angle-stable IM nails brings to this area new quality of reduction and osteosynthesis. 5 years long experience changed in our department strategy of the treatment of these fractures.

Materials and methods: To prospective study were involved 211 patients with proximal humerus fracture type 11 after AO ASIF classification, treated with proximal humerus nail Targon $\mathrm{PH}$ (Aesculap) inserted from deltoid-splitt approach. Average age of the patients was 67 years. Fractures were classified according to AO ASIF and Neer classification. Patients were examined clinically and radiologically after standard period of 1, 2, 4, 6 and 12 months after injury. Relative Constant score was evaluated after 6 and 12 months. Objective evaluation criteria were: time of the healing, $\mathrm{x}$-ray quality of the reduction and osteosynthesis, functional result-relative Constant score-after 6 and 12 months, and rate of complications especially humeral head necrosis and loosening of the implant.

Results: 201 fractures healed (95\%). Average time of healing was 38 days. The average relative Constant score was 72 pts. after 6 months and 83 pts after 12 months. Two pseudoarthroses of the surgical neck and two cases of deep infection were observed. Partial or total humeral head necrosis was observed in 15 cases (7\%), mainly in group 11C AO classification.

Conclusion: Osteosynthesis of the proximal humerus fractures with proximal humeral nail Targon PH is safe method, with low rate of complications. Deltoid-splitt approach allows new level of the quality and possibility of the reduction. Design of the nail allows then treatment of all types of proximal humerus fractures, including huge dislocated 4-part, head splitt fractures and fractures-dislocations. Rate of the loosening of reduction or implant failure is low. Very important for good functional result is quality and length of the physiotherapy, for best result performed most likely up to 1 year.

Disclosure: No significant relationships.

\section{9}

\section{INTRODUCTION OF A FRACTURE MODEL FOR THE PROXIMAL HUMERUS-PILOT STUDY}

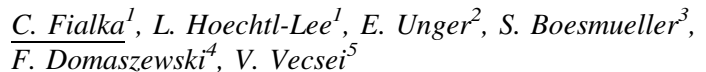

${ }^{1}$ Department of Traumatology, Medical University Vienna, Vienna, Austria, ${ }^{2}$ Applied Physics, Medical University of Vienna, Vienna, 
Austria, ${ }^{3}$ Department of Trauma Surgery, Medical University of Vienna, Vienna, Austria, ${ }^{4}$ Traumatology, Medical University Vienna, Austria, Vienna, Austria, ${ }^{5}$ Department Of Traumatology, Med University Vienna, Vienna, Austria

Introduction: Therapeutic algorithms for proximal humeral fractures are mainly based upon clinical data. Biomechanical studies on the other hand normally use osteotomy models, that do not allow to take the compression of the cancellous bone of the head fragment into account, when studying implant behavior, for example. The present Pilot Study tries to clear the mechanical properties of the different fracture types, using a cadaveric model. This model will allow to reproduce specific fracture types, that can be used to conduct further biomechanical examinations.

Materials and methods: Twenty two human shoulder joints were used to evaluate the acceleration and necessary energy to produce a fracture of the metaphyseal segment of the humerus. The custom made test machine allowed to create different joint position representing forward flexion (F) and sagital abduction (S). During impact all acting forces were monitored within a 3 dimensional pressure sensor system (measurement rate 2,000/s) and additionally documented using a high speed video camera.

Results: This pilot data shows that intra-articular fractures of the proximal humerus only could be produced in four glenohumeral positions ( $\mathrm{F} / \mathrm{S} 0^{\circ} / 90^{\circ}, 0^{\circ} / 60^{\circ}, 30^{\circ} / 60^{\circ}$ and $30^{\circ} / 90^{\circ}$, respectively). All other joint positions lead into glenoid fractures, no fractures or simple dislocations. Four part fractures were only seen in $0^{\circ}$ Flexion and $90^{\circ}$ Abduction, three part fractures only in the position $\mathrm{F} / \mathrm{S} 30^{\circ} / 60^{\circ}$, respectively.

Conclusion: These preliminary results show that it is possible to reproducibly create specific fracture types of the proximal humerus, if the necessary kinetic energy and direction of applied forces is used. Further studies will have to prove that this as reliable, if traction forces along the rotator cuff tendons are additionally applied. Disclosure: No significant relationships.

\section{0}

\section{PROXIMAL HUMERAL FRACTURES TREATED WITH TELEGRAPH ${ }^{\circledR}$ NAIL-A 47 CASES REPORT}

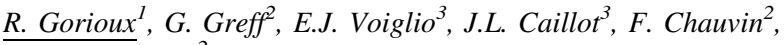 F. RONGIERAS}

${ }^{1}$ Service De Chirurgie Orthopédique, HIA Desgenettes, Lyon, France, ${ }^{2}$ Service De Chirurgie Orthopédique, HIA Desgenettes, LYON, France, ${ }^{3}$ Ucu, Centre hospitalier Lyon Sud, PIERRE BENITE, France

Introduction: This retrospective study is about the assessment of the clinical and radiological results of 47 proximal humeral fractures treated with TELEGRAPH ${ }^{\circledR}$ nail.

Materials and methods: This is a 4 years retrospective study, about 47 proximal humeral fractures treated with telegraph nail in lyon's military hospital, with a 20 month average stand back.

Results: $75 \%$ of the patients have been clinically assessed with a mean 20 month stand back. The average age was 67.5. Regarding two parts Neer's fractures, weighted Constant score were excellent $(m=90)$. Three and four parts Neer's fractures obtained average score $(\mu=83$ and $\mu=79)$. Regarding four parts Neer's fractures: in the under-sixties group, weighted Constant score were excellent $(\mathrm{m}=90)$ while they were poor in the over-sixties group $(\mathrm{m}<65)$. Conclusion: Three and four parts Neer's fractures treated with TELEGRAPH $^{\circledR}$ nail obtained a very satisfactory assessment. On the other hand, indication seems to be more debatable regarding four parts fractures among elderly osteoporotic people where arthroplasty should be debated.

Disclosure: No significant relationships.

\section{CENTRALIZATION CRITERIA}

\section{1}

\section{INTERNATIONAL, MULTICENTER FEASIBILITY STUDY OF CORE DATA COLLECTION IN SEVERELY INJURED PATIENTS USING A UNIFORM TRAUMA TEMPLATE}

\author{
K.G. Ringdal ${ }^{1}$, H.M. Lossius ${ }^{1}$, J.M. Jones ${ }^{2}$, J.M. Lauritsen ${ }^{3}$, \\ T.J. Coats ${ }^{4}$, R. Lefering ${ }^{5}$, S. Di Bartolomeo ${ }^{6}$, C.S. Palmer ${ }^{7}$, \\ D.J. Dries ${ }^{8}$, K. Søreide
}

${ }^{1}$ Department Of Research, Norwegian Air Ambulance Foundation, Drøbak, Norway, ${ }^{2}$ Mathematics Department, Keele University, Keele, UK, ${ }^{3}$ Odense University Hospital, Odense, Denmark, ${ }^{4}$ Leicester Royal Infirmary, Leicester, UK, ${ }^{5}$ Institute For Research In Operative Medicine, University Witten/Herdecke, Cologne, Germany,

${ }^{6}$ University of Udine, Udine, Italy, ${ }^{7}$ The Royal Children's Hospital, Melbourne, Australia, ${ }^{8}$ University of Minnesota, Minneapolis, MN, USA, ${ }^{9}$ Department Of Surgery, Stavanger University Hospital, Stavanger, Norway

Introduction: Global definitions for documenting, reporting and comparing data for severely injured trauma patients are lacking. We aimed to evaluate the feasibility of sampling core trauma data using a consensus template among several international trauma registries and institutions.

Materials and methods: International trauma registries and institutions representing three continents were invited to participate. Participants were asked to submit data on 50 trauma patients using the core Utstein Template during a preset period. All consecutively and directly admitted trauma patients with New Injury Severity Score (NISS) of $>15$ were eligible for inclusion. Main outcome variables were data completeness rate, rate of data differences, and rate of data collection difficulties.

Results: A total of 24 institutions from Europe $(n=20)$, North America $(\mathrm{n}=3)$, and Australia $(\mathrm{n}=1)$ submitted data on a total of 965 patients, of which $783(81 \%)$ were eligible for inclusion. Median age was 41.0 [interquartile range [IQR] 24-60], with men representing the majority $(\mathrm{n}=572 ; 73.1 \%)$. Median NISS was 27 (IQR $20-38)$, with blunt trauma predominating $(\mathrm{n}=713 ; 91.1 \%)$. Out of the 40 Utstein variables, 14 variables were collected by all participants. Eleven $(46 \%)$ institutions applied different outcome measures than the one recommended by the Utstein template. 17 (71\%) of the institutions used the Abbreviated Injury Scale (AIS) version recommended. Three data variables (Age, Gender, AIS) were completely documented in all 783 patients. Excellent completion (>90\%) was recorded for 20 of the variables, and good completion (81-90\%) for six variables. Poor completion $(<40 \%)$ was seen for one variable. The prehospital Revised Trauma Score variables were less complete than in-hospital data (80.0 vs. $92.8 \%)$.

Conclusion: Overall feasibility of documenting and sharing patient level data using the Utstein Template as a standard was achieved, with the majority of the core variables being $>90 \%$ complete. The template should allow data comparison across institutions and geographic boundaries.

Disclosure: No significant relationships. 


\section{2}

\section{IS A MAXIMUM REVISED TRAUMA SCORE A SAFE TRIAGE TOOL FOR HELICOPTER EMERGENCY MEDICAL SERVICES (HEMS) CANCELLATIONS?}

\author{
G.F. Giannakopoulos $^{1}$, T.P. Saltzherr ${ }^{2}$, W.D. Lubbers ${ }^{1}$, \\ H.M.T. Christiaans ${ }^{3}$, P. van Exter ${ }^{4}$, E.S.M. de Lange-de Klerk ${ }^{5}$, \\ F.W. Bloemers ${ }^{1}$, W.P. Zuidema ${ }^{1}$, J.C. Goslings ${ }^{2}$, F.C. Bakker ${ }^{1}$
}

${ }^{1}$ Trauma Surgery, VU University Medical Centre, Amsterdam, Netherlands, ${ }^{2}$ Trauma Unit Department Of Surgery, Academic Medical Center, Amsterdam, Netherlands, ${ }^{3}$ Anesthesiology, VU University Medical Centre, Amsterdam, Netherlands, ${ }^{4}$ Regional Medical Manager Of Ambulance Care, GGD, Amsterdam, Netherlands, ${ }^{5}$ Department Of Epidemiology And Biostatistics, VU University Medical Center, Amsterdam, Netherlands

Introduction: The RTS is used worldwide as a triage tool for trauma patients. Several studies have shown that the reliability of the RTS is high in trauma outcomes. In the Netherlands, HEMS are mostly used for delivery of specialized trauma teams on-scene. In our trauma system, the EMS crew performs triage on-scene and cancels the HEMS-dispatch if deemed unnecessary. In this study we assessed the ability of a maximum on-scene RTS $(=12)$ to be used as a triage tool for HEMS cancellation.

Materials and methods: All patients with a maximum on-scene RTS after blunt trauma who were presented in the Trauma Resuscitation room of two Level-1 trauma centers during a period of 6 months, were included. Information concerning vital parameters, severity and localization of the injuries, and the course were analyzed. Major trauma patients were classified using standard outcome parameters.

Results: Four-hundred and forty blunt trauma patients having a maximum RTS were included. Despite having a maximum RTS onscene, almost $16 \%$ concerned major trauma patients. Significant differences between major and minor trauma patients were detected in on-scene time, on-scene HEMS care, RTS deterioration and length of hospital stay. Major trauma patients showed significantly more scooter and motorcycle accidents, falls, injuries to the chest, abdomen, and lower extremities.

Conclusion: The RTS alone is not a reliable triage tool for HEMS cancellations in our trauma system and will lead to a considerable rate of undertriage. Other criteria based on physiological and anatomical parameters should be developed to minimize triage errors.

Reference: Champion, et al. A revision of the Trauma Score. J Trauma. 1989;29:623-9.

Disclosure: No significant relationships.

\section{3}

\section{NIRS (NEAR-INFRARED SPECTROSCOPY) MEASUREMENT OF PERIPHERAL TISSUE OXYGENATION USING THE NONIN/EQUANOX-7600 IS NOT DISTURBED IN HELICOPTER-EMS (HEMS) ENVIRONMENT}

\section{P. Schober, L.A. Schwarte}

Anaesthesiology, VUmc university medical center, Amsterdam, Netherlands

Introduction: Preservation of adequate peripheral tissue oxygenation remains crucial in treatment of critical patients. Traditional monitoring methods address systemic oxygenation, frequently not allowing conclusions on tissue oxygenation. (1) In addition, in HEMS-settings traditional monitoring may become disturbed by helicopter-inherent factors, e.g., cabin vibrations. The portable Nonin/Equanox-7600 NIRS-monitor appears attractive to measure peripheral tissue oxygenation in EMS, given the size, durability and suggestion that Equanox-technology is specifically artifact-resistant. To our knowledge, no data are available on the impact of a helicopter environment (e.g., vibrations) on NIRS-measurement of peripheral oxygenation $(2,3)$.

Materials and methods: The study was conducted at a level-1 university trauma-center (VUmc, Amsterdam, The Netherlands). To limit fluctuations in tissue oxygenation, the study was performed on healthy volunteers ( $n=6$ measurements; informed consent), positioned in the HEMS-helicopter patient cabin (EuroCopter; EC-135). NIRS was measured by a Nonin/Equanox-7600 monitor with 8000CA-probes placed on the forearm/thenar of the dominant arm. Protocol: After sufficient equilibration ("BASELINE"), helicopter engines were started subsequently ("ENGINES") and kept running for several minutes. After engine shut-down, subsequent "POST"data were collected.

Results: Inter-individual differences in "BASELINE" (rSO2range $\sim 75 \%-85 \%$ ) were noted based on sensor location, however intra-individual NIRS-measurements by Nonin/Equanox-7600 were not systematically affected by helicopter artifacts, e.g., cabin vibrations ("ENGINES"). In addition, no helicopter-instruments were apparently disturbed by the Nonin/Equanox-7600-monitor.

Conclusion: Our findings suggest that NIRS-measurements by Nonin/Equanox-7600 are not disturbed by HEMS-typical confounders, e.g., engines start-up/shut-down and helicopter vibrations. Therefore, the Equanox-technology based NIRS-system could become a valuable addition in HEMS-settings. Further studies will have to define how NIRS-monitoring may support (HEMS-)therapy and ultimately (HEMS-)outcome.

References: (1) Schwarte, et al. Br J Anaesth. 2010. (2) BurilloPutze, et al. Air Med J. 2002 (3) Schwarte et al. Euro J Anaesth. 2008. Disclosure: No significant relationships.

\section{4}

\section{PREHOSPITAL HYPOTENSION, TRIAGE AND MORTALITY: WHICH THRESHOLD?}

A. Volpi, P. Orlandi, C. Bricchi, S. Grossi, E. Sani, F. Martello, L. Cattani, M. Mergoni, D. De Matteis

1st Anaesthesia And Intensive Care, Azienda OspedalieroUniversitaria di Parma, Parma, Italy

Introduction: Early and accurate triage of trauma patients is critical to patient survival. The prehospital systolic blood pressure (PSBP) threshold of $<90 \mathrm{mmHg}$ has been suggested to identify haemodynamic unstable patients and determine the injury severity. A PSBP $<90 \mathrm{mmHg}$ is a late sign of hemorrhagic shock and little data exist to support this arbitrary value.

Materials and methods: A 4 years retrospective analysis of a Level I trauma centre registry was undertaken. All trauma patients admitted to the Intensive Care Unit were included. The thresholds of PSBP were: $<90,91-100,101-120,>120 \mathrm{mmHg}$, and the relationship between threshold and outcomes was explored.

Results: The mean age was 46 years and mean ISS 26. Patients with lower PSBP levels were more likely to be hypotensive on arrival to the ED, with worsening base deficit, and higher Injury Severity Score. The mortality was significantly higher in the group with PSBP $<90$ respect the other groups $(47,12.5,11.1,14.9 \%)$. An increase in 
mortality has been observed in the group with PSBP > $120(14.9 \%)$. This group was characterized from a significantly higher mean age (67 years).

Conclusion: The data suggest that the PSBP $<90 \mathrm{mmHg}$ in young adult trauma patients is an efficient threshold to identify those who need a prompt activation of trauma centre. A different higher threshold need to be identified for older patients .

Reference: Prehospital Hypotension Redefined. Bruns at al. J Trauma. 2008;65:1217-21. Hypotension begins at $110 \mathrm{~mm} \mathrm{Hg}$ : redefining "Hypotension" with Data. Eastridge et al. J Trauma. 2007;63:291-9.

Disclosure: No significant relationships.

\section{5}

\section{REPORT ON 566 WORK-RELATED INJURIES REQUIRING HOSPITALIZATION IN GREECE}

\section{E. Kleidi ${ }^{l}$, M. Riga ${ }^{l}$, P. Drimousis ${ }^{2}$, A. Larentzakis $^{2}$, M. Natoudi ${ }^{l}$, D. Theodorou ${ }^{3}$, S. Stergiopoulos ${ }^{4}$, S. Katsaragakis ${ }^{2}$}

${ }^{1}$ Surgical Intensive Care Unit, Hippocration General Hospital, Athens, Greece, ${ }^{2}$ Surgical Intensive Care Unit, Hippocration General Hospital, Athens, Greece, ${ }^{3}$ Surgical Intensive Care Unit, University of Athens, Athens, Greece, ${ }^{4}$ Surgical Clinic, Attikon General Hospital, Athens, Greece

Introduction: Work-related injuries constitute a significant part of all trauma incidents with considerable impact on overall morbidity and mortality. The aim of this study is to assess work-related injuries in Greece, where no trauma system is applied.

Materials and methods: Data from thirty health care facilities receiving trauma throughout the country were prospectively reported for 1 year time. Inclusion criteria were defined as trauma patients that required admission, transfer to another centre, arrived dead or died in the emergency department. Work related injuries were then discretely analyzed regarding patient and injury characteristics, hospital management and final outcome.

Results: In total 566 work-related injuries were reported which accounted for $6.4 \%$ of all 8,862 trauma patients. Of these $95.1 \%$ were male and $4.9 \%$ female with a mean age of 42.6 years old. The mean Injury Severity Score was 6.77 , whereas the mortality was $4.6 \%$. $17.1 \%$ of work-injured patients required transfer to another facility, $54.1 \%$ underwent a surgical operation and $1.4 \%$ required admittance to an intensive care unit. The mean length of hospital stay was 5.12 days. The majority of injuries $(61.7 \%)$ were at the extremities. Severe injuries concerned mostly the head (30.1\%), followed by thoracic trauma $(24.5 \%)$.

Conclusion: Work-related injuries do consist a significant part of trauma injuries. Their impact on the worker and on the health care system can be evaluated. Trauma registries provide data not available in other data sets and could be used for surveillance programs.

References: 1. Shannon HS, et al. How many injured workers' do not file claims for workers-compensation benefits? Am J Ind Med. 2002;42:467-73. 2. Alamgir $\mathrm{H}$, et al Epidemiology of work-related injuries requiring hospitalization among sawmill workers in British Columbia, 1989-1997. Eur J Epidemiol. 2007;22(4): 273-80. 3. Motamedi MH, et al. Assessment of severity, causes, and outcomes of hospitalized trauma patients at a major trauma center. J Trauma. 2009;66(2):516-8.

Disclosure: No significant relationships.
066

\section{ILLEGAL IMMIGRATION AND TRAFFIC ACCIDENTS. PARTICULAR PROBLEMS AND DIFFICULTIES IN MANAGEMENT OF SUCH MULTI-CASUALTY INCIDENTS IN A REGIONAL HOSPITAL}

D. Mavridis ${ }^{1}$, M. Athanassiou ${ }^{1}$, T. Mitkos ${ }^{2}$, I. Tzimagiorgis ${ }^{1}$,

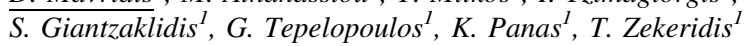

${ }^{1}$ 2nd Surgical Department, Kavala Hospital, Kavala, Greece, ${ }^{2}$ Kavala Hospital, Kavala, Greece

Introduction: Illegal immigration challenges our medical system, especially hospital emergency departments. Car accidents during illegal transportation of immigrants, are often in their effort to avoid prosecution, and they add additional stress in a usually overcrowded emergency department. Management of this multi-casualty incidents unveils specific problems, both medical and social, usually in a common pattern.

Materials and methods: Our aim is to discuss common trauma patterns and specific problems in this kind of multi-casualty incidents. From 2007 till 2010 there where five multi-casualty car accidents involving illegal immigrants, all in high speed conditions during prosecution. The total number of casualties where 38,5 where dead on arrival, 33 evaluated in the Emergency department and 30 where hospitalised. Five were admitted to ICU, five underwent emergency operation 4 required prolonged hospitalisation and two patients where transported to specialised centres for further treatment. Overall management of the incidents was evaluated through time to estimate the effect of the experience obtained by the personnel in timely and effective response.

Results: There where specific issues common in all cases, affecting procedures and outcome. Pattern and severity of injuries where reflecting unusual positions of the patients while in hiding, overcrowded vehicles, high speed driving, uncommon collisions with other cars. Communication difficulties and different legal status of patients impaired reliable retrieval of medical information, and previous relative experience proved critical in the effective management of casualties hospital resources and timely respond.

Conclusion: Although mass casualty accidents are a serious challenge for the overall operation of regional hospitals stressing their resources to their limits, knowledge of special issues involved, facilitate procedures and minimise the negative impact in emergency department workload.

Disclosure: No significant relationships.

\section{7}

\section{MULTIPLE CASUALTY INCIDENTS: A RARE CHALLENGE FOR TRAUMA CENTRES}

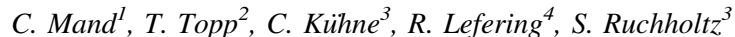

${ }^{1}$ Department Of Trauma, Hand And Reconstructive Surgery, University Hospital Giessen and Marburg GmbH, Marburg, Germany, ${ }^{2}$ University Hospital Giessen And Marburg Gmbh, Location Marburg, Department of Trauma, Hand and Reconstructive Surgery, Marburg, Germany, ${ }^{3}$ Trauma-, Hand- And Reconstructive Surgery, University of Marburg, Marburg, Germany, ${ }^{4}$ Institute For Research In Operative Medicine, University Witten/Herdecke, Cologne, Germany 
Introduction: While mass casualty incidents are seldom, a multiple casualty incident (2-4 patients; $\mathrm{MCI}$ ) occurs more often and has to be handled by a single hospital. The simultaneous treatment of more than one multiple injured patient is generally thought to adversely affect outcome and potentially overwhelm resources of a single trauma centre.

Materials and methods: Based on data derived from the TraumaRegistry of the German Trauma Society we investigated the frequency and outcome of multiple casualty incidents. Of 42,248 documented cases 29,203 were primarily treated, had an ISS $\geq 9$, and had their time of admission documented correctly. In 463 incidents two or more patients were admitted within $1 \mathrm{~h}$ (940 patients, 3.2\%). Two groups were formed, Group1 with the first MCI patient admitted $(\mathrm{n}=463)$ and Group2 with all patients following within $1 \mathrm{~h}$ $(\mathrm{n}=477)$. The remaining 28,263 patients were used as control group. Results: MCI patients were more often involved in road traffic accidents and therefore showed a different injury pattern (AIS head $\geq 3,45.1$ vs. $49.6 \%$, AIS Thorax $\geq 3,56.7$ vs. $51.6 \%$ ) compared to the control group. The duration of preclinical treatment and transport on average was 11 min longer for the Group 2 than it was for Group 1, and treatment in the shock room also took 5.7 min more for these patients (average $75.3 \mathrm{vs} .81 .0 \mathrm{~min}$ ). Overall lethality was $16.3 \%$ in MCI patients and $16.4 \%$ in solitarily treated patients. In cases where RISC prognosis was available we did not find great differences in predicted and observed mortality $(15.9 \% / 15.5 \%$ in Group 1, 16.9\%/15.8\% in Group 2,18.1\%/16.1\% in control).

Conclusion: Although a multiple casualty incident is a rare event in trauma care and may challenge the personnel and structural resources of any single trauma centre it does not seem to adversely affect outcome in simultaneously treated patients.

Disclosure: No significant relationships.

\section{8}

\section{ONLINE VICTIM TRACING AND TRACKING SYSTEM (VITTS)}

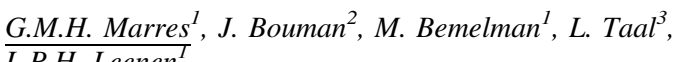
L.P.H. Leenen

${ }^{1}$ Surgery and Trauma, University Medical Centre Utrecht, Utrecht, The Netherlands, ${ }^{2}$ Major Incident Hospital, Utrecht, The Netherlands, ${ }^{3}$ University Medical Centre Utrecht, Utrecht, The Netherlands

Introduction: Major incidents require multiple organizations to coordinate an immediate and effective response with the allocation of various resources. Coordinating and communicating this over multiple geographical locations and organizations makes this a complex process. One of the greatest challenges is patient tracking and tracing. Often data concerning the amount of victims, their condition, location and transport is lacking. This hinders an effective response and causes public distress. Therefore we developed a victim tracing and tracking system (ViTTS).

Materials and methods: Development of an online Victim Tracing and Tracking System, based on a wireless network generated by routers on ambulances, with direct online registration of victims and triage-data through barcode injury cards. We tested the system for feasibility and usability during disaster drills.

Results: The conjured local radio-network of hotspots with mobile routers and connection over GPRS to the central database worked well and resulted in accurately stored data, on time availability (defined as available in the database within $1 \mathrm{~min}$ ), and a real-time overview of the patients. Through the central databus the system enabled interoperability between the different systems of the participants in the disaster relief chain.

Conclusion: The ViTTS provides a system for early unique, initially anonymous, registration and triage of victims close to the impact site. Personal identification and further medical details can be added at any time in the process. Online application and connection of the various systems used by the different chains in disaster relief enables patient tracking and tracing. It offers a real time overview of victims (quantity, seriousness of injury, whereabouts) to all involved disaster relief partners. This is necessary for the chain of command to conjure an adequate disaster response with optimized resource allocation (cost- and care-effective) and prevention of unnecessary public distress.

Disclosure: No significant relationships.

\section{VISCERAL TRAUMA 1}

\section{9}

\section{PROPHYLACTIC ANTIBIOTICS FOR TRAUMATIC THORACIC INJURIES REQUIRING TUBE THORACOSTOMY: A META-ANALYSIS}

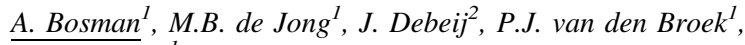 I.B. Schipper ${ }^{1}$}

${ }^{1}$ Trauma Surgery, D6-39, Leiden University Medical Centre, Leiden, The Netherlands, ${ }^{2}$ Clinical Epidemiology, Leiden University Medical Centre, Leiden, The Netherlands

Introduction: The aim of this study was to determine whether the prophylactic administration of antibiotics in patients undergoing tube thoracostomy for traumatic blunt or penetrating thoracic injuries reduces the incidence of surgical site- and pleural cavity infections.

Materials and methods: We conducted a meta-analysis based on a MEDLINE, EMBASE, Web of Knowledge and Cochrane Library search. Searches were not restricted by date, language or publication status. Only randomized controlled trials were included. Two reviewers screened the abstracts and extracted the data. A MantelHaenzel analysis was conducted.

Results: 11 of 326 articles were included, encompassing 1,234 patients $(1,241$ chest drains). $86 \%$ were male, with penetrating chest injuries as most common injury in $69 \%(\mathrm{n}=856)$, followed by blunt injuries in $25 \%(n=307)$. Our results show strong evidence that patients receiving antibiotic prophylaxis for tube thoracostomy after chest trauma are approximately at three times lower risk of developing empyema than patients not receiving antibiotic treatment (OR $0.3 ; 95 \%$ CI $0.2-0.6$ ). Regarding wound infections and pneumonia we found that antibiotic prophylaxis reduces the risk of infection development (OR 0.4; 95\%CI 0.1-2.2 and OR 0.6; 95\%CI 0.3-1.0).A subgroup analysis in patients suffering from penetrating chest injuries shows that antibiotic prophylaxis reduces the risk of infection development after tube thoracostomy (OR 0.3 ; 95\%CI $0.2-0.6$ ). Results for blunt thoracic injuries show no benefit from antibiotic prophylaxis (OR 1.6; 95\%CI 0.6-4.3).

Conclusion: Our results confirm that infectious complications, particularly empyema, are significantly less likely to develop when antibiotic prophylaxis is administered. Antibiotic prophylaxis specifically reduces the incidence of infectious complications after penetrating chest injuries. Causality for reduction of infectious complications in blunt injury patients could not be demonstrated. Our research preludes any comments on duration or intensity of treatment. Disclosure: No significant relationships. 


\section{0}

\section{CHANGES IN THE OUTCOMES OF SEVERE TRAUMA PATIENTS: STATISTICAL ANALYSIS FROM A SINGLE CENTER EXPERIENCE IN TRAUMA ICU (1996-2010)}

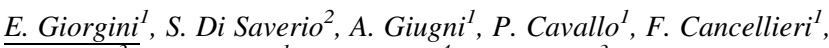

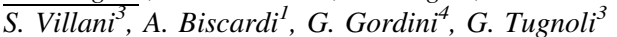

${ }^{1}$ Emergency and Trauma Surgery Unit (head Dr. G. Tugnoli), Maggiore Hospital Trauma Center, Bologna Local Health District, Bologna, Italy, ${ }^{2}$ Surgery, Emergency and Trauma Surgery Unit, maggiore Hospital Trauma Center, Bologna, Italy, ${ }^{3}$ Emergency And Trauma Surgery Unit, Maggiore Hospital Trauma Center, Bologna Local Health District, Bologna, Italy, ${ }^{4}$ Trauma Icu, Department Of Emergency, Maggiore Hospital Trauma Center, Bologna Local Health District, Bologna, Italy

Introduction: Recent safety regulations for RTA prevention, aimed to decrease mortality for trauma. We evaluated their effect during the last 15-years period.

Materials and methods: 60,247 trauma admissions (2,183 deaths) were recorded (1996-2010). 2,935 trauma patients (ISS > 16) were admitted to TICU and recorded in prospectively collected database. $97.1 \%$ were blunt trauma. A retrospective review of outcomes stratified for year was carried out; all available factors were included in multivariate analysis of prognostic value.

Results: Overall mortality was $17.2 \%$, respiratory morbidity was 23.3\%. A significant increase of trauma admissions occurred (before/after 2001, p < 0.01). Mean GCS (10.2) increased (test trend $\mathrm{p}<0.05)$. Mean age (44.12), ISS (24.83), mechanism didn't change significantly whereas mortality decreased showing two marked drops, from $25.8 \%$ in 1996 , to $18.3 \%$ in 2000 and again down to $10.3 \%$ in 2004 (test trend $\mathrm{p}<0.01$ ). TBI accounted for $58.4 \%$ of the causes of death, Hemorrhagic shock in $28.5 \%$ and MOF/Sepsis in $13.1 \%$. However TBI-related decreased with raise of MOF/Sepsis (test trend $p<0.05$ ). Significant predictors of mortality were year of admission $(\mathrm{p}<0.05)$, age, hemorrhagic shock and SBP, ISS and GCS and $\mathrm{pH}, \mathrm{BE}($ all $\mathrm{p}<0.01$ ). In the subgroup underwent emergency surgery the same factors remained significant, plus BloodUnits transfused $(\mathrm{p}<0.05)$. Surgical time (mean 71 min, showing significant trend to reduction) wasn't significantly associated with mortality $(\mathrm{p}=0.06)$

Conclusion: Mortality of severe trauma decreased significantly during the last 15 years as well as mean GCS whereas ISS remained stable. New safety regulations influenced incidence and severity of TBI and seemed to improve the outcomes. ISS seems better predictor of outcome than RTS.

Disclosure: No significant relationships.

071

\section{A NEW METHOD TO EVALUATE THE EXACT VOLUME OF HEMOPERITONEUM USING FAST OR CT SCAN}

\section{Massalou, P. Baqué}

Hôpital St Roch, Ucsu Chirurgie, Université de Nice/CHU de Nice, Nice, France

Introduction: In the trauma setting, the focused assessment for the sonographic examination (fast) accurately detects hemoperitoneum in trauma patients. Currently, only an approximation of the volume of free intraperitoneal fluid (FIPF) can ne done using ultrasound (US). We previoulsly reported a new method using a mathematic formula to evaluate the exact volume of FIPF on an experimental cadaveric study. The aim of this clinical prospective study was to confirm that this new method can be used in clinical practice.

Materials and methods: Twelve patients presenting with FIPF were included in the study: one ruptured extra-uterine pregnancy with hemoperitoneum, two patients with ascite, one diverticular peritonitis with purulent FIPF and eight patients with abdominal trauma with hemoperitoneum. All these patients had to be operated on using a surgical emergency procedure.

The measurement of the thickness of the FIPF was made preoperatively using US or CT scan in the hepatorenal pouch (morrisson recess) in a sagittal section plane and an axial section plane (in $\mathrm{mm}$ ). Results: The new formula: FIPF volume $(\mathrm{mL})=50 \times$ measurement $(\mathrm{mm})+500$ was used to preoperatively evaluate the volume of FIPF. During the emergency surgical procedure, we precisely quantified the volume of FIPF.

We founded a constant correlation beetween FIFP mesured during the radiologic procedure using this new formula and the direct peroperative measurement of the FIPF.

Conclusion: This new formula can be used to evaluate the exact volume of FIPF, specially during a FAST procedure for abdominal trauma. This evaluation can be helpful for surgical decision specially in cases of associated hemorrhagic lesions.

Disclosure: No significant relationships.

\section{2}

\section{STRATEGIES FOR CONDUCTING A MULTICENTER RANDOMIZED CONTROLLED TRIAL IN TRAUMA SURGERY: THE FAITH TRIAL EXPERIENCE}

\author{
S.M. Zielinski ${ }^{1}$, E.M.M. van Lieshout ${ }^{1}$, M.J. Heetveld ${ }^{2}$, \\ M. Swiontkowski ${ }^{3}$, M. Bhandari ${ }^{4}$, P. Patka
}

${ }^{1}$ Surgery-traumatology, Erasmus MC, University Medical Center, Rotterdam, The Netherlands, ${ }^{2}$ Surgery, Kennemer Gasthuis, Haarlem, The Netherlands, ${ }^{3}$ Orthopaedic Surgery, University of Minnesota Medical School, Minneapolis, MN, USA, ${ }^{4}$ Department Of Orthopeadic Surgey, McMaster University, Hamilton, ON, Canada

Introduction: Different strategies can be chosen when organizing a multicenter RCT. Surgeons in the Netherlands, Canada and the US participate in the Fixation using Alternative Implants for the Treatment of Hip fractures trial (FAITH). All Dutch sites are managed and visited by a single financed national trial coordinator. Most Canadian and US sites work with multiple local site coordinators and receive per patient payments. The aim of this study was to assess if these strategies affect trial performance.

Materials and methods: Details related to obtaining ethics approval, time to trial start-up, inclusion, and follow-up completeness were collected for each site and compared. Pre-trial patient screening data were compared with actual inclusion rates.

Results: Median trial start-up ranged from 136 days (P25-P75 104-243) in The Netherlands to 254 days (P25-P75 126-648) in Canada. Inclusion was highest in the Netherlands, with a median of 0.94 (P25-P75 0.4-2.2) patients per site per month, representing 35\% (P25-P75 24-63) of the total population. It was lowest in Canada: 0.14 inclusions, representing $3.9 \%(\mathrm{p}<0.001)$. Inclusion rates were much lower than expected from pre-trial screening; a decline of $48 \%$ (P25-P75 14-69) was noted for Dutch sites, versus 83\% (P25-P75 39-100) for Canadian and US sites $(\mathrm{p}=0.091)$. Follow-up completeness ranged from $84 \%$ (P25-P75 81-86) in The Netherlands to $67 \%$ (P25-P75 64-90) in the US. 
Conclusion: Centrally financed trial coordination contributes to higher inclusion rates per month and follow-up completeness compared with appointing individual site coordinators and per patient payments at sites. Pre-trial screening indicates good motivation for trial participation per site, but actual inclusion is lower than screening predicts.

Disclosure: No significant relationships.

\section{3}

\section{AUTOTRANSFUSION OF SHED INTRAPLEURAL BLOOD FOLLOWING THORACIC TRAUMA: A PROSPECTIVE STUDY}

\author{
T. Lustenberger, P. Talving, K. Inaba, L. Lam, B.C. Branco, \\ D. Plurad, D. Demetriades
}

Department of Trauma and Critical Care, Los Angeles County General Hospital (LAC + USC), Los Angeles, CA, USA

Introduction: Autotransfusion provides rapid access to warm, autologous blood and is widely utilized as an alternative to allogenic blood transfusion. However, autotransfusion may be complicated by bacterial contamination. Thus, the goal of the present study was to validate prospectively the microbiological profile of the autotransfused whole blood.

Materials and methods: Prospective study of patients admitted to a level 1 trauma center from 09/2008 through 12/2009 sustaining chest trauma and requiring chest tube insertion for the evacuation of a hemothorax with subsequent autotransfusion. Samples of the shed blood drained from the chest were collected prior to autotransfusion and were sent for gram stain and culture.

Results: A total of 40 patients were enrolled as a convenience sample. Average age was $36.3 \pm 16.1$ years, $90 \%(\mathrm{n}=36)$ were male, and mean ISS was $17.7 \pm 10.3$. The majority of patients $(72.5 \%, \mathrm{n}=29)$ suffered penetrating trauma (Stab wounds, $\mathrm{n}=12$; Gunshot wounds, $\mathrm{n}=16$; Crossbow injury, $\mathrm{n}=1$ ). Concomitant intraabdominal solid organ and hollow viscus injuries were found in $10(25 \%)$ and 5 $(12.5 \%)$ patients respectively. The combination of diaphragmatic and hollow viscus injury was diagnosed in 4 patients (10\%). Mean initial chest tube output was $813.8 \pm 478.8 \mathrm{~mL}$ and mean volume of blood autotransfused was $756.2 \pm 438.3 \mathrm{~mL}$. Overall, 1 culture $(2.5 \%)$ was positive (coagulase negative staphylococcus and streptococcus hemolyticus). This specific patient suffered a penetrating crossbow injury to the left chest without diaphragmatic and intraabdominal injury. Follow-up blood cultures were negative and the patient did not show any signs of infection or bacteremia during subsequent hospital course.

Conclusion: Autotransfusion of shed intrathoracic blood is safe even in instances of concomitant diaphragmatic injury and subdiaphragmatic contamination due to hollow viscus injury. Liberal use regardless of the injury pattern is advocated.

Disclosure: No significant relationships.

\section{4}

\section{COMPLICATIONS AND PREDICTORS OF MORBIDITY AND MORTALITY AFTER TRAUMA LAPAROTOMY}

\section{M.H. van Gool, F.C. Bakker, F.W. Bloemers, W.P. Zuidema}

Trauma Surgery, VU-University Medical Centre, Amsterdam, The Netherlands
Introduction: Trauma laparotomy (TL) is a potential lifesaving procedure after traumatic abdominal injury. Little is known about the morbidity and mortality rate of this procedure. The primary aim of this study is to describe the complications which may occur during and after TL. Secondary aim is to identify predictors for the morbidity and mortality.

Materials and methods: Retrospective descriptive study of 83 patients who received TL between January 2001 and December 2009 at our trauma center. Patient characteristics and complications related to the TL were measured. The collected data were analyzed with SPSS 16.0.

Results: Eighty -three patients received a TL at our institution. The study group consists of 60 men and 23 women. Mean age 40.6 years. Causes were traffic accident $(n=33)$, fall of height $(n=17)$, stabbing accidents $(\mathrm{n}=11)$, shooting accidents $(\mathrm{n}=11)$, other $(\mathrm{n}=11)$. The group harbors 26 penetrating and 56 blunt abdominal injury patterns. Mean ISS was 19,3. Twenty patients had in OR complication directly related to TL and 28 had post OR complications directly related to TL. Relaparotomy (RL) rate was $34.9 \%, 16$ patients needed more than one RL (19.3\%) with a maximum of $5 \mathrm{RL}$ in one patient. Most common indication for the first RL was persistent bleeding (12\%). In 34 patients a complication occurred without indication for a RL. Most common complication was a pulmonary infection $(8.4 \%)$. Overall $19.3 \%$ of patients died after TL.

Conclusion: This study described the variety of complications after $\mathrm{TL}$ and pre and per-operative predictive factors for the outcome of TL. Disclosure: No significant relationships.

\section{5}

\section{COMPLICATIONS OF HIGH GRADE LIVER TRAUMA: MANAGEMENT AND OUTCOME}

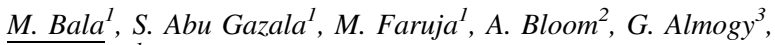 A. Rivkind ${ }^{l}$}

${ }^{1}$ General Surgery, Hadassah-Hebrew University Medical Center, Jerusalem, Israel, ${ }^{2}$ Interventional Radiology, Hadassah-Hebrew University Medical Center, Jerusalem, Israel, ${ }^{3}$ General Surgery And Trauma, Hadassah Ein Kerem Medical Center, Jerusalem, Israel

Introduction: Nonoperative management of high-grade liver injuries is associated with significant morbidity and correlates with grade of liver injury. Current attention needs to be focused on reducing hepatic-related morbidity. The purpose of this study was to define hepatic related morbidity in patients sustaining high-grade hepatic injuries that safely could be managed none operatively.

Materials and methods: This is a retrospective analysis of patients data with liver injury treated in Hadassah-Hebrew University Medical Center over a 10-year period. Collected data included the number and types of, hepatic-related complications and interventions in patients survived more than $48 \mathrm{~h}$ post injury. Grades $3-5$ were considered to be high-grade injuries.

Results: Of a total of 398 patients with liver trauma, $16 \%$ were found to have high grade (3-5) liver injuries. Mechanism of injuries were blunt trauma in 43 cases, penetrating-21. Operative treatment required 40 patients $(62 \%)$. Among survivors 20 patients $(50 \%)$ developed liver related complications that required additional interventional treatment. Arteriography and angioembolization performed in four patients for rebleeding. Bilomas and bile leaks were diagnosed in 16 cases post-injury. Seven patients required ERCP with biliary stent placement, 2-percutaneous transhepatic biliary drainage. There were 24 deaths $(37 \%)$ but only 3 hepatic-related mortalities due to liver necrosis, and hepatic artery pseudoaneurysm rebleeding. 
Conclusion: Complications in high grade liver injuries should be anticipated. Angioembolization, ERCP and temporary internal stenting, together with percutaneous drainage of intra-abdominal or intrahepatic bile collections, represent a safe and effective strategy for the management of complications following both blunt and penetrating hepatic trauma.

Disclosure: No significant relationships.

\section{6}

EVALUATING AND PROMOTING THE DEVELOPMENT OF TRAUMA SYSTEMS VIA TELECONFERENCE TO IMPROVE TRAUMA CARE IN LATIN AMERICA

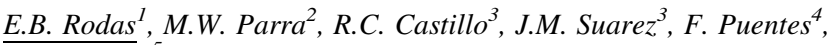 \\ R. Camacho
}

${ }^{1}$ Surgery, Division of Trauma and Critical Care, Broward General Medical Center/Nova Southeaster University, Fort Lauderdale, FL, USA, ${ }^{2}$ Surgery, Division of Trauma and Critical Care, Broward General Medical Center/Nova Southeastern University, Fort Lauderdale, FL, USA, ${ }^{3}$ Surgery, Division of Trauma and Critical Care, Broward General Medical Center/Nova Southeaster University, Plantation, FL, USA, ${ }^{4}$ Hospital San Félix, La Dorada, Colombia, ${ }^{5}$ Instituto Ecuatoriano de Seguro Social, Manta, Ecudaor

Introduction: Evaluation, development and implementation of trauma systems in Latin America is a challenging undertaking as no model is currently in place that can be easily replicated throughout the region. Use of teleconferencing has allowed overcoming other challenges in the medical field and improve medical care. We describe use of an international teleconference to evaluate and promote quality improvement and aid in the development of trauma care in the Latin American region.

Materials and methods: By creating an international trauma and critical care improvement project a monthly teleconference was implemented since February 2009 between US trauma surgeons and Latin American (LA) general surgeons, emergency physicians, and intensivists. In-depth discussions and prospective evaluations of each case presented were conducted by all participants based on resources available. Care rendered was divided in four stages: (1) pre-hospital setting, (2) emergency room or trauma room, (3) operating room, and (4) subsequent post-operative care. Furthermore, the participating institutions completed an electronic survey of trauma resources based on WHO/IATSIC guidelines.

Results: During a 17-month period a total of 15 cases were presented from a level I and level II US hospitals $(\mathrm{n}=3)$ and 6 LA hospitals $(\mathrm{n}=12)$. Presentations followed ATLS sequence in all US cases, while only in 3 of 12 LA cases. The following deficiencies were observed in cases presented from Latin America: pre-hospital communication was non-existent in all cases; pre-hospital services were absent in $60 \%$ of cases presented; lack of trauma team structure was evident in the emergency departments; during the initial evaluation and resuscitation ATLS protocol was followed one time and CRASH protocol in two occasions; it was determined that imaging resources were used adequately in half of the cases; the initial care was mostly provided by emergency room physicians; surgeon, operating room, and ICU were not readily available $83 \%$ of the time. The ease of patient flow was cumbersome due to a lack of a structured system for trauma care except for 1 academic urban center. Adequate trauma resources are present in less than $50 \%$ of the time. Multidisciplinary resources, quality improvement programs, protocols, and guidelines were deficient.

Conclusion: A well-structured International teleconference can be utilized as a dynamic window of observation to evaluate and identify deficiencies in trauma care in the Latin American Region. These findings can be employed to formulate specific recommendations based on local resources. Furthermore, by raising local awareness, leaders could be identified to become the executors of more efficient healthcare policies that can potentially affect trauma care.

Disclosure: No significant relationships.

077

\section{GUNSHOT INJURIES IN PAKISTAN; MANAGEMENT PROTOCOLS FOR DEVELOPING COUNTRIES}

\author{
H. Zafar, M.A. Moosa, A. Pardhan, A. Memon
}

Surgery, Aga Khan University Hospital, Karachi, Pakistan

Introduction: Morbidity and mortality due to firearms injuries remain a major public health problem in Pakistan. We present our experience of ten patients reviewed retrospectively who came with the gunshot injuries to trauma service at a tertiary care hospital to identify management protocols based on the need of a developing nation.

Materials and methods: We retrospectively reviewed ten consecutive cases presented to our hospital of gunshot injuries to torso during July-September 2010.

Results: There were no mortalities in these cases however their management forced us to think about our current problems. Patients included both single and multiple gunshot wounds, involving various regions of a human body. ICD $9 \mathrm{CM}$ classification of disease included from 800.00 to 959.0 . $80 \%$ of the patients were transferred from another acute care facility resulted in delayed presentation to our setup. All cases had good physiology on admission (RTS 12). Review of our treatment showed that in many patients with massive blood transfusion was not according the massive blood transfusion protocol as stated in literature. Three of our patients had renal injury resulted in a nephrectomy in one, and we were able to conserve the kidney in other two, a pseudo aneurysm was seen in one of the conserved kidney which urgently needed angioembolization and was then conservatively managed. Two of our patients developed violent behavior during their course resulting in a psychiatric evaluation which led us to see another aspect of trauma which is mental illness rather than physical. This review also led to development of a proper rehabilitation setup for trauma patients in our facility as rehabilitation was always been a problem for our trauma patients as expertise for this was scarce.

Conclusion: These ten patients presented with a variety of symptoms, their course was challenging and this led to us to review them one by one and identify issues which would make our trauma service better. These issues were (1) Development of pre-hospital setup (2) Establishment of massive blood transfusion protocol (3) Psychiatric problems with the patients (4) Establishment of trauma rehabilitation setup where patients can be slowly shifted back to their normal lives. Disclosure: No significant relationships.

\section{EMERGENCY SURGERY 5}

\section{8}

\section{MANAGEMENT OF URETERAL CALCULI AND MEDICAL} EXPULSIVE THERAPY IN EMERGENCY DEPARTMENTS

\section{S. Picozzi ${ }^{1}$, L. Carmignani ${ }^{2}$}

${ }^{1}$ Urology, IRCCS Policlinico San Donato, San Donato Milanese, Italy, ${ }^{2}$ Urology, IRCCS Policlinico san Donato, San Donato Milanese, Italy 
Introduction: Medical expulsive therapy (MET) has become routine in the treatment of obstructive ureteral calculi, and there is a large body of published data showing the efficacy of such therapy in increasing the expulsion rate and decreasing the expulsion time of stones, thereby reducing lost workdays, urological visits and stone removal procedures, even though this treatment did not substantially improve the studied outcomes in two recent trials.

Materials and methods: The aim of this systematic review and metaanalysis was to understand the role of MET in the treatment of obstructing ureteral calculi. A bibliographic search covering the period from January 1980 to March 2010 was conducted in PubMed, MEDLINE and EMBASE. This analysis is based on the 21 studies that fulfilled the predefined inclusion criteria.

Results: A metaregression analysis of expulsion time showed a statistically significant advantage in the experimental group. Analysis of the tamsulosin data base A total of 1,283 participants were included in the 17 studies. These studies showed that, compared to standard therapy or placebo, tamsulosin had significant benefits, being associated with both a higher stone expulsion rate and reduction of the expulsion time. Reductions in the need for analgesic therapy, hospitalization and surgery were also shown. Analysis of the nifedipine data base, a total of 488 participants were included in the six studies considered. Compared to standard therapy, the use of nifedipine significantly improved the spontaneous stone expulsion rate. The mean expulsion time was slightly, but not statistically significantly, different. A possible benefit of nifedipine, in terms of significantly reducing the doses of analgesics required, was reported in three studies.

Conclusion: These data raise concerns also with regards to potential cost savings. MET should be offered as a treatment for patients with distal ureteral calculi who are amenable to a waiting management. Disclosure: No significant relationships.

\section{9}

\section{ASSESSMENT OF DECREASED COMPARTMENT ELASTICITY BY PRESSURE RELATED ULTRASOUND: A CADAVER STUDY EXTENDING ELASTOGRAPHY}

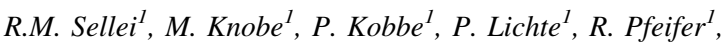 \\ S.J. Hingmann ${ }^{1}$, M. de la Fuente Klein ${ }^{2}$, F. Schmidt ${ }^{2}$, \\ S. Linke ${ }^{2}$, K. Radermacher ${ }^{2}, H$. Pape ${ }^{I}$
}

${ }^{1}$ Department of Orthopaedic Trauma, University Hospital of the RWTH AACHEN, Aachen, Germany, ${ }^{2}$ Chair of Medical Engineering, Helmholtz Institute for Biomedical Engineering, Aachen, Germany

Introduction: Overlooked compartment syndrome represents a devastating complication. Invasive compartment pressure measurement continues to be the gold standard. However, repeated measurements in uncertain cases may be difficult to achieve. We developed a new, noninvasive method to assess tissue firmness by pressure related ultrasound.

Materials and methods: Decreased tissue elasticity by means of rising compartment pressures was mimicked by infusion of saline directly into the anterior tibial compartment of six human cadavers. A transducer was located in a saline filled ultrasound probe head to allow a simultaneous recording of the probe pressure provoked by the user. The ultrasound images were generated at 5 and $100 \mathrm{mmHg}$ probe pressures to detect the tissue deformity by B-mode ultrasound. The fascial displacement was measured before and after compression $(\Delta \mathrm{d})$. Subsequently, increments of $5 \mathrm{mmHg}$ pressure increases were used to generate a standard curve $(0-80 \mathrm{mmHg})$, thus mimicking rising compartment pressures. The intra-observer reliability was tested using ten subsequent measurements. A correlation was determined between $\Delta \mathrm{d}$ and the simulated intacompartmental pressure (ICP) in the compartment. The Pearson correlation coefficient (r) was calculated. The reliability determined by the kappa value and a regression analysis was performed.

Results: With rising compartmental pressure, a concordant consistent correlation between $\Delta \mathrm{d}$ and ICP occurred. The Pearson coefficient was significant at $r_{S}=0.979$ and the intra-observer value kappa showed a statistically good reliability $\left(\kappa_{10}=0.73\right.$ and $\left.\kappa_{70}=0.79\right)$. Within a pressure ranging from 5 to $35 \mathrm{mmHg}$ there was an almost linear behavior, with decreasing elasticity and tissue movement in case of raising ICP.

Conclusion: Pressure related ultrasound emphasized as a reliable tool to determine the correlation between the measured compartmental displacement and the raising intracompartmental pressures. This may help to improve the clinical assessment of the compartment elasticity by the physician. Further studies are required to investigate whether the good correlation also applies for the clinical scenario. This may help to avoid delayed decision making.

Disclosure: No significant relationships.

080

\section{IS ABDOMINAL ULTRASOUND (US) USEFUL FOR DIAGNOSIS OF ACUTE APPENDICITIS?}

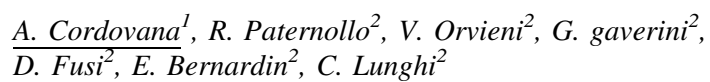

${ }^{1}$ General Surgery, A.O. FATEBENEFRATELLI, Milan, Italy, ${ }^{2}$ General Surgery, A.O. Fatebenefratelli, Milan, Italy

Introduction: In the last decade the number of abdominal ultrasounds (AUS) performed for suspected acute appendicitis has dramatically increased but there is no agreement about their real utility to improve the diagnosis.

Materials and methods: From March 2010 through September 2010, we applied US in 36 consecutive patients with suspected appendicitis. In all such patients, abdominal US was performed after surgical indications were given on the basis of symptoms, clinical signs and blood values. The aim of the study was to evaluate-on the basis of an intention to treat - the effective role of US in the diagnosis of acute appendicitis.

Results: In 14 patients signs, symptoms and clinical history, were specific and indications to surgery were given for suspected acute appendicitis. 22 patients didn't show peculiar signs and symptoms of acute appendicitis and surgical indication was given with an explorative intent. The results of the abdominal US were: 12 US images showed direct or indirect signs of appendicitis 14: other diagnosis 10 no signs of pathology After laparoscopy: When abdominal US showed images compatible with acute appendicitis, patients really had acute appendicitis (high positive value). The 12 patients with other diagnosis 5 have acute appendicites, 7 other pathoogies Out of the 10 with no US signs of pathology, 6 had an acute appendicitis, 4 other pathologies.

Conclusion: On the basis of our experience we consider abdominal US a useful instrument to improve diagnosis and to obtain more information in unclear and doubtful cases. However surgeons shouldn't base a surgical decision on it, the more so in those cases that show negative results.

References: [1] Toorewliet BR, et al. Routine ultrasound and limited computed tomography for diagnosis of acute appendicitis. World J 
Surg. 2010;34:2278-2285 [2] Rylaent JB et al. A prospective study of US in the diagnosis of appendicitis. N Engl J Surg. 1987;327:666-669.

Disclosure: No significant relationships.

\section{1}

\section{BOERHAAVE SYNDROME: ABOUT THREE CASES IN A ROW WITH DIFFERENT CLINICAL MANIFESTATIONS. WHEN CONSERVATIVE, WHEN SURGERY?}

\section{H. Kurihara ${ }^{I}$, D. Mariani ${ }^{I}$, A. Casamassima ${ }^{1}$, A. Ronsivalle ${ }^{2}$, F. Butti ${ }^{I}$, R.A. Foà ${ }^{l}$, M. Zago ${ }^{l}$}

${ }^{1}$ General and Emergency Surgery, Istituto Clinico Città Studi, Milano, Italy, ${ }^{2}$ Emergency Department, Istituto Clinico Città Studi, Milano, Italy

Introduction: Spontaneous perforation of the oesophagus (Boerhaave syndrome) is a rare condition with a persistent high mortality rates. An effective management and timing of prompt diagnosis and treatment are strictly linked with better survival rates, but there is no consensus regarding the best management option.

Materials and methods: From February 2010 to May 2010 three patients were admitted to our ED with a spontaneous oesophageal perforation. Case 1: a 88-year-old man, with severe comorbidities (APACHE II score: 22) came to ED with septic shock and massive left pleural effusion. A CT scan with oral contrast confirmed a perforation of the lower third of the oesophagus. Due to the expected adverse outcome, no therapeutic choice, beyond pleural drainage, was initially considered. After improvement of general conditions a suspended oesphageal stent was positioned. Case 2: a 65-year-old man (APACHE II score: 8 ) presented with acute thoracic pain, dysphagia, leucocytosis, fever, dysphonia and subcutaneous emphysema of the neck. A CT scan confirmed a perforation of the distal oesophagus and the patient underwent right thoracotomy. A two layers suture of a $3 \mathrm{~cm}$ laceration with a pleural flap was performed. Case 3: a 86 -yearold woman in healthy condition (admission APACHE II score: 6) presented with transitory acute thoracic pain and dysphagia and mild hemathemesis. A CT scan revealed pneumomedistinum with minimal closed oral contrast leak; endoscopy revealed a laceration of esofageal mucosal layer with no evidence of full thickness perforation. The patient underwent right thoracotomy. No lesion of the oesophagus could be detected but signs of mediastinitis were found and postoperative course was complicated by a persistent empyema that was treated by thoracic negative pressure wound therapy. The two patients treated surgically underwent surgery within $6 \mathrm{~h}$ from the arrival in the emergency department, while the case 1 patient underwent endoscopic stenting $60 \mathrm{~h}$ after admittance.

Results: All patients could be discharged with preserved digestive functions.

Conclusion: Treatment of oesophageal perforation should be chosen on an individual basis like in this 'three in a row' series. A prompt timing of diagnosis and treatment confirmed to be linked with positive outcome; immediate surgery should be considered also in those cases with no initial evidence of complications.

References: 1. Vallbohmer D et al (2010) Options in the management of esophageal perforation: analysis over a 12-year period. Dis Esophagus 23:185-190. 2. Schmidt SC et al (2010) Management of esophageal perforations. Surg Endosc 24:2809-2913. 3. de Schipper JP (2009) Spontaneous rupture of the oesophagus: Boerhaave syndrome in 2008. Literature review and treatment algorithm. Dig Surg 26:1-6.

Disclosure: No significant relationships.
082

\section{CAN EARLY APPLICATION OF NEGATIVE PRESSURE WOUND THERAPY PREVENT COMPLETE POST- LAPAROTOMY WOUND DEHISCENCE? PRELIMINARY EXPERIENCE OF A SINGLE INSTITUTION}

\author{
D. Mariani, M. Zago, H. Kurihara, A. Spreafico, M. Lucini, \\ R.A. Foà, F. Butti
}

General and Emergency Surgery, Istituto Clinico Città Studi, Milano, Italy

Introduction: Post-laparotomy wound dehiscence (PLWD) represents a serious complication; some patients require redo surgery with increased morbidity and costs. Several factors are involved: intraoperative factors (IF), local (LF), regional (RF), and systemic factors (SF). IF are represented by intestinal contamination, persistent hypotension, laparotomy time $>120 \mathrm{~min}$, vasoactive aminic support. Early and late LF include hematoma, seroma, hypotermia $\left(<34^{\circ} \mathrm{C}\right)$, perioperative hyperglicemia. Intra-abdominal hypertension is the RF, SF are advanced age, malnutrition, COPD, renal failure, obesity, diabetes, steroids, radio/chemotherapy. We designed a prospective study to identify criteria for an early treatment of patients at high risk of PLWD, to reduce the occurrence of a complete wound breakdown. Materials and methods: From January to September 2010 we enrolled 12 consecutive patients (pts) with PLWD. Seven pts (58\%) underwent emergency laparotomy, 5 (42\%) had elective surgery. We arbitrarily set the presence of at least two IF and two among LF/RF/ $\mathrm{SF}$ as positive predictive criteria for high risk PLWD. Wounds were daily checked, lastly through transparent dressing. The presence of local signs of inflammation or serosity in this subset of patients were considered indication for early NPWT. Seven out of 12 pts (58\%) fit with this definition and early NPWT (Vista ${ }^{\circledR}$, Smith and Nephew) was applied.

Results: Mean duration of NPWT was 7.5 days (range 2-18). In 6 out of 7 pts $(85,7 \%)$ skin closure was obtained at the end of the treatment. No fascial breakdown were observed. Mean follow-up is 3.8 months (range 1-10); no postincisional hernia or other late complications were observed.

Conclusion: Early NPWT appears effective in preventing complete PLWD in a particular subset of patients. NPWT could be helpful in reducing hospital stays and costs. To evaluate the real benefits of this approach, further prospectives controlled studies are needed.

Reference: 1. Ubbink DT (2010) Topical negative pressure for treating chronic wounds (review) The Cochrane Collaboration 2010. Disclosure: No significant relationships.

\section{3}

\section{DIFFERENT MANAGEMENT OF SURGICAL WOUNDS REDUCES THE RISK OF SSI}

\section{Fattori ${ }^{1}$, E. Colciago ${ }^{2}$, G. Pesenti ${ }^{3}$}

${ }^{1}$ Surgery Chirurgia Iii, OSP San Gerardo, Monza, Italy, ${ }^{2}$ Surgery Chirurgia Iii, Osp San gerardo, Monza, Italy, ${ }^{3}$ Surgery- Chirurgia Iii, Osp San Gerardo, Monza, Italy

Introduction: Surgical site infection (SSI) represents a risk for the safety of the patient. The SSI is associated with significant morbidity, and frequently lead to an increased length of hospital stay, pain and discomfort for the patient and engraves on the wealthy costs, so its 
reduction is a priority in Health Services. The colorectal surgery has a high incidence of risk of SSI because of the high risk of contamination. The aim of this study is to determine if a correct and standardized management of dressing is able to reduce the infection rate in surgical wounds.

Materials and methods: A prospective study was performed on 90 patients who undergone to colorectal surgery in 18 months: emergency was excluded. Patients were randomized into two groups statistically homogeneous: a control group with 50 patients who undergone to a standard dressing changing (first change on the third postoperative day and then every 2 days) and a study group which included 40 patients who undergone to a fully coded protocol dressing change on the seventh postoperative day unless the need of inspection of the wound or the saturation of the dressing. Patients were then followed up for 30 days postoperatively.

Results: In the control group the number of SSI was 10/50 (20\%) while in the study group was $1 / 40(2.5 \%) \mathrm{p}<0.01$.

Conclusion: The number of SSI is lower in the study group. The significative difference of infection rate between the two groups may be related to the "protocol effect" but also to a reduced but careful handling of the dressing of surgical wound. In conclusion, we can affirm that a higher level of vigilance and a higher awareness, necessary to apply the study protocol, associated with a reduced number of changes of dressing are important constituent to reduce the incidence of SSI.

Disclosure: No significant relationships.

\section{4 \\ ENDOSCOPIC RETROPERITONEAL NECROSECTOMY AND DEBRIDEMENT (ERND) FOR NECROTISING PANCREATITIS}

\author{
E. Sivrikoz, H. Yanar, S. Sarici, C. Ertekin, K. Günay, R. Güloğu
}

General Surgery, Istanbul Faculty of Medicine, İstanbul, Turkey

Introduction: Infected necrotising pancreatitis is drained surgically when patients' condition deteriorates or multiorgan failure ensues. An alternative drainage under local anesthesia and sedation employing endoscopic instruments may reduce mortality.

Materials and methods: Between November 2006 and October 2010, 87 patients with necrotizing pancreatitis presented to our department and nine were treated with endoscopic retroperitoneal necrosectomy and debridement (ERND). A $15 \mathrm{~mm}$ laparoscopic port was placed under CT-guidence in infected necrosis with left retroperitoneal fluid collections if suitable. A basket-catheter was used through a double-lumen gastroscope for necrosectomy and debridement. A 10-mm aspirative silicone drain was placed after ERND to abcess cavity. CT scans were obtained before and after ERND.

Results: Out of 87 patients, 10 patients underwent open surgical necrosectomy and debridement and mortality rate was $\% 40(n=4)$. ERND mortality rate was $\% 33(\mathrm{n}=3)$. All mortal ERND cases succumbed at ICU due to multi-organ failure. Post-procedure CT scans of all ERND patients demonstrated no residual necrotic material at pancreatic region. One patient developed a pancreatic fistula following ERND, which resolved after wirsungotomy. No patient sustained a hollow-organ perforation or vascular injury.

Conclusion: Surgical drainage of necrotizing pancreatitis results with high mortality. Every attempt to reduce the morbidity and mortality associated with drainage procedures is worth the effort. ERND seems beneficial, however timing and indications of the procedure need to be optimized.

Disclosure: No significant relationships.
V12

\section{FIRST ITALIAN MRMI: A NEW EDUCATIONAL EXPERIENCE}

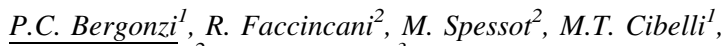
R. Sannicandro ${ }^{2}$, A. Rossodivita ${ }^{3}$

${ }^{1}$ Intensive Care Unit, San Raffaele Hospital, Milan, Italy, ${ }^{2}$ Deas, San Raffaele Hospital, Milan, Italy, ${ }^{3}$ Cardiosurgical Sub-intensive Care Unit, San Raffaele Hospital, Milan, Italy

Introduction: Education and training are key elements in increasing the preparedness for major incidents management. We offered to the Italian audience a new training opportunity: the Medical Response to Major Incidents (MRMI) course.

Materials and methods: A 3 days theoretical and practical MRMI course was organized at San Raffaele Hospital in Milan on 5-7 October 2010 in collaboration with ESTES Section of disaster and military surgery. Similarly to the program of the previous MRMI courses organized in other European Countries the trainees followed a full educational day with lectures on major incidents management and training-in-group on simulation method. In the last 2 days the trainees were engaged in real-time macro-simulations of major incidents with hundreds of people involved. We used the MACSIM simulation model created and developed by Prof Sten Lennquist. 52 people attended the course, within these a great variety of professions was represented: healthcare personnel (both doctors and nurses), Hospitals and Public Institution's directors and administrative staff, Civil Protection geologists, ecc. The course was conducted by an international faculty of experienced MRMI instructors with the cooperation of an Italian instructors team that had passed the first MRMI instructor course organized in Split (Croatia) on November 2009.

Results: We received extremely positive reviews from all participants regardless of the role they played during the simulations, their usual work and previous experience in major incidents management. The complete evaluation of pre- and post-course tests is in progress.

Discussion: The MRMI course is an appreciated, valuable and effective training opportunity for staff involved in each role of the Major Incidents management.

Disclosure: No significant relationships.

\section{VISCERAL TRAUMA 2}

\section{5}

\section{NON-OPERATIVE VERSUS SURGICAL TREATMENT IN HEMODYNAMICALLY STABLE PATIENTS SUSTAINING BLUNT SPLENIC INJURY}

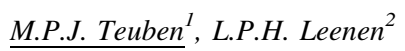

${ }^{1}$ Surgery, UMC Utrecht, Utrecht, The Netherlands, ${ }^{2}$ Department of Surgery, University Medical Centre Utrecht, Utrecht, The Netherlands

Introduction: During the past two decades, dramatic changes in the management of blunt splenic injuries (BSI) have been seen. Currently, non-operative management (NOM) is the treatment of choice in hemodynamically stable patients. This study evaluates whether there are differences in outcome between observationally and surgically treated hemodynamically stable patients.

Materials and methods: From our prospective trauma registry we selected all hemodynamically stable adult $(>15)$ patients with BSI, 
who were admitted to our level one trauma center during a 10 -year period ending in September 1, 2009.

They were divided into two groups according to the attempted type of treatment. Group I consisted of patients who received NOM and group II of those who received operative treatment. We compared patient characteristics and outcome of both treatment modalities. Furthermore, we compared outcome of patients who failed NOM and those who where initially treated operatively.

Results: A total of 69 patients were selected. Median age (IQR) was 36 (21-54) years. No differences were revealed in patient characteristics, ISS and admission hemodynamic parameters between groups. However, AIS of splenic injury was significantly higher in patients treated operatively $(\mathrm{P}=0.004)$. The median AIS-spleen (IQR) among NOM patients was $2.0(2.0-3.0)$, versus 4.0 (3.0-4.0) for patients subjected to operative management. Median ISS in group I was 18 (9-29), versus $25(17-30)$ in Group II $(\mathrm{P}=0.090)$.

In both groups no mortality occurred and no significant differences were seen in hospitalization time, ICU-stay and complications. Median length of stay (IQR) was 12 (8-27) in group I and 18 (13-28) in surgically treated patients $(\mathrm{P}=0.149)$.

Comparison of initially surgically treated patients and failed NOM patients showed no differences in outcome parameters.

Conclusion: NOM is a feasible treatment modality in hemodynamically stable patients. There are no significant differences in outcome between surgically and NOM treated patients.

Moreover, even if failure of non-operative management occurred, this was not associated with increased morbidity and mortality as compared with initially operated patients.

Disclosure: No significant relationships.

\section{6}

\section{AIS > 2 IN AT LEAST TWO BODY REGION: A POTENTIAL NEW ANATOMICAL DEFINITION OF POLYTRAUMA}

\author{
N.E. Butcher, Z. Balogh
}

Trauma Service, John Hunter Hospital, Newcastle, NSW, Australia

Introduction: Background: The term 'polytrauma' lacks a universally accepted, validated definition. In clinical trials the commonly applied injury severity based anatomical score cut-offs are ISS $>15$, ISS $>17$ and a recently recommended AIS $>2$ in at least two body regions ( 2 xAIS $>2)$. Purpose: To compare the outcomes of clinically defined polytrauma patients with those defined based on anatomical scores.

Materials and methods: A prospective observational study on all trauma team activation patients over a 7 -month period presenting at a level-1 trauma centre were included in the study. The prospective data collection included AIS in each body region, ISS, ICU length of stay (LOS), multiple organ failure (MOF) and mortality.

Results: 336 patients met inclusion criteria (age: $41 \pm 20,74 \%$ male, ISS: $15 \pm 11$, NISS: $19 \pm 15$, MOF: $3 \%$, mortality: $4 \%, 25 \%$ ICU admission). 131 patients (39\%) had an ISS $>15 ; 102(30 \%)$ had an ISS > 17; and $64(19 \%)$ had 2xAIS > 2. $44(13 \%)$ were categorised as polytrauma based on local expert opinion.

\begin{tabular}{llllll}
\hline & N & Polytrauma & Death & ICU & MOF \\
\hline Total & 336 & $44(13 \%)$ & $14(4 \%)$ & $85(25 \%)$ & $10(3 \%)$ \\
ISS $>15$ & 131 & $44(34 \%)^{*}$ & $13(10 \%)$ & $71(54 \%)$ & $10(8 \%)$ \\
ISS $>17$ & 102 & $40(39 \%)^{\#}$ & $11(11 \%)$ & $63(62 \%)$ & $10(10 \%)$ \\
2xAIS $>2$ & 64 & $37(58 \%)^{*, \#}$ & $8(12.5 \%)$ & $43(67 \%)$ & $9(14 \%)$ \\
\hline
\end{tabular}

Individual definitions performance on defining polytrauma

\begin{tabular}{lllllll}
\hline & Sens\% & Spec\% & PPV\% & NPV\% & Acc\% & Prec\% \\
\hline ISS $>15$ & 100 & 70 & 33 & 100 & 74 & 34 \\
ISS $>17$ & 91 & 78 & 39 & 98 & 80 & 39 \\
2 xAIS $>2$ & 84 & 91 & 58 & 98 & 90 & 58 \\
\hline
\end{tabular}

Conclusion: $2 x$ AIS $>2$ captured the greatest percentage of the worst outcomes and significantly larger $\%$ of the clinically defined polytrauma patients. 2xAIS $>2$ has higher accuracy and precision in defining polytrauma than ISS $>15$ and ISS $>17$. This simple, retrospectively also reproducible criteria warrants larger scale validation.

Disclosure: No significant relationships.

087

\section{SPLENIC ARTERY EMBOLIZATION IN BLUNT SPLENIC INJURIES. A HIGH RATE OF SUCCESS AGAINST A PROLONGED HOSPITALIZATION AND A HIGH RATE OF COMPLICATIONS}

N. de'Angelis ${ }^{1}$, M. Bertolani ${ }^{1}$, A. Hariscine ${ }^{I}$, A. Ranalli ${ }^{2}$, L. Buttarelli ${ }^{2}$, A. Volpi ${ }^{3}$, R. Dalla Valle ${ }^{l}$

${ }^{1}$ Surgery, University of Parma, Parma, Italy, ${ }^{2}$ Radiology, University of Parma, Parma, Italy, ${ }^{3} 1$ st Anaesthesia and Intensive Care, Azienda Ospedaliero-Universitaria di Parma, Parma, Italy

Introduction: Nonoperative management (NOM) of blunt splenic injury has become the preferred treatment in hemodynamically stable patients. The use of splenic artery embolization (SAE) in NOM is still controversial. The purpose of this study is to analyze the safety and effectiveness of SAE in patients who underwent NOM for splenic injury.

Materials and methods: Patients admitted with splenic injuries over a 4.6 year period at our trauma center were identified and retrospectively reviewed. We analyzed the initial management method (simple observation, SAE, or splenic surgery) Data collected for this study were age, gender, Injury Severity Score (ISS), grade of splenic injury, length of stay, NOM failures and complications.

Results: From January 2006 through September 2010, 89 patients were admitted for splenic injury, 63 men and 26 women with a mean age of 36.2 years and a mean Injury Severity Score of 30.1. Twelve patients $(13.4 \%)$ required immediate surgery (Operative management, OM). Twenty-four patients $(26.9 \%)$ were treated with SAE and $53(59.5 \%)$ were subjected to simple observation. Overall, 77 patients $(86.5 \%)$ qualified for NOM, which was successful in $76(98 \%)$. Patients treated with SAE had a longer length of stay (16 vs. 11 days). No post-operative complications were registered in patients who underwent splenectomy, whereas we noted 3 (12.5\%) major complications in patients treated with SAE (1 splenic abscess, 1 necrotizing pancreatitis and 1 prolonged dynamic ileus).

Conclusion: The increased use of initial SAE in blunt splenic injuries expanded the success of NOM at the expense of a higher rate of complications and a prolonged hospitalization.

Disclosure: No significant relationships. 


\section{8}

\section{PEDIATRIC POLYTRAUMA CARE IN SLAVONIA REGION, COUNTY GENERAL HOSPITAL VERSUS PEDIATRIC TRAUMA CENTRE}

\author{
I. Matic ${ }^{1}$, M. Jurjevic ${ }^{2}$, B. Hreckovski ${ }^{2}$, D. Danic ${ }^{2}$, \\ K. Sakic'Zdravčevic ${ }^{3}$, I. Mirkovic ${ }^{2}$
}

${ }^{1}$ Department of Anesthesiology and Intensive Care, Dr. Josip Benčević General Hospital, Slavonski Brod, Croatia, ${ }^{2}$ Dr. Josip Benčević General Hospital, Slavonski Brod, Croatia, ${ }^{3}$ Clinical Hospital Sveti Duh, Zagreb, Croatia

Introduction: Pediatric trauma centers (PTC) are still rare in developing countries. Care for pediatric polytraumatized patients must be available at County General Hospitals (CGH) equipped with an adequate Intensive care unit (ICU) as well as trauma surgeons and neurosurgeons. The goal of the research was to compare outcome parameters after injury for patients treated at PTCs or CGHs.

Materials and methods: A prospective randomized research was carried out at a multidisciplinary ICU over 30 months and included 69 patients under the age of 15 with ISS $>15$ and PTS $<8$. Basic patient data (age, sex) as well as cause of injury (fall, traffic accidents) and injury type (blunt or penetrating) were recorded. Severity of injury was asessed based on ISS, PTS, GCS, NISS and TRISS scores, that were calculated for each patient. Furthermore, we recorded mechanical ventilation duration as well as total ICU stay and ICU mortality. Data were compared with those from a PTC.

Results: Of the 69 patients, 40 had ISS 16-26, 25 had ISS $>26.12$ had PTS $<0.4$ patients were transferred to a PTC for treatment continuation, and were excluded from the study,11 patients died. Mechanical ventilation duration CGHvsPTC was 2.3 versus 2.1 days. Total ICU stay, CGH versus PTC was 6.4 versus 5.6 days. Mortality rates CGH versus PTC were 16 vs. $13 \%$.

Conclusion: PTCs present the golden standard of care for traumatized children, although due to the geographic and financial reasons they are not always available. CGHs should be able to provide sufficient quality of care, especially in mild to moderate injuries. Patients with ISS $<26$ and/or PTS $<0$ should be transffered to a PTC as soon as possible if the patient's condition is stable.

References: 1. Wetzel RC, Burns RC (2002) Multiple trauma in children: critical care overview. Crit Care Med 30:S468S477. 2. Thompson EC, Perkowski P, Villarreal D et al (2003) Morbidity and mortality of children following motor vehicle crashes. Arch Surg 138:142-145. 3. Letts M, Davidson D, Lapner P (2002) Multiple trauma in children: predicting outcome and long-term results. Can J Surg 45:126-131. 4. Schalamon J, v Bismarck S, Schober PH et al (2003) Multiple trauma in pediatric patients. Pediatr Surg Int 19:417-423. 5. Ott R, Kramer R, Martus P et al (2000) Prognostic value of trauma scores in pediatric patients with multiple injuries. J Trauma 49:729-736.

Disclosure: No significant relationships.

\section{9}

\section{FOR TRAUMATIC CRITICAL PATIENTS...TIME MEANS LIFE! THE IMPORTANCE OF DAMAGE CONTROL SURGERY}

\author{
S. Manfroni, T. Mastropietro, E.A. Adami, C. Cataldi,
} B.A.V. Truosolo, B. Benini, A. Roveran, D. Antonellis

Emergency and General Surgery, San Camillo Forlanini, Rome, Italy
Introduction: The optimization of the management of politraumatic patients during the first hour reduces mortality. In the prehospital stage is necessary reduce the time on the scene and respect the centralization criteria. Inside the hospital there has to be a trauma team and the adoption of management protocols.

Materials and methods: As we can see from the retrospective analysis of politraumatic patients, there are some point you can consider: the time to A\&E/operative room, haemodynamic conditions, the given surgical treatment and the mortality rate. The criteria for immediate surgery is : haemodynamic instability associated with a opened or closed trauma of thorax/abdomen/pelvis/limbs.

Results: During the years 2008-2010 we have treated 1,581 politraumatic patients: 176 received surgical treatment, 26 of them had an immediate surgery within $45 \mathrm{~min}$ from the arrival $\left(12^{\prime}-45^{\prime}\right.$, average $\left.28^{\prime}\right)$. Surgery treatment: 12 hepatic haemostasis ( 8 packing with 2 arterial embolization), 6 splenectomy, 1 suture of the right atrium, 1 resuscitative thoracotomy, 1 pneumonectomy, 3 thoracotomy, 2 aortic endoprotesis, 2 diaphragmatic sutures, 2 gastric sutures, 2 pulmonary sutures, 2 hemicolectomy, 1 nefrectomy, 1 ileum resection, 1 bladder suture, 1 splenic haemostasis, 1 epigastric artery haemostasis. 6 patients died on within $12 \mathrm{~h}$.

Conclusion: Quick and suitable treatment improves the survival of politraumatic patients reducing the avoidable deaths. It is fundamental the pre alert of the trauma team of trauma team, the centralization criteria, the organization of the trauma center, the adoption of the management protocols and the choise of damage control surgery.

References: 1. Johnson JW. Evolution in damage control for exsanguination penetrating abdominal injury. $\mathrm{J}$ Trauma. 2001;51:261-271. 2. Demetriades D. Preventable and potentially preventable mortality at a mature trauma center. J Trauma. 2007;63:1338-47.

Disclosure: No significant relationships.

090

\section{IS IT POSSIBLE TO TEACH OPEN CHEST CARDIOPULMONARY RESUSCITATION IN FRANCE? A 2 YEARS EXPERIENCE IN A MILITARY TEACHING HOSPITAL}

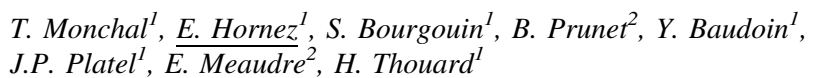

${ }^{1}$ Digestive Surgery, Sainte Anne Military Hospital, Toulon, France, ${ }^{2}$ Reanimation, Sainte Anne Military Hospital, Toulon, France

Introduction: During the past decade, an aggressive attitude for performing open chest cardiopulmonary ressucitation has developed in adjunct with the recognition of damage control surgical principles in major trauma. This procedure has become a part of resuscitative process in many anglo saxon urban trauma centers. Actually improvements in pre hospital cares system have allowed an increasing number of patients with haemorraghic shock in the emergency room. In France where the profile of injuries and the pre-hospital care are different from the anglo-saxon experience, the number of patients requiring an open chest cardiopulmonary ressucitation is limited. The goal of this retrospective study is to determine if the teaching of this procedure is possible and gainful in a medium-sized town as Toulon. Materials and methods: This study was a retrospective review of all cases where a thoracotomy was performed in the emergency department of the Sainte Military teaching hospital during a 2007-2009 period. The procedure was performed by a senior trauma surgeon assisted by a student for patient presenting a severe intra thoracic 
haemorrhage with witnesses cardiac arrest. The duration of cardiac arrest should be less than 5 min for blunt trauma and less than $15 \mathrm{~min}$ for penetrating trauma.

Results: During this period, 191 severe trauma were managed, 32 presented a haemorrhage and $12(6.5 \%)$ need an open chest resuscitation. There were no penetrating trauma; the median of duration of the pre hospital care was $90 \mathrm{~min}$. 2/12 patients have an isolated thoracic haemorrhage. 5/12 patients recovered a spontaneous cardiac activity. There were no survivor at the 24th $\mathrm{h}$.

Conclusion: According to the current literature, open chest resuscitation is predominately indicated in select patients after penetrating trauma and the training seems to be mandatory for the successful employment of this procedure. As shown in this study, the profile of injury, the duration of the pre hospital care and the low number of casualties does not promote the teaching of this procedure in France. This procedure is also time and team consuming for a poor survival even under the best conditions.

References: Nolan J, Deakin C, Soar J, Bottiger B, Smith G. European Resuscitation Council guidelines for resuscitation 2005. Section 4. Adult advanced life support. Resuscitation 2004;67:S39. Voiglio EJ, Coats TJ, Baudoin YP, Davies GD, Wilson AW. Thoracotomie transverse de reanimation. Ann Chir 2003;128: 728-733. Eckstein M. Termination of resuscitative efforts: medical futility for the trauma patient. Curr Opin Crit Care 2001;7: 450-454. Aihara R, Millham FH, Blansfield J, Hirsch EF. Emergency room thoracotomy for penetrating chest injury: effect of an institutional protocol. J Trauma 2001;50:1027-1030. Powell DW, Moore EE, Cothren CC, Ciesla DJ, Burch JM, Moore JB, et al. Is emergency department resuscitative thoracotomy futile care for the critically injured patient requiring prehospital cardiopulmonary resuscitation? J Am Coll Surg 2004;199:211-215. Rhee PM, Acosta J, Bridgeman A, Wang D, Jordan M, Rich N. Survival after emergency department thoracotomy: review of published data from the past 25 years. J Am Coll Surg 2000;190:288-298. Cothren CC, Moore EE. Emergency department thoracotomy for the critically injured patient: objectives, indications, and outcomes. World J Emerg Surg 2006;1:4

Disclosure: No significant relationships.

\section{1}

\section{IMPACT OF NEUROLOGICAL IMPAIRMENT ON OUTCOME OF OBSERVATIONAL TREATMENT IN BLUNT SPLENIC INJURY}

\author{
M.P.J. Teuben $^{\text {I }}$, L.P.H. Leenen ${ }^{2}$
}

${ }^{1}$ Surgery, UMC Utrecht, Utrecht, The Netherlands, ${ }^{2}$ Department of Surgery, University Medical Centre Utrecht, Utrecht, The Netherlands

Introduction: Non-operative management (NOM) is the preferred treatment in hemodynamically stable patients sustaining blunt splenic injury. In the beginning of the NOM era, altered mental status was a relative contraindication for this treatment modality. However, in the past decade we considered neurologically impaired patients are considered as appropriated patients for NOM.

The goal of this study was to determine whether outcome is poorer in neurologically impaired adult patients who had been treated nonoperatively for blunt splenic injury.

Materials and methods: Our prospectively maintained trauma database was utilized to identify all adult patients (age $>15$ years) who underwent initial non-operative therapy for blunt splenic injury, during a 10 year period. Subsequently, two groups of patients were formed on the basis of presence of neurological impairment.

Group I consisted of patients with maximal GCS-scores, and group II included patients with altered mental status (GCS $<15$ ).

Demographic and trauma characteristics as well as outcome were examined. Outcome analysis included mortality, failure of NOM, complications, length of hospital stay, ICU-stay.

Results: A total of 51 patients (median age 28) met the criteria, of whom fourteen patients had impaired mental status.

The groups were similar in patient characteristics and hemodynamic parameters on admission. As expected, median ISS was significant higher in the neurologically impaired group compared with group I (34 vs. $16, \mathrm{p}<0.01$ ). The median GCS (IQR) in group II was 9 (7-13).

Median length of stay ( 28 vs. 9 days; $\mathrm{p}<0.05$ ) and duration of ICU stay ( 13 vs. 0 days; $p<0.01)$ were significantly higher in patients with decreased GSC. No differences in failure of NOM and complications were found. Mortality was not seen in both groups.

Conclusion: According to our experience, the hemodynamically stable patient with impaired mental status can be safely selected for NOM. Reduced GCS-score on admission is associated with increased ICU-stay and hospitalization time. Although, morbidity and mortality is not increased.

Disclosure: No significant relationships.

\section{2}

\section{OPERATING ROOM EARLY TRAUMA DEATHS. WHOLE- BODY 16-MDCT IS ASSOCIATED WITH A THREEFOLD INCREASE IN THE TIME INTERVAL FROM HOSPITAL ACCESS TO EMERGENCY SURGERY IF COMPARED WITH A US-BASED PROTOCOL}

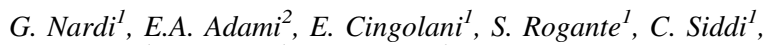

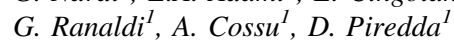

${ }^{1}$ Shock and Trauma Unit, S. Camillo Hospital, Roma, Italy, ${ }^{2}$ Department of Emergency and Surgery of Trauma, S. Camillo Hospital, Rome, Italy

Introduction: A retrospective analysis based on the data of the German Trauma Registry has shown a significant increase in the probability of survival in polytrauma patients submitted to whole body CT (WBCT). However even when the CT is installed in the Emergency Department, the time required to positioning the patient on the CT may significantly prolong the duration of CT diagnostic. Materials and methods: S. Camillo Hospital is a level 1 Trauma Center with a catchment population of 2.5 millions; there are $2 \mathrm{CT}$ scans based in the ED. All the severely injured patients are submitted to US on admission. WBCT is performed as the first line radiologic investigation in the haemodynamically stable patients or in the unstable patients with negative abdominal US and without a clear source of bleeding. Unstable patients with severe head trauma and lateral signs are also submitted to CT. To evaluate whether the use of WBCT in the severely unstable patients brings to significant delay in emergency surgery, we retrospectively analyzed all early trauma death that occurred during a 23 months' time-span (Jan 09-Nov 10). Results: In the study period 700 severe trauma patients (ISS $>15$ ) were brought in alive. 38 of them (5.4\%) died before ICU admission: 21 patients died in the shock room before any surgical intervention. 1 patient was submitted to thoracotomy and laparothomy in the shock room and died. One more died on the CT table. The remaining 15 patients, all severely hypotensive, were alive on admission to the OR. 
One of them was brought directly to the OR with no investigation because of massive bleeding from the extremities. All the other had US on admission. US was the only investigation for 7 of them; they all had a positive US showing important bleeding in the abdomen or in the chest. The other 7, who had no evidence of bleeding on the US, were submitted to WBCT. The mean time elapsed from hospital admission to OR entrance was $23^{\prime}\left(15^{\prime}-30^{\prime}\right)$ for patients who had only US and $70^{\prime}\left(52^{\prime}-90^{\prime}\right)$ for the WBCT group. The 7 patients who had only US, were all submitted to shock room decompressive minithoracothomy (5 bilateral) as part of the resuscitation process in the suspect of pneumothorax.

Conclusion: Recent reports suggest the implementation of multi-slice $\mathrm{CT}$ integrated into the resuscitation room, thus enabling resuscitation to be performed directly on the ER-CT. Unless this new technology is adopted, even the ED based CT still need an excessive time to be performed in the most unstable patients. In a cohort of patients who eventually died in the OR, a diagnostic process including ED based WBCT, was associated with a threefold increase in the time needed from hospital admission to surgery.

Disclosure: No significant relationships.

\section{3}

\section{PROSPECTIVE EVALUATION OF PENETRATING NECK INJURIES: PROPOSAL FOR A TREATMENT ALGORITHM}

\author{
O. van Waes $^{l}$, A. Nicol ${ }^{2}$, P. Navsaria ${ }^{3}$, E.M.M. Van Lieshout ${ }^{4}$, \\ J. Vermeulen
}

${ }^{1}$ Trauma Surgery, Erasmus University Medical Center Rotterdam, Rotterdam, The Netherlands, ${ }^{2}$ Surgery: Trauma, Groote Schuur Hospital, Cape Town, South Africa, ${ }^{3}$ Trauma Surgery, Groote Schuur Hospital, Cape Town, South Africa, ${ }^{4}$ Surgery-Traumatology, Erasmus MC, University Medical Center, Rotterdam, The Netherlands

Introduction: The incidence of penetrating neck injury (PNI) is increasing in the Netherlands, though experience in treatment of these patients is still limited. Goal: In order to improve care of these of patients, a study was done in a tertiary referral trauma center in which PNI is common.

Materials and methods: In a prospective cohort study all patients presented with PNI between September and November 2009, were included and analyzed for type of PNI, location, vitals, diagnostic work-up, treatment and outcome.

Results: A 78 patients were included: 62 patients with 67 stab wounds and 16 patients with 18 gunshot wounds (GSW). Vascular injury was found in 16 patients $(21 \%)$; in which active bleeding was controlled via a tamponading Foley catheter in de wounds, 2 patients (3\%) had esophageal injury; $12(15 \%)$ patients had neurological injury, of whom 6 showed paralysis. Injury of upper airways was seen once $(1 \%)$. 9 patients (12\%) underwent surgical intervention. Indications for surgery were: hemodynamic instability and vascular injury found on additional investigations. All other patients were treated conservatively. During follow up, no additional injuries were found. Three patients died, of whom 1 within $24 \mathrm{~h}$ due to injuries other than PNI.

Conclusion: A conservative treatment of selected patients with PNI is indicated, provided that it is based on careful physical examination and targeted investigations. Based our results we present a treatment algorithm for PNI.

Disclosure: No significant relationships.

\section{SPINAL INJURIES}

\section{4}

\section{D-BASED NAVIGATION AT INTERVENTIONS OF THE CERVICAL AND THORACIC SPINE: BENEFIT OR A WASTE OF TIME-RESULTS OF 451 PLACED SCREWS}

\author{
J.S. Jarvers $^{1}$, C. Schmidt ${ }^{1}$, S. Katscher ${ }^{1}$, S. Glasmacher ${ }^{1}$, A. Franck ${ }^{1}$, \\ T.R. Blattert ${ }^{1}$, C. Josten ${ }^{2}$
}

${ }^{1}$ Department of Traumatology, Plastic and Reconstructive Surgery, Spine Centre, University of Leipzig, Leipzig, Germany,

${ }^{2}$ Traumatology, University of Leipzig, Leipzig, Germany

Introduction: After good experiences with the CT-based navigation in the past this prospective study deals with our experiences with the intraoperative 3D-navigation at posterior stabilisations of the cervical and thoracic spine.

Materials and methods: A 3D-C-Arm (Vision Vario 3D, Ziehm) was connected with a navigation system (Vector vision, Brainlab) and since 10/2007 used for the placement of overall 451 Screws at 66 Patients. Of those 14 patients had to undergo operations in the posterior cervical spine, of 70 screws Judet $(\mathrm{n}=10)$, Massa lateralis $(n=37)$ and pedicle-screws $(n=23)$ were placed. Indications for instrumentation were traumatic fractures $(n=6)$, spondylodiscitis $(\mathrm{n}=1)$, multiple metastases with high-grade instability $(\mathrm{n}=6)$, and degenerative rheumatic stenosis of the spinal canal $(n=1)$. Concerning the high thoracic spine (T1-10) 53 interventions were made with the method, 381 pedicle-screws were implanted. Indications in this area were traumatic fractures $(\mathrm{n}=30)$, metastases $(\mathrm{n}=18)$ and spondylodiscites $(\mathrm{n}=5)$.

Results: Scan-time intraoperatively took $60 \mathrm{~s}$ on average, datatransfer to the navigation-system another $10 \mathrm{~s}$. Application-time including anti-collision-check needs approx. 6 min [5; 18]. In total $354 / 451(78.5 \%)$ screws could be inserted assisted with navigation, $272 / 451(60.3 \%)$ were controlled intraoperatively. Regarding the cervical spine in $87 \%(61 / 70)$ of the screws the navigation procedure was uneventful. Positioning of $64.2 \%$ (45/70) of the screws was checked immediately postoperatively. In the upper thoracic spine $77 \%$ (293/381) could be placed with navigation, 59.6\% (227/381) were controlled intraoperatively. In addition, we experienced technical problems (placement of reference base, unevenness of the ORfloor linked with stability problems of the C-arm). Correct placement was seen for each screw, thus correlating well with the intraoperative findings.

Conclusion: The application of the combination of intraoperative 3Dimaging and navigation for posterior instrumentation of the cervical and the upper thoracic spine is technically feasible and reliable in clinical use. User- and software-dependant sources of error could be solved during the first course of the series. In regard to time for operation, there is some prolongation, at the beginning of the study mostly caused by an initial learning curve. In case of an error free use of the C-arm, a scan can be accomplished within 6 min. Imagequality at the cervical spine is depending on individual bone density, and possible metal artifacts. With undisturbed visibility of the vertebral body, the reliability of 3D-based navigation at the cervical spine is comparable to that of CT-based procedures. Additionally, it has the advantage of skipping preoperative acquisition of data as well as the matching-process. Furthermore, exposure to radiation is reduced due to the possibility of sparing pre- and postoperative CT. Because of that, costs are being decreased.

Disclosure: No significant relationships. 


\section{5}

\section{A BIOMECHANICAL STUDY OF ANDERSON TYPE II ODONTOID FRACTURES IS THERE AN ADVANTAGE OF TWO SCREWS?}

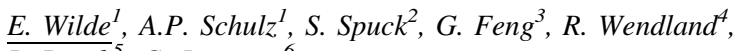 \\ $\overline{\text { L. Busch }}^{5}$, C. Juergens ${ }^{6}$
}

${ }^{1}$ Trauma + Orthopaedics, University Lübeck, Lübeck, Germany, ${ }^{2}$ Neurosurgery, University Hospital Luebeck, Luebeck, Germany, ${ }^{3}$ 2nd Affiliated Hospital, Orthopedic Department, Zhejiang University College of Medicine, Hangzhou, China, ${ }^{4}$ Trauma and Orthopaedics Lab. Biomechatronik, University Hospital Luebeck, Luebeck, Germany, ${ }^{5}$ Institute of Anatomy, University of Luebeck, Luebeck, Germany, ${ }^{6}$ BG Trauma Hospital Hamburg, Hamburg, Germany

Introduction: The odontoid fracture type II which is inherently unstable represents the most common type of odontoid fractures. Usually it is treated by anterior two screw fixation. Usage of only one screw is possible. Both possibilities have their advantages and problems. We wanted to objectify the difference in stability between one and two screw fixation.

Materials and methods: We used the axes of 14 fresh cadaver and randomized them into two groups and performed screw fixation with one or two screws following the usual techniques under radiographic control. The stability was measured before and after fixation in six directions using a universal mechanical testing machine (Zwick). The corresponding data was compared using SPSS v 16 after consultation of a biometric statistician.

Results: Between the one and two screw fixation no statistically difference could be shown in any direction. The results showed that the torque load transmitted to the odontoid by ligaments is around $1 / 3$ $(0.53 \hat{\mathrm{A}} \pm 0.38 \mathrm{~nm})$ of the maximum physiological load $(1.5 \mathrm{~nm})$ in axial rotation. The torque acting on the occipito-atlanto-axial complexes is dominated by the odontoid at smaller rotational angles. At larger rotational angles, the other ligaments that do not attach to the odontoid will join in and react against the torque more. The bone mineral density has statistical significant correlation with shear stiffness loading from anterior and posterior, torsional stiffness loading from left and right of intact specimen.

Conclusion: First we could show that either one or two screw fixation is not able to restore the normal shear and torsional stability so that the stiffness of the odontoid after one- or two-FCS fixation is much less than that of normal. But otherwise the stability with one or two screw anterior odontoid fixation produces similar stability concerning the shear and torsional stiffness after type II odontoid fractures.

Disclosure: No significant relationships.

\section{6}

DO MULTILEVEL SPINE FRACTURES IMPLICATE A MORE COMPLEX INJURY MECHANISM COMPARED TO SERIAL SPINE FRACTURES AND THEREFORE A MORE COMPLICATED PROCESS?

\section{S. Glasmacher ${ }^{1}$, J.S. Jarvers ${ }^{1}$, C. Schmidt ${ }^{2}$, A. Franck ${ }^{2}$, D. Task ${ }^{1}$, T.R. Blattert ${ }^{2}$, C. Josten $^{I}$}

${ }^{1}$ Department of Traumatology, Plastic and Reconstructive Surgery, Spine Center, University of Leipzig, Leipzig, Germany, ${ }^{2}$ Department of Traumatology, Plastic and Reconstructive Surgery, Spine Centre, University of Leipzig, University of Leipzig, Leipzig, Germany
Introduction: Definition and treatment of multilevel or serial injuries of the spine still remains controversal. For planning of an adequate therapy we investigated patients with this injuries taking radiological and clinical results into account.

Materials and methods: We defined at least 2 between the fractured vertebra placed vertebrae as multilevel and 2 fractured vertebrae with only one placed vertebra in between as serial. We investigated patient with traumatic fractures in the thoacolumbar region. Osteoporosis, pathologic fractures and spondylodiscites were excluded.

Results: In the time of 01.01.2005 till 01.01.2010 852 patients with fractures in the thoracolumbar region were treated operatively. The average age was $37 \mathrm{y}(18-65 \mathrm{y})$. The patients were between 18- and 65 -year-old. Of the remaining 341 patients $6.2 \%(\mathrm{n}=53)$ had multiple fractures ( $23 \%$ multilevel, $\mathrm{n}=12 ; 77 \%$ serial fractures, $\mathrm{n}=41$ ). All together 147 vertebrae were fractured. 16 patients were polytraumatised, 14 showed neurological deficits. The most frequent injury cause were traffic accidents and fall from height. The percentaged fraction of $\mathrm{B}$ and $\mathrm{C}$-type fractures was higher in the multilevel fractures. Overall $76 \%$ A, 11\% B and 13\% C-type fractures were seen.

Conclusion: With one exception all multilevel injuries were high velocity accidents. Apart from that the higher classification grade was found in these injuries. This shows, that multilevel fractures are caused by a more complex injury mechanism and a more complicated case can be expected, which also can be seen in the duration of the hospital stay.

Disclosure: No significant relationships.

\section{7}

\section{OPERATIVE TREATMENT OF FLAIL CHEST IN BLUNT THORACIC TRAUMA USING PRECONTOURED PLATES AND SPLINTS (MATRIXRIB): A SINGLE CENTRE EXPERIENCE}

\author{
M. Odermatt $^{l}$, C. Sommer ${ }^{2}$, M. Furrer ${ }^{1}$
}

${ }^{1}$ Surgery, Kantonsspital Graubünden, Chur, Switzerland, ${ }^{2}$ Chirurgie, Kantonsspital Graubuenden, Chur, Switzerland

Introduction: Surgical exposure and unsuitable implants are the main disadvantage of operative rib stabilisation of flail chest. We report on our experiences using specially designed precontoured plates and splints.

Materials and methods: Retrospective analysis of patients with extended flail chest due to blunt thoracic trauma. Inclusion criteria were severe rib cage deformity resulting in significant reduction of lung volume and/or destabilisation of the ipsilateral shoulder-girdle. Rib stabilisation by open access using MatrixRIB (Synthes). Technical handling and postoperative course were analysed.

Results: From August 2009 to September 2010, stabilisation of flail chest was performed in seven patients. Mean age was 62 (56-66). Mean Injury Severity Score was 26 (17-34). The mean number of serial multiple broken ribs on the operated side was 8 (5-10). Two patients with floating shoulder. Lung volume and normal position of the shoulder-girdle could be restored in all patients. Immediate postoperative extubation in 6 of 7 patients. Mean postoperative ICUstay was 3.2 days (1-8). Technical handling of MatrixRIB showed a steep learning curve. In the mean follow up of 6 months (2-12), there was dislocation of one screw and persistent pain in one patient.

Conclusion: Technically, rib stabilisation has become easier and short-term results are promising. However, the access trauma could not be minimized, which is the main disadvantage of the system and limits its application.

Disclosure: No significant relationships. 


\section{8}

\section{RIB FRACTURES FIXATION AFTER CHEST WALL TRAUMA, STRATOS SYSTEM}

\section{P. Moreno de la Santa Barajas ${ }^{I}$, M.D. Polo Otero ${ }^{I}$, C. Delgado Sanchez-Gracian', M. Choren Duran ${ }^{1}$, S. Leal Ruiloba ${ }^{3}$, M. Lozano Gomez ${ }^{1}$, C. Trinidad Lopez ${ }^{2}$, P. Magdalena Lopez ${ }^{4}$}

${ }^{1}$ Thoracic Surgery, Hospital Povisa, Vigo, Spain, ${ }^{2}$ Radiology, Hospital Povisa, Vigo, Spain, ${ }^{3}$ Critical Care, Hospital Povisa, Vigo, Spain, ${ }^{4}$ Statistic, Hospital Povisa, Vigo, Spain

Introduction: Rib fractures are very common in the blunt chest trauma. Most of these patients experience significant amounts of pain associated with cough and movements. The surgical stabilization of rib fractures can reduce pain; decrease ventilator requirements and short hospital stage.

Materials and methods: Between 2008 and 2010, 31 patients underwent open reduction and internal fixation of traumatic ribs. Indications included: (1) flail chest (16 patients), (2) acute pain with instability (10 patients) and (3) big chest wall defect (5 patients). Data were prospectively analysed. Age, trauma mechanism, thoracic and extrathoracic injuries, surgical data, complications and follow-up were assessed. We describe the surgical technique (video).

Results: 31 patients were admitted in this study: 18 patients were operated for flail chest, 9 for chronic pain or chest instability and 4 for chest wall defect. We show demographic, trauma mechanism, complications and outcome. In all groups the most frequent mechanism of trauma was motor vehicle accident $(55 \%)$, followed by fall $(30 \%)$. Most of patients were extubated immediately after surgery. All patients with pain and instability reported rapid subjective improvement or resolution. Five patients developed a minor complication after surgery: 4 wound infection requiring drainage and hemothorax. One patient died 33 days after surgery for MOF. After 3 months, lung function tests were normal in all patients. The $56 \%$ of patients back to work/usual activity, and $90 \%$ after 6 months. We described characteristics of each group.

Conclusion: Open reduction and internal fixation for rib fractures repair, in a select group of patients, its a good option. Morbidity is low and mortality its minimal. Titanium costal clips and plates produce good clinical results, they are easy to place and they have minimal complications.

Disclosure: No significant relationships.

\section{0}

\section{MONOSEGMENTAL ANTERIOR COLUMN RECONSTRUCTION AND FUSION IN INCOMPLETE BURST FRACTURES OF THE SPINE: EARLY RESULTS OF ANTERIOR VERSUS COMBINED OPERATIVE TECHNIQUES}

\section{A. Pizanis ${ }^{1}$, B. Reischmann ${ }^{1}$, S. Esch ${ }^{1}$, T. Pohlemann ${ }^{2}$}

${ }^{1}$ Trauma-, Hand- and Reconstructive Surgery, University of Saarland, Homburg, Germany, ${ }^{2}$ Department of Trauma, Hand and

Reconstructive Surgery, University of Saarland, Homburg, Germany

Introduction: Reconstruction of the anterior spinal column can be necessary after trauma induced compression and axial instability. This prospective designed study focuses on early results after minimal and less invasive techniques for shortest possible stabilization and fusion on thoracolumbar spine.

Materials and methods: Between 3/2005 and 3/2009 488 patients were operated for thoracolumbar spine injuries, 32 of which recruited for strictly monosegmental fixation and anterior fusion by monosegmental strut-graft transplant through anterolateral approach endoscopically/mini-open. Based on the technique used, 2 groups were compared: posterior monosegmental internal fixator $\left(\mathrm{USS}^{\circledR}\right)$ and anterior strut-grafting $(\mathrm{DV}, \mathrm{n}=17)$ and isolated monosegmental anterior fixation $\left(\right.$ Telefix $\left.{ }^{\circledR}\right)$ with strut-graft $(\mathrm{V}, \mathrm{n}=15)$. Clinical data from medical records as well as radiologic parameters were used to compare the results after combined and anterior surgery.

Results: Predominantly burst fractures treated of AO-Type A3.1A3.2 in both groups, for DV 4/17 B/C-Types, load sharing classification McCormack $7 \pm 1$. Similar initial conditions in both groups with vertebral body wedge $22-25^{\circ} \pm 6^{\circ}$ and height min. $50 \%$. Postop. significant correction of deformity in DV and $\mathrm{V}$, without significantly different reduction extent: sagittal Cobb angles $6^{\circ} \pm 7$ and $7^{\circ} \pm 5$ respectively. Spinal canal encroachment with both methods effectively reduced. Overall longer hospitalisation and operating-times for DV.

Conclusion: Both techniques allowed an effective reduction and fixation through the minimal invasive approaches used, allowing the shortest possible fusion reconstructing the anterior column. In selected cases depending on the injury and fracture type, monosegmental fixation and fusion can be preferable and especially with regard to A-type-fractures, anterior fixation and fusion by MIS become a valuable option.

Disclosure: No significant relationships.

\section{1}

\section{OUTCOME AFTER THORACOSCOPIC VENTRAL STABILIZATION OF THORACIC AND LUMBAR SPINE FRACTURES}

\section{R.Pfeifer ${ }^{1}$, R.M. Sellei ${ }^{2}$, M. Knobe ${ }^{2}$, P. Lichte ${ }^{3}$,H. Pape , $^{3}$ B. Schmidt-Rohlfing ${ }^{I}$}

${ }^{1}$ Klinik Für Orthopädie Und Unfallchirurgie, Schwerpunkt Unfallchirurgie, Universitaetsklinikum Aachen, Aachen, Germany, ${ }^{2}$ Department of Orthopaedic and Trauma Surgery, University Hospital of the Rwth Aachen, Aachen, Germany, ${ }^{3}$ Department of Trauma Surgery, University of Aachen, Aachen, Germany

Introduction: Thoracosopic-assisted ventral stabilization for thoracolumbar fractures has been shown to be associated with decreased recovery time and less morbidity. However, there is a limited number of studies evaluating late clinical and radiological results. We, therefore, performed an analysis of the late outcome of thoracolumbar fractures after minimal-invasive thoracoscopic ventral instrumentation.

Materials and methods: Between August 2003 and December 2009, 70 patients with thoracolumbar fractures (Th5-L2) have undergone ventral thoracoscopic procedure. Tricortical bone grafts or cage implants were used for stabilization. Assessment of outcome were: radiologic images (superior inferior endplate angle), Visual Analogue Scale (VAS), VAS Spine Score, patients satisfaction according to five choice satisfaction scale, and quality of life scores: SF-36 and ODI. Results: 47 patients $(67.1 \%)$ could be recruited for the follow up examination (range 64-2,405 days). At follow up mean VAS was 3.6 $( \pm 2.3)$ and mean VAS Spine Score $62.9( \pm 24.6)$. Around $67 \%$ of patients were very satisfied or satisfied with their results. Lower VAS Spine scores were observed in patients with concomitant injuries [54.5 $( \pm 27.4)$ vs. $63.1( \pm 22.1)]$ and in patients with intra- or postoperative complications $[44.7( \pm 16.7)$ vs. $65.8( \pm 24.5)]$. In addition, there was no difference in outcome between patients treated with bone graft versus cage implants.

Conclusion: Our results demonstrate good clinical results after ventral stabilization of thoracolumbar fractures. Stabilization methods 
(bone graft vs. cage implants) appear to not affect the long-term outcome. However, concomitant injuries and intra- or postoperative complications seem to be crucial.

Disclosure: No significant relationships.

\section{2}

\section{THE PREDICTIVE VALUE OF THE LOAD SHARING CLASSIFICATION OF THORACOLUMBAR FRACTURES}

\author{
M.J. Elzinga ${ }^{1}$, W.P. Zuidema ${ }^{2}$, F.W. Bloemers ${ }^{2}$, \\ E.S.M. de Lange-de Klerk ${ }^{3}$, F.C. Bakker ${ }^{2}$
}

${ }^{1}$ Trauma Surgery, VU University medical centre, Amsterdam, Amsterdam, The Netherlands, ${ }^{2}$ Trauma Surgery, VU University Medical Centre, Amsterdam, The Netherlands, ${ }^{3}$ Department of Epidemiology and Biostatistics, VU University Medical Center, Amsterdam, The Netherlands

Introduction: To predict failure of a short segment posterior stabilization for thoracolumbar fractures, McCormack et al. developed the Load Sharing Classification (LSC). They stated that fractures with a low LSC score can be managed with a posterior stabilization alone, whereas fractures with a high LSC score should have an anterior reconstruction to prevent failure of the posterior stabilization. We evaluated the LSC in a consecutive series of patients operated in our hospital. Thereby not only implant failure was considered but also loss of reduction without apparent implant failure.

Materials and methods: In this retrospective study, a consecutive series of patients with a posterior short segment stabilization for a thoracolumbar fracture of the spine was included. Loss of reduction was considered significant when it exceeded $10^{\circ}$. Implant failure was defined as bending, dislocation, or breaking of any part of the implant. 95\% Confidence intervals were calculated for differences in failure and loss of reduction between high and low LSC score fractures.

Results: 39 Patients were included in this study. Implant failure was seen in three fractures with a high LSC score, but this difference in failure between high and low LSC score fractures is not statistically significant. Fractures with a high LSC score showed significant more loss of reduction than fractures with a low LSC score (95\% confidence interval $=0.39 \pm 0.20$ ).

Conclusion: No difference in implant failure was seen between fractures with a low and a high LSC score in this small series of patients. However fractures with a high LSC score showed more loss of reduction. The LSC is a suitable adjunct for the selection of the type of surgical treatment for thoracolumbar fractures of the spine.

Reference: McCormack T, Karaikovic E, Gaines RW. The load sharing classification of spine fractures. Spine. 1994;19:1741-1744.

Disclosure: No significant relationships.

\section{EMERGENCY SURGERY 6}

\section{P17}

\section{PROMPT OR DELAYED APPENDECTOMY? A PROSPECTIVE STUDY IN 723 PATIENTS}

\section{G. Giraudo ${ }^{1}$, F. Baracchi $^{2}$, L. Pellegrino
H. SalCorso}

${ }^{1}$ Chirurgia Generale, ASO Santa Croce e Carle CUNEO, CUNEO, Italy, ${ }^{2}$ Chirurgia Generale, ASO Santa Croce e Carle, CUNEO, Italy, ${ }^{3}$ Chirurgia Generale, ASO Santa Croce e Carle, Cuneo, Italy
Introduction: The aim of the study was to compare, in a prospective trial, the clinical outcomes and histological findings in prompt and delayed appendectomy for acute appendicitis. It is debated in literature the safety of delayed appendectomy for suspected acute appendicitis. Materials and methods: From December 2001 to December 2007, the patients underwent appendectomy for histologically confirmed acute appendicitis were included in the study. Patients were divided into three groups according to the time of surgery after hospital admission: Early Appendectomy (EA) group within 12 h, Early Delayed Appendectomy (EDA) group between 12 and $24 \mathrm{~h}$ and Delayed Appendectomy (DA) group more than $24 \mathrm{~h}$. Perioperative data and pathologic state of appendix were considered and compared in each groups.

Results: 723 patients, with histological confirmed acute appendicitis, were included in the study: 518 patients in EA group, 140 patients in EDA group and 65 patients in DA group. Operative time were similar in each group. Post operative complications occurred in 49 patients $(6.8 \%)$ and resulted significantly higher in DA group compared to EA group $(\mathrm{p}=0.0012)$ and EDA group $(\mathrm{p}=0.003)$. Two patients $(3 \%)$ in DA groups died. No differences emerged in mean hospital stay. Phlegmonous and gangrenous appendicitis rates were significantly higher in DA group $(\mathrm{p}<0.05)$ compared to EA and EDA group.

Conclusion: The early administration of intravenous antibiotics followed by appendectomy within $24 \mathrm{~h}$ from presentation does not significantly increase the length of hospital stay or rate of complication References: Clyde C, Bax T, Merg A, MacFarlane M, Lin P, Beyersdorf S, McNevin MS. Timing of intervention does not affect outcome in acute appendicitis in a large community practice. Am J Surg. 2008. Ditillo MF, Dziura JD, Rabinovici R. Is it safe to delay appendectomy in adults with acute appendicitis? Ann Surg. 2006. Disclosure: No significant relationships.

\section{P20}

\section{SURGICAL MANAGEMENT OF PERFORATED PEPTIC ULCER: TRENDS IN DIAGNOSTICS AND INCREASED USE OF LAPAROSCOPIC REPAIR}

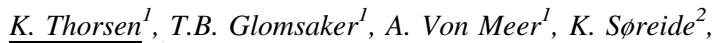 \\ $\overline{\text { J.A. Søreide }}{ }^{l}$
}

${ }^{1}$ Surgical Department, Stavanger University Hospital, Stavanger, Norway, ${ }^{2}$ Surgical, Stavanger University Hospital, Stavanger, Norway

Introduction: Prospective studies have demonstrated the feasibility and safety of laparoscopic management of patients with perforated peptic ulcer (PPU). However, routine clinical implementation has been rather slow and few studies report on the overall utility. The aim of this study was to evaluate the change in surgical management with outcomes in a defined population.

Materials and methods: Retrospective, single-institution, population-based review of all patients surgically treated for PPU during a 7-year period (2003-2009). Patient demographics, diagnostic workup, management and outcomes were evaluated.

Results: A total of 114 patients had surgery for PPU with a median age of 67 years (range 20-100). Women $(n=67,59 \%)$ were older $(\mathrm{p}<0.001)$, had more comorbidity $(\mathrm{p}=0.002)$, and a higher Boey risk score $(\mathrm{p}=0.036)$ compared to men. Perforation was located as gastric/pyloric $(72 \%)$ or duodenal $(28 \%)$. Pneumoperitoneum was diagnosed in $30(75 \%)$ of 41 patients after plain abdominal X-ray, in contrast to $76(99 \%)$ of 77 patients examined by abdominal CT $(\mathrm{p}<0.001)$. Laparoscopic treatment was intended in $48(42 \%)$ patients and completed in $36(75 \%)$ of these. Laparoscopically treated patients increased from 7 to $46 \%$ during the study period $(\mathrm{p}=0.02)$. Median operation time was shorter in the laparotomy group (70 $\mathrm{min}$ ), as compared to the laparoscopy group ( $82 \mathrm{~min})$, and the converted 
group $(105 \mathrm{~min})(\mathrm{p}=0.017)$. Postoperative complications were recorded in $56(49 \%)$ of the patients, and the overall 30-day postoperative mortality was $16 \%$. No statistically significant differences were found in morbidity and mortality between open or laparoscopic repair.

Conclusion: This study demonstrates an increased use of CT as the primary diagnostic tool for PPU and an increased use of laparoscopic repair in a surgical unit. Change in management is not associated with altered outcomes.

Disclosure: No significant relationships.

\section{P24}

\section{TRADITIONAL WEIGHT-BASED VANCOMYCIN DOSING IS INADEQUATE IN CRITICALLY ILL TRAUMA PATIENTS}

\section{K. Lunghi ${ }^{1}, \underline{\text { D.D. Yeh }}{ }^{2}$}

${ }^{1}$ Department of Clinical Pharmacy, University of California, San Francisco; San Francisco General Hospital, San Francisco, USA, ${ }^{2}$ Department of Surgery, San Francisco General Hospital, San Francisco, CA, USA

Introduction: Vancomycin is the most commonly used agent for empiric treatment of gram-positive pathogens. However, Vancomycin has poor lung penetration and current recommendations are to achieve serum trough levels of $15-20 \mathrm{mg} / \mathrm{L}$. Critically ill trauma patients are a unique population of patients with profoundly altered volume of distribution and renal clearance resulting from massive fluid shifts and physiologic insult.

Materials and methods: All injured ICU patients receiving intravenous Vancomycin at San Francisco General Hospital between May 1, 2004 and July 31, 2010 were identified through our Trauma Database and Pharmacy records. The initial weight-based dose was calculated and compared with Vancomycin trough levels. A true trough was defined as having been drawn within $30 \mathrm{~min}$ prior to administration of the next dose of Vancomycin; trough levels not meeting this definition were disregarded.

Results: 32 patients were identified who satisfied inclusion criteria. Only 12 patients $(37.5 \%)$ received adequate weight-based dosing (weight-based, $30 \mathrm{mg} / \mathrm{kg} /$ day). weight-based patients weighed significantly less than non-weight patients $(62.7$ vs. $84.0 \mathrm{~kg}$, $\mathrm{p}=0.0006)$. Weight-based patients were more likely to achieve therapeutic troughs than non-weight (58 vs. $30 \%, \mathrm{p}=0.15$ ). Of patients who were able to achieve therapeutic troughs, more weightbased patients achieved it initially than non-weight (33 vs. $5.0 \%$, $\mathrm{p}=0.05)$.

Conclusion: When prescribing commonly used dosing regimens, appropriate weight-based administration of Vancomycin occurred in only approximately one-third of patients. Those patients who did receive weight-based Vancomycin dosing were more likely to achieve therapeutic levels, both initially (33 vs. 5\%) and overall (58 vs. $30 \%$ ). Disclosure: No significant relationships.

\section{P37}

\section{ROLE OF BED SIDE SURGEON PERFORMED ULTRASOUND ON DIAGNOSIS AND TREATMENT OF APPENDICITIS IN YOUNG PATIENTS}

\section{Turati, G. Vignati, L. Pisoni, D.A. Merlini, E. Morandi}

Chirurgia Iv, Azienda Ospedaliera, Rho, Italy
Introduction: Surgical decision on appendicitis in young patients is always difficult due to difficulty with differential diagnosis.

Materials and methods: We prospective evaluate patients presenting to our Paediatric Department suspected for appendicitis. We collected data of the patient utilizing Alvarado Score (AS) adding Bed Side US Abdominal Evaluation done by a surgeon. We used a ESAOTE My Lab 25 with a 7.5-12.0 MHz Linear Transducer.

Results: 8 men and 10 women were observed. Age 7 to 17 -year-old (median age 12,3). 7 patients underwent surgical operation basing the decision on surgical evaluation (Alvarado Score) and Ultrasound Results. These patients had a median Alvarado Score (AS) of 5.86 (4-10). Of these patient only 5 had positive Ultrasound Evaluation and only these had a real appendicitis on surgical field and pathological microscopic examination. Median Alvarado Score for these 5 patient was 6.4 (4-10). Patients not undergoing surgical procedure were 11, median AS was 5.10 (3-8). All these patients were followed for 30 days to discover false negative cases. None of them underwent operation. Sensibility of Ultrasound was $100 \%$, Specificity was $83.33 \%$, PPV was $75 \%$ and PNV was $100 \%$. 7 patients had "Improbable Appendicitis", (AS 0-4) and of these 2 were operated due to symptoms (1 with negative US examination with no appendicitis on surgical field and another one had positive US and positive appendicitis). 7 patients had "Possible Appendicitis", (AS 5-6): 3 underwent operation ( 2 real appendicitis and 1 not, US evaluation was always accurated in diagnosis). 3 patient had "Probable Appendicitis", (AS 7-8), only 1 underwent operation and was positive either on surgical field or on US evaluation. Only 1 patient was "Almost Definite Appendicitis", (AS 9-10), and had positive US and real appendicitis.

Conclusion: In our study Alvarado Score $>5$ as a value to decide for operation led to a sensibility of $83.33 \%$ and specificity of $50 \%$, with a PPV of $45 \%$, NPV of $85.71 \%$. Data correlates with previous study. Authors propose CT scan to improve diagnosis but in our study ultrasound shows a sensibility of $100 \%$, a specificity of $83.33 \%$, with PPV of $75 \%$ and PNV $100 \%$.

References: 1. Alvarado A. Ann Emerg Med. 1986;15(5): 557-564. 2. Olivier Monneuse et al. Pain as the only consistent sign of acute appendicitis: lack of inflammatory signs does not exclude the diagnosis. World J Surg 2010;34:210-215.

Disclosure: No significant relationships.

\section{P58}

\section{VAC INSTILL THERAPY WITH ANTIBIOTIC IRRIGATION FOR THE TREATMENT OF A POSTOPERATIVE SEVERE DEEP WOUND INFECTION} I. Martínez-Casas, J. Sancho, M. Cáceres, E. Membrilla, N. Argudo,
M. Pons, L. Grande

Servicio De Cirugía General Y Digestiva, Hospital del Universitari Mar, Barcelona, Spain

Introduction: VAC-Instill is a system of synchronized intermittent vacuum therapy and irrigation. The World Union of Wound Healing Societies Consensus Document states that it can be used for the treatment of severe wound infections. To our knowledge, no cases of this therapeutic modality with antibiotic irrigation have been reported.

Materials and methods: Report a case of a severe deep wound infection in a surgical patient with prosthetic abdominal mesh, treated with VAC-Instill therapy irrigating with antibiotic. 
Results: A 45-year-old morbid obese female patient (BMI 40.6) was diagnosed of villoglandular cervix adenocarcinoma. During gynecological retroperitoneal laparoscopy for staging, conversion was required for intraoperative bleeding. An evisceration on the sixth postoperative day (POD) required a relaparotomy. An Ultrapro; mesh was placed in onlay position. The 10th POD the patient developed septic shock because deep wound and intrabdominal infections. A second relaparotomy was carried out to debride intrabdominal and deep wound abscesses. The mesh was taken out and a new one was placed to reinforce abdominal wall. Treatment with carbapenems was started and serial exudate cultures showed Bacteroides spp., S. epidermidis, $P$. aeruginosa, M. morgagnii, E. faecalis, and P. vulgaris. The wound was debrided many times and a conventional VAC; therapy was started after a normal abdominal CT-scan. Several antibiogram-guided intravenous antibiotic regimes were used. Nevertheless, evolution was torpid. On the 44th POD, conventional VAC; therapy was substituted by VAC-Instill; therapy irrigating with topic Piperacillin-Tazobactam $(2 \times 4 \mathrm{~g} / 125 \mathrm{mg} / 500 \mathrm{~mL}$ saline solution per cycle) guided by antibiogram results. Oral and parenteral antibiotics were discontinued. The wound was bedside debrided and the system was changed every 4th day. Subsequent wound cultures showed only $C$. albicans and oral fluconazol was given. After 25 days (POD 69) topic antibiotic therapy was discontinued and the patient discharged home with conventional VAC therapy. Three months later cosmetic results were excellent and there were no signs of mesh reinfection.

Conclusion: VAC-Instill Therapy with local antibiogram-guided antibiotic irrigation has been safe and effective in the treatment of a severe deep abdominal wound infection with a prosthetic mesh in place. This modality of treatment deserves further investigation.

Disclosure: No significant relationships.

\section{$\mathbf{P 1 7 3}$}

\section{DAMAGE CONTROL SURGERY IN ELDERLY WITH NON TRAUMATIC BLEEDING}

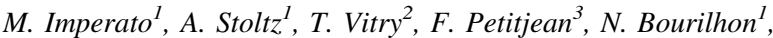 \\ J. Jarry ${ }^{I}$, P. Michel $^{I}$
}

${ }^{1}$ chirurgie Viscérale (Visceral Surgery), Hôpital D'instruction Des Armées Desgenettes (Military Hospital), Lyon Cedex 03, France, ${ }^{2}$ service De Radiologie (Radiology), Hôpital D'instructiond Des Armées Desgenettes, Lyon Cedex 03, France, ${ }^{3}$ service De Réanimation, Hôpital D'instruction Des Armées Desgenettes, Lyon Cedex 03, France

Introduction: Concept of damage control can also be used in non traumatic emergencies. This strategy is of great interest in elderly, specially with several comorbidities.

We report the case of an old woman with upper digestive acute bleeding. We use the concept of two steps surgery. We compare this case to literature's data.

Materials and methods: An 88-year-old ASA III women is admitted in ICU with melaena, tachycardy and haemorragic shock. Haemoglobine is $55 \mathrm{~g} / \mathrm{l}$. She had an oesogastroduodenal fibroscopy 15 days ago. It showed a huge gastric tumor $(10 \mathrm{~cm})$ with ulcerative lesion. An abdominal tomodensitometry shows an active bleeding from the tumor. Radiological embolization is not feasible. After blood transfusion (4 units), we decide to operate her.

Results: She has first a partial gastrectomy, without continuity restoration and with jejunostomy. After a complete resuscitation during 5 days, 2 nd surgery consists on a restoration of the digestive continuity with transmesocolic gastroenteral anastomosis.
The tumor is a stromal tumor with a high grade of malignancy. After surgeries, she presents arythmia with dysthyroïdy, and ARDS. A tomodensitometry does not show complications of the surgery. She died 41 days after the last surgery in a psychological shift syndrom. Conclusion: Surgery in 2 steps allows a survey for few more days in elderly patient with haemorragic shock even if comorbities are often a problem difficult to solve after surgery.

Reference: Stawicki, et al. Injury 2008;39:93-101. Hendrickson. Emerg Med Clin North Am. 2003;937-69. Finlay et al. BJS. 2004;91:83-85.

Disclosure: No significant relationships.

\section{P187}

\section{EARLY DIAGNOSIS CAN MAKE ISCHEMIC SMALL BOWEL CAUSED BY STRANGULATION AVOID RESECTION}

\section{T. Funabiki, S. Matsumoto, M. Shimizu, N. Hiroe,} M. Murakawa, T. Shibusawa, M. Yamazaki, M. Kitano

Department of Trauma and Emergency Surgery, Saiseikai Yokohamashi Tobu Hospital, Yokohama, Japan

Introduction: Strangulation needs to be operated as soon as possible. We have to diagnose correctly as strangulation immediately in ER. CT scan is essential to diagnose of acute abdomen. Multidetectorrow CT (MDCT) is known to be superior to conventional or single helical CT scan. As an early preoperative diagnosis, we can sometimes avoid resection of ischemic small bowel loop, just only get rid of the cause. Currently, strangulation were operated using a laparoscopic procedure instead of a laparotomy, especially in case of reversible small bowel ischemia. We hypothesis that small bowel ischemia/infarction can be divided into some phases. The present study retrospectively investigated some specific findings using 64-MDCT to diagnose strangulation.

Materials and methods: All 146 consecutive patients who were admitted to our hospital diagnosed as small bowel obstruction (SBO) between April 2007 and December 2008 were enrolled in the present study. All patients were performed CT scan, and were surgically or clinically confirmed. CT scans were obtained using 64-slice MDCT with or without contract material. All data were retrospectively reviewed by a board-certificated radiologist blinded to the patients' information and clinical details. The reader evaluated strangulation ileus or not. And also evaluated whether the findings, such as ascites, diminishment of contrast enhancement, closed loop, whirl sign, pneumatosis intestinalis, and peumoportogram are detectable or not. We separate these patients into two groups, strangulation group and not-strangulation group. And more, among the strangulation group, we separate into two groups, patients who were performed small bowel resection or not.

Results: Strangulation included 40 cases. The sensitivity, specificity, and accuracy of the MDCT were 87.5, 88.7, and 92.5\%. The specific finding was closed loop, and its sensitivity and specificity were 82.5 and $90.6 \%$. Among strangulation, 19 patients $(47.5 \%)$ were not performed to resect of ischemic small bowel loop because of reversible ischemia. The time from CT scan to surgery was no significant change whether small bowel resection or not ( 4.8 vs. $5.2 \mathrm{~h}$ ). In the patients who had whirl sign and did not have closed loop, 8 patients $(80 \%)$ were not performed small bowel resection.

Conclusion: When strangulation is diagnosed, operation could be needed. But if whirl sign is positive and closed loop sign is negative on MDCT, the resection of ischemic small bowel may be avoided. Disclosure: No significant relationships. 


\section{P194}

\section{PREDICTORS OF PERIOPERATIVE COMPLICATIONS ON LAPAROSCOPIC SURGERY FOR PERFORATED DUODENAL ULCER}

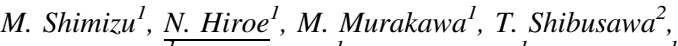 \\ S. Matsumoto ${ }^{T}$ T. Funabiki ${ }^{1}$, M. Yamazaki ${ }^{1}$, M. Kitano ${ }^{1}$
}

${ }^{1}$ Emergency Surgery, Saiseikai Yokohama-city Tobu Hospital, Yokohama, Japan, ${ }^{2}$ Emergency and Critical Care Medicine, Saiseikai Yokohama-shi Tobu Hospital, Yokohama, Japan

Introduction: Previous studies have demonstrated that about laparoscopic repair for perforated peptic ulcer, the predictors of postoperative complications are hemodynamic instability, APACHE II score, delayed diagnosis, and that conversion to open surgery are location or diameter of perforation. In terms of risk stratification, the indications for laparoscopic omental patch repair have limited to perforated duodenal ulcer without preoperative shock or organ failure. We investigated the predictors of postoperative complications and conversion to open surgery under our stratification.

Materials and methods: 85 patients who underwent laparoscopic surgery for perforated duodenal ulcer during a 14-year period were included in this study. We compared age, sex, APACHE II score, interval time between perforation and operation, diameter of perforation, between laparoscopic repair group (L) and conversion group (C). In group L, patients with complications were compared with those without complications.

Results: 11 of 85 cases (13\%) were converted to open surgery. Group $\mathrm{C}$ was older than group L (mean 59/46 year-old), took longer time to operation (mean 21/10 h), and had larger perforation (mean 8.7/ $4.5 \mathrm{~mm}) .8$ of $74(11 \%)$ cases in group L had postoperative complications, including leakage in 2 patients, and stenosis in 2 patients. Any risk factors such as hemodynamic instability and APACHE II score were not associated with postoperative complications.

Conclusion: In laparoscopic repair of perforated duodenal ulcer, larger perforation and longer time between perforation and operation increase the risk of conversion to open surgery. On the other hand, APACHE II score or interval time between perforation and operation are not associated with postoperative complications.

Disclosure: No significant relationships.

\section{OPEN FRACTURES: LOWER EXTREMITIES}

\section{3}

\section{MUSCULAR FREE FLAP FOR THE COVERAGE OF THE DISTAL THIRD OF THE LEG}

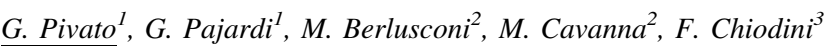

${ }^{1}$ Hand Surgery, Multimedica Hospital, Milan, Italy, ${ }^{2}$ Trauma Surgery Ii, Istituto Clinico Humanitas, Rozzano, Italy, ${ }^{3}$ Traumatology Ii, Instituto Clinico Humanitas, Rozzano, Italy

Introduction: The distal third of the leg is often affected by complex and high energy trauma, involving both the bone and soft tissues. Cutaneous ulcerations and bone or hardware exposure are frequent complications predisposing the patient to a high risk of infection and compromising the orthopedic treatment. Furthermore primary or secondary loss of soft tissue coverage are frequent after trauma or after damage control orthopedic surgery involving aggressive debridement of not viable or contaminated tissues. In this peculiar situations traditional coverage techniques are often inadequate in terms of dimensions, thickness and vascular supply to the affected part. Microsurgical techniques allow the transfer of richly vascularized tissues, without dimensional limits; in this way large debridement can be done. Muscular free flaps are a good option to provide at the same time coverage to the bone and hardware and to assure adequate blood supply. Furthermore bone stabilization and cutaneous coverage with free flap can be realized in emergency in one stage procedure. Our favorite flap is the free gracilis muscle: with a minimum damage at donor site level, it provides an optimal coverage of the loss of substance both from a superficial and volumetric point of view.

Materials and methods: From January 2007 and December 2009 we treated 12 patients with cutaneous loss of substance at the distal third of the leg level with free gracilis flap. All patients were investigated preoperatively with an arteriography in order to put in evidence the arterial blood supply. Patients with bone infection were studied with PET-TAC examination preoperatively and each 3 months after operation for 1 year.

Results: No major complications where observed. Skin closure occurred within 30 days.

Conclusion: In the treatment of fractures of the distal tibia with soft tissues loss of substance, the gracilis muscular free flap provides an optimal coverage.

Disclosure: No significant relationships.

\section{4}

\section{BIOMECHANICAL TESTING OF A NEW DEVELOPED PATELLAPLATE IN COMPARISON TO TENSION BAND WIRING}

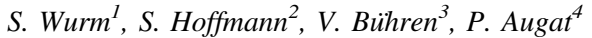

${ }^{1}$ Biomechanical Research Laboratory, Trauma Center Murnau, Murnau, Germany, ${ }^{2}$ Biomechanical Research Laboratory, Berufsgenossenschaftliche Unfllklinik Murnau e.V., Murnau, Germany, ${ }^{3}$ Trauma Surgery, Trauma Center Murnau, Murnau, Germany, ${ }^{4}$ Biomechanical Research Laboratory, Traumacenter Murnau, Murnau, Germany

Introduction: Due to the functional importance of the patella, accurate reduction and rigid fixation of a patellar fracture are required. Tension band wiring has been the standard treatment of patellar fractures for a long time, but there are several problems associated with the tension band wiring, e.g. loosening of the wires, dislocation of the fracture, poor outcome. Most of these problems could be avoided by employing a plating technique. Therefore, the aim of our study was to assess the biomechanical properties of a newly designed locking patellaplate.

Materials and methods: We tested a total of 12 dome-shaped Sawbone specimens (density $30 \mathrm{pcf}$ ) after osteosynthesis of a "transverse fracture": (1) plate osteosynthesis (6 specimens) (2) tension band wiring (6 specimens) The biomechanical test setup combined tension and bending to simulate the physiological load of the patella. The osteosyntheses were tested statically at $45^{\circ}$ of "knee flexion" to evaluate the load-to failure.

Results: The failure mechanism was a bone substitute failure occurring at $627 \mathrm{~N}(\mathrm{SD} 231 \mathrm{~N})$ using the tension band wiring and at $1,052 \mathrm{~N}(\mathrm{SD} 32 \mathrm{~N})$ using the plate $(\mathrm{p}=0.001)$. But using the tension band wiring, a fracture gap of $2.4 \mathrm{~mm}$ (=failure of the osteosynthesis) occurred already at $350 \mathrm{~N}$ and at $\mathrm{F}$ max the fracture gap extended to $5.1 \mathrm{~mm}$. In contrast, the fracture gap after plate osteosynthesis did not exceeded to $1.8 \mathrm{~mm}$ until the bone substitute failure $(1,052 \mathrm{~N})$.

Conclusion: In conclusion, the newly designed locking patellaplate provided significantly better biomechanical properties compared to 
the tension band wiring. In addition, its anatomical shape offered optimal fragment contact.

Disclosure: This study has been done in cooperation with Fa. Arthrex.

\section{5 \\ EXTERNAL FIXATION AS A DEFINITIVE TREATMENT FOR LONG BONE FRACTURES IN CHILDREN}

\section{F. Sala, M.A. Agus, F. Castelli, C. Guarnerio, D. Capitani}

Department of Orthopedic Surgery and Traumatology, Niguarda Ca Granda Hospital, Milano, Italy

Introduction: The accepted treatment for unstable displaced long bone fractures in adult is primary closed reduction and intramedullary nailing or plating. However this method poses a problem when treating young adolescents whose epiphyseal plates have not yet closed. We used the external fixation as a definitive method of treatment for these patients.

Materials and methods: 18 patients with displaced unstable humeral (1), tibial (9), and femoral (8) fractures (age 4-14 years) of which 2 were open (Gustilo II-III), were treated by this method from 2003 to 2009. All patients were allowed to weight bear from the first postoperative week. Physiotherapy was started immediately after operation and continued until normal knee and ankle function was regained. Dynamization was done in all cases 2 weeks before removal of frame.

Results: A good or excellent alignment with full ROM in the ankle and knee joint was obtained in all patients. There were no cases of delayed or non union. No cases of contractures or nerve injuries were reported. Superficial pin tract infection was seen in five patients, treated by antibiotics and local care. No case of osteomyelitis or deep infection occurred. Length of fixation was 8-20 weeks (mean 12 weeks).

Conclusion: This method permits fixation without danger of injury to the epiphysis in growing adolescents. The stability of the fixator allows early weight bearing and leaves the adjacent joints mobile. The healing time is relatively shorter than in other methods of the treatment and the complications rate was low in the presented series.

Disclosure: No significant relationships.

\section{6}

\section{PINS CONFIGURATION INFLUENCE IN BALANCE OF GENERAL TRANSVERSAL STABILITY OF LONG BONE FRACTURES EXTERNAL FIXATION}

\author{
M.M. Mitkovic ${ }^{1}$, M.B. Mitkovic ${ }^{1}$, I. Micic ${ }^{1}$, I. Kostic ${ }^{2}$, P. Stojiljkovic ${ }^{2}$ \\ ${ }^{1}$ Trauma, University Orthopaedic and Trauma clinic, Nis, Serbia, \\ ${ }^{2}$ University Orthopaedic and Trauma clinic, Nis, Serbia
}

Introduction: This study presents comparing of balances of general transversal stability between external fixation with pins in one axial plane and external fixation with pins in two axial planes.

Materials and methods: The investigation was performed by the use of two same long bone models made of wood. Each model was cut transversally at the middle area and was put an external fixator with two pins in each fragment. One model was fixed with all pins in the same axial plane and other was fixed with pins oriented in two perpendicular axial planes. There had been applied transversal force on these bone models. Intensity of the force was constant and the action of the force was realised in two different situations determinated by the direction of the force vector: direction in plane A and direction in perpendicular plane, plane B. There had been measured movement of long bone model segment during the action of transversal force in each of two situations. Relation of movements in each of two situations was parameter of the balance of general transversal stability of each long bone model fixation.

Results: The relation of average long bone fragment movement in fixation with pins oriented in one axial plane showed significant difference $(p<0,05)$ in relation to the relation of average long bone fragment movement in fixation with pins oriented in two perpendicular axial planes.

Conclusion: External fixation with pins oriented in different axial planes provide more balanced general transversal stability of long bone fractures what is more desirable feature according to biomechanical features of long bones.

References: 1. Windolf $\mathrm{M}$, et al. A novel nonbridging external fixator construct versus volar angular stable plating for the fixation of intraarticular fractures of the distal radius: a biomechanical study. Injury. 2010;41(2):204-209. 2. Rozbruch SR, Ilizarov S. Limb lengthening and reconstruction surgery, Informa, London, 2007.

Disclosure: No significant relationships.

\section{7}

\section{USE OF ILIZAROV-TYPE CIRCULAR EXTERNAL- FIXATOR IN TRAUMA AND POST TRAUMATIC RECONSTRUCTION. OUR EXPERIENCE AT NIGUARDA MILAN HOSPITAL}

\section{F. Sala, M.A. Agus, F. Castelli, D. Capitani}

Department of Orthopedic Surgery and Traumatology, Niguarda Ca Granda Hospital, Milano, Italy

Introduction: Truelok (TLK) fixator (Orthofix) is a multiplanar Ilizarov-type apparatus, we discuss our experience and the versatility of this device in management of trauma and post traumatic reconstruction.

Materials and methods: Over 24 months TLK was used in over 39 trauma and post traumatic reconstruction cases which were collected from our prospective external fixators data base. Classic TSK planning strategy was adopted and the TSK computer program used and in case of fractures indirect reduction of fractures was achieved by realigning one fracture end to the other through Ilizarov technique. Our series included acute fractures, neglected fracture, nonunions, malunions, osteomyelitis following internal fixation. Cases were followed until frame removal. Data base and radiographs were reviewed for demographic data, initial and residual deformity parameters, final correction, time in the TLK, and complications.

Results: Patients age ranged from 11 to 95 years. There were acute fractures, neglected fractures, nonunions (humeral and tibial), femoral malunion, and chronic osteomyelitis cases. Time in the TLK varied according to patient age indication of application. All patients achieved alignment within $5^{\circ}$ of normal. All patients had superficial pin site infections that resolved with oral antibiotics. Thirty patients were not experiencing any pain in their last follow up. All the fractures united. There were no loss of reduction or return to the operating room for remanipulation. Anatomic alignment was achieved in all patients. Conclusion: TLK system according to Ilizarov method is a valuable tool in the arsenal of the trauma surgeon. The use of TLK provides initial and definitive fracture stability.

Disclosure: No significant relationships. 


\section{8}

\section{CLINICAL OUTCOME OF OPEN GRADE III C LOWER EXTREMITY FRACTURES}

\author{
M. Mokawem $^{\text {I }}$, N.K. Kanakaris ${ }^{2}$, K. Tzafetta ${ }^{3}$, S. Kay ${ }^{3}$, \\ P. Giannoudis ${ }^{4}$
}

${ }^{1}$ Trauma and Orthopaedic, Leeds Teaching Hospitals NHS Trust, Leeds, UK, ${ }^{2}$ Trauma and Orthopaedics, Leeds Teaching Hospitals NHS Trusr, Leeds, UK, ${ }^{3}$ Plastic and Reconstructive Surgery, Leeds Teaching Hospitals NHS Trust, Leeds, UK, ${ }^{4}$ Academic Department of Trauma and Orthopaedics, Leeds Teaching Hospitals NHS Trust, Leeds, UK

Introduction: The experience of a regional tertiary referral centre for complex limb reconstruction and plastic surgery from managing open fractures of the lower extremities with associated vascular injuryGustilo IIIc is presented.

Materials and methods: Retrospective cohort of openIIIc lower extremity fractures, managed between 1991 and 2006. Data regarding demographics, inpatient hospitalisation, treatment, post-operative course and complications were collected referring to a mean period of follow-up of 25 months. ISS/AIS-05, MESS score, EuroQol-5D were used to quantify the parameters. SPSS-09 was used to perform linear regression, t-paired test and correlation analysis.

Results: Eighteen patients, 15 males, of a mean age 34.7 years, ISS $=13(9-45)$, MESS $=6.9(3-10)$, LOS $=24.5$ days $(5-105)$. Three ended to an amputation, 4 ex-fixed, 8 plated, 5 nailed. The mean number of repaired vessels was 1.6 , with most common the posterior tibialis. Rotational flaps were used in 3 , free flaps in 6 , while another 3 were closed primarily. Seven developed wound infections, and 4 nonunions. According to the EuroQol sub-parameters physical health $(\mathrm{p}=0.001)$ and depression $(\mathrm{p}=0.003)$ limited social activities in $61 \%$ of the cases, mobility was correlated to MESS $(\mathrm{p}=0.002)$.

Conclusion: OpenIIIc lower extremity fractures represent the most complex of open injuries requiring optimal initial management, and early referral to specialist tertiary centres. Multidisciplinary teams need to be involved early; the "fix and flap" protocol is feasible in these conditions, while MESS can be a predictor of functional outcome.

References: 1. Veliskakis KP. JBJS(Br) 1959;41:342-354. 2. Gustilo RB, et al. J Trauma 1984;24:742-746. 3. Gopal S, Giannoudis PV, et al. JBJS(Br). 2004;86:861-867. 4. Kanakaris NK, et al. Injury. 2007; 38:S9-S18. 5. Crowley, Kanakaris N, et al. Injury. 2007;38:879-889. 6. Helfet DL, et al. CORR 1990;256:80-86

Disclosure: No significant relationships.

\section{9}

\section{GRADE 3C OPEN FRACTURES: AN ANALYSIS OF 47 REVASCULARIZED CASES}

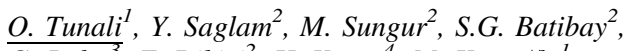

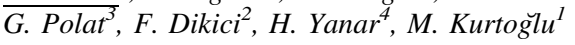

${ }^{1}$ Department of Orthopaedics and Traumatology, Istanbul University School of Medicine, Istanbul, Turkey, Istanbul University İstanbul Medical Faculty, İstanbul, Turkey, ${ }^{2}$ Orthopedics and Traumatology, Istnbul Tip Fakultesi, Istanbul, Turkey, ${ }^{3}$ Department of Orthopaedics and Traumatology, Istanbul University School of Medicine, Istanbul, Turkey, İstanbul University İstanbul Medical Faculty, İstanbul,
Turkey, ${ }^{4}$ General Surgery, Istanbul University, Medical Faculty, Istanbul, Turkey

Introduction: The fractures with major vascular injuries carry great risk of limb loss. The purpose of this study is to analyse the treatment results of grade 3C open fractures in our clinic between 2006 and 2009.

Materials and methods: A retrospective study of 59 fractures with major vascular injuries which were treated in our clinic between 2006 and 2009 years was executed. Twelve of the patients had no indication for revascularization due to major soft tissue injuries (autoamputation) and amputation was applied without trying vascular reconstruction. Forty-seven patients with a mean age of 31.08 (7-56) years were treated with vascular reconstruction and stabilization of the fractured bone. The locations of fractures were 13 humeri (27.6\%), 13 tibiae (27.6\%), 18 femora $(38.2 \%)$, and 3 radii $(6.3 \%)$. The vascular injury happened in 14 brachial arteries (29.7\%), 11 superficial femoral arteries (23.4\%), 9 tibialis posterior arteries (19.1\%), 11 popliteal arteries $(23.4 \%), 1$ ulnar artery $(2.1 \%), 1$ radial $(2.1 \%)$ artery.

Results: Vascular repair included 38 interpositional vein grafts $(80.8 \%), 9$ end to end anastomoses $(19.2 \%)$, ( 2 of them reimplantation). For fracture stabilization, 39 patients were treated with external fixation (82.9\%; 30 uniplanar, 8 multiplanar, 1 hybrid), and 8 patients with internal fixation $(17.1 \%$; 4 Kirschner wires, 3 plates, 1 intramedullary nail). Amputation was performed in 8 patients $(17.0 \%)$ after revascularization. Limb salvage was achieved in 39 patients (82.9\%). Eighteen of 39 patients $(46.1 \%)$ needed further operation because of pseudoarthrosis $(23.0 \%)$, infection $(7.7 \%)$, deformity $(2.5 \%)$ and to revise temporary fixation to definitive fixation $(12.8 \%)$. Twenty-one of all patients $(44.6 \%)$ needed no further operation and union was achieved.

Conclusion: A well organised multidisciplinary approach is needed to improve the outcomes of fractures with vascular injuries. Overall prognosis of these trauma patients is dependent on fast and sufficient diagnostics and therapy.

Reference: Gäbel G, Pyrc J, Hinterseher I, Zwipp H, Saeger HD, Bergert H. Arterial injuries combinated with open fractures-management and therapy. Zentralbl Chir. 2009;134(4):292-297 (Epub 2009 Aug 17. German).

Disclosure: No significant relationships.

\section{0}

\section{OPEN EXTREMITY FRACTURES: IMPACT OF DELAY IN OPERATIVE DEBRIDEMENT AND IRRIGATION}

A.K. Malhotra, S. Goldberg, N. Martin, M. Willis, V. Mounasamy, K. Guilford, T. Duane, M. Aboutanos, R. Ivatury

Department of Surgery, Medical College of Virginia, Virginia Commonwealth University, Richmond, VA, USA

Introduction: Early $(<8 \mathrm{~h})$ operative debridement and irrigation (D\&I) of open fractures is considered essential to reduce the risk of deep infection. With advent of powerful anti-microbials this axiom has been challenged. The current study evaluates the rates of deep infections of open fractures in relation to the time to first D\&I.

Materials and methods: A list of all blunt open extremity fractures was obtained from the trauma registry. Patients were evaluated for age, injury severity (ISS), physiologic derangement (SBP, Lactate, RTS), and fracture type (Gustilo). Time to first D\&I was calculated. All patients received appropriate prophylactic anti-microbials. Infection rates were calculated and correlated to the time to first D\&I ( $<8$ vs. $>8$ h). 
Results: Over 48-month study period, 248 patients presented with 251 open extremity fractures (upper 83 , lower 168). Two groups, $<8$ and $>8$-h were matched though age was lower and ISS higher in the $>8$-h group. Rates of infection were $23 / 203(11 \%<8$-h) and 10/48 $(21 \%>8-\mathrm{h})-\mathrm{p}>0.05$. Infection rates by Gustilo type were: I-2/48 (4\%), II-6/89 (7\%) and III-25/114 (22\%) - p $<0.05$ I and II versus III. When fractures were sub-grouped by extremity, for lower extremity, delay ( $>8-h)$ and higher Gustilo type, both correlated with development of infection. In upper extremity, only higher Gustilo type correlated and delay did not increase the incidence of infection (Table).

\begin{tabular}{llllll}
\hline Gustilo & \multicolumn{2}{l}{$\%$ Infection } & & \\
\cline { 2 - 3 } & Upper & & & Lower & \\
\cline { 2 - 3 } & $<8-\mathrm{h}$ & $>8-\mathrm{h}$ & & $<8-\mathrm{h}$ & $>8-\mathrm{h}$ \\
\hline I & 11 & 0 & 0 & 0 \\
II & 0 & 12 & 7 & 11 \\
III & 22 & 14 & 15 & 54 \\
\hline
\end{tabular}

Conclusion: Delay ( $>8-\mathrm{h}$ ) to first D\&I for open fractures of the lower extremity increases the likelihood of infection, but not for the upper extremity. Higher Gustilo type open fractures have a higher incidence of infection for both upper and lower extremities. The results have important implications in an era of decreasing surgeon availability, especially in off hours.

Disclosure: No significant relationships.

\section{VISCERAL TRAUMA 3}

\section{1}

\section{PAEDIATRIC TRAUMA MORTALITY IN NORWAY}

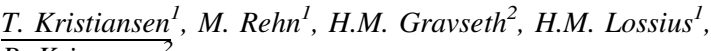 \\ P. Kristensen
}

${ }^{1}$ Department of Research, Norwegian Air Ambulance Foundation, Drøbak, Norway, ${ }^{2}$ National Institute of Occupational Health, Oslo, Norway

Introduction: Trauma is the leading cause of death among children and adolescence. Population-based epidemiological data are crucial for both preventive measures and the development of trauma systems tailored to provide optimal paediatric care [1]. We present a 10-year population-based register study of paediatric trauma deaths in Norway.

Materials and methods: All traumatic deaths $<16$ years of age in Norway from 1998 to 2007 were extracted form the Cause of Death Registry. 2002 was used as index-year for denominator data from Statistics Norway. Rates were compared between Norway's 19 counties. Percentages, median ages with interquartile range (IQR) are presented. Results: A total of 462 children died in the 10 -year period giving a rate of 4.81 per 100,000 per year. The median age was 8 years (3-13) and $60.4 \%$ were boys.

Nationally, 179 (38.7\%) deaths involved transport accidents and 32\% of deaths were caused by head injuries. In the most sparsley populated and northern county, Finnmark, there were 24 deaths, giving a mortality rate of 14.4 per 100,000 per year, almost three times the national average. The median age of death in Finnmark was 1.5 years over the national average. Fifteen $(62.5 \%)$ of deaths in this area were caused by head injuries and $17(71 \%)$ of patients were involved in transport accidents.

Conclusion: The national rates, injury characteristics and circumstances are congruent with other Scandinavian studies [2]. Mortality rates in Finnmark were almost three times national levels in this area and transport accidents and head injuries were also more common. This study identifies a high-risk area and describes the injury characteristics. Paediatric deaths occur soon after injury. This stresses the need for effective preventive measures as well as organized trauma systems that provide rapid prehospital care. Population-based epidemiological data are prerequisites for optimal resource allocation and preparedness.

References: 1. World Health Organization 2006. 2. Kristiansen $\mathrm{T}$, et al. Injury 2010;41(5):444-452.

Disclosure: No significant relationships.

0112

\section{EFFECT OF PREHOSPITAL FOCUSED ABDOMINAL SONOGRAPHY FOR TRAUMA (P-FAST) ON TRAUMA CARE AND MANAGEMENT: RESULTS OF A PROSPECTIVE, MULTICENTER STUDY}

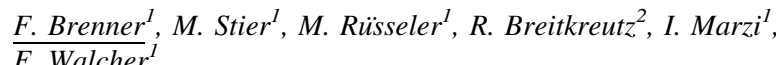

${ }^{1}$ Department of Trauma, Hand and Reconstructive Surgery, Hospital of the Johann Wolfgang Goethe-University Frankfurt am Main, Frankfurt am Main, Germany, ${ }^{2}$ Department for Anesthesiology, Hospital of the University of Saarland, Homburg/Saar, Germany

Introduction: P-FAST offers the possibility to detect an abdominal injury directly on trauma scene. We investigated the potentially benefit of this examination on management and outcome in multiple trauma patients.

Materials and methods: In a prospective, multicenter study we compared conventionally physical examination of the abdomen and such advanced by P-FAST in univariate/bivariate analysis. Means were compared using Student's $t$ test and analysis of variance and categorical variables using $\mathrm{chi}^{2}$.

Results: 243 patients were included. P-FAST was performed in 143 cases, physical examination in $100.12 .3 \%$ patients had a documented intraabdominal hemorrhage. In case of a prehospital diagnosis of intraabdominal hemorrhage the mean time from performing P-FAST to arriving the emergency department (ED) $(28 \pm 10 \mathrm{~min})$ was significantly lower than the time from performing clinical examination to arrival at ED ( $40 \pm 13 \mathrm{~min})$. In those cases when P-FAST showed positive findings prehospital care was changed in $45 \%$ (physical examination 50\%), prehospital management in $90 \%(60 \%)$ and the choice of receiving hospital in $80 \%(60 \%)$. In $12 \%$ hospital management was changed because of P-FAST, in no case because of clinical examination.

Conclusion: P-FAST improves an optimized prehospital and hospital trauma care and management of multiple injured patients.

References: Clarke $\mathbf{J}$ et al. Time to laparotomy for intra-abdominal bleeding from trauma does affect survival for delays up to $90 \mathrm{~min}$. J Trauma. 2002;52:420-425. Blaivas M, et al. Significant hemoperitoneum in blunt trauma victims with normal vital signs and clinical examination. Am J Emerg Med 2002;20:218-221. Walcher F, et al. Prehospital ultrasound imaging improves management of abdominal trauma. Br J Surg 2006;93:238-242.

Disclosure: No significant relationships. 


\section{3}

\section{PREDICTORS FOR DEVELOPMENT OF POST TRAUMA LAPAROTOMY ABDOMINAL COMPARTMENT SYNDROME}

\author{
D.L. van Imhoff ${ }^{1}$, E.M.M. Van Lieshout ${ }^{2}$, S. d'Amours ${ }^{3}$, O. van Waes ${ }^{4}$
}

${ }^{1}$ Trauma Surgery, Erasmus University Medical Center, Rotterdam, The Netherlands, ${ }^{2}$ Surgery-Traumatology, Erasmus MC, Rotterdam, The Netherlands, ${ }^{3}$ Liverpool Hospital, Sydney, Australia, ${ }^{4}$ Trauma Surgery, Erasmus University Medical Center Rotterdam, Rotterdam, The Netherlands

Introduction: Abdominal Compartment Syndrome (ACS) is well recognized and dreaded complication after a trauma laparotomy (TL). Discriminating pre-operative risk factors for developing ACS after trauma laparotomy would supply the trauma surgeon with information to support the decision whether or not to close the abdomen.

Materials and methods: From a prospective database of over 10 years, those patients were analyzed who received a trauma laparotomy within $24 \mathrm{~h}$. Univariate analysis was done to extract risk factors for developing ACS after a TL.

Results: 559 patients received a TL. The mean age was 31 years. $8.4 \%$ of the patients developed IAP greater then $20 \mathrm{mmHg}$ (intra abdominal pressure), of whom 45 per cent ACS. These patients were older (49 vs. 30 years), sicker (ISS 34 vs. 20), and suffered more from blunt then penetrating injury ( 83 vs. $64 \%$ ). ACS patients were preoperatively more acidotic (base excess -9.5 vs. $-5.0 \mathrm{mEq} / \mathrm{L}$ ) and coagulopathic (PTT 41 vs. $32 \mathrm{~s}$ ). ACS patients received significantly more crystalloids ( 6 vs. $4.3 \mathrm{~L}$ ) and units of RBC (16.5 vs. 2.0), but less units of whole blood ( 2 vs. 4 ). Mortality was higher for patients with ACS (23 vs. $11 \%)$

Conclusion: The "deadliest triad" of coagulopathy, acidosis and ACS should be combatted prior to trauma laparotomy, via resuscitation with less crystalloids and more whole blood.

Disclosure: No significant relationships.

\section{4}

\section{ROUTINELY USE OF LAPAROSCOPY IN STABLE PATIENTS WITH PENETRATING ABDOMINAL TRAUMA}

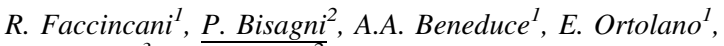 \\ V. Tomajer $^{3}, M$. Carlucci $^{2}$
}

${ }^{1}$ Deas, San Raffaele Hospital, Milan, Italy, ${ }^{2}$ Deas, IRCCS San Raffaele, Milan, Italy, ${ }^{3}$ Deas, IRCCS San Raffaele, Milano, Italy

Introduction: Trauma is the first cause of death in the first four decades in developed countries. Penetrating trauma are increasing in Europe. Routinely use of laparotomy in penetrating abdominal trauma with anterior fascia violation has lead to a wide number of non therapeutic laparotomies in certain report [1-2].

Materials and methods: Aim: Introduce of routinely laparoscopic approach in the treatment of stable penetrating trauma in a single center Methods: From July 2007 indications for laparoscopy in penetrating abdominal trauma were: stable patients, demonstrated anterior fascia violation. Contraindications were: haemodynamic instability and evisceration which both mandated laparotomy; evident superficial trauma (with no fascia violation) which usually did not require any surgery. All elected patient underwent general anesthesia, and $2-3$ trocars approach to abdominal laparoscopy with open periumbelical Hasson trocar positioning. In case of peritoneal violation we went on looking for visceral injuries and in case of hollow viscus perforation we usually converted in an open surgical procedure.

Results: We performed 18 laparoscopies for penetrating abdominal trauma haemodynamically stable with anterior fascia violation. In 3 $(16.7 \%)$ cases this approach avoided a non therapeutic laparotomy (2 cases no visceral or major vascular injuries, 1 case no peritoneal violation). In $1(5.5 \%)$ case we repaired a sigmoid mesenteric laceration without colonic involvement during the laparoscopy. In 1 (5.5\%) case we decided to convert to open surgery in presence of haemoperitoneum without finding any visceral injury at laparotomy. All the other $13(72.3 \%)$ cases underwent therapeutic laparotomies. Conclusion: Minimally invasive technique seems to be a valid tool in the diagnostic and probably therapeutic approach to penetrating abdominal trauma avoiding non therapeutic laparotomies. More studies are needed to confirm the role of laparoscopy in the trauma patients.

References: 1. Heng-fu L, et al. World J Surg. 2010;34: 1653-1662. 2. Uranus et al. Eur J Trauma Emerg Surg. 2010;36: 19-24.

Disclosure: No significant relationships.

\section{5}

\section{EXTRAPERITONEAL PACKING: DAMAGE CONTROL FOR THE HEMODYNAMIC UNSTABLE PATIENT}

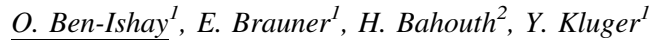

${ }^{1}$ Department of General Surgery, Rambam Health Care Campus, Haifa, Israel, ${ }^{2}$ Acute Care Service, Rambam Health Care Campus, Haifa, Israel

Introduction: Extraperitoneal packing (EPP) is reserved for the unstable trauma patients suffering pelvic fractures too unstable to undergo angiography. The objective of the study was to assess the impact and outcome of patients subjected to EPP in our institution.

Materials and methods: Charts of all of blunt trauma patients who were rushed to the operating theater for exploratory laparotomy due to hemodynamic instability from July 2007 through October 2010 were reviewed retrospectively. Patients who underwent EPP were identified. Data collected included: age, gender, ISS, Mechanism of injury, results of FAST or DPL, the use of ER Pelvic fixation, need for blood transfusion, length of stay in the ICU (LOS) and in the hospital (LHS) and associated injuries. Morbidity and 30 days mortality rate were recorded.

Results: 84 patients had exploratory laparotomy for hemodynamic instability. $8(9.5 \%)$ patients underwent EPP. Mean age was 43.5 years (25-73), $63 \%$ were males (n-5), $75 \%$ were involved in an MVA, mean ISS was $53.37 \%(n-3)$ had a positive FAST, and $25 \%$ (n2) had DPL performed that was negative in both. $50 \%(n-4)$ had an ER external pelvic fixation. $37 \%$ had a post packing angiography performed and only $12.5 \%$ (n-1) had an active arterial bleeding that resulted in embolization. Mean number of packed cells (PC) units used was 11 and fresh frozen plasma (FFP) 10. Mean LOS and LHS were 22 and 32 respectively. $25 \%$ of the patients had EPP without laparatomy. $100 \%$ had associated chest and orthopedic injury, head injury was diagnosed in $75 \%$. 30 days survival rate was $75 \%$ and no major complications directly related to the EPP were observed.

Conclusion: EPP is a life saving procedure reserved for patients in extreme. The procedure is safe, easy to perform and has low morbidity. EPP does not need to be followed by angiography if the patient remains stable after the surgical intervention.

Disclosure: No significant relationships. 


\section{6}

\section{SEVERE PELVIC INJURIES: MANAGEMENT AND HEMORRHAGE CONTROL. A RETROSPECTIVE STUDY}

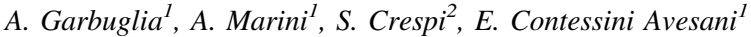

${ }^{1}$ General and Emergency Surgery, Fondazione IRCCS Cà Granda, Ospedale Maggiore Policlinico, Milano, Milan, Italy, ${ }^{2}$ Radiology, Fondazione IRCCS Cà Granda, Ospedale Maggiore Policlinico, Milano, Milan, Italy

Introduction: The aim of this study is to analyse the relationship between Hoffmann' s external fixation and selective arteriography with embolization in the management of unstable pelvic fractures and severe hemorrhage.

Materials and methods: We reviewed 330 patients with severe pelvic injuries admitted to our hospital from 1986 to the first 9 months of 2010 (155 male and 175 female; average age 40 years). At the admission in the emergency room, unstable pelvic ring fractures was present in 54\% of patients; hemorrhagic shock in $40 \%$ of patients. When CT showed pelvic bleeding, patient underwent external pelvic ring fixation or selective arteriography and embolization.

Results: In the first period (1986-1993) we performed only external fixation to control hemorrhage ( 8 cases). In the intermediate period (1994-2000) we performed both procedures, external fixation (4 cases) and embolization ( 8 cases). In the last period (2000-2010) only embolization was performed to control bleeding (12 cases): external fixation was a delayed (36-48 h) procedure to only stabilize pelvic ring ( 13 cases). One patient died in the first group. Two patient died in the second group (one underwent embolization, the other both procedures). Nobody died in the last group.

Conclusion: These results suggest us that embolization is a correct procedure to control pelvic hemorrhage if it is performed by an interventional radiologist with wide experience in this technique supported by an intensive care unit with a wide experience in trauma care. Hoffmann's pelvic fixation may be a complementary delayed procedure or may be performed in a damage control laparotomy. T-POD (Trauma-Pelvic Orthotic Device), or similar device, is a reliable and easy use device complementary to embolization in the acute care of pelvic trauma patient.

Reference: Garbuglia A, et al. Severe pelvic injuries: management and techniques of skeletal fixation. Eur J Trauma Emerg Surg. 1999;XXII (3): 13 .

Disclosure: No significant relationships.

\section{7}

\section{LAST 3 YEARS EXPERIENCE WITH POLITRAUMA AT TRAUMA CENTER OF CESENA: COMPARISON WITH REGIONAL MAJOR TRAUMA REGISTRY}

\section{P. Ruscelli, F. Buccoliero}

Emergency, Trauma Center Cesena, Cesena, Italy

Introduction: The purpose of this paper is to compare the results obtained in the patients treated in the Trauma Center of Cesena, with those obtained in the homogeneous group stored in the Regional Major Trauma Registry.

Materials and methods: We analyzed a population of 4,550 major trauma patients. They were divided into two Groups: Group A included 3,803 patients selected for ISS $>15$, treated in ICU or dead on arrival in shock room and stored in the Regional Major Trauma
Registry of Emilia Romagna. Group B included 747 patients treated in the ICU of the Cesena Trauma Center, in the last 3 years. The group B was treated according to precise diagnostic and therapeutic protocols developed and rewieved by the Trauma Service of our Trauma Center.

Results: In the Group A the overall hospital mortality was $12.5 \%$, while in Group B was $11,9 \%$. Mortality at discharge from intensive care was $12.8 \%$ in Group A and $10.6 \%$ in Group B. The total average hospital stay was 26.7 and $21.2(-2.2 \%)$ days respectively. Average ICU stay was respectively 8.8 and $7.6(-13.6 \%)$. 2,200 (57.8\%) patients underwent surgical procedure in Group A and 559 (74.8\%) in Group B $(+17 \%)$. $40 \%$ of Group A patients undergoing diagnostic level II with multislice CT while $36 \%$ in the Group B; a total of respectively $4.4 \%$ and $8.2 \%(+3.8 \%)$ of patients underwent angiography. In the Group B 24 patients $(3.2 \%)$ underwent embolization.

Conclusion: The results in comparison with the Regional Major Trauma Registry seem to demonstrate a reduction in overall mortality, average hospital and ICU stay, and a less need of multislice CT. At the same time the comparison shows a higher rate of surgical procedure and angiographies implemented.

Disclosure: No significant relationships.

\section{6}

\section{MORPHOLOGICAL EVALUATION OF AREAS OF DAMAGE IN BLUNT CARDIAC INJURY AND INVESTIGATION OF TRAFFIC ACCIDENT RESEARCH}

\section{Y. Sakamoto ${ }^{1}$, N. Kutsukata $^{1}$, K. Mashiko ${ }^{2}$, H. Yokota ${ }^{3}$, S. Tominaga ${ }^{4}$, T. Nishimoto 5}

${ }^{1}$ Emergency Medical Center, Emergency Disaster Medicine, Saga University Hospital, Saga, Japan, ${ }^{2}$ Shock and Trauma Center, Nippon Medical School Chibahokusoh Hospital, Chiba, Japan, ${ }^{3}$ Critical Care Medicine, Nippon Medical School, Tokyo, Japan, ${ }^{4}$ College of Science Technology, Nihon University, Tokyo, Japan, ${ }^{5}$ College of Engieering, Nihon University, Fukushima, Japan

Introduction: This study investigated the relationship between cause of injury and areas of damage in blunt cardiac injury and, in a micro study using engineering-based medicine, examined traffic accident cases for injury prevention.

Materials and methods: Of the 2,673 subjects transported to our facility within the 15-month period from 2009 to 2010.33 underwent emergency thoracotomy. Among them, 12 patients had perforated cardiac injury. To determine the severity of injury in each case, survival probability rate was calculated using the Abbreviated Injury Scale. Furthermore, in collaboration with the Department of Engineering, we conducted a micro study on circumstances surrounding the traffic accident and vehicle damage in two of the cases.

Results: Subjects were 8 males and 4 females (mean age 64 years). Cause of injury was 9 traffic accidents, 2 falls, and 1 labor accident. The mean ISS was $54.0 \pm 19.6$, and the probability of survival was 0.147 . The actual survival rate was $16.7 \%$. Lesion sites in the 12 cases (total of 21 sites) were: right atrium in 8 cases, superior or inferior vena cava in 5 cases, right ventricular free wall in 4 , excluding the left ventricle and ascending aorta. The 2 cases that were further studied both involved a compact car accident while driving at relatively low barrier conversion speed: $41,25 \mathrm{~km} / \mathrm{h}$ ).

Conclusion: Blunt cardiac injury was more common on the right cardiac chambers, especially to the right atrium. The survival rate was extremely close to the calculated survival probability. Proper seat belt usage is important for the prevention of blunt cardiac injury.

Disclosure: No significant relationships. 


\section{TECHNICAL AND NON-TECHNICAL SKILLS}

\section{8}

\section{EMERGENCY SURGERY EDUCATION AND TRAINING IN PORTUGAL}

\section{C.A. Mesquita, E.S. Steering Group}

\section{Surgery Iii, Coimbra University Hospital, Coimbra, Portugal}

Introduction: The Portuguese Medical Association (Ordem dos Médicos, OM) is the official entity that regulates all the medical and surgical activities in Portugal, being his duty to protect the public interest. In Portugal there are three official ways to differentiate: Specialty (vertical), Subspecialty (vertical) and Competence (transversal). Doctors may access to a subspecialty or a competence as a second step, after a specialty.

Materials and methods: Doctors must be registered with to practise medicine or surgery. OM also sets the standards and outcomes for basic medical education. After graduating from medical school and completing their foundation training, doctors usually complete a third and even a fourth stage of postgraduate training, whose standards are set by the Colleges. These are responsible for promoting the development of postgraduate medical education and training for all, establishing standards and requirements and making sure they are met across the country.

Results: Emergency Medicine exists as a competence since 2002 and goes behind the pre-hospital acute care. This College strongly supports the development of an autonomous College of Competence on Emergency Surgery (trauma surgery included) and it exists, since 2007, a national Steering Group on Emergency Surgery Education (Grupo de Trabalho para a Formação Específica em Cirurgia de Emergência), with 13 representatives from general surgery (7), neurosurgery (1), orthopaedics (1), thoracic (1), vascular (1), urological (1) and paediatric surgery (1). All general surgeons are IATSIC members and representatives of the existing trauma and emergency surgery societies: Sociedade Portuguesa de Cirurgia, Grupo Trauma, Associação Lusitana de Trauma e Emergência Cirúrgica and Sociedade Portuguesa de Trauma. Those who are DSTC instructors also lead the National Steering Committee for DSTC, after a memorandum of understanding signed with IATSIC in 2008. The other members are representatives of the surgical specialties Colleges relevant in this field.

Conclusion: The main objective of this multidisciplinary steering group is the development of an autonomous College to rule, in a next future, emergency surgery education and training in Portugal and act as national representative near the UEMS and other international institutions.

Reference: Ordem dos Médicos, Colégio de Competência em Emergência Médica, Grupo de Trabalho para a Formação Específica em Cirurgia de Emergência. Coimbra, 2009

Disclosure: No significant relationships.

\section{9}

\section{EVALUATION OF TRAUMA CARE IN A MATURE LEVEL I TRAUMA CENTER IN THE NETHERLANDS}

\section{K. Lansink ${ }^{1}$, A.C. Gunning ${ }^{2}$, L.P.H. Leenen ${ }^{2}$}

${ }^{1}$ Department of Surgery, Trauma and Surgical Intensive Care Unit, University Medical Centre Utrecht, Utrecht, The Netherlands, ${ }^{2}$ Department of Surgery, University Medical Centre Utrecht, Utrecht, The Netherlands
Introduction: Trauma centers are associated with improved survival rates and outcomes in trauma patients [1]. In 2001 our hospital officially became a Level I trauma center. The implementation of trauma centers showed a significant reduction in mortality and hospital length of stay in our hospital and trauma region [2]. The aim of this prospective database study is to present the outcomes of patients in the further maturation of a Level I trauma center.

Materials and methods: We included and analyzed all adult trauma patients admitted to our trauma center during the years 2003 through 2005 (period 1) and 2007 through 2009 (period 2).

Results: A total of 3,479 patients were included; 1,721 in period 1 and 1,758 in period 2. Mean ISS increased from 12.4 to 14.1 $(p<0.001)$. ICU admittances increased from 17.7 to $19.5 \%$ $(\mathrm{p}=0.176)$. Penetrating injuries increased from $67(3.9 \%)$ to 125 $(7.1 \%)(\mathrm{p}<0.001)$. More head $(+7.0 \%)$ spine $(+3.5 \%)$ and rib/lung $(+3.9 \%)$ injuries and less injuries to extremities $(-6.0 \%)$ were seen period 2. Mortality, adjusted for age and ISS, was lower in period 2 (OR $0.742, \mathrm{p}=0.039$ ). In period 2 , more patients relatively died of CNS injuries ( 58.3 vs. $66.0 \%, \mathrm{p}=0.172$ ) and less of MOF (9.0 vs. $7.2 \%, \mathrm{p}=0.561)$. Adjusted for age, ISS and survival fewer patients were admitted to the ICU (OR 0.684, p < 0.001) and hospital and intensive care unit stay shortened (OR 1.178, $\mathrm{p}<0.001$; OR 1.252, $\mathrm{p}=0.006)$.

Conclusion: As the trauma center matured, patient outcomes improved. Mortality reduced, ICU admittances decreased, and hospital and ICU stay shortened. Further, mean ISS increased indicating admittances of patients with worsening injuries.

References: 1. Lansink KWW, Leenen LPH. Do designated trauma systems improve outcome? Curr Opin Crit Care 2007;13(6): 686-690. 2. Spijkers ATE, et al. Mortality decreases by implementing a Level I Trauma Center in a Dutch Hospital. J Trauma 2010;68:1138-1142.

Disclosure: No significant relationships.

\section{0}

TRAUMA TEAM ACTIVATION CRITERIA IN MANAGING TRAUMA PATIENT AT EMERGENCY ROOM IN THAILAND

\section{P. Wuthisuthimethawee}

Emergency Medicine, Prince of Songkla University, Hatyai, Thailand

Introduction: Trauma team activation criteria was used to select the patients who need immediate decision making by most experience surgeons. The objective of this study was to determine the effect of trauma team activation criteria after applied in emergency room.

Materials and methods: This was 1 year prospective longitudinal study. The patients age $>18$-year-old who met inclusion criteria were eligible. The demographic and physiologic data. The injury severity score (ISS) was calculated and multiple logistic regression was used to determine factors affecting on mortality rate. Institutional review board approval was obtained from the Prince of Songkla University. Results: A total of 80 patients were eligible. Shock, penetrating torso injury and pulse rate $>120$ beats per minute were three most common criteria for consultation. The median response time was $5 \mathrm{~min}$. The overall median ISS was 21.0 (1-75). At the emergency room; 9 patients were dead, 30 patients needed immediate operation and 41 patients were admitted. All of arrest patients were died. In immediate operation patients; $53.3 \%$ survived. For admitted patients; $65.9 \%$ survived $(\mathrm{p}=0.026)$. All patients in ISS $<9$ were survived and 29 patients in ISS $>25: 18$ patients were dead $(\mathrm{p}=0.032)$. The median time of ERLOS was improved from 184 to $85 \mathrm{~min}$. The parameters which increased mortality were presenting with arrest, and ISS. When considered all parameters, the ISS is the sole predictor of dead. 
Conclusion: Trauma team activation criteria can improve emergency trauma care and decreased ERLOS. The patients presenting with arrest and high ISS tend to have high mortality rate. Only the ISS is the predictor of mortality.

References: 1. Sava J, Alo K, Velmahos GC, Demetriades D. All patients with truncal gunshot wounds deserve trauma team activation. J Trauma. 2000;52:276-279. 2. Tinkoff GH, O'Connor RE. Validation of new trauma triage rules for trauma attending response to the emergency department. J Trauma. 2002;52:1153-1158.

Disclosure: No significant relationships.

\section{1}

\section{ESTES POSTGRADUATE TRAINING IN MEDICAL RESPONSE TO MAJOR INCIDENTS (MRMI): EXPERIENCES FROM THE FIRST FIVE COURSES}

K. Lennquist Montán ${ }^{1}$, M. Bemelman ${ }^{2}$, B. Dobson ${ }^{3}$, B. Hreckowski $i^{4}$, P. Fischer ${ }^{5}$, A. Khorram-Manesh ${ }^{6}$, C.H. Montan ${ }^{7}$, P. Ortenwall ${ }^{8}$, S. Lennquist ${ }^{9}$

${ }^{1}$ Department of Surgery, Centre for Prehospital and Disaster Medicine, Gothenburg, Sweden, ${ }^{2}$ Surgery Trauma, University Medical Center Utrecht, Utrecht, The Netherlands, ${ }^{3}$ Education, LAS, London, UK, ${ }^{4}$ Orthopaedics and Trauma Surgery, University of Split, Slavonski Brod, Croatia, ${ }^{5}$ Orthopaedics and Trauma Surgery, University of Bonn, Bonn, Germany, ${ }^{6}$ Department of Surgery, Centre for Prehospital and Disatser Medicine, Gothenburg, Sweden,

${ }^{7}$ Surgery, University of Lund, Helsingborg, Sweden, ${ }^{8}$ Department of Surgery, Centre for prehospital and disaster medicine, Gothenburg, Sweden, ${ }^{9}$ Department of Surgery, University of Linkoping, Linkoping, Sweden

Introduction: Within the section of Disaster and Military Surgery, a new model for postgraduate training was developed in collaboration between representatives from different member states. The training should:

- Cover the whole chain of management, from the scene to the hospital, including coordination, communication and command.

- Include training in triage and treatment of patients.

- Be fully interactive.

- Have a strictly standardised design, making it possible to establish in different countries with the same set-up and with reproducible results.

Materials and methods: A new simulation system, originally developed for scientific evaluation of different methods in major incident response, was used. With this system, based on injuries from real scenarios, it was possible to numerically describe the outcome of a response expressed in preventable mortality and complications related both to trauma scores (RTS, ISS) and to available resources of all kinds.

Results: Between April 2009 and November 2010 (at the time for abstract submission) five courses were run, at the time for presentation expanded with additional three. In the five courses run so far, totally 247 delegates participated, all active in their real positions during the exercises. The participants perception of their own qualifications for major incident response was documented on a scale 1-6 where $1=$ very well and $6=$ insufficient. Before the course $36.5 \%$ of this very experienced audience answered "well" or "very well" and $17.4 \%$ "poor" or "insufficient". After the course the corresponding figures were 78.2 and $0 \%$, respectively.

Conclusion: The results indicate that the developed model of training fulfilled the given prerequisites and can be further promoted as a standardised training model for this purpose in any European country. References: Lennquist S. Education and training in disaster medicine. Scand J Surg. 2005;94:300-310

Disclosure: The MRMI courses are run by a Board of ESTES members without any commercial interest; no part of the course fees goes to the board and no licence has to be paid. One of the authors (SL) has the copyright for the simulation system (MACSIM) used in the course.

\section{3}

\section{ENHANCING TRAUMA MANAGEMENT THROUGH SYSTEM-BASED LEARNING: THE TRAUMA ORIENTATION PROGRAM EXPERIENCE}

\section{A. Vijayan}

General Surgery, Tan Tock Seng Hospital, Singapore, Singapore

Introduction: Major trauma management is highly complex requiring individual clinical competency, knowledge of system-based practice and team work. In our institution, clinical competency in trauma care is developed through the ACS Advanced Trauma Life Support Course and the IATSIC Definitive Surgical Trauma Care Course. Prior to 2007, there was no training for standardized systembased practice. Such knowledge was acquired thorough on-the-job training resulting in variations in trauma management. To minimize variations, a system-based biannual Trauma Orientation Program (TOP) was introduced in 2007, targeting doctors- in-training from disciplines involved in the management of major trauma. The impact of TOP on patient's Time in Emergency Department (TED) from resuscitation through multidisciplinary evaluation to final disposition was measured.

Materials and methods: Doctors from General Surgery, Emergency Medicine, Anesthesia and Intensive Care, Orthopedic Surgery and Neurosurgery participated in the TOP. TOP consisted of didactic sessions, multidisciplinary group discussions on the presentation and clinical management, emphasizing system and human factors in the clinical micro-system and meso-system of trauma management continuum. As the largest variation in system practice was in the TED, this was used as a surrogate marker of system knowledge. Data was prospectively obtained for all trauma patients seen in ED with ISS of 3-75. Pre TOP course TED data for the year 2007 was compared with post TED data in the year 2009 .

Results: There were 882 trauma patients in 2007 and 1,152 in 2009, who were grouped into five ISS groups; $1-15,16-30,31-45,46-60$ and 61-74. There were significant $(5-50 \%)$ decreases in TED in all groups. Major decreases occurred in the ISS 31-45 Group (50\%), followed by the ISS $46-60$ Group and the ISS 61-74 Group. Overall trauma mortality also decreased by $10 \%$.

Conclusion: Formal training in clinical micro and meso systems within the trauma continuum is necessary for improving trauma outcomes.

Disclosure: No significant relationships. 


\section{4}

\section{IMPROVING CONFIDENCE AND COMPETENCE AMONGST JUNIOR DOCTORS}

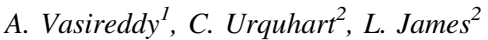

${ }^{1}$ Department of Orthopaedics, University Hospital Lewisham, London, UK, ${ }^{2}$ Orthopaedics, University Hospital Lewisham, London, UK

Introduction: In the United Kingdom, junior doctors interested in a particular specialty were appointed to relevant posts. However, the structure of post-graduate medical training has changed. Therefore, doctors appointed to Surgical Posts may not have any interest in pursuing a surgical career and thus their surgical knowledge may be limited [1]. Therefore, at our institute, we decided to implement an intensive training program aimed at providing doctors with the knowledge and skills required to manage emergency orthopaedic patients.

Materials and methods: Within our department, each junior doctor was given a questionnaire when they commenced their post in order to assess their subjective level of confidence and competence (visual analogue score out of 10) when dealing with a variety of common Orthopaedic scenarios and post-operative problems. A strict daily programme of teaching was implemented and led by a Senior Surgeon on a daily basis for 10 days. After 3 months, the questionnaire was redistributed and doctors asked to score their subjective level of competence and confidence.

Results: 13 doctors out of a possible 18 participated in the survey. When initially assessed, there was a low level of subjective confidence and competence (average VAS score of 4 out of 10). After 3 months, results from the survey revealed an increase in the scores of both subjective confidence and competence (average VAS score of 7-8 out of 10).

Conclusion: When on-call, junior doctors may find it difficult to manage surgical patients because of their limited experience. A specific teaching programme aimed at instructing junior doctors led to an improvement in their subjective level of confidence and competence. The results of this study support the need for a focused teaching programme to be implemented in order to help junior doctors work more effectively.

Reference: Griffiths JR, Roberts N. Do junior doctors know where to insert chest drains safely? Postgrad Med J 2005;81:3.

Disclosure: No significant relationships.

\section{5}

\section{INTERNATIONAL COLLABORATIONS AND TRAINING IN TRAUMA RESEARCH}

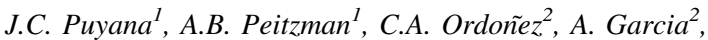
$\overline{\text { M.I. Gutierrez }}{ }^{2}$, M. Roberts ${ }^{1}$, T.R. Billiar ${ }^{1}$

${ }^{1}$ Surgery, University of Pittsburgh, pittsburgh, United States of America, ${ }^{2}$ Surgery, Universidad del Valle, Cali, Colombia

Introduction: The NIH-sponsored Fogarty International Center (FIC) promotes international collaborations by funding training grants in global health. For the first time, in 2006 an International Collaborative
Trauma and Injury Research Training Program was offered and later awarded to our university.

Materials and methods: We designed an integrated trauma research program combining mentored apprenticeship training with a didactic educational curriculum. We present the academic accomplishments and research productivity of the trainees during the first 4 years of the grant. Results: Nine individuals have been trained to date. Four have enrolled in long term training programs, and 4 in short term training. Trainee-initiated research endeavors have generated, 5 manuscripts published in peer review journals, 7 manuscripts submitted for publication, 6 publications in textbooks and local journals and 17 abstracts. These abstracts have been accepted for either oral or poster presentations at ten international Injury/Trauma and Acute Care Societies/Meetings. Research topics included injury surveillance in alcohol, traffic and violence-related events, trauma registry and policy development, disaster response in low and middle income countries (LMIC), damage control techniques and long term functional outcome of critically ill trauma patients. Additional projects have been completed on pre-hospital care, landmine explosion injuries, traumatic brain injury and penetrating trauma.

Conclusion: Creating a strong international partnership provides the necessary environment to build productive and successful research programs. Multidisciplinary collaborations between health professionals from a high-income country and LMIC have resulted in significant trauma research contributions relevant to LMICs as well as enhanced awareness of trauma as a global health priority.

Disclosure: No significant relationships.

0182

\section{'CONSULTANT OF THE WEEK' AND PLASTIC SURGERY JUNIOR DOCTORS' TRAINING}

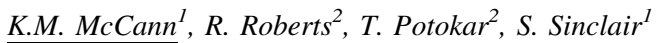

${ }^{1}$ Plastic and Maxillofacial Surgery Department, Ulster Hospital, Dundonald, Belfast, UK, ${ }^{2}$ University of Wales, Swansea, UK

Introduction: Effective postgraduate training is paramount to ensuring high levels of service provision. The aim of this study was to analyse the impact of the introduction of a 'Consultant of the Week' (COW) system on plastic surgery junior doctors' training.

Materials and methods: (1) the application of a retrospective questionnaire to both trainees and trainers, before and after the changes, to obtain data on the subjective view of the change in system; (2) a prospective, consecutive study of zone two flexor tendon laceration repairs, during each system, which facilitated assessment of level of access to a key trauma procedure and the impact of such changes on patient outcome.

Results: No change in trainees' evaluation of training experience $(\mathrm{p}=0.09)$ across the change, but a significant improvement in the subjective opinion of trainers $(\mathrm{p}=0.031)$. Access and supervision of zone II flexor tendon repairs significantly increased with the COW system $(p=0.004 /<0.005)$. Functional outcome was significantly reduced with the new system $(\mathrm{p}=0.003-0.05)$, but this was not attributable to increased input from juniors $(\mathrm{p}>0.005)$.

Conclusion: Although trainee assessment of training did not change, trainers' assessment and objective assessment favoured the 'consultant of the week' system, without negatively impacting on patient outcome.

Disclosure: No significant relationships. 


\section{3}

\section{TRAUMA AND DSTC IN PORTUGAL: RESULTS EVALUATION}

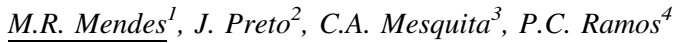

${ }^{1}$ Cirurgia 2.6, Hospital S. A. Capuchos-Chlc, 050 Lisboa, Portugal, ${ }^{2}$ Cirurgia 2, Hospital De São João, 319 Porto, Portugal, ${ }^{3}$ Surgery Iii, Coimbra University Hospital, Coimbra, Portugal, ${ }^{4}$ Surgery, Hospital Nelio Mendonça Funchal, Funchal, Portugal

Introduction: The Portuguese Society of Surgery SPC brought the ATLS $^{\circledR}$ Course, in 1999. Until 2010, SPC ran 150 courses involving 2,400 doctors. A recent interest around trauma and emergency training, led to other thematic societies such SPT, GT and ALTEC, this one affiliated to ESTES. The Portuguese Medical Association OM created, in 2002, the College of Competence on Emergency Medicine CCEM and, subsequently, steering groups for trauma and emergency surgery. Concerning education in trauma surgery and surgical decision making, in 2003 GT established a protocol with RACS, which led to the introduction of DSTC and the satellites DPNTC and DATC courses, all related with IATSIC.

Materials and methods: In 2008, a OM National Steering Committee NSC was created for the development of DSTC in Portugal, in cooperation with the OM Working Group for Emergency Surgery (GTFCE). A "MOU" has acknowledged that completed courses and future ones are in accordance with the requirements established by IATSIC. The NSC has assured the maintenance of high standards in Courses, Faculty and Participants selection. Since 2010 a Portuguese translated Manual, from the original 2nd edition, has been adopted. Results: Up to now, ten DSTC were done, involving 230 surgeons: four in Porto, four in Coimbra, one in Lisbon and one in Funchal. The AA evaluate the results attained.

Conclusion: The importance of these training models towards a future programme for a title of Competence in Emergency Surgery, including trauma, is now under evaluation by the CCEM steering group.

References: 1. Boffard KD Manual of definitive surgical trauma care. Internayional Association for Trauma Surgery and Intensive Care. Arnold, London (2nd edn 2006). 2003. 2. Mesquita C. Formação Específica em Cirurgia de Emergência e Trauma-Do ATLS ao DSTC. Separata ALTEC. Coimbra, Maio. 2008. 3. Mesquita C. Trauma, cirurgia de urgência e cirurgia vascular. Tempo Medicina, Lisboa. 2010.

Disclosure: No significant relationships.

\section{OPEN FRACTURES: UPPER EXTREMITIES}

\section{6}

\section{DOUBLE-COLUMN PLATING THOUGH EFFECTIVE TECHNIQUE: DOES IT PRODUCE GOOD OUTCOME? A PROSPECTIVE COHORT}

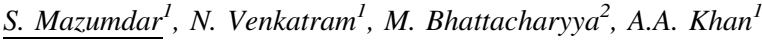 \\ ${ }^{1}$ Orthopaedics, University Hospital Lewisham, London, UK, ${ }^{2}$ Orthopaedics, Lewisham Hospital London, London, UK}

Introduction: The purpose of our study was to determine the functional outcome of fixation of displaced intra-articular distal humeral fractures with use of a standardized evaluation methodology consisting of objective testing of muscle strength and use of patient-based questionnaires.
Materials and methods: We identified sixteen patients (12 male and 4 female), who had an isolated, closed, displaced, intercondylar, intraarticular fracture of the distal part of the humerus treated operatively through a posterior approach and fixed with plates on both the medial and the lateral column. During follow up all patients were evaluated for range of motion, infection, nerve injury, myositis ossificans, varus/ valgus deformity, pain, instability, weakness, and degenerative, radiographic examination, and completion of a limb-specific questionnaire [Disabilities of the Arm, Shoulder and Hand (DASH)].

Results: The minimum follow-up was 12 months. Four patients had post operative nerve injury (one radial nerve, one median nerve and two ulnar nerve). Two patients had reoperation for failure of fixation and one for non union. The flexion contracture range, $15^{\circ}-65^{\circ}$ and the arc of flexion-extension was range $55^{\circ}-140^{\circ}$. Muscle strength was decreased when compared with that on the normal side were seen in both elbow flexion measured at $90^{\circ}$ and elbow extension measured at $45^{\circ}$. The mean DASH score was 35 points, indicating mild to moderate esidual impairment.

Conclusion: Postoperative nerve dysfunction after open reduction and plate and screw fixation of the distal humerus, is highlighted in this small cohort of patient. Although, the dual plating is able to provide rigid fixation for the humeral intercondylar fractures and allow early functional exercise after operation, overall functional result and the related complications showed poor outcome in our series.

Disclosure: No significant relationships.

\section{7}

\section{EFFECTS OF BOTULINUM TOXIN A ON FRACTURE HEALING IN RATS: AN EXPERIMENTAL STUDY}

\author{
A. Aydin ${ }^{1}$, K. Memisoglu ${ }^{2}$, A. Cengiz $^{3}$, B. Muezzinoglu, \\ S.U. Muezzinoglu ${ }^{2}$
}

${ }^{1}$ Orthopaedics and Traumatology, Igdir State Hospital, Igdir, Turkey, ${ }^{2}$ Orthopaedics and Traumatology, Kocaeli University School of Medicine, Kocaeli, Turkey, ${ }^{3}$ Mechanical Education, Kocaeli University School of Technical Education, Kocaeli, Turkey, ${ }^{4}$ Pathology, Kocaeli University School of Medicine, Kocaeli, Turkey

Introduction: Fracture healing is a complex process influenced by intrinsic and extrinsic factors. The aim of the present study was to evaluate the effects of botulinum toxin $\mathrm{A}$, which causes transient flaccid paralysis by blocking acetylcholine release at the presynaptic nerve terminal, on fracture healing.

Materials and methods: Following induction of bilateral standard closed femoral fractures and relative fixation in 18 Wistar-Albino rats, $8 \mathrm{IU}$ of botulinum toxin A was injected into the right femoral region. After 28 days, all of the rats were sacrificed and the diameter of the callus was measured and fracture healing was assessed by biomechanical and histopathologic evaluation.

Results: While an increase in biomechanical and histopathologic healing was noted on the side of the botulinum toxin A injection, a decrease in callus diameter was observed. Botulinum toxin A administration increases the healing power in a relatively fixated fracture and decreases the callus diameter as if rigid fixation had been performed.

Conclusion: The beneficial effect of botulinum toxin A on fracture healing might be associated with increased fixation rigidity.

References: 1. Huang W, Foster JA, Rogachefsky AS. Pharmacology of botulinum toxin. J Am Acad Dermatol. 2000;43: 249-259. 2. Bonnarens F, Einhorn TA. Production of a standard closed fracture in laboratory animal bone. J Orthop Res. 
1984;2:97-101. 3. Aoki KR. A comparison of the safety margins of botulinum neurotoxin serotypes $\mathrm{A}, \mathrm{B}$, and $\mathrm{F}$ in mice. Toxicon. 2001;39:1815-1820. 4. Heiple KG, Goldberg VM, Powell AE, et al. Biology of cancellous bone grafts. Orthop Clin North Am. 1987;18:179-185. 5. Buckwalter JA, Einhorn TA, Marsh JL. Bone and joint healing. In: Bucholz RW, Heckman JD (eds) Fractures in adults. vol 1, 5th ed. Lippincott, Philadelphia, pp 245-271; 2001.

Disclosure: No significant relationships.

\section{8}

\section{NON-OPERATIVE MANAGEMENT OF RADIAL HEAD FRACTURES: A CLINICAL DECISION MAKING ALGORITHM}

\section{J. Waddell}

Division of Orthopaedic Surgery, St. Michael's Hospital, Toronto, ON, Canada

Introduction: Isolated radial head fractures are relatively common and can pose a treatment dilemma because of the intra-articular nature of the fracture and the potential for elbow stiffness.

Materials and methods: All patients with intra-articular radial head fractures are assessed by clinical and X-ray examination. Clinical examination focuses on range of motion and crepitus; X-ray assessment determines the degree of displacement and the number of fracture fragments. Patients with associated elbow injuries are treated surgically; patients without elbow instability or associated fractures are referred for conservative management. These patients are managed with early active exercise.

Results: 34 patients (20 males, 14 females; mean age 31.5 years) have been treated according to protocol. Patients with no mechanical block to motion and no crepitus on examination of the elbow are enrolled in a vigorous physiotherapy program with intermittent splinting for pain relief only. Assessment is by range of motion and functional activity score (DASH). All patients were assessed at 1 year following injury. Range of motion demonstrated a mean loss of $2^{\circ}$ of pronation, $6^{\circ}$ of supination, $5^{\circ}$ of flexion, $2^{\circ}$ of extension as compared to the opposite elbow. The mean DASH score was 7 with a range of 0-60. Radiographs showed union in all cases.

Conclusion: A near normal range of motion with minimal pain and excellent function is possible with this technique in appropriately selected patients.

Disclosure: No significant relationships.

\section{9}

\section{ORIF FOR THE TREATMENT OF COMPLEX FRACTURES OF THE PROXIMAL HUMERUS}

F. Chiodini ${ }^{1}$, D. Marchettini ${ }^{2}$, I. scarabello ${ }^{1}$, M. Berlusconi ${ }^{1}$,

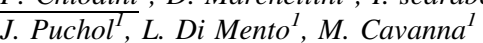

${ }^{1}$ Traumatology Ii, Instituto Clinico Humanitas, Rozzano, Italy, ${ }^{2}$ Trauma Surgery Ii, Istituto Clinico Humanitas, Rozzano, Italy

Introduction: Complex Fractures of the Proximal Humerus (CFPH) have a widely accepted surgical indication, but there is no consensus on the best surgical procedure. There is a big difference in the results reported for both ORIF and hemiarthroplasty in terms of function, pain, head avascular necrosis and number of additional surgery. ORIF seems to give better function but is exposed to the risk of avascular necrosis and hardware failure. Since hardware failure is often related to technical errors and to the surgeon's experience and head necrosis may gives mild symptoms, we postulate that for this kind of fractures the first choice is the ORIF.

Materials and methods: At our institution from 2005 to 2009 we operated on 116 fractures of the proximal humerus. 36 of them were complex fractures: 21 females, mean age was 50 years. The fractures were treated with ORIF using PHLCP or PHILOS plates through a deltopectoral approach. The patients have been revaluated at a mean follow up of 17 months clinically by the DASH score and radiologically. Results: All the fractures united within 4 months with a mean DASH score of 35. There were four cases of head necrosis with screw protrusion, two patients was reoperated on with a total reverse arthroplasty, but two of them, suffering of mild pain, refused further surgery. There were three cases of partial necrosis not requiring further intervention. One patient sustained a deep infection was treated successfully by revision surgery. In five patients hardware removal was indicated because of subacromial impingement of the plate. Age, severe osteoporosis and head dislocation were related to a higher incidence of complication and lower functional score.

Conclusion: ORIF for CFPH is a complex but feasible choice. The results are strongly related to the age of the patient, bone quality and dislocation of the humeral head.

Disclosure: No significant relationships.

\section{0}

\section{FIXATOR-ASSISTED NAILING IN FRACTURES OF THE FOREARM SHAFT IN ADULTS}

\section{A. Chelnokov, A. Lazarev}

Orthopaedic Trauma, Ural Scientific Institute of Traumatology and Orthopaedics, Ekaterinburg, Russian Federation

Introduction: Closed nailing is dominating in surgical treatement of shaft fractures of long bones but not in forearm fractures where plating is conventional technique. Lack of enthusiasm in forearm nailing is caused by difficulties of closed restoration of anatomy. Main problems are rotational alignment of the radius and resotation of radial bow to prevent functional deficit. Aim of our study was development of surgical technique of closed fixator-assisted forearm nailing.

Materials and methods: We treated 144 patients with forearm fractures (120) and nonunions (24). There were lesions of both bones in 57, ulna: 54, radius: 33 .

For fixator assisted nailing we used a device consisted of a half-ring proximally and a ring distally. The distal part of the forearm was fixed in neutral rotation regardless of the fracture level. For rotational control of the proximal fragment of the radius a joystick K-wire was used.

Interlocking and non-locking nails were used. The ulna was nailed first.

Results: Patients demonstrated fast functional recovery. Full range of motions occurred in 1 month after the surgery in $78 \%$ of patients, and in 2 months in $97 \%$. DASH functional score improved from 21 in 1 month to 3 in 2 months.

Nonunion of the ulna occurred in 5 cases $(3.4 \%)$, of the radius in 1 case $(0.7 \%)$. Exchange reamed nailing was performed in ulna and plating with bone grafting in the radius nonunion.

Conclusion: The technique provides good alignment of both bones which results with quick functional recovery and high union rate. Closed nailing can be treatment of choice in forearm shaft fractures. Disclosure: No significant relationships. 


\section{1}

\section{STABILIZATION OF DISTAL RADIUS FRACTURES WITH DISTRACTION AND COMPRESSION HEADLESS SCREW SYSTEM}

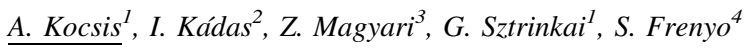

${ }^{1}$ Iv. Trauma Department, Trauma Center, Péterfy S. Hospital, Budapest, Hungary, ${ }^{2}$ Department 3, Trauma Center, Budapest, Hungary, ${ }^{3}$ Department 3, National Institute of Traumatology, Budapest, Hungary, ${ }^{4}$ Department 1 , Trauma Center, Budapest, Hungary

Introduction: Utilizing the advantages of headless screw system used in calcanear fractures, we worked out a new intracutaneous minimalinvasive method to treat distal radius fractures.

Materials and methods: The common method to fix distal radius fractures is K-wiring. During the development of our technique we wanted to avoid the most common complications of wiring methods: the redislocation of fracture, the migration of implants, soft tissue damages and irritations caused by the ends of K-wires. Beside of eliminating the complications we pursued to keep the operation time as short as possible and to keep percutaneous method.

The operation steps are very similar to K-wire technique: we use extension devices to reduce the fracture, then we fix the intraarticular components with compression HLS screw, after having right joint surface we implant distraction HLS screws from the processus styloideus to maintain the length of radius. Because of the special design of screw threads, we managed to intensify the stability and reached lesser casting times.

Results: In the last 3 years we treated 36 patients with the new screws. We achieved greater stability, lesser casting time and better postoperative functional results of the wrist. Redislocation of implants occured in one case only. Because of the percutaneous and fullyintraosseal technique we did not recognised any soft tissue damages or complaints.

Conclusion: The brand new method is easy to learn. By the help of these screws the number of complications and the casting time can be decreased. Because of the intraosseal placement of screws implant removal is not necessary.

In our presentation we would like to introduce the principals of distraction screw system and the follow-up results of the patients.

Disclosure: No significant relationships.

\section{2}

\section{TYPE C INTERCONDILAR FRACTURES OF DISTAL HUMERUS TREATED THROUGH EXTENSOR MECHANISM-SPARING PARATRICIPITAL POSTERIOR APPROACH}

\section{F. del Prete, A. Petrini, S. Longo, G. Alberti Lepri}

Chirurgia U.o Ortopedia E Traumatologia, ASF 10 Firenze, Ospedale San Giovanni di Dio, Firenze, Italy

Introduction: We evaluated the outcome after internal fixation of articular distal humerus fractures treated through Extensor mechanism-sparing para tricipital posterior approach. Internal fixation was achieved with LCP distal Humerus plates.

Materials and methods: 12 patients, managed by a single surgeon with this tecnique, between January 2008 and June 2010, clinically and radiologically evaluated after a mean follow up of 20,5 months; 2 $\mathrm{C} 1,3 \mathrm{C} 2$ e $7 \mathrm{C} 3$ (2 with $\mathrm{I}^{\circ}$ exposition) according the AO classification, all without anterior displaced fragments, from 14 to 82 , mean 51 years.

Results: 9 patients (75\%) achieved good or excellent clinical scores, 2 patients $(16.6 \%)$ fair, and $1(8.3 \%)$ poor using Mayo Elbow Performance Score. No finding of neurological deficit, delayed or nonunion and infection.

Conclusion: The Extensor mechanism-sparing paratricipital posterior approach is appropriate for $\mathrm{C} 1$ e $\mathrm{C} 2$ fractures of distal Humerus. In some kinds of $\mathrm{C} 3$ fractures, too, without anterior displaced fragments, it may represent an interesting alternative to traditional approaches, that can, nevertheless be carried out, if the reduction was not obtained.

References: Schildhauer TA, Nork SE, Mills WJ, Henley MB. Extensor mechanism-sparing paratricipital posterior approach to the distal humerus. J Orthop Trauma. 2003;17(5):374-378. Alonso-Llames M. Bilaterotricipital approach to the elbow. Acta Orthop Scand 1972;43:479-490. Ali AM, Hassanin EY, El-Ganainy AE, Abd-Elmola $T$ Management of intercondylar fractures of the humerus using the extensor mechanism-sparing paratricipital posterior approach. Acta Orthop Belg. 2008;74(6):747-752. Ek ET, Goldwasser M, Bonomo AL. Functional outcome of complex intercondylar fractures of the distal humerus treated through a triceps-sparing approach. J Shoulder Elbow Surg. 2008;7(3):441-446 (Epub 2008 Feb 20).

Disclosure: No significant relationships.

\section{BRAIN AND SPINAL TRAUMA}

\section{3}

\section{INJURY SEVERITY AND MORTALITY FOR THREE TYPES OF MOTORIZED TWO-WHEELED VEHICLE CRASH VICTIMS IN THE NETHERLANDS}

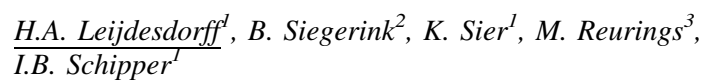

${ }^{1}$ Trauma Surgery, Leiden University Medical Center, Leiden, The Netherlands, ${ }^{2}$ Clinical Epidemiology, Leiden University Medical Center, Leiden, The Netherlands, ${ }^{3}$ Road Safety Analysis, Institute For Road Safety Research (SWOV), Leidschendam, The Netherlands

Introduction: This nationwide study analyzed in what way type of motorized two-wheeled vehicle (MTV) influenced injury severity and mortality upon hospitalisation.

Materials and methods: Data from the Institute for Road Safety Research (SWOV) Trauma Database were analyzed. All MTV crash victims admitted to hospitals from 1993 to 2008 were included. Logistic regression analysis was performed on gender, age, type of MTV, Injury Severity Score, Abbreviated Injury Scale, to identify and evaluate independent risk factors for injury severity and mortality. Motorcyclists were used as reference group.

Results: Among 33,495 MTV crash victims admitted to hospitals, 10,607 were motorcyclists, 19,708 moped riders and 3,180 light-moped riders. The median age per group was respectively 33,18 and 30 years. Head injury, especially severe head injury (AIS $\geq 3$ ), was most common in the light-moped rider group. Severe poly trauma and mortality was highest in the light-moped rider group $(17.1,4.2 \%)$. Compared to motorcyclists, light-moped riders were more likely to die and sustain severe poly trauma when admitted to a hospital (OR 1.46, 1.71). 
Mortality was twofold higher in the light-moped rider group older than 55 years compared with motorcyclists of the same age (OR 2.14). Conclusion: Hospitalized light-moped riders show more severe head injuries, severe poly trauma and higher mortality than moped riders and motorcyclists. Young motorcyclists and elderly light-moped users are the most vulnerable groups in daily MTV traffic, with highest chances of severe injury and mortality. Continuing improvement of the MTV safety is urgent; an enforced helmet law for light-mopeds should be introduced in The Netherlands.

Disclosure: No significant relationships.

\section{4}

\section{THE RISK FACTORS FOR SEAT BELT ABDOMINAL INJURY AS JUDGED BY A COMPUTER SIMMULATION SYSTEM: SEAT RECLINING ANGLE, FRICTION COEFFICIENT, BODY SIZE}

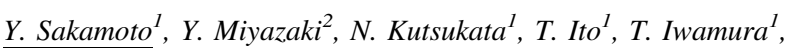
A. Nakashima $^{l}$, M. Yahatal

${ }^{1}$ Emergency Medicine, Saga University Hospital, Saga, Saga, Japan, ${ }^{2}$ Kanazawa University, Kanazawa, Japan

Introduction: Recently, the injury form of motor vehicle driver changed according to improvement of motor vehicle. In spite of any abdominal injury reports with seat belt, the form of the seat belt was not revised too much. And our clinical data showed that all seat belt injury cases suffered the seat belt associated contusion in not applicable seat belt point of abdomen.

Materials and methods: In order to clarify the effects of both the reclining angle of an occupant seat and slipping between hip and the seat in frontal collision, computer simulations were carried out using multi-body models of whole-body of a human. We analyzed difference of body size, difference of seat angle, difference of friction coefficient.

Results: Computer simulation showed that seat belt portion moves to slightly upper position in the pattern 1 (reclining) and pattern 2 (slip seat) compared with pattern 3 (normal) and position moves to more upper position in pattern 4 (reclining and slip seat) in normal body size. Computer simulation showed that risk of seat belt injury rise so the angle of seat belt becomes high, and risk of seat belt injury rise too so the friction coefficient become low. In addition, small body size was high risk factor of seat belt injury.

Conclusion: We showed that compression of the abdomen occurs due to upward sliding of the lap belt with "submarine", in the cases of lower friction coefficient between the hip and more backward recline of the seatback. And body size is very important factor for seat belt restriction.

Disclosure: No significant relationships.

\section{5}

\section{CONTRAST NEPHROPATHY IN TRAUMA PATIENTS: IS IT A PROBLEM?}

A. Volpi, C. Bricchi, L. Cattani, D. De Matteis, E. Sani, F. Martello, S. Grossi, M. Mergoni

1st Anaesthesia and Intensive Care, Azienda OspedalieroUniversitaria di Parma, Parma, Italy
Introduction: Radiologic investigations with contrast have become a mainstay in the assessment of trauma patients (TP). Whether the advantages of this technique justify its use against cost, radiation exposure and risk of contrast nephropathy $(\mathrm{CN})$ is controversial. In clinical practice its use is increasing during the early resuscitation phase, even in haemodynamically unstable patients.

Materials and methods: A 6-month retrospective study was undertaken on TP admitted to the ICU of the University Hospital of Parma. Serum creatinine $(\mathrm{Cr})$ levels were measured before the procedure and up to 5 days after. The highest value of $\mathrm{Cr}$ was compared with the baseline measure. $\mathrm{CN}$ defined by an increase in serum creatinine of more than $25 \%$ or more than $0.5 \mathrm{mg} / \mathrm{dL}$ within $48 \mathrm{~h}$ of admission were recorded. Compliance with institutional regulations for clinical research were followed.

Results: 77 patients were enrolled (ISS 26). The ICU length of stay was 8.7 days, mortality $10.5 \%$. 1 patient with renal injury developed renal failure and required dialysis. Compared with the baseline, no increase in $\mathrm{Cr}$ levels after contrast was observed in all patients and in the subgroup with risk factors for renal failure.

Conclusion: Administration of nonionic contrast in severe TP were not associated with $\mathrm{CN}$, supporting its safe use in these patients.

References: Velmahos et al. A prospective study on the safety and efficacy of angiographic embolization for pelvic and visceral injuries. J Trauma. 2002;53:303-308. Rieger H et al. Modern CT diagnosis of acute thoracic and abdominal trauma. Anaesthesist. 2002;51:835.

Disclosure: No significant relationships.

\section{6}

\section{BICYCLE-RELATED INJURIES OF HOSPITALIZED PATIENTS IN A HIGH-INCOME DEVELOPING COUNTRY}

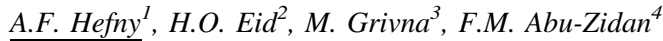

${ }^{1}$ General Surgery, Faculty of Medicine and Health Sciences, UAE University, Al-Ain, UAE, Al-Ain, United Arab Emirates,

${ }^{2}$ Department of Emergency Medicine, Tawam Hospital, Al-Ain, United Arab Emirates, Al-Ain, UAE, ${ }^{3}$ Community Medicine, Faculty of Medicine, UAE University, Al-Ain, UAE, ${ }^{4}$ Surgery, Department of Surgery, UAE University, Al-Ain, UAE

Introduction: Bicycle-related injuries depend on the mechanism and severity of trauma. We aimed to study the anatomical distribution, severity, and outcome of hospitalized bicycle-related injured patients in Al-Ain Hospital, United Arab Emirates in order to improve preventive measures.

Materials and methods: All patients with bicycle-related injuries who were admitted to Al-Ain Hospital for more than $24 \mathrm{~h}$ or who died in the hospital after arrival were studied. Data were prospectively collected over a period of 6 years (March 2001-February 2007). Demography of patients, Glasgow Coma Scale (GCS), Injury Severity Score (ISS), hospital stay, and mortality were analyzed.

Results: There were 130 patients (126 males). Mean (SD) age was 27.1 (14.5) years. 17.7\% were United Arab Emirates (UAE) nationals. None of the patients was wearing a helmet. The percentage of UAE nationals of less than 15 years old was significantly higher $(65.2 \%)$ compared with non UAE nationals $(14.3 \%)$ ( $p<0001$, Fisher's Exact Test). The most common mechanism of injury for UAE nationals was falling from a bicycle $(73.7 \%)$ while for non UAE nationals was hitting a moving vehicle $(66.7 \%)$. the median (range) ISS was 4 (1-41) and the median (range) GCS was 15 (3-15). The mean (range) hospital stay was 8.35 (1-95) days. 17 patients (13.1\%) were admitted to the Intensive Care Unit (ICU). Two patients died due to head injury (overall mortality was $1.5 \%$ ). 
Conclusion: Mechanism of injury differs for UAE national and non UAE national bicycle riders. Legislation for compulsory helmet use by bicycle riders should be adopted with other preventive measures. Disclosure: No significant relationships.

\section{7}

\section{BRAIN PERFUSION CT IN SEVERE TRAUMATIC BRAIN INJURY: AN OBSERVATIONAL PILOT STUDY?}

C. Bendinelli ${ }^{1}$, S.D. Nebauer ${ }^{2}$, M. Parsons ${ }^{3}$, N. Spratt ${ }^{3}$, C. Abel ${ }^{3}$, M. Edger ${ }^{3}, P . \mathrm{Saul}^{3}$, N. Enninghorst ${ }^{3}, \mathrm{~K}_{\text {. Sisak }}{ }^{3}$, Z. Balogh $^{4}$

${ }^{1}$ Surgery, John Hunter Hospital, Newcastle, Australia, ${ }^{2}$ Medicine, University of Newcastle, Callaghan, NSW, Australia, ${ }^{3}$ John Hunter Hospital, Newcastle, NSW, Australia, ${ }^{4}$ Trauma Department, John Hunter Hospital, Newcastle, Australia

Introduction: Perfusion CT (PCT) in stroke patients quantifies the penumbra and allows targeted thrombolysis [1, 2]. Following severe traumatic brain injury (STBI) PCT may predict functional outcome better than non contrast CT (NCCT) by identifying areas of abnormal perfusion not seen on NCCT [3]. The aim of this study is to compare the information obtained from simultaneous NCCT and PCT.

Materials and methods: Ongoing prospective observational study of adult STBI patients (GCS $<9$, not requiring craniotomy at admission). PCT was obtained with 320 -slice scanner at the time of the first follow-up NCCT $(<48 \mathrm{~h})$.

Results: From March to November 2010, 19 blunt trauma patients met inclusion criteria and 14 had PCT (age: $31 \pm 15,86 \%$ males ISS: $29 \pm 8$, AIS-head: $5 \pm 1$, GCS pre-intubation $6 \pm 2$ ). Six $(43 \%)$ had ICP monitoring or intraventricular drain (ICP: $25 \pm 13 \mathrm{mmHg}$ ). The short term outcomes were: $8 \pm 5$ ICU days and 2/14 (14\%) mortality. PCT was normal in 8/14 (57\%) patients. In $4(29 \%)$ patients the PCT showed regions of reduced cerebral blood flow and volume. In three of these, the abnormally perfused regions on PCT were not apparent on the concomitant NCCT, but evolved in hypodense regions on subsequent NCCT. One of these patients had an occult carotid dissection.

Conclusion: Based on this preliminary assessment PCT provides additional and earlier diagnostic information to NCCT in almost $30 \%$ of STBI patients. Its role in this population warrants further studies. References: 1. Parsons MW. Perfusion CT: is it clinically useful? Int J Stroke. 2008;3(1):41-50. 2. Parsons MW, Pepper EM, Bateman GA, Wang Y, Levi CR. Identification of the penumbra and infarct core on hyperacute noncontrast and perfusion CT. Neurology. 2007;68(10):730-736. 3. Wintermark M, van Melle G, Schnyder P, Revelly JP, Porchet F, Regli L, Meuli R, Maeder P, Chioléro R. Admission perfusion CT: prognostic value in patients with severe head trauma. Radiology. 2004;232(1):211-220.

Disclosure: No significant relationships.

\section{8}

\section{HELMETS REDUCE MOTORCYCLE-RELATED DEATH: A GLOBAL EVALUATION}

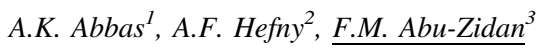

${ }^{1}$ Trauma Group, Faculty of Medicine, Al-AIn, UAE, ${ }^{2}$ Trauma Group, Faculty of Medicine, Al Ain, UAE, ${ }^{3}$ Surgery, Department of Surgery, UAE University, Al-Ain, UAE
Introduction: The high risk of injury and death of motorcycle riders is a major global health problem. This study aimed to evaluate factors affecting motorcycle-related death rates worldwide.

Materials and methods: Data for motorcycle riders were collected from 70 countries. These data included motorcycle-related death rates, helmet non-compliance percentage, Gross National Income per capita (GNI), number of registered motorized 2-3 wheelers, the effectiveness of law enforcement, and whether there was standards for helmets use or not. Correlations between studied variables were done using Pearson correlation. Multiple linear regression model was used to define factors affecting motorcycle-related death rates.

Results: Motorcycle-related death rate has a strong trend for correlation with helmet non-compliance percentage $(\mathrm{p}=0.056)$ and strong correlation with motorcycle per person ratio $(\mathrm{p}=0.005)$. Helmet non-compliance percentage was significantly correlated with GNI $(\mathrm{p}<0.0001)$ and effectiveness of the law $(\mathrm{p}<0.0001)$. Countries with helmet standards had significantly higher GNI $(\mathrm{p}=0.001)$, motorcycle per person ratio $(\mathrm{p}=0.012)$, and motorcycle-related death rates $(\mathrm{p}=0.05)$. A multiple linear regression model has shown that helmet non-compliance percentage $(\mathrm{p}=0.003)$, motorcycle per person ratio $(\mathrm{p}=0.01)$ and the presence of helmet standards $(\mathrm{p}=0.05)$ predicted motorcycle-related death rates.

Conclusion: Helmet non-compliance percentage was the most significant factor defining motorcyclists' death rate. Wearing a motorcycle helmet is an important and effective measure to reduce the risk of death in road traffic crashes. Enforcement of motorcycle helmet laws should be effectively supported by motorcycles safety programs.

Disclosure: No significant relationships.

\section{9}

\section{IMPACT OF SECONDARY TRANSFER ON PATIENTS WITH SEVERE TRAUMATIC BRAIN INJURY}

\author{
P. Joosse $^{\text {I }}$, T. Saltzherr ${ }^{\text {I }}$, W.V. Lieshout ${ }^{1}$, P. van Exter ${ }^{2}$, K.J. Ponsen ${ }^{3}$, \\ P. Vandertop ${ }^{4}$, J.C. Goslings ${ }^{3}$
}

${ }^{1}$ Trauma Unit Department of Surgery, Academical Medical Center, Amsterdam, The Netherlands, ${ }^{2}$ Regional Medical Manager of Ambulance Care, GGD, Amsterdam, The Netherlands, ${ }^{3}$ Trauma Unit Department of Surgery, Academic Medical Center, Amsterdam, The Netherlands, ${ }^{4}$ Neurosurgical Center Amsterdam, Amsterdam, The Netherlands

Introduction: The purpose of this study was to assess the effects of secondary transfer to a center with neurosurgical experience on clinical outcomes in trauma patients requiring emergency neurosurgical intervention.

Materials and methods: From 1-1-2006 to 31-12-2009 all patients were included who sustained a severe traumatic brain injury (TBI) and required a neurosurgical intervention within $6 \mathrm{~h}$ after admission to the Amsterdam Medical Center (AMC). Patients were classified into two groups: direct presentation in the AMC (group A) or requiring secondary transport after having been diagnosed for neurosurgical intervention in other hospitals (group B). Outcomes were mortality, Glasgow Outcome Scale (GOS) and several time intervals.

Results: In total, 80 patients were included for analyses. The 24 patients in group B who were secondarily transported had a better GCS on-scene but a higher 30-day mortality compared to patients who were primarily presented in the AMC ( 33 vs. $27 \% ; \mathrm{p}=0.553$ ). Group B also had a worse GOS during follow up. In group B, time to operation was $304 \mathrm{~min}$ compared with $151 \mathrm{~min}$ in group A 
$(\mathrm{p}<0.001)$. For group B most of the time spent before arrival in the trauma center was during the initial trauma evaluation and the interval between the first $\mathrm{CT}$ and the transfer ambulance departure at the referring hospital.

Conclusion: Patients requiring an emergency neurosurgical intervention had a clinically relevant worse outcome after secondary transfer to a neurosurgical service. Therefore, patient care can probably be improved by better triage on-scene and standardized procedures in case of a secondary transfer.

Disclosure: No significant relationships.

\section{0}

\section{POLYTRAUMA FATALITIES AND HEAD INJURIES: AN ANALYZE OF 46 CASES}

\author{
Z. Korac ${ }^{1}$, N. Simic Korac ${ }^{2}$, A. Jankovic ${ }^{1}$
}

${ }^{1}$ Traumatology, General Hospital Karlovac, Karlovac, Croatia,

${ }^{2}$ Anesteziology and ICU, General Hospital Karlovac, Karlovac, Croatia

Introduction: Mortality rates in large trauma patient studies are driven by severe head injury more than by any other organ system. An analysis of various factors influencing upon fatal outcome can improve results and treatment.

Materials and methods: We analyzed data on polytraumatized patients which died in General Hospital Karlovac (level II Trauma Center), Croatia, in 5 years period (January 1, 2004-December 31, 2008). Only the patients older than 18 years with fully available data were included in the study. Dead on arrival were excluded.

Results: We analyzed data on 46 patients, 37 (80.4\%) male and 9 $(19.6 \%)$ female. The median age was 47.1 years (range 18-85). Dominant injury was more frequent in the region of head: $27(58.7 \%)$, than chest 11 (23.9\%), abdomen $4(8.7 \%)$ and extremities including pelvis $4(8.7 \%)$. Main causes of injuries were traffic accidents in 33 patients $(71.7 \%)$, followed by falls from the height in 10 patients $(21.7 \%)$. Twenty two patients died in the first $24 \mathrm{~h}$ after admission. All patients with head injuries were taken to the CT scanner. Autopsy findings showed that in $4(8.7 \%)$ cases there is a difference between clinical/CT and autopsy diagnosis. In these cases pathologist found signs of brain contusion which were not described by radiologist. Despite this fact, these deaths were not recognized as preventable according to opinion of a panel discussion.

Conclusion: Analysis of mortality rates is one of the most important methods of collecting epidemiology data and recognition of weaknesses in trauma care systems. Severe head injury in polytraumatized patients, especially in combination with a chest injury, is often related to a worse prognosis and is a challenge to treat in any trauma center. Reference: 1. Lefering R, et al. Head injury and outcome: what influence do concomitant injuries have? J Trauma. 2008;65:1036-44. Disclosure: No significant relationships.

\section{1}

\section{BLUNT CEREBROVASCULAR INJURIES: 5 YEARS EXPERIENCE IN A SINGLE INSTITUTION}

\section{E. Brauner ${ }^{1}$, J. Amsalem $^{2}$, O. Ben-Ishay ${ }^{1}$, Y. Kluger ${ }^{\text {, H. H. Bahouth }}{ }^{3}$}

${ }^{1}$ Department of General Surgery, Rambam Health Care Campus, Haifa, Israel, ${ }^{2}$ Institute of Interventional Neuroradiology, Rambam Health Care Campus, Haifa, Israel, ${ }^{3}$ Acute Care Surgery Service, Rambam Health Care Campus, Haifa, Israel
Introduction: Blunt Cerebrovascular Injuries (BCVI) are potentially devastating injuries. They are under diagnosed and there is no agreement of their treatment. In this study we present our experience with the diagnosis, treatment and outcome of BCVI.

Materials and methods: Charts of all patients diagnosed with BCVI from January 2006 through September 2010 were reviewed retrospectively. Data Collected included Age, gender, mechanism of injury, ISS, side of injury, associated injuries, time to diagnosis and treatment, type of treatment, length of stay in the ICU (LOS) and hospital (LHS), in-hospital morbidity and mortality were recorded.

Results: 14,036 patients with blunt trauma were admitted to our institution during the study period. $28(0.2 \%)$ BCVI were diagnosed in 21 patients. All were depicted in CTA. Mean age of the injured was 38.8 years (range 10-75), 57\% were males (n-12). Mean ISS was 35, $57 \%$ were injured on the left side and $14 \%$ had a bilateral injury, mechanism of injury was MVA in $76 \%$ (n-16), Fall from height in $10 \%$ and direct blow in $14 \%$ (n-3). Associated facial fractures were diagnosed in $57 \%(\mathrm{n}-12)$, cervical spine fractures in $48 \%(\mathrm{n}-10)$, Chest injuries in $71 \%(\mathrm{n}-15)$, head injuries in $52 \%(\mathrm{n}-11)$ and orthopedic injuries in $43 \%$ (n-9). Mean time to diagnosis and treatment was 9.6 and $53.3 \mathrm{~h}$ respectively. Mean time to ICU arrival was $6.2 \mathrm{~h}$. Treatment elected was endovascular in $9.5 \%$ (n-2) and conservative with anticoagulation or anti aggregation medication in the remaining patients. Heparin was used in 57\%, LMWH in $9.5 \%$, 5-ASA in $9.5 \%$ and clopidogrel in $9.5 \%$. Close surveillance only was elected for $9.5 \%$ of patients. Mean LOS and LHS were 9.6 and 24 days respectively. $19 \%$ of patients (n-4) suffered symptomatic CVA, 1 under full heparinization. 3 patients developed CVA and eventually were diagnosed with BCVI. Mortality was $9.5 \%$ (n-2).

Conclusion: BCVI is rare, high index of suspicion and strict adherence to diagnostic protocols is recommended for early detection and treatment initiation. Treatment modalities are diverse and should not be delayed if no contraindication exists. Strict clinical observation is mandatory.

Disclosure: No significant relationships.

\section{MAJOR INCIDENTS AND DISASTERS}

\section{2}

\section{EMERGENCY MEDICAL INFORMATION SYSTEM (EMIS) AND THE STRATEGY OF DISASTER MEDICINE IN ACUTE PHASE}

\section{Y. Koido, H. Kondo}

Institute for Clinical Research, National Disaster Medical Center, Tokyo, Japan

Introduction: The Japanese disaster medical system had started to develop after the Grate Hanshin-Awaji Earthquake. The lesson to learn from this earthquake is that maximum effort of each hospital could not satisfy the excess medical needs in disaster. This time, we report the strategy for this problem established through experiences and trials.

Materials and methods: We analysis the disaster cases after the Grate Hanshin-Awaji Earthquake and develop the strategy through these cases.

Results: In disaster, it is needed whole medical recourses construct as organization. Information shearing is essential for organization. Emergency Medical Information System (EMIS) include the information about hospital damage, patient reception and activity disaster medical assistant team (DMAT). EMIS has the role of information shearing. For organization of whole hospitals and effective use of EMIS, specialists of disaster medicine will be needed. DMAT bear the role of disaster specialist. First priority of DMAT activity in disaster acute phase is recovery function of the disaster base hospital. 
Second priority is establishment the system of information shearing and transportation among general hospital and disaster base hospital. Third priority is establishment the system of transportation disaster base hospital to non affected area.

Conclusion: In disaster, we established the strategy for whole medical resources organization trough the work of EMIS and DMAT. In Japanese we have the problem of the difficulty to hospitalization emergency patients. The process of whole medical resource organization in disaster has the possibility to apply for solution such problem on emergency medicine.

Disclosure: No significant relationships.

\section{3}

\section{HAITI EARTHQUAKE RESPONSE: LESSONS LEARNED FROM THE FIELD}

\author{
M.T. Cibelli ${ }^{1}$, N. Blagojevic ${ }^{2}$, S. Bossi ${ }^{2}$, S. Bozzini ${ }^{2}$, A. Briguglio ${ }^{2}$,

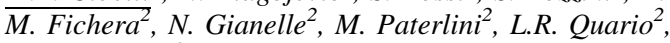 \\ R. Faccincani ${ }^{3}$
}

${ }^{1}$ Icu, IRCCS San Raffaele, Milano, Italy, ${ }^{2}$ IRCCS San Raffaele, Milano, Italy, ${ }^{3}$ Deas, San Raffaele Hospital, Milano, Italy

Introduction: The 2010 earthquake in Haiti caused major distress in Port-au-Prince. All hospitals in the capital had collapsed except the Saint Damien Paediatric Hospital, built in collaboration with NPH-Italia Fondazione Rava. San Raffaele Emergency Team reached Haiti on February 18, 6 days after the tremor and was immediately operational. Materials and methods: In 1 month, five medical teams (13 people) have been rotating to support activities at the Saint Damien Hospital. The two main areas of interventions were: trauma surgery and $\mathrm{MCH}$. We provided crucial assistance to cover needs in the two operating theatres, for non stop emergency, orthopaedic and abdominal surgery and then plastic and reconstructive surgery. A significant contribution was also given in assisting women in labour and newborn babies, by rapidly setting up a special $\mathrm{MCH}$ Unit.

Results: Saint Damien Hospital was one of the very few functioning health facilities in the immediate post-earthquake phase, thanks to: infrastructure built in accordance with earthquake-proof standards; strong voluntary network already created before the disaster by NPH and Fondazione Rava. All these allowed a prompt deployment of the resources needed and provided immediate assistance in order to guarantee the survival of victims and leading to the possibility of building the foundations for a rehabilitation process.

Conclusion: The success factors in health interventions during major incidents are rapidity and efficient logistics to quickly be operative. It is essential to organise, during "peace time", health teams with appropriate qualifications and ready for a prompt intervention in difficult situations. This is what San Raffaele Emergency Team is aiming at. Disclosure: No significant relationships.

\section{4}

HEALTH STATUS CASUALTY MODEL FOR SIMULATION
OF CRISIS MANAGEMENT ACTIVITIES (SICMA PROJECT)

M. Di Mugno ${ }^{1}$, S. Magalini ${ }^{1}$, A. De Gaetano ${ }^{2}$, D. Sermoneta ${ }^{3}$, C. Lodoli ${ }^{3}$, C. Gaz ${ }^{2}$, G. La Posta ${ }^{4}$, F. De Padova ${ }^{4}$, D. Gui ${ }^{3}$

${ }^{1}$ Surgery, Università Cattolica del Sacro Cuore, Rome, Italy, ${ }^{2} \mathrm{CNR}$ IASI, Rome, Italy, ${ }^{3}$ Università Cattolica del Sacro Cuore, Rome, Italy, ${ }^{4}$ SELEX Sistemi Integrati, Rome, Italy
Introduction: The European Project SICMA (Simulation of Crisis Management Activities) provides a modeling of the behavior of the entire Health Service System during field emergency operations, as well as the rules it operates by. The first step toward chain procedure modelling in the management of major emergencies is the representation of a traumatized patient whose health status can be followed in time during simulation. Since management of the trauma patient follows criteria of stabilization of main physiological functions, a trauma patient model was developed based on fundamental pathophysiological functions independently of specific lesion characterization.

Materials and methods: Each patient's health status was modelled according to five parameters (ATLS): A (airway), B (breathing), C (circulatory), D (disability), E (exposure). Patient samples are extracted from a 10,000,000 patient database, generated by considering real anatomical lesions compatible with type and severity of considered scenarios (explosion, building collapse, fire, gunfight). Simulated lesion characteristics were then converted to pathophysiological parameters. Each physiological compensation parameter was represented by: (1) baseline value expressed as percentage of altered function; (2) function reduction rate over time, obtained by a mathematical approximation of clinical worsening. From level of function, rate of worsening and functionspecific death thresholds, estimated time-to-death according to sustained damage is computed.

Results: This model allows simulation of evolution of patient health status both in absence of medical care, but also under therapy, in terms of immediate increment of each single parameter ("temporary" treatment), and of reduction or zeroing of parameter decrement rate ("definitive" treatment).

Conclusion: This model, based on evaluation of physiological parameters, presents an advantage over the consideration of single lesions, because simulating logical procedures that guide treatment choice in real situations can provide a more accurate assessment of casualities for those actors assigned to management of major emergencies.

References: SICMA project website: http://www.sicmaproject.eu Advanced Trauma Life Support (ATLS) (American College of Surgeon, Committee on Trauma) website: http://www.facs.org/trauma/ atls/index.html.

Disclosure: No significant relationships.

\section{5}

\section{MILITARY MODEL OF DISASTER RESPONSE TO EARTHQUAKE IN BAM AND HEALTH LESSONS LEARNT}

\section{H.C. Talan}

Orthopaedic, Defence Complex Hospital (MIN of Defence) Sanaa, Sanaa, Yemen

Introduction: On Friday 26th December 2003 at 0530 hours an earthquake hit Bam city and surrounding area. The city and the surrounding village were completely destroyed. According to WHO, devastation of Bam was deadliest in last decade. Aim (1) to established disaster relief hospital in Bam and provide definite care to victims.

Materials and methods: On 28th December 2003, Indian government was requested to provide humanitarian aid to earthquake victims. Indian army medical cops was entrusted to establish 75 bedded hospital in Bam. 65 personnel were dispatched in transport plane. Team consisted of orthopedic surgeon, general surgeon, anesthesiologist, physician, pediatrician and radiologist whose 
hospital was in tentage accommodation. Tentage accommodation blew off. Trade containers were improvised and used as wards, operating theater and lab.

Results: In our stay in Bam total OPD attendance was 51,204 patients, hospital admission 597, lab investigation 14,478, ultrasound 435. Total surgeries perform was 5,769 which includes 307 major, and minor 5,492. Various departments were established like orthopedic, general surgery, medicine and pediatrics. Hospital gain popularity because of large numbers of orthopedic operations. Major surgery were upper limb 75, pelvis and lower limb, 113 and others 113. Health lesson learnt: triage principle in disaster require entirely different approach for evaluation and care. Training tool such as incident command system in hospital command system affect coordinated response.

Conclusion: Disaster management focused on coordination of multiple agencies in planning of mass casualty is practised.

Reference: WHO community 29 Feb 2004 of Damage in BamSchnitez \& BRIGG US Medical response in Bam, New England. J Med:1-5.

Disclosure: No significant relationships.

\section{6}

\section{MILITARY MODEL OF THE DISASTER RESPONSE TO THE EARTHQUAKE VICTIMS IN BAM AND HEALTH LESSONS LEARNT FROM THE EXPERIENCE}

\section{H.C. Talan}

Orthopaedic, Defence Complex Hospital (MIN of Defence) Sanaa, Sanaa, Yemen

Introduction: On Friday, 26th December 2003, earthquake measuring scale of 8.6 ritcher scale hit BAM city. The city and the surrounding area were completely destroyed. According to the WHO, devastation of BAM was deadliest in the last decade, the key impact was 43,200 people died and 3,000 injured. Aim was to established disaster relief hospital in BAM and provide definite care to victims. Materials and methods: On 28th December 2003, Indian government was requested to provide humanitarian need to the victims. Indian army medical corp was entrusted to establish 75 bed hospital. 65 personals were dispatched in transport plane. Team consisted of orthopedic surgeons, general surgeons, anesthesiologists, physicians, pediatricians, and radiologists with supporting paramedics in the ambulance. Hospital was established in an open field. Whole hospital was in tentage accommodation. OPD's of different departments were established. Emergency room, operation theater, lab and radiology were established in tents. Tentage accommodation blew off. Trade containers were improvised and used as hospital.

Results: In our stay in BAM, total OPD attendance was 51,204 patients, hospital admissions was 597. Total surgeries performed were 5,769, which includes 307 major and minor 5,492. Hospital gained popularity because of large number of orthopedic operations were performed. Team also provide consultation for paraplegic and 100 bedded relief camp. Major surgery were upper limb 75, pelvis and lower limb 113, other 113. 100 cases of broncho pneumonia with respiratory distress were treated in pediatric ICU. Health lesson learnt: (1) Triage principle requires different approach for evaluation and care. Training tool such as incident command system and hospital command system help to affect coordinated response. (2) Guideline for field hospitals were ignored must be updated and practiced.
Conclusion: In response to disaster, proper planning of mass casualities must be practiced and updated for any future event.

Reference: WHO communique February 29, 2004. Damage in BAM. Disclosure: No significant relationships.

0147

\section{MRMI COURSE IN SLOVENIA}

$\underline{\text { R. Komadina }}^{1}$, A. Strahovnik ${ }^{2}$, M. Zajc ${ }^{3}$

${ }^{1}$ Department of Traumatology, General and Teaching Hospital Celje, Celje, Slovenia, ${ }^{2}$ Traumatology, General Hospital Celje, Celje, Slovenia, ${ }^{3}$ Ministry of Health Republic of Slovenia, Ljubljana, Slovenia

Introduction: Medical response to major injuries course was established by ESTES to enable member countries of ESTES to educate health providers to react efficiently and uniformly in mass casualty incidents.

Materials and methods: First group of MRMI instructors from Slovenia was educated during the Croatian MRMI course in 2009. They forwarded the information to the Ministry of health.

Results: From February 1-5, 2011 the first course dedicated to ESTES system of education in MRMI took place in Portoro $\AA^{3 / 4}$, Slovenia, under the sponsorship of the Ministry of health. The faculty staff consisted of international and Slovenian instructors provided knowledge of uniform reacting to MCIs to representatives of Slovenian public health institutions on primary and secondary level.

Conclusion: Periodical education of senior health providers in Slovenian public health institutions will spread the knowledge of MRMI skills to major important organizers of health system.

Reference: Lennquist S. Manual for instructors, MACSIM simulation system. 2010. Lennmed AB. Söderköping, Sweden; 2010.

Disclosure: No significant relationships.

\section{8}

\section{TRIAGE AFTER THE 2009 TURKISH AIRLINES CRASH NEAR SCHIPHOL}

I.L.E. Postma ${ }^{1}$, H. Weel ${ }^{2}$, M.J. Heetveld ${ }^{3}$, F.W. Bloemers ${ }^{4}$, I. $v d$ Zande $^{5}$, T.S. Bijlsma ${ }^{6}$, J.C. Goslings ${ }^{7}$

${ }^{1}$ Trauma Unit, Department of Surgery, Academic medical Centre, Amsterdam, The Netherlands, ${ }^{2}$ VU Medical Center, Amsterdam, The Netherlands, ${ }^{3}$ Kennemergasthuis, Haarlem, The Netherlands,

${ }^{4}$ Trauma Surgery, VU University Medical Centre, Amsterdam, The Netherlands, ${ }^{5}$ GGD kennemerland, Haarlem, The Netherlands,

${ }^{6}$ Spaarne ziekenhuis, Hoofddorp, The Netherlands, ${ }^{7}$ Trauma Unit

Department of Surgery, Academic Medical Center, Amsterdam, The Netherlands

Introduction: Triage is an important aspect of the management of disasters and Mass Casualty Events (MCE). In this study we evaluated the triage after the Turkish Airlines crash near Schiphol Airport on February 25, 2009. How were the casualties triaged? How was triage related to Injury Severity Score (ISS) and emergency interventions? What role did trauma mechanism have in triage and spinal immobilisation on transportation?

Materials and methods: Retrospective analysis of official investigational reports, ambulance forms, and medical charts of 126 
surviving victims of the crash. Outcomes were (pre-hospital/in-hospital) triage classification according to Major Incident Medical Management and Support (MIMMS), ISS, need for emergency intervention according to the 'Baxt criteria', and spinal immobilisation during transport.

Results: Minimal documentation of pre-hospital triage was found. According to the in-hospital triage $28 \%$ casualties were 'immediate', $10 \%$ had an ISS $\geq 16$ and $3 \%$ met the Baxt criteria for emergency intervention. Forty percent was triaged 'delayed', $72 \%$ had an ISS $\leq 8$ and $63 \%$ was discharged from the emergency department. There was up to $89 \%$ over triage but 'critical mortality rate' was $0 \%$. Twenty five percent of all victims was transported with spinal immobilisation, $22 \%$ of all victims with diagnosed spinal injury were not transported with spinal immobilisation.

Conclusion: After the crash documentation of triage was minimal. There seemed to have been a lot of overtriage which is undesirable in MCE's. There has been too little consideration for the high energy trauma mechanism with regard to spinal immobilisation during transport.

Disclosure: No significant relationships.

\section{9}

\section{TSUNAMI RESPONSE FROM EMERGENCY TO RECONSTRUCTION: AN INTERESTING EXPERIENCE}

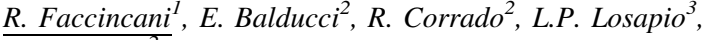 M. Carlucci $^{2}$}

${ }^{1}$ Deas, San Raffaele Hospital, Milano, Italy, ${ }^{2}$ Aispo, IRCCS San Raffaele, Milano, Italy, ${ }^{3}$ Gimc, ANA-Ospedale da Campo, Bergamo, Italy

Introduction: Early after the 2004 Tsunami ANA-Bergamo Field Hospital moved to Kinnya, in the north-east of Sri-Lanka, for an emergency relief mission. The intervention continued through a jointventure between the Field Hospital and Aispo-San Raffaele, gradually transforming an emergency into a development operation.

Materials and methods: From January to June 20058 successive teams of physicians, nurses and logisticians ran the Field Hospital. From July 2005 to March 2007 health activities have been assigned to AISPO-San Raffaele, while ANA-Bergamo provided only logistic support. In the meanwhile AISPO-San Raffaele has been running a project for the reconstruction of Kinnya District Hospital. The hospital was completed and furnished on August 2008, when health activities can finally move from tents to the new building. Up to August 2009 AISPO-San Raffaele has been supporting the Kinnya District Hospital with shipments of consumables, supply of equipment and exchange of personnel for on job training.

Results: This experience represents a valid answer to the question how relief humanitarian missions can move from emergency to reconstruction. This goal was achieved because since the beginning health personnel of AISPO-San Raffaele have been part of the ANA-Bergamo's Field Hospital missions. Thus the end-over of activities from the emergency to the reconstruction phase was natural and came to be a continuous action, avoiding lethal interruption of activities or the fearful risk of construction of "cathedrals in the desert".

Conclusion: We suggest this as a model for relief humanitarian operations where emergency has a vision towards development and cooperation between different organizations gains maximum from individual expertise.

Disclosure: No significant relationships.

\section{0}

\section{The ROLE OF THE FIELD SURGICAL TEAM: EXPERIENCE FROM AFGHANISTAN}

\author{
A. Strahovnik ${ }^{1}$, K. Blair $^{2}$, R. Komadina ${ }^{3}$
}

${ }^{1}$ Traumatology, General Hospital Celje, Celje, Slovenia, ${ }^{2}$ FST, US Military, 09382, Afghanistan, ${ }^{3}$ Department of Traumatology, General and Teaching Hospital Celje, Celje, Slovenia

Introduction: Field surgical theatre has some specific attribute. I would like to present structure of the so cold Field Surgical Team (FST) which upgrade ROLE1 and do not demand logistic support as ROLE2. Distinctions of FST are quick response and fast mobility. Patients are treated as ambulatory, even those with major trauma and damage. Principles of Damage Control Surgery and Advanced Trauma Life Support are used. Premedication and postoperative care is limited up to $24 \mathrm{~h}$. FST is structured of OR, ICU, Ambulatory, Communication centre and Storage with pharmacy included. All the personnel are polyvalent and constitute of surgeons (2), anestesiologists (2), or nurses (3) ICU and ambulatory staff (4), and some logistic crew (6). In field theatre is demanding because of non-stop schedule with burning out of the personnel treat. 2-4 elective operations per day were performed, 5-10 emergency operations per week were performed. FST is highly effective and logistically less demanding as ROLE2.

Materials and methods: Patients are treated as ambulatory, even those with major trauma and damage. Principles of Damage Control Surgery and Advanced Trauma Life Support are used. Premedication and postoperative care is limited up to $24 \mathrm{~h}$. FST is structured of OR, ICU, Ambulatory, Communication centre and Storage with pharmacy included. 2-4 elective operations per day were performed, 5-10 emergency operations per week were performed.

Results: Field Surgical Team is highly effective in war zone because of fast mobility and not so demanding as ROLE2 unit. Surgeons are skilled in ATLS and DCS standards and are polyvalent in their skills. Conclusion: Field surgical theatre has some specific attribute. I would like to present structure of the so cold Field Surgical Team FST which upgrade ROLE1 and do not demand logistic support as ROLE2. Distinctions of FST are quick response and fast mobility. Disclosure: No significant relationships.

\section{COMPLEX PELVIC RING}

\section{1}

\section{'REAMER IRRIGATOR ASPIRATOR' (RIA) REAMINGS VERSUS ILIAC CREST ASPIRATE: A COMPARATIVE ASSESSMENT OF MESENCHYMAL STEM CELLS (MSC) AND ENDOTHELIAL PROGENITOR CELLS (EPC)}

\section{Henrich, C. Seebach, E. Sterlepper, C. Tauchmann, I. Marzi, J. Frank}

Department of Trauma Surgery, University of Frankfurt, Frankfurt, Germany

Introduction: Iliac crest derived autologous bone grafts are the gold standard for treating atrophic non-unions. However, the amount of bone obtainable from the iliac crest is limited and the resulting donor site morbidity is a problem. Autologous bone grafts can also be harvested from the femoral bone cavity using a RIA. The purpose of 
this study was to compare the concentration and differentiation potential of MSC and EPC harvested from the iliac crest and from the RIA technique.

Materials and methods: RIA aspirate was collected from 26 patients undergoing intramedullary femur fracture nailing. Iliac crest aspirate was collected from 38 patients undergoing bone graft transplantation. Patient's age, gender or pre-existing health conditions did not significantly differ. Concentrations of MSC and EPC were measured using MSC colony assay, EPC culture assay and flow cytometry (CD34, CD133, VEGF-R2), respectively. Differentiation potential of MSC's was measured using van Kossa staining. Statistics: Wilcoxontest, $\mathrm{p}<0.05$ is significant, results are presented as median values. The study was approved by our local ethics committee.

Results: When compared to iliac crest derived bone grafts, RIA aspirates contained significantly higher; percentage of CD34+ progenitor cells ( 3.7 vs. $1.4 \%$ ), MSC (15 vs. 9 colonies), early EPC (43 vs. 16 cells/microscopic field) and calcium deposition (16 vs. $6 \%$ van Kossa positive area).

Conclusion: Our results indicate that RIA technique yields more MSC and EPS with greater differentiation potential than iliac crest. This, together with limited volume and donor site morbidity could make RIA a better source of donor bone grafts for treating atrophic non-unions.

Disclosure: No significant relationships.

\section{2}

INTRAMEDULLARY NAILING OF FEMORAL SHAFT FRACTURES: DOES THE PROCEDURE HAVE AN IMPACT ON THE CARDIOPULMONARY- AND INFLAMMATORY RESPONSES IN SEVERELY INJURED PATIENTS?

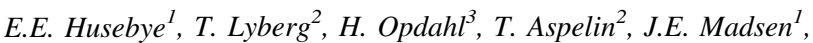 \\ R. Støen ${ }^{I}$, O. Røise
}

${ }^{1}$ Department of Orthopaedics, Oslo University Hospital, Ullevaal, Oslo, Norway, ${ }^{2}$ Center For Clinical Research, Oslo University Hospital, Ullevaal, Oslo, Norway, ${ }^{3}$ National Resource Centre for Nuclear, Biologic and Chemical Medicine, Oslo University Hospital, Ullevaal, Oslo, Norway, ${ }^{4}$ Division of Critical Care, Oslo University Hospital and Faculty of Medicine, University of Oslo, Oslo University Hospital, Oslo, Norway

Introduction: Early intramedullary nailing (IMN) of long bone fractures in severely injured patients has in the literature both been evaluated as beneficial, but has also been associated with increased inflammation, multi organ failure (MOF) and morbidity. This study was initiated to evaluate to what extent cardiopulmonary and inflammatory responses in the traumatized and already inflammatory activated patient is further affected by early IMN.

Materials and methods: Twelve adult patients with femoral shaft fractures were included. Blood was sampled for evaluation of coagulation-, fibrinolytic-, and cytokine activation. A pulmonary artery catheter was inserted in $8 / 12$ patients. Cardiopulmonary function parameters were registered per- and postoperatively. Systemic inflammatory response syndrome (SIRS), acute lung injury (ALI), adult respiratory distress syndrome (ARDS), pneumonia, sepsis and/ or wound infection were monitored and recorded daily.

Results: ISS was 31. No additional procedure-related effect on coagulation- and fibrinolysis activation was evident. TNF- $\alpha$ increased significantly from $6 \mathrm{~h}$ post procedure to the third postoperative day. IL-6 increased from the first to the third postoperative day. IL-10 peaked on the first postoperative day. A procedure-related transient hemodynamic response was observed at $30 \mathrm{~min}$ to $2 \mathrm{~h}$ post procedure. 11/12 patient had SIRS, 7/12 pneumonia, 3/12 ALI, 3/12 ARDS, $3 / 12$ sepsis, $0 / 12$ wound infection.
Conclusion: The additional IMN-related trauma was modest when compared to the total extent of injury. The coagulation- and fibrinolysis activation seems to "drown" in the extensive general trauma response. IMN could participate in the cytokine response. A transient cardiopulmonary response was demonstrated.

Disclosure: No significant relationships.

\section{3}

\section{TREATMENT OF RECTAL INJURIES ASSOCIATED TO PELVIC RING DISRUPTIONS}

F. Castelli $^{1}$, S. cimbanassi $^{2}$, S. Andreani ${ }^{3}$, D. Capitani $^{1}$

${ }^{1}$ Dea, Niguarda Ca Granda, Milan, Italy, ${ }^{2}$ Emergency, Ospedale Niguarda Ca'Granda, Milano, Italy, ${ }^{3}$ Trauma Team, General Surgery, Niguarda Hospital Milan, Milano, Italy

Introduction: Open pelvic fractures are rare. Rectum injuries in association with pelvic ring disruption are thought to be result of a fracture fragment piercing the rectum in case of lateral compression or injury is on the anterior rectal wall as a result of pure open book injury without fracture. We describe the epidemiology and fracture pattern associate to rectal injuries and discuss when fecal diversion is necessary.

Materials and methods: Retrospective analysis of data on pelvic fractures injuries from October 2002 to October 2008 in a level 1 Trauma Center in Milan. Demographics, mechanism, fracture pattern, ISS, resuscitation data and outcomes were recorded.

Results: Results In the study period, 11 patients suffered from open pelvic fracture. Males represented $72.7 \%$ of case. Population mean age was $39.2 \hat{\mathrm{A}} \pm 12.06$ years, mean ISS $36.8 \hat{\mathrm{A}} \pm 14.07$. Mechanisms of injuries were car crashes in 1 case, motorvehicle accidents 6 cases, cycle 1 , pedestri strucked 1 , fall from height 1 , gunshot wound 1 case. Injuries determined 1 vaginal laceration, 5 rectal lacerations, 4 perineal extended lacerations and 1 laceration of anal sphincter. Temporary colostomy in the right upper quadrant was performed in all cases of rectal lacerations, in presence of vaginal injury and in 2 cases of extended perineal involvement. Anal sphincter damage was directly sutured, lacerations of soft perineal tissues were treated by in vacuum assisted closure in two cases. Survivors accounted for $90.9 \%$ of cases. Conclusion: With the availability of advanced wound management techniques (VAC) the gold standard treatment of the open pelvic ring disruption needs to be re-evaluated. Our limited experience show that pelvic fracture without a rectal injuries can be successfully treated without faecal diversion. Fecal diversion is mandatory in rectal injuries in association with pelvic ring disruption.

Disclosure: No significant relationships.

\section{4}

\section{BIOMECHANIC INVESTIGATION OF COMPRESSIVE FORCE AND CONTACT AREA IN PLATE FIXATION FOR PELVIC SYMPHYSIS RUPTURE}

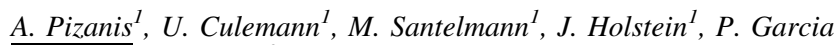 \\ Caso $^{\text {, T. T. Pohlemann }}{ }^{2}$
}

${ }^{1}$ Trauma-, Hand- and Reconstructive Surgery, University of Saarland, Homburg, Germany, ${ }^{2}$ Department of Trauma, Hand and

Reconstructive Surgery, University of Saarland, Homburg, Germany

Introduction: Plate fixation is the treatment of choice for traumatic symphysis rupture. This experimental study compares different plate geometry and fixation techniques in order to assure clinical healing and lessen implant failure. 
Materials and methods: Simulation of AO-B1 injuries in pelvis 4060 (Synbone ${ }^{\circledR}$ ) to investigate the compressive force and resulting contact area of the symphysis after reduction and fixation using I-Scan sensors $\left(\right.$ Tekscan $\left.{ }^{\circledR}\right)$. Parameters: compressive Force $(\mathrm{N})$, contact area $(2 \mathrm{~mm})$, center of force position and reduction profile. Study groups with steel plates: $\mathrm{DCP} 0^{\circ}$ w/o prebending, prebended $\mathrm{DCP} 10^{\circ}$, symphysis-plate prebended $\left(\mathrm{SCP} 10^{\circ}\right)$, combi-hole $(\mathrm{SC} /$ LP10 ${ }^{\circ}$ and locking-screws (SLP). Statistics by SigmaStat, significances at $\mathrm{p}<0.05$.

Results: Most effective significant compression achieved by prebended plates in $\mathrm{DCP} 10^{\circ}$ and $\mathrm{SCP} 10^{\circ}$. $\mathrm{DCP} 0^{\circ}$ fell under initial reduction levels. Pure locking screw effect in SLP did not preserve initial compressive force at reduction, but allowed maximal contact in symphyseal lesion. All symphyseal plates groups revealed anatomic contact areas, whereas DCP groups showed slight $\left(\mathrm{DCP} 10^{\circ}\right)$ and severe $\left(\mathrm{DCP} 0^{\circ}\right)$ losses in contact areas $(\mathrm{p}<0.05)$. The center of force differed significantly depending on the type of plate used and the effect of pre-bending: only those with symphysis-plate fixation showed centered forces in the symphyseal gap. The DCP Platereduction induced pronounced effects near implants, while symphysis plates allowed even contact for the whole extent of the gap.

Conclusion: Under experimental conditions, symphysis plate fixation depends on prebending to increase compressive forces and contact areas in a symphyseal rupture model. Best results can be achieved with the anatomic contoured symphysis plates in combined configuration "central DC and peripheral locking screws".

Disclosure: No significant relationships.

\section{5}

\section{COMBINED PELVIC AND ACETABULAR FRACTURE PATTERNS}

D. Blanco Díaz ${ }^{1}$, P. Caba Doussoux ${ }^{1}$, J.L. León Baltasar ${ }^{1}$, J.L. Muñoz Vives ${ }^{2}$, C. Resines Erasun ${ }^{1}$

${ }^{1}$ Orthopedic Trauma Unit, Hospital Universitario 12 de Octubre, Madrid, Spain, ${ }^{2}$ Orthopedic Trauma Unit, Hospital Universitari Doctor Josep Trueta, Girona, Spain

Introduction: Treatment of combined pelvic and acetabular fractures is a surgical challenge. We analyzed a case series of patients with complex pelvic injuries with special attention to fracture pattern and clinical outcome.

Materials and methods: Hospital Trauma Registry review. Inclusion criteria: ISS $>10$, combined pelvic and acetabular fractures. We searched the Trauma Hospital Registry between 2000 and 2010. Radiologic assessment, according to classification and postoperative reduction, was performed by detailed blind rewiew of three experienced pelvic surgeons. Data analysis included: demographic data, injury mechanism, fracture classification (AO-OTA), surgical approach, complications and functional outcome.

Results: We reviewed 221 patients with pelvic injuries, 28 of them met the inclusion criteria. Average age was 32.5 years. Mechanism of injury: falls from height in 16, RTA in 10 . We found two main patterns: displaced transverse fracture of the acetabulum associated with unilateral complete posterior ring fracture in 12 cases, and both column acetabular fractures associated with complete posterior injury in 6 cases. Six patients were treated by external fixation alone. Eleven patients were treated with ilioinguinal approach and percutaneous fixation of the posterior ring. One patient needed a combined approach and another patient a KL approach. Four cases were treated by percutaneous methods. Complication rate was low (one deep infection, one intraoperative iliac vein bleeding). Congruent reduction was achieved in $80 \%$ of cases.
Conclusion: Most patients with complex pelvic ring and acetabulum fractures can be treated in supine with a combination of anterior approach and percutaneous techniques with a low complication rate. Main combination fracture pattern was posterior complete injuries with displaced transverse fractures or both column fractures.

Reference: Porter SE. Acetabular fracture patterns and their associated injuries. J Orthop Trauma, 2008;22:165-170. Cano-Luis P. Fracturas del anillo pélvico asociadas a fracturas acetabulares. Rev Orthop Traumatol. 2004;48(3):83-90.

Disclosure: No significant relationships.

\section{6}

\section{DEFINITIVE FIXATION OF THE FRACTURES OF THE PELVIS IN TRAUMA PATIENTS}

\section{Rizzi, C. Castelli}

Orthopaedics and Trauma, Ospedali Riuniti di Bergamo, Bergamo, Italy

Introduction: Fractures of the pelvis are a rare lesion, less than 5\% of the traumatic skeletal lesions, that involves the thee bones of the pelvis, nerves, vessels and its viscera. Initial management in trauma patients with pelvic fracture is aimed at saving life and identify and treat life-threatening injuries in order to priority. Definitive fixation of these lesions seeks to fix unstable lesions and to restore pelvic anatomy in order to avoid deformities and pain due to chronic instability and non-union $[1,2]$. We report our experience during the last 6 years.

Materials and methods: 54 patients were treated with anterior and/or posterior internal fixation for the injured pelvic ring between 2005 and 2010. The modified Tile classification was applied for evaluation of the fracture of the pelvis. Follow-up at average 46 months included both radiological and clinical examination.

Results: Patients treated for a B-type lesion had $80 \%$ of excellent and good results and healed anatomically. C-type fractures had a less percentage of anatomical reduction and only $33 \%$ of excellent and good results.

Conclusion: Fractures of the pelvis have a high incidence of poor results due to the residual pain. In our experience we found a poor correlation between clinical and radiological results in C-type fractures because of associated traumatic lesions and due to high energy injuries. Our data suggest that the better clinical results are related to the more stability of the fixation of the posterior pelvic ring. Anatomical reduction of the pelvic ring and internal fixation of the disruption of the pelvis is the goal standard in the treatment of these lesions.

References: 1. Matta JM, Saucedo T. Internal fixation of pelvic ring fractures. Clin Orthop 242:83-97. 2. Tile M. Fractures of pelvis and acetabulum. Williams and Wilkins, Baltimore; 1984.

Disclosure: No significant relationships.

\section{7}

\section{MANAGEMENT OF DELAYED VERTICALLY UNSTABLE PELVIC FRACTURES}

\section{O. Farouk, Y. Khalifa}

Orthopaedic Department, Assiut University Hospitals, Assiut, Egypt

Introduction: Current treatment protocols recommend early fixation of pelvic fractures within few days after trauma following stabilization 
of the patient. For some reasons, patients may be referred to level one trauma centers late after trauma, and surgery imposes difficulties to the patient and the surgeon. This study aims at evaluating the results of surgical management of neglected vertically unstable type-C pelvic fractures.

Materials and methods: The study is a case series of 16 patients with vertically unstable pelvic fractures who presented to the authors' institution 3 weeks or more late after the initial trauma during a period of 5 years (2005-2009). Time since injury ranged from 3 to 32 weeks. There were 14 males and 2 females. Age of the patients ranged from 14 to 48 years. There were twelve $\mathrm{C} 1$, one $\mathrm{C} 2$, and three C3 fractures. Seven patients had associated neurological injuries. Fixation of posterior and anterior pelvic fracture components was done in nine patients. Posterior pelvic segment fixation alone was done in seven patients. Radiographic assessment was done using the grading system of Matta and Tornetta, and functional outcome assessment was done using the score system of Majeed.

Results: All fractures healed. Complications included transient L5 palsy $(\mathrm{n}=1)$, and ilio-femoral DVT $(\mathrm{n}=1)$. Fracture reduction was considered excellent in 11 patients $(69 \%)$, good in four patients $(25 \%)$, and fair in one patient $(6 \%)$. Functional result was excellent in 12 patients $(75 \%)$, good in three patients $(18.75 \%)$ and fair in one patient $(6.25 \%)$.

Conclusion: This study supports surgical interference for delayed vertically unstable pelvic fractures with expected good functional results.

Disclosure: No significant relationships.

\section{8}

\section{PARARECTAL APPROACH FOR INTRAPELVIC MANAGEMENT OF ACETABULAR FRACTURES: ANATOMIC EXAMINATION IN HUMAN CADAVERS}

\section{Keel, T.M. Ecker, L. Büchler, K.A. Siebenrock, J.D. Bastian}

Department of Orthopaedic Surgery, Inselspital, University of Bern, Bern, Switzerland

Introduction: The ideal approach for surgical treatment of displaced acetabular fractures involving the anterior column and the quadrilateral surface should expose as much as needed for anatomic restoration with respect to the pelvic anatomy in order to reduce access morbidity. We describe a new anterior, intrapelvic, extraperitoneal approach with focus on the feasibility of secure and optimal instrumentation of the pelvis.

Materials and methods: Anatomic examinations were conducted on five human cadaver full torso specimen. The skin incision is directed along the palpable lateral border of the rectus abdominis muscle, the so-called "pararectal" approach. The extraperitoneal space is entered by incision of the rectus sheath to establish four "windows" for instrumentation. Cadavers were analyzed radiographically for the appropriate placement of the instrumentation.

Results: The pararectal approach facilitates sufficient extraperitoneal access to the inner surfaces of the entire false and true hemi pelves. Neurovascular structures at risk during dissection are visualized for damage control reasons in a clinical setup. The radiographic analysis confirmed secure and optimal instrumentation of the pelvis.

Conclusion: The clear limitation of the study is due to its anatomic character. The clinical implementation has to prove whether the pararectal approach might be beneficial compared to established approaches in terms of anatomic fracture reduction and patient morbidity. The pararectal approach has the potential to facilitate proper reduction of complex acetabular fractures as multi-directional placement of screws and various plate configurations are feasible without vast retraction of the soft-tissues.

Disclosure: No significant relationships.

\section{VISCERAL TRAUMA 5}

\section{9}

\section{SURGICAL MANAGEMENT OF FLAIL CHEST IN MULTIPLE TRAUMA PATIENTS}

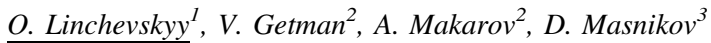

${ }^{1}$ Multiple Trauma, Clinical hospital \# 17, Kyiv, Ukraine, ${ }^{2}$ National Medical Academy of Postgraduate Education, Kyiv, Ukraine,

${ }^{3}$ Clinical hospital \# 17, Kyiv, Ukraine

Introduction: Indications, techniques of operative rib fixation in flail chest remain still debatable. The objective of this research was to study the results of flail chest surgery in multiple trauma patients. Materials and methods: We retrospectively studied a series of 40 consecutive patients with polytrauma, who have undergone operative flail chest fixation: 33 men and 7 women, with a median age of 46 (27-79) years. Median ISS was 22, range 16-34. Indications for surgery were paradoxal chest movement, required prolonged ventilation and/or significant chest wall deformity causing volume loss of hemithorax. Operative muscle preserving approach was performed depending on maximal ribs dislocation. We used Kirschner wires for intramedullary rib splinting despite fracture localization.

Results: Median time from admission to operation was 2.5 (range 0-20) days. Median number of fixed ribs was 4 (range 1-6) additional sternal fixation was done in 4 patients. Thoracotomy due to underlying lesions was performed in 10 cases. The operating time was 110 (60-190) min. Stable fixation of flail segment was achieved in 39 cases, 1 patient required reoperation. There was no mortality among operated patients, median lengths of hospital stay was 15 (6-57) days. Among chest related morbidity we observed 14 cases of pleural effusions, required punctions, 2 pneumonia, 1 empyema, 1 anemia. Conclusion: Surgical stabilization of flail chest can be safely performed in multiple trauma patients on early stages. K-wire rib fixation is feasible and efficient for stable fixation of any flail segment of chest wall. No specialized rib plates are needed.

References: Fitzpatrick D, Denard P, Phelan D, Long W, Madey S, Bottlang M. Operative stabilization of flail chest injuries: review of literature and fixation options. Eur J Trauma Emerg Surg. 2010;36:427-433. Athanassiadi K, Theakos N, Kalantzi N, Gerazounis M. Prognostic factors in flail-chest patients Eur J Cardiothorac Surg. 2010;38:466-471.

Disclosure: No significant relationships.

\section{0}

\section{WHOLE BODY CT SCAN IN TRAUMA PATIENT MANAGEMENT}

\section{Fattori ${ }^{1}$, M. Oldani $^{2}$, G. Pesenti ${ }^{3}$}

${ }^{1}$ Surgery Chirurgia Iii, OSP San Gerardo, Monza, Italy, ${ }^{2}$ SurgeryChirurgia Iii, san gerardo hospital, Monza, Italy, ${ }^{3}$ Surgery- Chirurgia Iii, Osp San Gerardo, Monza, Italy

Introduction: In severely injured trauma patients imaging is highly coded during initial assessment. Radiography and ultrasounds are 
usually performed. Imaging is frequently supplemented by wholebody CT-scan. The ability of multislice-CT to detect trauma lesions more accurately than alternative methods might profoundly affect the management of trauma patients and orient angiographic embolization. Although unstable trauma patients would theoretically benefit the most from a CT diagnostic program, the clinical conditions don't allow wasting too much time far from a safe area. In these patients X-rays and ultrasounds play an important role in the diagnostic screening. Otherwise patients may be resuscitating for all over the work-up also in the CT-scan suite to save time.

Materials and methods: Patients data (June 2008-June 2010) were selected from the local trauma registry. A complete time registration was performed. Data on severe trauma patients (ISS $>15$ ) were reviewed focusing on contrast-enhanced CT-scan. Minor cranial trauma was excluded according to a specific algorithm. An extensive use of CT in hemodynamically stable or marginally-stabilized patients was recommended. FAST was liberally performed before CT. Prediction of death was defined by TRISS.

Results: 208 patients were evaluated. No patient died during CTscan or during transport to and from CT-suite. Overall mortality was $9 \% .89 \%$ of the severe trauma patients were diagnosed by CTscan (mean time $52 \mathrm{~min}$ ) and $2 \%$ were scanned immediately. In the first semester only $33 \%$ were scanned in the first $40 \mathrm{~min}$. In the the last year $50 \%$ of severely traumatized patients were scanned in 40 min. $14 \%$ were scanned even in temporarily stabilized conditions. CT-report modified radically therapeutical pathway in these patients.

Conclusion: CT-scan during the early diagnostic workup in severe trauma patients is safe even in temporary unstable ones in which a whole body CT-scan algorithm may lead to earlier and more accurate diagnosis Current imaging algorithms before CT might be reassessed. Disclosure: No significant relationships.

\section{1}

\section{ARTERIOGRAPHY IN OPERATING ROOM ALLOWS AN IMPORTANT REDUCTION IN THE TIME TO CONTROL BLEEDING IN SEVERE TRAUMA}

\author{
E. Cingolani, T. Mastropietro, S. Manfroni, C. Cataldi, \\ L. Riccioni, D. Antonellis, G. Nardi
}

Emergency and General Surgery, San Camillo Forlanini, Rome, Italy

Introduction: Uncontrolled bleeding is among the most important cause of death for patients with major injuries. The rapid management of bleeding is one of the major challenges at the beginning of trauma phase care. Bleeding control may be obtained through surgical interventions or transcatheter angiographic embolization (TAE) when indicated. To be effective TAE must be performed as soon as possible after admission before the onset of trauma induced coagulopathy, as a preserved clotting function is a key issue to allow TAE to be effective. Data in the literature show that when TAE is delayed over $3 \mathrm{~h}$ from admission, mortality increases significantly. Unfortunately surgical control of bleedings and TAE are usually performed in separate locations.

Materials and methods: One year (2009) data of all major trauma (MT) patients (ISS > 15) hemodynamically unstable who required emergency TAE, were prospectively entered in the local Trauma Registry and retrospectively analyzed. MT patients admitted in 2009 (angiography in the OR) were compared with MT patients admitted during the same period of 2005 (angiography in the IR suite).
Results: From January 1, 2005 to December 31, 2005, 286 MT patients were admitted to the ED and $22(7.7 \%)$ required emergency TAE. The average time interval between hospital admission and TAE was 3 h 30'. From Jan 1, 2009 to Dec. 31, 2009343 MT patients were admitted to the ED. Thirty required TAE (8.7\%): 22 were submitted to TAE in the OR. The mean interval time from hospital admission was $1 \mathrm{~h} 12^{\prime}$ for the patients treated in OR, with a three time reduction if compared with 2005.

Conclusion: A portable angiograph may allow to successfully perform TAE in the OR in the unstable trauma patients, with a sharp reduction in the time interval to control haemorrhage.

Reference: Misselbeck TS. Hepatic angioembolization in trauma patients: indications and complications. J Trauma. 2009;67:769-773. Disclosure: No significant relationships.

\section{2}

\section{MECHANICAL VENTILATION AFTER LUNG CONTUSION CAUSES MORE INFLAMMATION THAN VENTILATION ALONE IN A BLUNT CHEST TRAUMA MODEL}

\author{
K.J.P. van Wessem ${ }^{1}$, M. Heeres ${ }^{1}$, M.P. Hennus ${ }^{2}$, T.J. Blokhuis ${ }^{3}$, \\ L. Koenderman ${ }^{4}$, L.P. $\overline{\text { H. Leenen }}{ }^{5}$
}

${ }^{1}$ Department of Trauma Surgery, University Medical Centre Utrecht, Utrecht, The Netherlands, ${ }^{2}$ Intensive Care, Wilhelmina Children's Hospital, Utrecht, The Netherlands, ${ }^{3}$ Surgery, University Medical Center Utrecht, Utrecht, The Netherlands, ${ }^{4}$ Immunology, University Medical Centre Utrecht, Utrecht, The Netherlands, ${ }^{5}$ Department of Surgery, University Medical Centre Utrecht, Utrecht, The Netherlands

Introduction: Posttraumatic lung contusion is common after blunt chest trauma and patients often need ventilatory support. Lung contusion induces an inflammatory response signified by primed polymorph neutrophil granulocytes (PMNs). Mechanical ventilation (MV) itself can also cause an inflammatory response. The aim of this study is to develop an animal model to investigate inflammatory response in blunt chest trauma.

Materials and methods: Twenty-three male Sprague-Dawley rats were randomized for MV with (17 rats) or without (6 rats) lung contusion. Three rats were used as controls. Lung contusion was produced by a 'blast generator', a device releasing a single pressure blast wave centered on the chest. The distance between 'blast generator' and rat varied between 3.5 and $6 \mathrm{~cm}$. After termination, lungs were harvested for lung-body weight ratio (LBWR), PMN percentage in broncho-alveolar lavage fluid (BALF) and myeloperoxidasis (MPO) analysis.

Results: Fifteen rats survived the experiment (69\%). Survival was correlated to the distance to the 'blast generator' (39\% in $3.5-4.5 \mathrm{~cm}$ vs. $83 \%$ in $6 \mathrm{~cm}, \mathrm{P}=0.05$ ). LBWR was significantly increased in contused ventilated rats $\left(13.9 \times 10^{3}\right)$ compared to ventilated only $\left(8 \times 10^{3}\right)$ and control rats $\left(7.3 \times 10^{3}, \mathrm{P}=0.005\right)$. All ventilated rats had more PMNs in BALF than control rats (48 vs. $0 \%, \mathrm{P}=0.03$ ). MPO analysis showed a significant increase in contused ventilated rats compared to ventilated only rats (5.33 vs. 2.60 units/g lung tissue, $\mathrm{P}=0.04)$.

Conclusion: The 'blast generator' generates reproducible, isolated blunt chest trauma in rats and is suitable to study inflammatory response after lung contusion. MV after lung contusion induces a larger inflammatory response than MV alone.

Disclosure: No significant relationships. 


\section{3}

\section{THE ROLE OF OXIDATIVE STRESS IN OUTCOME OF PATIENTS WITH MULTIPLE BLUNT TRAUMA}

\author{
O. Sogut ${ }^{1}$, H. Kaya ${ }^{2}$, M.T. Gokdemir ${ }^{2}$, M. Cevik ${ }^{3}$, M.B. Sayhan ${ }^{4}$, \\ M.A. Dokuzoglu', M.E. Boleken ${ }^{3}$
}

${ }^{1}$ Emergency Medicine, University of Harran, Sanliurfa, Turkey,

${ }^{2}$ Emergency Medicine, Harran University, Sanliurfa, Turkey,

${ }^{3}$ Pediatric Surgery, Harran University, Sanliurfa, Turkey, ${ }^{4}$ Emergency

Medicine, Trakya University, Edirne, Turkey

Introduction: In all types of critical illness, such as sepsis, trauma or burn injury, the occurrence of increased oxidative stress or a reduced antioxidative status is related to a poor prognosis [1].

The objective in this prospective hospitals based study is to determine the early oxidative changes for the outcome of patients with multiple blunt trauma by evaluating serum total oxidant status (TOS), total antioxidant status (TAS) and oxidative stress index (OSI) levels.

Materials and methods: Venous blood samples were obtained from 52 multiple blunt injury patients within $24 \mathrm{~h}$ of trauma onset. The patients were divided into two groups; the survival and non-survival groups. The TOS and TAS of the plasma were assessed using a novel automated measurement method developed by Erel [2]. For every patient, the Revised Trauma Score (RTS) and Injury Severity Score (ISS) scores were calculated.

Results: The overall mortality rate was $32.7 \%$. Mortality was negatively correlated with RTS ( $<<0.001)$. TOS levels were significantly higher in non-survivors $(\mathrm{n}=17)$ as compared with survivors $(\mathrm{n}=35)(\mathrm{p}<0.05)$. However, TAS and OSI levels were not significant in non-survivors when compared with survivors $(\mathrm{p}=0.374$, $p=0.344$, respectively). There was a statistically significant, positive correlation between mortality and ISS $(\mathrm{p}<0.001)$.

Multiple injury severity (ISS) was positively correlated with TOS levels $(\mathrm{p}=0.002)$ (TAS and OSI, not significantly related). RTS was negatively correlated with TOS levels $(\mathrm{p}=0.009)$ (TAS and OSI, not significantly related).

Conclusion: ISS and RTS were significant independent predictors of mortality. During the early post-traumatic period, TOS may be an objective alternative criterion to the both ISS and RTS in the management of patients with multiple blunt trauma.

References: 1. Roth E, Manhart N, Wessner B. Assessing the antioxidative status in critically ill patients. Curr Opin Clin Nutr Metab Care. 2004;7:161-168. 2. Erel O. A new automated colorimetric method for measuring total oxidant status. Clin Biochem. 2005;47:119-129.

Disclosure: No significant relationships.

\section{4}

\section{END-TO-SIDE ANASTOMOSIS FOR LIMB SALVAGE IN THE SINGLE ARTERY OF THE TRAUMATIZED EXTREMITY}

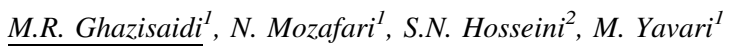

${ }^{1}$ Plastic and Reconstructive Surgery, 15 Khordad Hospital, Tehran, Iran, ${ }^{2}$ Burns Department, Zanjan University of Medical Sciences, Zanjan, Iran

Introduction: In free flap transfer, the recipient's artery is often sacrificed to provide the pedicle anastomosis. Since the recipient artery is necessary for distal circulation, preserving its patency is also important (especially in the traumatized extremities). Decision-making is a difficult task in choosing among the following types of anastomoses for a single artery extremity reconstruction: $\mathrm{T}, \mathrm{Y}$ arterial shape, vein graft or end-to-side arterial anastomosis. These techniques can be used to preserve the recipient's flow beside other clinical purposes depending on the recipient's vessel condition.

Materials and methods: To this end, angiography was performed on all the patients who were planned for free flap and a single artery was confirmed in 11 cases. End-to-side anastomosis was applied for limb salvage in single-artery extremity reconstruction, such as preserving recipient's flow and preserving donor's flow in 11 patients. The anastomosis arteries included the popliteal artery $(n=3)$, the posterior tibial artery $(n=2)$, the peroneal artery $(n=5)$ and radial $\mathrm{n}=1$.

Results: Finally, the results show that free flap transfer by end-to-side anastomosis for limb salvage with a single artery is a safe procedure. Conclusion: Consequently, a vein graft, $\mathrm{T}$ and $\mathrm{Y}$ shape anastomosis are not necessary.

Reference: There are 14 references for this study.

Disclosure: No significant relationships.

\section{5}

\section{THE DEPENDENCY OF A TRAUMATIC AORTIC INJURY FROM THE AORTIC STRAIN COEFFICIENT. A CASE SERIES AND BIOMECHANICAL SIMULATION}

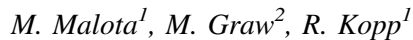

${ }^{1}$ Abteilung Für Chirurgie, Krankenhaus München Harlaching, Munich, Germany, ${ }^{2}$ Institut Für Rechstmedizin, Ludwig-Maximilians Universität München, Munich, Germany

Introduction: Traumatic aortic injuries are rare, but extremely lethal. The understanding of the trauma mechanism is fundamental for patient treatment and injury prevention. Especially the question, which factors lead to traumatic aortic injuries, is of essential importance.

Materials and methods: A database with all patients in South Bavaria that died from a traumatic thoracal vascular lesion was created during a 2-year period, including all clinical findings, anthropometric patient data and technical analysis of the course of death. Using a computer based, biomechanical method (the "finite element method, FEM"), selected accidents were simulated and the application of the force on the thoracal skelet and the aorta was analysed.

Results: Selected victims with a definite traumatic aortic injury were taken for further examinations. By reconstructing the accident, we were able to calculate the relative coefficient of the strain in the thoracal aorta. During the simulations we found evidence that neither the velocity nor the shear force are essential for an aortic injury, but the aortic strain coefficient, which was furthermore associated to internal and external cofactors (structure of the aortic wall, distribution of the forces, muscular tension, stability of osseous structures). These cofactors can be modified in the simulation, so that the direct influence on the injuries can be shown.

Conclusion: Using biomechanical simulations with the FEM, it is possible to reconstruct an accident and to calculate the individual strain coefficient of the traumatised aorta. The additional influence of internal and external factors on the relative strain of the thoracal aorta can be modified selectively in the simulations.

Disclosure: No significant relationships. 


\section{5}

PACS LAPAROSTOMY: 2 YEARS OF EXPERIENCE IN THE CESENA TRAUMA CENTER

\section{P. Ruscelli, F. Buccoliero}

\section{Emergency, Trauma Center Cesena, Cesena, Italy}

Introduction: In the last few years laparostomy has become an even more useful option for the surgeon due to the development of the experience in the critical abdominal trauma and in the severe surgical urgencies as PANE and peritonitis with the introduction of damage control as surgical strategy to prevent the abdominal compartimental syndrom (ACS). After a laparostomy the surgeon needs to provide a delayed fascial closure (DFC) to achieve the best outcome for the patient. The aim of this paper is to illustrate the experience and the results after the introduction of a modified laparostomy technique in our surgical activity.

Materials and methods: Protection of intestinal content, aspiration under sub-athmospheric pressure, control in quality and quantity of the drainage and skin temporary closure, rappresent the four principles of the PACS tecnique. In order to evaluate the rate of delayed fascial closure and the rate of ACS onset, we have retrospectively studied 31 patients over a period of 2 years treated with open abdomen with an amount of 36 PACS performed. Routinely IAP measurament was also performed.

Results: ACS was developed in no patients. IAP was measured on average at 19. DFC was achieved in 33 PACS of $36(91.7 \%)$ after 5 days on average ( $20 \mathrm{~h}$ as minimum; 20 days as maximum). Aspected mortality (SAPSII) in ICU for these patient was $76.5 \%$ on average. On 31 patients, $20(64.5 \%)$ died and $11(35.5 \%)$ survived.

Conclusion: PACS seems to be an effective, easy and low cost technique for the management of open abdomen.

Disclosure: No significant relationships.

\section{OPEN PELVIC FRACTURES}

\section{6}

\section{A NEW PROSTHETIC IMPLANT FOR THE TREATMENT OF} ACUTE FRACTURES OF THE ACETABULUM IN ELDERLY

\section{H. Resch}

Traumatology and Sports Injuries, University Hospital Salzburg, Salzburg, Austria

Introduction: Fractures of the acetabulum in elderly patients pose a major problem as osteosynthesis may not provide sufficient stability due to poor bone quality. Prosthetic replacement would be desirable in the acute phase in these patients. Unfortunately, commonly available prosthetic implants do not allow stable fixation of the acetabulum component.

Materials and methods: A new implant for the stable fixation of the acetabulum component in a fractured acetabulum was developed. The key element is a metallic titanium ring with an integrated plate enabling the stable fixation of the ring on the ilium bone with numerous angle stable screws. The direction of the screws provide high primary stability. In the metallic ring a commonly available polyethylene socket is cemented. So far six patients with an average age of 75 years (71-87) were treated with this implant acutely within 1 week after injury. All patients were suffering from an acute acetabulum fracture with both columns destroyed (Transverse- and T-fractures).

Results: Five of the six patients could be mobilised with weight bearing at least 2 weeks after surgery. One patient has died. After a FU time of 4-25 min no signs of loosening were observed. In four of the six cases bony healing around the metallic ring was found. In one case the time period is too short for the assessment.

Conclusion: Although the number of cases is small the preliminary results are very encouraging. This implant offers the opportunity of early mobilisation despite a fracture which is disastrous for old patients. Disclosure: No significant relationships.

\section{7}

\section{EXTERNAL FIXATION OF FEMORAL FRACTURES FOR DAMAGE CONTROL ORTHOPAEDICS: 52 CONSECUTIVES PATIENTS}

V. Rodriguez Vega ${ }^{1}$, P. Caba ${ }^{2}$, V. Guimera ${ }^{3}$, J.L. Leon ${ }^{2}$, $\overline{J . A . ~ Z a f r a ~ J i m e ́ n e z ~}^{4}$, A.A. Jorge Mora ${ }^{5}$, D. Blanco Díaz, I. Auñón ${ }^{3}$, C. Resines

${ }^{1}$ Traumatlogia Ii, Hospital 12 de Octubre, Madrid, Spain, ${ }^{2}$ Servicio De Cirugia Ortopedica Y Traumatologia Ii, Hospital 12 De Octubre, Madrid, Spain, ${ }^{3}$ Orthopaedics and Trauma Surgery, Hospital 12 De Octubre, Madrid, Spain, ${ }^{4}$ Orthopaedic and Trauma Surgery, Hospital Universitario 12 De Octubre, Madrid, Spain, ${ }^{5}$ Cot Ii, Hospital 12 De Octubre, Madrid, Spain, ${ }^{6}$ Traumatologia Ii, Hospital Universitario 12 De Octubre, Madrid, Spain

Introduction: Damage control orthopaedics (DCO) seeks to minimize surgical impact while treating all long bone and pelvic fractures in an emergency basis although it is not clear which is the effect on complications and mortality. Temporary external fixation is the preferred method for DCO in polytrauma care. Some recent papers point some doubts about the efficacy of DCO. We examine our experience in the treatment of femur fractures by DCO in a group of severe politrauma patients.

Materials and methods: Retrospective analysis of data trauma registry data. We studied the clinical outcome of a consecutive group of politrauma patients with femoral fractures treated by DCO concepts. We analyzed the clinical outcomes in terms of systemic complications and mortality measured by TRISS methodology. Inclusion criteria: Age $>15$, ISS $>22$, external fixation in first $24 \mathrm{~h}$ for femoral fractures.

Results: 52 patients met the inclusion criteria. Average age was 33 years, means ISS 32, means NISS 37; most patients suffered road traffic accidents and high falls. We performed 60 temporary external fixators in fifty two patients. Systemic complication rate was high. Four patients died. We found a reduction in mortality between probability of survival by TRISS and real mortality of $0.13(0,79$ Ps vs. 0,92 real survival).

Conclusion: External fixation for early fixation of long bone fractures in polytrauma patient is a safe and successful treatment. DCO seems to reduce mortality in severe polytrauma patients with multiple fractures.

References: Taeger G, Ruchholtz S, Waydhas C et al. Damage control orthopedics in patients with multiple injuries is effective, time saving, and safe. J Trauma. 2005;59:408-415. Pape H, Hildebrand F, Pertscht $\mathrm{S}$, et al. Changes in the management of the femoral shaft fractures in polytrauma patients: from early total care to damage control orthopedic surgery. J Trauma. 2002;53:452-462

Disclosure: No significant relationships. 


\section{8}

\section{THE EFFECT OF HOLLOW-REAMER AT MAKING INSERTION HOLE OF SHORT FEMORAL NAIL IN FEMORAL TROCHANTERIC FRACTURE}

\section{E. Shoda, K. Ouchi, S. Maruyama, S. Kitada, A. Sakurai}

Orthopaedci Surgery, Hyogo Prefectural Nishinomiya Hospital, Nishinomiya, 0918, Japan

Introduction: Short femoral nail is the most popular instrumentation for femoral trochanteric fracture. However, displacement of proximal fragment or increasing the fracture gap with insertion of the nail is pointed out. We use the Hollow-Reamer for making the insertion hole of Gamma 3 nail (G3) and PFNA-II in order to prevent these complications. In this study, we introduce the new technique and evaluate the effect of this Hollow-Reamer compared with awl at making the insertion hole of the nail.

Materials and methods: Forty four patients who had the surgery using G3 and 26 patients treated with PFNA-II were investigated. In eleven cases of G3 (male 3, female 8, average age 86.2-year-old), insertion hole of the nail was made by awl in ordinary method (Group G3-A). Otherwise, in 33cases (male 4, female 29, average age 85.8), hole was made by Hollow-Reamer (Group G3-HR). In all patients who treated with PFNA II (male 7, female 19, average age 80.4), insertion hole was made by Hollow-Reamer (Group PFNA-II). The diameter of Hollow-Reamer is 15.5 and $16.5 \mathrm{~mm}$, same as proximal diameter of these nails. In new technique for making the insertion hole of the nail, $3.0 \mathrm{~mm} \mathrm{~K}$-wire is inserted to intramedullary space from the top of the greater trochanter at first. After confirmation of the exact position of this $\mathrm{K}$-wire by image intensifier, insertion hole is made by overdrilling with Hollow-Reamer. Intraoperative complications were investigated in three groups.

Results: Intraoperative complications occurred in eight cases. Medial displacement of proximal fragments with insertion of the nail was three cases only in Group G3-A. Another one case in Group G3-A was excessive reaming of lateral wall of greater trochanter. Other two cases were displacement or rotation of proximal fragment with the insertion of lag screw in Group G3-HR. In Group PFNA-II, inferior displacement of proximal fragment was seen in one case and another one case was not able to use because the reamer hit the iliac crest.

Conclusion: This study showed that the use of Hollow-Reamer prevented displacement of proximal fragment or increasing the fracture gap at nail insertion. However, additional reaming was necessary to insert the nail in three cases of Group G3-HR. Size or design of the Hollow-Reamer should be considered.

Disclosure: No significant relationships.

\section{9}

\section{ANALYSIS OF OUTCOMES AND COMPLICATIONS IN COMBINED PELVIC AND ABDOMINAL TRAUMA}

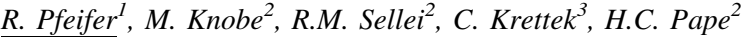

${ }^{1}$ Department of Trauma Surgery, University of Aachen Medical Center, Aachen, Germany, ${ }^{2}$ Department of Orthopaedic and Trauma Surgery, University Hospital of the Rwth Aachen, Aachen, Germany, ${ }^{3}$ Department of Trauma, Medizinische Hochschule Hannover, Hannover, Germany

Introduction: Patients with pelvic injuries are at high risk to die due to uncontrolled haemorrhage from either the pelvis or abdominal solid organs. It is still unclear whether severe pelvic injuries, concomitant abdominal injuries or both contribute to worse outcome. We, therefore, analysed the outcome and the occurrence of posttraumatic complications in severely injured patients with pelvic trauma, abdominal trauma and combined pelvic and abdominal trauma.

Materials and methods: 5,353 records of severely injured trauma patients treated between 1993 and 1999 were reviewed. The German Trauma Registry (GTR) was used as the source of data. Inclusion criteria: ISS $>16$, pelvic and/or abdominal trauma, primary admission to a trauma center, and AIS head $<3$. Mortality rate, associated complications, and treatment strategies were recorded.

Results: A total 871 patients, pelvic injuries $(n=267)$, abdominal injuries $(\mathrm{n}=343)$, and combined pelvic and abdominal trauma ( $\mathrm{n}=261)$ met the inclusion criteria. The overall mortality rate was $20.4 \%$ (13.5\% pelvic injuries; $20.4 \%$ abdominal injuries; $27.6 \%$ both injuries). The majority ( $84 \%)$ of patients with abdominal trauma underwent a laparotomy. Early definitive pelvic osteosynthesis was chosen in $48.3 \%$ of patients with pelvic injuries. Complications like ARDS (16.6\%), MOF (20.1\%) and sepsis (17.4\%) were common in all groups.

Conclusion: Patient's sustaining combined pelvic and abdominal injuries were associated with increased injury severity and higher mortality rates. Moreover, this injury pattern places these patients at an increased risk to develop posttraumatic complications. Early surgical intervention has been shown to be advantageous in all groups. Disclosure: No significant relationships.

\section{0}

\section{ANGIOGRAPHIC EMBOLIZATION AND SEVERE PELVIC FRACTURES: SINGLE CENTRE RESULTS}

\author{
W. Metsemakers ${ }^{1}$, S. Nijs ${ }^{2}$, S. Heye ${ }^{3}$, P. Vanderschot ${ }^{1}$, G. Maleux ${ }^{4}$
}

${ }^{1}$ Trauma Surgery, University Hospitals Leuven, Leuven, Belgium, ${ }^{2}$ Department Of Traumatology, University Hospitals Leuven, Leuven, Belgium, ${ }^{3}$ Interventional Radiology, University Hospitals Leuven, Leuven, Belgium, ${ }^{4}$ Interventional Radiology, University Hospitals Leuven, leuven, Belgium

Introduction: The management of hemodynamically unstable patients with severe pelvic fractures remains a challenge. Various treatment strategies have been advocated. Our study describes the management and outcome of this patient population, with emphasis on the use of angiographic embolization (AE).

Materials and methods: Between January 2002 and July 2010 we retrospectively identified patients with unstable pelvic fractures from the University of Leuven trauma registry. In this group there were 14 hemodynamically unstable patients with severe pelvic fractures who underwent angiographic embolization.

Results: The median age in the angiographic embolization-group was 59 years and the median ISS (Injury Severity Score) was 36 . Of the 14 patients, $12(86 \%)$ received initial external fixation. In total 3 (21\%) patients died during the period of hospitalization, none of them because of uncontrolled hemorrhage. Complication rate due to angiographic embolization was extremely low.

Conclusion: Angiographic embolization is a save and lives saving procedure for the hemodynamically unstable patient with pelvic ring disruption. Early identification of patients who would benefit from this procedure is of primordial importance. In our opinion primary surgical treatment remains the strategy of choice, followed by angiographic embolization for patients who do not respond to the initial therapy. Disclosure: No significant relationships. 
0171

\section{MANAGEMENT OF HAEMODYNAMICALLY UNSTABLE PELVIC FRACTURES IN FRENCH ARMY FORWARD SURGICAL TEAM: INTEREST OF THE PRE-PERITONEAL PACKING}

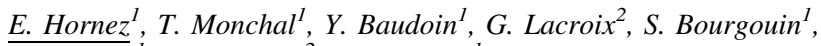

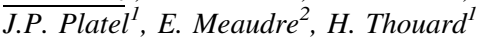

${ }^{1}$ Digestive Surgery, Sainte Anne Military Hospital, Toulon, France, ${ }^{2}$ Reanimation, Sainte Anne Military Hospital, Toulon, France

Introduction: Despite a multidisciplinary approaches in the treatment of people with haemorragic pelvic fractures, mortality from pelvic trauma remains high, ranging from 40 to $60 \%$. Exsanguinating haemorrhage has been identified as the major cause of death during the first $24 \mathrm{~h}$. The optimal treatment strategy in these cases remains controversial, with the focus on emergency pelvic angiography and external stabilisation of the pelvis.

Materials and methods: The management of these casualties on a battlefield is a challenge because of the poor medical environment and the need to evacuate the casualties to a secured zone sometime with uncontrolled delay. French Army forward surgical team are small surgical structure composed with a trauma surgeon, a digestive surgeon, an anesthetist and three nurses. They are deployed close to the front line. They are self-sufficient for $48 \mathrm{~h}$ and designed to manage ten surgical casualties per day. Blood transfusions are possible but limited. The focal assessment sonogram for trauma is commonly done. If the stabilisation of the pelvis is easily done with an external fixator, at this day embolisation is never available in any conflict in wich French army is engaged. A technique of direct retroperitoneal pelvic packing has been recently described by German trauma team with the objective to be an alternative to the embolisation. Actually, the source of bleeding is often multifactorial and may be difficult to ascertain. Angiography does nothing to address the potentially major venous bleeding that comprises more than $85 \%$ of the haemorrhage associated with unstable pelvic fracture.

Results: A review identifies three major studies done to evaluate safety and efficacy of this technique: they showed a decrease in the mean transfusion rate. Early mortality ranged from 7 to $29 \%$. The need to a therapeutic embolisation was estimated at $80 \%$ in the pilot study but decrease from 10 to $15 \%$ in the most recent studies. Also the procedure is short $(20 \mathrm{~min})$ and don't need a specific surgical material.

Conclusion: Considering all these parameters, pelvic packing as a part of a damage control protocol seems to be a potentially aid in early intrapelvic bleeding control, especially in French Army forward surgical team. The specific objectives of this procedure realized very early are: to provide a definitive hemostasis in the best situation to decrease the blood transfusion rate to provide haemodynamic stability and time for a strategic evacuation to a structure providing a capacity of embolization.

References: Tötterman A, Madsen JE, Skaga NO, Røise O. Extraperitoneal pelvic packing: a salvage procedure to control massive traumatic pelvic hemorrhage. J Trauma. 2007;62(4):843-852. Cothren CC, Osborn PM, Moore EE, Morgan SJ, Johnson JL, Smith WR. Preperitonal pelvic packing for hemodynamically unstable pelvic fractures: a paradigm shift. J Trauma. 2007;62(4):834-839 (discussion 839-842). Suzuki T, Smith WR, Moore EE. Pelvic packing or angiography: competitive or complementary? Injury. 2009;40(4):343-353. Smith WR, Moore EE, Cothren CC, Morgan SJ, Williams AE, Stahel PF, Osborn PM. Direct retroperitoneal pelvic packing versus pelvic angiography: a comparison of two management protocols for haemodynamically unstable pelvic fractures. Injury. 2009;40(1):54-60 (Epub 2008 Nov 30). Verbeek D, Sugrue M,
Balogh Z, Cass D, Civil I, Harris I, Kossmann T, Leibman S, Malka V, Pohl A, Rao S, Richardson M, Schuetz M, Ursic C, Wills V. Acute management of hemodynamically unstable pelvic trauma patients: time for a change? Multicenter review of recent practice. World $\mathrm{J}$ Surg. 2008;32(8):1874-1882.

Disclosure: No significant relationships.

\section{2}

\section{PROTOCOL-DRIVEN APPROACH OF BLEEDING ABDOMINAL AND PELVIC TRAUMA}

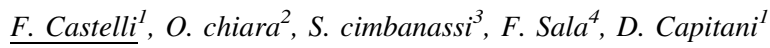

${ }^{1}$ Dea, Niguarda Ca Granda, Milan, Italy, ${ }^{2}$ Emergency, Ospedale Niguarda Ca' Granda, milano, Italy, ${ }^{3}$ Emergency, Ospedale Niguarda Ca'Granda, Milano, Italy, ${ }^{4}$ Department of Orthopedic Surgery and Traumatology, Niguarda Ca Granda Hospital, Milano, Italy

Introduction: Control on a priority basis of predominant site of hemorrhage in multitrauma patients with pelvic disruption may be critical for survival. Purpose of this study was to evaluate prospectively a work-up based on initial pelvic radiograph, abdominal ultrasound (US) and contrast CT (CESCT).

Materials and methods: In the present study 87 patients with abdominal-pelvic trauma meeting the study criteria have been selected in 16 months period. Thirthy-seven patients $(42.5 \%)$ had an unstable and 50 a stable pelvic fracture. Unstable fractures were classified APC II or III in 12 cases, LC II or III in 16, VS in 6 and a combination of previous patterns in 3 .

Results: Results Pelvic fracture pattern derived from initial screening radiograph correctly predicted in UPF patients a significant retroperitoneal hemorrhage in 32 of 37 cases, while in SPF patients correctly indicated a significant extra-pelvic source of bleeding in 41 of 50 . Therefore, sensitivity was found to be $78.04 \%$, specificity $89.13 \%$, PPV $86.48 \%$, NPV $82 \%$, and overall accuracy $83.9 \%$.

Conclusion: Conclusion In summary, this study suggest: (a) pattern of pelvic fracture, even roughly classified by a screening radiograph, is suggestive of a significant pelvic bleeding in the majority of patients who survive initial emergency room treatment; (b) early application of measures of temporary pelvic closure,(PIB o C-Clamp) when needed, should be considered a completion of the initial resuscitation protocol; (c) once excluded the need for emergency celiotomy with US or DPL, CESCT is the best diagnostic tool to choice the appropriate way, angiography or fixation, to manage bleeding pelvic injuries; (d) CESCT is the best diagnostic tool to indicate the need of abdominal angiographic embolization and to diagnose initially missed hollow viscus injuries; (e) availability of equipped CT scan and angiographic suites closed to the emergency room and of short respons.

Disclosure: No significant relationships.

\section{3}

\section{SEVERE PELVIC BLEEDING: THE ROLE OF PRIMARY INTERNAL FIXATION}

\section{E. Varga $^{l}$, B. Erdöhelyi ${ }^{2}$}

${ }^{1}$ Department of Trauma Surgery, University of Szeged, Szeged, Hungary, ${ }^{2}$ Department of Medical Imaging and Computer Graphicstics and, University of Szeged, Szeged, Hungary 
Introduction: Pelvic ring injuries form part of the spectrum of polytrauma and must be considered as a potentially lethal injury with mortality rates of $10-20 \%$. The stabilization of the unstable pelvic ring in acute resuscitation of multiply injured patients is now conventional wisdom.

Materials and methods: We aimed: (1) to design a new iliosacral (IS) screw, (2) to prove the clinical advantages of this new implant, and (3) to work out the optimal surgical strategy using this implant. Taking the demands of the data mentioned above into account, a $10 \mathrm{~mm} 2.8 \mathrm{~mm}$-cannulated iliosacral screw seemed to be optimal for the special requirements. Finite element analysis (FEA) was performed before industrial production to find out whether these screws would be strong enough to stabilize the posterior pelvic ring alone. Clinical experience led to the modification of the set of instruments, which finally yielded handy tools and implants. Increase surgical skills and experiences gained (by the surgeons and the O.R. personnel), we increased our capacity to perform more and more immediate pelvic fixations.

Results: Emergency pelvic stabilizations were performed in patients with pelvic injuries who had hemodynamic instability, despite immediate shock management during the diagnostic period. During the last 11 years, 244 patients with Tile B3 and C pelvic injuries have been stabilized with $10-\mathrm{mm}$ diameter cannulated IS screws percutaneously posteriorly. Forty-eight hemodynamically unstable patients were stabilized in the first $2 \mathrm{~h}$ with iliosacral screw fixation. The percutaneous pelvic ring stabilization with $10 \mathrm{~mm}$ cannulated screws proved strong enough in bothersome cases as well.

Conclusion: Emergency iliosacral fixation of unstable pelvic ring fractures could be performed within $2 \mathrm{~h}$, even in severely shocked patients with multiple injuries. This surgery did not lead to increased rates of transfusion, mortality. Furthermore, all these parameters showed a tendency toward benefit, compared with a staged approach. References: Agolini SF, Shah K, Jaffe J, Newcomb J, Rhodes M, Reed JF III. Arterial embolisation is a rapid and effective technique for controlling pelvic fracture hemorrhage. J Trauma. 1997;43: 395-399. Varga E, Gorczyca JT (1988) Pelvic fracture related hemorrhage. Should they be immediately, definitively fixed? Surgery of the pelvis and acetabulum. In: 4th International Consensus, Birmingham. Erdohelyi B, Varga E, Kuba A. Surgical planning tool with bio- mechanical simulation. Int J Comput Assist Radiol Surg. 2007; 2(Supp1):S262-S263.

Disclosure: No significant relationships.

\section{VISCERAL TRAUMA 6}

\section{4}

\section{THE TRAUMATIC DIAPHRAGM RUPTURE: A DIAGNOSTIC CHALLENGE?}

\author{
S. Lederer, R. Bogner, A. Auffarth, S. Karpik, G. Korn, M. Mayer, \\ H. Resch
}

Unfallchirurgie Und Sporttraumatologie, Universitätsklinik Salzburg, Salzburg, Austria

Introduction: Traumatic diaphragmatic ruptures are usually caused by blunt trauma or penetrating injuries to the thorax or the abdomen. The incidence of this injury has been reported to range from 0.8 to $5.0 \%$ in polytraumatised patients. Depending on the type of diaphragmatic rupture every method of imaging bears advantages as well as pitfalls.
Materials and methods: In a retrospective trial from 2003 to 2009 we identified 17 patients with a traumatic diaphragm rupture admitted to either the Department of Traumatology or the Department of General Surgery at the Paracelsus Medical University Salzburg. Every patient had a chest $\mathrm{x}$-ray, an abdominal sonography and a CTScan on admission.

Results: The cause of the injury was a motor vehicle accident in 11 cases, a gun shot in 4 and a stab in 2 . It was diagnosed by the CT-scan in 11 cases and was accidentally seen in 4 cases of surgery for pneumoperitoneum or an organ lesion. Fifteen of the ruptures laid on the left and 2 on the right side A direct suture or placement of a vicrylnet was performed in all patients. In 8 cases laparotomy was performed. The remaining 9 procedures started laparoscopic. A switch to a laparotomy was necessary in 5 of these 9 cases.

Conclusion: Traumatic diaphragmatic ruptures are rare but serious injuries. Even modern imaging does not always reveal this diagnosis. In blunt abdominal trauma a rupture of the diaphragm has to be thought on. Even if not suspected, during surgery for traumatic lesions of an abdominal organ, the diaphragm must carefully be inspected.

Disclosure: No significant relationships.

\section{6}

\section{BEST TIMING OF ENDOVASCULAR TREATMENT FOR BLUNT AORTIC INJURY}

\author{
$\underline{\text { Y. Kurimoto }}^{1}$, N. Kawaharada ${ }^{2}$, T. Ito $^{3}$, T. Koyanagi $^{3}$, T. Maeda $^{3}$, \\ A. Watanabe $^{3}$, T. Higami ${ }^{3}$, T. Shimizu ${ }^{4}$, I. Takahashi ${ }^{4}$, K. Tanno $^{1}$, \\ K. Mori $^{l}$, Y. Asai ${ }^{1}$ \\ ${ }^{1}$ Traumatology and Critical Care Medicine, Sapporo Medical \\ University, Sapporo, Japan, ${ }^{2}$ Sapporo Medical University, Sapporo, \\ Japan, ${ }^{3}$ Thoracic and Cariovascular Surgery, Sapporo Medical \\ University, Sapporo, Japan, ${ }^{4}$ Teine Keijinkai Hospital, Sapporo, \\ Japan
}

Introduction: Although the timing of open surgery for blunt aortic injury (BAI) had been controversial because of multiple injuries, thoracic endovascular aortic repair (TEVAR) overcame this problem and made it simpler. We evaluate about the timing of TEVAR for BAI.

Materials and methods: TEVAR has been performed using surgeon's hand-made fenestrated or non-fenestrated stent graft except for recent 2 cases using TAG or Talent device. Twenty-five, excluding 5 patients who underwent TEVAR later than 2 weeks after BAI, are the subjects of this study. Early and mid-term clinical results were compared between the immediate group (within $48 \mathrm{~h}$ after BAI) and the urgent group.

Results: There was no difference between the immediate group $(\mathrm{n}=18)$ and the urgent group $(\mathrm{n}=7)$ in mean age $(53.2$ and 66.1$)$, gender, injury sites, mean injury severity score (34.9 and 38.6), mean revised trauma score (6.63 and 6.97) and mean probability of survival $(67.2$ and $62.8 \%)$. Early mortality rates of the immediate and the urgent groups were 11.1 and $0 \%(\mathrm{p}=0.358)$, and survival rates at 3 years after BAI were 84 and $100 \%(p=0.466)$, respectively. All causes of death were not aorta-related. Although the length of hospital stay was not different, the length of ICU stay was shorter in the immediate group (7.0 and 15.9 days, $p=0.001)$. The incidence of ventilator-associate pneumonia (VAP) was lower in the immediate group $(12.5$ and $57.1 \%, \mathrm{p}=0.025)$. 
Conclusion: Although the timing of TEVAR for BAI had no influence on early and late mortalities, TEVAR for BAI had better be performed as early as possible to reduce the length of ICU stay and the incidence of VAP.

References: Kurimoto Y, et al. Initial experiences in management of blunt aortic injury taking associated brain injury into consideration. Circ J. 2006;70(2):198-201. Kurimoto Y, et al. Fenestrated stent-graft facilitates emergency endovascular therapy for blunt aortic injury. J Trauma 2009;66(4):974-978.

Disclosure: No significant relationships.

\section{7}

\section{PREDICTIVE FACTORS OF MORBIDITY AND MORTALITY IN GRADE IV AND V LIVER TRAUMA UNDERGOING PERIHEPATIC PACKING: SINGLE INSTITUTION 14 YEARS EXPERIENCE AT EUROPEAN TRAUMA CENTER}

\author{
S. Di Saverio ${ }^{1}$, E. Giorgini ${ }^{2}$, C. Coniglio ${ }^{3}$, S. Villani ${ }^{4}$, A. Giugni $^{3}$,

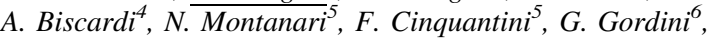 \\ G. Tugnoli ${ }^{2}$
}

${ }^{1}$ Surgery, Emergency and Trauma Surgery Unit, Maggiore Hospital Trauma Center, Bologna, Italy, ${ }^{2}$ Emergency and Trauma Surgery Unit (head Dr. G. Tugnoli), Maggiore Hospital Trauma Center, Bologna Local Health District, Bologna, Italy, ${ }^{3}$ Trauma Icu, Department Of Emergency, Maggiore Hospital Trauma Center, Bologna Local Health District, Bologna, Italy, ${ }^{4}$ Emergency and Trauma Surgery Unit, Maggiore Hospital Trauma Center, Bologna Local Health District, Bologna, Italy, ${ }^{5}$ Interventional Radiology Unit, Department of Radiology, Maggiore Hospital Trauma Center, Bologna Local Health District, Bologna, Italy, ${ }^{6}$ Trauma Icu, Department of Emergency (head Dr. G. Gordini), Maggiore Hospital Trauma Center, Bologna Local Health District, Bologna, Italy

Introduction: Major liver trauma in polytrauma patients leads to significant morbidity and mortality.

Materials and methods: 293 patients were admitted in TICU for liver trauma (1996-2009), 30\% had grade IV-V AAST injury. A retrospective review of 39 patients (grade IV-V) treated with perihepatic packing was carried out. Morbidity, mortality, univariate, multivariate analysis of prognostic factors were calculated.

Results: 34 patients were hemodynamically unstable; mean BE and RTS were -9.66 and 5.9. 10 patients undergone additional angioembolization. Overall mortality was $51.3 \%$, liverrelated mortality $23.1 \%$, after respectively 84 and $10 \mathrm{~h}$. Overall and liver-relatedmorbidity rates were 89.7 and $28.2 \%$, intra-abdominal septic collections incidence $23.1 \%$. GCS, systolic BP (SBP), respiratory rate (RR), $\mathrm{pH}$, RTS and TRISS, PRBC transfused, amount of fluids infused in ER and time duration before ICU admission are significantly associated with mortality; after multivariate analysis only RTS remained significant. Shorter the time to ICU admission lower the morbidity. Lower GCS and RR, better pH and BE, worse RTS or TRISS, angioembolization, lower amount of PRBC, higher fluids load volume, early OR and ICU admission are associated with significant decrease of 24-h mortality. GCS, RTS and pH remained significant after multivariate analysis. SBP and BE are significant predictors of liver hemorrhage-related mortality. PRBC transfused in the first $24 \mathrm{~h}$ and high $\mathrm{BE}$ value are independent predictors of re-bleeding and need for re-packing.

Conclusion: RTS, hemodynamic instability and indicators of tissue perfusion ( $\mathrm{pH}, \mathrm{BE}$ ) confirmed their prognostic predictive value. Early, rapid, effective damage control and early ICU admission are associated with less complications and shorter ICU stay.

Disclosure: No significant relationships.
0178

\section{GENETIC VARIATION IN INNATE IMMUNITY CONTRIBUTES TO INFECTIOUS COMPLICATIONS IN POLYTRAUMATIZED PATIENTS}

M.W.G.A. Bronkhorst, E.M.M. Van Lieshout, P. Patka

Surgery-Traumatology, Erasmus MC Rotterdam, Rotterdam, The Netherlands

Introduction: Polytrauma patients are at serious risk for developing infectious complications during hospital stay which may add significantly to mortality. Single Nucleotide Polymorphisms (SNPs) in innate immunity genes may influence the risk for developing complications in polytraumatized patients. The MBL2, FCN2 and MASP2 genes are key genes in activation of the lectin pathway and subject to genetic variation. The aim of this study was to determine if SNPs in these genes predispose severely injured trauma patients to infectious complications.

Materials and methods: In a prospective cohort of polytraumatized patients with Injury Severity Scores of 16 or higher admitted to a Level I Trauma Center genomic DNA was isolated from venous blood samples. After PCR the genotype of MBL2, FCN2 and MASP2 was determined using High Resolution Melting Analysis (HRMA) on a LightScanner.

Results: Genotyping the MBL2, FCN2 and MASP2 genes was performed for 147 patients. Median age was 44 years and $82 \%$ were male. Median ISS was 25 . For MBL2 exon 1, 33\% carried the heterozygous $\mathrm{A} / 0$ genotype and $5 \%$ were homozygous for $0 / 0$. The mutant genotype seems to be more susceptible to develop positive wound cultures $(p=0.048)$. For FCN2 T236 M, 42\% carried the heterozygous T/M genotype and $8 \%$ were homozygous for variant M/M. No statistically significant association with a positive culture was found. For the FCN2 A258S, 77\% carried the A/A wildtype and $23 \%$ were heterozygous $\mathrm{A} / \mathrm{S}$ carriers. The total number of positive cultures was $48 \%$ for wildtype A/A and $62 \%$ for variant A/S (NS). Positive wound cultures were $12 \%$ in A/A wildtype and $29 \%$ in A/S variants $(\mathrm{p}=0.028)$.

Conclusion: Variant genotypes in key genes of the lectin pathway of complement activation predispose severely injured trauma patients to developing positive wound cultures.

Disclosure: No significant relationships.

\section{9}

\section{BLUNT RENAL ARTERY INJURY}

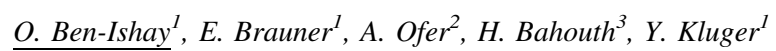

${ }^{1}$ Department of General Surgery, Rambam Health Care Campus, Haifa, Israel, ${ }^{2}$ Radiology Institute, Rambam Health Care Campus, Haifa, Israel, ${ }^{3}$ Acute Care Surgery, Rambam Health Care Campus, Haifa, Israel

Introduction: Blunt renal artery injury (BRAI) is rare. Treatment of this injury is dependent on overall trauma complexity. The objective is to summarize our experience with BRAI and to suggest algorithm for this injury evaluation and management.

Materials and methods: Charts of patients diagnosed with BRAI from January 2002 through October 2010 were reviewed retrospectively. Data collected included age, gender, mechanism and associated injuries, injury side, ISS, time to angiography, treatment, ICU (LOS) and hospital (LHS) stay, complications, renal function surveillance and artery patency. 
Results: 26,136 patients were admitted with blunt injury during the study period. $9(0.034 \%)$ were diagnosed with BRAI, 8 by CT and 1 at surgery. Mean age was $30.70 \%$ were males. $78 \%$ had left kidney injury. Mean ISS was 25. Associated injuries were: chest $78 \%$, orthopedic 56\%, head 44\%. 67\% were involved in MVA. Mean LOS in the ICU was 11.8 and LHS 17.3 days. Angiography was performed in $78 \%(n-6)$. Conservative management was elected in $22 \%(n-2)$ and nephrectomy in $11 \%(\mathrm{n}-1)$. Average time from injury to angiography was $4 \mathrm{~h}$ and angiography resulted in embolization in $50 \%(\mathrm{n}-3)$ and stenting in $33 \%(\mathrm{n}-2)$. Failure of angiography resulted in loss of kidney function in one patient. Rate of revascularization was $67 \%$. Overall Loss of kidney function was recorded in $22 \%$ (n-2). Average follow-up was 42 months. Dimercaptosuccinic acid (DMSA) scan $(44 \%)$ or US-Doppler $(56 \%)$ revealed normal flow and kidney function in $78 \%$.

Conclusion: BRAI are rare and associated with severe injuries (ISS > 16). CT scan is the main diagnostic tool. Angiography with endovascular treatment is the first line of therapy and should be attempted in the hemodynamic stable patient.

Disclosure: No significant relationships.

\section{0}

\section{THE MANAGEMENT OF A PNEUMOPERICARDIUM AFTER PENETRATING CHEST TRAUMA: A PROSPECTIVE ANALYSIS}

\section{A. Nicol, P. Navsaria}

Surgery: Trauma, Groote Schuur Hospital and University of Cape Town, Cape Town, South Africa

Introduction: A pneumopericardium presenting after penetrating chest trauma is a rare event in the emergency room and the surgical management of this clinical sign has not been well documented in the current literature. The current recommendations range from mandatory emergency surgery to conservative management if the ultrasound is normal. The aim of this study was to suggest a protocol for the management of a pneumopericardium after penetrating chest trauma. Materials and methods: A prospective analysis of patients presenting to Groote Schuur Hospital Trauma Centre with a pneumopericardium on chest X-ray after penetrating trauma between October 2001 till February 2009 with respect to their presentation and surgical management.

Results: There were 27 patients in total with a mean age of 25 years (range 17-36). The mechanism of injury was a stab wound to the chest in 26 patients and a single patient had sustained multiple low velocity gunshot wounds. The mean revised trauma score was 7.566 (range 4.094-7.841). Six patients (22\%) were unstable and required emergency surgery. One of these patients presenting with a tension pneumopericardium. Twenty-one patients were initially stable but two of these $(10 \%)$ later developed a tension pneumopericardium within 24-h and were taken to theatre. The remaining 19 patients were managed with a subxiphoid pericardial window (SPW) at between 24 and $48 \mathrm{~h}$ post admission. Ten of these 19 patients $(52 \%)$ were positive for blood. Only 4 of the 19 underwent a sternotomy and only two of these had cardiac injuries that had sealed. There were no deaths in this series.

Conclusion: Patients with a penetrating chest injury with a pneumopericardium that are unstable require emergency surgery. A delayed tension pneumopericardium developed in $10 \%$ of patients that were initially stable and it is our recommendation that all stable patients with a pneumopericardium after penetrating chest trauma should undergo a SPW. A sternotomy is not required in stable patients unless there is evidence of active bleeding at SPW.
References: Demetriades D, Van der Veen B. Penetrating injuries of the heart: experience over two years in South Africa. J Trauma. 1983;23:1034-41. Demetriades D. Cardiac wounds: experience with 70 patients. Ann Surg. 1986;203:315-317. Spotnitz A, Kaufman J. Tension pneumopericardium following penetrating chest injury. J Trauma 1987;27:806-8.

Disclosure: No significant relationships.

\section{1}

\section{VASCULAR SURGERY FOR PENETRATING NECK TRAUMA}

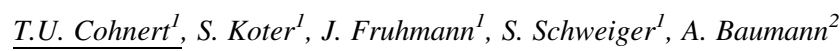

${ }^{1}$ Graz Medical University, Department of Vascular Surgery, Graz, Austria, ${ }^{2}$ Department of Vascular Surgery, Graz Medical University, Graz, Austria

Introduction: Operations for treatment of traumatic lesions are rare in vascular surgical practice with $0.3-4 \%$. The current mortality for civilians with penetrating neck injuries ranges from 3 to $6 \%$. Aim of this study was to analyze the results of vascular surgery in emergency procedures for penetrating neck trauma.

Materials and methods: Between 1.1.1998 and 31.10.2010 a total of 80 patients (pts) underwent vascular surgical operations for penetrating trauma. Prospectively collected data were analyzed retrospectively.

Results: In 17 pts $(17 / 80=31.3 \%)$ surgery was necessary for vascular lesions due to penetrating trauma of the neck (14 male, 3 female, mean age $45.8+19.6$ years). 2 patient pts died 0 and 7 days postoperatively (mortality $2 / 17=11.8 \%$ ). In 4 pts the lesion was self inflicted, in 3 pts a second person had injured the patient intentionally. There were 8 arterial and 9 venous lesions. Site of lesion: common carotid artery in 2, internal carotid artery in 2, external carotid artery in 2 and thyroid artery in 2 pts. Reconstruction was performed by venous graft (1), venous patch plasty (1) and direct suture (6 pts). Internal jugular vein injury was present in 6 and external jugular in 3 pts. In the 4 attempted suicides, patients had lacerated the internal jugular vein and 1 patient the internal carotid artery.

Conclusion: Penetrating neck trauma is a potentially lethal injury. Emergency surgery by an experienced vascular surgeon can help to determine the extent of trauma and its treatment. In trauma to the carotid arteries complete intraoperative exposure with external and internal inspection of the vessel including the intimal layer is recommended.

Disclosure: No significant relationships.

\section{VISCERAL TRAUMA 7 \& EDUCATION 2}

\section{4}

\section{THE NEED FOR MANDATORY TRAINING IN ENDOVASCULAR HAEMORRHAGE CONTROL FOR ALL SURGEONS}

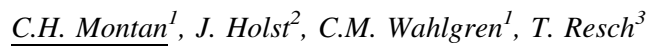

${ }^{1}$ Vascular Surgery, Karolinska Intitute, Stockholm, Sweden,

${ }^{2}$ Vascular Centre Malmo, Lund University, Malmö, Sweden,

${ }^{3}$ Vascular Centre Malmo, Lund University, Malmo, Sweden 
Introduction: Some traumatic or surgical bleedings are very difficult to control by open surgery alone. Endovascular approach is an accepted surgical technique and offers a complement to open surgery. Still many surgeons are not aware of its simplicity and potentials in treating massive haemorrhage. It is therefore important to increase endovascular awareness and training among general, emergency and trauma surgeons.

Materials and methods: Endovascular techniques of haemorrhage control include either intraluminal temporary balloon occlusion of the bleeding or proximal and/or distal vessel, or exclusion of the bleeding site by a covered stent. Temporary occlusion facilitates repair of the injury with minimal blood loss. This technique is simple and fast and requires either an open or percutaneous approach to the vessel. A standard $\mathrm{C}$-arm for X-ray guidance is often sufficient. There is no mandatory need for using contrast as massively bleeding patients should be surgically explored.

By percutaneous femoral artery or vein approach, the aorta or the vena cava and their main branches can easily be reached and controlled. By open approach almost all vessels can be reached and occluded by this technique.

Covered stents carries risks of complications and requires extensive training and should be kept in the hands of vascular specialists.

Results: Numerous reports describe successful outcome in extremely difficult cases managed partly by endovascular techniques. A few emergency surgery courses for general surgeons in Sweden are teaching simple endovascular techniques as fundamental skills.

Conclusion: Surgeons have had a long time to adopt the endovascular techniques for temporary bleeding control, but many have failed to do so. This might have led to the loss of potentially salvable lives. The use of simple endovascular techniques for the control of massive haemorrhage should be taught as fundamental surgical skills to all surgeons involved in the treatment of patients with lethal bleedings.

Disclosure: No significant relationships.

\section{5}

\section{A SINGLE TRAINING SESSION WITH FIRST TIME PELVIC C-CLAMP USERS. WHAT TO EXPECT?}

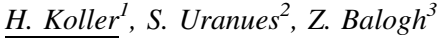

${ }^{1}$ Department of Surgery, Medical University of Graz, Graz, Austria, ${ }^{2}$ Department of Surgery, Medical University Graz, Graz, Austria,

${ }^{3}$ Trauma Department, John Hunter Hospital, Newcastle, Australia

Introduction: Unstable pelvic fractures with associated haemodynamic instability are major contributors of mortality and morbidity after blunt trauma. The Pelvic C-Clamp is favored by many authors for the initial stabilization of the pelvic ring. The application of this device is taught on courses, still it is seldom used. The safety and efficiency of C-clamp application by trained first time users is unknown. We hypothesised that the pelvic C-clamp cannot be safely and efficiently positioned on a training model after one training session.

Materials and methods: The Pelvic TrainerTM was used for the accuracy and time of pelvic C-clamp application. The standard posterior pin placement was taught and practiced with a study population of 27 participants with different levels of experience. A theoretical and a practical session were held before the actual study (11.2 Â \pm 4.4 days).

Results: 20 (70.4\%) of all participants were able to place both pins inside the safe area, while $81.5 \%$ of all pins were positioned safe. The average distance to the optimal target was $9.15 \hat{\mathrm{A}} \pm 7.86 \mathrm{~cm}$. The time needed for assembly was $99.7 \hat{\mathrm{A}} \pm 39.7 \mathrm{~s}$, for placement it averaged at $133.9 \hat{\mathrm{A}} \pm 74 \mathrm{~s}$.

Conclusion: After one training session the pelvic C-clamp can be rapidly applied on a training manikin with over $80 \%$ safely positioned pins. The risks associated with the $20 \%$ suboptimal pin positioning even in controlled environment within 2 weeks of training require careful assessment in view of the potential benefits of the C-clamp.

References: 1. Pohlemann T, Braune C, Gansslen A, Hufner T, Partenheimer A. Pelvic emergency clamps: anatomic landmarks for a safe primary application. J Orthop Trauma. 2004;18(2): 102-5. 2. Burkhardt M, Culemann U, Seekamp A, Pohlemann T. Strategies for surgical treatment of multiple trauma including pelvic fracture. Review of the literature. Unfallchirurg. 108(10):812, 4-20. 3. Ertel W, Keel M, Eid K, Platz A, Trentz O. Control of severe hemorrhage using C-clamp and pelvic packing in multiply injured patients with pelvic ring disruption. J Orthop Trauma. 2001;15(7):468-74. 4. Quintero AJ, Tarkin IS, Pape HC. Case report: the prone reduction of a sacroiliac disruption with a pelvic C-clamp. Clin Orthop Relat Res. 2009;467(4):1103-6. 5. Richard MJ, Tornetta P, 3rd. Emergent management of APC-2 pelvic ring injuries with an anteriorly placed C-clamp. J Orthop Trauma. 2009;23(5):322-6. 6. Sadri H, Nguyen-Tang T, Stern R, Hoffmeyer $\mathrm{P}$, Peter R. Control of severe hemorrhage using C-clamp and arterial embolization in hemodynamically unstable patients with pelvic ring disruption. Arch Orthop Trauma Surg. 2005;125(7):443-7. 7. Reynolds JH, Attum B, Acland RJ, Giannoudis P, Roberts CS. Anterior versus posterior pin placement of pelvic C-clamp in relationship to anatomical structures: a cadaver study. Injury. 2008;39(8): 865-8. 8. Archdeacon MT, Hiratzka J. The trochanteric C-clamp for provisional pelvic stability. J Orthop Trauma. 2006;20(1): 47-51. 9. Pohlemann T, Culemann U, Tosounidis G, Kristen A. Application of the pelvic C-clamp. Unfallchirurg. 2004;107(12): 1185-91.

Disclosure: No significant relationships.

\section{6}

\section{APPLICATION OF THE TERTIARY SURVEY IN THE INITIAL MANAGEMENT OF TRAUMA PATIENTS}

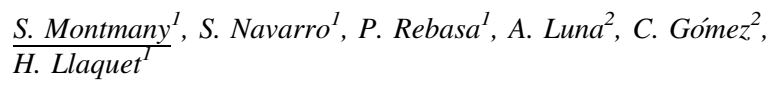

${ }^{1}$ General Surgery, Corporació Sanitària Parc Taulí, Sabadell, Spain, ${ }^{2}$ General Surgery, Corporació Sanitària Parc Taulí, Sabadell, Spain

Introduction: Despite the detailed and methodical treatment defined by the primary and secondary surveys of The Advanced Trauma Life Support (ATLS), some injuries go unnoticed. The application of the Tertiary Survey can reduce the incidence of missed injuries and the morbi-mortality of trauma patients.

Materials and methods: Prospective study including trauma patients older than 16-year-old admitted at critical area and excluding patients who died within the first $24 \mathrm{~h}$, to which tertiary survey couldn't be applied. We compared patients with tertiary survey with an historical group registered prospectively in the same circumstances without this procedure. We registered the incidence of missed injuries and clinically significant missed injuries. We analyzed the main mistakes related with missed injuries and their inevitable risks. We studied the mortality of both groups and their complications.

Results: We compared 119 trauma patients with the tertiary survey from March 2009 to April 2010, with 117 trauma patients without it from March 2006 to January 2007. Both groups were homogeneous. When the tertiary survey was applied the incidence of missed injuries 
varied from 40.17 to $15.13 \%$ and the incidence of clinically significant missed injuries varied from 17.09 to $3.36 \%$. The main missed injuries (i.e. fractures) were reduced with the tertiary survey application and column and craneocerebral missed injuries disappeared. The rate of mortality decreased from 10.25 to $4.25 \%$, disappearing deaths from multiorganic failure. The rate of complications was also reduced from 52.99 to $43.7 \%$. With the application of tertiary survey radiological error decreased and communication and surgical errors were completely eliminated. The main risk factors detected were blood pressure, the number of injuries and, as the more relevant factor demonstrated through logistical regression, the application of the tertiary survey.

Conclusion: The application of the tertiary survey would have to be mandatory in the initial management of trauma patients.

References: J Trauma. 1998;44(6). J Trauma 2003;54(1). J Trauma 2004;57(1). J Trauma. 1990;30:666-670. J Trauma Nursing. 2006;13(3):89-95.

Disclosure: No significant relationships.

\section{7}

\section{IMPROVING CLINICAL DOCUMENTATION: HOW MINIMAL INTERVENTION CAN LEAD TO AN IMPROVEMENT IN PERFORMANCE}

\section{Urquhart ${ }^{1}, \underline{\text { A. Vasireddy }}{ }^{2}$, T. Gorst $^{I}$, L. James $^{I}$}

${ }^{1}$ Orthopaedics, University Hospital Lewisham, London, UK, ${ }^{2}$ Dept of Orthopaedics, University Hospital Lewisham, London, UK

Introduction: Clinical documentation is critical to good medical practice. It also plays an important role in medico-legal issues, audit and handover. The importance of accurate documentation is emphasised by all Medical Associations. The 'CRABEL' scoring system ${ }^{1}$ provides a reproducible framework for evaluating the quality of clinical documentation. The score is a percentage with $100 \%$ being the ideal.

Materials and methods: For each of the eight surgical teams within our department, two sets of case notes from elective surgery patients and two from trauma surgery patients were randomly selected and evaluated using the CRABEL scoring system in August 2010. The results of this study were then presented to all members of staff during a departmental clinical governance day. Areas for improvement and areas of excellence were described and recommendations made for improving documentation. After a period of 2 months, the study was repeated with the same protocol and new case-notes were assessed.

Results: During the first part of the study, the average CRABEL score was $80.9 \%$ (range $72-95 \%$ ) with regards to the quality of trauma patient case-note documentation. With regards to elective case-note documentation, the average CRABEL score was $86.2 \%$ (range $82-92 \%)$. After the implementation of recommendations, the average CRABEL scores were $87.6 \%$ (range $78-97 \%$ ) and $87.5 \%$ (range $81-91 \%$ ) for trauma case-note and elective case-note documentation respectively. The overall improvement in CRABEL score was from 83.5 to $87.5 \%$.

Conclusion: In our study, the initial CRABEL scores are higher than the values reported in the literature indicating an above average standard. By increasing awareness of documentation standards amongst staff, our results show an improvement in performance. To further improve the CRABEL score, integrated care pathway proformas have been considered but these have their own limitations.

Reference: 1. Crawford JR et al. CRABEL Score-a method for auditing medical records. Ann R Coll Surg Engl. 2001;83:65-68.

Disclosure: No significant relationships.

\section{8}

PHTLS: Positive Effects On Competence In Trauma After Team Training

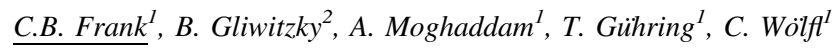

${ }^{1}$ Trauma and Orthopedic Surgery, BG Trauma Center Ludwigshafen, Ludwigshafen, Germany, ${ }^{2}$ PHTLS Germany, Aachen, Germany

Introduction: To evaluate the purpose and acceptance of PHTLS (Pre-Hospital Trauma Life Support) courses, using participant surveys.

Materials and methods: PHTLS course participants were surveyed with written questionnaires at the start and end of the course. Participant profession, satisfaction with their professional training and continuing education, and opinions about the course were requested, along with questions aimed at obtaining a personal estimate of each participant's knowledge and competence in trauma care.

Results: Over 100 participants were surveyed. Half were physicians (residents, interns, and senior physicians) and half were emergency medical technicians and paramedics. All professions identified deficits in their continuing education programs, deficits which often only become evident on scene at a trauma. Theoretical knowledge was rated positively on the surveys, however, the absence of a practical knowledge exchange was rated most negatively. After the course, all participants reported feeling better prepared to deal with prehospital trauma. The general course design was given positive ratings, and even skeptics were convinced in the end. The course was able to compensate for deficits in the participants ${ }^{\mathrm{TM}}$ previously received formal training. $88 \%$ of the participants are unsatisfied with the continuing education courses offered to them, and $95 \%$ desire the PHTLS course to be implemented into their formal training to cover the deficits.

Conclusion: PHTLS course participants can compensate for formal education deficits, and through this course, competence in pre-hospital trauma can be achieved. PHTLS courses should be integrated into all continuing education courses for all medical professionals.

References: 1. Rubiano AM, Sanchez AI, Guyette F, Puyana JC. Trauma care training for National Police Nurses in Columbia. Prehosp Emerg Care. 2010;14(1):124-30. 2. Esteri RM Jr, Henzi DL, Cohn SM. Senior medical student "Boot Camp": can result in increased self-confidence before starting surgery internships. Curr Surg. 2006;63(4):264-8. 3. Brunt LM, Halpin VJ, Klingensmith ME, Tiemann D, Matthews BD, Spitler JA, Pierce RA. Accelerated skills preparation and assesment for senior medical students entering surgical internship. J Am Coll Surg. 2008;206(5):897-904.

Disclosure: No significant relationships.

\section{9}

\section{THE TEAM COURSE: PLANNING, DEVELOPMENT AND RESULTS AFTER 59 COURSES SINCE 1999 IN THE CENTRAL REGION OF PORTUGAL}

\section{C.A. Mesquita, A.L. Trauma Emergency Surgery}

Surgery Iii, Coimbra University Hospital, Coimbra, Portugal

Introduction: It is clear, over recent years, a reinforced interest around emergency training in general and trauma in particular. In this context was the creation in 2002, by the Portuguese Medical Association (Ordem dos Médicos), of the College of Competence on Emergency Medicine. As to the Portuguese Society of Surgery (SPC), 
naturally interested in trauma, that brought to Portugal, in 1999, the ATLS (Advanced Trauma Life Support) course of the American College of Surgeons (ACS). Since then, under its aegis, about 150 courses have been organized in nine diferent centres around the country for 2,400 doctors of different specialties. The TEAM (Trauma Evaluation and Management, ACS) course is a scaled down version of ATLS for students.

Materials and methods: In its original form the TEAM course is a scaled down version of ATLS for students. It consists in a theoretical session supported by a manual and a video. In Portugal the Center Region pioneered in supporting this initiative, having TEAM accompanying the regional start of the ATLS in 1999-2000. In subsequent years, with the new format proposed by the ATLS Commission of SPC, it began also in the South and the North Regions. In addition to the lecture and video session, there are also skill stations (airway/ventilation, shock/imobilisation and others). Teaching is done by ATLS instructors, supplemented by colleagues that have done the ATLS course. With this new model, in the central region of Portugal-Coimbra, Covilhã and Viseu (with Piaget University of Luanda, Angola) - 59 TEAM courses have been frequented by more than 1,400 students.

Results: This pedagogical experience presented based on evaluations and feedback from participants collected anonymously. More specifically, applied for an overall assessment and an assessment of the various sessions, on a scale of $0-3$ (bad, reasonable, good and very good), as well as additional comments. Results: lecture2.71/video-1.65/airway-2.91/shock-2.75/imobilisation-2.81/case presentations - 2.85/global assessment-2.89. Critics: lack of time $(51 \%)$, educational in English (22\%), poor quality of the video (15\%), insufficient practical material $(07 \%)$, lack of pictures $(02 \%)$.

Conclusion: Results are extremely rewarding for organizers and teachers. It has be taken into account the weak classification of the video, the criticism to the lack of time and the need for portuguese language editions.

References: 1. ATLS Student Course Manual, 8th edition. Chicago 2008. 2. TEAM Student Study Guide, 2nd edn. Chicago 2005.

Disclosure: No significant relationships.

\section{0}

\section{TRAUMA REGISTRIES: THE FIRST STEP IN THE "BUILDING BRIDGES" PROCESS?}

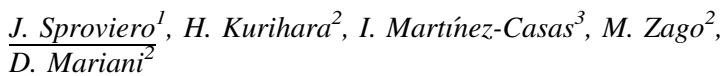

\author{
${ }^{1}$ Emergency, Hospital Santojanni, Olivos, Argentina, ${ }^{2}$ General and \\ Emergency Surgery, Istituto Clinico Città Studi, Milano, Italy, \\ ${ }^{3}$ Servicio De Cirugía General Y Digestiva, Hospital del Mar, \\ Barcelona, Spain
}

Introduction: Penetrating trauma (PT) is associated with high morbidity and mortality rates, but its hospitalization frequency, differs all over the world. Our aims were to perform a trauma registry (TR) for stab wounds patients (SWP) and to analyze its uses as a scientific society data banking.

Materials and methods: Information regarding SWP were agreed between three hospitals to perform a digital TR. Patient's hospitalization was considered a main criteria for registration. Among this population, the following clinical features were retrospectively analyzed: demographics, scoring, organ injuries, treatment and outcome. Results: Between 01/06 and 31/10/2010, 144 trauma patients were hospitalized in all facilities, 43/144 presented PT (29.8\%) and 24/43 because of SW (55.8\%), which represent our study group. Men were affected in 23/24 cases and the mean age was 28.6-year-old. Mean TSR and ISS was 11.2 and 12.4 respectively. Of the 38 organ injuries observed, hemopneumothorax was diagnosed in 7/38 cases, followed by wounds in small bowel, heart and liver. Surgery was performed in 21 patients and mortality was $2 / 24$.

Conclusion: In our series, SW represented $55.8 \%$ among the PT population and was registered in young men. Trauma scores showed lower severity values and correlated well with the mortality. Accepting trauma as a public health problem in the world, sharing information related to it, should be considered crucial between trauma centers. Behind distances, languages and infrastructure differences, TRs are proper tools for educational, organizational and management proposals and should be supported by those Scientific Societies with interest in trauma management.

Disclosure: No significant relationships.

\section{MINIMALLY INVASIVE OSTHEOSYINTHESIS}

\section{1}

\section{TRAUMART: A COMPUTER BASED PLANNING AND EDUCATIONAL SYSTEM FOR ORTHOPEDIC-TRAUMA SURGERY}

\author{
E. Varga $^{1}$, B. Erdöhelyi ${ }^{2}$, E. Varga jr. ${ }^{2}$
}

${ }^{1}$ Department of Trauma Surgery, University of Szeged, Szeged, Hungary, ${ }^{2}$ Humnan Morphology Services, AO Research Institute, Davos, Switzerland

Introduction: Highly complex fractures require surgical interventions which mandate specific experience and proficiency. The preparation of a mechanical model [using a Finite Element Analysis (FEA)] before surgery is feasible to promote optimal surgical solution. A computerized system (TraumArt) is presented to facilitate the process above.

Materials and methods: The system builds up from different modules with special tasks. The first module segments the bone from the gray scale CT scans. Then the $3 \mathrm{D}$ structure is constructed from the segmented volume model. A mesh simplification algorithm is developed to eliminate the complexity of the surface. A surgical planner is created in the fourth module, where distinct procedures can be tested. The 3D editing functions like slicing, drilling and implant insertion are visualized. The surgeon applies forces to the model and exports the data to the FEA system. Multicenter evaluations of the TraumArt system were performed. MedSys-JMed systems were applied to evaluate the program.

Results: The implemented system is well functioning. The average time to create geometric and mechanical models is approximately 5 min including the user interactions. FEA takes 6 min (pelvis 3D volume study, $2 \mathrm{GHz}$ PC, with $1.5 \mathrm{~GB}$ RAM). The system international evaluation will be discussed.

Conclusion: TraumArt could avoid several complications, while more scrupulous and prompt assessment capabilities could be provided for surgeons. This method offers new possibilities that complement current visual analytic methods and gives great perspective in the postgraduate education, preoperative planning and implant design.

References: Gunilla Borgefors. Distance transformations in arbitrary dimensions. Computer Vision, Graphics, and Image Processing. 1984;27(3):321-345.Jason Brownlee. Oat: the optimization algorithm toolkit. Technical Report 20071220A, Complex Intelligent Systems Laboratory (CIS), Centre for Information Technology Research (CITR), Faculty of Information and Communication Techno- logies 
(ICT), Swinburne University of Technology, December 2007.Balazs ErdA'helyi, Endre Varga, and Attila Kuba. Surgical planning tool with bio- mechanical simulation. In: Proceedings of the International Conference on Computer Assisted Radiology and Surgery (CARS), vol. 2 Suppl. 1 of International Journal of Computer Assisted Radiology and Surgery, p. S262-263, 2007.Michael Garland and Paul S. Heckbert. Surface simplification using quadric error metrics. In SIGGRAPH ${ }^{\mathrm{TM}_{9}}$ 97: Proceedings of the 24 th annual conference on Computer graphics and interactive techniques, p. 209-216, New York, NY, USA, 1997.ACM Press/Addison-Wesley Publishing Co. William E. Lorensen and Harvey E. Cline. Marching cubes: a high resolution 3D surface construction algorithm. SIGGRAPH Comput Graph. 1987;21(4):163-169.

Disclosure: No significant relationships.

\section{2}

\section{TROCHANTERIC FRACTURES: A MINIMALLY INVASIVE TECHNIQUE USING DYNAMIC HIP SCREW}

T. Aguiar, P. Amaral, L. Pinheiro, L. Tavares, J. Raposo, R. Soares, A. Rebelo

Orthopaedic, Hospital Divino Espirito Santo, Ponta Delgada, Portugal

Introduction: Trochanteric fractures are the most common fractures of the proximal femur. Fixation of these fractures by using dynamic hip screw (DHS) is well accepted as a treatment method for stable type of fracture (31-A1 e 31 A2.1). Recent studies have shown the benefit of minimal invasive plate osteosynthesis (MIPO). Objective: Evaluate MIPO in the treatment of stable trochanteric fractures.

Materials and methods: Retrospective study conducted between January 2004 and December 2009 in 172 patients with a stable fracture, subjected to fixation with DHS. Epidemiological data of patients were collected: age, gender, comorbidities, mean length of skin incision, time of surgery, blood work changes, and postoperative complications. Imaging evaluation was performed. We collected data regarding fracture classification, fracture reduction, tip-apex-distance (TAD) and screw position. Statistical treatment performed with SPSS v17.0. Results: In our Hospital were operated 31 patients using MIPO. Mean age 78.6 years, $87.1 \%$ female. $100 \%$ of comorbidities. Mean length of skin incision $3.7 \mathrm{~cm} \mathrm{(3-4.5).} \mathrm{Surgical} \mathrm{time} \mathrm{averaged} 41 \mathrm{~min}(35-68)$. Postoperative hemoglobin mean decrease $0.5 \mathrm{~g} / \mathrm{dl}(0.2-0.9)$. Complications: 1 implant failure. Imaging Evaluation: 76.2\% 31-A1 and 23.8\% 31-A2.1. Compared with the group in which the conventional technique was used (control group) there were no statistically significant differences on the reduction of the fracture, TAD and screw position.

Conclusion: This technique is simple and less invasive. It can be performed effectively with very minimal soft tissue dissection, minimal blood loss and with no disturbance of the fracture area. Compared to standard technique no differences were observed regarding the proper placement of the implant, surgical time and implant failures. Disclosure: No significant relationships.

\section{3}

\section{A NEW METHOD TO TREAT ANKLE-FRACTURES: THE INTRAMEDULLAR HEADLESS SCREW SYSTEM: 5 YEARS EXPERIENCE}

A.Kocsis $^{\text {I }}$, I. Kádas ${ }^{2}$, Z. Magyari ${ }^{3}$, G. Sztrinkai ${ }^{\text {I, S. Frenyo }}{ }^{4}$

${ }^{1}$ Iv. Trauma Department, Trauma Center, Péterfy S. Hospital, Budapest, Hungary, ${ }^{2} 3$, National Institute of Traumatolgy, Budapest,
Hungary, ${ }^{3}$ Dept 3, National Institute of Traumatology, Budapest, Hungary, ${ }^{4}$ Department 1 , Trauma Center, Budapest, Hungary

Introduction: Objective: by the help of compression and distraction HeadLess Screw System used to fix articular fractures, we worked out a new method to treat medial and lateral malleolar fractures. During the development the main consideration was, to reduce the time of operation and surgical invasion while maintaining the stability of fixation.

Materials and methods: Plates are the current most accepted methods to cure ankle fractures. As this technique requires open approach, it is associated with longer time of surgical intervention, greater incision, finally: more complications. We developed a new method to avoid open approaches: we reduce the fracture and verify the situation with intraoperative X-ray. Keeping the right position of the axis, we ream the intramedullar space of the fibula, then we use the type of screw according to the level of fracture: compression or reduction screw. With these screws we can avoid the most common redislocations of fractures: in case of Weber $\mathrm{B}$ fractures the shortening, in case of Weber A the lengthening of fibula.

Results: In the last 5 years we treated more than 100 patients with the new method and numerous trauma centers have joined to our research. Septic complication occured in one case, we significantly reduced the time of intervention and surgical invasion. We mobilized the patients after the operation with full weight-bearing and used 3 weeks of casting.

Conclusion: Comparing to plating methods: a new minimal-invasive closed technique was born. We managed to reduce operation time and surgical invasion. We decreased the casting time and achieved better functional results.

In our presentation we would like to introduce the results of the new technique: intraoperative videos and detailed X-ray measurements of follow-up examinations.

Disclosure: No significant relationships.

\section{4}

\section{FIXATOR ASSISTED NAILING IN PROXIMAL THIRD TIBIA FRACTURES}

\section{A. Chelnokov, D. Bekreev}

Orthopaedic Trauma, Ural Scientific Institute of Traumatology and Orthopaedics, Ekaterinburg, Russian Federation

Introduction: Proximal third fractures of the tibia can be difficult to nail using conventional technique. Typical problems include valgus malalignment, antecurvation and posterior shift of the distal fragment. Different methods of avoiding these pitfalls have been suggested, but use of the Ilizarov technique seems to be underestimated. The aim of our study was to refine the technique of closed interlocking nailing in fractures of the proximal tibia introducing an Ilizarov-based small wire external distractor.

Materials and methods: Over a 7-year period, 56 proximal tibia fractures and nonunions were treated with closed interlocked nailing. All nailings were performed on a flat radiolucent table. Reduction was provided by a distractor consisting of two half-rings connected with three telescopic rods, with a support for controllable knee flexion. Solid titanium nails were used in all cases with minimal reaming. Results: Only $7 / 56$ proximal fractures had angulation of $5^{\circ}$ or greater in the frontal or sagittal plane. In 4/56 proximal cases there was partial loss of fixation. All of them occurred at the initial period of the study. In 7/56 cases healing was reached after exchange nailing. Conclusion: The technique allows low-invasive nail insertion in knee hyperflexion. It renders unnecessary many conventional measures like 
lateral point of insertion, knee semiextension, extended incision, Poller screws, reduction clamps, and provisional buttress plating.

Disclosure: No significant relationships.

\section{5 \\ HWX: A NEW PROXIMAL HUMERAL NAIL FOR FRACTURE TREATMENT}

\section{Z. Detre}

Buda Trauma Center, St János Hospital, Budapest, Hungary

Introduction: Treatment of the fractures of the proximal end of the humerus is still an important problem, especially at the geriatric patient population. The basic goal of our treatment is to provide proper stability of fixation in order to obtain early restoration of motion and furthermore to assure the use of walking aids for the elderly patients. The surgeons choice among the different treatment options is influenced by the anatomy of the fracture, the quality of the bone, the expected deterioration of the blood supply of the humeral head and the task to obtain sufficient stability of fixation.

Materials and methods: In order to comply with the above requirements we developed an implant that might be inserted through a minimally invasive approach to spare the circulation of the headand also provides sufficient fixation stability. Intramedullary devices give higher stability than plates. Angular stable locking screws give more reliable fixation in the bones with poor quality. The combination of these two resulted in a new versatile device called HWX nail. The special targeting arm of the nail is a patent. The new nail was used in 15 cases, average age 76 years. 10 cases of 3 -part fractures and 5 cases of 4-part fractures were operated on. The operative technique as well as the clinical results are presented following a 6,12 and 20 weeks long follow up.

Results: The patients tolerated the new device well. All fractures consolidated. Technical failures as nail protrusion (3 cases), deep sitted nail (1 case) and screw protrusion (1 case) were reported. No infection, delayed union, or head AVN were encountered. Early good function was quickly obtained. The average Constant score were 70, 79 and 81 respectively.

Conclusion: Our results are promising. They show that HWX nailing - a combination of intramedullary technique with angular stable interlocking screws gives higher stability and earlier functional recovery. Head circulation is spared by using a minimally invasive approach.

Disclosure: No significant relationships.

\section{6}

\section{NEW SELFDYNAMISABLE INTERNAL FIXATOR AND MINIMALLY INVASIVE METHOD IN FEMORAL FRACTURES TREATMENT}

\section{$\frac{\text { M.B. Mitkovic }^{1}, \text { S. Milenkovic }^{2}, \text { D. Mladenovic }}{}{ }^{3}$, I. Micic $^{4}$,}

${ }^{1}$ Orthopaedic and Traumatology Clinic, Orthopaedic and Traumatology clinic, Nis, Serbia, ${ }^{2}$ University Orthopaedic and Traumatology Clinic, Clinical Center of Nis, Nis, Serbia, ${ }^{3}$ Trauma, University Orthopaedic and Trauma clinic, Nis, Serbia, ${ }^{4}$ Orthopaedic Surgery And Traumatology, Clinical Center, Faculty of Medicine, Nis, Serbia
Introduction: Axial dynamisation is recognized method which encourages bone union. Because of that many doctors routinely remove one screw from interlocking nail, two months after primary operation to provide axial dynamisation. Dynamisation is happened in about $15-25 \%$, according to the literature, but we still can not predict which patient or fracture will need dynamisation. The aim of this study is to present one new selfdynamisable implant and method for internal fixation of different femoral fractures.

Materials and methods: Between 2000 and 2008, 849 patients with 871 fractures receiving selfdynamisable internal fixator developed by Mitkovic, for proximal, diaphyseal and distal femur fractures were included in the study.

Results: The average operative time was $44 \min (23-119)$, average fluoroscopy time was $12 \mathrm{~s}(6-92)$ while average blood loss of $90 \mathrm{ml}(60-250 \mathrm{ml})$ when minimally invasive technique used. None of the patients developed complications during the intraoperative period. Healing time was 3.9 months (3-9). Healing was achieved in $99.1 \%$. Superficial infection developed after 7 fixations $(0.9 \%)$ while deep infection developed in 4 patients $(0.5 \%)$. The screw breaking occurred 6-18 weeks after 19 fixations (2.6\%). Cut out phenomenon happened in 24 cases. Spontaneous axial dynamisation was observed in seventy-one patient $(23.8 \%), 5 \mathrm{~mm}$ on average $(2-12 \mathrm{~mm})$

Conclusion: SIF is one effective method for the treatment of femoral fractures. This method particularly valuable for treatment of comminuted fractures with regard to minimally invasive surgery.

References: [1]. Mitkovic M. External fixation in TraumatologyDevelopment and application of author's devices. 1st ed. Nis (Serbia): Prosveta; 1992. [2] Mitkovic M et al. New biological method of internal fixation of the femur. Acta Chir Iugosl. 2005;52:113-6.

Disclosure: No significant relationships.

\section{7}

\section{OUTCOME OF AO/OTA 31-A3 FRACTURES OF THE PROXIMAL FEMUR TREATED WITH EITHER SHORT OR LONG PROXIMAL FEMORAL NAIL}

$\underline{\text { G. Okcu }^{1}, N . \text { Ozkayin }^{2}, \text { K. Aktuglu }}{ }^{3}$

${ }^{1}$ Orthop and Traumatology, Celal Bayar University, Izmir, Turkey, ${ }^{2}$ Orthop and Traumatology, Ege University, Izmir, Turkey,

${ }^{3}$ Department of Orthopedics and Traumatology, Ege University, Faculty of Medicine, Department of Orthopedics and Traumatology, Izmir, Turkey

Introduction: A prospective randomized trial was conducted to compare the outcome of reverse oblique fractures of the proximal part of femur treated with either short or long proximal femoral nail.

Materials and methods: All consecutive patients presenting with radiological diagnosis of an unstable trochanteric femoral fracture, classified as 31-A3.1 to A3.3 according to the AO/OTA classification were randomly allocated into two groups during one-year period in two university hospitals. Fifteen patients in Group 1 were treated with short version of the proximal femoral nail while 18 patients in Group 2 were managed with the long version of the same nail. The two groups were comparable with regard to demographic variables. The minimum follow-up was 1 year. Clinical outcome and complications were compared between them.

Results: Union time averaged 19.5 weeks in Group 1 and 17.7 weeks in Group $2(\mathrm{p}=0.093)$. Average tip-apex distance was 23.8 and $24.5 \mathrm{~mm}$, respectively $(\mathrm{p}=0.79)$. Average Harris hip score was 78 in Group 1 and 81 in Group $2(\mathrm{p}=0.32)$ There was one cutting out of lag screw in Group 2. Average sliding of the blade and change of the 
neck shaft angle was not significant between two groups. Local and general complications was not different either between two groups. Conclusion: The treatment of reverse oblique fractures of the proximal femur are still challenging. Although the study group is small to conclude definitely, long version of the nails should be preferred for this type of fractures because of shorter healing time.

Reference: 1. Min WK, Kim SY, Kim TK, Lee KB, Cho MR, Ha YC, Koo KH. Proximal femoral nail for the treatment of reverse obliquity intertrochanteric fractures compared with gamma nail. J Trauma. 2007;63:1054-60.

Disclosure: No significant relationships.

\section{8}

\section{PERCUTANEOUS PEDICULAR FIXATION COMBINED WITH KYPHOPLASTY FOR THE TREATMENT OF THORACOLUMBAR BURST FRACTURE. A NOVEL TECHNIQUE}

\author{
P.G. Katonis ${ }^{1}$, J. Aggelidakis ${ }^{2}$, K. Alpantaki $^{3}$, S. Lianoudakis ${ }^{3}$, \\ M. Marketakis ${ }^{3}$, A. Karantanas ${ }^{3}$ \\ ${ }^{1}$ Orthopaedics and Traumatology, Medical School of Crete, \\ Heraklion, Greece, ${ }^{2}$ Medical school of Crete, Heraklion, Greece, \\ ${ }^{3}$ Medical School of Crete, Heraklion, Greece
}

Introduction: The aim of this prospective study was to assess the efficacy and safety of balloon kyphoplasty with calcium phosphate after posterior percutaneous short-segment reduction and fixation for the treatment of acute traumatic thoracolumbar fractures.

Materials and methods: Twenty-five consecutive patients with an average age of 36 years who sustained thoracolumbar A3 burst fracture (T11-L1) without neurological deficit were included in this prospective study. All patients were treated between 2 and 4 days after trauma using the same technique. First step was the percutaneous short segment pedicle screw and rod reduction and fixation that was performed to realign the adjacent vertebral bodies anatomically and restored the segmental kyphosis by ligamentotaxis. The second step of our surgical strategy was Balloon kyphoplasty with biological cement, calcium phosphate, allowing some fine tuning of the endplate reduction.

Results: With a mean average follow up 15 months, all the patients were evaluated by clinical and radiological parameters and considered as healed after a 6 months control. The clinical evaluation by using VAS scale (back pain) was 1.8 points at the final follow up $(\mathrm{P}<0.001)$. The domain bodily pain ranged from 28 preop to 93 postop $(\mathrm{P}<0.001)$ and the domain role physical improved from 42 before surgery to 95 after surgery $(\mathrm{P}<0.001)$. No further changes in VAS and Sf-36 scores were reported after 6 months. Radiographic evaluation showed a significant improvement in the post-operative period (segmental kyphosis $18 \pm 12^{\circ}$ preop, $1 \pm 7^{\circ}$ postop, vertebral body height $0.56 \pm 0.20$ preop $0.97 \pm 0.09$ postop). We evaluated 5 patients with consecutive QCT measurements and our data suggest that osseous integration occur within the calcium phosphate implants. No intra and postoperatively complications were observed.

Conclusion: Percutaneous pedicular fixation plus balloon kyphoplasty with calcium phosphate cement provide excellent immediate functional outcome. There was not observed instrumentation failure and cement leakage while QCT showed continuity between calcium phosphate cement and vertebral bone.

References: [1] Palmisani M et al. Minimally invasive percutaneous fixation in the treatment of thoracic and lumbar spine fractures. Eur Spine J. 2009. [2] Verlaan et al. JJ. Balloon vertebroplasty in combination with pedicle screw instrumentation. Spine. 2005.

Disclosure: No significant relationships.

\section{VISCERAL TRAUMA 8}

\section{9}

\section{WARSHAW TECHNIQUE FOR PANCREATIC INJURY}

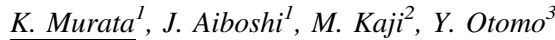

${ }^{1}$ Shock Trauma and Emergency Medical Center, Tokyo Medical and Dental University Hospital, Tokyo, Japan, ${ }^{2}$ Shock Trauma and Emergency Medical Center, Tokyo Medical and Dental University hospital, Tokyo, Japan, ${ }^{3}$ Shock Trauma and Emergency Medical Center, Tokyo Medical and DentaTokyo Medical and Dental University hospital of Medicinel University hospital, Tokyo, Japan

Introduction: Warshaw et al. reported the effectiveness of spleen preserving distal pancreatectomy with his own technique. The technique is mainly indicated to elective operation of benign pancreatic disease. We had provided three patients those suffering from brunt pancreatic injury with Warshaw technique successfully. We look at why the technique is influential to pancreatic injury.

Materials and methods: Case 1: A 19-year-old man transported to our ER by motorcycle accident. Though he was diagnosed as renal injury and tibia fracture, he complained of an abdominal pain after $12 \mathrm{~h}$ of injury. Enhanced CT and intra-operative study revealed main pancreatic ductal injury. Case 2: A 18-year-old girl jump down from upstairs window. A stump gave her a smash on abdomen. Abdominal CT scan showed abdominal wall hernia and pancreatic injury. Case 3: A 35-year-old woman was struck on her abdomen. She had abdominal pain after $24 \mathrm{~h}$ and transported to our ER urgently. Enhanced abdominal CT scan revealed transection of the pancreas.

Results: All of the cases we confirmed normality of short gastric artery. Splenic artery and vein were ligated at hilum, and the pancreas tale was resected with auto-suture devices. All of patients had no complication, and discharged within 30 days.

Conclusion: Spleen preserving distal pancreatectomy usually needs complicated procedure for ligation of splenic vessels. Warshaw technique is a simple procedure and excellent at hemostasis. In trauma surgery, we think this technique is very reasonable.

References: [1] Management of pancreatic trauma. Injury. 2008;39:21-9. [2] Management of Blunt Major Pancreatic Injury. J Trauma. 2004;56:774-8. [3] Distal pancreatectomy with splenic preservation revisited. Surgery. 2007;141:619-25.

Disclosure: No significant relationships.

\section{$\mathbf{O 2 0 0}$}

\section{ENDOVASCULAR TREATMENT OF ACUTE BLUNT THORACIC AORTIC INJURIES}

\section{E.M. Marone, A. Kahlberg, Y. Tshomba, R. Chiesa}

Vascular Surgery, San Raffaele Hospital, Milano, Italy

Introduction: Endovascular repair of the thoracic aorta (TEVAR) has been recently considered an appealing alternative to open treatment of traumatic aortic injuries $[1,2]$. However, the use of this technique in emergency is often limited by patient's hemodynamic instability, severe associated lesions and unavailability of adequate materials. Dedicated stent-grafts are not currently available [3]. We report our results in treating blunt traumas of the thoracic aorta using different commercially available stent-grafts.

Materials and methods: Between 2003 and 2010, 26 patients (20 males, mean age $39.2 \pm 12.1$ years) underwent TEVAR for a 
traumatic aortic lesion. Fourteen patients $(54 \%)$ were hemodynamically unstable at presentation, and 18 patients (69\%) presented severe associated lesions. A contrast enhanced body computed tomography (CT) was performed in all cases to establish the diagnosis of aortic rupture and evaluate associated injuries. After TEVAR, patients were followed-up with CT angiography of the chest before discharge from the hospital, at 6 months and yearly thereafter.

Results: Twenty-three patients were treated emergently, whereas 3 patients underwent prior clinical stabilization of severe associated injuries. Primary technical success rate was 100\%. No patient required conversion to open thoracic surgical repair. No paraplegia or stroke were observed. Procedure-related complications included an external iliac artery lesion during introducer sheath removal. The left subclavian artery was intentionally covered in 7 cases (27\%), and revascularized in two hemodynamically stable patients prior to stentgraft deployment. Two patients died perioperatively due to multiorgan failure, for a total in-hospital mortality of $7.7 \%$. Twenty-two patients ( $92 \%$ of survivors) adhered to the follow-up protocol (mean $24.2 \pm 16.5$ months), and they are all well without instances of reintervention.

Conclusion: In our experience, endovascular treatment of acute traumatic thoracic aortic injuries using different commercially available stent-grafts allows to obtain satisfactory short term results. References: [1] Midgley PI, Mackenzie KS, Corriveau MM, et al. Blunt thoracic aortic injury: a single institution comparison of open and endovascular management. J Vasc Surg. 2007;46(4):662-8. [2] Demetriades D, Velmahos GC, Scalea TM, et al. American Association for the Surgery of Trauma Thoracic Aortic Injury Study Group. Operative repair or endovascular stent graft in blunt traumatic thoracic aortic injuries: results of an American Association for the Surgery of Trauma Multicenter Study. J Trauma. 2008;64(3):561-70. [3] Rosenthal D, Wellons ED, Burkett AB et al. Endovascular repair of traumatic thoracic aortic disruptions with "stacked" abdominal endograft extension cuffs. J Vasc Surg. 2008;48(4):841-4.

Disclosure: No significant relationships.

\section{1}

\section{MANAGEMENT OF BLUNT RENAL INJURY IN A DUTCH LEVEL 1 TRAUMA CENTER IN VIEW OF THE EUROPEAN GUIDELINES}

\author{
D.C. Olthof ${ }^{1}$, C.H. van der Vlies ${ }^{2}$, O.M. van Delden ${ }^{3}$, T.M. de Reijke ${ }^{4}$, \\ J.C. Goslings ${ }^{2}$
}

${ }^{1}$ Trauma Unit Department of Surgery, Academical Medical Center, Landsmeer, The Netherlands, ${ }^{2}$ Trauma Unit Department of Surgery, Academic Medical Center, Amsterdam, The Netherlands, ${ }^{3}$ Radiology, Academic Medical Center, Amsterdam, The Netherlands, ${ }^{4}$ Urology, Academical Medical Center, Amsterdam, The Netherlands

Introduction: Debate continues about the optimal management strategy for patients with blunt renal injuries.

Materials and methods: To report the management of our patients with blunt renal injury and to compare them to the guidelines of the European Association of Urology.

Design, setting and participants: A historical cohort of all consecutive patients with blunt renal injury, during 5 years in an academic level 1 trauma center.

Measurements: The management of all these patients was retrospectively analysed to determine evaluation and management strategies. Furthermore, the medical records and radiology reports were analyzed to assess if the treating physicians presence of renal injury.
Results: Results and limitations: Median age and ISS of the 186 included patients were 40 and 17 years respectively. The management of haemodynamically stable patients with microscopic haematuria was consistent with the guidelines, but in $64 \%$ of these patients (all grade 1 and 2 injuries) renal injury was not formally documented during hospital admission. Although the guidelines dictate a CT scan for haemodynamically stable patients with gross haematuria only $60 \%$ of these patients underwent CT scanning. Patients with Grade 1-4 renal injury were treated conservatively, in accordance with the guidelines. Additionally, two patients with Grade 3-4 renal injury received angiography and embolization (A\&E). One patient with grade 5 injury underwent renal exploration (recommended) and two A\&E. Four of the 8 haemodynamically unstable patients underwent emergency laparotomy (consistent with the guidelines) and in 4 patients A\&E was performed.

Conclusion: The treatment of patients with blunt renal injury was not completely in line with the guidelines. Particularly, A\&E was more frequently performed in patients with high grade renal injuries. Strikingly, grade 1-2 renal injuries were often not documented during admission. The treating physicians were often not aware of renal injury in patients with microscopic haematuria. Disclosure: No significant relationships.

\section{$\mathbf{O 2 0 2}$}

\section{EFFECT OF PRE-EXISTING MEDICAL CONDITIONS ON IN-HOSPITAL MORTALITY: ANALYSIS OF TRAUMA PATIENTS IN 5 YEARS}

\author{
T. Shoko ${ }^{1}$, A. Shiraishi ${ }^{2}$, M. Kaji ${ }^{2}$, Y. Otomo ${ }^{3}$
}

${ }^{1}$ Shock Trauma And Emergency Medical Center, Tokyo Medical and Dental University Hospital, Tokyo, Japan, ${ }^{2}$ Shock Trauma And Emergency Medical Center, Tokyo Medical and Dental University hospital, Tokyo, Japan, ${ }^{3}$ Shock Trauma And Emergency Medical Center, Tokyo Medical and DentaTokyo Medical and Dental University hospital of Medicinel University hospital, Tokyo, Japan

Introduction: The average life expectancy of Japanese individuals is the longest in the world. The mortality rate from injury is increasing among older people. We had reported on the relationship between pre-existing co-morbidities and trauma mortality from 2004 to 2007 in this last congress. In this report, we added the data of 2008 and analyzed the data of Japan Trauma Data Bank (JTDB) among elderly people for 5 years.

Materials and methods: We conducted a retrospective analysis using cases recorded in the JTDB from 2004 to 2008. The subjects were 19,027 hospital inpatients 16 years of age or older. The selected subjects were divided into three age groups for analysis: young subjects (16-49 years of age), middle-aged subjects (50-74 years of age), and elderly subjects (over 75 years of age). Subjects were also divided into three groups by ISS severity for analysis: minor injury (ISS of 1-15), moderate injury (ISS of 16-24), and severe injury (ISS of over 25). A logistic regression analysis was conducted for the relation between 23 pre-existing medical conditions (PMCs) and inhospital mortality.

Results: Overall in-hospital mortality was 10.1 and $18.1 \%$ for people 75 years of age and older. The incidence of PMC was 7,782 (40.9\%). Patients with PMCs of dementia/mental retardation [odds ratio(OR) 1.8, 95\% confident interval $(95 \% \mathrm{CI}) 1.3-2.6]$, chronic obstructive pulmonary disease (OR 2.7, 95\% CI 1.4-5.4), stroke (OR 1.6, 95\% CI 1.2-2.1), coronary heart disease (OR $1.5,95 \%$ CI 1.0-2.1), congestive heart failure (OR 2.0, 95\% CI 1.2-3.1), cirrhosis (OR 6.6, 95\% CI 4.3-10.2), dialysis (OR 2.1, 95\%CI 1.2-3.6), active cancer (OR 2.5, 
95\%CI 1.6-4.2), or anticoagulation drugs (OR 2.4, 95\%CI 1.4-4.2) had higher in-hospital mortality. The existence of PMCs increased mortality. The existence of two or more PMCs in the 50-74 years age group and in the minor injury group strongly affected the odds ratio for mortality. Conclusion: The existence of certain PMCs increases in-hospital mortality from injury. This effect is particularly conspicuous in middle-aged patients and people with minor injuries, but was not found to be a problem among elderly people. The increased mortality from injury in elderly people in Japan is therefore not affected by the existence of PMCs.

References: 1. Japan Trauma Data Bank. Available at: http://www. jtcr-jatec.org/traumabank/dataroom/dataroom.htm. Accessed 31 Octorber 2010. 2. Hollis S, Lecky F, Yates DW, et al. The effect of pre-existing medical conditions and age on mortality after injury. J Trauma 2006;61:1255-60. 3. Wutzler S, Maegele M, Marzi I, et al. Association of preexisting medical conditions with in-hospital mortality in multiple-trauma patients. J Am Coll Surg 2009;209: $75-81$.

Disclosure: No significant relationships.

\section{3}

\section{PROSPECTIVE EVALUATION OF A REFINEMENT IN THE TECHNIQUE OF PERIHEPATIC PACKING: A SAFE AND EFFECTIVE SURGICAL HEMOSTASIS AND MULTIDISCIPLINARY APPROACH CAN IMPROVE THE OUTCOME OF SEVERE LIVER TRAUMA}

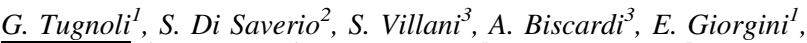

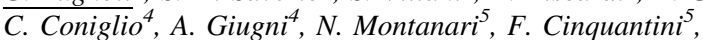 \\ G. Gordini ${ }^{4}$, F. Baldoni
}

${ }^{1}$ Emergency And Trauma Surgery Unit (head Dr. G. Tugnoli), Maggiore Hospital Trauma Center, Bologna Local Health District, Bologna, Italy, ${ }^{2}$ Surgery, Emergency and Trauma Surgery Unit, maggiore Hospital Trauma Center, Bologna, Italy, ${ }^{3}$ Emergency And Trauma Surgery Unit, Maggiore Hospital Trauma Center, Bologna Local Health District, Bologna, Italy, ${ }^{4}$ Trauma Icu, Department Of Emergency, Maggiore Hospital Trauma Center, Bologna Local Health District, Bologna, Italy, ${ }^{5}$ Interventional Radiology Unit, Department Of Radiology, Maggiore Hospital Trauma Center, Bologna Local Health District, Bologna, Italy

Introduction: Since 2005 we refined the technique of perihepatic packing including complete mobilization of the right lobe, packing around the posterior paracaval surface, lateral right side, anterior and postero-inferior surfaces

Materials and methods: Two groups of patients with grade IV/V liver trauma, underwent perihepatic packing respectively before and after 2005. The study group included 12 patients treated with the new technique. The control group included 23 patients treated with the old technique.

Results: All the 13 patients but one who died within $24 \mathrm{~h}$, were treated with the old technique. Overall survival rate was $75 \%$ in the patients treated with new technique (vs. 30.4\%, p < 0.02); liver-related mortality was 8.3 versus $34.8 \%(\mathrm{p}=\mathrm{ns})$. Mean survival time in ICU was longer in the latest group ( 39.4 vs. 22.3 days; $p=n s$ ). The incidence of re-bleeding requiring re-packing was $16.7 \%$ in patients underwent new packing versus $45.5 \%(\mathrm{p}=\mathrm{ns})$. Overall ( 81.8 vs. $100 \%, \mathrm{p}=\mathrm{ns})$ and liver-related morbidity rate ( $18.2 \mathrm{vs} .41 .7 \%, \mathrm{p}=\mathrm{ns})$ and the incidence of abdominal sepsis (9.1 vs. $41.7 \%, \mathrm{p}=\mathrm{ns}$ ) decreased.

Conclusion: Our refined technique of perihepatic packing seems to be safe and effective in achieving a fast and reliable hemostasis. The requirement of resuscitative fluids, PRBC and FFP transfusions decreased. The new technique seems to reduce the incidence of rebleeding and need for repacking. By reducing the operative time and shortening the time to ICU admission, it can also contribute to decrease morbidity and mortality. Such a safe and effective surgical hemostasis and multidisciplinary approach can improve the outcome of severe liver trauma.

Disclosure: No significant relationships.

\section{4}

\section{WHOLE-BODY MSCT VERSUS CONVENTIONAL RADIOGRAPHY +ORGANSPECIFIC CT: ONE YEAR'S PROSPECTIVE STUDY}

\author{
T. Bodzay ${ }^{1}$, E. Papp ${ }^{2}$, G. Nardai ${ }^{3}$, T. Gall ${ }^{4}$, I. Flóris ${ }^{1}$, J. Szita ${ }^{5}$ \\ 13/k, Trauma Center Péterfy Hospital, Budapest, Hungary, \\ ${ }^{2}$ Radiology, Trauma Center Péterfy Hospital, Budapest, Hungary, \\ ${ }^{3}$ Icu, Trauma Center Péterfy Hospital, Budapest, Hungary, ${ }^{4} 5 / \mathrm{k}$, \\ Trauma Center Péterfy Hospital, Budapest, Hungary, ${ }^{5} 6 / \mathrm{k}$, Trauma \\ Center Péterfy Hospital, Budapest, Hungary
}

Introduction: The use of whole-body multislice CT scan for the examination of multiple-trauma patients is spreading worldwide. The largest multicenter study by Huber-Wagner, accounts of 4,621 multiple-trauma cases according to the DGU polytrauma register. The infrastructural reconstruction of our hospital in the beginning of 2009 (shock room built next to the CT, installation of a new 16 slice Siemens Emotion CT) allowed us to start performing this type of examination.

Materials and methods: We analyzed patients treated in our department from 1 April 2009 until 1 April 2010. Only those patients were included in the study, who received their primary treatment in the shock room. After airway and intravenous line management, physical examination, and FAST (focused assessment sonography in trauma) were performed, the patient either was further examined with conventional radiography followed by organ focused CT scan or MSCT and the necessary additional radiographs were performed. There were no selection criteria, the choice of examination method was left to be determined by the trauma team leader on duty.

Results: We analyzed 1 year's amount of cases according to two aspects: first, from the time of arrival to the shock room until the transportation to the operating room (i.e. the time spent for diagnostic examination) and secondly, the amount of overlooked injuries, which were unnoticed on the day of admission to our department, only to be later diagnosed. We developed a CT protocol for the examination as well as which patients were to be indicated for the full-body multislice CT scan.

Conclusion: The whole-body multislice CT scan is much faster; in our study, the time spent on diagnostic examination was 20 min less than using the conventional diagnostic algorithm.

Disclosure: No significant relationships.

\section{5}

\section{CIVILIAN POPLITEAL ARTERY INJURIES: A TEN YEAR AUDIT IN AN URBAN TRAUMA CENTRE}

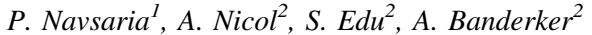

${ }^{1}$ Surgery: Trauma, Groote Schuur Hospital and University of Cape Town, Cape Town, South Africa, ${ }^{2}$ Surgery: Trauma, Groote Schuur Hospital, Cape Town, South Africa 
Introduction: The aim of this study is to identify factors associated with limb loss in patients with popliteal artery injuries.

Materials and methods: Retrospective chart review of a prospectively collected data base of all patients with popliteal artery injuries presenting to the Groote Schuur Hospital Trauma Centre over 10 years.

Results: One hundred and thirty six patients with popliteal artery injuries were identified. Penetrating trauma accounted for $81(59.5 \%)$ injuries. Injury level: above the knee (39), at the knee (47), and below the knee (34). Associated injuries: fractures in 66 (48.5\%), knee dislocation in $29(21.3 \%)$ and popliteal vein injury in $59(43.4 \%)$ patients. Limb viability on admission was assessed being ischaemic in $85(64.4 \%)$ patients. Treatment of the arterial injury involved reversed vein grafting in 68 , primary anatomises in 33 , and prosthetic graft insertion in 11. The overall amputation rate was $37.5 \%$. Mechanism of injury $(\mathrm{p}=0.684)$, concomitant venous injury $(\mathrm{p}=0.701)$, fracture $(\mathrm{p}=0.183)$, knee dislocation $(\mathrm{p}=0.784)$, and anatomical level of arterial injury $(\mathrm{p}=0.393)$ were not associated with increased amputation rates. Delay between injury and definitive repair of more than $7 \mathrm{~h}$ in patients with ischaemic limbs $(\mathrm{p}=0.0236)$ and the presence of a compartment syndrome $(\mathrm{p}=0.003)$ were the only factors significantly associated with an increased amputation rate. Conclusion: In the current series, the amputation rate was $37.5 \%$. The most significant factors associated with this finding were an ischaemic time of more than $7 \mathrm{~h}$, and the presence of a compartment syndrome. Disclosure: No significant relationships.

\section{7}

\section{VASCULAR TRAUMA OF THE LIMBS: ELEVEN-YEAR MONOCENTRIC EXPERIENCE}

\author{
E.M. Marone, D. Logaldo, A. Kahlberg, Y. Tshomba, R. Chiesa
}

\section{Vascular Surgery, San Raffaele Hospital, Milano, Italy}

Introduction: Vascular trauma of the limbs is associated with significant mortality and morbidity rates. Aim of this article is to analyze results of surgical treatment of vascular trauma of the upper or lower limbs in a high volume Vascular Center.

Materials and methods: A retrospective review of data collected between 1998 and 2009 was performed. Patients' details, including cause and pattern of vascular injury, diagnostic modality, intervention, limb-salvage rate and long-term survival were recorded.

Results: Between January 1998 and December 2009, 154 patients (mean age 43 years; range 9-76 years) presented to our Institution with vascular trauma of the upper $(n=41 ; 26.7 \%)$ or lower limbs $(\mathrm{n}=106 ; 68.8 \%)$ or both $(\mathrm{n}=7 ; 4.5 \%)$. The most common cause of vessel damage was road accident (52\%), followed by workrelated $(26 \%)$, self-inflicted $(14 \%)$, recreation-related $(6 \%)$ and other causes $(2 \%)$. Thirty-six patients $(23.4 \%)$ presented with major associated lesions. Primary amputation rate was $8.4 \%$. Treatment included direct suture (35\%), patch repair (20\%), bypass grafting $(41 \%)$ and endovascular treatment $(4 \%)$. Vascular shunts were used in $7.3 \%$ of patients; fasciotomies were performed in $33 \%$ of patients. After limb revascularization, eight patients died because of major associated injuries. Overall limb salvage rate in this study was $82.9 \%$. Survival rate of patients following peripheral vascular intervention was $96.4 \%$.
Conclusion: Vascular trauma of the limbs represents a clinical entity for which immediate diagnosis and treatment is essential. To ensure the best possible outcomes, algorithms of care will need to guarantee the actual availability of diagnostic tools and surgeons. The current management approach, including use of angiography and prompt surgical management, results in successful outcomes after extremity arterial injuries. The use of shunt and fasciotomies in complex cases improves early results.

References: 1. Mommsen P, Zeckey C, Hildebrand F, Frink M, Khaladj N, Lange N, Krettek C, Probst C. Traumatic extremity arterial injury in children: epidemiology, diagnostics, treatment and prognostic value of Mangled Extremity Severity Score. J Orthop Surg Res. 2010;5:25. 2. Burkhardt GE, Cox M, Clouse WD, Porras C, Gifford SM, Williams K, Propper BW, Rasmussen TE. Outcomes of selective tibial artery repair following combat-related extremity injury. J Vasc Surg. 2010;52(1):91-6. 3. Haddock NT, Weichman KE, Reformat DD, Kligman BE, Levine JP, Saadeh PB. Lower extremity arterial injury patterns and reconstructive outcomes in patients with severe lower extremity trauma: a 26-year review. J Am Coll Surg. 2010;210(1):66-72.

Disclosure: No significant relationships.

\section{Video Presentations}

\section{EMERGENCY SURGERY 1}

\section{V1}

\section{LAPAROSCOPIC MANAGEMENT OF PERFORATED APPENDICEAL DIVERTICULITIS: A RARE CASE WITH VIDEO PRESENTATION}

\section{G.O. Kucuk}

General Surgery, Samsun Vezirkopru Public Hospital, Samsun, Turkey

Introduction: Appendiceal diverticulitis is a rare entity with clinical and pathological differences from acute appendicitis. It usually presents with atypical abdominal signs and it has a more rapid progression to perforation. In this case, a 40-year-old female with perforated appendiceal diverticulitis was described.

Content: A 40-year-female patient was admitted to our hospital with a 3-day history of right lower-quadrant pain, anorexia and vomiting. The physical examination showed localized tenderness in the right lower quadrant of the abdomen. She said that she did not feel epigastralgia or pain shifting to the right lower abdomen. No rebound tenderness was observed. Abdominal ultrasonography demonstrated an enlarged swollen appendix with a cross section diameter of $11 \mathrm{~mm}$ and pouch-like projection filled with fluid in $7 \mathrm{~mm}$ diameter. Wall thickening of the appendix was $3 \mathrm{~mm}$ and it was more prominent than that seen in typical appendicitis. Laparoscopic appendectomy was performed. The resected appendix was $7 \mathrm{~cm}$ long, $1.0 \mathrm{~cm}$ in diameter with multiple diverticula, of which one was perforated. Microscopic study revealed a diverticulum with severe inflammation and perforation in its wall. She had an uneventful recovery and discharged 3 days after surgery.

Discussion: Appendiceal diverticulum is a rare clinical entity with an incidence of $0.2-1.5 \%$ in surgical pathologic specimens. Diverticulum of the appendix may ultimately develop diverticulitis and it has a 
high perforation rate. Laparoscopy with appendectomy is an important final diagnostic procedure for appendiceal diverticulitis because of difficulties in diagnosis.

Disclosure: No significant relationships.

\section{EMERGENCY SURGERY 2}

\section{V3}

\section{THERE WAS ONCE AN ACUTE APPENDICITIS..}

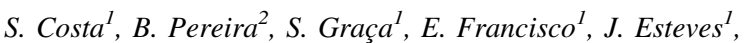

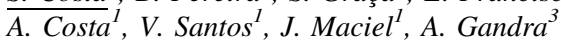

${ }^{1}$ Surgery, CHVNG/E, VNG, Portugal, ${ }^{2} \mathrm{CHVNG} / \mathrm{E}, \mathrm{VNG}$, Portugal, ${ }^{3}$ Surgery, Centro Hospitalar Vila Nova de Gaia e Espinho, EPE, VNGaia, Portugal

Introduction: The possibility of combining a diagnostic and therapeutic procedure grant the exploratory laparoscopy an increasingly important role in emergency surgery, that keeps growing, as the experience of surgeons develops.

Content: We report the case of a 22 years old patient, healthy, who went to the ER because of a sudden abdominal pain in the epigastrium and hypogastrium without any other symptoms. The patient was afebrile, with soft and depressible abdomen, with tenderness in the right iliac fossa and hypogastrium, with a positive Blumberg sign. Analysis revealed leukocytosis and chest and abdomen radiograph did not show significant alterations. Exploratory laparoscopy was proposed and accepted, for differential diagnosis of ruptured ovarian cyst and acute appendicitis. Laparoscopy identified purulent peritonitis with Ileocecal appendix, uterus, fallopian tubes and ovaries of normal characteristics. At exploration, a perforated pyloric ulcer was identified. We performed suture of ulcer, omentoplasty and profuse washing of the abdominal cavity with sub-hepatic drain placement, laparoscopically. The postoperative period progressed uneventfully, and she restarted working at the 3rd postoperative week.

Discussion: As shown in the present case, the advantages of a laparoscopic approach in emergency surgery are: the versatility of incisions and consequent aesthetic result, the excellence of the peritoneal toilette and shorter length of hospitalization and recovery.

Disclosure: No significant relationships.

\section{EMERGENCY SURGERY 3}

\section{V4}

\section{LAPAROSCOPIC MANAGEMENT OF APPENDIX CARCINOID PRESENTED WITH COMPLICATED APPENDICITIS: A RARE CASE WITH VIDEO PRESENTATION}

\section{G.O. Kucuk}

General Surgery, Samsun Vezirkopru Public Hospital, Samsun, Turkey

Introduction: An increasing number of surgeons are performing laparoscopy in the management of acute appendicitis. In this a case a
26 year-old-male who was diagnosed as carcinoid tumour after pathological examination of resected appendix was discussed.

Content: A 26-year-old male presented to the emergency department with a 4-day history of intermittent right lower quadrant abdominal pain, nausea, and vomiting. Upon further questioning, he stated that his abdominal pain had started after physical exercise in the right lower quadrant. The following morning his symptoms had subsided. Two days later pain recurred in the periumbilical region and localized to the right lower quadrant. In his history, he said that he felt similar abdominal pain 1 month ago. On physical examination, there was rebound tenderness in the right lower quadrant. Laboratory data revealed a white blood cell account of $17,200 / \mathrm{mm}^{3}$. Abdominal ultrasound demonstrated perforated appendicitis with a cross section diameter of $12 \mathrm{~mm}$. The patient was taken for laparoscopy. Laparoscopic appendectomy with periappendicular abscess drainage was performed. The patient had an uneventful postoperative course and was discharged on postoperative day 4 . Histologic examination of the appendix revealed carcinoid tumour of the appendix.

Discussion: Inflammatory complications of appendix carcinoid may mimic acute appendicitis. Careful macroscopic and pathologic examination of the specimen is crucial to ensure appropriate definitive treatment. Disclosure: No significant relationships.

\section{V5}

\section{SUBDIAGNOSED CASE-UPPER INTESTINAL OBSTRUCTION IN ADULT BY INTETSINAL MALROTATION}

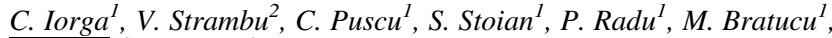 \\ A. Manta ${ }^{2}$, F. Popa ${ }^{2}$
}

${ }^{1}$ General Surgery, st pantelimon hospital, bucharest, romania, bucharest, Romania, ${ }^{2}$ General Surgery, Sf Pantelimon Clinical Emergency Hospital, Bucharest, Romania

Introduction: A 19 years old female patient presented with the fourth episode of epigastric pain, nausea, vomiting.

Content: Material and method: During 2005-2007 the patient was admitted three times in our clinic for epigastric pain, nausea, vomiting, the diagnosis was considered each time to be acute pancreatitis. This fourth episode, the most severe of all, did not improve after medical treatment, the general status of the patient worsened, the values of blood amylase raised. We performed an abdominal CT scan (not conclusive result), abdominal Doppler ultrasound (suggested the presence of vascular abnormalities), mesenteric arteriography which established the probable diagnosis of intestinal malrotation. Results: Once the probable diagnosis established we performed an open laparotomy and confirmed the diagnosis: intestinal malrotation, obstruction of the third part of the duodenum (Ladd bands) and we continued with Ladd's procedure (described in children): section of the bands and appendectomy. The postoperative evolution was excellent, with spectacular improvement of the patient's condition.

Discussion: Conclusions: Intestinal malrotation is usually diagnosed in children in the first months of life; the condition in rarely seen in adults (incidence $0.2 \%$ ). Adults are generally asymptomatic, the anomaly is discovered during laparotomy imposed by obstructive complications. The preoperative diagnosis is difficult, in our case arteriography was the most suggestive exam.

Disclosure: No significant relationships. 


\section{SHOULDER FRACTURE-DISLOCATION}

V6

\section{MINIINVASIVE PROXIMAL HUMERUS FRACTURE OSTEOSYNTHESIS USING THE LCP PERCUTANEOUS AIMING SYSTEM FOR PHILOS}

A. Skackov $^{1}$, M. Krivohlavek ${ }^{2}$, R. Lukas $^{2}$

${ }^{1}$ Trauma And Orthopeadic Centre, Liberec Regional Hospital, Liberec, Czech Republic, ${ }^{2}$ Trauma And Orthopaedic Centre, Liberec Regional Hospital, Liberec, Czech Republic

Introduction: Conventional open plate osteosynthesis of the proximal humeral fractures remains one of the basic treatment options. Miniinvasive plate ostheosynthesis (MIPO) introduced to this region is an enhancing alternative to deltopectoral approach. The use of LCP Percutaneous Aiming system for PHILOS simplifies the whole procedure. This system has been first tested and used at author's department in Czech Republic. The whole step-by-step MIPO technique procedure is presented in a video lecture with the eldest patient from the series - 78 year old woman with the fracture type AO 11-C2. Content: Material : The series contains 12 patients -8 women and 4 men with age average 63.5 year (range 39-78). Fracture types range from A3, B2 to C2 fractures. MIPO technique principles were followed. Results: The patient presented in the video is currently 6 months after surgery with almost full range of motion and CS of 96 points. Despite 1 malunion, 1 loss of reduction and 1 supraspinate tendon calcification, no other complication occurred.

Discussion: MIPO technique for this region represents stable fixation focused on soft tissue and blood supply preservation with very optimistic outcomes even in $\mathrm{C} 2$ types of fracture. The use of the aiming device makes this procedure accessible to all trauma and orthopaedic surgeons. As an exclusion criteria we consider the malunions, revision surgeries, fractures with humeral head dislocation, fractures where the use of hemiarthroplasty might be an option.

Disclosure: No significant relationships.

\section{SKELETAL TRAUMA VIDEO SESSION 1}

V9

\section{THE PIROGOFF AMPUTATION}

\author{
G. de Klerk, L.P.H. Leenen
}

Trauma Surgery, UMC Utrecht, Utrecht, Netherlands

Introduction: Indication: The Pirogoff amputation results in excellent weight bearing without prosthesis and is indicated in severe forefoot injuries. Technique: A skin incision is made distal to the talocrural joint on the dorsum of the foot and continued in a fish mouth shape to the plantar aspect at the level of the calcaneocuboid joint. All tendons and nerves are sharply divided. Vessels are treated by electrocauterization or ligation. A disarticulation in Chopart is performed, followed by removal of the talus and the cartilage bone of the distal tibia and fibula. An osteotomy of the calcaneus is done just behind the calcaneocuboid joint to diminish the loss of leg length because of removal of the talus. Performing a $70^{\circ}$ osteotomy decreases the tension on the Achilles tendon after rotation of the calcaneus. Nevertheless, lengthening of the Achilles tendon is often necessary, which can be done percutaneously. There are several techniques to fixate the calcaneus to the tibia. An external fixator results in the best compression, but with high risk of pin tract infection. Internal screw fixation with large fragment parallel cancellous screws is another option. The skin should be closed in a two layer technique. Aftercare: Weight bearing is not allowed for 6 weeks. Rehabilitation is supervised by a physiotherapist and a rehabilitation-officer, who should already be consulted preoperatively.

Content: abstract for video presentation

Discussion: see abstract above

Disclosure: No significant relationships.

\section{V10}

TIBIOCALCANEAL NAILING OF THE ANKLE

\section{J. Stimac, D. Seligson, J. Statton}

Orthopaedic Surgery, University of Louisville, Louisville, KY, USA

Introduction: Tibiocalcaneal nailing can be used for fractures and also for post-traumatic arthritis. Tibiocalcaneal nailing avoids many of the complications of open reduction and internal fixation. The nailing allows immediate weightbearing, which is useful in patients who may not be compliant with non-weightbearing instructions.

Content: The video shows the surgical technique. The patient is positioned prone on a radiolucent table. The $\mathrm{C}$-arm is positioned perpendicular to the non-operative leg. It is essential to obtain alignment of the ankle prior to making the entry point in the calcaneus. The position for the foot is in neutral ankle dorsiflexionplantarflexion, $5^{\circ}$ of hindfoot valgus and $5^{\circ}$ of external rotation. The skin incision and starting point are located at the intersection of the longitudinal axis of the calcaneus and the tibia. A K-wire is inserted to the level of the distal tibia and acts as a guide for the cannulated starting reamer. A ball tip guide-wire is inserted. The canal is reamed with flexible reamers. The nail is placed and is locked with posterioranterior and lateral-medial calcaneal locking screws using the targeting arm. We typically use a long nail $(300 \mathrm{~mm})$. The proximal locking screws can be placed free hand.

Discussion: Tibiocalcaneal nailing is an excellent option for the treatment of acute tibial pilon fractures with significant comminution that are not amenable to plate fixation. It avoids complications of open reduction and fixation and allows the surgeon to control alignment of the tibiotalar joint.

Disclosure: No significant relationships.

\section{V28}

\section{UNILATERAL LCP OSTEOSYNTHESIS OF THE PROXIMAL TIBIA}

\section{Nerlich, J. Zellner, F. Baumann}

Department of Trauma And Reconstructive Surgery, University Hospital of Regensburg, Regensburg, Germany

Introduction: Fractures of the proximal tibia can be difficult to treat. A bone deficiency of the tibial head like in multifragmentary fractures reduces stability and narrows anchoring possibility of the osteosythetic implants.

This report is about a 53 year old female who experienced a complex knee trauma in a ski accident. Computertomographic imaging showed a multifragmentary depression type fracture of the proximal tibia classified as 41-B3 according to AO classification.

Content: Our video demonstrates open reduction and internal fixation with a LCP osteosynthesis of a multifragmentary proximal tibia fracture via an antero-lateral approach. 
The massive depression of the lateral articular surface is a real challenge. The ruptured lateral meniscus needs to be fixed.

The crucial point is to reconstruct the massive lateral depression fracture with a voluminous iliac crest bone graft to correct alignment of the articular surface in both planes. The fragments are adjusted and temporarily fixed with K-wires. Anatomic alignment of the joint is assured under fluoroscopy.

The LCP plate is adapted to the shape of the proximal tibia and fixed to the tibial shaft with lag screws. Angular stable bolts are applied to avoid a secondary loss of axis. Fixation of the torn lateral meniscus is an important element to achieve a good long term result.

Discussion: Unilateral LCP osteosynthesis of the proximal tibia is a viable method to fix multifragmentary tibial fractures. LCP offers a combination of angular stability and lag screws to achieve anatomic reconstruction of the joint line. It can be easily applied via an anterolateral approach.

Disclosure: No significant relationships.

\section{VISCERAL TRAUMA VIDEO SESSION}

\section{V13}

\section{SURGICAL REPAIR OF A TRAUMATIC RIGHT-SIDED DIAPHRAGMATIC HERNIA USING A VIDEO-ASSISTED OPEN TECHNIQUE}

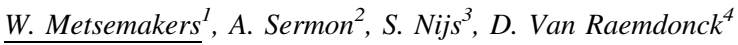

${ }^{1}$ Trauma Surgery, University Hospitals Leuven, Leuven, Belgium, ${ }^{2}$, University Hospitals Leuven, Leuven, Belgium, ${ }^{3}$ Department Of Traumatology, University Hospitals Leuven, Leuven, Belgium, ${ }^{4}$ Thoracic Surgery, University Hospitals Leuven, Leuven, Belgium

Introduction: We present a case of a surgical repair of a traumatic right sided diaphragmatic hernia using a video-assisted (VATS) open technique.

Content: A 45-year old female patient was brought to the emergency department after a motor vehicle crash. She complained of chest pain. Clinical evaluation showed a seat-belt sign. Plain X-ray examination revealed slight elevation of the right hemidiaphragm. A computed tomografic (CT) scan showed right lower lobe collaps and again elevation of the right hemidiaphragm. Due to suspicion of a diaphragmatic hernia we performed a VATS-approach for diagnosis and treatment. The postoperative course was uneventful.

Discussion: In case of a diaphragmatic hernia there are no specific findings that suggest the diagnosis. A high index of suspicion is of primordial importance. VATS is useful in thoracic trauma patients not only for diagnosis but also for treatment.

Disclosure: No significant relationships.

V14

\section{MANAGEMENT OF DUODENAL RUPTURE DUE TO BLUNT ABDOMINAL TRAUMA: PRIMARY REPAIR AND PYLORIC EXCLUSION}

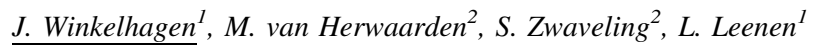

${ }^{1}$ Traumasurgery, university medical center Utrecht, Utrecht, Netherlands, ${ }^{2}$ Pediatric Surgery, university medical center Utrecht, Utrecht, Netherlands
Introduction: An 11 years old boy was transferred from a rural hospital to our level-one traumacenter $6 \mathrm{~h}$ after sustaining blunt abdominal injury due to a crash with his motorcross bike. His bloodpressure was $110 / 60 \mathrm{mmHg}$, his pulse was $110 / \mathrm{min}$. Physical examination showed guarding in the upper abdomen. CT-scan with enteral en intravenous contrast showed a rupture of the descending part of the duodenum and a grade III liver laceration.

Content: Emergency laparotomy was performed, a grade IV duodenal rupture was diagnosed.

Discussion: Our video shows the technique of primary repair and pyloric exclusion by gastrotomy and gastroenterostomy.

Disclosure: No significant relationships.

\section{V16}

\section{PATIENT WITH SEVERE SEPSIS_MEDICAL EMERGENCY}

\section{Omerbegovic}

Clinic of Anaesthesia and Intensive Care, University Clinical Centre Sarajevo, Sarajevo, Bosnia and Herzegovina

Introduction: Despite many improvements in understanding the pathophysiology of sepsis syndrome, the mortality rate of this condition is at disturbingly high level.

Content: Reviewing the abundant literature on the diagnosis and therapy revealed that late diagnosis of the sepsis syndrome, delayed antibiotic therapy and misinterpretation of the severity of the condition have been recognized as main casues of suboptimal therapy. In the last decade the large multinational initiative under the name of Surviving Sepsis Campaign gathered different groups of clinicians and investigators to analyse the abundant literature and give evidencebased recommendations on the therapy with main aim to decrease the overall mortality rate of sepsis. Strong emphasis was put to timely antibiotic therapy after obtaining cultures, and timely recognition of vital organ dysfunctions with adequate support, what was suggested in the therapeutic bundles for the first 6-h and the first $24 \mathrm{~h}$ after admission.

Discussion: The importance of early-goal directed therapy targeting haemodynamic and respiratory stabilization of the septic patient and prompt administration of antibiotic therapy together with the need for intervention of eradication of the source of infection should be recognized and initiated in the emergency department.

The importance of recognition of septic syndrome in the emergency department and the necessary measures for improving the therapeutic approach, stabilization and following of the patients are described according to recommendations from literature.

Disclosure: No significant relationships.

\section{V17}

\section{THE NATURAL COURSE AND MODERN SURGERY OF EXTRAPLEURAL HEMATOMA AFTER CAROTID ARTERY INJURY}

$\underline{\text { M.A. Rashid }}$

Surgery, 1 Division of Vascular and Thoracic Surgery, Department of Surgery, The Southern Norwegian Hospital of Kristiansand, Kristiansand, Norway. 2 Scandinavian Cardiovascular Surgery Center, Gothenburg, Sweden, Kristiansand, Norway 
Introduction: The Natural Course and Modern Surgery of Extrapleural Hematoma After Carotid Artery Injury Moheb A. Rashid, MD, PhD,1,2 1 Division of Vascular and Thoracic Surgery, Department of Surgery, The Southern Norwegian Hospital of Kristiansand, Kristiansand, Norway. 2 Scandinavian Cardiovascular Surgery Center, Gothenburg, Sweden. Objective: Hemothorax is an old malady with clear guidelines of management. How-ever, bleeding located into the chest but outside the parietal pleura recently termed ex-trapleural hematomal still poses critical issues in its management even in expert hands.

Content: Methods and Results: Our case is a 63-year-old man who was subjected to insertion of central vein catheter (CVC). The right carotid artery was accidentally penetrated causing serious and huge bleeding into the right chest. The pathognomonic sign of "parietal pleura-fat layer" described by Rashid 19992 in trauma patients was seen in CT scans of the chest, but was not observed due to the common attention obviously drawn by the more common type of bleeding "œhemothorax". Chest tube drainage was ineffective. He was operated on using a transaxillary mini-thoracotomy. Extrapleural massive coagu-lated blood was evacuated, the redundant thickened parietal pleura was excised, and the chest wall was meticulously examined for any bleeders. The operative and postoperative course was smooth, fast track, and uneventful.

Discussion: Conclusions: We conclude that, the diagnosis and correct management of extrapleural hematoma is yet to be known for several clinicians in spite of their excellent clinical experience. The pathognomonic sign is an ideal tool to make the diagnosis and a mod-ern minimal invasive surgery is to be recommended in huge hematoma. A review of the world literature and an operative video of the current case will be presented.

References: 1. Rashid MA, Wikström, T, Ortenwall P. Nomenclature, classification, and significance of traumatic extrapleural hematoma. J Trauma. 2000;49:286-290. 2. 2 Rashid MA. Value of video-assisted thoracic surgery in traumatic extrapleural hematoma. Thorac Car-diovasc Surg 1999;47:255-7.

Disclosure: No significant relationships.

\section{SKELETAL TRAUMA 2 VIDEO SESSION}

\section{V18}

\section{SINGLE MIDLINE INCISION AND EXTRA PERITONEAL APPROACH FOR TYPE C PELVIC FRACTURES}

\author{
K. Lansink ${ }^{1}$, M. Bemelman ${ }^{2}$, L.P.H. Leenen ${ }^{2}$
}

${ }^{1}$ Department Of Surgery, Trauma And Surgical Intensive Care Unit, University Medical Centre Utrecht, Utrecht, Netherlands, ${ }^{2}$ Surgery And Trauma, University Medical Centre Utrecht, Utrecht, Netherlands

Introduction: Internal fixation of type $\mathrm{C}$ pelvic fractures is a very challenging operation. Often a combination of approaches is necessary for adequate exposure to achieve reduction and fixation. During combined approaches the patient must be turned on the operating table, which is time consuming, and can be dangerous for the patient. There may be contraindications to lateral positioning because of other injuries. Repositioning of fracture elements can be impossible if other fragments of the pelvic ring have not yet been reduced or if other fragments have already been stabilised through another incision. A single midline incision and extra peritoneal approach, described by Biert et al. in JACS in 1995, can be used to explore the pelvic ring from the pubic symphysis to the sacrum.
Content: We show a singe midline incision, and extra peritoneal (Stoppa) approach in a patient with a type $\mathrm{C}$ pelvic ring fracture. The patient is positioned supine. A midline incision is made from the umbilicus to the pubic symphysis. The peritoneum is left intact. A Stoppa approach is used to reach the left SI joint. Reduction and fixation is performed with two dynamic compression plates, followed by reduction and plate fixation of the bilateral fractures of the superior ramus of the pubic bone.

Discussion: Advantages of this approach are: it is less invasive, the lateral femoral cutaneous nerve is hardly ever damaged, and there is no need to turn the patient.

Disclosure: No significant relationships.

\section{V19}

\section{ANTERIOR EXTERNAL FIXATION AND SACROILIAC PERCUTANEOUS FIXATION FOR COMPLEX PELVIC RING INJURIES}

\author{
A. Aprato, A. Massè, G. Governale, A. Stucchi
}

Pelvic Unit, San Luigi Hospital of Orbassano, Orbassano, Italy

Introduction: The emergency fixation of the pelvis significantly improves the outcome of the hemodynamically unstable patients. Complex pelvic injuries, such as lesions unstable on both horizontal and vertical planes, should be fixed anteriorly and posteriorly. In the first part of the video we present the surgical technique to perform an anterior external fixation that could be performed also in emergency. Content: The anterior fixation is based on four screws placed in the iliac crest and in the supra-acetabular region to obtain a good stability of the ExFx. Screws can be placed even without image intensifier. In the second part a posterior fixation with a percutaneous sacroiliac screw is presented: one or two screws are inserted percutaneously to close the sacroiliac joint or the sacral fracture using the image intensifier.

Discussion: If these two techniques are done in two stages, the ExFx could be removed and a common anterior pubic internal fixation could be performed as definitive treatment.

Disclosure: No significant relationships.

\section{V20}

\section{THORACOSCOPIC VENTRAL SPONDYLODESIS OF TRAUMATIC THORACOLUMBAR SPINE FRACTURES}

\section{R.J. Derksen, F.W. Bloemers, W.P. Zuidema, F.C. Bakker}

Traumasurgery, VU University Medical Center Amsterdam, Amsterdam, Netherlands

Introduction: The operative treatment of unstable thoracolumbar spine fractures can comprise of either posterior stabilization alone or combined with an anterior (ventral) stabilization. The necessity of additional ventral stabilization is dependent on type of fracture, degree of comminution, and associated ligamentous injury, all of which contribute to the amount of instability of the injury.

Content: In case of a spine fracture between level Th5 and L3 that needs a ventral spondylodesis, a thoracoscopic approach is the preferred approach. In comparison to a dorsolateral thoracotomy or a thoracophrenicolaparotomy, the added trauma due to the operation is markedly reduced. 
Patients experience less postoperative pain and have a shorter rehabilitation period. The use of a distractable cage in these procedures leads to a high percentage of patients in which the achieved correction is maintained both in the short and the long term.

Discussion: Both the indications, technique, pitfalls and complications of the thoracoscopic approach are dealt with in a comprehensive video.

Disclosure: No significant relationships.

\section{V21}

\section{CT GUIDED SACROILIAC OSTEOSYNTHESIS WITH THE USE OF OWN AIMING DEVICE}

\author{
M. Krivohlavek, S. Taller, R. Lukas, J. Sram
}

Trauma and Orthopaedic Centre, Liberec Regional Hospital, Liberec, Czech Republic

Content: Material: Series of 178 patients between the years 1993-2010 with the average age of 40.7 years and the follow up from 2 months to 3 years. Indications are fractures of the sacrum (uni- and bilateral) and displacement of the SI joint including six patients with sacral pseudoarthrosis and chronic sacroiliac instabilities. Methods: CT was used to plan the exact position of the implant and, during the procedure, to guide its precise insertion. All procedures were carried out as percutaneous technique with the use of the CT aiming device and digital angle gauge. Results: In all cases, correct implant insertion and complete bony healing was achieved. Only one serious intraoperative complication occurred-guidewire deviation from its planned direction without this being shown by CT scan. One late superficial infection was recorded. Implants temporally immobilizing the SI joint were removed at 6-9 months after the operation with no record of secondary dislocation.

Introduction: $\mathrm{CT}$ guided osteosynthesis of the sacroiliac region with the use of our own CT aiming device. The method and device are used since 1993 with 178 patient treated. The use of our CT aiming device is presented.

Discussion: Conclusions: The CT-guided procedure assesses the shape, course of fracture lines and distance between the injured structures. It also enables us to plan the direction, angle and length of the sacroiliac screw in three planes. CT aiming device is used to apply precise measures from $\mathrm{CT}$ into the screw application and thus placing it into the narrow "safe zone" of this region.

Disclosure: No significant relationships.

\section{MINIMALLY INVASIVE SURGERY}

\section{$\mathbf{V 2 3}$}

\section{MINIMALLY INVASIVE SURGERY IS SAFE AND EFFECTIVE FOR URGENT AND EMERGENT COLON OBSTRUCTION: A CASE OF ACUTE OBSTRUCTED HIRSCHSPRUNG'S DISEASE IN A YOUNG ADULT}

\section{N. Balalaa, N. Balalaa}

\section{Surgery, Alain hospital, Alain, United Arab Emirates}

Introduction: Acute large bowel obstruction by suspicious of Hirschsprung's disease (HD) in the Adolescence is a challenging matter in terms of establishing the diagnosis, obstruction release, fast recovery as well of cosmetic issues. The video presentation aims to show a multistep laparoscopic procedure on emergency management of acute obstructed HD.

Content: A 17-year-old woman who had suffered from severe constipation since 2 years of age. She developed significant abdominal distension and peritonismus. A computed tomographic scan confirmed significant fecal loading of the entire colon and rectum. An anal manometry revealed normal rectoanal inhibitory reflex, but a gastrographin enema showed spastic rectosigmoid segment. Rectal and colonic biopsies were not able to confirm the suspicious HD. Because of the severe fecal retention that was refractory to conservative management, a three step approach was necessary. In the emergency phase diagnostic laparoscopy with full wall biopsy from the rectosigmoid junction as well as laparoscopic diversion transverse colostomy with full wall biopsy of transverse colon has been performed as a first step procedure. After receiving the histopathology laparoscopic anterior resection with pouch anal anastomosis was done as a second step followed by closure of the diverting colostomy as a third step. Her postoperative course was uneventful with complete resolution of the symptoms.

Discussion: Minimally invasive colectomy by experienced surgeons appears to be safe and effective for appropriately selected patients with emergent and urgent conditions of the large bowel.

Disclosure: No significant relationships.

\section{V25}

\section{PERFORATED GASTRIC VOLVULUS}

\section{A. Fabregues ${ }^{1}$, T. Sánchez ${ }^{2}$, C. Camarero $^{2}$, C. Rey Valcárcel $^{2}$,

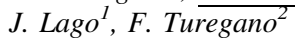

${ }^{1}$ General Surgery, Hospital Gregorio Marañon, Madrid, Spain, ${ }^{2}$ Surgery, Gregorio Marañón Hospital, Madrid, Spain

Introduction: Acute gastric volvulus is an unusual condition caused by the rotation of the stomach around one of its axes. Clinical suspicion should lead to an early treatment, thus preventing potential life-threatening complications.

Content: A 78-year-old female patient was brought in the ED with epigastric pain of $10 \mathrm{~h}$ duration, associated to nausea, with no vomiting. Her medical history included a paraesophageal hernia. On physical exam there were no peritoneal signs. X-rays showed a huge hiatal hernia and a full stomach. An unsuccessful attempt was made to place a nasogastric (NG) tube. CT-scan confirmed a huge herniation of the stomach leading to obstruction with signs of vascular compromise of the gastric wall. Emergency laparoscopy was undertaken which confirmed a strangulated paraesophageal hernia with partial necrosis of the gastric wall. A "sleeve-like" gastrectomy and Dor fundoplication were performed.

Discussion: Acute gastric volvulus is an unusual condition which can occasionally complicate a big hiatal hernia. Classically its clinical diagnosis is suspected by the triad of epigastric pain, retching and failure to pass an NG tube. CT scan is recommended to confirm this diagnosis and rule out potential complications. Initial treatment in uncomplicated cases consists of devolvulation by a NG tube or endoscopy. In complicated or refractory torsions a surgical approach is warranted, with or without resection, depending on the viability of the gastric wall. In any case, an antireflux technique is recommended, and the whole procedure can be carried out laparoscopically depending on the patient's condition.

Disclosure: No significant relationships. 


\section{V26}

\section{LAPAROSCOPIC TREATMENT OF SMALL BOWEL OBSTRUCTION}

\section{G. Tomasch, S. Uranues}

\section{Department Of Surgery, Medical University Graz, Graz, Austria}

Introduction: The most common etiologies of small bowel obstruction (SBO) in the Western world are adhesions after one or more laparotomies $(93 \%)$, abdominal wall hernia $(3.1 \%)$ and malignancy (2.9\%). SBO was previously considered to be a relative contraindication for laparoscopic management. As experience grows, more surgeons are treating SBO with laparoscopy. This presentation aims to show it can be applied safely in selected cases.

Content: This video presentation will show three selected cases with SBOs of different genesis.

Discussion: Each of these three cases had clinical symptoms and intraoperative findings typical for one of the most common etiologies of SBO. Criteria for safety were met and there were no complications during or after surgery. Conclusion: Laparoscopic treatment is feasible, safe and effective for SBO. The benefits are the well known advantages of the laparoscopic approach.

Disclosure: No significant relationships.

\section{SKELETAL TRAUMA 8 VIDEO SESSION}

V29

\section{FIXATION OF PILON (AO 43C) FRACTURES WITH AN ANTERO-LATERAL APPROACH}

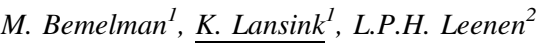

${ }^{1}$ Surgery Trauma, University Medical Center Utrecht, Utrecht, Netherlands, ${ }^{2}$ Trauma Surgery, UMC Utrecht, Utrecht, Netherlands

Introduction: Open reduction and internal fixation of pilon fractures (AO 43C) remain a challenging operation. Due to the intra-articular character, anatomic reduction with a stable fixation is mandatory. This makes the MIPO techniques less attractive for precise reduction is only achievable through an open procedure. The antero-medial approach, which is slightly more popular, has the disadvantage of relative poor soft tissue coverage, making this procedure at risk for wound problems and thus infection.

Content: Using the antero-lateral approach creates two major advantages. The first step in reconstructing a pilon, osteosynthesis of the fibula, can be done through the same approach and secondly a thick anterior flap is created with vital muscle giving a better coverage of the osteosynthesis.

Discussion: In this video presentation we present the anterolaterale approach for pilon fractures, explaining all crucial steps of the approach, reduction and highlighting the difficulties in this slightly technical more demanding technique.

Disclosure: No significant relationships.
V30

\section{TECHNIQUE FOR SPRING PLATE FIXATION FOR LABRUM AND ACETABULAR RECONSTRUCTION IN PIPKIN IV FRACTURE DISLOCATION}

\section{F. Castelli}

Dea, Niguarda Ca Granda, Milan, Italy

Introduction: Background This technique is infuenced by the trochanteric slide osteotomy described by Mercanti in 1972 and modified by Ganz et al. [5] as to reduces the probability of AVN femoral head iatrginacally with modified use of Spring Plate advocated by Mast et al. [6] in 1989 to stabilise periarticular acetabular fragments.

Content: Technique The patient is positioned in the lateral position. The approach preserves and protects the profendus branch Of the MFCA, which becomes intracapsular at the level of the superior gemellus muscle. The gluteus medius and vastus latrralis remain attached to the trochanteric fragment thus creating a digastric muscle. The entire flap, including gluteus minimus is retracted anteriorly and superiorly to expose the superior capsule. The capsule is first incised anterolaterally along the long axis of femoral neck to prevent injury to deep branch of the MCFA. The goal of surgery was to obtained reduction of acetabular wall with reattachment of labrum with fixation of femoral head without further compromising the blood supply of femoral head. We reviewed seven patients retrospectively without any case of AVN

Discussion: Conclusion The advantage of this technique include simultaneous exposure and fixation of femoral head, acetabulum and labrum without further compromising blood supply of femoral head. Spring plate increases the ultimate strength of fixation of acetabular fragment. Spring plates provide an alternative to the extensive soft tissue dissection and stripping necessary to place a large pelvic reconstruction plate as a buttress and can provide fixation for too small fracture fragments as showed in this technique.

References: 1. Letournal E, Judet R Fractures of the Acetabulum. Berlin: Springer; 1993. 2. Matta JM Fractures of the Acetabulum: accuracy of reduction and clinical results in patient managed operatively within three weeks after injury. J Bone Jt Surg 1996;78A:1632-45. 3. Tile M Fractures of the pelvis and acetabulum. Baltimore: Williams and Wilkins; 1995. 4. Leunig M, Sledge JB, Gill TJ, Ganz R Traumatic labral avulsion from the stable rim: a constant pathology in displaced transverse acetabular fractures Arch Orthop Trauma Surg. 2003;123:392-5. 5. Ganz R, Gill TJ, Gautier E, Ganz K, Krugel N, Berlemann U. Surgical dislocation of the adult hip. A technique with full acess to femoral head and acetabulum without the risk of avascular necrosis. J Bone Joint Surg 2001; 83-B;1119-24. 6. Mast J, Jakob R, Ganz R. Planning and Reduction Technique in FractureSurgery. New York: Springer; 1989. 7. Rashleigh-Belcher HJC, Cannon SR. Recurrent dislocation of hip joint with aBankart-type lesion. J Bone Jt Surg. 1986;68B: 398-399. 8. Richter H, Huston JJ, Zych G. The use of spring plates in the internal fixation of Acetabular Fractures. J Orthop Trauma. 2004;18:179-181.

Disclosure: No significant relationships. 


\section{V31}

\section{TREATMENT OF A TIBIA ATROPHIC NON UNION}

\section{F.D. Wagner}

Septische Chirururgie, Berufsgenossenschaftliche Unfallklinik Murnau, Murnau, Germany

Introduction: The treatment of a long bone non union follows the principles of the "Diamond Concept". All necrotic bone has to be removed, the stabilisation has to be rigid, the bone biologie should be enhanced using bone morphogenic proteines. Remaining bone gaps are filled by spongeous bone transplantation and the patients conditions are optimised. In the following case presentation the five steps of treatment are shown.

Content: We present case of a 20 year old male. He suffers from a $2^{\circ}$ open fracture of the lower limb. Primary treaten by intramedullary nail he underwent a spongeous bone transplantation after 4 months for delayed bone healing. 6 Months later the patient was referred. We detect a wealthy young smoking worker. A atrophic non union was clinical and radiological shown. A bony side infection was excluded by biopsy. For reconstruction of the tibia we performed a segmental $2 \mathrm{~cm}$ resection, shortening and dynamic compression nailing by T2 Nail. The biology was enhanced unsind bone morphogeneic proteine 7 and reaming debris. The postoperative treatment runs with full weight bearing. He did no more smoking. 6 weeks later the bone shows radiological signs of healing, the patients was painless weight bearing. Thee months later he was back to work. The length deficit was treated by elevation of his shoe. The video shows the operation steps and the clinical results.

Discussion: In the video are the five steps of bone reconstructive surgery shown: Remove of dead bone, stability, optimise of perfusion, cell activation by mone morphogenic protein, autologous scaffold.

Disclosure: The author earns support by traveling and reembursement for lectures from Medtronic, Stryker, AO and AIOD. There is no influence in the scientific work of the author.

\section{Posters}

\section{ACUTE CARE SURGERY}

\section{P1}

\section{ACUTE APPENDICITIS IN PREGNANCY}

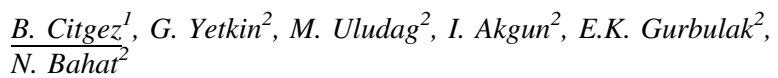

${ }^{1}$ General Surgery, Sisli Etfal Training and Research Hospital, Istanbul, Turkey, ${ }^{2}$ Sisli Etfal Training and Research Hospital, Istanbul, Turkey

Introduction: Acute appendicitis is one of the most common nonobstetric surgical pathology. Pregnancy continues to obscure the accurate diagnosis of acute appendicitis due to gestational physiological changes. The aim of the study is to evaluate the diagnostic methods and treatment options of pregnant acute appendicitis among the patients who admitted to general surgery clinic.

Materials and methods: Ten cases were diagnosed as acute appendicitis and underwent appendectomy during pregnancy, were evaluated between January 2005 and September 2010. All patients had preoperative ultrasonography and laboratory examination. Fetal heart rate and viability were followed with ultrasonography in perioperative period.
Results: Seven patients (70\%) were in 3rd trimester, two patients (20\%) were in 2nd and one patient $(10 \%)$ was in first trimester in admission. All patients had abdominal tenderness and the patients were preoperatively diagnosed as acute appendicitis by ultrasonography examination. Ten patients underwent appendectomy. Seven of ten $(70 \%)$ were acute appendicitis, two were perforated appendicitis, and one patient had the appendix without any pathology. The mean postoperative hospital stay was 3.8 days. There were not any maternal mortality and morbidity after operation.

Conclusion: Timely diagnosis of acute appendicitis in pregnancy can be difficult. In most cases a correct diagnosis can be arrived at on the basis of a history and physical examination with supportive routine laboratory tests. The delay in the diagnosis and the presence of different clinic signs of acute appendicitis due to physiologic changes during pregnancy leads to maternal and fetal morbidity and mortality. Recognition is important because early diagnose and prompt surgical intervention can reduce maternal and fetal mortality and morbidity in acute appendicitis.

Disclosure: No significant relationships.

P2

\section{ANALYSIS OF THE SIGNIFICANT DIAGNOSTIC SIGNS AND PROGNOSTIC FACTORS OF GANGRENOUS ISCHEMIC COLITIS}

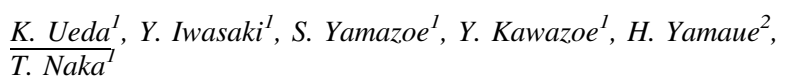

${ }^{1}$ Critical Care Medicine, Wakayama Medical University, School of Medicine, Wakayama, Japan ${ }^{2}$ Second Department Of Surgery, Wakayama Medical University, School of Medicine, Wakayama, Japan

Introduction: Gangrenous ischemic colitis occurs in about $15 \%$ of patients with acute ischemic colitis and requires surgery as soon as possible. However, it cannot almost be diagnosed easily in the elderly due to the lack of clear signs of necrotic inflammation and the mortality rate is about $60 \%$. We clarify the significant diagnostic signs and prognostic factors of gangrenous ischemic colitis.

Materials and methods: We reviewed 24 patients who underwent an emergency operation for gangrenous ischemic colitis between 2002 and 2010.

Results: The average age is 77.4 years in 24 patients. Before operation, $100 \%$ had some underlying atherosclerotic diseases and SIRS signs and $87.5 \%$ had abnormally elevated Lactate, whereas only $66.7 \%$ had peritoneal irritation sign. Furthermore, 45.8, 37.5, 70.8 and $29.1 \%$ in patients had DIC, shock vital, metabolic acidosis and perforation of colon, respectively. A Hartmann's operation which resected perfectly necrotic colon was performed in 23 patients and a colectomy followed by primary anastomosis was performed in one patient, and the survival rate was $58.3 \%$. Next, to examine the risk factors of survival in those patients, univariate and multivariate analyses using logistic regression were performed. It was only independent prognostic determinants of survival that the time from the onset of this disease to an operation was less than $24 \mathrm{~h}$.

Conclusion: To get the accurate diagnosis, SIRS sign, elevated Lactate and underlying atherosclerotic diseases in the elderly are very useful, although the peritoneal irritation sign is poor. And, the emergency operation within $24 \mathrm{~h}$ from the onset should be performed in order to improve the prognosis.

Disclosure: No significant relationships. 
P3

\section{ANASTOMOTIC COVERAGE AND DUODENAL DECOMPRESSION ARE THE ESSENTIAL FACTORS TO PREVENT ANASTOMOTIC LEAKAGE IN TRAUMATIC DUODENAL INJURY}

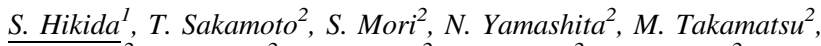

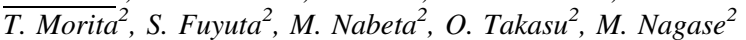

${ }^{1}$ Life Saving Emergency \& Critical Care, Kurume Univeristy, Hikida Clinic, Kurume City, Japan, ${ }^{2}$ Life Saving Emergency \& Critical Care, Kurume University Hospital, Kurume City, Japan

Introduction: In spite that the early diagnosis and the progress of surgical device has become possible in traumatic duodenal injury, safe and established surgery has not been established yet. Especially, the anastomotic leakage, once it occurs, becomes intractable and sometimes fatal. The aim of this study is to know what the risk factors of anastomotic leakage in traumatic duodenal injury are.

Materials and methods: We studied fourteen duodenal injury cases. They were divided into the uneventful group (nine cases) and the leakage group (five cases). The injury severity score (ISS), the revised trauma score (RTS), and the prognostic survival (PS) as the severity factors of trauma and the Apache 2 score and the time from the onset to operation as the preoperative risk factors were studied. As the operative factors, the additional coverage of the anastomotic site with adjacent tissue, the direct decompression with nasogastric or gastric tube, and the influence of choledochojejunostomy accompanied with bile duct injury or pancreatojejunostomy at pancreatoduodenectomy were analyzed.

Results: There were no statistical significant differences in the severity factors of trauma or in the preoperative factors. As to the operative factors, both the simple closure without coverage and the no duodenal decompression were significant risk factors. In the bile duct or pancreatic duct reconstruction case, duodenal or gastro-jejunal anastomotic leakage occurred following the leakage of choledochojenunotomy or pancreato-jejunostomy.

Conclusion: The coverage of anastomois, direct duodenal decompression, and safe anastomosis of bile duct or pancreatic duct to digestive tract are the essential factors to prevent anatomotic leakage in duodenal injury.

Disclosure: No significant relationships.

\section{P4}

\section{ASSESSMENT OF CHEST TRAUMA IN PATIENTS ADMITTED TO ACADEMIC MEDICAL CENTERS OF IRAN}

\section{Hossein Pour}

Trauma Research Center, Kashan University of Medical Science, 87196-74591, Iran

Introduction: Chest trauma is responsible for one fourth of deaths in accidents, so its early diagnosis and proper management is important. The aim of this study was evaluation of various aspects of chest trauma in patients admitted to trauma centers in Isfahan.

Materials and methods: One hundred patients with chest trauma referred to hospitals affiliated to Isfahan Medical University were enrolled to this cross sectional study. Data including age, sex, accident time, trauma mechanism, organ injury, hospitalization length, complications, and outcomes were all recorded and analyzed with Chi square and $t$ tests.
Results: Eighty percent of patients were male. The most common time of injury was between 12 p.m. and 1 a.m. (62\%). Blunt trauma was the common type of injury. The most common age range was 21-30 years. The main complication of trauma was hemothorax $(41 \%)$ and it was more common in penetrating injuries $(45 \%)$. Hospitalization length was below 10 days in $47 \%$, ten days in $12 \%$ and more than 10 days in $41 \%$ of patients. Associated head and abdominal injuries were found in 25 and $4 \%$ of patients, respectively. Death rate was $5 \%$.

Conclusion: Further consideration of chest injury is recommended in any case of trauma especially in blunt injuries. Epidemiologic studies in other parts of Iran can help in establishing appropriate criteria for chest trauma diagnosis and management in our country.

References: 1. Nirula R, Pintar FA. Identification of vehicle components associated with severe thoracic injury in motor vehicle crashes: a CIREN and NASS analysis. Accid Anal Prev 2008;40:137-141. 2. Vodicka J, Spidlen V, Safránek J, Simánek V, Altmann P. Severe injury to the chest wall-experience with surgical therapy. Zentralbl Chir. 2007;132:542-6. 3. Devitt JH, Pagliarello G, Simons J. The involvement of anesthetists in critical care medicine. Can J Anesth. 1990;37:119.

Disclosure: No significant relationships.

P5

\section{BLUNT LIVER AND SPLEEN TRAUMA: EPIDEMIOLOGY AND MANAGEMENT IN A TERTIARY HOSPITAL OF WESTERN GREECE}

\author{
A. Zygomalas, C. Spyropoulos, D. Karavias, M. Stavropoulos, \\ I. Maroulis, D. Karavias
}

General Surgery, University Hospital of Patras, Patras, Greece

Introduction: Liver and spleen are the most commonly injured organs in blunt abdominal trauma. Depending on trauma grade, conservative non-surgical approaches are the mainstay of treatment nowdays. The purpose of our work is to study the epidemiology and management of liver and spleen trauma in a population of Southwestern Greece.

Materials and methods: The records of all patients admitted to the Department of Surgery of our hospital with blunt abdominal trauma during the last decade (2000-2009) were reviewed and analyzed.

Results: In a total of 869 trauma patients, blunt liver trauma was revealed in 149 (Group A) and splenic trauma in 126 (Group B), while 24 patients had both types of injuries (Group C). There was no statistically significant difference in ISS score among the above patient groups. Male predominance was common in all cases. A conservative approach was undertaken for $114(76.5 \%)$ patients of Group A, 69 (54.8\%) of B, and 18 (70.8\%) of Group C. There was statistically significant difference in hospital stay between patients operated and managed non-operatively in both Groups A and B $(\mathrm{p}<0.0001, \mathrm{p}<0.0001)$. The mortality rate was low $(2,1.6 \%$ in groups A, B respectively and null in group $\mathrm{C}$ ).

Conclusion: Conservative management of hepatic and splenic injuries is associated with low overall morbidity and mortality rates and does not increase total hospital stay. Non-operative management is a safe approach for patients with liver or splenic trauma, depending on patient's hemodynamic status, in centers provided with the appropriate infrastructure.

Disclosure: No significant relationships. 


\section{P6}

\section{BLUNT SPLENIC TRAUMA IN CHILDREN: WHICH FACTORS INFLUENCE THE CHOICE OF TREATMENT?}

\author{
M.P.J. Teuben ${ }^{\text {, W. Wramer }}{ }^{2}$, L.P.H. Leenen ${ }^{3}$
}

${ }^{1}$ Surgery, UMC Utrecht, Utrecht, Netherlands, ${ }^{2}$ Wilhelmina Children's Hospital Utrecht, Utrecht, Netherlands, ${ }^{3}$ Department Of Surgery, University Medical Centre Utrecht, Utrecht, Netherlands

Introduction: Non-operative management (NOM) of blunt splenic injury in children has become the treatment of choice in hemodynamically stable patients. Although, we found by evaluating our pediatric population that nearly 20 percent of patients are treated operatively. As in most institutions, our treatment guidelines were revised over time. Therefore, our objective was to identify factors that affect the choice of treatment of splenic injury in children.

Materials and methods: All pediatric patients admitted with the diagnosis of blunt splenic injury to our level one trauma center, between 1 January 2000 and 1 September 2009 were selected from a prospectively filled trauma database. Multivariate analysis was performed to identify which factors predisposed for operative management.

Results: A total of 49 patients met the criteria for inclusion in the study. The median age(IQR) was 13(9-16). Injuries were caused by traffic accidents in half of the patients. A median Injury Severity Score(IQR) of 16(9-27) was found. Eight patients were treated operatively. Two of those patients developed hemodynamic instability during CT-scanning.

Multivariate analysis showed injury grade as independent predictor for surgical intervention. For every grade increase in splenic injury, the odds of operative treatment increases by a factor of 13.5. Remarkably, admission hemodynamic parameters demonstrated in a multivariate model analysis no significant relationship with surgical intervention

Conclusion: The decision to treat pediatric patients operatively in our institution can be predicted by injury grade and not by admission hemodynamic parameters. This is not in line with the current opinion to attempt NOM in all hemodynamic stable patients despite grade of splenic injury, but reflects current practice.

Disclosure: No significant relationships.

\section{P7}

\section{BODY PACKERS; A PLEA FOR CONSERVATIVE TREATMENT}

J.K. de Bakker, P.W.B. Nanayakkara, L.M.G. Geeraedts jr., E.S.M. de Lange, M.O. Mackintosh, H.J. Bonjer

\section{Surgery, Vu MC, Amsterdam, Netherlands}

Introduction: The incidence of smuggling and transporting of illegal drugs by internal concealment also known as body packing is increasing in the western world. The objective of this study was to determine the outcome of conservative and surgical approaches in body packers.

Materials and methods: Clinical data on body packers admitted to our hospital from January 2004 until December 2009 were collected. The protocol for body packers required surgery when packets were present in the stomach for more than $48 \mathrm{~h}$. Outcomes of the conservative and surgical group were assessed and analyzed. Morbidity and mortality were assessed in body packers with drug packets present in the stomach for less than $48 \mathrm{~h}$ and in those with gastric packets for more than $48 \mathrm{~h}$.

Results: During the study period more body packers were treated conservatively. Mortality was $2 \%$ in all patients and was due to intoxication. There were no significant differences of mortality, hospital admission time and ICU admission time in the compared groups with drug packets in the stomach for less or more than $48 \mathrm{~h}$. In $24 \%$ (4/17) of the patients with bad package material, a ruptured drug packet was found during surgery. In only one patient this resulted in death of the patient.

Conclusion: Drug packets in the stomach for more than $48 \mathrm{~h}$ are not an indication for surgery. We recommend that surgery should only be performed in body packers with signs of intoxication or ileus and reserve conservative treatment for all other patients.

Disclosure: No significant relationships.

P8

CAREER DEVELOPMENT FOR FEMALE SCIENTISTS IN MEDICINE-EXPERIENCE WITH THE TANDEMPLUSMED MENTORING PROGRAMME FROM A TRAUMA SURGICAL POINT OF VIEW

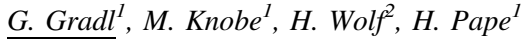

${ }^{1}$ Trauma Surgery, University Hospital Aachen, Aachen, Germany, ${ }^{2}$ Faculty of Medicine, RWTH Aachen, Aachen, Germany

Introduction: TANDEMplusMED was established at the University of Aachen in 2006. The programme has been designed specifically for female high potentials who intend to advance in their scientific career and qualify as a professor. TANDEMplusMED is a modular mentoring and career development scheme that consists of three elements: mentoring, coaching, networking. Participants are paired with a mentor of international scientific repute and are expected to actively establish and cultivate the relationship. Coaching topics include career planning, leadership skills and conflict management. The networking module aims towards the creation of a network of highpotential scientists from various special fields within the domain of medicine.

Materials and methods: Analysis of influence of the programme on career development of former and current participants in terms of occupational and scientific progress utilizing a personal questionnaire. Results: 69\% registered a change in their careers. 94\% related their occupational advancement to the programme.

Conclusion: Goals and benefits of the described programme concerning hospitals and research facilities include the retention of highly qualified female scientists, the promotion of gender equality in professional cultures as well as rising competitiveness and locational advantage due to innovative professional training strategies and career boosting measures. Benefits concerning the participants are strengthening of personal and professional competencies, obtainment of a better knowledge of structures and rules in the scientific community and useful skills to build up scientific, professional and personal networks.

References: Wolf, H. Hilfe zum Aufstieg durch Mentoring. Das Projekt TANDEMplusMED an der Medizinischen Fakultät Aachen. Hauptstadtkongress 2007. Medizin und Gesundheit; 2007. Bühren A. Zukunft der Orthopädie-Chirurgie: work-life-balance UND Karriere? DGU und DGOOC; 2007.

Disclosure: No significant relationships. 
P9

\section{EVALUATION OF SYSTEMIC INFLAMMATORY RESPONSE SYNDROME (SIRS) SCORE AS A PREDICTOR OF MORTALITY AND LENGTH OF HOSPITAL STAY IN TRAUMA PATIENTS}

\section{Hossein Pour}

Trauma Research Center, Kashan university of medical sciences, 87155.111, Iran

Introduction: The aim of this study is to evaluate of the relation between systemic inflammatory response syndrome (SIRS) score and mortality and length of hospital stay in trauma patients

Materials and methods: Seventy-three trauma patients admitted to Al- Zahra hospital in Isfahan medical university were enrolled to this study. The injury severity score and SIRS of each patient were calculated. In all patients, length of hospital stay, age, sex, trauma mechanism, and vital signs were recorded and blood samples were obtained. Patients were followed until their discharge or death. Logistic regression test was used for statistical analysis.

Results: Forty three percent of 73 patients had positive SIRS markers. Mean age of patients was $25.09 \pm 14.35$ years. Trauma mechanism was blunt in $84 \%$ of patients. Mean of Injury severity and SIRS were $12.09 \pm 10.15$ and $1.3 \pm 0.07$ respectively. Mean length of hospital stay was $5.6 \pm 5.2$ days. There is a significant correlation between SIRS and length of hospital stay $(\mathrm{p}=0.03)$, and SIR Ù S and death $(\mathrm{P}=0.01)$. WBC count has a significant correlation with length of stay $(\mathrm{p}=0.04)$ and mortality $(\mathrm{p}=0.02)$.

Conclusion: Our findings suggest that admission SIRS score of trauma patients may be used as a predictor of outcome.

References: 1. Bone R, Balk SA. Definitions/or sepsis and organ failure and guidelines for the use of innovative therapies. The ACCP/ SCCM consensus conference committee: American college of chest physicians/societyof critical care medicine. 1992;101:164455. 2. Faist E, Wichmann MW. Immunology in the severely injured. Chirurg. 1997;68:1066-70. 3. Schinkel C, Sendtner R, Zimmer S, Faist E. Functional analysis of monocyte subsets in surgical sepsis. J Trauma. 1998;44:743-8. 4. Moore FA, Moore EE. Evolving concepts in the pathogenesis of postinjury multiple organ failure. Surg Clin North Am. 1995;75:257-7.

Disclosure: No significant relationships.

\section{$\mathbf{P 1 0}$}

\section{GENDER SPECIFIC EVALUATION OF STUDENT'S CAREER PLANNING IN THE ANGULAR FIELD OF ORTHOPEDIC TRAUMA}

\section{S.C. Mooij, M. Holschen, R. Pfeifer, H. Pape, M. Knobe}

Department Of Trauma Surgery, University of Aachen, Aachen, Germany

Introduction: Major national reforms of medical education have been taken place during recent years caused by reasons as an increasing lack of fellowship trainees in operative medicine, particularly in orthopedics/traumatology. The purpose of this study was to evaluate the reasons of career decision as well as the motivations and expectations of medical students during the phases until individual decision for residency und career choice is made-especially under the gender aspect.

Materials and methods: Evaluation was conducted among 370 medical students between 2007 and 2010 [male (m)/female (f) $126 / 244 ; 23.6$ years; term 3-12]. The questionnaire consisted of 150 questions organized in: motivation, perception of learning/ teaching aspects especially in traumatologic lessons, future career plans-divided into: discipline of greatest interest (WF), discipline probably chosen with regard on personal resources (RF) especially for operative disciplines (oWF/oRF) and orthopedics/traumatology (tWF/ tRF) under the gender aspect.

Results: Overall response rate was $87.3 \%[\mathrm{n}=323, \mathrm{~m}=98(30.1 \%)$, $\mathrm{f}=228(69.9 \%) ; 23.6$ years; mean 6.3 terms]. 206 students $(63.8 \%)$ had a WF, the percentage increased significantly $(\mathrm{p}=0.048)$ within the first four terms; RF increased significantly $(\mathrm{p}=0.001)$ from $41.2 \%$ (1st term) up to $77.8 \%$ (last term). $53.9 \%(\mathrm{~m}=46.3 \%$, $\mathrm{f}=56.6 \%$ ) of the students had an oWF. Significant more females as males had an oRF $(n=40.9 \%, m=28.0 \%, f=45.8 \%, p=0.029)$. Both gender showed a damage of interest in traumatology $(\mathrm{tWF}=29.1 \%, \quad \mathrm{~m}=29.6 \%, \quad \mathrm{f}=29.0 \% \quad$ vs. $\quad \mathrm{tRF}=19.2 \%$ $\mathrm{m}=20.0 \% \mathrm{f}=19.1 \%) .41 .7 \%$ of the female students with oRF declared becoming a trauma surgeon whereas $58.3 \%$ were represented by visceral-/vessel-/plastic surgery and gynecology. Main reasons for this lack were high workload (13.4\%) and family planning $(18.2 \%$, $98.1 \%$ female opinion).

Conclusion: Our results show that during medical education the existing structures should be modified and the charm of combining theory and practical skills should be highlighted in order to enhance especially female student's perception of the discipline. Especially positive role models of female surgeons could encourage young interested female students to follow their interests.

Disclosure: No significant relationships.

\section{P11}

\section{IMMEDIATE 'TOTAL BODY' COMPUTED TOMOGRAPHY IN MULTI-TRAUMA PATIENTS-A SYSTEMATIC REVIEW}

\author{
J.C. Sierink, T.P. Saltzherr, J.B. Reitsma, J.C. Goslings
}

Trauma Unit Department Of Surgery, Academic Medical Centre, Amsterdam, Netherlands

Introduction: The aim of this systematic review was to assess the value of immediate 'total body' computed tomography (TBCT) during the primary survey of trauma patients compared with conventional radiographic imaging supplemented with selective CT. Materials and methods: A systematic search of the literature published between 1966 and 2010 was performed in the MEDLINE, EMBASE and Cochrane databases. Reports were eligible if they contained original data comparing immediate TBCT to conventional imaging supplemented with selective CT in trauma patients. The outcomes of interest were total mortality rate and time to diagnosis. Results: Four studies were included [1-4], describing a total of 5470 patients. The median sample size was 344. All four studies were observational studies with retrospective data collection. Mortality was reported in three studies [1-2,4]. Although the absolute height of mortality varied substantially across studies, mortality between TBCT and conventional radiographic imaging supplemented by CT was comparable within studies (pooled odds ratio $0.91,95 \%$ confidence interval 0.79 to 1.05 ). Time to diagnosis was described in three studies and in each of the studies these periods were significantly shorter in patients who underwent immediate TBCT (differences in medians ranging from 23 to $105 \mathrm{~min}$ ) [2-4].

Conclusion: This review shows differences in time to diagnosis in favour of TBCT during primary trauma survey compared with conventional radiographic imaging supplemented with selective $\mathrm{CT}$, but no difference in mortality rates. The substantial reduction in time to diagnosis is a promising feature of TBCT, but how this will translate 
to clinical outcomes requires well-designed and larger randomized studies.

References: 1. Huber-Wagner S et al. Effect of...multicentre study. Lancet. 2009;373(9673):1455-61. 2. Weninger P, et al. Emergency room...trauma center. J Trauma. 2007;62(3):584-591. 3. Wurmb TE, et al. Whole-body multislice...on time. J Trauma. 2009;66(3):658-65. 4. Wurmb TE, et al. Whole-body multislice...multiple trauma. Emerg Med J. 2010;28(4):300-4.

Disclosure: No significant relationships.

P12

\section{ISOLATED NECROSIS OF CECUM: A RARE CAUSE OF RIGHT-SIDED INFERIOR ABDOMINAL QUADRANT PAIN}

\author{
I. Lintzeris ${ }^{1}$, X. Agrogianni ${ }^{2}$, A. $^{\text {Lintzeri }}{ }^{3}$ \\ ${ }^{1}$ Surgery, General Hospital of Tripolis, Tripolis, Greece, \\ ${ }^{2}$ Postgraduate Student Of Medical School, University of Athens, \\ Athens, Greece, ${ }^{3} 3$ rd Hospital of IKA, Athens, Greece
}

Introduction: To the contradiction of ischemic necrosis of colon which is rather common in elderly population, isolated necrosis of cecum is an infrequent entity. The aim of our essay is to report such a rare case of necrotic cecum.

Materials and methods: A 70 year old man was admitted to our hospital because of intense abdominal pain localized in right lower quadrant. Clinical examination and laboratory testing revealed tenderness of abdomen, rebound and increase of leukocytes. Abdominal computed tomography indicated signs of inflammation in the right lower quadrant. The patient $\mathrm{s}$ medical history involved coronary disease. Therefore, he was receiving the appropriate medication.

Results: The patient underwent a surgical operation which revealed necrosis of cecum without any subnormal findings in appendix. Histological examination of surgical specimen confirmed the diagnosis. Conclusion: Isolated necrosis of cecum is a rare entity that occurs in patients with chronic diseases, heart disease or patients in shock. Its clinical manifestations are similar to those of acute appendicitis. However early diagnosis and surgical treatment promise a good prognosis for these patients compared to the most unfavourite prognosis of those suffering from general ischemic necrosis of colon that is associated with high rate of recurrence and morbidity.

References: 1. Dirican A, Unal B, Bassulu N, Tatli F, Aydin C. Isolated cecal necrosis mimicking acute appendicitis: a case series. J Med Case Reports, 2009;3:7443. 2. Perko Z, Bilan K, Vilovic K, Druzijanic N, Kraljevic D, Juriicic J, Srsen D, Pogorelic Z, Tomic S. Partial cecal necrosis treated by laparoscopic partial cecal resection. Coll Antropol. 2006;30(4):937-9. 3. Rist CB, Watt JC, Lucas RJ. Isolated ischemic necrosis of the cecum in patients with chronic heart disease. Dis Colon Rectum. 1984;27(8):548-51.

Disclosure: No significant relationships.

\section{P13}

\section{MEDICAL EVALUATION OF HOSPITALIZED DEADLY INJURED PATIENTS OF ROAD ACCIDENTS}

\section{T. Bogosi, P. Gergely, G. Merenyi}

Department of Traumatology, Karolyi Sandor Hospital, Budapest, Hungary
Introduction: The aim of this study is to make an assessment based on the data available about how can the time of locate and transportation of patient influence the outcome of injuries among those injured in road accidents. It is a question if the injured person is taken to hospital earlier the critical injuries could be eased or life could be saved.

Materials and methods: Retrospective analysis has been carried out among patients who sustained injuries in road accidents. The polytraumatised cases have been listed who were brought to the trauma department and died at the department no later than 35 days after their injuries.

Results: In all the data of 36 injured patients have been processed. The average age is 52 years, the average time of survival 3.4 days. Most of them (67\%) died within 24 h. $44 \%$ were pedestrians hit by car. The average time of transportation is $58 \mathrm{~min}$, by which the time between notification and reception at the hospital is meant. In each case autopsy was performed in the forensic medical institute of the local medical university. The injured have been grouped on the basis of their ISS (average ISS 52.1!) The clinical diagnoses also had been compared with the results of autopsy findings.

Conclusion: The average ISS of the injuries is very high, the state of the majority was critical. The factor of time has crucial importance regarding rescue, especially the cases involving bleeding and certain skull-brain injured. According to the reports of forensic autopsies each injured received the expected optimal treatment according to the medical rules. In cases of major bleeding, especially hemorrhage in a body cavity, the earliest possible hospitalization can influence the final outcome. The majority of brain injuries are not effected with the earlier possible hospital treatment.

Reference: bogosi.dr@gmail.com

Disclosure: No significant relationships.

\section{P14}

\section{OCCULT HEMOPEUMOTHORAX IN CHEST TRAUMA: CLINICAL SIGNIFICANCE-PROSPECTIVE STUDY}

\author{
I.Y. Mahmood ${ }^{\text {, }}$ Z.S. Tawfik ${ }^{2}$, S.R. Khoschnau ${ }^{2}$, A. Almadany, \\ S. Nabir ${ }^{3}$, K.I. Maull ${ }^{4}$
}

${ }^{1}$ Surgical Department, Trauma Section, Hamad Medical corporation, DOHA, Qatar, ${ }^{2}$ Surgical Department, Hamad medical corporation,

Doha, Qatar, ${ }^{3}$ Radiology, Hamad medical corporation, Doha, Qatar, ${ }^{4}$ Truama Surgery, Hamad medical corporation, Qatar, Qatar

Introduction: The increasing use of thoracic computed tomography (CT) has led to the recognition of intrapleural blood and air which is not initially evident on admission supine chest $x$-ray, defining the presence of occult hemopneumothorax. The clinical significance of occult hemopeumothorax and the role of tube thoracostomy is not clearly defined.

Materials and methods: During the recent 24 months period ending July 2010, data on 73 trauma patients with occult hemopneumothorax were recorded to determine whether tube thoracostomy was needed and, if not, to define the consequences of non-drainage. Data included hemothorax and pneumothorax size, demographics, injury severity, need for mechanical ventilation, indications for tube thoracostomy, complications and outcome.

Results: Tube thoracostomy was successfully avoided in 60 patients $(82 \%)$. Indications for placement of a chest tube in 13 patients included x-ray evidence of hemothorax progression (10) and respiratory compromise with oxygen desaturation (3); one patient suffered a parenchyma lung injury from tube placement and underwent thoracotomy. Mechanical ventilation was required in 19 patients. 
Patients with a maximum intrapleural fluid thickness of more than $1.5 \mathrm{~cm}$ and those with high injury severity score (ISS) were more likely to require tube thoracostomy.

Conclusion: Occult hemopneumethorax can be successfully managed without tube thoracostomy in most cases. Patients with high ISS score and CT detected fluid collection measuring more than $1.5 \mathrm{~cm}$ increased the likelihood of need for tube thoracostomy.

References: 1. Hill SL, et al. The occult pneumothorax: an increasing diagnostic entity in trauma. Am Surg. 1999;65(3):254-8.

2. Bilello JF, Davis JW, Lemaster DM. Occult traumatic hemothorax: Am J Surg. 2005; 190:841-4.

\section{P15}

\section{OPINION OF THE MEDICAL STAFF ON INTRAOSSEOUS ACCESS DURING EMERGENCY CARE}

\section{H. Turkan}

Anesthesia and Reanimation, Kasımpasa Military Hospital, Istanbul, Turkey

Introduction: Intravascular access is a vital component of emergency care and resuscitation. Success rate and time need for vascular access is crucial in the emergency patient under resuscitation. Intraosseous access (IO) has recently been revived in adults as an alternative when conventional intravenous access may be difficult or impossible. The aim of our study were both the determine opinion of the medical staff on IO with a survey and use of a battery driven device (EZ-IO system) for IO access by the doctors, nurses and paramedics on a bone model. Materials and methods: 60 doctors from several branch, nurses and paramedics were included in this study. All participants were inexperienced on IO access and saw EZ-IO system first time. After $30 \mathrm{~min}$ lecture and hands-on training, ease of insertion, number of attempts, and insertion time were recorded.

Results: There were $53(88 \%)$ successful placements of the EZ-IO system. The average time taken to place the EZ-IO system was $12 \mathrm{~s}$. At the end of the insertion, 55 (91\%) of participants stated that it was significantly easier and less noninvasive to insert the EZ-IO than they thought before attending to the research. Also they report that they can consider its use from now on.

Conclusion: The EZ-IO system has high success rates of insertion with inexperienced participants. If it is learned, the use of IO access will be increased during resuscitation. Therefore, education and training of the EZ-IO system for emergency and trauma team should be generalized.

Reference: 1. Mahajan R, Nazir R, Mehta S. An overview of intraosseous access. Anesth Analg. 2010;111(3):825-6.

Disclosure: No significant relationships.

\section{P16}

\section{OUR EXPERIENCE WITH PLASTRONE APPENDICITIS}

$$
\begin{aligned}
& \text { B. Citgez } \\
& \text { F. Ferhatoglu } \\
& \text { Fetkin }
\end{aligned}
$$

${ }^{1}$ General Surgery, Sisli Etfal Training and Research Hospital, Istanbul, Turkey, ${ }^{2}$ Sisli Etfal Training and Research Hospital, Istanbul, Turkey

Introduction: Acute appendicitis is a table of the distension and inflammation of the appendix vermiformis as a result of blockage of the appendix lumen by a variety of reasons. In some cases, distended lumen content can cause peritonitis by perforating wall of the inflamed tissue and spreading surrounding mesenteric tissue, or can cause plastron by wrapping inflammation by surrounding tissues. Physical examination reveals a palpable mass in the right lower quadrant. These cases are applied medical treatment and then undergo surgical treatment after 6-8 weeks.

Materials and methods: In our hospital, 15 patients admitted with abdominal pain and diagnosed plastrone appendicitis between 01.01.2006 and 01.10.2010 were reviewed retrospectively. The features of patients were reviewed, such as age, gender, duration of symptoms, period between diagnosis and surgery, hospital stay, radiologic images.

Results: There were 11 male and 4 female cases. The mean age was 34 (22-45). The mean duration of symptom was $7.5(5-12)$ days. The first ultrasound findings revealed a mass in $7.2(5-12) \mathrm{mm}$ diameter, compatible with plastrone appendicitis. All patients were given broadspectrum antibiotic therapy following hospitalization. Ultrasound was performed for all patients at the time of discharge, and found that masses were significantly decreased. None of patients needed emergency care. Patients were operated in their 6th to 8th weeks, and no complication occurred.

Conclusion: The reported incidence of recurrent appendicitis after conservative treatment of an appendiceal mass ranges from 0 to $20 \%$. Plastrone appendicitis should be treated conservatively, and operated later in case of recurrence of appendicitis will not occur.

Disclosure: No significant relationships.

\section{P17}

Moved to Oral Presentations

\section{P18}

\section{SAFETY AND EFFICACY OF FUROSEMIDE ADMINISTRATION IN FULLY RESUSCITATED TRAUMA PATIENTS}

D.D. Yeh ${ }^{1}$, J. Tang $^{2}$

${ }^{1}$ Department Of Surgery, San Francisco General Hospital, San Francisco, CA, USA, ${ }^{2}$ Department Of Anesthesia, University of California, San Francisco, San Francisco, CA, USA

Introduction: Massive fluid administration in injured patients may be required to maintain perfusion initially. Once hemostasis is achieved, however, additional net fluid gain may be detrimental. Forced diuresis with furosemide is an option not universally exercised by trauma surgeons.

Materials and methods: All injured patients admitted to the intensive care unit (ICU) at San Francisco General Hospital (SFGH) during a 2 year period were reviewed for receipt of furosemide. Data collected included demographics, physiologic data, labs, and medications. Fully resuscitated was defined as: no vasopressor requirements, mean arterial pressure $(\mathrm{MAP})>45$, heart rate $(\mathrm{HR})$ $<130$, and base deficit (BD) $<-6$.

Results: 22 patients received furosemide during the first 14 days of ICU stay. Mean age was 39 years (19-65) and mean ISS was 20 (4-34). The median day of first diuretic use was 3; mean cumulative fluid balance upon initial diuresis was 18.61 (0.2-44.8). Compared with the previous day, furosemide administration was associated with significantly higher urine output ( 3.0 vs. $2.21, \mathrm{p}=0.049)$ and less 24-h fluid gain $(1.28$ vs. $3.321, p=0.03)$. There was no significant 
change in HR, MAP, CVP, creatinine, Hct, BD, anion gap, or potassium related to furosemide administration.

Conclusion: Administration of furosemide in fully resuscitated, significantly fluid-positive critically ill trauma patients is safe and associated with an average of $36 \%$ increase in urine output and $61 \%$ decrease in fluid gain.

Reference: Lowell JAS, et al. Postoperative fluid overload: Not a benign problem. Crit Care Med. 1990;18(7):728-733.

Disclosure: No significant relationships.

\section{P19}

\section{SOCIOECONOMIC OUTCOME IN PATIENTS WITH MULTIPLE INJURIES 10+ YEARS AFTER TRAUMA}

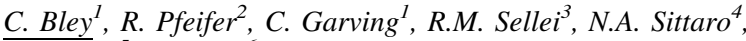 \\ C. Probst ${ }^{5}$, H. Pape
}

${ }^{1}$ Unfallchirurgie, Universitätsklinikum der RWTH Aachen, Aachen, Germany, ${ }^{2}$ Klinik Für Orthopädie Und Unfallchirurgie, Schwerpunkt Unfallchirurgie, Universitaetsklinikum Aachen, Aachen, Germany, ${ }^{3}$ Department Of Orthopaedic And Trauma Surgery, University Hospital of the RWTH AACHEN, Aachen, Germany, ${ }^{4}$ Hannover Reinsurance, Hannover ReInsurance, Hannover, Germany, ${ }^{5}$ Department Of Trauma And Orthopaedics, Medizinische Hochschule Hannover, Hannover, Germany, ${ }^{6}$ Department Of Trauma Surgery, University of Aachen, Aachen, Germany

Introduction: For trauma patients the impact on their socioeconomic status is important. Trauma patients often cannot be reintegrated into their work life, suffer financial losses or are compromised in their social activities. The aim of this study was to investigate the socioeconomic outcome of patients with multiple injuries after trauma.

Materials and methods: Patients with multiple injuries treated at a level I trauma center were re-evaluated to obtain functional and socioeconomic long-term outcome data following trauma. Inclusion criteria: minimum follow-up of 10 years, age between 3 and 60 years, injury severity score (ISS) $>16$, extremity fractures. Exclusion criteria: amputation and paraplegia. Patients were divided in three groups I-III (3-18, 19-50 and >50 years) and were invited for a physical examination and a battery of questionnaires.

Results: All study inclusion criteria were matched by 510 out of 637 recorded trauma patients. At time of re-examination financial losses were reported in $20 \%$ of patients in age group I $(n=140)$, in $44.3 \%$ of patients in age group II $(\mathrm{n}=341)$, and $37.9 \%$ in age group III $(\mathrm{n}=29)$, $(20$ vs. $44.3 \%, \mathrm{p}<0.0001 ; 20$ vs. $37.9 \%, \mathrm{p}=0.06 ; 44.3$ vs. $37.9 \%, \mathrm{p}=0.4$ ). Younger patients (group I) reported in $82.1 \%$ that their number of friends had decreased, whereas older patients less frequently reported a loss of friendship (group II 29.9\%, group III $6.9 \%)$, $(82.1$ vs. $29.9 \%, \mathrm{p}<0.0001 ; 82.1$ vs. $6.9 \%, \mathrm{p}<0.0001 ; 29.9$ vs. $6.9 \%, \mathrm{p}=0.022)$

Conclusion: A trauma with multiple injuries has a severe impact on the socioeconomic outcome of these patients. Older patients complain more about financial losses whereas younger patients report losses of friendship more frequently.

Disclosure: This study was supported by Hannover-Re Insurance. There are no further conflicts of interest.

\section{P20}

Moved to Oral Presentations
P21

\section{THE IMPERIAL COLLEGE LONDON SURGICAL SOCIETY INTERNATIONAL UNDERGRADUATE TRAUMA CONFERENCE 2010}

\section{Y.J. Chong, C.T. Cheong, A. Logeswaran, K. Bhatt}

Imperial College School of Medicine, Imperial College, London, UK

Introduction: On the 30th and 31st of October 2010, the Imperial College London Surgical Society hosted the largest international trauma conference for undergraduate medical students in the UK. This annual conference is modelled after the Advanced Trauma Life Support (ATLS) Course. It was first organised in 2006 to provide trauma education to undergraduate medical students at Imperial College London. In 2010, the conference expanded to 280 medical students from 15 countries across Europe and Asia who attended it in London.

Materials and methods: The conference featured talks by surgeons such as Professor Kenneth Boffard the President of the International Society of Surgery and Worldwide Director of the Definitive Surgical Trauma Skills (DSTS) Course, as well as 12 other eminent surgeons from the UK on various trauma management topics. Delegates had the opportunity to engage in 8 trauma related practical stations. 60 junior doctors and registrars from the UK participated as demonstrators for the practical stations. There was also a poster presentation competition for delegates to present trauma related case reports and research to surgeons as well as to other delegates. In addition, the conference had a showcase of an inflatable surgical theatre that simulated trauma surgery pioneered by Professor Roger Kneebone at Imperial College London.

Results: The results are a component of the conclusion.

Conclusion: With a conference fee of Â£50 compared to the ATLS fee of Â£525, this conference provides an accessible form of trauma education to undergraduate medical students. The popularity of this conference is such that it sells out every year. The model of this conference could be used to complement trauma education at the undergraduate level in various medical schools, providing medical students with exposure to the importance of ATLS principles in the management of patients.

Disclosure: No significant relationships.

\section{P22}

\section{THE PERCEPTION OF JUNIOR DOCTORS WITH AN INTEREST IN TRAUMA, ON THE CURRENT LEVEL OF EDUCATION AND MANAGEMENT OF TRAUMA IN THE UNITED KINGDOM}

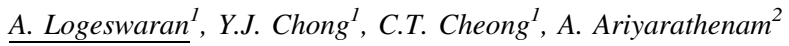

${ }^{1}$ Imperial College School Of Medicine, Imperial College, London, UK, ${ }^{2}$ Surgery, Royal Cornwall Hospital, Cornwall, UK

Introduction: Audit data by the National Confidential Enquiry into Patient Outcome and Death (NCEPOD 2007) identified severe shortfalls in the management of trauma in the UK. Our research was conducted at the Imperial College Surgical Society Trauma Conference in October 2010, which is the largest undergraduate trauma conference in the UK. The conference attracted 280 medical students from 13 different countries and 60 doctors from the UK as demonstrators. 
Materials and methods: We provided questionnaires to the 60 junior doctors who were demonstrating at the conference. All questionnaires were completed and returned during the conference.

Results: We had a response rate of $78 \%$. Our data showed that $87 \%$ of doctors felt that they were required to perform trauma skills as junior doctors. However, only $66 \%$ of respondents had attended ATLS before at some point during their career. $51 \%$ of those who completed the ATLS course did so during foundation training in spite of the course being recommended for first and second year of specialist training by the Royal College of Surgeons. 77\% had been part of a trauma team as junior doctors during night shifts, with $21 \%$ as most senior members on site. Only 57\% thought that most or all trauma cases were well handled in the UK. Perception of trauma education was poor, with $79 \%$ giving a rating of average and below average.

Conclusion: Our research highlights the general perception that trauma is still poorly taught and managed in the UK. This highlights the need for an earlier reassessment and re-audit of trauma care in the UK especially in view of the changes the National Health Services is undergoing.

Reference: NCEPOD 2007.

Disclosure: No significant relationships.

\section{P23}

\section{THE RESULTS OF OUR NONOPERATIVE APPROACH TO THE BLUNT ABDOMINAL TRAUMA}

\author{
M. Altıntaş ${ }^{1}$, A. çevik ${ }^{2}$, N. Bildik ${ }^{2}, \underline{\text { T. Yücel }}{ }^{2}$
}

${ }^{1}$ Second Surgical Clinic, Dr. Lütfi Kirdar Kartal Education and Training Hospital, Istanbul, Turkey, ${ }^{2}$ Second Surgery Clinic, Dr. Lütfi Kirdar Kartal Education and Training Hospital, Istanbul, Turkey

Introduction: Blunt abdominal trauma as a result of the studies related to the hemodynamic stability and absence of hollow organ injuries, non-operative treatment of solid organ injuries, the condition that the two will not change is accepted by everyone today. These two conditions ensure the existence of solid organ injuries; non-operative treatment creates the most difficult and most important part of our aim in this study, solid organ injuries in blunt abdominal trauma and underwent non-operative treatment protocol, developing practices and the approach to examine this issue.

Materials and methods: In our study, between January 2000 and October 2010 Dr. Lütfi Kirdar Kartal Training and Research Hospital. General Surgery, Emergency Service what, admitted after blunt abdominal trauma and subsequent follow-up and isolated solid organ injuries were determined retrospectively in 208 patients treated in the group of patients treated with all admissions. Conservatively seven drawn between the control and the lesions on CT with possible changes 9. uncu tested for comparison purposes, and radiological findings are shown in the previous status of the injury. The clinical and radiological improvement in patients 15-20 days after the occurrence and the rest by giving control abdominal CT scan 1 month after discharge were called to the control of the clinic.

Results: Developing isolated solid organ injuries after blunt abdominal trauma admitted 140208 patients were treated operatively and 68 conservatively. 127 male cases (61\%) and 81 (39\%) were female. The oldest member is 78 years old, the youngest 3 years old, mean age was 28.3. The mechanism of blunt abdominal trauma is examined, 133 patients $(64 \%)$ after the traffic accident (81

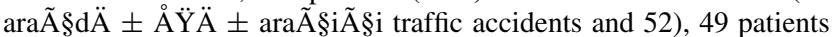
$(23.5 \%)$ after a fall from a height, and 26 patients $(12.5 \%)$, beaten were injured after the admission of 68 patients treated conservatively and 31 had worked in isolated liver after CT (44.5\%), isolated from the spleen in $23(34 \%), 11$ with liver and spleen (16\%), 3 'third of the liver, spleen and kidney with $(0.5 \%)$ injuries were identified and US'da observed in 3 patients CT demonstrated splenic injury. Ranked 7 of 42 grade I liver injury, injury by CT (17\%), 17 grade II (40\%), 12 grade III (29\%) and 6 of the grade IV (14\%), respectively. 6 of 37 grade I splenic injury is still rated by CT (19\%), 15 grade II (46\%), 7 grade III $(21 \%)$ and 4 of grade IV (14\%), respectively. Present in three patients with grade I and II renal injuries, respectively. One of these three patients, non-operative treatment, operative urology perop team is one of the patients were crossed, but an aid, was made. The other two patients after intravenous urography in the urology team worked, did not have urine leakage, and any aid, was made. Total of 89 patients with concomitant injuries, 37 head injuries $(42 \%)$, thoracic trauma in $21(24 \%)$, pelvic trauma, $6(6.5 \%), 15$ with head and chest trauma (16\%), with thorax and pelvis trauma (8\%) and 2 patients had head, chest and pelvic injuries, together with $(1.5 \%)$ respectively. Increase in diagnostic methods were analyzed by years of conservative treatment. Conservatively treated group of patients was calculated as the average length of hospital stay 8.3 days. This group of 38 patients $(56 \%)$ had to blood transfusion and the mean blood transfusion was $2.4 \mathrm{IU}$. These patients, systolic blood pressure $108 \mathrm{mmHg}$ on admission to the emergency department, and the mean diastolic blood pressure $65 \mathrm{mmHg}$, pulse $86 / \mathrm{min}$ and the mean hematocrit 33.4 Conservatively monitored and treated six patients in the development of hemodynamic instability despite appropriate fluid and blood replacement hepatoraphy and three of them were operated on, two to one in hepatoraphy and splenoraphy and splenectomy. Transition rate of non-operative treatment, operative treatment was approximately $8 \%$ of patients who were operated and these patients were included in the group 140 patients were operated in our group, 8 of them had died (6.3\% mortality). Associated organ injury is present in all of these patients (5 patients with head trauma and chest injuries, 2 cases of skull, pelvis and chest injuries, and 1 patient with pelvic and chest injuries were together), these patients were found to be lost for reasons other than abdominal surgery. Mortality rate was $0 \%$ for conservative treatment of non-operative treatment, operative treatment also did mortality in the last six patients. Conclusion: In this study, hospital emergency surgical ward, which develops after blunt abdominal trauma in the field of solid organ injury diagnosis and treatment has been applied with success to date by following the approaches.

Disclosure: No significant relationships.

P24

Moved to Oral Presentations

\section{CENTRALIZATION CRITERIA AND PRE-HOSPITAL CARE}

P25

\section{"REVISION" OF THE REVISED TRAUMA SCORE- CHANGES IN GLASGOW COMA SCALE BEFORE AND AFTER ARRIVING AT HOSPITAL AFFECT TRAUMA MORTALITY}

\section{A. Shiraishi, Y. Otomo}

Shock Trauma and Emergency Medical Center, Tokyo Medical and Dental University Hospital, Tokyo, Japan 
Introduction: Aims of the study were to assess relation between temporal changes in vital signs before and after arriving at hospital and trauma mortality and to "revise" the Revised Trauma Score (RTS) in reflecting temporal changes in vital signs.

Materials and methods: We selected records from Japan Trauma Databank with complete dataset to estimate RTS before and after arriving at hospital. We included RTS and temporal improvement in or temporal exacerbation of each RTS code as predictors into logistic regression model to predict in-hospital death. Based on the analysis, we modified the triage RTS (TRTS) to include changes in vital signs (modified-TRTS) and compared it with conventional-TRTS using receiver operating characteristics (ROC) analysis.

Results: A total of 11,937 records matched the selection criteria. Any exacerbations of coded Glasgow Coma Scale (cGCS), coded systolic blood pressure (cSBP) and coded respiratory rate (cRR) affected trauma mortality after adjustment for hospital RTS as OR (odds ratio) $1.52,95 \% \mathrm{CI}$ (95\% confident interval) [1.21-1.90], OR $1.06,95 \% \mathrm{CI}$ [0.82-1.38] and OR 1.20, 95\%CI [0.95-1.52], respectively. Similarly, any improvements in cGCS, cSBP and cRR affected trauma mortality as OR 2.14, 95\% CI [1.78-2.56], OR 1.16, 95\%CI [0.83-1.64] and OR 1.26 , 95\%CI [1.00-1.59], respectively. Changes in cGCS, even improvements in cGCS, significantly related to worsened trauma mortality, we therefore designed modified-TRTS to use worst cGCS (before or after arriving at hospital) instead of hospital cGCS. ROC analysis comparing conventional- and modified-TRTS showed similar area under curve of 0.89 and 0.89 , respectively, and sensitivity/ specificity of $0.90 / 0.71$ and $0.92 / 0.65$ at score 11 , respectively.

Conclusion: Both temporal exacerbation of and temporal improvement in GCS related to poor trauma outcome. Modification of the triage RTS in light of our results achieved good sensitivity with acceptable specificity in screening trauma patients, however needed further verification with another cohort.

Disclosure: No significant relationships.

\section{P26}

\section{CHILD AND ADOLESCENT MORTALITY DUE TO EXTERNAL CAUSES IN A BRAZILIAN CITY}

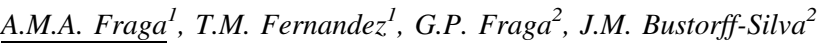

${ }^{1}$ Pediatrics, University of Campinas, Valinhos SP, Brazil, ${ }^{2}$ Division Of Trauma Surgery, State University of Campinas, Campinas, SP, Brazil

Introduction: Injury is the leading cause of death and long term disability in children worldwide. This is an analysis of all injuryfatalities in children between ages zero and 17 years, examined between January 2001 and December 2008, occurred in Campinas city, state of São Paulo, Brazil.

Materials and methods: Information was collected from the City of Campinas Medical Examiner's database. External causes were selected and grouped by age and mechanism. Demographics, injury mechanism, location and time of death were described.

Results: There were 662 medico-legal examinations in age $<17$ years who died of external causes, grouped in: $<1,1-4,5-9,10-14$, and 15-17 years of age. The most prevalent mechanism of injury leading to death was firearms injury $(41.9 \%)$, followed by road-traffic accidents $(28.2 \%)$ and drowning $(12.1 \%)$. Among the children younger than 5 years of age, the main cause of death was asphyxia. Pedestrian running over was an important mechanism of death (29.8\%) in children 5-14 years old. Penetrating trauma was the cause of death in $63.4 \%$ of $15-17$ age group. Death occurred at the scene in 270 cases $(49.2 \%)$. One hundred seventy two patients $(31.4 \%)$ died in the first $24 \mathrm{~h}$.

Conclusion: External causes remain an important cause of death in children in Campinas city. Specific strategies to decrease firearms and road-traffic accidents must be developed and implement to reduce the burden of childhood injury-related deaths.

Reference: Fraga AM, Fraga GP, Stanley C, Costantini TW, Coimbra R. Children at danger: injury fatalities among children in San Diego County. Eur J Epidemiol. 2010;25(3):211-7.

Disclosure: No significant relationships.

\section{P27}

\section{CRITERIA FOR CANCELLING HELICOPTER EMERGENCY MEDICAL SERVICES (HEMS)—DISPATCHES.}

\author{
G.F. Giannakopoulos ${ }^{1}$, F.W. Bloemers ${ }^{1}$, H.M.T. Christiaans ${ }^{2}$ \\ P. van Exter ${ }^{3}$, E.S.M. de Lange- de Klerk ${ }^{4}$, W.P. Zuidema ${ }^{5}$, \\ J.C. Goslings ${ }^{6}$, F.C. Bakker ${ }^{1}$
}

${ }^{1}$ Trauma Surgery, VU University Medical Centre, Amsterdam, Netherlands, ${ }^{2}$ Anesthesiology, VU University Medical Centre, Amsterdam, Netherlands, ${ }^{3}$ Regional Medical Manager Of Ambulance Care, GGD, Amsterdam, Netherlands, ${ }^{4}$ Department Of Epidemiology And Biostatistics, VU University Medical Center, Amsterdam, Netherlands, ${ }^{5}$ Trauma Surgery, VU-University Medical Centre, Amsterdam, Netherlands, ${ }^{6}$ Trauma Unit Department Of Surgery, Academic Medical Centre, Amsterdam, Netherlands

Introduction: In the Netherlands there is no consensus about standardized criteria for cancelling HEMS dispatches. In this study we assessed the ability of the primary HEMS dispatch criteria to identify major trauma patients. Furthermore we evaluated the predictive power of other early prehospital parameters in order to design a safe triage model for HEMS dispatch cancellations.

Materials and methods: All trauma-related dispatches of the HEMS during a period of 6 months were included. Data concerning prehospital information and inhospital treatment were collected. Patients were divided in two groups (major and minor trauma) according to the following criteria: ISS $\geq 16$, emergency intervention, ICU admission and inhospital death. Logistic regression analysis was used to design a prediction model for early identifying major trauma patients.

Results: In total, 420 trauma-related dispatches were evaluated, of which 155 concerned major trauma patients. The HEMS was more often cancelled for minor trauma patients ( 57.7 vs. 20.6\%). Overall, HEMS dispatch criteria had a sensitivity of $87.7 \%$ and a specificity of $45.3 \%$ for identifying major trauma patients. Significant differences were found for vital sign abnormalities, anatomical components and several parameters of the mechanism of injury. A triage model was designed for safely cancelling HEMS. This model correctly identified major trauma patients (sensitivity $99.4 \%$ ) with an acceptable figure of overtriage $(25.2 \%)$

Conclusion: The accuracy of our HEMS dispatch criteria is relatively low, resulting in high cancellation rates and low predictability for major trauma. The new HEMS cancellation triage model identified all major trauma patients with an acceptable overtriage and will probably reduce unjustified HEMS assists.

Disclosure: No significant relationships. 
P28

\section{EMERGENCY DEPARTMENT CARDIOPULMONARY RESUSCITATION IN PATIENTS WITH CARDIAC ARREST FOLLOWING CLOSED INJURIES. DOES IT MAKE SENSE?}

\author{
G.C. Georgiou ${ }^{1}$, V. Popko ${ }^{2}$, N. Vlassis $^{2}$, A. Kambouris ${ }^{2}$,
}

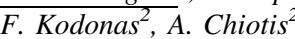

${ }^{1}$ Surgical Department, Xanthi General Hospital-Greece, Xanthi, Greece ${ }^{2}$ Surgical Department, Xanthi General Hospital-Greece, Xanthi, Greece

Introduction: To bring our experience and discuss the effectiveness of Emergency Department (ED) Cardiopulmonary Resuscitation (CPR) on patients sustained cardiac arrest, following blunt trauma.

Materials and methods: This is a retrospective study using our hospital's ED records from January 2005 to June 2010. Seventy four victims of blunt trauma were expired, of which 63 in the scene, 5 allegedly in transit and 6 in the ED. Cardiopulmonary Resuscitation was attempted in 12 patients $(12,6 \%)$. Data concerning patient's gender, age, type of injuries and cause of accident, result of the CPR and autopsy reports were examined. Physicians attended the cases were also interviewed.

Results: Cardiopulmonary Resuscitation was instituted in 10 males and 2 females, mean age 39.5 years (range 5-71 years). Cause of injury was determined as motor vehicle accident in 6 cases, crushing in 2 cases and fall from height in 1 case. On 2 patients ages 5 and 6 years old, CPR succeeded to restore heart function and peripheral pulses but did not revive the cerebrum. Both patients finally expired after 240 and $70 \mathrm{~min}$ respectively. In both cases cause of death was severe head injury. On four patients CPR temporarily brought back cardiac activity for less than $15 \mathrm{~min}$. Six patients did not respond to CPR.

Conclusion: The perspectives of successful CPR upon cardiac arrest after blunt trauma are dismal. We did not detect any factors influencing positively the outcome although it was noticeable that young individuals temporarily respond in CPR. Patients with severe blunt or thoracic injuries and imminent cardiac arrest should undergo emergency surgical intervention and resuscitation in the theatre over long CPR attempts in the ED. An often omitted aspect of the issue concerns the patients' next of kin, who must be early informed on CPR's effectiveness, to avoid mistaken expectations.

Disclosure: No significant relationships.

P29

\section{EMERGENCY SURGERY AND TRAUMATOLOGY IN EXTREME CONDITIONS}

\section{Bertocco $^{1}$, A. $^{\text {Adduci }}{ }^{2}$, C. Viglino $^{3}$, D. Colombero $^{4}$}

${ }^{1}$ General And Emergency Surgery Doctor, Azienda Ospedaliera e Polo Universitario Luigi Sacco, Moncalieri, Italy, ${ }^{2}$ Rianimazione Del Pronto Soccorso, Ospedale San Giovanni Battista, Turin, Italy, ${ }^{3}$ Orthopedic Doctor, Istituto Cellini, Turin, Italy, ${ }^{4}$ Orthopedic Doctor, Ospedale San Luigi, Orbassano, Italy

Introduction: The last great competition in the land of Africa runs through the western desert of Egypt, for more than 3,000 km: the Pharaohs. This is the FIM Rallies 'All Terrain' World Championship.
Materials and methods: In the land where the medicine was born, a group of specialized and trained doctors take care of the life of more than 500 peoples, including competitors, team supporters, organizers, staff, journalists, cameramen and photographers. The major traumas are the orthopaedic cases, emergency surgery and medicine surgery. On disposition no more than diagnostic procedures as like our experiences, some medical instruments, supported by five $4 \times 4$ offroad cars, two helicopters, one medical center in a big truck and one overland ambulance.

Results: We collected a great variety of diseases, from the diarrea fever to the death. We have to approach the patients in extreme conditions, such us high temperature, sand, dehydration, without proper diagnostic instruments etc.... In most cases, the first aid is following the rules of ATLS.

Conclusion: Because the speed is the most important thing for the competitors, the dynamic of all the traumas is always comparable to the normal red code in the "classic" ER in a normal Hospital. The most intriguing cases will be described. Disclosure: No significant relationships.

P30

HOW HELPFUL ARE THE USE OF AIS, ISS, RTS, TRISS, ETC., IN ASSESSMENT, TREATMENT AND PROGNOSIS OF POLYTRAUMA.

\section{A.S. Dogjani ${ }^{1}$, G.S. Zikaj ${ }^{2}$, B.E. Hasanaj ${ }^{2}$, S.L.CEKA ${ }^{2}$, K.D. Subashi ${ }^{2}$}

${ }^{1}$ General Surgery, Military University Central Hospital, Tirana, Albania, ${ }^{2}$ Kirurgji, Military Central Universitary Hospital, Tirana, Albania

Introduction: There are many classification systems traumatized patients have been published so far they include classifications for all levels. Relevant examples like physiological trauma scores (GCS, RTS, APACHE), anatomic, injury pattern based scores (ISS, NISS, ICISS), biological aspects, mixed scoring systems (PTS, TRISS, ASCOT). These scores are explained in detail and discussed as to their practicability.

Materials and methods: The study has a retrospective character and it has been realized within a period of time January 2009 till June 2009. This study consists of 50 cases all patients admitting and treating in NTC, which belongs to UCMH.

Results: Value of AIS is variable from 3 to 27 . Value of ISS is variable from 5 to 57 . In this study is evident that value of RTS has indirect relationship with value of ISS, that depending on trauma mechanism, situation of traumatic patient in coming by hospital, age of patient is very important.

Conclusion: The scoring systems have been used as a method to assess the injury severity of multi-system trauma. They have been used as a tool for triage, for emergency-room quality management, for educational reasons or, nowadays, in order to evaluate the cost effectiveness of either a complete hospital.

References: 1. Boyd CR, et al. Evaluating trauma care: the TRISS method. J Trauma. 1987;27:370-8. 2. Copes WS, Sacco WJ, Champion HR, Bain LW. Progress in Characterising Anatomic Injury. In: Proceedings of the 33rd Annual Meeting of the AAAM, Baltimore, MA, USA, pp 205-218. 3. Champion HR, et al. A revision of the trauma score. J Trauma. 1989;29:623-9.

Disclosure: No significant relationships. 


\section{P31}

\section{INTER-HOSPITAL TRANSFER IN JAPAN}

T. Fujita, M. Kitamura, M. Yamazaki, R. Yamaguchi, K. Nakazawa, Y. Uchida, H. Ikeda, T. Sakamoto

Trauma and Resuscitation Center, Teikyo University, Tokyo, Japan

Introduction: There is no protocol for an inter-hospital transfer in Japan. However, about $15 \%$ of traumatized patients was transferred for definitive care. The purpose of this study is to determine whether the inter-hospital transfer is acceptable or not.

Materials and methods: Twenty three thousand six hundred thirty one cases of ambulance transport were registered on Japan Trauma Data Bank (JTDB) 2004-2008. Ten thousand fifty one cases had data deficit and the remaining 13,580 are used to calculate the probability of survival (Ps) by TRISS method. We divided into direct transport group (DG) and inter-hospital transferred group (IG) and analyzed the unexpected death rate and unexpected survival rate. Unexpected death defines as the mortality of the patients who had over 50\% of Ps. Unexpected survival defines as the survival of the patients who had under $50 \%$ of Ps.

Results: The number of male patients was $8,189 / 11,716(=0.70$, SD $0.46)$ for DG versus $1,289 / 1,863(=0.70,0.46)$ for IG. The mean age (SD) was 51.3 (24.5) for IG versus $46.5(22.5)$ for DG $(\mathrm{p}<0.001)$ The mean injury severity score (SD) was 16.7 (10.9) for IG versus 17.8 (14.0) for DG $(\mathrm{p}<0.001)$ Unexpected death rate (SD) by TRISS method was $3.6(18.7) \%$ in IG and $5.2(22.2) \%$ in DG (p < 0.001). Unexpected survival rate (SD) was $30.8(35.5) \%$ in IG and 14.8 (46.4)\% in DG $(\mathrm{p}<0.001)$.

Conclusion: Inter-hospital transfer reduced the unexpected trauma death rate and improved the unexpected survival rate. The referral facilities with good outcome should designate the higher hierarchy in Trauma system in Japan.

Disclosure: No significant relationships.

\section{P32}

\section{THE ELDER PATIENT WITH HYPERTENSION AND HEAD INJURY RELATED TO IN-HOSPITAL MORTALITY}

\section{K. Morishita, A. Shiraishi, Y. Otomo}

Acute Critical Care And Disaster Medicine, Tokyo Medical and Dental University, Tokyo, Japan

Introduction: To assess characteristics and in-hospital mortality in trauma patients with systolic hypertension.

Materials and methods: From trauma patients' records of Japan Trauma Data Bank updated in 2008, we included patients with complete sets of revised trauma score (RTS) and numeric data of systolic blood pressure (SBP) on arrival at emergency departments. In modifying RTS, we divided score 4 in SBP subcategory into score $4 \mathrm{a}$ $(90-179 \mathrm{mmHg})$ and score $4 \mathrm{~b}(\geq 180 \mathrm{mmHg})$ and defined as categorical variables. Logistic regression analysis including all the parameters of RTS as explanatory variables predicted the in-hospital death. A stratified analysis by age, Glasgow coma scale (GCS), and Abbreviated Injury Scale (AIS) on head region was performed for the in SBP $4 \mathrm{~b}$ group patients.

Results: A total of 29,435 records matched the inclusion criteria. Those scored of $0,1,2,3,4 \mathrm{a}$ and $4 \mathrm{~b}$ in SBP subcategory consisted of $1,468,308,527,679,15,832$ and 1,612 patients, respectively. Inhospital mortality of those subcategory were $99,70,40,26,7$, and 17, respectively. After adjustment for all of RTS parameters, odds ratios for the in-hospital death of those subcategory were 1058.5, 32.0, 9.4, 4.8, 1.0 (reference) and 2.8, respectively. Stratified analysis showed that elder in age, GCS $\leq 5$, and AIS $\geq 3$ on head region were all found to be significant predictor of death in SBP $4 \mathrm{~b}$ group patients. Conclusion: A trauma patient with systolic hypertension $\geq 180 \mathrm{mmHg}$ scored four points in SBP category in RTS, however exposed to higher mortality similar to patients with three points in SBP category. The elder patient with hypertension and head injury related to mortality.

Disclosure: No significant relationships.

\section{P33}

\section{THE PRACTICALITY OF INCLUDING THE SYSTEMIC INFLAMMATORY RESPONSE SYNDROME IN THE DEFINITION OF POLYTRAUMA}

\author{
N.E. Butcher ${ }^{1}$, Z. Balogh ${ }^{2}$
}

${ }^{1}$ Trauma Service, John Hunter Hospital, Newcastle, NSW, Australia, ${ }^{2}$ Department Of Traumatology, John Hunter Hospital and University of Newcastle, Newcastle, NSW, Australia

Introduction: Background: The systemic inflammatory response syndrome (SIRS) is advocated as a predictor of outcome and a surrogate for physiological response to trauma. Some authors recommend the inclusion of SIRS into the definition of polytrauma. The practicality of daily collection $(0-72 \mathrm{~h})$ of SIRS data is unknown. Purpose: Assess the availability of SIRS variables in patient records in real time.

Materials and methods: A prospective observational study was performed on consecutive trauma team activation patients over a 7-month period presenting at a level-1 trauma centre. SIRS variables (white cell count WCC, temperature TEMP, pulse and respiratory rate $\mathrm{RR})$ were collected at 24,48 and $72 \mathrm{~h}$ post-injury.

Results: 336 patients met inclusion criteria (age $41 \pm 20,74 \%$ male, ISS $15 \pm 11$, NISS $19 \pm 15$, MOF $3 \%$, Mortality $4 \%$, 25\% ICU admission). In $155 / 336$ patients (48\%) it was not possible to calculate serial SIRS scores due to missing data. The table summarizes the missing data:

\begin{tabular}{lc}
\hline Variable & Total data missing \\
\hline $24 \mathrm{~h}$ & \\
WCC & $23 / 336(7 \%)$ \\
TEMP & $9 / 336(3 \%)$ \\
PULSE & $1 / 336(0.3 \%)$ \\
RR & $4 / 336(1 \%)$ \\
$48 \mathrm{~h}$ & \\
WCC & $190 / 336(57 \%)$ \\
TEMP & $29 / 336(9 \%)$ \\
PULSE & $27 / 336(8 \%)$ \\
RR & $54 / 336(16 \%)$ \\
$72 \mathrm{~h}$ & \\
WCC & $183 / 336(54 \%)$ \\
TEMP & $74 / 336(22 \%)$ \\
PULSE & $72 / 336(21 \%)$ \\
RR & $101 / 336(30 \%)$ \\
\hline
\end{tabular}


Conclusion: Calculating SIRS scores prospectively based on real time bedside data is a challenge due to a high proportion of missing data. The practicability of including SIRS as a physiological component of polytrauma definition is questionable even in prospective fashion.

Disclosure: No significant relationships.

\section{CLINICAL ULTRASOUND IN ACUTE CARE SURGERY}

\section{P34}

\section{ACUTE CHOLECYSTITIS IN THE ELDERLY: CONSERVATIVE TREATMENT, ULTRASOUND MONITORING AND TREATMENT OPTIONS}

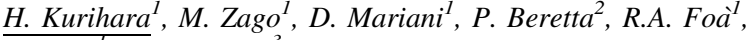 F. Butti ${ }^{I}$, A. Biloslavo}

${ }^{1}$ General And Emergency Surgery, Istituto Clinico Città Studi, Milano, Italy, ${ }^{2}$ Endoscopy And Gastroenterology Unit, Istituto Clinico Città Studi, Milano, Italy, ${ }^{3}$ Emergency Department, Ospedali Riuniti di Trieste, Trieste, Italy

Introduction: Laparoscopic cholecystectomy (LC) is the standard procedure in patients with acute cholecystitis (AC). In an ageing society, an increasing number of patients are not suitable for urgent surgery due to severe comorbidity. Predictive criteria for failure of conservative treatment could help in the management of these patients.

Materials and methods: From January 2010 to October 2010, 43 elderly patients $(23 \mathrm{M}, 20$, mean age 71$)$ were admitted with AC. Twenty-six patients underwent early LC (conversion rate $0 \%$ ). Seventeen patients (ten male, seven female) underwent conservative treatment due to severe comorbidity (mean APACHE II score 20). Median age was 81 (range 74-96). A daily clinical and ultrasound bedside evaluation (comprehensive of gallbladder transverse diameter and wall thickness) was performed in all cases. Four patients underwent endoscopic sphincterotomy and stone extraction. One patient developing septic signs and ultrasound worsening underwent; sphincterotomy and naso-cholecystic drainage, managed through ultrasound assisted daily lavages. Two patients underwent surgery due to development of septic signs and ultrasound signs of gallbladder necrosis.

Results: Simple conservative treatment was successful in 11 out of 17 patients, with a median LOS of 8 days. Patients who underwent endoscopic stone extraction (4/17) needed no further treatment. The patient with naso-cholecystic drainage showed an immediate relief and was discharged 4 days after the procedure. Patients requiring surgery had WBC counts (more than 20,000), diabetes mellitus and gallbladder diameter more than $6 \mathrm{~cm}$. Both were approached laparoscopically; one patient underwent laparotomic conversion. Second patient in 4th postoperative day required laparotomy and i.o. ERCP for a cystic duct leakage due to a preampullary stone not revealed by preoperative MRI.

Conclusion: Bedside ultrasound monitoring, high WBC counts, diabetes mellitus and gallbladder transversal diameter more than $6 \mathrm{~cm}$ could be negative predictive factors for conservative treatment of acute cholecystitis, but further prospective studies are mandatory. Endoscopic gallbladder drainage seems to be a valid method to avoid surgery or cholecystostomy, but case control studies are needed to evaluate the real benefit and limits of the method.
References: 1. Bakkaloglu H, Yanar H, Guloglu R, Taviloglu K, Tunca F, Aksoy M, Ertekin C, Poyanli A. Ultrasound guided percutaneous cholecystostomy in high-risk patients for surgical intervention World J Gastroenterol. 2006;12(44):7179-82. 2. Mutignani M, Iacopini F, Perri V, Familiari P, Tringali A, Spada C, Ingrosso $M$, Costamagna $G$. Endoscopic gallbladder drainage for acute cholecystitis: technical and clinical results. Endoscopy. 2009;41(6):539-46. 3. Barak O, Elazary R, Appelbaum L, Rivkind A, Almogy G. Conservative treatment for acute cholecystitis: clinical and radiographic predictors of failure. Isr Med Assoc J. 2009;11(12):739-43.

Disclosure: No significant relationships.

\section{P35}

\section{DIAGNOSTICS OF BLUNT ABDOMINAL INJURY IN POLYTRAUMA PATIENTS. ULTRASOUND VS. CT SCAN}

\author{
F. Urbán ${ }^{1}$, B.L. Szeferinkin ${ }^{1}$, M. Kovács ${ }^{2}$
}

${ }^{1}$ Department of Traumatology and Hand Surgery, Debrecen University, Kenézy Hospital, Debrecen, Hungary, ${ }^{2}$ Central Radiology Diagnostics, Kenézy Hospital, Debrecen, Hungary

Introduction: In the last years there is a trend to start the diagnostics of polytrauma patients with whole body CTscan. In the examination of blunt abdominal injury it requires contrast material, takes some time, and puts a high dose irradiation on the patient. The authors checked the specificity, sensitivity and accuracy of the ultrasound in these cases.

Materials and methods: Retrospective analysis of 33 polytrauma cases treated between 01-07-2009 and 30-06-2010. Eight of the patients were female, and 25 male. The youngest was 7 , the oldest 96 years of age, 42.7 as an average. The average ISS was 36 .

Results: All the patients with one exception had urgent ultrasound performed by radiologist trained in sonography. One had emergent whole-body CT scan. There was one false-negative ultrasound finding in the case of a renal parenchyma lesion. The sensitivity of sonography was $96.9 \%$, the specificity $84.4 \%$, the accuracy was $81.8 \%$.

Conclusion: The ultrasound is a cheap, fast and repeatable investigation but it needs well trained specialist It is preferable in emergency cases and completely replaced peritoneal lavage/1/. The accuracy and specificity of sonography is limited in the diagnostics of retroperitoneal injuries. In hemodynamically stable patients CT scan with contrast material has to be preferred. Especially precise renal evaluation requires additional CT examination/2/. The findings of picture imaging diagnostics always have to be appreciated together with the physiological symptoms and the results of the laboratory tests. Ultrasound is a highly sensitive and accurate test for the detection of injuries requiring intervention in the hemodynamically unstable patient with blunt abdominal trauma. Given the potential for rapid deterioration in these patients, CT scan be problematic or contraindicated $/ 3 /$.

References: 1. The value of sonography in traumatology and orthopedics : Part 2: emergency diagnostics in blunt abdominal and thoracic trauma. 2008;111(12):958-64, 966-7. 2. Pasławski M. 2004;59(2):328-34. 3. Navid F, et al. Hypotensive patients with blunt abdominal trauma: performance of screening US1. Radiology 2005;235:436-43.

Disclosure: No significant relationships. 


\section{P36}

\section{RELIABILITY OF ULTRASONOGRAPHY FOR DIAGNOSING ACUTE APPENDICITIS}

\author{
A.H. Gökce ${ }^{1}$, A. Aren ${ }^{1}$, F.S. Gökçe $e^{2}$, N. Dursun ${ }^{3}$, A.Y. Barut ${ }^{4}$ \\ ${ }^{1}$ General Surgery, İstanbul Education and Research Hospital, \\ Istanbul, Turkey, ${ }^{2}$ General Surgery, Balıklı Rum Hospital, Istanbul, \\ Turkey, ${ }^{3}$ Pathology, İstanbul Education and Research Hospital, \\ Istanbul, Turkey, ${ }^{4}$ Radiology, İstanbul Education and Research \\ Hospital, Istanbul, Turkey
}

Introduction: Background: Abdominal ultrasonography is the most commonly used diagnostic tool for diagnosing acute appendicitis, which is one of the most common causes of acute surgical abdomen. In this study, we examined the reliability of ultrasonography for diagnosing acute appendicitis.

Materials and methods: Methods: In this prospective study we performed abdominal ultrasonography on 235 patients admitted to our surgical emergency department and diagnosed as acute surgical abdomen according to the physical examination and laboratory findings during 2007. These patients were surgically treated by appendectomy and the materials were pathologically examined.

Results: 235 patients were admitted to this study. 193 of these patients $(82.1 \%)$ were diagnosed as acute appendicitis, and $42(17.9 \%)$ of them diagnosed differently.133 (88.67\%) of 150 patients diagnosed as acute appendicitis on ultrasonography examinations were reported as acute appendicitis on histopathological examination. 60 of 85 patients diagnosed differently on ultrasonography examination were reported as acute appendicitis on histopathological examination.

Conclusion: The sensitivity of abdominal ultrasonography for diagnosing acute appendicitis $69 \%$. We calculated that the specificity is $60 \%$, positive predictive value is 0.89 , negative predictive value 0.30 , accuracy is 0.67 . Abdominal ultrasonography is a helpful diagnostic tool for diagnosing acute appendicitis. However, it should not be seen superior to anamnesis and physical examination findings.

References: 1. SCOAP Collaborative, Cuschieri J, et al. Negative appendectomy and imaging accuracy in the Washington State Surgical Care and Outcomes Assessment Program Ann Surg. 2008; 248(4):557-63. . 2. Ohmann C, Franke C, Yang Q, et al. Clinical benefit of diagnostic score for apandicitis. Arch Surg. 1999;134: 993-6.

Disclosure: No significant relationships.

P37

Moved to Oral Presentations

P38

ULTRASONOGRAPHIC EXAMINATION OF THE RADIAL AND ULNAR NERVES AFTER PERCUTANEOUS CROSSWIRING OF SUPRACONDYLAR HUMERUS FRACTURES IN CHILDREN: A PROSPECTIVE, RANDOMIZED CONTROLLED STUDY

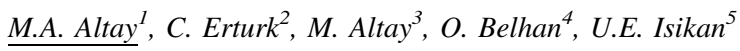

${ }^{1}$ Orthopaedics and Traumatology, Harran University Medical Faculty, Sanliurfa, Turkey, ${ }^{2}$ Orthopaedics And Traumatology, Harran University Medical Faculty, Sanliurfa, Turkey, ${ }^{3}$ Radiology, Private
Dunya Hospital, Sanliurfa, Turkey, ${ }^{4}$ Department Of Orthopaedic Surgery, Firat University Faculty of Medicine, Elazig, Turkey ${ }^{5}$ Orthopaedics And Traumatology, Harran University Faculty of Medicine, Sanliurfa, Turkey

Introduction: Iatrogenic nerve injury is still an important problem in pediatric supracondylar humerus fractures [1]. In this prospective, randomized controlled study, we used ultrasonographic imaging to evaluate the radial and ulnar nerves. We investigated the effects of the medial and lateral pins in a traditional cross-wiring technique as well as the effects of proximal and distal pins in a lateral cross-wiring technique.

Materials and methods: Twenty-nine consecutive children with completely displaced Gartland type III supracondylar humerus fractures were treated with the traditional (group T) or lateral (group L) cross-wiring technique. Group T consisted of the operated elbows of 15 children (11 males and 4 females) with average age 7.2 (range 3-12) years, while group L consisted of the operated elbows of 14 children ( 9 males and 5 females) with an average age of 8.5 (range 3-13) years. The contralateral unaffected elbows of the children were classified as controls (group T/C and group L/C). Ultrasonographic features of the radial and ulnar nerves as well as clinical outcomes were assessed.

Results: The mean follow-up period was 14.6 months (13-18 months) for group $\mathrm{T}$ and 13.9 months (12-19 months) for group $\mathrm{L}$. Our findings showed that despite the theoretical risk, ultrasonographic features of the radial nerve were not changed in group $\mathrm{T}$ or group $\mathrm{L}$. Ulnar nerve movement was reduced, and the diameter of the ulnar nerve's major axis during elbow flexion was larger $(p=0.040)$ than in elbow extension in the group $\mathrm{T}$ but not in the group $\mathrm{L}$.

Conclusion: In the lateral cross-wiring technique, ultrasonographic features of the radial and ulnar nerves were not affected. This technique provides satisfactory functional and cosmetic results that are comparable to outcomes from the traditional cross-wiring technique. Reference: 1. Belhan O, Karakurt L, Ozdemir H. Dynamics of the ulnar nerve after percutaneous pinning of supracondylar humeral fractures in children. J Pediatr Orthop B. 2009;18:29-33.

Disclosure: No significant relationships.

P39

\section{ULTRASONOGRAPHY AS PRIMARY IMAGING MODALITY IN PATIENTS WITH SUSPECTED APPENDICITIS}

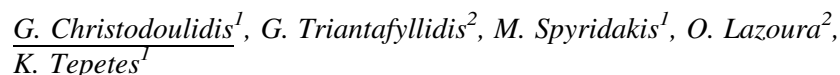

${ }^{1}$ General Surgery, University hospital of Larissa, Larissa, Greece,

${ }^{2}$ Radiology, University Hospital of Larissa, Larissa, Greece

Introduction: Preoperative imaging has been demonstrated to improve diagnostic accuracy in appendicitis. To evaluate the efficacy and accuracy of triaging patients with symptoms that suggest appendicitis by using ultrasonography.

Materials and methods: One hundred twenty two patients with suspected appendicitis were included in this study. After clinical examination, the patients were assigned to undergo ultrasound examination. Diagnosis of appendicitis or perforated appendicitis at time of operation was based on macroscopic findings. Radiologic findings were correlated with surgical, histopathologic and clinical follow-up findings.

Results: The median age was 21 years old. All of the 122 patients were evaluated by ultrasonography. In 74 patients the diagnosis of 
appendicitis was confirmed by ultrasound and clinical examination. The 74 patients were subjected to appendectomy. In four patients (5.4\%) the surgical findings revealed a normal appendix despite the positive diagnosis by ultrasonography. From the 48 patients with negative ultrasound examination 5 were operated and in 2 patients the histological findings proved a diagnosis of appendicitis. The sensitivity and specificity of ultrasonography was 85 and $96 \%$.

Conclusion: The use of ultrasound as the primary imaging modality seems to be accurate for diagnosis in patients with findings suggestive of appendicitis. The great advantage is that ultrasonography is noninvasive and does not use ionizing radiation.

Disclosure: No significant relationships.

\section{P40}

\section{UNSTABLE HEMODYNAMICALLY TRAUMA PATIENTS WITH HEMOPERITONEUM: WHY SHOULD WE GO TO CT SCAN FIRST?}

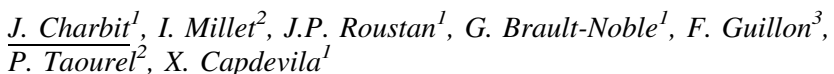

${ }^{1}$ Département Anesthésie Réanimation Lapeyronie, Réanimation Traumatologique, Hôpital Lapeyronie, CHU Montpellier, Montpellier, France ${ }^{2}$ Département De Radiologie, Hôpital Lapeyronie, CHU Montpellier, Montpellier, France ${ }^{3}$ Service De Chirurgie Viscérale Et Traumatologique, Hôpital Saint-Eloi, CHU Montpellier, Montpellier, France

Introduction: With unstable hemodynamically trauma patients, a laparotomy for hemostasis should be decided in the presence of free intraperitoneal effusion by Focus Assessment with Sonography for Trauma (FAST), but what is the incidence of peritoneal active bleeding $(\mathrm{PAB})$ and extraperitoneal active bleeding $(\mathrm{EPAB})$ in these cases?

Materials and methods: We have reviewed all trauma patients who were consecutively admitted in our level I regional trauma center with moderate or abundant hemoperitoneum associated with a systolic blood pressure $\leq 90 \mathrm{mmHg}$ within the first $15 \mathrm{~min}$ of their admission. The active bleeding $(\mathrm{AB})$ were defined as lesions which required intervention of hemostasis.

Results: On 671 patients admitted for major trauma, 110 patients met the inclusion criteria; 99 (90\%) underwent a whole body CT scan after initial resuscitation and $11(10 \%)$ immediate exploratory laparotomy for hemostasis. Thirty-seven patients (34\%) had PAB, 34 $(31 \%)$ EPAB, $20(18 \%)$ both associated and $19(17 \%)$ had no AB. An abundant hemoperitoneum was independent predictor of PAB [odd ratio $(\mathrm{OR})$ to $13.6,95 \% \mathrm{CI} 5.2-35.5(\mathrm{P}<0.001)]$ with $16 \%$ of falsepositives and $25 \%$ of false-negatives. The absence of exteriorized $A B$ was also significantly associated with PAB [OR 4.6, 95\% CI 1.1-22.2 $(\mathrm{P}=0.05)]$. Conversely, lactic acid, base deficit levels and resuscitation requirements (fluid therapy, vasopressor infusion or early transfusion) can not predict the presence of PAB.

Conclusion: Only $52 \%$ of unstable hemodynamically trauma patients with hemoperitoneum had PAB and $49 \%$ had EPAB; CT scan is an essential aid to define all the $\mathrm{AB}$ and to decide the adequate treatment for these patients.

Disclosure: No significant relationships.
DIFFICULT WOUND MANAGEMENT_VISCERAL TRAUMA

\section{P41}

\section{A NEW TECHNIQUE FOR DEFINITIVE TENSION-FREE CLOSURE OF LAPAROSTOMY}

M.C.W. Reis, C.T. Almeida, D.P. Santana, B.D.L. Rodrigues, E. Celso Santos, A.F.Z.B. de Andrade

General Surgery, Hospital da Baleia, Belo Horizonte, Brazil

Introduction: We describe a simple and definitive tension-free technique of abdominal wall reconstruction after laparostomy using prosthesis over the granulation tissue.

Materials and methods: From January 1998 to January 200515 patients were treated. Data were recorded prospectively including reason and timing for laparostomy, duration of the surgical procedure and postoperative course. The epithelium of the granulation tissue of the scar was completely scraped with scalpel. An incision was made along the border of the scar, including all scraped scar and the granulation tissue. The skin and subcutaneous fat was dissected off the anterior rectus sheath on each side, so the edges of the skin were brought to the midline tension-free. A monofilament polypropylene mesh was placed over the sanded surface and overlying the exposed anterior rectus sheath by $8 \mathrm{~cm}$. The mesh was secured to the aponeurotic tissue with interrupted sutures. Follow up was 45 months.

Results: Operating time ranged from 55 to $190 \mathrm{~min}$. Two patients developed wound infection. Mean postoperative length of hospital stay among patients without postoperative surgical wound infection was 2.1 days. There were no deaths or abdominal compartment syndrome related and no evidence of incisional hernia.

Conclusion: The technique described in this study provides adequate protection for the intra-abdominal viscera and reliable support for the abdominal wall, and incisional hernia has not occurred up to now. Disclosure: No significant relationships.

P42

\section{ANDROLOGIC EMERGENCIES: HOW TO TREAT AND SOLVE THEM. OUR EXPERIENCE}

\section{G. Cozzi, F. Mantovani}

Clinica Urologica I, Fondazione IRCCS Ospedale Maggiore Policlinico- $\mathrm{Ca}$ ' Granda, Milano, Italy

Introduction: We present the major andrologic emergencies and the different treatment options.

Materials and methods: Emergencies involving the penis are: frenulum laceration, paraphymosis, dorsal vein thrombosis, hematomas, skin avulsion, contusion or fracture of the cavernous bodies, and priapism. Imaging (ultrasonography, color-Doppler, urethrography MRI and contrast-enhanced CT) can be used in differential diagnosis. Frenulum laceration treatment can be conservative or interventional (frenuloplastic). Paraphymosis can be treated in local anesthesia; if needed, edema can drained with fine needle. Dorsal 
vein thrombosis is treated conservatively with heparinoid gel and antibiotic therapy in case of septic thrombosis. Treatment of traumas, hematomas and skin avulsion is dependent on their extension and on the presence of bleeding. Small hematomas can be treated conservatively with local ice, analgesics and anti-inflammatories. Skin avulsion treatment is surgical, in one or more times. Suspecting a cavernous bodies lesion, ultrasonography, cavernosography, or MRI can be useful. High-flow priapism often resolves by itself; if not, it can be treated with ice, compression, cavernous aspiration and irrigation with alpha-antagonists. Low-flow priapism can be treated with alpha-agonists intracavernous injection or with cavernous-glandular or cavernous-spongiosum shunts. Emergencies involving the scrotum can be septic (abscess, gangrene), traumatic (skin laceration, testis contusion or fracture), or vascular (torsion of the testis or of the annexes). The implied imaging consists of ultrasonography, color-Doppler, and, seldom, contrast-enhanced CT. Treatment can be conservative, hyperbaric, interventional and then, if possible, reconstructive. Especially in case of testis torsion, surgery is mandatory because within the first hours it can save up to $90 \%$ of the testes.

Results: Whatever is the chosen treatment option, it is mandatory to consider the possible evolution of the condition, with special regards to invalidating scars, potency, and fertility.

Conclusion: Andrologic emergencies evaluation and treatment must be tailored on the single situation in order to solve to emergency and prevent its consequences.

Disclosure: No significant relationships.

\section{P43}

\section{CONSERVATIVE SURGERY VERSUS NONOPERATIVE MANAGEMENT OF SPLENIC INJURIES IN POLYTRAUMA PATIENT}

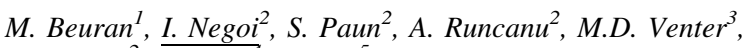

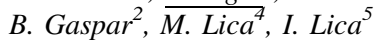

${ }^{1}$ General Surgery, Clinical Emergency Hospital of Bucharest, Bucharest, Romania, ${ }^{2}$ General Surgery, Emergency Hospital of Bucharest, Bucharest, Romania, ${ }^{3}$ Surgery, Emergency Hospital Bucharest, Bucharest, Romania, ${ }^{4}$ General Surgery, Clinical Emergency Hospital Bucharest, Bucharest, Romania, ${ }^{5}$ General Surgery, Clinical Emergency Hospital, Bucharest, Romania

Introduction: Although the selective nonoperative management represents the standard of care for splenic lesions, there is still a lack of standardization regarding polytrauma patients.

Materials and methods: Retrospective study of patients admitted in Emergency Hospital of Bucharest during the last 5 years. The selection criteria were: (1) Injury Severity Score (ISS) $\geq 17$; (2) splenic injury; (3) no splenectomy during initial management.

Results: Out of 60 patients there were 36 nonoperatively managed (NOM) and 24 with a splenic salvage surgical procedure (SSSP). Mean age was 36.9 (NOM) and 30.7 (SSSP) (p > 0.05). There were $51 \%$ traffic related injuries, $21 \%$ falls and $13 \%$ human aggressions. Mean ISS was 26.9 (NOM) and 30.04 (SSSP) ( $>$ > 0.05). Mean Revised Trauma Score was 6.29 (NOM) and 5.73 (SSSP) (p > 0.05). Out of 24 SSSP there were 10 local hemostatic applications, 3 splenoraphies and 2 partial splenectomies. There were $15 \%$ unnecessary/nontherapeutic laparotomies. Need for transfusion was 2.17 $(\mathrm{NOM})$ and $4.58(\mathrm{SSSP})(\mathrm{p}=0.05)$. Mean in-hospital stay was 10.5 (NOM) versus $16.6(\mathrm{SSSP})(\mathrm{p}>0.05)$. There were 7 early and 13 late deaths, most of them due to cerebral trauma (MNO vs. SSSP, $\mathrm{p}>0.05)$.
Conclusion: No additional early morbidity and mortality were noted, for polytrauma patients, related to splenic surgical management. Disclosure: No significant relationships.

\section{P44}

\section{DAMAGE CONTROL IN PENETRATING CHEST TRAUMA}

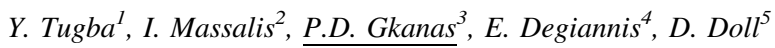

${ }^{1}$ Department of Surgery, University Hospital Of Ankara, Ankara, Turkey, ${ }^{2}$ Department Of Surgery, The General Hospital, Nafplion, Greece, ${ }^{3}$ Department Of Surgery, The General Hospital Of Nafplion, Nafplion, Greece, ${ }^{4}$ Chris Hani Baragwanath Hospital, Chris Hani Baragwanath Hospital, Johannesburg, South Africa ${ }^{5}$ Department Of Visceral Vascular And Thoracic Surgery, Philipps-University Of Marburg, Marburg, Germany

Introduction: Damage control procedure is well established and worldwide acceptable in abdominal trauma. Controversially, there are very few cases reports of chest packing in the medical literature.

Materials and methods: At the $\mathrm{CH}$ Baragwanath Hospital more than 2,000 emergency thoracotomics/sternotomies have been performed for penetrating trauma over the last 10 years but the present case is the only one that packing of the pleural cavity was performed.

Results: A 27 year old man was brought to the Trauma Resuscitation Area of the C.H Baragwanath Hospital, after he was stabbed at the right chest. On arrival he was in distress with GCS 13/15, BP 90/50, PR 132/min and RR 38/min. Immediate administration of 21 crystalloids started on admission and an intercostal drain was inserted in the right pleural cavity. There were $1,100 \mathrm{ml}$ of blood immediately drained. Because of this large amount of drainage and the fact that patients blood pressure and pulse rate did not improve a right anterolateral thoracotomy was done in theatre. Torrential bleeding was seen due to a stab wound of the right hilum and an intercostal artery. A formal right middle lobe lobectomy was performed in order to control the bleeding from the hilum and then we focused again on the intercostal artery's injury. This failed although all methods described were attempted. In desperation of not being able to control the bleeding and the patient getting further physiologically unstable it was decided to pack the pleural cavity with abdominal swabs. The patient was transferred ventilated to Trauma ICU and he returned to theatre in $48 \mathrm{~h}$. The bleeding had stopped and after 21 days he was discharged in good condition.

Conclusion: Packing of the pleural cavity although rare, can be a method of salvaging patients in extremies who sustain penetrating chest injury.

Disclosure: No significant relationships.

\section{P45}

\section{DEGLOVING INJURY, PATTERNS AND CLINICAL CLASSIFICATION}

\section{R. Martens, F.W. Bloemers, H.G. Licher, F.C. Bakker, W.P. Zuidema}

Trauma Surgery, VU-University Medical Centre, Amsterdam, Netherlands

Introduction: In degloving injuries there is an avulsion of the skin off the underlying tissue caused by a high energy deforming force. The soft-tissues primarily absorb the forces, although it can be accompanied by various types of fractures. 
Materials and methods: We retrospectively evaluated a series of deglovement injuries treated in our centre between January 2005 and July 2010. The skin lesion had to be larger than $5 \mathrm{~cm}$ and isolated hand and or feet injuries were excluded. Data were analyzed using SPSS $^{\circledR} 16.0$.

Results: The study group consisted of 60 patients with a total of 71 injured extremities. The average age was 52.6 years (range 19 till 98 years). There were 36 men and 24 female. Sixty-five percent had a lower extremity injury. The causes were $26.7 \%$ traffic accidents, in $8.3 \%$ machinery accidents. The soft-tissue injuries were classified according to the new Vudis score: type 1-abrasion more than $5 \mathrm{~cm}$, type 2-partial circular deglovement, type 3A-circular deglovement and type $3 \mathrm{~B}$ - circular deglovement with muscle injury. Four patients had a secondary amputation. Associated fractures showed four tibial, three femur and one humeral fracture. Differentiated treatment according to type was carried out. Excision of devitalised tissue followed by soft-tissue reconstruction with degreasing of the skin was used in $16.7 \%$ of patients. In four patients a VAC system was used. Fifty patients could be transferred to their home environment.

Conclusion: Deglovement injuries have a high impact on patient's psychology and physiology and require long term management by a dedicated team.

Disclosure: No significant relationships.

\section{P46}

\section{EFFECTIVE COMBINED VAC AND MESH TREATMENT IN MASSIVE ABDOMINAL LOSS OF DOMAIN}

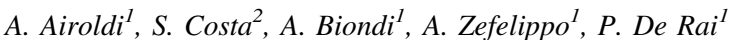 \\ ${ }^{1}$ General And Emergency Surgery, IRCCS, H. Ca' Granda,} Policlinico, Milano, Milano, Italy, ${ }^{2}$ General And Emergency Surgery, IRCCS, H. Ca' Granda, Policlinico, Milano, Italy

Introduction: The inability to close the abdomen due to tissue loss, lost domain or extreme visceral swelling are a mandatory indication for laparostomy in order to prevent abdominal compartment syndrome. Failure to close the fascia is one of the major drawbacks of laparostomy. A staged management of abdominal closure in case of massive lost domain by means of Vacuum Assisted Closure (VAC; $\mathrm{KCI}$ International) and MESH is presented.

Materials and methods: A 50-year old obese male patient, affected by huge inveterate post-traumatic diaphragmatic hernia underwent mesh repair by thoracic approach with incomplete reduction of the viscera. The patient needed an early postoperative urgent surgical reintervention for displaced rightward mediastinum related to postoperative ileus. The diaphragmatic defect was repaired through laparotomy by plication of the mesh. At the end of the procedure, abdomen closure was impossible due to massive lost domain. We applied a temporary polypropylene mesh sutured to the fascial edges under VAC dressing (125 mmHg).

Results: Every 48 h, VAC dressing was changed and mesh gradually tightened by running suture. The mesh prevented fascial retraction allowing fascial approximation while maintaining intra-abdominal pressure within normal limits. The progressive reduction of the defect allowed a delayed primary closure with use of an absorbable mesh after 40 days. The VAC fluid collection decreased from 1,000 to $50 \mathrm{ml} /$ day at the end of treatment. The patient needed mechanical ventilation and 57 days ICU stay. No technical or systemic complications were observed.

Conclusion: Combined VAC and mesh management method is an effective option to allow a safe and fast closure in case of massive abdominal lost domain.
Reference: Petersson U. Vacuum-assisted wound closure and meshmediated fascial traction-novel technique for late closure of the open abdomen. World J Surg. 2007; 31:2133-37. Leppäniemi AK. Laparostomy: why and when? Crit Care 2010;14:216

Disclosure: No significant relationships.

\section{P47}

\section{ELECTRIC DECONTAMINATION OF THE SKIN IN THE PRESENCE OF FOREIGN BODIES}

\section{P. Reynders-Frederix}

Traumatology, University Hospital Gasthuisberg Leuven, Leuven, Belgium

Introduction: Investigation of the possibility of preventing contamination of metal pins passing through the skin with flexible silicone membranes coated with carbon for the sake of electric conductivity. Materials and methods: ATCC S. Aureus; P. Aeruginosa. We used two semicircular conductive carbon electrodes with a diameter of $48 \mathrm{~mm}$ and a thickness of $0.5 \mathrm{~mm}$. The direct current was provided by an adjustable current source for the agar petri dish and custom made constant current source for the goat model. Experimental Agar Model. There were three different setups, each with two conductive carbon electrodes that were placed on a Mueller-Hinton II Agar (Oxoid) that was incubated with S. Aureus McFarland 0.5 with a $1 \mathrm{~cm}$ gap between the electrodes. We applied $(4 \mathrm{~V} ; 1 \mathrm{~mA}),(3.5 \mathrm{~V}$; $500 \mu \mathrm{A}),(2.3 \mathrm{~V} ; 82 \mu \mathrm{A})$. An identical setup was created for a Pseudomonas aeruginosa experimental animal model. Saanen Goats were used. In every goat, we placed three percutaneous screws on both sides of the spine in the processus transversus. The two carbon conductive pads were placed underneath a cylindrical transparent polyethylene bio-incubator.

Results: Agar Model Correlation between the current intensity and inhibition zone around the carbon pads. ATCC Staphyloccocus aureus generated a larger inhibition zone than Pseudomonas aeruginosa. Goat Model We measured the non-appearance of bacterial growth as success and the appearance as failure. Early bactericidal effect was seen. All cultures, which were taken later, came back positive.

Conclusion: Direct, low amperage, current is not able to prevent bacterial growth in a pin-skin interface in goats.

Reference: van der Borden AJ. Biomaterials. 2007;28(12):2122-6. Disclosure: No significant relationships.

\section{P48}

\section{HEMOSTATIC SPONGE TACHOSIL AS A TREATMENT OPTION FOR PERFORATED PEPTIC ULCER. AN EXPERIMENTAL ANIMAL STUDY}

\section{G. Christodoulidis $^{1}$, M. Spyridakis ${ }^{1}$, D. Simeonidis ${ }^{2}$, A. Diamantis $^{2}$, E. Polychronopoulou ${ }^{2}$, K. Tepetes ${ }^{1}$}

${ }^{1}$ General Surgery, University Hospital of Larissa, Larissa, Greece, ${ }^{2}$ University Hospital Of Larissa, Larissa, Greece

Introduction: Gastrointestinal tract perforation is an emergent condition that requires prompt surgery. Tachosil is the only ready-to-use fixed combination of a patch sponge coated with a dry layer of the human coagulation factors fibrinogen and thrombin. The aim of our study is to evaluate the safety and efficacy of Tachosil as an alternative treatment option in patients with perforated peptic ulcer disease. 
Materials and methods: Twelve white New Zeeland rabbits were used in this study. A peptic ulcer creation model was performed and all the animals were subjected to laparotomy. At the site of the ulcer a small hole, $2 \mathrm{~cm}$ in diameter, was created. The Tachosil was applied down to the wound area. The covered area included a surface $2-3 \mathrm{~cm}$ beyond the opening in the surface of the stomach.

Results: Two rabbits died due to peritonitis the 3rd and the 5th day. In the rest nine rabbits the hole was fully covered by the sponge without any sign of leakage. The histopathological results revealed a mild inflammatory response at the site of application.

Conclusion: Despite the fact that more studies are needed, the first results indicate that the hemostatic sponge tachosil seems to be an effective alternative treatment option for perforated peptic ulcer disease.

Disclosure: No significant relationships.

\section{P49}

\section{INNOVATIVE APPROACH FOR PRIMARY CLOSURE OF RUPTURED LARGE INCISIONAL HERNIA WITH COMPLETE EVISCERATION}

\section{J. Rodrigues ${ }^{1}, \underline{\text { S. Mallidu }}{ }^{2}$}

${ }^{1}$ Department Of Surgery, Goa Medical College, Panaji, India, ${ }^{2}$ Department Of Surgery, Goa Medical College, Panjim, India

Introduction: Incisional hernias are challenging complications of abdominal surgeries. It occurs in about $2-11 \%$ of laparotomies. Spontaneous rupture of hernial sac is dreaded complications of the incisional hernias occurring in less than $1.4 \%$ of incisional hernias. The contributing factor for rupture include gender, obesity, smoking, malnutrition, wound infection and steroids. The management of ruptured incisional hernias aimed at preventing abdominal compartment syndrome. A staged closure is preferred in such situations [3], with expensive aids which are not available and unaffordable by patients in third world countries. Hence management of such cases is still a challenge to surgeons.

Materials and methods: we are presenting a rare case of ruptured chronic incisional hernia with psoriasis with complete evisceration of small and large bowel for a duration of $11 \mathrm{~h}$. The defect was primarily closed by giving release at bilateral external oblique aponeurosis lateral to rectus abdominis muscle and release incision of the thickened peritoneum and transversus abdominis muscle in the paracolic gutter [1]. The defect between the two recti is closed using prolene mesh and the skin defect is closed by a rotation flap [2].

Results: With the above mentioned method we were able to close a large abdominal wall defect without patient going into abdominal compartment syndrome at a cost affordable by patients in the third world countries as one stage procedure.

Conclusion: The above method is an innovative technique is cost effective and very useful in third world countries requiring minimum surgical skill with regular operation theatre setup.

References: 1. Massive abdominal wall hernia reconstruction with expanded external/internal oblique and transversals musculofascia. Plats Reconstruct Surg 1997;100;326-335. . 2. Sliding myofascial flap of the rectus abdominus muscles for the closure o f recurrent ventral hernias. Plast Reconstr Surg 1996;98 464-469. 3. Upper abdominal wall defects: immediate or staged reconstr uction? Plast Re constr Surg 199086 281-286. 5. The Burstabdomen J.R. Hampton ClinicalStudent, theRadcliffeInfirmary, Oxford.

Disclosure: No significant relationships.
P50

\section{NEGATIVE PRESSURE WOUND THERAPY FOR PERSISTENT THORACIC EMPYEMA AFTER SPONTANEOUS OESOPHAGEAL PERFORATION}

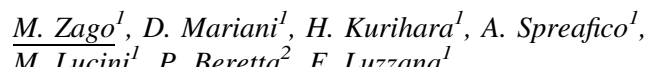

${ }^{1}$ General And Emergency Surgery, Istituto Clinico Città Studi, Milano, Italy, ${ }^{2}$ Endoscopy And Gastroenterology Unit, Istituto Clinico Città Studi, Milano, Italy

Introduction: Persistent thoracic empyema is a difficult problem complicating elective or emergency thoracic surgery and medical diseases. Standard treatments (open window thoracostomy, long lasting drainage) are invasive and discomfortable for the patients. Negative-pressure wound therapy (NPWT) was rarely applied in this field and reveals promising.

Materials and methods: Two patients with persistent thoracic empyema complicating spontaneous oesophageal perforation were treated with NPWT. NPWT $\left(V\right.$ ista ${ }^{\circledR}$, Smith\&Nephew) with gauzefiller was applied and renewed every 2-4 days. Proper position of the filler and evolution of the empyematous cavity was monitored with basal focused low-dose CT slices.

Results: The first pt (88 year-old, Alzheimer disease, admission Apache II score 22) was not operated on and treated with a suspended oesphageal prosthesis and thoracic drainage. Chronic empyema developed after drainage removal, resumption of oral intake and discharge, requiring readmission. NPWT (Vista ${ }^{\circledR}$, Smith\&Nephew) with gauze-filler was applied. Focused CT slices at day 7 and day 14 were performed, NPWT removed after 10 days. Pt is well 8 months after. The 2nd pt (86 year-old, healthy, admission Apache II 6), was operated on. An empyema developed after removal of the thoracic drainage. NPWT with Vista ${ }^{\circledR}$ gauze-filler was employed successfully, removed after 8 days. No CT scan was needed. The pt is well 6 months after. Conclusion: A few reports are available on NPWT in thoracic empyema. No reports after oesophageal perforation were found. When compared with conventional management, NPWT seems to accelerate wound healing, increase pt compliance, ameliorate nursing and recovery. The gauze-filler appears easier to apply than classical sponge filler. References: 1. Ann Thorac Surg. 2010;90(1):266-70. 2. Ann Thorac Surg. 2009;88(4):1131-6.

Disclosure: No significant relationships.

P51

\section{PENETRATING CARDIAC TRAUMA}

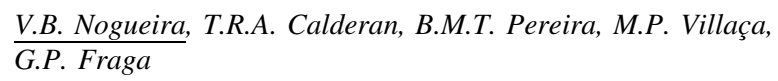

Division of Trauma Surgery, State University of Campinas, Campinas, SP, Brazil

Introduction: Injuries caused by gunshots and stab wounds are the major causes of cardiac trauma. The objective is to describe and compare the variables between patients with penetrating cardiac trauma in the past 20 years, identifying risk factors for complications and death.

Materials and methods: Review of trauma registry data followed by descriptive statistical analysis comparing the periods 1990-1999 (group 1, 54 cases) and 2000-2009 (group 2, 39 cases). 
Results: There was a reduction in the incidence of such injuries in recent years. Comparing the two groups, they were similar for age, mechanism of trauma (gunshot $\times$ stab), grade of cardiac injury and Injury Severity Score. Patients in group 1 were admitted in worse physiologic conditions than those in group 2, with lower systolic blood pressure (SBP) on admission (mean 87 vs. $109 \mathrm{mmHg}$, respectively), Glasgow coma scale (12.9 vs. 14.1), Revised Trauma Score-RTS (6.4 vs. 7.3), more complex cardiac lesions (74 vs. $48.7 \%$ of grade IV$\mathrm{V}$ lesions), and lower probability of survival ( $0.83 \mathrm{vs} .0 .93)$. The major risk factors for death were gunshot (13 times greater risk than stab), $\mathrm{SBP}<90 \mathrm{mmHg}$, Glasgow $<8$, RTS lower than 7.84, associated injuries, grade IV-V injury, and ISS $>25$. Although not significant, there was a reduction in mortality from 20.3 to $10.3 \%$.

Conclusion: In the last decade patients began to be accepted in better physiological condition with tendency to lower mortality.

References: Fraga GP, Espinola JP, Mantovani M. Pericardial window used in the diagnosis of cardiac injury. Acta Cir Bras. 2008;23(2):208-215.

Disclosure: No significant relationships.

\section{P52}

\section{POSTOPERATIVE INFECTIONS AFTER FEMORAL HEAD FRACTURES, TREATED WITH VACUUM ASSISTED TECHNIQUES.}

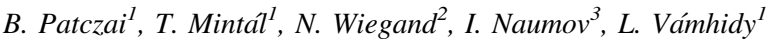

${ }^{1}$ Department of Traumatology And Hand Surgery, Institute of Musculoskeletal Surgery University Pécs, Pécs, Hungary, ${ }^{2}$ Department of Traumatology And Hand Surgery, Institut of Musculoskeletal Surgery University Pécs, Pécs, Hungary, ${ }^{3}$ Department Of Traumatology And Hand Surgery, Institute of Musculosceletal Surgery University Pécs, Pécs, Hungary

Introduction: Our department performs numerous operations resulting from either fractures or OA of the hip. Since the incidence is increasing every year, we are faced with more complications. One of the most severe is deep infection, propagating the implant.

Materials and methods: We introduced the KCI V.A.C. system to solve this problem in 2009. We followed up 27 patients in a 2-year-period.

Results: The postoperative hospitalization time was significantly reduced in all cases. We lost a patient on the 6th day following surgery, due to co-morbidity. In the other cases we reached good results. On two patients we could even perform prosthesis-retaining operations.

Conclusion: Based on our results the use of negative pressure wound care techniques is highly recommended not only in the hip region, but also in other fields of surgery.

Disclosure: No significant relationships.

\section{P53}

\section{SCIN DEFECT IN SURGICAL TREATED CALCANEAL FRACTURES, CASE REPORTS}

\section{Z. Todorović}

\section{Orthopaedics, General Hospital, Leskovac, Serbia}

Introduction: Purpose. The calcaneus is the most frequently fractured tarsal bone. Calcaneus fractures may occur as a result of falls from heights or from twisting injuries or through a pathologic process such as osteoporosis, cysts, and tumors. Calcaneus fractures are bilateral in $5-9 \%$ of patients; they are associated with compression fractures of the lumbar and/or dorsal spine in $10 \%$. These fractures are complicated by a compartment syndrome in $10 \%$ of cases, with half of these involving developments of clawing of the lesser toes or other chronic problems, such as stiffness and neurovascular dysfunction. We will demonstrate one unusually case scin defect in surgical treated calcaneal fractures. It is necessary to be great careful and care scin flap, according respect all principles operating treatment. It is necessary too, good preoperation planning in treatment fractures of calcaneus. With scin problems it is essential collaboration by plastic surgery.

Materials and methods: Methods; From 1998 to 2008, during 10 years in General Hospital Leskovac, Departement of Orthopaedics and Traumatology we operated 44 patients, with 50 intraarticular calcaneal fractures. The mean age of the patients was 51 years (22-78) and $80 \%$ of the fractures occured in men. And six patients the fracture were bilateral. One patient was operated with open calcaneal fracture. The most common injury mechanism was a fall from height. The mean period between injury and surgery was 7 days. The intraarticular fractures were classified according to Essex-Lopresti type II/ B Surgery was performed using the lateral extensile approach, as described by Benirschke and Sangeorzan. Basic target during operation has been acquirement more reconstruction subtalar joint and normalisation of anatomy of Bohlers angle.

Results: Patient male, 50 years old with calcaneal fracture after subsequently minor damage, downfall from height about $50 \mathrm{~cm}$. It has done open repozition and fiksation by calcaneal plate. Postsurgery has not put vacuum drainage. Third day after operation has been appeared completely necrose of whole skin flap. Necrectomy until helth tissue has done and has been continuing by dressing. After everydays debridmen of wound and defect scin treatment with plastic surgery consultation, 50 days after operation, we taken out osteosintetic material. Using suggestion of plastic surgery defect of soft tissue has not solved in surgery way. It has left to per secundum accreted. Treatment of wound finished after 5 months from contusion. The scar and movements has been good. Only partial problem has occured with peroneale ligament. Conclusion: Through therapy of heel bone it is necessary serious preoperative planning. Of course, if has appeared skin problem consultation with plastic surgery is obligatory. Well done preoperative preparation including: RTG and CT scan, planning of operation immediately after receiving in hospital or into 10-14 days after quit down soft tissue. Vacum drainage is obligatory way by well intraoperative hemostasis.

References: 1. Steven YW, Operatively treated calcaneus fractures: to mobilize or not tomobiliz. Univ $\mathrm{Pa}$ Orthop $\mathrm{J}$; 2001. 2. Keith A, Heier Md, Anthony F, Infante D. Open fractures of the calcaneus: soft-tissue injury determines outcome. J Bone Jt Surg. 2003. 3. Roy Sanders, MDC. Urrent concepts review displaced intra-articular fractures of the calcaneus, Tampa, Florida, 2000. 4. Paul M. Fractures of the calcaneum. J Bone Jt Surg [Br]. 2004;86-B:1142-5. 5. Jain V, Kumar R, Mandal Dk. Osteosynthesis for intra-articular calcaneal fractures. J Orthop Surg. 2007;15(2): 144-8.

Disclosure: No significant relationships.

\section{P54}

\section{SUCCESSFUL TECHNIQUE FOR MANAGEMENT OF ENTERO-ATHMOSPHERIC FISTULA IN OPEN ABDOMEN WOUND}

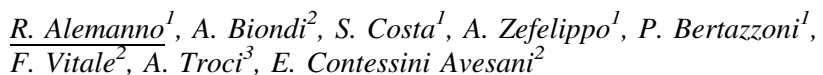

${ }^{1}$ Surgery, Fondazione IRCCS, Milano, Italy, ${ }^{2}$ Surgery, Fondazione IRCCS "Ca' Granda" Policlinico Maggiore, Milano, Italy, ${ }^{3}$ Fondazione IRCCS, Milano, Italy 
Introduction: Entero-atmospheric fistulas arising in open abdomen wounds are fistulas without formal fistula tract, since they lack surrounding soft tissue and skin. In entero-athmospheric fistulas collection of effluent and wound management represent the major challenges. A successful management by vacuum assisted closure therapy (VAC; KCI International) is presented.

Materials and methods: In December 2009, a 73-year-old woman with a history of multiple surgeries for Crohn's disease underwent stricturoplasty and extensive adhesiolysis for small bowel obstruction. Postoperatively, a suture leak occured and several unsuccessful attempts to leak closure were performed. An high output enteral fistula $120 \mathrm{~cm}$ distal from Treitz's ligament within a open abdominal wound ensued. An integrated wound management by VAC therapy dressing was attempted.

Results: The wound surrounding the fistula was covered with petrolatum gauzes and with a VAC device. The VAC foam was fitted around the fistula opening and then the polyurethane drape was placed on the whole wound. $75 \mathrm{mmHg}$ continuous negative pressure was applied. The drape was open directly over the fistula and ostomy appliance was placed over the drape. Dressings were changed approximately every 3 days. In May 2010 a split-thickness skin graft was performed and the VAC was further used to control fistula effluent, and to improve graft survival as well. In June 2010, when skin graft definitively took, ostomy appliance was placed without VAC dressing.

Conclusion: Customized VAC therapy is a viable option in management of entero-athmosferic intestinal fistulas in open abdominal wounds.

References: Goverman, et al. The "Fistula VAC," a technique for management of enterocutaneous fistulae arising within the open abdomen: report of 5 cases. J Trauma. 2005;60:428-31. Becker, et al. Small bowel fistulas and the open abdomen. Scand J Surg 2007;96:263-71.

Disclosure: No significant relationships.

\section{P55}

\section{SURGICAL ANATOMY OF PANCREATIC BRANCHES OF SPLENIC VESSELS PERTINENT TO SPLEEN-PRESERVING DISTAL PANCREATECTOMY WITH PRESERVED SPLENIC ARTERY AND VEIN: CADAVER STUDY AND CASE REPORT}

\author{
N. Kaneko ${ }^{1}$, Y. Kobayashi ${ }^{2}, H$. Terayama $^{3}$, M. Itoh $^{3}$
}

${ }^{1}$ Department Of Emergency And Critical Care Medicine, Tokyo Medical University, Shinjuku, Japan ${ }^{2}$ Anatomy, National Defense Medical College, Tokorozawa, Japan, ${ }^{3}$ Anatomy, Tokyo Medical University, Shinjuku, Japan

Introduction: Distal pancreatectomy usually proceeds with splenectomy. Although spleen-preserving distal pancreatectomy (SPDP) has recently been advocated, most SPDP has sacrificed the splenic artery and vein (SAV) while maintaining splenic blood circulation by preserving the short gastric vessels and only few have preserved the $\mathrm{SAV}$. The reason for this is that the pertinent anatomic variations of the pancreatic branches of the SAV are not well understood.

Materials and methods: The location and number of branches were investigated in 50 cadavers.

Results: Number of SA branches: average 3.0; range 1-8. Number of SV branches: average 4.3; range 1-10. The SA branches emerged only at the inferior side of the SA in $48(96 \%)$ cadavers, additionally the superior in $1(2 \%)$, and also the anterior in $1(2 \%)$. However, the entering directions to the SV varied widely: $26(52 \%)$ cadavers had anterior branches. No correlation was found between the number of SA branches and SV branches. Alongside the SA run the splenic branch of vagus nerve.

Case report: A 10-year-old boy sustained pancreatic damage during a bicycle accident. Pancreatic laceration was identified via incision of the left half of the gastrocolic ligament at laparotomy. The distal pancreas was mobilized without touching the spleen, and SPDP with preserved SAV was achieved.

Conclusion: SPDP with preserved the SAV is a safe way to totally conserve the splenic vascular and nervous system. The key issue in the procedure is the disconnection between the SV and the pancreas at the anterior face of the SV.

Disclosure: No significant relationships.

\section{P56}

\section{THE FLESH EATING BACTERIA}

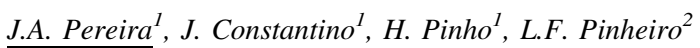

${ }^{1}$ Cirurgia 1, Hospital São Teotónio, Viseu, Portugal, ${ }^{2}$ Cirurgia Geral, Hospital de São Teotónio, Viseu, Portugal

Introduction: The necrotizing fascitis is a poorly understood pathologic entity, despite its poor prognosis, fast clinical evolution and high mortality rate. Among the survivors, few escape to a high and disabling morbidity, including various degrees of limb amputation. So, only a prompt diagnosis, aggressive surgical approach and intensive care support can change the very dismal prognosis of this disease.

Materials and methods: The Authors present a case of a 35 year old male, presenting with a recent onset edema of the lower left limb, evolving in the next few hours to a necrotizing fasceitis with sepsis syndrome. Its prompt identification allowed that, with an aggressive surgical approach, the patient survived with a degree of mutilation inferior to that documented in the world literature.

Results: The bacteriological results of the specimens collected, showed a soft tissue infection caused by a Lancefield's Group A beta hemolitic Streptococcus, sensible to the Penicilin G, which was instituted once this results were available. After the treatment of the infectious state, the patient was grafted with skin mesh, with full coverage of the debrided areas after four surgical sessions. He needed intense rehabilitation to recover the pre-illness physical status.

Conclusion: The necrotizing fasceitis has an unpredictable and often fulminant course. Only a prompt recognition and aggressive approach can change the dismal prognosis of this disease.

References: Treatment of Complicated Skin and Soft Tissue Infections; Addison May, Renae Stafford, Eileen Bulger, Daithi Heffernan, Oscar Guillamondegui, Grant Bochicchio and Soumitra Eachempati Surgical Infections, Volume 10, Number 5, 2009.

Disclosure: No significant relationships.

\section{P57}

\section{URGENT AND EMERGENCY SURGERY IN NECK AND MAXILLOFACIAL TRAUMA}

C. Camarero ${ }^{1}$, J. Martín Gil ${ }^{2}$, T. Sanchez-Rodriguez $z^{3}$, Y. M. Al-lal ${ }^{4}$, M. Burneo $^{l}$, A. Kulyapina ${ }^{5}$, L.E. Bernardos ${ }^{1}$, C. Rey Valcárcel $^{6}, F$. Turegano

${ }^{1}$ Surgery, Gregorio Marañon Hospital, Madrid, Spain, ${ }^{2}$ CirugÍa General Ii, Hgu- Gregorio Marañón, Madrid, Spain, ${ }^{3}$ Surgery, 
Gregorio Marañon Hospital, Madrid, Spain, ${ }^{4}$ Gregorio Marañon Hospital, Madrid, Spain, ${ }^{5}$ Maxilofacial Surgery, Gregorio Marañon Hospital, Madrid, Spain, ${ }^{6}$ Surgery, Hospital General Universitario Gregorio Marañón, Madrid, Spain, ${ }^{7}$ General And Emergency Surgery, University Hospital Gregorio Maranon, Madrid, Spain

Introduction: Neck and maxillofacial trauma is a part of the secondary survey in the assessment multiple trauma patients. Injuries to these areas can pose a life-threatening risk. We describe the incidence, diagnostic, therapeutic methods, mortality from severe neck and maxillofacial trauma in our hospital.

Materials and methods: Retrospective descriptive study during a period of 14 years (July 1993-July 2007). We focused on those patients with neck and maxillofacial injuries, and assessed the severity by means of the trauma scores (GCS, RTS, ISS and NISS).

Results: 156 patients had severe injuries to the maxillofacial and/or cervical area. Anatomic distribution of major lesions: midfacial 105 (33\%), mandibular $22(14 \%)$, cervical $29(18,6 \%)$, minor lesions in 37 (24\%) patients. $90 \%$ of all cases were due to blunt trauma. 28 presented shock on arrival (SBP $<90 \mathrm{mmHg}$ ), 69 required OTI, and 5 needed cardiac massage. A GCS $\geq 14$ was observed in 81 cases, an ISS $>15$ in 114(73\%), and the average NISS was of 26.50 patients were operated on, $24(48 \%)$ of them on an emergency basis. 25 interventions were in the midfacial region (7 urgent), 9 in the mandible ( 2 urgent), and 16 in the cervical area (15 urgent). 7 interventions for bleeding control, 6 tracheostomies, 3 explorations under anesthesia, and 8 cases of soft tissues reconstruction, as well as 26 reconstructive deferred surgeries were carried out. Overall mortality was of $17 \%$ (26 patients), 15 of them with midfacial trauma. Head injury was the most frequent cause of death.

Conclusion: Neck and Maxillo-facial trauma is an uncommon reason for urgent surgery in the trauma patient population. The most frequent intervention involves the neck region, aimed at securing the airway and stopping the bleeding. Associated brain injury is the main cause of death

Disclosure: No significant relationships.

\section{P58}

Moved to Oral Presentations

\section{P59}

\section{VATS IN BLUNT THORACIC TRAUMA}

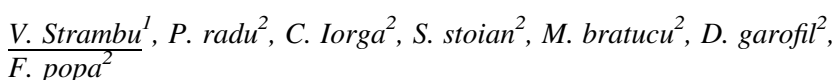

${ }^{1}$ General Surgery, St Pantelimon Hospital, Bucharest, Romania, Bucharest, Romania, ${ }^{2}$ General Surgery, St Pantelimon Hospital, Bucharest, Romania, Bucharest, Romania

Introduction: VATS is increasingly used in diagnosis, and in therapeutic management in stables patients with thoracic injuries. This study was designed to evaluate the effectiveness of this method in case of chest trauma, in short term reduction of pain, dosage of analgesia used, duration of hospitalization, in restoring lung function and reducing complications.

Materials and methods: The study was conducted on 70 patients with chest trauma (penetrating or closed).

Results: The various thoracoscopic procedures were performed: the evacuation of remaining clots, pleural lavage, electrocoagulation, lung parenchymal stapling repair, repair of the diaphragm). Pain relief $(p<0.001)$, improvement in dyspnea $(p<0.05)$, rapid restoration of pulmonary function and radiological improvement were statistically significantly more favorable in 7-day treatment group with less length of hospital stay $(\mathrm{p}<0.001)$.

Conclusion: VATS is a better option in management of traumatic thoracic injuries with lower complication rate and possibly shorter duration of stay in hospital.

Disclosure: No significant relationships.

\section{FRACTURES/DISLOCATIONS}

\section{P60}

\section{A NEW ANATOMIC SHAPED TIBIA NAIL-FIRST EXPERIENCES AND RESULTS}

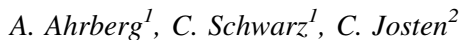

${ }^{1}$ Department of Traumatology And Reconstructive Surgery, University of Leipzig, Leipzig, Germany, ${ }^{2}$ Department Of Traumatology, Plastic- And Reconstructive Surgery, Spine Center, University of Leipzig, Leipzig, Germany

Introduction: Anatomic shaped intramedullary nails allow treatment also of proximal and distal tibia fractures and have become the implant of choice in most tibia fractures. In this study we present the results of a new anatomic shaped tibia nail (Zimmer NaturalNail, ZNN) in our first 21 patients.

Materials and methods: We included all patients having been treated with the new nail in tibia fractures (AO42). There were 11(50.4\%) A, $6(28.6 \%) \mathrm{B}$ and $4(19 \%) \mathrm{C}$ fractures, all together 4(19\%) open fractures. All nails were reamed. Average diameter of the shaft was $10.3 \mathrm{~mm}$ (9.3-12), average length was $345 \mathrm{~mm}$ (300-400). In $5(23.8 \%)$ there had been an previous operation (two external fixations, two other nails with non-union, one plate in a proximal fracture).

Results: Average duration of operation was $90 \min (34-263)$. There was one fracture of the proximal shaft when inserting the nail, otherwise no intraoperative complications or problems reported. One nail had to be changed to a shorter nail because of patellar tendon problems. There were $2(9.5 \%)$ compartment syndromes that had to be operated and $2(9.5 \%)$ infections, one after an $\mathrm{II}^{\circ}$ open fracture treated with external fixation before and one after changing from another nail to ZNN because of non-union.

Conclusion: The new anatomic shaped nail is a safe procedure for the treatment of fractures in all segments if the tibia, even very distal and proximal ones. No implant specific problems occurred. It can also be used for the treatment of non-unions after other nails/plates. There is an increased risk of infection when changing implants and after open fractures.

Disclosure: No significant relationships.

P61

\section{A NEWLY DESIGNED INTRAMEDULLARY NAIL FOR FOREARM FRACTURES IN ADULTS}

\section{F. Akpinar ${ }^{1}, \underline{\text { G. Saka }}{ }^{2}$, F. Küçükdurmaz ${ }^{3}$, C. $\ddot{\text { zzer }}^{1}$, U. Bakır ${ }^{1}$}

${ }^{1}$ Umraniye Research and Educational Hospital, Istanbul, Turkey,

${ }^{2}$ Umraniye Training Hospital, Istanbul, Turkey, ${ }^{3}$ Orthopedics And Traumatology, Umraniye Research and Educational Hospital, Istanbul, Turkey

Introduction: Although plate-screw ostheosyntesis is standard procedure in forearm fractures in adults, however the complications like 
incision scar, nounion, delayed union, infection and risk of refracture following the hardware removal are still high. In this study, we present follow-up results of two new design intramedullary nail (IMN) for radius and ulna.

Materials and methods: The results of surgically treated 59 forearm fractures in 43 adult patients ( 14 both forearm, 17 ulna, 14 radius) between May 2008 and October 2009 are evaluated retrospectively. Mean age was 37 (18-65) with 11 female and 32 male patients.

Results: Mean operation time was $25 \mathrm{~min}$ (range 20-45 min) and 20 min (range15-32 min.) for ulna and radius respectively. Mean flouroscopy time was $1.2 \mathrm{~min}$ (range $10 \mathrm{~s}-1.5 \mathrm{~min}$ ) for both. Mean union time was 13 weeks (range 10-14 weeks) and 12 weeks (range 10-13 weeks) for ulna and radius respectively. All patients allowed full range of motion as they can tolerate the day after surgery without any type of external support. There was no neurovascular injury, deep infection, radioulnar synosytosis or nonunion.

Conclusion: Our new design IMNs might require flouroscopy just for confirmation of entry point or the implantation. They allow to implant in very short surgical time and obtain high primary stability especially at ulna with its controlled compression function. Very early postoperative motion, day after surgery, without need for external support is important advantage. Single incision is enough for implantation and proximal interlocking in ulna and without external guide. The distal interlocking at ulna do not require any flouroscopy use, because of its design. For radius one incision is enough for implementation and distal interlocking. The result of this study demonstrate that, our new design IMN is superior to currently available ones and is an considerable alternative to plate-screw fixation for forearm fractures in adults.

References: 1. Lee YH, Lee SK, Chung MS, Baek GH, Gong HS, Kim KH. Interlocking contoured intramedullary nail fixation for selected diaphyseal fractures of the forearm in adults. JBJS Am. 2008;90:1891-8. 2. Beaupre GS, Csongradi JJ. Refracture risk after plate removal in the forearm. JOT. 1996;10:87-92.

Disclosure: No significant relationships.

\section{P62}

\section{A NEWLY DESIGNED INTRAMEDULLARY NAIL WITH DISTAL INTERLOCKING SYSTEM FOR TIBIA FRACTURES IN ADULTS}

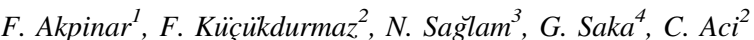

${ }^{1}$ Umraniye Research and Educational Hospital, Istanbul, Turkey, ${ }^{2}$ Orthopedics And Traumatology, Umraniye Research and Educational Hospital, Istanbul, Turkey, ${ }^{3}$ Istanbul Medipol University, İstanbul, Turkey, ${ }^{4}$ Umraniye Education And Research Hospital, Umraniye Education and Research Hospital, Istanbul, Turkey

Introduction: The gold standard method of surgical treatment for fractures of the tibia in adults is closed intramedullary nailing (IMN). Nevertheless, the rates of non-union, delayed union, malunion are still high in tibia. We developed a new IMN with distal interlocking (DI) system, namely Distal Supportive Bolt Locking Screw, DSBLS ${ }^{\mathrm{TM}}$, which may improve construct and fracture stability.

Materials and methods: The results of surgically treated 50 tibia (4 tibia, 46 tibia + fibula) fractures (22 tibia shaft, 28 distal tibia) in 50 adult patients between May 2008 and May 2010 are evaluated retrospectively. Mean age was $39(\min 17, \max 80)$

We used unreamed DSBLS ${ }^{\mathrm{TM}}$. We took standard AP and Lat views weekly and recorded radiologic callus formation for each view and assumed union if there is callus in both views without pain in fracture site. We recorded the flouroscopy time for DI. All patients allowed full weight bearing and range of motion the day after surgery without any type of external support.

Results: Mean flouroscopy time was $18 \mathrm{~s}(\min 10$, max 30). Mean union time was 9 weeks $(\min 6, \max 12)$. There was no neurovascular injury, deep infection, malunion, delayed union, nonunion.

Conclusion: The complications following tibia IMN is found to be associated with interfragmentary movements and may be decreased by modifying DI options. DSBLSTM provides absolute stability between DI bolt screw and nail. By this system the stability of whole construct increases and provides important advantages in clinical setting.

Although various attempts, none of the currently available IMN systems provide sufficient stability to allow full weight bearing the day after surgery. However, although we use system, full weight bearing without any need for external support the day after surgery, is the goals of DSBLS ${ }^{\mathrm{TM}}$.

Another advantage of DSBLS ${ }^{\mathrm{TM}}$ is minimum requirement of flouroscopy.

The result of this study demonstrates that, our new design system is superior to currently available IMN systems.

References: 1. Penzkofer R, et al. Influence of intramedullary nail diameter and locking mode on the stability of tibial shaft fracture fixation. AOTS. 2009;129(4):525-31. 2. Horn J, et al. Angle stable interlocking screws improve construct stability of intramedullary nailing of distal tibia fractures: a biomechanical study. Injury. 2009;40(7):767-71.

Disclosure: No significant relationships.

\section{P63}

\section{AGREEMENT BETWEEN INITIAL CLASSIFICATION AND SUBSEQUENT RECLASSIFICATION OF FRACTURES OF THE DISTAL RADIUS IN A PROSPECTIVE COHORT STUDY}

\section{R.H. van Leerdam, A. Lindenhovius, S. Souer, D. Ring}

Hand And Upper Extremity Department, Massachussetts General Hospital, Harvard Medical School, Harvard University, Boston, MA, USA

Introduction: We tested the hypothesis that the original surgeoninvestigator classification of a fracture of the distal radius in a prospective cohort study would have moderate agreement with the final classification by the team performing final analysis of the data.

Materials and methods: The initial post-injury radiographs of 621 patients with distal radius fractures from a multicenter international prospective cohort study were classified according to the Comprehensive Classification of Fractures, first by the treating surgeoninvestigator and then by a research team analyzing the data. Correspondence between original and revised classification was evaluated using the Kappa statistic at the type, group and subgroup levels.

Results: The agreement between initial and revised classifications decreased from Type (moderate; Îštype $=0.60$ ), to Group (moderate; Ǐšgroup $=0.41$ ), to Subgroup (fair; Îšsubgroup $=0.33$ ) classifications (all $\mathrm{p}<0.05$ ).

Conclusion: There was only moderate agreement in the classification of fractures of the distal radius between surgeon-investigators and final evaluators in a prospective multicenter cohort study. Such variations might influence interpretation and comparability of the data. The lack of a reference standard for classification complicates efforts to lessen variability and improve consensus.

Disclosure: No significant relationships. 
P64

\section{AMERICAN COLLEGE OF CARDIOLOGY \& AMERICAN HEART ASSOCIATION PERIOPERATIVE ASSESSMENT GUIDELINES FOR HIP SURGERY: A PROSPECTIVE STUDY}

\section{S. Smeets $^{1}$, M. Poeze $e^{2}$, J. Verbruggen ${ }^{3}$}

${ }^{1}$ Surgery, Maastricht Universitair Medisch Centrum, Maastricht, Netherlands, ${ }^{2}$ Department Of Surgery, Division Of Trauma, Maastricht University Medical Center, Maastricht, Netherlands, ${ }^{3}$ Surgery, Maastricht University Medical Centre, Maastricht, Netherlands

Introduction: The purpose of this study was to investigate the use of the American College of Cardiology (ACC) and the American Heart Association (AHA) guidelines for perioperative assessment of orthopaedic patients with acute hip fracture.

Materials and methods: This abstract includes preliminary data of more than 50 elderly patients with acute hip fracture. Preoperative cardiac consultations were analyzed with respect to content and following ACC/AHA guidelines. In accordance with these guidelines patients were divided in low, intermediate and high risk categories. The study population was characterized by using the American Society of Anesthesiologists (ASA) score for physical status, the Barthel index for functional evaluation, Metabolic Equivalent of Task (MET) score, Mini Mental State Examination (MMSE) and the Palmer \& Parker mobility score. Furthermore the type of housing in relation with functional and mental status was investigated.

Secondary output measurements were perioperative complications and delay to surgery.

Results: $80 \%$ of patients received correct preoperative cardiac screening in the low risk group versus $80 \%$ in the intermediate group and $86 \%$ in the high risk group. Only $29 \%$ of consultations in the low and $40 \%$ in the intermediate risk group were correctly in comply with the ACC/AHA guidelines versus $86 \%$ in the high risk group. The most frequent reason for incorrect preoperative cardiac screening was overscreening $(>95 \%)$. People in nursing homes have significant worse ASA, Barthel, MET, MMSE and Palmer \& Parker scores than patients who live independently. Not all analysis are completed because of a still ongoing study.

Conclusion: In the low and the intermediate risk group there is, respectively, 29 and $40 \%$ unnecessary preoperative cardiac consultations. The ACC/AHA guidelines maybe helpful to optimize a patient for operation, reduce the use of preoperative recourses and prevent further delay.

Reference: Fleisher LA, et al. ACC/AHA 2007 guidelines on perioperative cardiovascular evaluation and care for noncardiac surgery. Circulation. 2007; 116(17):e418-99.

Disclosure: No significant relationships.

\section{P65}

\section{AN ASSESSMENT OF CLINICAL OUTCOME AFTER REAMED INTRAMEDULLARY NAILING IN PATIENTS OVER 60 YEARS COMPARED TO YOUNGER PATIENTS WITH A TIBIAL SHAFT FRACTURE}

\author{
T.W.A. Koedam ${ }^{I}$, G.F. Giannakopoulos ${ }^{2}$, F.C. Bakker ${ }^{1}$ \\ F.W. Bloemers ${ }^{l}$, W.P. Zuidema
}

${ }^{1}$ Trauma Surgery, VU-University Medical Centre, Amsterdam, Netherlands, ${ }^{2}$ Trauma Surgery, VU University Medical Centre, Amsterdam, Netherlands
Introduction: Elderly patients may require special management due to co-morbidities and low bone-stock compared to young patients with tibial shaft fractures.

Materials and methods: A retrospective study from 2005 to 2008 included 76 consecutive patients ( $>16$ years) with a tibial shaft fracture treated with intramedullary nailing. After variable analysis, the elderly patients (Group I; $>60$ years) were matched with the younger patients (Group II; $<60$ years) based on sex, type of fracture (AO) and compound/closed fracture. Data were analyzed using SPSS 16.0.

Results: Group I consists of 6 male and 6 female and a mean age of 70.4 years and average ISS of 9.2. Group II was made up of 25 men and 13 women and mean age 31.9 years with an average ISS of 7.6. The fractures in group I and group II were mainly caused by traffic $83 \%$ and falling $68 \%$. More than $75 \%$ of the patients in group I had co-morbidities and increased use of medication. Union rates within 12 months were 83 versus $50 \%$. Fifteen percent needed additional surgery in group II. Removal rate of osteosynthesis was $8.3 \%$ in group I and $63 \%$ in group II due to pain. The total complication rate was $38.5 \%$ in group I and $30.4 \%$ in group II.

Conclusion: Elderly patients with tibia shaft fractures treated with intramedullary nailing have an increased risk of complications. Union rates are better than in young patients probably due to the lower energy transfer at time of accident. Also elderly have less additional operations or removal of hardware.

Disclosure: No significant relationships.

\section{P66}

\section{AN INVESTIGATION OF THE FACTORS ASSOCIATED WITH THE PREOPERATIVE D-DIMER VALUES IN TRAUMA PATIENTS}

\author{
N. Takeuchi ${ }^{1}$, M. Hashizume ${ }^{2}$, Y. Iwamoto ${ }^{1}$
}

${ }^{1}$ Orthopaedic Surgery, Kyushu University, Fukuoka, Japan, ${ }^{2}$ Critical Care Center, Kyushu University, Fukuoka, Japan

Introduction: The purpose of our study was to examine factors that are associated with the preoperative $\mathrm{D}$-dimer values for trauma patients with fractures in the spine, pelvis or lower extremities. Materials and methods: Twenty patients (male 13, female 7) were treated in the Critical Care Center in Kyushu University Hospital from April 2008 to December 2009. The mean age was 47.1 (16-79) years. All patients sustained at least one severe injury [Abbreviated Injury Scale (AIS) $\geq 3$ ] in the lumber, pelvis or lower extremities. D-dimer was examined on days 1, 3, and 7 after admission, and 2 days before definitive surgery. The maximum D-dimer value after day 2 was defined as Max D-D. The Injury Severity Score (ISS) ranged from 10 to 35 (mean 20.8). Body mass index (BMI) ranged from 18.2 to 36 (mean 25.4), and the period until the definite surgery ranged from 5 to 16 days (mean 11.1). The study examined the correlation between Max D-D and four factors; age, ISS, BMI, and the period until the definitive surgery. A multiple regression analysis was used for the statistical analysis and values of $p<0.05$ were considered to be significant.

Results: Max D-D had a significantly positive correlation with the ISS $(p=0.03, r=0.55$, Max D-D $=0.563 \times$ ISS + 2.322). Max D-D was not significantly correlated with age, BMI, or the period until the definite surgery $(\mathrm{p}=0.54,0.78,0.90$, respectively).

Conclusion: D-dimer values have been shown to be related with the appearance of deep venous thrombosis. The current study indicated that the ISS can predict the preoperative maximum D-dimer values for trauma patients.

Disclosure: No significant relationships. 
P67

\section{AUDIT ON COMPLICATION RATE OF TROCHANTERIC ANTEGRADE NAIL (TRIGEN) IN TREATING UNSTABLE INTERTROCHANTERIC FRACTURES OF NECK OF FEMUR}

\section{A.M. Saad, I.A. Ali, A.G. Cobb}

Trauma \& Orthopaedics, Epsom \& St. Helier University Hospitals NHS trust, Carshalton, Surrey, UK

Introduction: Background: The treatment of intertrochanteric fractures using intramedullary nails in our centre is routine. All our patients are treated within $48 \mathrm{~h}$ from admission according to the national guidelines. We tend to observe increase in the complications in such fracture fixation method. Hence, the inception of this study Aim: To review the complication rate of Intramedullary Nailing of unstable Intertrochanteric fractures of Neck of Femur and to identify areas of improvement of such fixation method.

Materials and methods: We collected retrospective data from medical notes and radiological images of patients, who underwent Trochanteric Antegrade Nails (Trigen) for unstable Intertrochanteric neck of Femur fractures between August 2008 and October 2009 in St. Helier University Hospital trauma Centre. Patient demographics, site and anatomical location of fractures, classification, comorbities, time from admission to surgery, prophylaxis methods, grade of surgeons, duration of hospital stay, complications and outcome were assessed. The results were compared with the treatment of Intertrochanteric fractures from current worldwide published evidence.

Results: Total of 17 patients were identified. $76 \%$ of patients were female and only $24 \%$ were male with the mean age of $84.82 \%$ of patient underwent surgery within $48 \mathrm{~h}$ from admission to hospital and only 3 patients (18\%) were delayed due to significant medical conditions, which required optimization prior to surgery. 11 cases were operated by specialist registrars of different level of experience, and 6 cases by consultants Orthopaedic surgeons 11 patients $(64 \%)$ had no complications with the metalwork, and 6 patients $(36 \%)$ developed post operative complications The mean hospital stay was 23 days and all patients received prophylactic antibiotics and LMWH. All patients had pre-operative and post-operative radiographs, which were analysed for fracture classification, adequacy of intra-operative reduction and position of metalwork, as well as post-operative areas of failure. Conclusion: The fixation of unstable Intertrochanteric Fractures is challenging. Trigen IM Nailing is an inadequate fixation device. The complications of Trigen IMN could be significantly reduced by adequate intra-operative reduction of fracture.

Disclosure: No significant relationships.

\section{P68}

\section{BENEFIT OF CORRECTION-OSTEOTOMY IN MALUNITED DISTAL FRACTURES OF THE RADIUS}

\section{H.K. Widhalm ${ }^{1}$, G. Vekszler ${ }^{I}$, S. Hajdu ${ }^{I}$, M. Chochole ${ }^{2}$ \\ ${ }^{1}$ Trauma Surgery, Medical University of Vienna, Vienna, Austria, \\ ${ }^{2}$ Orthopedics, Herz-Jesu-Hospital, Vienna, Austria}

Introduction: Conservative as well as surgical therapies of radius fractures are often accompanied by malunion and malposition. Most often there are distinctions of the niveau, malposition of the axis with a tilting and an intraarticular displacement. As a consequence patients show painful reduction of motion, especially of the forearm rotation. Aim of this retrospective study was to evaluate the benefit of radius- correction-osteotomy in malunited radius fractures according to reduction of pain, strength and postoperative extent of motion. Materials and methods: 12 patients ( 7 men, 5 women) with an average age of 37.9 years (17.4-63.5 years) have been operated within 3 years. In all cases a correction-osteotomy with or without cortico-spongiose iliac crest, followed by volar plating has been performed. Radiological evaluation was made by comparison of the pre- and postoperative X-rays, whereas the axis and the proportion of the niveau in the distal radio-ulnar joint have been assessed. The function was measured by goniometer, the strength by dynamometer and the pain intensity was calculated by VAS-score. DASH-score was used for subjective appraisement of the arm function.

Results: The average follow up time was 2 years (0.9-3.5 years). Postoperatively there were no deviations of the axis. In 11 of 12 cases the niveau could have been compensated to $\pm 1 \mathrm{~mm}$. The rotation of the forearm could have improved by 31 in average. Pain intensity measured in VAS could have been reduced from 3.5 to 2.0, confirmed by average DASH-score values of $22(2.2-54.5)$.

Conclusion: The operative therapy of painful, malunited distal radius fracture with deviation of the axis, dislocations and reduction of the motion according to subjective and objective evaluation is very effective.

Disclosure: No significant relationships.

\section{P69}

\section{BIOMECHANICAL ANALYSIS OF PERCUTANEOUS ALL LATERAL PINNING (DORGAN'S TECHNIQUE) TECHNIQUE FOR SUPRACONDYLAR HUMERUS FRACTURES IN CHILDREN.}

\section{K. Memisoglu ${ }^{I}$, R. Musaoglu ${ }^{I}$, A. Cengiz ${ }^{2}$, C.C. Kesemenli ${ }^{l}$}

${ }^{1}$ Orthopaedics and Traumatology, Kocaeli University School of Medicine, Kocaeli, Turkey, ${ }^{2}$ Mechanical Education, Kocaeli University School of Technical Education, Kocaeli, Turkey

Introduction: We tried to compare biomechanical properties of all lateral pinning (Dorgan's technique) with other pinning configurations used for humerus supracondylar fractures in children. In all lateral pinning technique iatrogenic nerve injury risk is minimized with secure fixation compared with other pin fixation configurations. Materials and methods: Biomechanical testing was performed on 50 synthetic humeral bones (Keklikoglu. Transverse supracondylar humeral fractures were made with a hand-held saw above the olecranon fossa. Five different pin configurations were selected for fracture stabilization: 2 lateral pins and 1 medial pin ( 3 crossed), 2 laterally divergent pins, 2 crossed pins, 2 all laterally crossed pins (Dorgan's technique), 2 laterally parallel pins. All the pin configurations were fluoroscopically confirmed. The fractures were stabilized with 0.062 inch Kirschner wires. Biomechanical tests were performed by torsional load producing machine with internal rotation of the distal fragment. All the models were subjected to torsional loads for 10 times between 5 and $50 \mathrm{~N}$ and rotation in degrees and corresponding torques were recorded for statistical analysis.

Results: The differences of the stiffness of the pin configurations [2 lateral pins and 1 medial pin ( 3 crossed), 2 laterally divergent pins, 2 crossed pins, 2 all lateral crossed pins (Dorgan's technique), 2 laterally parallel pins] were found respectively $0.06,0.043,0.039,0.012$ and $0.013 \mathrm{Nm} /$ degree.

Conclusion: All lateral crossed pins (Dorgan's technique) showed biomechanically equal properties to the medio-lateral, lower to the 2 lateral and 1 medial pin, and superior to the other pin configurations. All lateral crossed pins do not jeopardize the ulnar nerve during 
insertion of the pins percutaneously and fix the fracture with low learning curve.

References: Cheng JC, Shen WY. Limb fracture pattern in different pediatric age groups: a study of 3350 children. J Orthop Trauma. 1993;7:12-22. Farnsworth CL, Silva PD, Mubarak SJ. Etiology of supracondylar humerus fractures. J Pediatric Orthop. 1998;18:38-42. Disclosure: No significant relationships.

\section{P70}

\section{BIOMECHANICAL TESTING OF A TENSION BAND PLATE FOR OLECRANON FRACTURES}

\section{A. Spiegel ${ }^{1}$, J. Schonhardt ${ }^{2}$, N. Pochlatko ${ }^{1}$}

${ }^{1}$ Research/Testing, Medartis AG, Basel, Switzerland, ${ }^{2}$ Business Unit Trauma, Medartis AG, Basel, Switzerland

Introduction: Olecranon fractures with interfragmentary support (or osteotomies) are usually treated by tension band wiring (TBW) [1]. This method presents some disadvantages though as soft tissue problems have been reported [2]. Medartis has therefore developed a tension band plate (TBP) that offers more stability while being less bulky. A biomechanical fatigue test setup was developed to compare this TBP with TBW.

Materials and methods: A 3D ulna CAD model (Sawbones) was used to obtain anatomically correct fixtures; samples were tested using a Zwick load frame. Fatigue testing was performed following a modified Locati approach: samples were tested at periodically increasing loads until mechanical failure. Load and deformation were recorded.

Results: The TBP had a fatigue strength of $197 \pm 42 \mathrm{~N}$ compared to the TBW's fatigue strength of $143 \pm 31 \mathrm{~N}$. Deformation of the TBP (roughly $1.5 \mathrm{~mm}$ at $140 \mathrm{~N}$ ) was also lower than the TBWs $(2.0 \mathrm{~mm})$. Also, the wiring typically failed in a catastrophic way (fracture of the wire) while the plate construct retained some mechanical strength through the screws even after plate fracture.

Conclusion: A novel tension band plate (TBP) was compared to tension band wiring (TBW) for the treatment of olecranon fractures with interfragmentary support. Fatigue testing showed that the TBP offers both lower deformation and higher fatigue strength. Since the plate is also less bulky it should reduce soft tissue problems and mitigates the problem of K-wire migration offering the surgeon an interesting alternative to TBW.

References: 1. Weber, et al. Z Unfallmed Berufskr. 1963;56:90. 2. Macko, et al. J Bone Joint Surg Am. 1985;67:1396.

Disclosure: No significant relationships.

P71

\section{BIPOLAR HEMIARTHROPLASTY FOR THE TREATMENT OF FEMORAL NECK FRACTURES AND THE EFFECT OF SURGICAL APPROACH ON FUNCTIONAL RESULTS}

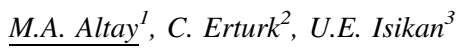

${ }^{1}$ Orthopaedics and Traumatology, Harran University Medical Faculty, Sanliurfa, Turkey, ${ }^{2}$ Orthopaedics and Traumatology, Harran University Medical Faculty, Sanliurfa, Turkey, ${ }^{3}$ Orthopaedics and Traumatology, Harran University Faculty of Medicine, Sanliurfa, Turkey
Introduction: The aim of this retrospective study was to evaluate the postoperative functional results of patients operated with cemented bipolar hemiarthroplasty via anterior versus posterior approach and to evaluate the effect of surgical approach on functional results.

Materials and methods: Between 2000 and 2008, among 196 patients over the age of 65 and treated with cemented bipolar hemiarthroplasty for femoral neck fracture 76 patients followed up for at least 1 year were evaluated. All fractures were Garden's type III or IV. In this study, the average follow-up period was 18.7 months (range 12-40 months), and mean age was 72.9 years (range 65-94 years). The male/female gender distribution was 39/37. During the surgical process, the posterior approach was performed in 52 cases; the anterior approach was performed in 24 cases. Cements were applied with the help of hand in 62 cases and with cement gun in 14 cases.

Results: Mean Harris scores were $84.7 \pm 10.3$ in posterior approach group and $85.8 \pm 7.1$ in the anterior approach group. According to the Harris scoring system, results in 26 cases were very good; in 40 cases were good; in 6 cases were fair; and in 4 cases were poor. While early dislocation was observed in $9.6 \%$ of patients with the posterior approach, it was not observed in any patients treated with the anterior approach. There was no statistically significant difference with regard to functional scores or early dislocations between patients treated with the posterior as compared to the anterior approach $(\mathrm{p}>0.05)$.

Conclusion: Based on our results, bipolar hemiarthroplasty is an appropriate and effective treatment option for patients with femoral neck fracture to obtain early return to daily activity. The surgical approach does not affect the functional results. Although not statistically significant, it was concluded that anterior approach is more reliable for early dislocation.

Disclosure: No significant relationships.

\section{P72}

\section{CABLE PLATE FIXATION OF VANCOUVER TYPE-B\& C PERIPROSTHETIC FEMORAL FRACTURE IN OCTAGENERAIAN.}

\author{
M. Bhattacharyya $^{\text {I }}$, N. Venkatram $^{2}$, S. Mazumdar $^{2}$, B. $_{\text {Gerber }}^{2}$
}

${ }^{1}$ Orthopaedics, Lewisham Hospital London, London, UK, ${ }^{2}$ University Hospital Lewisham, London, UK

Introduction: Cable plating system using wire or unicortical screw that lock into the plate allowing multiple points of unicortical fixation. The purpose of this study was to analyze clinically and radiographically a group of patients with multiple co morbidity ASA 3, a Vancouver type-B \& C periprosthetic femoral fracture treated with open reduction and internal fixation with use of a cable plating system.

Materials and methods: Fourteen consecutive patients (14 hips) with a Vancouver type-B \& C periprosthetic femoral fracture were treated with a cable plating system. There were five men and nine women with an average age of 79 years at the time of fracture. All of the fractures occurred after a uncemented hemi or total hip arthroplasty performed with cement, and three of the arthroplasties were revisions. In addition to the plate, cancellous allografts were used to facilitate union of five fractures. The patients were assessed clinically and radiographically.

Results: The average duration of follow-up was 20 months. Eight fractures healed uneventfully at an average of 5.4 months. None of constructs failed after surgery. 
Conclusion: On the basis of the high success rate in this cohort of high risk patients, cable plating system do appear to offer advantages and improve mobility in the treatment of complex periprosthetic femoral fractures without revision arthroplasty. Despite the potential to preserve the cement mantle, the proximal unicortical screws did appear to offer good pullout resistance in this fracture type. We believe that supplementation with strut allografts may not be used routinely if this type of construct is selected to treat these fractures in octagenerians.

Disclosure: No significant relationships.

\section{P73}

\section{CANNULATED COUNTERSINKABLE COMPRESSION SCREW FOR MEDIAL MALLEOLAR FRACTURES}

\section{J.A. Bekken ${ }^{I}$, G.R. Roukema ${ }^{2}$}

${ }^{1}$ Algemene Heelkunde, Maasstad Ziekenhuis, Rotterdam, Netherlands, ${ }^{2}$ Surgery, Maasstad Ziekenhuis, Rotterdam, Netherlands

Introduction: Fractures of the medial malleolus are a common operatively treated skeletal injury, for which open reduction and internal fixation is generally recommended. In our hospital, we used two different techniques: Placement of two lag screws, perpendicular to the fracture line, and tension-band wiring (Zuggurtung-osteosynthesis). Both of these lead to osteosynthesis-material protruding out of the medial malleolus.

Materials and methods: In concordance with literature, our patients often complain of ankle-pain after surgery. Prominent lag screws or Kirschner-wires may be one source of this pain, with influencing factors being subcutaneous positioning, bone impingement and irritation of deeper soft-tissue structures. These complaints lead to resurgery to remove the osteosynthesis-material.

Results: A relatively new type of bone screw is the Cannulated Countersinkable Compression Screw, which allows the head of the screw to be buried below the bone surface. They have shown promising results in the treatment of fractures of small bones such as those of the hand and foot. Because of its ability to sink its head in the cortex, with no protruding osteosynthesis-material, we started using the screw for internal fixation of medial malleolar fractures in relatively young patients.

Conclusion: Since December 2009, we have treated 6 patients with medial malleolar fractures with two Cannulated Countersinkable Compression Screws. We place these screws in the same way as we would place regular lag screws. After a medial follow-up period of 6 months, all of these patients have shown a stable fixation of the fracture, no complaints of ankle pain, and therefore no re-surgery has yet been necessary.

Disclosure: No significant relationships.

\section{P74}

\section{COMMINUTED FRACTURE OF RADIAL HEAD- OSTEOSYNTHESIS OR PROSTHESIS}

\section{Forman ${ }^{1}$, D. Kusy ${ }^{2}$, M. Carda ${ }^{3}$}

${ }^{1}$ Traumatology, PKN a.s., Pardubice, Czech Republic,

${ }^{2}$ Traumatology, PKN a.s, Pardubice, Czech Republic, ${ }^{3}$ Traumatology, PKN a.ss, Pardubice, Czech Republic

Introduction: We would like to introduce the change of our opinion of treatment the isolated comminuted radial head fracture Mason 3 type-either isolated or with combination of proximal ulna fracture (Monteggi fracture), elbow dislocation or Essex Lopresti lesion.

Materials and methods: Since 2006 we have treated a group of 25 patients with comminuted radial head fracture. For osteosynthesis we use screws and plates from small instrumentarium or Headless Bone Screws. For replacement of radial head we use the ExploR ${ }^{\circledR}$ Modular Radial Head Prosthesis. The surgical approach depends on type of injury.

Results: For measurement outcomes after treatment of radial head fracture we use Mayo Elbow Performance Score. We have fair and poor middle term results after excision of radial head. We have good and excellent short term results after osteosynthesis and good middle term results after osteosynthesis, but sometimes was necessary the second operation with hardware removal for radial head necrosis. And we have excellent short term results and good or excellent middle term results after using the radial head prosthesis. We have never needed to extract radial head prosthesis.

Conclusion: Our recent opinion of treatment the comminuted radial head fracture is that both methods are possible, but implantation of prosthesis has a little bit better results and less complications. This is the reason why we use more often radial head prosthesis in this time. Reference: Stannard JP, Schmidt AH, Kregor PJ. Surgical treatment of orthopedic trauma, Thieme Rockwood and Green's, fractures in adults. 7th ed.

Disclosure: No significant relationships.

\section{P75}

\section{COMPARISON OF INTRAOPERATIVE FLUOROSCOPY TIME AND CLINICAL-RADIOLOGICAL RESULTS IN THE TREATMENT OF PEDIATRIC FEMUR SHAFT FRACTURES WITH MINI-OPEN VERSUS CLOSED REDUCTION AND TITANIUM ELASTIC NAIL}

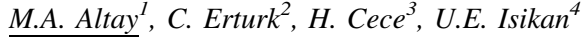

${ }^{1}$ Orthopaedics and Traumatology, Harran University Medical Faculty, Sanliurfa, Turkey, ${ }^{2}$ Orthopaedics And Traumatology, Harran University Medical Faculty, Sanliurfa, Turkey, ${ }^{3}$ Radiology, Harran University Medical Faculty, Sanliurfa, Turkey, ${ }^{4}$ Orthopaedics and Traumatology, Harran University Faculty of Medicine, Sanliurfa, Turkey

Introduction: Titanium elastic nails (TEN) are commonly used for the treatment of pediatric femur fractures in children [1]. We aimed to compare intraoperative fluoroscopy time and clinical-radiological results in the treatment of pediatric femur shaft fractures treated with the mini-open "blind-hand" technique versus closed reduction and TEN, retrospectively.

Materials and methods: Study participants included 87 children (18 girls and 69 boys) who underwent surgical treatment for femur shaft fractures with TEN. Patients were divided into two groups. Group 1 consisted of 42 patients (mean age $8.3 \pm 2.7$ years) treated with the mini-open "blind-hand" technique and TEN. Group 2 consisted of 45 patients (mean age $8.8 \pm 2.6$ years) treated with the closed reduction technique and TEN. Surgical period and intraoperative fluoroscopy time were recorded in both groups. Clinical and radiologic results were assessed using the TEN scoring system after mean follow-up periods of $21.3 \pm 5.8$ and $19.3 \pm 5.6$ months in group 1 and group 2 , respectively.

Results: The mean surgical periods were $31.7 \pm 7.6$ and $52.1 \pm 14.4 \mathrm{~min}$, and the mean fluoroscopy times were $32.9 \pm 22.1$ and $75.1 \pm 31.5 \mathrm{~s}$ in group 1 and group 2 , respectively. Both surgical period and fluoroscopy time were longer in group $2(\mathrm{p}<0.001)$. There was no significant difference between the two groups in terms of clinical and radiological results. All fractures healed with solid 
union, and there was no complication that was expected to cause permanent disability. Although successful clinical and radiologic results were obtained with both techniques, intraoperative fluoroscopy time and surgical period were significantly higher in group 2 than in group 1.

Conclusion: We suggest the "blind-hand" technique as an alternative to closed reduction to prevent extensive intraoperative radiation exposure and to decrease the length of the surgical period.

References: 1. Flynn JM, Hresko T, Reynolds RA, et al. Titanium elastic nails for pediatric femur fractures: a multicenter study of early results with analysis of complications. J Pediatr Orthop. 2001;21:4-8.

Disclosure: No significant relationships.

P76

\section{COMPARISON OF REAMED INTRAMEDULLARY COMPRESSION NAILS VS. REAMED INTRAMEDULLARY NAILING WITHOUT COMPRESSION IN TIBIA SHAFT FRACTURES}

\section{F. Högel ${ }^{1}$, P. Augat ${ }^{2}$, V. Bühren ${ }^{3}$}

${ }^{1}$ Trauma, Berufsgenossenschaftliche Unfllklinik Murnau e.V., Murnau, Germany, ${ }^{2}$ Biomechanical Research Laboratory, Traumacenter Murnau, Murnau, Germany, ${ }^{3}$ Trauma Surgery, Trauma Center Murnau, Murnau, Germany

Introduction: Modern intramedullary implants provide the option to perform compression to the fracture gap in long bone fractures by a compression screw-mechanism. The aim of this study was to asses whether the application of interfragmentary compression can increase the union rate in tibia fractures treated with intramedullary nailing. Materials and methods: All 61 patients between 2003 and 2008 who suffered from an acute tibia fracture of AO-type 42-A3 and who were treated by reamed intramedullary nailing were included in this study. 23 patients obtained dynamic interlocking without compression and 38 patients obtained compression nailing. Healing was assessed by radiological evaluation until completion of healing or secondary intervention. Non unions were defined by absence of radiological union and persistence of clinical symptoms after 6 months.

Results: In the compression group 38 patients were included. In 19 cases open tibia fractures and in further 19 cases closed tibia fractures occurred. In the non compression group 25 patients were included with 10 cases of closed soft tissue and 13 cases of open soft tissue. Non unions occurred in 2 cases in the compression group (5\%) while in the non-compression group 5 cases of non unions (20\%) were detected.

Conclusion: By reamed nailing of tibia shaft fractures with additional compression of the fracture gap the healing rate can be increased. Therefore, in simple transverse fractures compression of the fracture gap provides the option to improve healing outcome.

Disclosure: No significant relationships.

P77

\section{COMPLICATED PATELAR TENDON RUPTURES TREATMENT WITH BTB ALOGRAFT}

\section{Kecojevic ${ }^{1}$, S. Kecojevic ${ }^{2}$, I. Lalic $^{3}$, Z. Gojkovic ${ }^{1}$, M. Stankovic ${ }^{1}$, V. Harhaji ${ }^{4}$ S. Ninkovic ${ }^{4}$, R. Matijevic ${ }^{1}$}

${ }^{1}$ Orthopaedic Surgery and traumatology, Clinical Center, Novi Sad Serbia, ${ }^{2}$ Ambulance, Public Health, Novi Sad, Serbia, ${ }^{3}$ Ortopedics, KCV, Novi Sad, Serbia, ${ }^{4}$ Clinical Center, Novi Sad, Serbia
Introduction: Management of old, unhealed or open rupture of patelar tendon is difficult task for trauma surgeon. Loss of tissue, infection and quadriceps muscle stretching are main problems in treatment. We tried to improve the outcome of surgery using BTB patelar allograft as a good and strong graft.

Materials and methods: From 2007 we operated 7 patient with complicated patelar tendon ruptures. All seven were mail, average age 47 (from 43 to 63 ). In 5 cases we have rerupture after primary surgery or after conservative treatment, in 2 cases there were open ruptures. In one case of open rupture we have infection after primary suturing. In all cases we used BTB patelar allograft for ligamentoplasty. After debridement of fibrous and necrotic tissue we made cut of a tibial tuberosity bone block cut, and then we made a tunnel in the patella in the same manner as in the femoral tunnel in ACL surgery, using tibial tunnel guide for ACL reconstruction under X-ray control. Patelar bone block we secure it with two cortical screws. Finally we fix tibial part using two cortical 4.5 screws in position that allowed full range of motion. After that we sutured remaining ligament, and secure it with metal wire placed through base of patella and secure for one cortical screw placed beneath tibial tuberosity.

Results: We made X-rays control in 6 week, 3, 6 and 12 months. After 6 months we examine IKDC, Tegner and Lyscholm scores. We found all result were good, no one excellent, and no pure results. We have one superficial infection that heeled by treatment. We have no rerupture of allograft.

Conclusion: In our small group we find patellar BTB allograft is satisfactory and safe solution for complicated cases of patellar tendon reruptures or open ruptures with loose of ligament tissue.

Disclosure: No significant relationships.

\section{P78}

\section{DIAGNOSIS AND TREATMENT CRITERIA FOR TIBIAL PILON FRACTURES}

\section{G. Tomoaia, C.A. Bardas}

Ortopedie Si Traumatologie Ii, Spitalul Clinic "Prof. O. Fodor”, ClujNapoca, Romania

Introduction: Tibial pilon fractures are severe injuries, often comminuted, difficult to treat, no matter the chosen method. Representing $3-10 \%$ of all tibial fractures and less than $1 \%$ of all lower limb fractures, about $10-30 \%$ of these fractures are open fractures, with badly damaged soft tissues. Diagnosing requires a CT examination, besides the radiologic examination, for a correct asses of the articular surfaces.

Materials and methods: This article analyses the problems related to tibial pilon fractures, as reflected by the authors experience. Between 2004 and 2009 we had 145 patients with tibial pilon fractures, 20 patients presented open fractures. The methods we have used to treat the tibial pilon fractures were: intramedullary nails (10 cases), plates and screws (25 cases), external fixators (12 cases), K-wires and screws (64 cases) and nonsurgical methods (34 patients).

Results: Minimally displaced fractures were treated with Plaster immobilisation (26 patients) or skeletal traction (8 patients). The severely displaced fractures required open reduction and stable internal fixation (111 patients). Active mobilization without loading of the ankle was permitted for avoiding joint stiffness and algic osteoporosis. In most cases we have obtained good functional outcomes with a low malunion and nonunion rates. In case of vicious consolidation with marked joint osteoarthritis, ankle arthroplasty or joint fusion were necessary.

Conclusion: Tibial pilon fractures occur on a poorly vascularized bone and have severe functional consequences for the tibiotarsian 
joint. The frequent involvement of the surrounding soft tissues led to important changes in managing these fractures, varying from rigid internal fixation to less extensive, even percutaneous fixation techniques.

References: 1. Canale ST. Campbell's operative orthopaedics. 10th ed, vol 3. Philadelphia: Mosby; 2003. p. 2741-54. 2. Browner BC, Jupiter JB. Skeletal trauma. 3rd ed. Philadelphia: Saunders; 2003. p. 2257-306. 3. Miller MD Review of orthopaedics. 5th ed. Philadelphia: Saunders, Elsevier; 2008. p. 621-6.

Disclosure: No significant relationships.

\section{P79}

\section{DIAGNOSIS OF CONCOMITANT FEMORAL NECK FRACTURE IN PATIENTS WITH FEMORAL SHAFT FRACTURE}

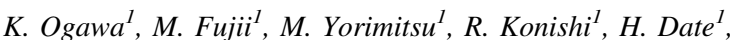 \\ K. Munetomo ${ }^{1}$, S. Arataki ${ }^{1}$, T. Noda ${ }^{2}$, T. Ozaki \\ ${ }^{1}$ Division of Orthopaedic Trauma, Fukuyama City Hospital \\ Emergency Medical Center, Hiroshima, Japan, ${ }^{2}$ Department of \\ Orthopaedic Surgery, Okayama University Medical School, \\ Okayama, Japan
}

Introduction: An ipsilateral femoral neck fracture is seen in association with $1-9 \%$ of femoral shaft fractures, and $20-50 \%$ of these injuries are missed initially. Although preoperative bone window of CT scan is helpful to diagnose the neck fracture, but there are cases in which fracture line can not visible preoperatively. We assumed that the window of abdominal CT scan around femoral neck may help to diagnose "occult" femoral neck fracture.

Materials and methods: Forty nine femoral shaft fractures were taken preoperative CT scan and were treated with intramedullary nailing. Four $(8.2 \%)$ of them had an associated femoral neck fracture. Two of them were diagnosed by preoperative radiograph and bone window of CT scan, and the other two cases were diagnosed by intraoperative fluoroscopy despite negative finding on preoperative bone window of CT scan. No other femoral neck fractures were diagnosed during follow-up period.

We retrospectively measured the width of anterior capsule and posterior soft tissue around femoral neck using soft tissue window of CT scan in all cases.

Results: Nine cases had soft tissue widening around hip joint. Three of femoral neck fractures was included, but one had no widening. 4 of them had associated acetabular fractures, hip dislocation, or pubic rami fracture which reaches hip joint. Remaining 2 cases had no intraarticular fractures but had quite severe high energy injury.

In summary, one of the cases of preoperatively unrecognized femoral neck fracture had soft tissue widening around the hip joint on the preoperative soft tissue window of CT scan, but the other had no widening. Conclusion: Assessment of preoperative soft tissue window of CT scan around the hip joint might help to estimate occult femoral neck fracture and it helps to decide preoperative planning. However, as iatrogenic fracture might potentially be generated, postoperative anterior and lateral radiographs of the hip also should be taken to evaluate the fracture in the operation room before the patient is awakened.

References: 1 . Tornetta et al. Diagnosis of femoral neck fractures in patients with a femoral shaft fracture. Improvement with a standard protocol. J Bone Joint Surg Am. 2007;89(1):39-43. 2. Yang et al. Fracture of the ipsilateral neck of the femur in shaft nailing. The role of CT in diagnosis. J Bone Joint Surg Br. 1998;80(4):673-8.

Disclosure: No significant relationships.
P80

\section{DISTAL TIBIA AND PILON FRACTURES TREATED WITH THE EXTERNAL FIXATION METHOD}

\section{S.S. Milenkovic}

Orthopaedic and Traumatologic Clinic, Clinical centre of Nis, Nis, Serbia

Introduction: There is no universal method in treating distal tibial fractures. We present a series of operated patients with distal tibia and pilon fractures. The patients were operated on using, the external fixation method and the method of external fixation with a minimal internal fixation.

Materials and methods: Thirty-seven patients were operated on. Type A fractures were operated on using the method of external fixation, whereas type $\mathrm{B}$ and $\mathrm{C}$ fractures were operated on using the methods of external fixation and minimal internal fixation. In the type $\mathrm{B}$ and $\mathrm{C}$ fractures, a rigid fixation was transformed into a dynamic external fixation 1 month post-surgery.

Results: A retrospective study analysed 37 patients. Their average age was 42.81 (from 21 to 60). The average follow-up was 21.86 (from 12 to 48) months. The percentage of union was $94.59 \%$ (35), nonunion $2.70 \%$ (1) and malunion $2.70 \%$ (1). The mean to fracture union was 14 weeks (range 12-20 weeks). There were $10.81 \%$ (4) infections around the pins of the external fixator and $2.70 \%$ (1) of deep infections. The ankle joint arthrosis as a late complication appeared in $13.51 \%$ (5). All arthroses appeared in patients who had the type $\mathrm{C}$ fracture. The final functional results based on the AOFAS score were excellent-56.75\% (21), good $29.72 \%$ (11), average $10.81 \%$ (4) and bad $2.70 \%$ (1).

Conclusion: The external fixation of distal tibial and pilon fractures as a one-step procedure is a good method for treating all types of fractures. In the type $\mathrm{B}$ and $\mathrm{C}$ fractures, a dynamic external fixation allows early mobility in the ankle joint.

References: 1. Anglen JO. Early outcome of hybrid external fixation for fracture of the distal tibia. J Orthop Trauma. 1999;13:92-7. 2. Mitkovic M, Bumbasirevic M, Lesic A, Golubovic $Z$. Dynamic external fixation of comminuted intra-articular fractures of the distal tibia (type $\mathrm{C}$ pilon fractures). Acta Orthop Belg. 2002;68:508-14.

Disclosure: No significant relationships.

\section{P81}

\section{DO GENDER DIFFERENCES INFLUENCE THE OUTCOME OF ELDERLY HIP FRACTURE PATIENTS DURING HOSPITAL STAY?}

P.C. Johannesma ${ }^{1}$, F.W. Bloemers ${ }^{2}$, F.C. Bakker ${ }^{2}$, E.S.M. de Lange de Klerk ${ }^{3}$, W.P. Zuidema ${ }^{2}$

${ }^{1}$ Department of Trauma Surgery, VU Academic Medical Center, Amsterdam, Netherlands, ${ }^{2}$ Trauma Surgery, VU University Medical Centre, Amsterdam, Netherlands, ${ }^{3}$ Department Of Epidemiology And Biostatistics, VU University Medical Center, Amsterdam,

Netherlands

Introduction: The annual incidence of hip fracture is known to rapidly expand within western society. Hip fractures mainly concern advanced elderly patients with a high degree of co-morbidity and therefore susceptible to morbidity and mortality. Previous studies found a gender bias favouring women. The aim of this study was 
therefore to evaluate the gender differences in elderly hip fractures patients during hospitalization.

Materials and methods: All consecutive patients operated for a hip fracture at our hospital between 2005 and 2009 were included. Data were retrospectively retrieved and reviewed from patients records. Exclusion criteria were age $<65$ years, polytrauma and pathologic fractures. Primary outcomes were postoperative complications, in hospital length of stay and in hospital mortality. Male and female hip fracture patients were compared.

Results: In total 618 patients were included, of which $76.7 \%$ were women. The mean age for male was 81 years (range 65-96) and for women 83 years (range 65-99). Male gender was associated with significant more pulmonal morbidity $(\mathrm{p}<0.05)$, malignancy $(\mathrm{p}<0.006)$, postoperative pneumonia $(\mathrm{p}<0.005)$, wound infections $(\mathrm{p}<0.046)$ and an increased in hospital death $(\mathrm{p}<0.001)$. Female gender was significant associated with earlier hip surgery. No significant differences between male and female gender were found in time to OR and length of stay in the hospital.

Conclusion: Hip fractures in male gender led to significant more postoperative complications and increased number of in-hospital death. No increased length of stay was detected. This outcome might need us to reconsider the organisation of surgery, nursing and rehabilitation in male gender hip fracture patients during hospitalization. Disclosure: No significant relationships.

\section{P82}

\section{DOES FRACTURE LOCATION INFLUENCE FUNCTIONAL OUTCOME IN CONSERVATIVELY TREATED SCAPHOID FRACTURES?}

\author{
S.J. Rhemrev ${ }^{1}$, R.H. van Leerdam ${ }^{2}$, D. Ootes ${ }^{1}$, M. Otoide ${ }^{3}$, \\ F. Beeres ${ }^{4}$, S.A.G. Meylaerts
}

${ }^{1}$ Surgery, Medisch Centrum Haaglanden, The Hague, Netherlands, ${ }^{2}$ Surgery, Medisch Centrum Haaglanden, The Hague, Netherlands, ${ }^{3}$ Landsteiner Institute, Medisch Centrum Haaglanden, The Hague, Netherlands, ${ }^{4}$ Surgery, Medisch Centrum Haaglanden, The Hague, Netherlands, ${ }^{5}$ Surgery, Medical Centre Haaglanden,

The Hague, Netherlands

Introduction: Functional outcome after scaphoid fracture treatment is often valued using clinical examination and radiological consolidation. However, this surpasses the importance of functional outcome, indicating the success of fracture treatment from the patient's perspective. This is the first study that examines whether fracture location influences functional outcome.

Materials and methods: In this study we evaluated 60 patients with a conservatively treated scaphoid fracture. We questioned whether the location of the fracture influences functional outcome. Functional outcome was assessed by the Disabilities of the Arm and Hand questionnaire (DASH).

Results: Trauma mechanism, treatment modality, diagnostic modalities, duration of cast immobilization and complications were analyzed. Median DASH score was 6 (26-75 percentile 3-15) for 13 distal pole fractures and 5 (26-75 percentile 0-21.5) for 44 mid-waist fractures $(p=0.70)$. No significant differences were measured between cast duration (median 6 weeks) and age.

Conclusion: Functional outcome by conservative treatment was good and no significant differences were measured between mid-waist and distal pole scaphoid fractures. We believe future studies should focus on proximal scaphoid fractures, thus being a separate subgroup requiring different treatment

Disclosure: No significant relationships.
P83

\section{EARLY ASSESSMENT AND EMERGENCY DEPARTMENT MANAGEMENT OF UNSTABLE ANKLE INJURIES SHORTENS HOSPITAL ADMISSION}

\section{E. Battaloglu, A. Lakdawala, T. Thangarajah, A. Tillu}

Trauma and Orthopaedic Surgery, Sandwell and West Birmingham Hospitals NHS Trust, 4HJ, UK

Introduction: To evaluate, in patients presenting with unstable ankle fracture, whether a correlation lies between the duration of delay to initial fracture management with the duration of hospital admission. Materials and methods: A retrospective review of the first 100 patients in 2010 requiring operative management (manipulation under anaesthetic + open reduction and internal fixation) for unstable ankle fractures. Operative date and hospital stay were collated with duration of emergency department wait and time to radiological assessment in plaster.

Results: Of the 100 patients assessed, 91 patients whose hospital admissions were uncomplicated by co-morbid injuries or illness, had a mean inpatient stay of 5 days. Comparison of patients with similar bony injury, based on AO classification, shows an increased hospital stay in patients whose assessment and initial management was delayed over 90 min after presentation to the emergency department. This is seen most evidently within patients presenting with associated joint dislocation.

Conclusion: Assessment and instigation of early initial management through adequate fracture reduction, immobilisation and elevation is crucial in the course to early operative fixation and reduced in-patient stay for patients presenting with unstable ankle injuries. It is theorised that aggressive initial management for ankle injuries in trauma is likely to lead to greater soft tissue care, thus allowing early fixation of associated bony injuries. Further evaluation into the validity for the utilisation of a rapid triage system to prioritise ankle injuries would be recommended.

Disclosure: No significant relationships.

\section{P84}

\section{ELASTIC STABLE INTRAMEDULLARY NAILING (ESIN) COMPARED WITH PLATE OSTEOSYNTHESIS IN DISLOCATED CLAVICLE FRACTURES, RESULTS OF A RETROSPECTIVE COHORT STUDY}

\author{
R. van den Berg ${ }^{1}$, P.van Rutte ${ }^{2}$, G. van Montfort ${ }^{2}$, A. van der Veen ${ }^{2}$ \\ ${ }^{1}$ General Surgery, Catharina Ziekenhuis, Eindhoven, Netherlands, \\ ${ }^{2}$ General Surgery, Catharina Hospital Eindhoven, Eindhoven, \\ Netherlands
}

Introduction: Until the late 90s most clavicle fractures were treated conservatively. Because of high rates of non- and mal-union nowadays plate osteosynthesis is the gold standard [1] in treating dislocated clavicle fractures. An alternative surgical treatment is intramedullary nail fixation [2]. The aim of this study was to evaluate the results of ESIN in dislocated clavicle fractures and compare them with plate osteosynthesis and the results described in literature.

Materials and methods: All patients operated for a dislocated clavicle fracture from January 2005 to August 2010 were reviewed. Data of interest were duration of operation, type of reduction, complications, fracture consolidation and the functional DASH and Constant Shoulder Score. 
Results: 72 patients were operated for a dislocated midshaft fracture. 51 patients were treated with ESIN, 21 patients with plate osteosynthesis.

For both groups there was at least $95 \%$ fracture consolidation with an excellent functional recovery with the DASH and CSS. Big difference was a much higher complication rate in the ESIN group, 63 versus $30 \%$ with plate osteosynthesis. This difference is mainly caused by migration of the nail and consequently pain at the medial site of the clavicle. Complaints resolved in all patients after nail removal, an uneventful procedure in all cases.

Conclusion: Mid-shaft clavicle fractures should be classified carefully to choose the right indication for ESIN or plate osteosynthesis. A prospective randomized controlled trial is necessary to draw any definitive conclusions.

References: 1. Smekal V, et al. Elastic stable intramedullary nailing versus nonoperative treatment of displaced midshaft clavicular fractures - a randomized, controlled, clinical trial. J Orthop Trauma. 2009;23(2):106-12. 2. Canadian Orthopaedic Trauma Society. Nonoperative treatment compared with plate fixation of displaced midshaft clavicular fractures. A multicenter, randomized clinical trial. 2007;89(1):1-10.

Disclosure: No significant relationships.

\section{P85}

\section{EPIDEMIOLOGY OF STERNAL FRACTURES: WHERE WE GOING?}

P. Moreno de la Santa Barajas ${ }^{1}$, M.D. Polo Otero ${ }^{l}$, C. Delgado $\overline{\text { Sanchez-Gracian }}{ }^{2}$, P. Magdalena Lopez ${ }^{3}$, M. Choren Duran ${ }^{1}$, C. Trinidad Lopez ${ }^{2}$, M. Lozano Gomez ${ }^{1}$, S. Leal Ruiloba ${ }^{4}$

${ }^{1}$ Thoracic Surgery, Hospital Povisa, Vigo, Spain, ${ }^{2}$ Radiology, Hospital Povisa, Vigo, Spain, ${ }^{3}$ Statistic, Hospita Povisa, Vigo, Spain, ${ }^{4}$ Critical Care, Hospita Povisa, Vigo, Spain

Introduction: The epidemiology of sternal fractures has been poorly described. The objective of this study was to examine the demographics, outcomes and injuries associated with sternal fractures.

Materials and methods: Medical records from Hospital POVISA were retrospectively reviewed to identify all patients with sternal fractures over a 20 years period. Demographic data collected included age, gender, mechanism of injury, and injury severity score. Patients were analyzed according two periods of time; Group I (1991-2000), 43 patients and Group II (2001-2010) 170 patients.

Results: During the 20 years study period, a total of 2,775 patients were admitted with thoracic trauma. Of these, $170(6.1 \%)$ had a sternal fracture. The average age was 51 years (17-85), with $63.5 \%$ being male. The most common mechanism of injury was motor traffic accident 135 (79.4\%) followed by falls 28 (16.5\%). In 101 patients had only chest was. Associated rib fractures occurred in $50(29.4 \%)$ of the population and flail chest $14(8.3 \%) \%$. Incidence of sternal fractures was highest in group II (3.7 vs. $8.5 \%$; P).

Conclusion: Sternal fractures are a rare complication from thoracic trauma. Associated injuries are common, including other chest lesions, in particular the flail chest. The incidence of sternal fractures is increasing, but a best management of trauma patients keep the mortality rates low. The majority of patients diagnosed with a sternal fracture following trauma can be discharged to home.

Disclosure: No significant relationships.
P86

\section{FAST TRACKING OF PROXIMAL FEMUR FRACTURES! WHO IS FIT?}

\author{
Z. Khan ${ }^{1}$, M.U. Khattak ${ }^{2}$, N. Bennett ${ }^{2}$, I.R. Chambers $^{2}$
}

${ }^{1}$ Trauma and Orthopaedics, Kettering General Hospital, 8UZ, UK,

${ }^{2}$ Trauma and Orthopaedics, Scunthorpe General Hospital, 7BH, UK

Introduction: Many hospitals in the UK based on the audit commission report in 1995 , adopted a locally viable fast track scheme to admit patients with fractured neck of femur. Fast tracking of these patients from $A \& E$ helps initiates early cares plans and avoid the $4 \mathrm{~h}$ breaches in A\&E. With this there comes a risk of missing other problems which can be potentially life threatening.

Materials and methods: After approval from the local quality evaluation and clinical audit department, we prospectively recruited 61 patients over the period of 2 months presenting to our local Accidents and Emergency Department with hip fractures. The medical notes of these patients were assessed against a pre prepared evaluation tool by two investigators (ZK and MUK). Following the results of this audit, a fast tracking checklist was created in liaison with the A\&E department to eradicate the flaws and was implemented locally. 2 months after the implementation of the checklist, this practice was re audited prospectively.

Results: In the initial audit 14 percent of the patients were inadequately fast tracked, including 2 under the age of 60,1 each with a missed wrist fracture and shoulder dislocation and 2 with significant cardiac problems. Varying incidences of delay in delivery of analgesia and fluid resuscitation were also noted. Since the introduction of the checklist, a marked improvement in practice was noted in our re audit.

Conclusion: Fast tracking of hip fractures is a beneficial tool for all involved, but it should not compromise proper clinical evaluation in accidents and emergency department.

Disclosure: No significant relationships.

\section{P87}

\section{FEMORAL SHAFT FRACTURE IN ELDERLY POPULATION}

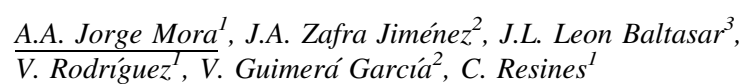

${ }^{1}$ Cot Ii, Hospital 12 de Octubre, Madrid, Spain, ${ }^{2}$ Orthopaedic and Trauma Surgery, Hospital Universitario 12 de Octubre, Madrid, Spain, ${ }^{3}$ Orthopaedic Surgery and Traumatology II., Hospital 12 de Octubre., Madrid, Spain

Introduction: Femoral shaft fracture is an uncommon type of fracture in the elderly population with different behaviour than in younger people, and with different lesional pattern.

Materials and methods: Our current study makes a retrospective review of all femoral shaft fractures in people older than 65 years old seen in our hospital from 1998 to 2008. The fractures were classified in terms of Müller AO Classification, age, gender, previous functional status and dependence, Glasgow come scale (GCC) at the emergency room and lesional mechanism. We also registered the surgical technique used, the average of survival within 2 years of minimum follow up, the need for blood transfusion, the consolidation rates, the delayed union, nonunion, and malunion, the functional outcomes and the 
associated injuries. All early and late complications and their consequences were recorded. We also compare this outcomes with those seen in intertrochanteric fractures in this group of population.

Results: With all the data registered we performed a statistical analysis to find out significant relations with survival and functional outcomes in the group of study.

Conclusion: Femoral shaft fractures have final outcomes comparable with intertrochanteric fractures according to survival but higher rates of complications. Previous situation is a predictor of functional result.

References: Specific features associated with femoral shaft fractures caused by low-energy trauma. J Trauma. 1997;43(1):117-22. Patterns and complications of femur fractures below the hip in patients over 65 years of age. J Orthop Trauma. 1992;6(2):167-74. Basic epidemiology of fractures of the upper and lower limb among Americans over 65 years of age. Epidemiology. 1996;7(6):612-8. Outcome after femoral shaft fractures in the elderly: the effects of altitude. Bull Hosp Joint Dis. 2006;63(3-4):117-22. Outcome of femoral shaft fractures in the elderly. Clin Orthop Relat Res. 1996;(332):105-9.

Disclosure: No significant relationships.

\section{P88}

\section{FIRST RESULTS: FIXATION DEVICE RELATED ROTATIONAL INFLUENCES IN HIP FRACTURES, A RADIO STEREOMETRIC ANALYSIS}

\section{G.S. Stollenwerck $^{1}$, D. van Embden ${ }^{1}$, B. Kaptein ${ }^{2}$, R.G. Nelissen ${ }^{2}$, I.B.} Schipper $^{I}$

${ }^{1}$ Trauma Surgery, Leiden University Medical Center, Leiden, Netherlands, ${ }^{2}$ Orthopaedic Surgery, Leiden University Medical Center, Leiden, Netherlands

Introduction: Several fixation devices have been developed for treatment of proximal femur fractures. Still, treatment of these fractures suffers from high complication rates. For treatment of trochanteric fractures extramedullary DHS and intramedullary nail fixations are commonly used. Stable femoral neck fractures are treated with a DHS or three-cannulated screws, when preservation of the femoral head is intended. These types of implants are related to complications like cutout of the implant and nonunion. Some of these complications may be accounted for by rotation of the femoral head fragment. The amount of rotational instability in hip fractures, related to the fracture type and current implants, has never been objectified. This study has been performed to investigate this particular phenomenon.

Materials and methods: Micromotion across the fracture site (rotation and translation) determines stability of the type of osteosynthesis. This micromotion is best determined three-dimensionally by radiostereometric analysis (RSA).

Results: Three patients, with an AO31-A2 type trochanteric fracture were treated with a DHS and two patients with a Gamma-nail. Four patients with a non-displaced femoral neck fracture were treated with three-cannulated screws. Results show very interesting rotational and translational shifts of the fracture fragments along the axes. The different types of hip fractures and fixation methods tend to show different micromotion patterns.

Conclusion: This case series provides more insight into the biomechanical properties of these complex fractures and will help to optimize current treatment concepts. Currently, a randomized controlled trial is performed to objectify the above mentioned trend in micromotion patterns between different fracture types and fixation methods.

Disclosure: No significant relationships.
P89

\section{FREEZING OF RAT TIBIAE AT $-20^{\circ} \mathrm{C}$ DOES NOT AFFECT MECHANICAL PROPERTIES OF INTRAMEDULLARY BONE/IMPLANT-INTERFACE IN PULL-OUT TESTING} M. Diefenbeck ${ }^{1}$, S. Zankovych ${ }^{2}$, J. Bossert ${ }^{2}$, C. Schrader

${ }^{1}$ Klinik Für Unfall- Hand- Und Wiederherstellungschirurgie, Friedrich-Schiller-Universität Jena, Jena, Germany, ${ }^{2}$ Insitut Für Materialwissenschaften, Friedrich-Schiller-Universität Jena, Jena, Germany, ${ }^{3}$ Innovent Technology Development, Jena, Germany, ${ }^{4}$ Königsee Implantate $\mathrm{GmbH}$, Aschau, Germany

Introduction: Orthopaedic implants rely on osseointegration to achieve mechanical fixation to the host bone, which is a prerequisite for a good clinical outcome. As the rat tibial implant model is used more frequently to assess osseointegration, it is important to define optimum conditions and procedures for all parts of a trial, from anaesthesia via implantation procedures to storage of tissue samples and mechanical testing. So far, biomechanical testing was performed under at least three different conditions: fresh explanted bone, frozen bone or dehydrated and rehydrated bone. In many other reports, the condition of the bone during testing is not mentioned.

The purpose of the present study was to determine, if freezing and storage of bones with implants at $-20^{\circ} \mathrm{C}$ does have an effect on the biomechanical behaviour of the fragile bone/implant-interface during mechanical pull-out tests in a rat model.

Materials and methods: Implants

Twenty TiAl6V4 rods $(\varnothing 0.8 \mathrm{~mm}$, length $10 \mathrm{~mm})$.

Procedure

10 rats underwent bilateral tibial implantation of TiAl6V4 rods. After 8 weeks, all animals were sacrificed and the tibiae harvested for biomechanical testing. Eight tibiae were frozen and stored at $-20^{\circ} \mathrm{C}$ for 14 days, while the remainder were evaluated immediately after harvesting. Pull-out tests were used to determine maximum force and interfacial shear strength.

Results: There were no significant differences between the fresh and the frozen-thawing group in maximum force or in interfacial shear strength (Figs. 1, 2) at the 95.0\% confidence level [Fisher's least significant difference (LSD) procedure].

Conclusion: Freezing and storage at $-20^{\circ} \mathrm{C}$ of rat tibiae containing an implant does not affect mechanical properties of bone/implantinterface in biomechanical testing.

Reference: Gabet Y, Kohavi D, Voide R, Müller TL, et al. Endosseous implant anchorage is critically dependent on mechanostructural determinants of peri-implant bone trabeculae. J Bone Miner Res. 2010;25:575-83.

Disclosure: No significant relationships.

\section{P90}

HOW RELIABLE IS THE COMPREHENSIVE AO/OTA-CLASSIFICATION OF ADULT LONG-BONE FRACTURES IN A BUSY ORTHOPAEDIC DEPARTMENT?

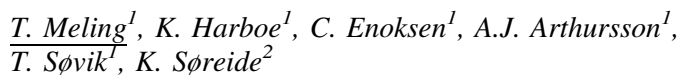

${ }^{1}$ Orthopaedic, Stavanger University Hospital, Stavanger, Norway,

${ }^{2}$ Surgical, Stavanger University Hospital, Stavanger, Norway 
Introduction: The comprehensive classification of long-bone fractures (Müller, AO, OTA) is accepted worldwide yet proper validation has not been performed. The aim of this study was to test the interand intra-rater reliability and accuracy of the scoring system as used among orthopaedic trauma surgeons.

Materials and methods: All long-bone fractures at Stavanger University Hospital treated in hospital (for year 2008) were classified in a fracture registry. The fractures were re-classified by the same surgeon after at least 11 months for the intra-rater reliability. An average experienced resident re-classified $50 \%$ of the fractures for the interrater reliability. A reference standard (by two expert orthopaedic trauma surgeon) dataset was developed for the blinded and nonblinded accuracy calculation. Both Kappa statistics and proportion of agreement were used.

Results: Analysis of the classification system using four AO-signs, the Kappa agreement (and proportion of agreement) was calculated for the intra-rater at $0.67(69 \%)$, for the inter-rater at $0.67(67 \%)$, for the non-blinded accuracy at $0.76(78 \%)$, and for the blinded accuracy at $0.68(70 \%)$. The disagreement related to the blinded accuracy was more related to the fracture seriousness and the fractured segment than to the raters' experience and the age and gender of the patient. Conclusion: When using four signs of the comprehensive classification of long-bone fractures in a in hospital fracture registry the overall Kappa value was close to 0,70. Proportion of agreement of no more than $70 \%$ should be expected. This is of importance when comparing studies across institutions.

Disclosure: No significant relationships.

\section{P91}

\section{HOW SEVERE ARE INITIALLY UNDETECTED INJURIES TO THE KNEE ACCOMPANYING A TIBIAL SHAFT FRACTURE?}

\author{
S. Lederer, A. Auffarth, R. Bogner, M. Mayer, S. Karpik, \\ G. Korn, H. Resch
}

Unfallchirurgie Und Sporttraumatologie, Universitätsklinik Salzburg, Salzburg, Austria

Introduction: Fractures of the tibia are severe injuries that quickly attract the physician's attention. Previous reports have shown that injuries to the ipsilateral knee can occur. In most cases, such injuries were diagnosed on delay. Excluding cases in which a knee injury was apparent already at admission, we sought to investigate the number and severity of initially undetected lesions to the knee concomitant with a tibial shaft fracture and give an overview of the literature referring to these combined injuries.

Materials and methods: Charts and X-rays of patients treated for a tibial shaft fracture from January 2002 until September 2009 were reviewed. Patients, in whom any other injury of the affected limb apart from a tibial diaphyseal fractures. Was initially diagnosed, were excluded. Also patients, in whom an injury to the knee had been diagnosed at admission, were excluded.

Results: 304 patients with tibia fractures were available for analysis. An injury to the knee was diagnosed in ten cases. There were partial tears of the cruciate ligaments and lesions of the medial meniscus. All lesions were conservatively treated without any after-effects.

Conclusion: Physical examinations under anesthesia, arthroscopy and magnetic resonance imaging have shown lesser correlation among each other than one would expect. More severe injuries to the knee with tibial shaft fractures are more likely to be detected early, than minor ones. Pain about the knee communicated by the awake patient should be the indication for further apparative examination by magnetic resonance imaging or arthroscopy.

Disclosure: No significant relationships.

P92

\section{IMPACT OF OPENING A SKATE PARK ON THE INCIDENCE AND SEVERITY OF INJURIES PRESENTING TO A DISTRICT HOSPITAL}

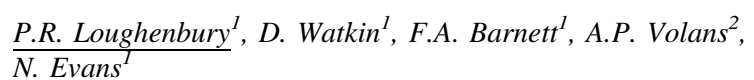

${ }^{1}$ Department Of Trauma And Orthopaedic Surgery, Scarborough Hospital, Scarborough, UK, ${ }^{2}$ Department Of Emergency Medicine, Scarborough Hospital, Scarborough, UK

Introduction: The opening of dedicated Skate Parks has been linked to an increase in the incidence of paediatric injuries in some units. This study provides a retrospective review of the effect of a Skate Park opening close to a district hospital.

Materials and methods: The number and pattern of admissions to the emergency department were investigated in the first month following opening of the Skate Park. The operative workload of the orthopaedic department was also examined. These were compared with the same time period in the previous year.

Results: In the first month after opening of the Skate Park 393 patients attended the ED following minor trauma (234 limb injuries, 126 head injuries, 33 contusions/lacerations). 55 (14\%) required inpatient treatment. In the previous year 392 patients attended the ED following minor trauma (218 limb injuries, 145 head injuries, 29 contusions/lacerations). 54 (14\%) required inpatient treatment. There was no difference between the number and pattern of ED attendances during these two time periods. In the month after the Skate Park opened 28 paediatric patients required operative fixation of limb injuries (19 manipulations under anaesthetic $+/-\mathrm{k}$-wire fixation; 9 open procedures). In the previous year, 23 paediatric patients required operative fixation of limb injuries (21 manipulations under anesthetic $+/-$ k-wire fixation, 2 open procedures). $32 \%$ of cases required open reduction following opening of the Skate Park compared with $9 \%$ in the previous year $(\mathrm{p}=0.0168$, Chi squared test).

Conclusion: In our unit the opening of a Skate Park did not alter the number or pattern of ED attendances. However, a larger number of paediatric injuries required operative fixation and a greater proportion of these were open procedures. Although the number of injuries did not change, the severity of paediatric injuries increased following opening of the Skate Park.

Disclosure: No significant relationships.

\section{P93}

\section{IMPACT OF SNOW ON TRAUMA WORKLOAD IN A DISTRICT GENERAL HOSPITAL}

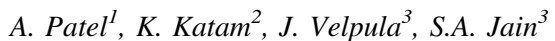

${ }^{1}$ General Paediatrics, Birmingham Children's Hospital, Birmingham, UK, ${ }^{2}$ Trauma And Orthopaedics, Heartlands Hospital,

Birmingham, UK, ${ }^{3}$ Trauma And Orthopaedics, Good Hope

Hospital, Birmingham, UK 
Introduction: From 16th December 2009 to 20th January 2010 the United Kingdom experienced the coldest winter since 1978, with lowest recorded temperatures of $-22^{\circ} \mathrm{C}$ in some areas. Average snowfall up to $61 \mathrm{~cm}$ was recorded in some parts. This caused severe disruption and economic costs of $£ 700 \mathrm{~m}$ to UK taxpayers. There was widespread transport disruption, school closures, power failures, cancelled sporting events and over 25 people died. We studied the impact of the recent snowfall on the workload of an Orthopaedic department. We also analysed its financial implications and made suggestions on how to deal with possible similar situations in future. Materials and methods: We compared the number of admissions, cancellations, theatre delays and fracture patterns during the heavy snow period (16 December 2009-20 January 2010) to December 2008. Data was collected retrospectively from accident and emergency records, clinic bookings, ward admissions, and theatre records. The hospital intranet (PAWS, PACS, i-Care) was used to retrieve the data.

Results: The number of patients attending the emergency department increased by 350 from the previous year. A $15 \%$ increase in fracture clinic attendance was found. A total 30\% increase in inpatient admissions, with increased bed occupancy of $30 \%$ in orthopaedic and $80 \%$ in non-orthopaedic wards. There were more complex pelvic and lower limb fractures. There was an average $12 \mathrm{~h}$ delay to operation time compared to the previous year. Elective procedures were cancelled due to shortage of inpatient beds and theatre space. There was a delay in patient discharge by an average of 3.5 days.

Conclusion: The overall financial impact of this heavy snowfall was $£ 260,573$. There was also the burden of rescheduling costs and inconvenience to patients which were harder to quantify. This audit highlighted the importance of awareness and future planning to allocate extra resources and manpower to avoid severe disruption to health services.

Disclosure: No significant relationships.

\section{P94}

\section{IMPLANT REMOVAL IN THE DUTCH PRACTICE. RESULTS OF A SURVEY}

\author{
D.I. Vos ${ }^{1}$, B. Hanson ${ }^{2}$, M. Verhofstad ${ }^{3}$
}

${ }^{1}$ Trauma Surgery, Amphia Hospital Breda, Breda, Netherlands, ${ }^{2}$ AO Clinical Investigation and Documentation, Dübendorf, Switzerland,

${ }^{3}$ General and Trauma Surgery, St. Elisabeth Hospital, Tilburg, Netherlands

Introduction: The indication for implant removal is not always well defined as part of routine clinical protocol and there is ongoing discussion concerning this issue worldwide. In the Netherlands alone, about 18,000 operations for implant removal after fracture healing are performed each year. The aim of this survey study was to evaluate the current opinion and practice of Dutch trauma and orthopaedic surgeons in the removal of implants after fracture healing.

Materials and methods: A web-based questionnaire consisting of 44 items was sent to all members of the Dutch Trauma Society and Dutch Orthopaedic Trauma Society to determine their habits and opinions about implant removal.

Results: Dutch surgeons (95\%) commonly remove implants only in the case of elastic nails in children, yet this is not advocated by all $(66 \%)$ for young adult $(<40$ years) patients. Orthopaedic and trauma surgeons differ in their opinion about this subject $(\mathrm{p}=0.002)$. Most of the implants are generally not removed. Infection of the implant or bone and specific complaints made by the patient were the main reasons for removing hardware $(>90 \%)$, while performing the same service to make money was motivation for only $1 \%$. Titanium plates are more difficult to remove than stainless steel hardware.

Conclusion: This survey indicates that there is no general opinion about implant removal after fracture healing and a lack of policy guidelines in the Netherlands. Surgeons practice what they think is best and patient complaints play an important role in the decision making.

Disclosure: No significant relationships.

P95

\section{INCIDENCE OF UPPER EXTREMITY INJURIES IN THE US}

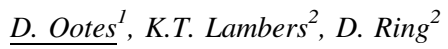

${ }^{1}$ Orthopaedic Hand Service, Massachusetts General Hospital, Boston, MA, USA, ${ }^{2}$ Hand and Upper Extremity Department, Massachussetts General Hospital, Harvard Medical School, Harvard University, Boston, MA, USA

Introduction: The epidemiology of upper extremity injuries presenting to Emergency Departments in the United States is not well studied. The purpose of this study is to estimate the incidence rates of upper extremity injuries presenting to Emergency Departments.

Materials and methods: The National Electronic Injury Surveillance System (NEISS) - a database of Emergency Department visits based on a sample of hospitals selected and weighted to represent the entire US population in order to allow estimates of overall incidence-was queried for all upper extremity injuries presenting to US emergency departments in 2009. Injury types were analyzed for each region of the upper extremity and incidence rates were calculated based on population estimates from the US Census.

Results: A query of the NEISS resulted in 92,601 records of upper extremity injury treated at an Emergency Department in the United States in 2009, which translates to an estimated total of $3,468,996$ such injuries that year. This corresponds to an incidence of 1,130 upper extremity injuries per 100,000 persons per year. The most common region injured was the finger $(38.4 \%)$. The most common upper extremity injury was a fracture $(29.2 \%)$. Specific injuries with high incidence rates (all per 100,000 per year) included finger lacerations (221), wrist fractures (72), finger fractures (68) and lower arm fractures (64). Home is the most common setting for an upper extremity injury.

Conclusion: The NEISS provides useful estimates of the incidences of upper extremity injuries that may be useful for public health initiatives.

Disclosure: No significant relationships.

\section{P96}

\section{INFECTIONS AFTER ANKLE FRACTURES-RARE BUT COMPLICATED?}

\author{
A. Ahrberg $^{1}$, N. Höde ${ }^{l}$, C. Josten ${ }^{2}$
}

\begin{abstract}
${ }^{1}$ Department of Traumatology and Reconstructive Surgery, University of Leipzig, Leipzig, Germany, ${ }^{2}$ Department of Traumatology, Plastic and Reconstructive Surgery, Spine Center, University of Leipzig, Leipzig, Germany
\end{abstract}

Introduction: Ankle fractures are frequent and seem to be easy to treat. Yet, also these fractures can lead to infections that must be carefully managed. What are the risk factors? What treatment options 
do we have? What are the consequences and results compared to noninfected cases?

Materials and methods: 82 patients (average age 52.4 years) were included operated on a B or C ankle fracture. In $9(10.9 \%)$ there was an infection. Final Results were evaluated by AOFAS. We looked for risk factors, treatment and microbiology results.

Results: In the infected cases 4 (44.4\%) were smokers, 2 (22.2\%) with additional alcohol abuse. Average stay in hospital was 39.6 days (9-95). 5 (55.5\% had additional operations due to infection, in average 5.4 per patient (1-10) with $3(33.3 \%)$ early implant removals, $1(11.1 \%)$ vacuum sealing and 1 intramedullary antibiotic spacer. Pathogenes identified were St aureus (22.2\%), MRSA (11.1\%), MRSA plus Proteus mirabilis $(11.1 \%)$, in 5 cases $(55.5 \%)$ no pathogen could be identified. There was no ICU stay, no plastic surgery (e.g. a flap) needed. All fractures finally showed bony healing. In follow up, the average AOFAS was 76.5 compared to 89.4 of all 82 patients.

Conclusion: In spite of usually good outcomes after ankle fractures, an infection in this area can be a serious problem for both patient and surgeon. Consequent therapy including early implant removal, vacuum dressings or spacersif necessary as well as the right antibiotics are needed to control the infections. Problems remaining are the functional outcome and wound closure in this sensitive area. Disclosure: No significant relationships.

\section{P97}

\section{INTERNAL FIXATION IN FEMORAL NECK FRACTURES}

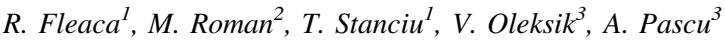

${ }^{1}$ Orthopaedics and Trauma Surgery, University of Medicine Sibiu, Sibiu, Romania, ${ }^{2}$ Orthopedic Surgery And Trauma, University of Sibiu, Sibiu, Romania, ${ }^{3}$ Engineering Faculty, University of Sibiu, Sibiu, Romania

Introduction: This work is a retrospective study whose purpose is to evaluate the results on medium term follow-up in patients treated with internal fixation for femoral neck fractures in the Emergency Hospital Sibiu.

Materials and methods: In the Department of Orthopaedic Trauma Sibiu there have been treated for femoral neck fractures 962 patients between January 2000 and June 2009, 342 of them with internal fixation fulfilled the inclusion criterias. End point of the study: control at 2 year fu, reintervention due to early redisplacement postop, nonunion or avascular necrosis of the femoral head, later comorbidity that influence patient's status, decease, lost of follow up. The evaluation has been done by two trauma surgeons using the Harris Hip Score and hip roentgenograms in anteroposterior and lateral view done before surgery, postoperative and at control or prior to reintervention. We have evaluated the quality of the reduction, the functional outcome and the complications rate. There were 58 patients lost of fu, 26 patients with comorbidities that influenced the functional outcome and 30 deceased.

Results: 182 patients came to the 2 year follow up control. Maximum Harris score was 100 points, minimal 38.72 and average 77.7. 228 patients were evaluated clinical and radiological (control, reinterventions), 136 fractures were consolidated. There were complications in $40.4 \%$. Reinterventions were needed for 23 patients. There was no significant statistical difference between different types of internal fixation. Delayed treatment was associated with higher rate of avascular necrosis.

Conclusion: The number and positioning of the screws does not influence the functional outcome, however this will be influenced by the reduction quality and the age of the patient. The high complication rate sustains the necessity of a reevaluation of indications for this method of treatment.

Disclosure: No significant relationships.

\section{P98}

\section{IS AN OPERATIVE TREATMENT OF RIB FRACTURES BENEFICIAL "FEASIBILITY STUDY FOR A MRCT"}

\section{F. Struik, L. Poelhekke}

Department of Surgery, Division of Trauma Surgery, Radboud University Nijmegen Medical Centre, Nijmegen, Netherlands

Introduction: It is hypothesized that acute surgical fixation of (multiple) rib fractures diminishes pain, ICU stay, duration on the ventilator and the chance of suffering pneumonia. Before participating in a Dutch MRCT, we evaluated our current practice of nonoperative treatment, in particular length of ICU stay and the incidence of pneumonia.

Materials and methods: Retrospective closed cohort study including all patients, in a level 1 trauma center, admitted with blunt chest trauma, between 2005 and 2009. Number of days on the mechanical ventilator is registered as primary and length of hospital and ICU stay and pneumonia as secondary outcome measures.

Results: Total number of patients admitted was 619, of which 406 with a IIS $\geq 16,70 \%$ male, mean age 47.9 years. The mean hospital stay was 16.8 days. When admitted to the ICU $(52.9 \%)$ the mean hospital stay was 25.1 days, with a mean of 9.8 days on the ICU. Of the ICU patients $50.4 \%$ was ventilated, with a mean of 8.2 days on the ventilator. $26.3 \%$ of the ventilated patients suffered a pneumonia, non-ventilated ICU patients just 6.9\% (p<0.01), and non-ICU patients $7.4 \%(\mathrm{p}<0.01)$.

Conclusion: There will probably be patient benefits from an operative treatment of rib fractures when it diminishes hospital and/or ICU stay and more specifically the number of days on the ventilator. Shorter hospital and ICU stay might also give economic benefits. However, it can be debated if, taking the incidence of possible post-operative complications into account, including pneumonia, a significant reduction of pneumonia can be achieved. In order to make this study feasible and to get maximum results, we advise to include all admitted patients with rib fractures in the upcoming Dutch MRCT.

Disclosure: No significant relationships.

\section{P99}

\section{IS IT POSSIBLE FOR THE USE OF EXTERNAL FIXATION TO REDUCE FAILURES OF STANDARD ENDER NAILING IN INTERTROCHANTERIC FRACTURES?} A COMPARATIVE INTERVENTION STUDY

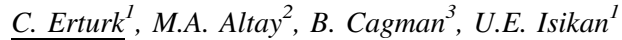

${ }^{1}$ Orthopaedics and Traumatology, Harran University Medical Faculty, Sanliurfa, Turkey, ${ }^{2}$ Orthopaedics and Traumatology, Harran University Medical Faculty, Sanliurfa, Turkey, ${ }^{3}$ Orthopaedic Surgeon, MH Av. Cengiz Gokcek State Hospital, Gaziantep, Turkey

Introduction: The use of Ender nails in intertrochanteric fractures was very popular in the 1980s. However, this method has fallen out of use due to insufficient rotational stability, leg shortening, external rotation failure, and post-operative distal nail migration. Therefore, 
we have applied a new method. We used a unilateral external fixator to support Ender nails. The purpose of the present study was to compare the results of fractures treated with those two methods of skeletal fixation (Ender nailing with or without an external fixator).

Materials and methods: Group 1 (Ender nailing with an external fixator) had 36 patients $(16 \mathrm{~m} / 26 \mathrm{f}$, mean age $71.6 \pm 11.3$ years $)$, and Group 2 (standard Ender nailing) included 12 patients $(6 \mathrm{~m} / 6 \mathrm{f}$, mean age $66.7 \pm 6.6$ years). Unilateral external fixator was used 6-8 week in Group 1. We compared a prospective series of Group 1 with a retrospective series of Group 2.

Results: The mean time to union was 9.3 weeks in Group 1 and 10.6 weeks Group 2. Rates of shortening and external rotation deformities were higher in the standard Ender nailing group. However, seven patients $(20 \%)$ in the fixator group had pin track infections. The rates of nail penetration of the femur head, loss of reduction, and knee irritation due to distal nail migration did not differ between the groups.

Conclusion: Although the treatment groups are not fully comparable, the results indicate that Ender nailing with external fixation is superior to standard Ender nailing in the treatment of unstable intertrochanteric fractures. According to our results, we think that this method may be a viable, alternative solution to prevent complications related to only using Ender nails in unstable intertrochanteric fractures.

Reference: 1. Eren OT, Küçükkaya M, Tezer M. Treatment of intertrochanteric fractures of the femur with Ender nails in patients over the age of 65 years. Acta Orthop Traumatol Turc. 2003;37(2): 102-6.

Disclosure: No significant relationships.

\section{$\mathbf{P 1 0 0}$}

\section{IS THE MÜLLER CLASSIFICATION OF LONG-BONE FRACTURES RELIABLE IN CHILDREN'S FRACTURES}

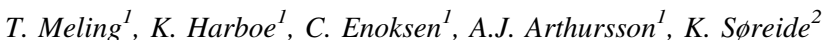

${ }^{1}$ Orthopaedic, Stavanger University Hospital, Stavanger, Norway, ${ }^{2}$ Department of Surgery, Stavanger University Hospital, Stavanger, Norway

Introduction: A new classification system is introduced by AO/ASIF for children's long-bone fractures. We still classify both fractures among children and adults according to the more familiar and simpler Müller's classification. The aim of this study was to test the inter- and intra-rater reliability and accuracy of the scoring system as used among orthopaedic trauma surgeons in children's fractures.

Materials and methods: All inhospitally treated long-bone fractures at Stavanger University Hospital (for year 2008) were classified in a fracture registry. The fractures were re-classified by the same surgeons at least 11 months later for the intra-rater reliability measurement. An average experienced resident re-classified $50 \%$ of the fractures for the inter-rater reliability analysis. A reference standard (by two expert orthopaedic trauma surgeons) dataset was developed for the blinded and non-blinded accuracy calculation. Subanalysis of the three most frequent bone segments was performed. Both Kappa statistics and proportion of agreement were used.

Results: According to four sign of the classification (fracture group) Kappa agreement (K) (95\% confidence interval) and proportion of agreement (PA) for the intraobserver classification were calculated to $\mathrm{K}=0.75(0.68-0.82)$ and $\mathrm{PA}=79 \%$, for the interobserver; $\mathrm{K}=$ $0.71(0.61-0.81)$ and $\mathrm{PA}=77 \%$, for the blinded accuracy; $\mathrm{K}=0.72$
(0.65-0.79) and $\mathrm{PA}=76 \%$ and for the non-blinded accuracy; $\mathrm{K}=0.84(0.79-0.89)$ and $\mathrm{PA}=87 \%$. The distal humeral fractures had a proportion of agreement of $83 \%$ (20 out of 24 ), the forearm shaft; $90 \%$ (47/52) and the distal forearm; 67\% (48/72). Twelve of the 72 distal forearm fractures were misclassified as forearm shaft fractures.

Conclusion: Considering reliability measurements applied to fractures in adults, the reliability of the Müller classification for longbone fractures in children seems very good. However, the determination of the bone segment involved is difficult. It might be easier if the definition of the segment involved from the children's classification system is adapted.

Disclosure: No significant relationships.

\section{P101}

\section{LEG LENGTH DISCREPANCY AFTER CEMENTED HIP HEMIARTHROPLASTY}

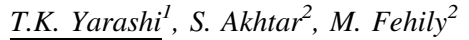

${ }^{1}$ Trauma And Orthopaedics, Royal Bolton Hospital, Bolton, UK,

${ }^{2}$ Royal Bolton Hospital, Bolton, UK

Introduction: Leg Length discrepancy (LLD) is a well known complication following hip arthroplasty. There are many papers looking at LLD after total hip replacement however there are none looking at LLD after hip hemiarthroplasty for fracture neck of femur. Our aim was to accurately assess leg length discrepancy following cemented hip hemiarthroplasty.

Materials and methods: Sixty consecutive patients who underwent cemented hip hemiarthroplasty for fracture neck of femur between 2008 and 2009 were selected for the study. Patient details including implant sizes, were collected from theatre records. We used the trauma cad software to accurately calculate LLD after appropriate calibration. We measured pre and post-operative femoral position to calculate the degree of LLD. The LLD's were calculated by two observers to look for any inter-observer differences. We excluded cases where the xray was too externally rotated, missed out the greater trochanter or no post-op X-rays were available.

Results: Sixteen cases were excluded for not meeting the inclusion criteria. In the remaining forty four patients, the mean leg length discrepancy was a $5.6 \mathrm{~mm}$ increase in length with a range of $5.3 \mathrm{~mm}$ shortening to $20.7 \mathrm{~mm}$ lengthening. The $95 \%$ confidence intervals was $1.8 \mathrm{~mm}$ above and below the mean. Pearson's correlation coefficient between pre and post operative leg length showed no significant length discrepancy with a coefficient of 0.42 . There was no significant inter observer error.

Conclusion: Many studies looking at LLD after total hip arthroplasty had a mean lengthening of $9 \mathrm{~mm}$. Our study has shown that hip hemiarthroplasties have marginally better results. However, some LLD still exists and we feel that this could have been due to the absence of pre-op templating, inaccurate femoral cut and the inability of the implant to accommodate variations in patient femoral neck angle. We believe that correcting these issues could reduce LLD, accurately restore normal anatomy and reduce the potential complications of low back pain, nerve palsy and abnormal gait associated with LLD.

References: 1. Konyves A, Bannister GC. The importance of leg length discrepancy after total hip arthroplasty. J Bone Joint Surg Br. 2005;87(2):155-7. 2. Maloney W, Keeney JA. Leg length discrepancy after total hip arthroplasty. J Arthroplasty. 2004;19(4): 
108-10. 3. Weng WJ, et al. Leg length discrepancy after total hip arthroplasty: impacts on postoperative function and patients' satisfaction. Zhongguo Gu Shang. 2009;22(12):906-8. 4. Clark CR, Huddleston HD, Schoch EP, Thomas BJ. Leg-length discrepancy after total hip arthroplasty. J Am Acad Orthop Surg. 2006;14(1): 38-45. 5. Edeen J, Sharkey PF, Alexander AH. Clinical significance of leg-length inequality after total hip arthroplasty. Am J Orthop. 1995;24(4):347-51. 6. Abraham WD, Dimon JH. Leg length discrepancy in total hip arthroplasty. Orthop Clin North Am. 1992;23(2):201-9. 7. Williamson JA, Reckling FW. Limb length discrepancy and related problems following total hip joint replacement. Clin Orthop Relat Res. 1978;134:135-8.

Disclosure: No significant relationships.

$\mathbf{P 1 0 2}$

\section{LESSONS LEARNED FROM $>5$ YEAR ELASTIC STABLE INTRAMEDULLARY NAILING (ESIN) AS OPERATIVE TREATMENT FOR DISLOCATED MIDSHAFT CLAVICLE FRACTURES. A RETROSPECTIVE COHORT STUDY}

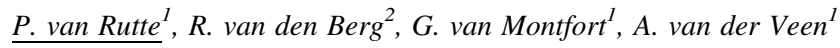

${ }^{1}$ General Surgery, Catharina Hospital Eindhoven, Eindhoven, Netherlands, ${ }^{2}$ General Surgery, Catharina Ziekenhuis, Eindhoven, Netherlands

Introduction: Elastic stable intramedullary nailing (ESIN) of displaced mid-shaft clavicle fractures is reported to be an easy procedure with good functional results, low complication rates and fast return to daily activities [1]. The aim of this study was to evaluate retrospectively the results of elastic stable intramedullary nailing in midshaft clavicle fractures.

Materials and methods: All patients operated with ESIN from January 2005 to August 2010 in the Catharina Hospital in Eindhoven were reviewed. Data of interest were fracture type, duration of operation, type of fracture reduction, complications, number of reoperations, fracture healing, nail removal and the functional DASH score and Constant Shoulder Score (CSS).

Results: In total 51 patients, were treated with ESIN. All procedures were performed by one experienced surgeon. Open reduction was performed in 34 cases. This was mainly necessary due to multiple fragments of the fracture. 25 patients complained of pain at the entry point at the medial site of the clavicle, 6 of them needed a re-operation for this cause. Other complications included re-fractures [2] and brachial plexus palsy [1]. There was a high rate of fracture consolidation $(98 \%)$ with excellent functional recovery. The mean DASH-score was 6.9 and the mean CSS was 97.9.

Conclusion: ESIN for displaced mid-shaft clavicle fractures showed good fracture healing and good functional results. However, there was a relatively high complication rate mainly caused by nail migration, wherefore in $11.8 \%$ a re-operation was necessary. Therefore midshaft clavicle fractures should be classified by criteria of contact and number of fragments to choose the right indication for ESIN. Further prospective research is necessary to determine best surgical procedure.

Reference: 1. Smekal V et al. Elastic stable intramedullary nailing versus nonoperative treatment of displaced midshaft clavicular fractures-a randomized, controlled, clinical trial. J Orthop Trauma. 2009; 23(2):106-12.

Disclosure: No significant relationships.
P103

\section{LOW INTENSITY PULSED ULTRASOUND ACCELERATES ENDOCHONDRAL OSSIFICATION IN AGED MOUSE FEMUR FRACTURE MODEL}

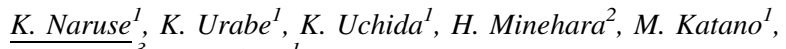 M. Itoman ${ }^{3}$, M. Takaso}

${ }^{1}$ Orthopedic Surgery, Kitasato University, Kanagawa, Sagamihara, Japan, ${ }^{2}$ Orthopaedic And Trauma Surgery, Kitasato university school of medicine, Sagamihara, Japan, ${ }^{3}$ Kyushu Rosai Hospital,

Kitakyushu, Japan

Introduction: The mechanism of action whereby endochondral ossification is promoted has yet to be clarified and detailed studies on shortening of the fracture healing period have not yet been conducted. Therefore, we examined the effects of LIPUS on endochondral ossification in the fracture healing process using a model of agingrelated delayed union fractures consisting of aged mouse with femur fractures.

Materials and methods: Fracture healing effects of LIPUS were assessed by comparing 40- and 8-week-old mice using X-ray imaging analysis and histomorphological and immunohistological evaluations. We also quantitatively studied cell migration using primary cultured human endothelial cells to examine whether neovascularization contributes at least in part to the mechanisms of action underlying healing promotion.

Results: In 8-week-old mice with femur fractures without LIPUS exposure, endochondral ossification, hard callus formation, and bone remodeling were observed 10,14 , and 21 days after the fracture, respectively. In 40-week-old mice with femur fractures without LIPUS treatment, however, fracture healing was delayed significantly. On the other hand, LIPUS exposure resulted in significant neovascularization that was histologically observed in the VEGF-expressing soft tissues around the fracture 10 days after the fracture in 40-weekold mice. In addition, hard callus formation was observed 21 days after the fracture. We analyzed the sequential migratory profiles using $\mathrm{NIH}$ imaging software during the subsequent $24 \mathrm{~h}$, and found that the human vascular endothelial cells differed significantly from the control groups.

Conclusion: We conclude that LIPUS may induce migration of vascular endothelial cells, which, in turn, accelerates endochondral ossification by neovascularization.

Disclosure: No significant relationships.

\section{P104}

\section{MANAGEMENT OF DISTAL FEMORAL FRACTURES WITH LOCKING PLATES}

K.I. Papagiannakos ${ }^{1}$, G. Sinnis ${ }^{1}$, I.K. Akrivos ${ }^{2}$, E.T. Charalampidis ${ }^{2}$,

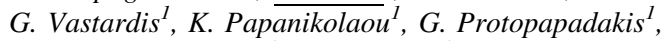
P. Papagiannopoulos ${ }^{1}$, P. Kalantzis ${ }^{1}$

${ }^{1}$ 2nd Orthopaedic Dep., "Evangelismos" General Hospital of Athens, Athens, Greece, ${ }^{2}$ 2nd Orthopaedic, "Evangelismos" General Hospital of Athens, Athens, Greece

Introduction: The aim of this study is to evaluate the outcomes of stabilization of distal femoral fractures using locking plates.

Materials and methods: Between 2004 and 2010, 25 patients (9 men, 16 women) who sustained distal femoral fractures were treated with internal fixation with locking plates. Among them, 12 fractures 
were periprosthetic. The mean age was 59.8 years (18-88 years). Mobilization of the knee started on the second to sixth postoperative day. We studied the following parameters: time of union, range of motion, limb alignment, articular congruity_post-traumatic arthritis and infection rate.

Results: Post-operative follow up ranged from 1 to 6 years. The results were evaluated with Lysholm Knee Score (LKS) and Knee Society Score (KSS). Fracture union was achieved at an average time of 4.5 months (3-6.8 months).Range of motion of the knee was on average $0^{\circ}-105^{\circ}\left(90^{\circ}-125^{\circ}\right)$. We encountered one superficial infection with concomitant skin necrosis and peroneal nerve palsy, all in the same patient.

Conclusion: The use of locking plates in all types of distal femoral fractures is a safe procedure with good results, provided careful preoperative planning and surgeon's experience with this technique. This leads to fracture stabilization and permits early mobilization of the patient.

Disclosure: No significant relationships.

\section{$\mathbf{P 1 0 5}$}

\section{MAY THE NON-FRACTURED FEMUR BE USED AS A STANDARD FOR ROTATION CONTROL OF THE FRACTURED FEMUR IN INTRAMEDULLARY NAILING?}

\author{
D. van Embden ${ }^{1}$, G.R. Roukema ${ }^{2}$
}

${ }^{1}$ Trauma Surgery, Leiden University Medical Center, Leiden, Netherlands, ${ }^{2}$ Surgery, Maasstad Ziekenhuis, Rotterdam, Netherlands

Introduction: Femoral malrotation after interlocked intramedullary nailing of a femoral shaft fracture is a clinical significant problem. In this study we evaluate whether or not, the femoral anteversion of the unfractered leg may be used as a standard, in the prevention of malrotation deformity after intramedullary nailing. Furthermore, the reliability of MR Imaging for measurement of femoral anteversion is assessed.

Materials and methods: The femoral anteversion of 36 pairs of femurs was measured by two observers using MR Imaging. Pearsontests, linear regression plots and T-tests were performed to assess the reliability of the use of the rotation of the unfractured femur as a standard control for the fractured femur. Furthermore, an intra-class correlation was calculated. The reliability of the MRI method was assessed by calculating the inter-class correlation coefficient.

Results: The Pearson's correlation showed to be 0.675 and 0.724 for the observers. Both T-tests failed to show significance. The intra-class correlation of both observers showed to be 0.713 . The reliability of the MRI showed an interclass reliability of $\alpha 0.96$ and $\alpha 0.94$.

Conclusion: The unfractured femur can be used as rotation control to prevent malrotation with interlocked intramedullary nailing of femoral shaft fractures. MR Imaging is very reliable for the measurement for femoral rotation.

Disclosure: No significant relationships.

\section{P106}

\section{MEDIAL FEMORAL CONDYLE FRACTURES}

\section{$\underline{\text { T. Randl }}$}

Traumatology, University Medical Centre Ljubljana, Ljubljana, Slovenia

Introduction: Medial femoral condyle fractures occur in the sagittal or frontal plane and result from axial compression or angular strains at the knee joint. Treatment consists of anatomic reduction and stable fixation. We compared the success of osteosynthesis in 2 cases regarding different bone quality in a similar fracture pattern.

Materials and methods: 1st patient: 27 years, female, high energy trauma, fracture type AO 33-B1. Percutaneous osteosynthesis with 3 cancellous lag screws and washers was performed. 2nd patient: 77 years, male, low energy trauma, fracture type AO 33-B2. 3 cancellous lag screws were used for condyle fixation. After 6 weeks osteosynthesis failed. Reosteosynthesis with additional LCP was performed. After 12 weeks osteosynthesis failed, again. We removed lag screws and used another plate to buttress the condyle. The fracture healed in 3 months.

Results: 1st patient: the osteosynthesis with 3 cancellous bone screws and washers was sufficient. No additional surgery was necessary. Full weightbearing was allowed after 8 weeks. Knee ROM was full. 2nd patient: because of poor bone quality and less stable fracture pattern the fixation with only 3 screws proved to be insufficient. Buttress plate and two more operations were needed. Time of treatment was prolonged to 8 months. There was $20^{\circ}$ of flexion deficit of the knee. Conclusion: The shape of fracture and bone quality determine the type of osteosynthesis needed for medial femoral condyle fixation. Wrong decisions lead to osteosynthesis failure, additional surgical procedures and extended time of treatment.

Disclosure: No significant relationships.

\section{P107}

\section{OCCULT FRACTURES AROUND THE WRIST: INCIDENTAL FINDINGS LOOKING FOR OCCULT SCAPHOID FRACTURES}

\author{
S. Woltz ${ }^{1}$, R.H. van Leerdam ${ }^{2}$, A.D. de Zwart $^{3}$, F.J.P. Beeres ${ }^{4}$, \\ S.A.G. Meylaerts ${ }^{5}$, S.J. Rhemrev
}

${ }^{1}$ Surgery, Medisch Centrum Haaglanden, The Hague, Netherlands, ${ }^{2}$ Surgery, Medisch Centrum Haaglanden, The Hague, Netherlands,

${ }^{3}$ Surgery, MCH Haaglanden, Amsterdam, Netherlands, ${ }^{4}$ Surgery, Medical Centre Haaglanden, The Hague, Netherlands,

${ }^{5}$ Surgery, Medical Center Haaglanden, The Hague, Netherlands, ${ }^{6}$ Surgery, Medical Centre Haaglanden, Den Haag, Netherlands

Introduction: The wrist is the most commonly fractured joint in the body. Adequate treatment is important to prevent complications like non-union and to maintain good function. It is not known how often a fracture around the wrist is missed on conventional radiographs. A prospective study on occult scaphoid fractures incidentally showed that many other fractures were missed on initial radiographs. To evaluate the amount and type of fractures around the wrist that were found on CT while looking for occult scaphoid fractures.

Materials and methods: The population of a prospective study on occult scaphoid fractures was analyzed. Included were 140 patients presenting at the emergency department who were clinically suspected to have a scaphoid fracture but who had no scaphoid fracture shown on conventional radiography. All patients had undergone computed tomography. We analyzed the type and amount of other fractures incidentally found on CT.

Results: $37(26.4 \%)$ of 140 patients with a normal radiograph were found to have a total of $39(27.9 \%)$ occult fractures around the wrist on CT. 12 Fractures were of the scaphoid and 27 other fractures were incidentally found: eight of the triquetrum, seven of the distal radius, three of the hamatum, three of the trapezium and one fracture of each of the following: capitatum, pisiforme, lunatum, trapezoid, metacarpal 1 and metacarpal 3 .

Conclusion: In 140 patients, up to 39 (27.9\%) bone fractures of the wrist are missed with conventional radiography only. This is an 
outcome we did not expect; the clinical consequence, however, is unknown. A CT should always be considered if a clinical suspicion of a fracture exists.

Disclosure: No significant relationships.

\section{P108}

\section{OSTEOPOROSIS IS NOT A RISK FACTOR FOR THE DEVELOPMENT OF NONUNION: A COHORT NESTED CASE-CONTROL STUDY}

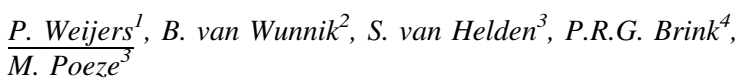

${ }^{1}$ Surgery, Division of Traumatology, Maastricht University Medical Centre, Maastricht, Netherlands ${ }^{2}$ Surgery, Maastricht University Medical Centre, Maastricht, Netherlands, ${ }^{3}$ Department Of Surgery, Division Of Trauma, Maastricht University Medical Center, Maastricht, Netherlands, ${ }^{4}$ Traumatology, MUMC, Maastricht, Maastricht, Netherlands

Introduction: Osteoporosis (OP) is one of the most prevalent metabolic bone disorders worldwide and it is associated with a higher incidence of fractures. The aim of this study was to identify OP as a risk factor for the development of nonunion and less favourable clinical outcome.

Materials and methods: In a prospective database all patients aged $>50$ years with an acute fracture were screened for osteoporosis from September 13, 2004 till February 9, 2009. Bone mineral density measurements (T-scores, Z-scores and absolute values in $\mathrm{g} / \mathrm{cm}^{2}$ ) were performed. The selected patients were matched (1:2 ratio) to control patients based on gender, age $( \pm 5$ years $)$ and type of fracture according to the AO-criteria. Other parameters including diabetes mellitus, corticosteroid use, rheumatoid arthritis, smoking, alcohol use, and body mass index were recorded. Follow-up for the patients in the matched group was at least 1 year.

Results: This study included a total of 1,498 patients who were screened for the presence of osteoporosis. In total 40 patients were treated for non-union. After 1:2 matching this resulted in a total number of 120 patients for analysis. Logistic regression analysis including all covariates in the model demonstrated no correlation between the standardized regression coefficients and the development of nonunion $\left(\mathrm{r}^{2}=0.14, \mathrm{p}=0.4\right)$. The patients that developed an atrophic nonunion, according to radiographic results, were analyzed separately and compared to the matched control patients. The presence of osteoporosis, osteopenia and normal bone density and the related independent BMD measurements did not differ significantly between the atrophic nonunion group and the matched controls.

Conclusion: We conclude that although bone quality may be diminished in the elderly this does not influence the occurrence of nonunion. These results indicate that the use of BMD measurements preoperatively to identify osteoporosis as a possible risk factor of nonunion has no clinical value.

Disclosure: No significant relationships.

\section{P109}

\section{OUR EXPERIENCES WITH NAVIGATION SYSTEM IN TRAUMATOLOGY}

\section{T. Kozák, R. Hart}

Ortop., Traum., Nemocnice Znojmo, Znojmo, Czech Republic
Introduction: We have used navigation system BrainLAB/fluoroscopic/for the treatment of fractures of long bones, pelvis and spine since 2006. The purpose of usage of the navigation system is our endeavour for improvement of results/rotation and axis deviation, difference in length/, and reduction of radiation time for patients and for operation team.

Materials and methods: This navigation system works on passive infrared transmission principle. There are three functional parts: camera, computer with touch screen, and special markers which have to be placed firmly on the bone surface. The system calculates real time position of the markers and creates $2 \mathrm{D}$ or $3 \mathrm{D}$ bone model. This procedure reduces radiation during operations. Material 42 patients 27 males 15 females Indication close fractures age 13-83 years median 56 years location femur, tibia, pelvis, spine.

Results: The mean operation time was $75-154 \mathrm{~min}, 96 \mathrm{~min}$ in average rotation and axis deviation in femur and tibia fractures less than $5^{\circ}$ in all cases mean radiation time $106 \mathrm{~s}$ range $65-153 \mathrm{~s}$

Conclusion: The navigation is a significant improvement in traumatology. A quarter of femur diaphysis fractures treated by nailing have the rotation deviation greater than $15^{\circ}$. In tibia fractures $10^{\circ}$ rotational and axial deviation is very often. The trauma navigation reduces the risk of such malunions and reduces radiation exposure for a patient and staff of the operating theatre.

Disclosure: No significant relationships.

\section{P110}

\section{OUTCOMES OF DIAGNOSTIC ULTRASOUND SCANS FOR COLLECTIONS AROUND HIP WOUNDS IN FRACTURE} NECK OF FEMUR PATIENTS OVER ONE CALENDAR YEAR

\section{K. Katam, G. McNeela, S. Malik, S. Zaman, M. Jenkins, J. Ramos}

Trauma and Orthopaedic Surgery, Heart of England NHS Trust, Birmingham, UK

Introduction: Musculoskeletal ultrasound scans (USS) are a lowcost, non-invasive way of evaluating many conditions. USS is particularly useful in the field of trauma and orthopaedics, especially in the post-operative period when metal prosthesis can make CT or MRI images difficult to interpret.

Surgical site infection (SSI) remains an important complication of hip surgery and the effects on patient morbidity and mortality are well documented. Our study looked at outcomes of ultrasound requests for hips in one calendar year with special focus on postoperative fracture neck of femur (NOF) patients. Our aim was to evaluate the operative versus conservative approach in these patients and the benefit of USS.

Materials and methods: Data was collected for hip USS requests over a 1 year period and indications, clinical diagnosis and sonologists impressions were reviewed. Outcomes of scans were analysed to evaluate its use in determining patient management plans.

Results: Out of 157 USS, 36\% were done in patients with prosthetic hip joints. Of these 23 had implants secondary to a fractured NOF. Only 14 patients had clinical signs of infection with 6 patients undergoing surgical intervention while all others were managed conservatively.

Conclusion: Our study has illustrated that although USS may be used as an adjunct in determining post operative collections it cannot be used as a determinant factor for surgery. Of the 6 patients that underwent revision surgery only 1 patient had positive intra-operative cultures hence we would suggest that USS guided aspiration would be a more valuable tool to avoid high risk operative management of SSI's. 
References: Acklin YP et al. Unexpectedly increased rate of surgical site infections following implant surgery for hip fractures: problem solution with bundle approach. Injury. 2010. doi:10.1016/j.injury. 2010.09.039. Jodra VM, et al. Excess length of stay attributable to surgical site infection following hip replacement: a nested casecontrol study. Infect Control Hosp Epidemiol. 2006;27:1299-303. Disclosure: No significant relationships.

\section{P111}

PARALLEL LAG SCREWS ACROSS A MEDIAL MALLEOLUS FRACTURE, A RARITY OR THE NORM?-A TECHNICAL TIP

M. Wijeratna ${ }^{l}$, E.M. Prempeh $^{l}, \underline{\text { J.Oni }}{ }^{2}$

${ }^{1}$ Trauma and Orthopaedics, Addenbrookes University Hospital, Cambridge, UK, ${ }^{2}$ Orthopaedics, Addenbrookes Hospital, Cambridge, UK

Introduction: To achieve even compression across a uniplanar fracture with two lag screws, parallel screws are the optimal configuration. The most common indication for this is during fixation of a fracture of the medial malleolus. The usual method of achieving this is by freehand insertion of the screws [1] but this often results in screws that are inserted obliquely to each other. The technique described results in parallel screws on a regular basis without multiple attempts at screw insertion. Use of the $2.0 \mathrm{~mm}$ triple-drill guide that is found in the AO small fragment set (but rarely used) ensures favourable screw position. This technique is particularly suitable for fixation of Salter-Harris IV fractures of the medial malleolus where further disruption of the epiphysis is undesirable.

Materials and methods: Once the fracture is exposed and reduced, the $2.0 \mathrm{~mm}$ triple-drill guide is used to exert axial pressure across the fracture site and stabilise the fracture. One $2.0 \mathrm{~mm}$ Kirschner-wire is then passed through the first hole to hold the reduction. The position of this wire is checked under image-intensifier and if satisfactory, the $2.0 \mathrm{~mm}$ drill bit is then passed through the third hole. The use of the triple-drill guide ensures that the Kirschner-wire and drill bit are parallel. The drill bit is removed and a suitable small fragment screw passed across the fracture. The outer cortex can be tapped with the $4.0 \mathrm{~mm}$ cancellous tap if needed. The $2.0 \mathrm{~mm}$ Kirschner-wire is removed and the same process repeated for the second screw. Final position is checked under image-intensifier.

Results: No results

Conclusion: This technique is simple to replicate and once mastered, will result in parallel lag screws if the medial malleolus fragment is large enough to accommodate two screws. If not, then a tension-band technique is used to provide fixation.

Reference: 1. Rüedi TP, Murphy WM. AO principles of fracture management. 2000. p. 573-5.

Disclosure: No significant relationships.

\section{P112}

\section{PARTIAL ACL LESIONS}

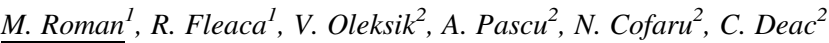

${ }^{1}$ Orthopedic Surgery and Trauma, University of Sibiu, Sibiu, Romania, ${ }^{2}$ Engineering Faculty, University of Sibiu, Sibiu, Romania
Introduction: Partial ACL tear is a rare condition, often unrecognized, difficult to diagnose and treat.

Aim: Evaluate the value of single bundle procedure in partial ACL lesions.

Materials and methods: Out of 285 ACL reconstructions, 14 cases (12 AM, 2 PL) were partial lesions. Diagnosis was made by MRI, clinical examination, arthroscopy. Reconstruction of only the ruptured bundle (STG graft $3 \times$ ) was performed in 6 cases, the rest required replacement of both. Fixation was performed with bioabsorbable interference screws on both sides (tibial side-hybrid fixation was performed). Rehabilitation protocol was similar to total ACL reconstruction. Postoperative assessment: clinical tests; IKDC2000 Score, Lysholm Score; Functional tests.

Results: Clinical diagnosis under anesthesia and MRI should always be performed. Arthroscopy may offer the correct diagnosis. Evaluation of the functional capacity of the nonruptured bundle remains challenging. Decision making was made intraoperatively and went towards saving the intact PL bundle and replacing the torn AM bundle. Clinical evaluation showed good results regarding AP and rotational stability. Lysholm score was over 97 points for all cases. Conclusion: Partial ACL tear is a rare condition which is difficult to diagnose. Indication/decision should be made after a careful arthroscopic evaluation. Single bundle reconstruction should be performed (restores/preserves normal ACL function). Paper supported by a national investigation grant (CNCSIS nr. 698 19. 01. 2009)

Disclosure: No significant relationships.

\section{P113}

\section{PERCUTANEOUS AUTOLOGOUS BONE MARROW INJECTION FOR DELAYED OR NON UNION: IS IT PRACTICABLE AT DISTRICT HOSPITAL LEVEL?}

\author{
A.K. Singh ${ }^{1}$, J.J. Saraswathy ${ }^{2}$, S. Shetty ${ }^{2}$, A. Sinha $^{2}$
}

${ }^{1}$ Department of Trauma and Orthopaedics, King's College Hospital, 9RS, UK, ${ }^{2}$ Trauma and Orthopaedics, Glan Clwyd Hospital, 5UJ, UK

Introduction: The process of fracture healing is complex, and any disturbance can result in delayed or non-union. We present our method of treating delayed or non union.

Materials and methods: Twelve patients with delayed or non-union of various bones, treated between March 2004 and August 2010 were included in the study. The group had 6 male and 6 female patients, with a median age of 42 years (15-70 years). The median time of presentation was 9 months (5-53 months). There were 6 ulna, 3 femur, 2 humerus, and 1 metacarpal fractures.

The procedure was performed in similar manner as done by the haematologists and oncologists for the bone marrow transplant [1]. We do two injections of 30-40 $\mathrm{ml}$ for children and adolescents, while three for adults. The interval between the injections is 6-8 weeks.

Results: Eleven $(91.6 \%)$ of twelve fractures achieved union with injections. The median time for callus formation was 4 weeks (3-10 weeks), clinical union was 6 weeks (3-12 weeks) and radiological union was 14 weeks (10-24 weeks). The only failure was a 56 year old, with right proximal humerus non-union following multiple operative interventions including plating with bone grafting; who presented to us 4.5 years post initial injury.

Conclusion: Bone marrow injection is less invasive, virtually complication free, day surgery procedure for delayed or non-union. The administration of adequate bone marrow volume, at frequent intervals can be successfully practiced with good results at a district hospital. Reference: 1. Thomas ED, Storb R. Technique of human marrow grafting. Blood. 1970;36:507-15.

Disclosure: No significant relationships. 


\section{P114}

\section{PLATE FIXATION OF HUMERAL HEAD FRACTURES WITH A TELESCOPING MECHANISM (DHS-PRINCIPLE)}

\author{
G. Gradl ${ }^{1}, H$. Pape $^{2}$, G. Gradl
}

${ }^{1}$ Trauma Surgery, University Hospital Aachen, Aachen, Germany, ${ }^{2}$ Department Of Trauma Surgery, University of Aachen, Aachen, Germany, ${ }^{3}$ Abteilung Für Unfall- Und Wiederherstellungschirurgie, Universität Rostock, Rostock, Germany

Introduction: Objectives: Biomechanical evaluation of a new fixation device for proximal humeral fractures using the DHS-principle (H-DHS-plate) in comparison to a regular locking plate (PHILOS). Materials and methods: In four paired fresh-frozen human cadaver humeri (age $>70$ years) a Neer IV/3 fracture was created with a $5 \mathrm{~mm}$ osteotomy gap simulating metaphyseal comminution. Limbs were randomly assigned to receive $\mathrm{H}$-DHS plate fixation and on the contralateral limb Philos plate fixation before biomechanical evaluation (MTS-Bionix 858.2).

Results: The mean stiffness $(300.9 \pm 28.8 \mathrm{~N} / \mathrm{mm})$ in the H-DHS plate proved to be significantly higher than in the Philos plate group $(184.2 \pm 23.4 \mathrm{~N} / \mathrm{mm} ; \mathrm{p}=0.006)$. The H-DHS plate also resisted higher loads in terms of fixation failure with loss of reduction at $290 \pm 58.6 \mathrm{~N}$ in comparison to $205 \pm 8.6 \mathrm{~N}$ for the Philos plate $(\mathrm{p}=0.2)$. Displacement of the greater tubercle occured in no case of the H-DHS group and in two out of four cases in the Philos group. Conclusion: The H-DHS plate provides high fixation stability in an in vitro humeral head fracture model.

Disclosure: No significant relationships.

\section{P115}

\section{PREDICTION OF THE MORTALITY IN GREEK PATIENTS WITH HIP FRACTURES USING THE NITTGHAM HIP FRACTURE SCORE}

\author{
D. Kotzamitelos ${ }^{1}$, R. Mavropoulos ${ }^{2}$, G. Drosos ${ }^{2}$, A. Ververidis ${ }^{1}$ \\ K. Kazakos ${ }^{l}$, A. Hatzigiannakis ${ }^{1}$, D.A. Verettas $^{1}$, K. Tilkeridis $^{2}$
}

${ }^{1}$ Orthopaedic, University Hospital of Alexandroupolis, Alexandroupolis, Greece, ${ }^{2}$ University Hospital of Alexandroupolis, Alexandroupolis, Greece

Introduction: Hip fractures (HF), usually refers to older people and are associated with high mortality rate. Nottingham Hip Fracture Score (NHFS) is designed to predict the mortality rate in patients with HF within the first 30 post traumatic days. Our aim is to validate the NHFS, in Greek patients.

Materials and methods: All patients (129) admitted in our department with a HF the period December 2009 to October 2010 were included in this study. The predicted mortality rate in the first 30 days has been calculated according to NFHS. All patients were followed up for at least 30 days and the NHFS prediction compared with the mortality rate of the patients.

Results: The mortality rate of this group of patients, within the first 30 days of the fracture, was $12.40 \%$ (16 patients). The NFHS predicted a $16.53 \%$ mortality rate for the same period of time. Our mortality rate according to the sex was $4.9 \%$ for the women $(5$ of the $102)$ and $40.7 \%$ for the men (11 of the 27 ).

Conclusion: Our study showed that there is correlation between the predicted rate by NHFS and the actual mortality rate in Greek patients with HF. The higher mortality rate in men seems to be in line with the NHFS that recognize the male sex as risk factor. We believe that NFHS is a useful tool to predict the mortality of the patients with these type of fractures. This study is going on in order to include a large number of patients for more accurate validation of the NHFS in Greek patients.

References: Court-Brown CM, Caesar B. Injury. 2006;37:691-7. Wood DJ, Ions GK, Quinby JM, Gale DW, Stevens J. J Bone Joint Surg [Br]. 1992;74-B:199-202. Maxwell MJ, Moran CG, Moppett IK. Br J Anaesth. 2008;1-7. doi:10.1093.

Disclosure: No significant relationships.

P116

\section{PREOPERATIVE PLANNING PROGRAM TOOL IN TREATMENT OF ARTICULAR FRACTURES: PROCESS OF SEGMENTATION}

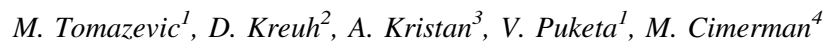

${ }^{1}$ Department of Traumatology, University Medical Centre Ljubljana, Ljubljana, Slovenia, ${ }^{2}$ Ekliptik Ltd, Researcher, University Medical Centre Ljubljana, Ljubljana, Slovenia, ${ }^{3}$ Dpt. of Traumatology, UMC Ljubljana, Ljubljana, Slovenia, ${ }^{4}$ Traumatology, Univ. Clinical Centre Ljubljana, Ljubljana, Slovenia

Introduction: Pre-operative planning is important in trauma orthopaedic surgery. It is difficult and time consuming to do good and useful planning from plain radiographs. That is why many surgeons do not do it [1]. Computer programs were introduced to help planning, but the segmentation process was either done by some other institution, or was time consuming [2]. A developing computer program for preoperative planning of articular fractures is presented where segmentation can be easily done on PC with semiautomatic process.

Materials and methods: Fracture segmentation was done using preoperative planning program on regular PC in 30 consecutive articular fracture cases that were admitted to our department where CT was indicated and done. DICOM images were used. Process of segmentation was recorded and type of fractures were described according to AO classification [3].

Results: 7 acetabulum, 7 proximal humerus, 6 proksimal tibia, 3 distal humerus, 3 spine, 2 pelvis, 1 pilon and 1 midfoot fractures were segmented. In all fractures all segmentation processes were done semiautomatically. The program found fracture lines between two points that were set by the planner. None fracture needed to be marked by hand. AO classification was correct in all cases according to plain radiographs.

Conclusion: With preoperative fracture planning program segmentation process can be done easily and on PC that we use every day. Latter the reposition and fixation can be carried out virtually. After a good preoperative planning, real operation seems just like another rehearsal, and time spent by planning is time saved in the operating room.

References: 1. Wade RH, Kevu J, Doyle J. Pre-operative planning in orthopaedics: a study of surgeons' opinions. Injury. 1998;29(10): 785-6. 2. Cimerman M, Kristan A. Preoperative planning in pelvic and acetabular surgery: the value of advanced computerised planning modules. Injury. 2007;38(4):442-9. 3. Ruedi TP. AO principles of fracture management. 2nd ed. Thieme Medical Publishers. 2007.

Disclosure: No significant relationships. 
P117

PRIMARY CARE SPORTS MEDICINE SPECIALIST FOR ACUTE MUSCULOSKELETAL INJURY WILL REDUCE THE DEMAND ON ORTHOPEDISTS FOR NONSURGICAL TREATMENT

\section{Bhattacharyya}

Orthopaedics, Lewisham Hospital London, London, UK

Introduction: Integration of sports medicine in the accident and emergency and within primary care appears as evolving practice. Managed care organizations are increasingly incorporating primary care sports medicine providers into their organizations. This study analyses whether integration of physician with special interest in sports medicine will lead to better utilization of the resources. Primary care sports medicine specialist for acute musculoskeletal injury will reduce the demand on orthopaedists for nonsurgical treatment.

Materials and methods: A descriptive longitudinal observational analysis was made of patient clinical notes at the time of consultation at fracture clinic with a secondary care system. This study describes patient information including demographic data, referral source, primary diagnosis, specialized diagnostic testing, and subsequent specialty consultation.

Results: A total of 1986 patients referred to secondary care randomly were analyzed. All patients were referred by accident and emergency department physician and primary care emergency nurse practitioner. The majority of patient visits were for acute musculoskeletal injuries (95.4\%); the most frequently involved injury sites were: knee $(26.5 \%)$, shoulder $(18.2 \%)$, back (14.3\%), and ankle $(10 \%)$. The most common types of injury were: tendinitis $(21.3 \%)$, chronic anterior knee pain $(10.6 \%)$, and ligament sprains (9.9\%). Specialized testing was requested for $8 \%$ of all patients. The majority of patients were treated non operatively with further physiotherapy referral.

Conclusion: This study demonstrates the need for primary care physicians with special interest in sports medicine. They may offer accurate assessment of injury and possibly avoiding unnecessary orthopedic consultation while maintaining a good medical clinical practice. This practice may reduce cost of treating acute musculoskeletal injury. This study supports the complementary nature of primary care sports medicine and orthopedics, with the primary care sports medicine physician reducing the demand on orthopedists for nonsurgical treatment.

Disclosure: No significant relationships.

\section{P118}

\section{PROMISING RESULTS IN TREATMENT OF OLECRANON FRACTURES WITH A MODIFIED ONE-THIRD TUBULAR HOOK-PLATE OSTEOSYNTHESIS}

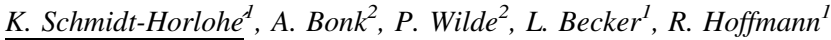 \\ ${ }^{1}$ Unfallchirurgie Und Orthopädische Chirurgie, \\ Berufsgenossenschaftliche Unfallklinik, Frankfurt am Main, \\ Germany, ${ }^{2}$ Berufsgenossenschaftliche Unfallklinik Frankfurt am \\ Main, Frankfurt am Main, Germany
}

Introduction: Operative treatment of olecranon fractures by tensionband wiring is considered to be the most popular technique and mostly good clinical results can be obtained. However, a complication rate up to 50 percent is reported in literature. For this reason we use a custom-made one-third tubular hook-plate osteosynthesis in olecranon fracture treatment.

Materials and methods: In a prospective cohort study over a period of 14 month 34 patients (mean age 52 years, 29-83) with 34 olecranon fractures were treated with the above described modified fixation device after open reduction. After a mean follow-up of 8 months (6-9) the outcome was evaluated using the Mayo Elbow Performance Scores (MEPS), the Visual Analogue Scale (VAS) and the Disabilities of the Arm, Shoulder and Hand (DASH) Score. Radiological examinations were conducted.

Results: In 33 patients (97\%) the aim of a primary stable osteosynthesis to allow early initiation of physiotherapy was gained. According to the MEPS predominantly excellent (14 cases/41\%) and good results (19 cases/56\%) were obtained. Only one patient was judged to have a fair result. Mean MEPS was 92 points (65-100) and mean DASH-Score was found to be 16.8 points (0-39). No complications occurred intraoperatively and in the postoperative course. Conclusion: Treatment of olecranon fractures with a custom-made one-third tubular hook-plate provides promising results. The one-third tubular plate needed for the presented osteosynthesis is widely used and can easily be customized to both, patient's anatomy and the fracture pattern.

Disclosure: No significant relationships.

\section{P119}

\section{PROSPECTIVE CLINICAL EVALUATION OF A FIRST GENERATION LOCKED "WAVE PLATE" IN COMPLEX FEMORAL NON-UNION}

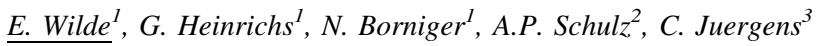

${ }^{1}$ Trauma and Orthopaedics, University Hospital Luebeck, Luebeck, Germany, ${ }^{2}$ Ortopedics \& Trauma, University Hospital Luebeck, Luebeck, Germany, ${ }^{3}$ BG Trauma Hospital Hamburg, Hamburg, Germany

Introduction: Since the first results in the treatment of femoral pseudarthrosis with a wave plate published in 1990 by Blatter and Weber we reached to a commercially produced wave plate called â€œDruckplattenfixateur interne â€" DPFâ€ (Litos, Hamburg, Germany). This development was made by using Finite Element Studies in our Institution and led to a multidirectional angular stable (or so called â€œlockedâ€) plate. Aim of this study was to clinically evaluate the results of this plate.

Materials and methods: During a period of 10 years we treated 76 patients of persisting femoral non-union with the DPF. The Indications for treatment were:

- Femoral non-union previously treated with ORIF (plate and screws) that was found loose or broken

- Atrophic non-union requiring bone grafting

- Non-union presented after treatment with external fixator

- Non-union after treatment with intra-medullary nailing with failed exchange of nail and over-reaming

- Non-union after treatment with intra-medullary nailing without exchange of nailing because of atrophic non-union

The patient group consisted of 76\% (58 patients) male with an average age at initial trauma of 40.3 years (5.5 to 79). In 58 cases the patients were referred from other hospitals, only 18 patients were primary treated in the BG Trauma Hospital in Hamburg. The left limb was affected in 43 cases $(57 \%)$. We used an 8 hole locked wave plate (Pressure Plate Fixator/PPF, Litos, Hamburg, Germany) made of 
titanium. The locking mechanism is achieved by a (second) pressure plate applied over screw heads after the osteosynthesis has been performed with the main plate. Prior to this the oval screw holes allow compression or distraction by the recognized AO technique. In contrast to other locked implants the screws can be applied in a variable angle up to $30 \AA$. Two lengths are available $(234 \mathrm{~mm} / 250 \mathrm{~mm})$ and a slightly flattered and curved alternative for application near to the trochanter and femoral condyles. Bone grafts were only performed to improve bone healing in 72 cases.

Results: In $85.3 \%$ (64 cases) we reached bony healing after application of the PPF. Full weight bearing could be achieved at an average of 4.2 months (1 day to 12 months), radiological consolidation was found at a mean time of 7.3 months (3-19 months). In 8 cases occurred delayed union which made a second cancellous bone graft necessary (4-10 months). Positive microbiology was documented in 2 cases, positive histology in 4 cases. In 3 patients a failure of the plate (break) happened at 6 weeks, 3 and 5 months after surgery. Positive microbiology (Staph. epidermidis) in 2 cases and histological signs of chronic infection in another case were found. One patient had a rotational deformity of $25 \AA$ and required revision. All patients finally gained full weight bearing. Three of them needing a stick regularly, another 5 patients only occasionally. Retirement from work were necessary in $9.4 \%$ ( 7 patients) due to health reasons. Pain was only experienced in one case at rest, 21 patients mentioned pain on long walking distances and stairs and 51 patients were pain free. The functional deficit compared with the non-injured limb showed over the hip extension/flexion $-7 \AA /-17 \AA$ and over the knee extension/flexion $-2 \AA /-12 \AA$ with an average limb length discrepancy of $-9 \mathrm{~mm}$. Conclusion: In this prospective study we could show that the PPF is not only a recommendable treatment option for complex femoral nonunion when nailing is not possible but also a further surgical option for the treatment of complex fractures of the femur. The advantage lies in the combination of a biological blood supply preserving implant with a high level of stability.

Disclosure: No significant relationships.

\section{P120}

\section{PROSPECTIVE COHORT STUDY INTO THE USE OF REGIONAL NERVE BLOCK IN THE EMERGENCY MANAGEMENT OF FEMORAL NECK FRACTURES}

\author{
P.R. Loughenbury ${ }^{1}, \underline{\text { F.A. Barnett }}^{1}$, N. Pennington ${ }^{2}$, A.P. Volans $^{1}$, \\ J.P. Livesey ${ }^{I}$
}

${ }^{1}$ Department of Trauma and Orthopaedic Surgery, Scarborough Hospital, Scarborough, UK, ${ }^{2}$ Dewsbury and District Hospital, Dewsbury, UK

Introduction: Femoral neck fractures are the most common injury affecting older people and approximately 76,000 cases occur a year in the UK. Attention to analgesic requirements is an essential part of emergency management. This study aimed to determine whether regional nerve block provides better pain relief than oral and intravenous analgesia alone following femoral neck fractures.

Materials and methods: Consecutive patients admitted with a femoral neck fracture over a 4-month period. Patients were given an Iliacus block ( $30 \mathrm{ml} 0.5 \%$ levo-bupivocaine) on admission whenever an appropriately qualified medic was present. The control group did not receive a regional nerve block. Oral and intravenous analgesia were given for breakthrough pain. Pain scores and analgesic requirements were recorded over the first $24 \mathrm{~h}$.

Results: 69 patients were included -46 received a regional nerve block and 23 did not. Mean age was 80.8 years (55-101). The mean sum of pain scores was 3.04 in the nerve block group and 6.98 in the control group (95\% CI 2.72-5.15; p < 0.0001). Pain scores were significantly lower in the nerve block group on admission and at 4,8 and $12 \mathrm{~h}(\mathrm{p} 0.005)$ and at $24 \mathrm{~h}(\mathrm{p}=0.01)$. In the first $24 \mathrm{~h}$ after admission patients in the nerve block group required less codeine (mean 21 vs. $114 \mathrm{mg}$; 95\% CI 49.4-137.1; p < 0.0001), tramadol (mean 54.4 vs. $142.4 \mathrm{mg}$; 95\% CI $13.7-162.4 ; \mathrm{p}=0.02$ ) and morphine (mean 1.3 vs. $5.7 \mathrm{mg} ; 95 \%$ CI $2.98-5.8 ; \mathrm{p}<0.0001$ ). The mean length of stay was 9.91 days in the nerve block group and 12.6 days in the control group, although this was not statistically significant $(\mathrm{p}=0.068)$.

Conclusion: In this study the use of a regional block lead to significantly lower pain scores throughout the first $24 \mathrm{~h}$ and significantly lower oral and intravenous analgesic requirements. These results support the use of regional nerve blocks as an effective method of providing analgesia in the emergency management of femoral neck fractures.

Disclosure: No significant relationships.

\section{P121}

\section{PROSPECTIVE STUDY OF TRIPLE ERADICATION THERAPY FOR MRSA IN PATIENTS ADMITTED WITH FRACTURED NECK OF FEMUR}

\author{
C.U. Menakaya ${ }^{1}$, S.B. Roberts ${ }^{2}$, B. Ilango $^{2}$
}

${ }^{1}$ Trauma and Orthopaedics, Aintree University Teaching Hospital, 7AL, UK, ${ }^{2}$ Orthopaedic Surgery, Fairfield General Hospital, Fairfield, UK

Introduction: Patients admitted to hospital with a hip fracture are at risk of MRSA infection. Chlorhexidine mouthwash, mupirocin and triclosan are effective treatments for preventing MRSA infection. The benefit of using all three agents simultaneously has not yet been investigated. We conducted a prospective study to determine whether or not triple therapy with chlorhexidine, mupirocin and triclosan improves eradication of MRSA colonisation and prevention of postoperative MRSA infection and bacteraemia in patients admitted with fractured neck of femur.

Materials and methods: From 1st January 2009, all patients admitted to the trauma ward with fractured neck of femur were screened for MRSA with swabs taken from nose, throat, groin, skin lesions and any indwelling lines or catheters. On admission, triple therapy (chlorhexidine, mupirocin, and triclosan) was commenced for each patient and continued until a set of swabs were reported negative, with re-screening swabs performed every 5-days. We studied the incidence of MRSA colonisation, infection and bacteraemia in these patients from 1st January 2009 to 1st July 2009.

Results: Screening swabs were MRSA-positive on admission for 6 $(8.5 \%)$ of 71 patients admitted. Of these 6 patients, none had postoperative wound problems or developed deep surgical site infection, and 3 patients were MRSA-negative after triple therapy treatment. MRSA could not be confirmed as eradicated in the remaining 3 patients due to: persistent MRSA-positive chronic skin ulcer, patient death from intra-abdominal sepsis, and patient discharged before rescreening swabs required. MRSA wound infection occurred in 1 patient $(1.4 \%)$ who was MRSA-negative on admission, and who required revision surgery and prolonged admission: the infection was eradicated with triple therapy and intravenous antibiotics. No patients developed MRSA bacteraemia.

Conclusion: Our study demonstrates that triple therapy helps in the prevention of MRSA wound infection and bacteraemia. We strongly recommend triple therapy for all patients admitted with fractured neck of femur. 
References: 1. Biant LC, Teare EL, Williams WW, et al. Eradication of methicillin-resistant. Staphylococcus aureus by "œringfencing" of elective orthopaedic beds. $\mathrm{Br}$ Med J. 2004;329: 149-51. 2. Bed linen Carnicer-Pont D, Bailey KA, Mason BW, et al. Risk factors for hospital-acquired MRSA bacteraemia: a casecontrol study. Epidemiol Infect. 2006;134:1167-73. 3. Espehaug B, Engesaeter LB, Vollset SE, et al. Antibiotic prophylaxis in total hip arthroplasty. J Bone Joint Surg [Br]. 1997;79-B:590-5. 4. Fascia DTM, Singanayagam A, Keating JF. Methicillin-resistant Staphylococcus aureus in orthopaedic trauma. J Bone Joint Surg [Br]. 2009;91-B:249-52. 5. Guidelines for the prophylaxis and treatment of MRSA infections in the UK. J Antimicrob Chemother. 2006;57: 589-608.

Disclosure: No significant relationships.

\section{P122}

\section{REAMER IRRIGATOR ASPIRATOR SYSTEM: EARLY EXPERIENCE}

\section{N.K. Kanakaris ${ }^{1}$, M. Mokawem $^{2}$, S. Gudipati ${ }^{2}$, D. Morell ${ }^{2}$, P. Giannoudis ${ }^{3}$}

\begin{abstract}
${ }^{1}$ Trauma and Orthopaedics, Leeds Teaching Hospitals NHS Trusr, Leeds, UK, ${ }^{2}$ Trauma and Orthopaedic, Leeds Teaching Hospitals NHS Trust, Leeds, UK, ${ }^{3}$ Academic Department of Trauma and Orthopaedics, Leeds Teaching Hospitals NHS Trust, Leeds, UK
\end{abstract}

Introduction: The indications of the Reamer/Irrigator/Aspirator system-Synthes include acute cases (minimisation of second hit), osteomyelitis (debridement of intramedullary canal), autologous grafting (harvesting of morsesiled autograft), oncology (intramedullary lesion biopsy and prophylactic nailing).

Materials and methods: Since January 2008, data from the cohort of patients where the RIA system was used in a single centre were prospectively collected. Epidemiological, in-hospital, post-discharge follow-up data, and intraoperative direct medical costs were included in this series stratified as to its 4 basic indications.

Results: RIA was used in 70 patients in 77 different occasions [mean age 53 years, (19-80)]. These included 11 polytrauma with thoracic and/or neurosurgical associated injuries, 11 pathological fractures/ pending fractures, 21 osteomyelitis for debridement, and 27 for graft harvesting (atrophic nonunions). At those where the by-products of reaming were collected, for diagnostic or grafting purposes, the mean volume was $60 \mathrm{ml}(40-90 \mathrm{ml})$. One intraoperative complication was recorded (dismantling of the reamer head from the RIA tube assembly attributed to user's error). In the limited follow-up period of this series no fractures/hematomas/compartment syndrome/postoperative pain/ limitation of patients' mobility associated to the RIA technique were recorded. The mean direct medical cost of its use was $£ 355$ (312-740). Conclusion: The RIA system was proven versatile, with short learning curve, and highly effective especially for the cases of bone harvesting and intramedullary debridement. Further evidence is anticipated to elicit its safety and efficacy to the impressing variety of its indications.

References: 1 . Giannoudis PV, et al. Injury. 2009;40:1231-6. 2. Kobbe $\mathrm{P}$, et al. Injury. 2008;39:796-800. 3. Pape HC, et al. JBJS Am. 2005;87:2515-22.

Disclosure: Synthes has provided clinical research funds to the Department regarding a clinical study on the inflammatory response of polytrauma patients treated with the system during closed IMN of the femur.

\section{$\mathbf{P 1 2 3}$}

\section{RELIABILITY OF PREDICTORS FOR SCREW CUTOUT IN PERITROCHANTERIC HIP FRACTURES}

\author{
K. De Bruijn ${ }^{1}$, W.E. Tuinebreijer ${ }^{2}$, D. den Hartog ${ }^{3}$, G.R. Roukema ${ }^{4}$
}

${ }^{1}$ Traumatology, Maasstad Hospital Rotterdam, Rotterdam,

Netherlands, ${ }^{2}$ Epidemiology, freelance epidemiologist, Wijk aan Zee, Netherlands, ${ }^{3}$ Surgery-Traumatolgy, Erasmus MC, University Medical Center Rotterdam, Rotterdam, Netherlands, ${ }^{4}$ Surgery, Maasstad Ziekenhuis, Rotterdam, Netherlands

Introduction: Failing osteosynthesis has a major impact on mortality and morbidity in the elderly population with peritrochanteric hip fractures. Tip apex distance (TAD), fracture classification, position of the screw in the femoral head and fracture reduction are known predictors for cutout. We investigated whether they were related to cutout in our study group. In addition, we investigated reliability of determining TAD, position of the screw and fracture reduction.

Materials and methods: We evaluated 215 peritrochanteric hip fractures using AO-classification, measuring TAD and determining fracture reduction and position of the screw. This was performed using pre- and postoperative radiographs in PACS (picture archiving and communication system).

Results: TAD and cutout were related $(\mathrm{P}=0.005)$. TAD measurement was reliable $(\mathrm{ICC}=0.81)$. The agreement with regard to screw position and fracture reduction was moderately reliable (Kappa = 0.46 and 0.48 , respectively). A3 fractures were at 14 times more risk of cutout $(\mathrm{OR}=14.24, \mathrm{P}=0.004)$. Poor fracture reduction was significantly related with higher incidence of cutout $(\mathrm{OR}=5.19$, $\mathrm{P}=0.017)$. Central-inferior and anterior-inferior positions were significantly protective $(\mathrm{OR}=0.08, \mathrm{OR}=0.07$, respectively).

Conclusion: To decrease risks of cutout, TAD needs to stay small and the screw needs to be placed central-inferiorly or anterior-inferiorly. A3 fractures require even more attention. TAD measurement is reliable and therefore a safe method in reducing risks for cutout.

References: Baumgaertner MR, Curtin SL, Lindskog DM, Keggi JM. The value of the tip-apex distance in predicting failure of fixation of peritrochanteric fractures of the hip. J Bone Joint Surg Am. 1995;77: 1058-64.

Disclosure: No significant relationships.

\section{P124}

\section{RESULTS AFTER OPERATIVE TREATMENT OF DISPLACED FRACTURES OF THE ACETABULUM: A 12-MONTH TO 6-YEAR FOLLOW-UP INVESTIGATION}

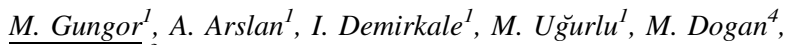 \\ A. Ocguder ${ }^{3}$
}

${ }^{1}$ Orthopaedics and Traumatology, Ataturk Education and Research
Hospital, Ankara, Turkey

Introduction: To review our experience with 33 surgically treated displaced acetabular fractures over a 5-year period.

Materials and methods: Thirty-three consecutive displaced acetabular fractures were consecutively operated on from January 1, 2005 through December 31, 2010, and 33 were available for review with a minimum of 12-month follow-up. Clinical (Merle D'Aubigné modified by Matta et al.) and radiographic (Matta) outcomes were evaluated. Complications and secondary operative procedures were 
documented. According to the classification of Letournel and Judet, 24 fractures (73\%) were simple and $9(23 \%)$ were of compound type. Central and posterior dislocations occurred in 3 and $36.3 \%$ of fractures, respectively.

Results: Anatomical reduction was achieved in $61 \%$ of the patients. At follow-up examination 12-72 months postoperatively (mean 38.5 months), clinical results were satisfactory in 25 patients $(76 \%$ of the cases), with 12 excellent and 13 good results, and roentgenographic results were satisfactory in 23 patients (69\% of the cases). Complications included a $11 \%$ incidence of sciatic nerve palsy, a $6 \%$ incidence of infection, a $15 \%$ incidence of Brooker et al. heterotopic ossification, a $18 \%$ incidence of posttraumatic osteoarthritis, and a $12 \%$ incidence of avascular necrosis of the femoral head. Statistical analyses revealed that significant prognostic factors included mean period between the trauma and operation, damage to the femoral head and the quality of anatomic reduction.

Conclusion: Operative treatment is an effective method for the management of displaced acetabular fractures. Clinical and roentgenographic results correlated closely with an anatomic reduction. Low complication rate can be expected if adequate preoperative assessment and planning is performed. Strategies to minimize the risk of thromboembolism and heterotopic ossification on the basis of prophylaxis and respectively, are reliable techniques for these injuries. References: Wright R, Barre K, Christie MJ, et al. Acetabular fractures: long-term follow-up of open reduction and internal fixation. J Orthop Trauma. 1994;8(5):397-403. Korovessis P, Stamatakis M, Sidiropoulos, et al. Treatment protocol, results and complications of operative treatment of displaced acetabular fractures. Eur J Orthop Surg Traumatol. 2000;10:99-106.

Disclosure: No significant relationships.

\section{$\mathbf{P 1 2 5}$}

\section{RESULTS OF OSTEOSYNTHESIS WITH HOOK-PIN FIXATION AND ARTHROPLASTY IN 132 FEMORAL NECK FRACTURES}

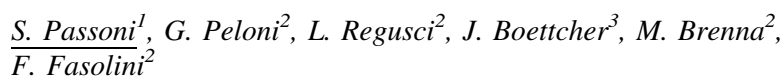

${ }^{1}$ Chirurgia, Ospedale Beata Vergine, Mendrisio, Switzerland, ${ }^{2}$ Chirurgia, Ospedale Regionale Mendrisio, Mendrisio, Switzerland, ${ }^{3}$ Chirurgia, Ospedale Beata Vergine, Mendrisio, Switzerland

Introduction: Evaluate the surgical outcome of 132 consecutive femoral neck fractures.

Materials and methods: We consider two groups of patients: the first $(\mathrm{N}=67)$ was treated with hook-pin fixation. In the second $(\mathrm{N}=65)$, the undisplaced fractures were treated with hook-pin while displaced fractures with arthroplasty, except for aged patients with general compromise, who received hook pin fixation. The main outcome was hip complication rate and health related quality of life after intervention.

Results: In the first group the F/M ratio was 3.2 with a mean age of 78 years. Garden classification was: I $24 \%$, II $18 \%$, III $36 \%$, IV $22 \%$. In the second group the F/M ratio was 3.1 with a mean age of 79 years. Garden classification was: I 17\%, II 17\%, III 17\%, IV $49 \%$. In the first group the operation time was $42,97 \mathrm{~min}$ in the second and blood transfusion rate was respectively 7 and $26 \%$. Hospitalization was similar, respectively 13 and 15 days. After hospital discharge in the first group $56.7 \%$ of patients returned to previous living conditions, 55.3\% in the second group. In the first group total healing complications were $19.4 \%$ and perioperative mortality was $4.5 \%$. In the second group total healing complications were $13.8 \%$ : $17.6 \%$ in the hook pin and $9.6 \%$ in the arthroplasty.

Conclusion: Arthroplasty for displaced fractures is the better intervention for fit patients older than 65 years. Otherwise for undisplaced fractures, for patients younger than 65 years and for patients with a displaced fractures with a general compromise, the hook-pin fixation still represents a good intervention.

Disclosure: No significant relationships.

\section{P126}

\section{RESULTS OF OSTEOSYNTHESIS WITH HOOK-PIN FIXATION IN 67 CONSECUTIVE FEMORAL NECK FRACTURES.}

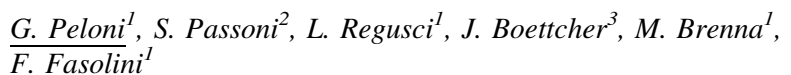

${ }^{1}$ Chirurgia, Ospedale Regionale Mendrisio, Mendrisio, Switzerland, ${ }^{2}$ Chirurgia, Ospedale Beata Vergine, Mendrisio, Switzerland,

${ }^{3}$ Chirurgia, Ospedale Beata Vergine, Mendrisio, Switzerland

Introduction: To evaluate the outcome of closed reduction and hookpin fixation in acute femoral neck fractures.

Materials and methods: 67 consecutive patients with femoral neck fracture treated with closed reduction and hook-pin fixation from December 2004 to March 2007 were prospectively studied. The main outcome measurements were hip complication rate, reoperation rate and health-related quality of life.

Results: The F/M ratio was 3.2. Mean age was 78 years. The Garden classification was: I $24 \%$, II $18 \%$, III $36 \%$, IV $22 \%$. The mean time between admission and operation was $32 \mathrm{~h}$. All patients under 65 years were operated within $6 \mathrm{~h}$. The mean time of operation was $42 \mathrm{~min}$. The blood transfusion rate was $7 \%$. The mean hospital time was 13 days. Weight bearing was forbidden in patients younger than 65 years $(n=9)$, while direct unrestricted weight bearing was encouraged in all the others. Following treatment, 38 patients $(56.7 \%)$ returned in the previous living conditions. Total healing complications were $19.4 \%$ : 5 redisplacement, 3 avascular necrosis, 3 trochanteric fractures and 2 non-union. In undisplaced fractures (Garden I-II) total complications were $10.8 \%$. In displaced fractures (Garden III-IV) total complications were $28.7 \%$. Total reoperations were $20.8 \%, 2$ osteosynthesis and 8 hemiarthroplasty. In Garden I-II reoperations were $6.9 \%$; in Garden III-IV reoperations were $21 \%$. Overall mortality was $4.5 \%$.

Conclusion: Closed reduction and hook-pin fixation is a good surgical option for undisplaced femoral neck fractures (Garden I-II) and in patients younger than 65 years. In older and fit patients with displaced fractures (Garden III-IV) arthroplasty provides a better outcome.

Disclosure: No significant relationships.

\section{P127}

\section{RETROGRADE NAILING FOR 32-C3 AND 33 A\&B FRACTURES. SURVEY OF 42 NAILS}

\section{P. Reynders-Frederix}

Traumatology, University Hospital Gasthuisberg Leuven, Leuven, Belgium 
Introduction: The authors retrospectively investigated the results of 42 retrograde femoral nails in 40 patients for Traumatic lesions.

Materials and methods: 42 retrograde nails of the same company were used between 2004 and 2009. These nails represented $33.6 \%$ of the implants we used in the same period for these injuries. Group A (30 patients) consisted of osteoporotic (23) and periprosthetic (7) \# Group B (10 patients) consisted of 33 C-2 (3) and 32 C3 (7) \# Mean age was 76 years in group A and 32 years in group B. Subsidence of the blade plate, implant failure, knee pain, walking ability and bone healing time were recorded.

Results: All except one fracture healed. Quality of healing was periosteal. The non-union was seen with a patient 79 years. 2 years after sustaining a periprosthetic knee fracture. No subsidence of the blade plate was seen into the knee joint No instability of the nail-bone construct was seen. Knee pain was recorded in 3 out of 10 patients in group B. In group A only 10 patients were able to walk independently with a walking aid. The other patients in group A were wheelchair bound after the injury. No infection was seen; Proudness of the implant in the knee joint was seen in two cases making implant removal necessary.

Conclusion: Retrograde nailing is a good option for complex 32-C fractures with good periosteal healing. Healing is somehow slower after retrograde nailing after periprosthetic fracture. No subsidence of the blade plate was seen, even in osteoporotic fractures.

Reference: Papadokostakis. Injury. 2005;36.

Disclosure: No significant relationships.

\section{P128}

\section{RETROGRADE NAILING VERSUS ANGULAR STABLE PLATE OSTEOSYNTHESIS IN PROXIMAL HUMERUS FRACTURES-BIOMECHANICAL ASPECTS}

\author{
S. Dietz ${ }^{1}$,W. Sternstein ${ }^{2}$, P.M. Rommens ${ }^{2}$
}

${ }^{1}$ Center of Trauma Surgery and Orthopedics, Mainz University, Mainz, Germany, ${ }^{2}$ Center Of Trauma And Orthopaedic Surgery, University Medical Center Mainz, Mainz, Germany

Introduction: Since the incidence of proximal humerus fractures is rising there is interest for a save, easy-to-use and biomechanical stable implant. Angular stable plate osteosynthesis and antegrade nailing are standard procedures for proximal humerus fractures. Both procedures are linked to method immanent disadvantages. A retrograde nail has been developed to avoid opening of the fracture side and to avoid penetrating the rotator cuff. The aim of our biomechanical study was to evaluate if retrograde nailing of proximal humerus fractures is as stable as locking plate osteosynthesis.

Materials and methods: The biomechanical properties of 2 implants were tested in 11 human fresh frozen cadaveric humeri pairs. The retrograde nail (Retron nail) and the angular stable plate (Philos plate) were implanted after osteotomy. All specimens were suspected to axial and torque load for 1,000 cycles in a servo pneumatic testing apparatus.

Results: Even though the Philos plate had greater torsion stiffness than the Retron nail and the Retron nail had greater axial stiffness our findings were not statistically significant.

Conclusion: Our study showed, that there are no significant differences between a retrograde nail and locking plate osteosynthesis for proximal humerus fractures concerning axial and torsion deformities. From the biomechanical point-of-view the retrograde nail is a suitable alternative for fixation of proximal humerus fracture.

Disclosure: No significant relationships.

\section{P129}

\section{RISK FACTOR ANALYSIS FOR COMPLICATIONS AFTER BLUNT CHEST TRAUMA WITH RIB FRACTURES}

\author{
H. el Jilali ${ }^{1}$, F.C. Bakker ${ }^{2}$, P.A.M. van Leeuwen ${ }^{2}$, F.W. Bloemers ${ }^{2}$, \\ W.P. Zuidema ${ }^{2}$
}

${ }^{1}$ Trauma Surgery, VU-University Medical Centre, Amsterdam, Netherlands, ${ }^{2}$ Trauma Surgery, Vrije Universiteit Medisch Centrum, Amsterdam, Netherlands

Introduction: Rib fractures are a common injury sustained following blunt chest trauma. The purpose of this study is to determine how often pulmonary associated complication, especially pneumonia, pneumothorax or lung contusion occurred after a rib fracture. Furthermore an analysis was made to identify the subgroup with the highest risk to develop complications.

Materials and methods: Retrospectively a group of 301 patients above the age of 16 years were analysed. The patients were divided by sex and in three age groups, group I: $16-60$ years, group II: 60-80 and group III: 80 years and older.

Results: The mean age was 51 years (range 16 till 99). There were $206(68.4 \%)$ male and $95(32.6 \%)$ female patients. Age group I consisted of $164(79.2 \%)$, group II of $58(19.2 \%)$ and group III of 36 patients (12\%). The main cause of rib fractures was a traffic accident in $49.8 \%$. Most frequent there were one or two rib fractures. A combination with pneumothorax was present in $36.5 \%$. Lung contusion was seen in $27.2 \%$. Admission time showed a mean of 13.7 days. Five or more rib fractures led in $78 \%$ to a pulmonary associated complication. Pneumonia occurred in $14.5 \%$. The overall mortality rate was $6.6 \%$.

Conclusion: Female patients above 80 years, who developed pneumonia, had a highly prolonged stay and the highest mortality rate of $14 \%$. The deceased patients in this age group were all female. There is an almost linear relation between the amount of rib fractures and the risk of developing a pulmonary associated complication in all groups. Disclosure: No significant relationships.

\section{P130}

\section{RISK FACTORS FOR EARLY POSTOPERATIVE COMPLICATIONS AFTER OPEN REDUCTION AND INTERNAL FIXATION OF CALCANEAL FRACTURES}

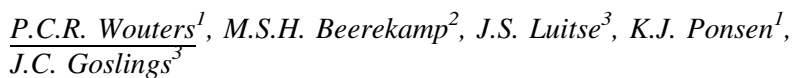

${ }^{1}$ Trauma Unit, Dept of Surgery, Amsterdam Medical Center, Amsterdam, Netherlands, ${ }^{2}$ Trauma Unit, Department of Surgery, Academic Medical Center, Amsterdam, Netherlands, ${ }^{3}$ Trauma Unit Department of Surgery, Academic Medical Center, Amsterdam, Netherlands

Introduction: Postoperative complications within 30 days after an open reduction and internal fixation (ORIF) of calcaneal fractures using an extended lateral approach occur in $3-30 \%$ of the patients. The current literature is unclear which patient-related, trauma mechanism or logistic factors influence the occurrence of these complications. Aim of this study was to define risk factors which correlate with the occurrence of early postoperative complications after ORIF of calcaneal fractures.

Materials and methods: In a retrospective case study, all patients between January 1997 and March 2010 undergoing an ORIF of unilateral or bilateral calcaneal fractures were included. An early local 
postoperative complication was defined as a superficial or deep wound infection, wound dehiscence, or nervus suralis dysfunction within 30 days after ORIF. Anticipated patient-related risk factors (diabetes mellitus, cardiovascular-, or psychiatric disease, neurological impairment, immunocompromised, smoking, and substance abuse), trauma mechanism, and logistic factors (time to operation, duration of operation, and hospital stay in days) were evaluated. Statistical analysis was performed using uni- and multivariate logistic regression.

Results: We included 237 calcaneal fractures in 208 patients. $60 \%$ was male, with a mean age of 43.3 years $(12-77) .29$ patients $(14 \%)$ developed an early postoperative complication. Superficial woundinfection occurred in 22 patients, deep woundinfection in 6 patients. Wound dehiscence was observed in 15 patients, 14 preceded an woundinfection. Nervus suralis dysfunction did not occur. Four patients sustained an open fracture, [OR 4.51 (1.20-17.10)]. Trauma mechanism, diabetes mellitus, smoking, substance abuse and logistic factors did not correlate with a higher risk.

Conclusion: Postoperative complications after ORIF of calcaneal fractures occured in $14 \%$ of the patients. Although clinically associated, Diabetes Mellitus and smoking were not correlated in this study, in contrast to an open fracture and immunocompromised status of the patient. Counselling of the patient when considering surgical treatment is necessary when patients have these risk factors.

Disclosure: No significant relationships.

\section{P131}

\section{RISK FACTORS FOR PERI-PROSTHETIC FRACTURES OF THE FEMUR-A NEW SCORING SYSTEM TO PREDICT RISK}

\section{S. Kale}

Trauma and Orthopaedics, East and North Herts NHS Trust, Welwyn Garden City, UK

Introduction: Periprosthetic fractures of the femur are a serious complications following hip arthroplasty. It is important to identify factors both intra-operative and post-operative to delineate the risk of these fractures occurring. Presently there is no scoring system to effectively predict an impending fracture and thus there is no current consensus on proactive or preventive treatment. Scoring the risk factors initiated at surgery and at follow up will help to identify the majority of these patients and initiate treatment before it occurs.

Materials and methods: Periprosthetic fractures in 35 patients in one DGH were studied operated on by 5 surgeons. We studied age, sex, surgical approaches and intra-operative adverse events, radiological grades for osteopaenia and events surrounding injury. Taking all these factors into consideration we have compiled a simple scoring which gives us good predictive value for identifying the patients at risk. We believe these sub-group of the patient population needs early or proactive prophylactic management either revision and/or fixation or both depending on the status and the score.

Results: This scoring identifies the arthroplasty population at risk for suffering the fracture. We are confident that this may help in smarter selection of the candidate for surgery and quantify risk before an eventuality.

Conclusion: In our opinion routine use of this scores initiated at surgery including every follow-up will help identify candidates at risk of fractures.

References: Beals RK. Periprosthetic fractures of the femur: an analysis of 93 fractures. CORR. 1996;(327):238-46. Kale et al. A proposed new addition to the Vancouver classification for periprosthetic fractures of the femur. Chicago: AAOS meeting presentation. 2006.

Disclosure: No significant relationships.

\section{P132}

\section{SCAPULA TRAUMA FIXATION IN PATIENTS WITH FLAIL CHEST}

P. Moreno de la Santa Barajas ${ }^{I}$, M.D. Polo Otero ${ }^{1}$, M. Lozano Gomez ${ }^{I}$, C. Delgado Sanchez-Gracian ${ }^{2}$, S. Leal Ruiloba ${ }^{3}$, C. Trinidad Lopez ${ }^{2}$, M. Choren Duran ${ }^{1}$

${ }^{1}$ Thoracic Surgery, Hospital Povisa, Vigo, Spain, ${ }^{2}$ Radiology, Hospital Povisa, Vigo, Spain, ${ }^{3}$ Critical Care, Hospital Povisa, Vigo, Spain

Introduction: A scapula fracture is an uncommon injury. Scapular body fractures are the most common type of scapula fracture. These injuries seldom require any specific treatment more than a simple arm sling. The important fact is that scapular body fractures are commonly (80-90\%) associated with other injuries such as lung and chest injuries.

Materials and methods: We present a series of 9 patients with flail chest and scapular body fracture. All patients underwent open reduction and internal fixation (ORIF) using titanium plates (STRATOS System $\left.\hat{A}^{\circledR}\right)$. We compared two groups: Group a: Internal fixation of scapular body (4 patients). Group b: conservative treatment of scapular body fracture (5 patients). Patients from surgical group were approached by a posterior way, extending the thoracotomy incision. We stabilized the scapula with locking scapula plate system (Acumed $\hat{\mathrm{A}}^{\circledR}$ ).

Results: Since 2008, nine patients were admitted for flail chest and scapular body fracture. The study is prospective and comparative. Both groups were compared; age, sex, ISS, transfusion, complication, and hospital stage were similar in both groups. We follow up the patient at least during 6 months. All patients survive. All patient form the surgical group back to normal and working life, and shoulder mobility were normal. In the conservative group 3 patients had reduced shoulder mobility at least a $20 \%$, and one was unable to comeback to work. The outwork period was 174 versus 277 days.

Conclusion: This is a small series, but we recommended perform an ORIF in scapular body fractures when they are associated with flail chest stabilized surgically. The recovery is shortened and there are not increments in complications or hospital stay.

Disclosure: No significant relationships.

\section{P133}

\section{SOCIETAL CONSEQUENCES OF FALLS IN THE OLDER POPULATION: INJURIES, HEALTHCARE COSTS AND LONG TERM REDUCED QUALITY OF LIFE}

K.A. Hartholt ${ }^{1}$, E.F. van Beeck ${ }^{2}$, S. Polinder ${ }^{2}$, N. van der Velde ${ }^{3}$, E.M.M. van Lieshout ${ }^{1}$, M. Panneman ${ }^{4}$, T.J.M. van der Cammen ${ }^{3}$, P. Patka ${ }^{l}$

${ }^{1}$ Surgery-traumatology, Erasmus MC, University Medical Center, Rotterdam, Netherlands, ${ }^{2}$ Public Health, Erasmus MC, University Medical Center, Rotterdam, Netherlands, ${ }^{3}$ Internal Medicine, Section Geriatrics, Erasmus MC, University Medical Center, Rotterdam, 
Netherlands, ${ }^{4}$ Injury Research, Consumer and Safety Institute, Amsterdam, Netherlands

Introduction: Fall incidents are a major cause of morbidity and mortality in older adults. The aim of this cohort study was to determine the incidence, costs and quality of life for fall-related injuries in the older Dutch population presenting at the Emergency Department (ED).

Materials and methods: Data on fall-related injuries in persons $\geq 65$ years were retrieved from the Dutch Injury Surveillance System (DISS). The DISS is based upon a representative sample of 14 geographically distributed hospitals in the Netherlands. It contains data on all patients with injuries treated at the ED as well as patient followup survey sent out between 2003 and 2007. Injury incidence, discharge rates, healthcare costs, and quality of life measures were extrapolated to full national coverage.

Results: The incidence rate of fall-related injuries was age-related, and increased from 17.7 (per 1,000 persons) in persons aged 65-74 years to 105.0 in persons $>95$ years. The majority of fallrelated injuries involved the upper (36\%) or lower limb (40\%), and consisted mainly of fractures $(60 \%)$, superficial injuries $(21 \%)$, and open wounds (8\%). Falls led to a total healthcare cost of $€ 474.4$ million, which represents $21 \%$ of total healthcare expenses due to injuries. Both admitted and non-admitted patients reported a reduced quality of life up to 9 months after the injury.

Conclusion: Fall-related injuries in older adults are age- and genderrelated, leading to high healthcare consumption, costs, and long-term reduced quality of life. Further implementation of falls prevention strategies is needed in order to control the burden of fall-related injuries in the aging population.

Disclosure: No significant relationships.

\section{P134}

\section{SOFT TISSUE IRRITATION AND IMPLANT REMOVAL CAN BE DISASTROUS WITH LOCKING COMPRESSION PLATE (LCP) FIXATION}

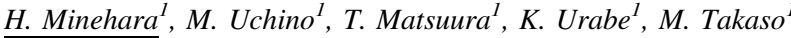 \\ ${ }^{1}$ Orthopaedic and Trauma Surgery, Kitasato Univ. School of \\ Medicine, Sagamihara, Japan
}

Introduction: The recent widespread introduction of LCP has led to the concept of biological fracture healing by preservation of blood supply with angular stability especially for the treatment of osteoporotic bones. However, there have been several reports about the problems related to LCP. In this study, we reviewed fracture cases treated with LCP in our facility to investigate clinical outcomes and complications.

Materials and methods: 191 cases and 182 patients (110 male and 72 female) were included in this study. Average age at the time of injury was 47 years old and plate removal was performed in 55 cases. Distribution of plates, numbers of MIPO (minimal invasive plate osteosynthesis), peri-operative and post-operative complications were investigated.

Results: Distribution of plate fixations was as follows: clavicle 12 cases, humerus 35 cases, radius 64 cases, ulna 8 cases, pelvis 5 cases, femur 20 cases, tibia 38 cases, fibula 9 cases. MIPO was performed in 47 cases. Complications during operations included screw breakage: 2 cases, implant removal difficulty: 10 cases. Post-operative complications were infection: 3 cases, delayed union: 4 cases, screw loosening: 2 cases, soft tissue problems: 7 cases.

Conclusion: Correct indications for fracture fixation should be considered very carefully when applying LCP. Troublesome implant removal and soft tissue problems due to its bulky profile seemed distinctive complications of LCP $[1,2]$. Thorough pre-operative planning and understanding of biomechanical principles of osteosynthesis are essential.

References: 1. Namazi H, Mozaffarian K. Awful considerations with LCP instrumentation: a new pitfall. Arch Orthop Trauma Surg. 2007;127(7):573-5. 2. Schmuts B, Wullschleger ME, Kim H, Noser H, Schütz MA. Fit assessment of anatomic plates for the distal medial tibia. J Orthop Trauma. 2008;22(4):258-63.

Disclosure: No significant relationships.

\section{P135}

\section{STABILITY OF INTERNAL FIXATION FOR DISTAL CLAVICULAR FRACTURES}

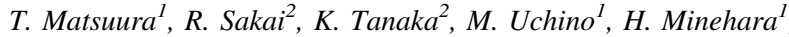 \\ T. Suzuki ${ }^{3}$, M. Itoman ${ }^{4}$, K. Mabuchi ${ }^{2}$, M. Takaso $^{1}$
}

${ }^{1}$ Orthopaedic Surgery, Kitasato University School of medicine, Sagamihara, Japan, ${ }^{2}$ Biomedical Engineering, Kitasato University School of Allied Health Science, Sagamihara, Japan, ${ }^{3}$ Traumatology and Emergency Medicine, Kitasato University School of Medicine, Sagamihara, Japan, ${ }^{4}$ Orthopaedic Surgery, Kyusyu Rosai Hospital, Kitakyusyu, Japan

Introduction: Strong internal fixation to maintain reduction of distal clavicular fractures is difficult because the distal bone fragment is small and the fracture site is adjacent to the acromioclavicular joint. The purpose of this study was to experimentally compare stability of internal fixation methods having different characteristics.

Materials and methods: The internal fixation methods used were non-locking arm plate fixation, locking hook plate fixation, and tension band wiring, and they were applied to simulated clavicle bones. Then distal clavicular fractures were created. A load tester was used to perform mechanical tests on the distal clavicular fracture models, and the amounts of displacement, fixation stiffness, and stiffness were determined at the fracture site of each distal clavicular fracture model by using the above fixation methods, and the results obtained were compared.

Results: No significant differences were found in either the amounts of displacement or the fixation stiffness between the non-locking arm plate group and locking hook plate group. The amount of displacement in the tension band wiring group was significantly greater than in the other 2 groups, and fixation stiffness was significantly lower. The difference in stiffness between the non-locking arm plate group and locking hook plate group was not significant, but stiffness was significantly lower in the tension band wiring group than in the other 2 groups.

Conclusion: Stability of fixation with the non-locking arm plate and the locking hook plate was similar, and it was stronger than fixation by tension band wiring.

Disclosure: No significant relationships. 


\section{P136}

\section{SURGICAL TREATMENT OF NEGLECTED HUMERUS FRACTURES}

\author{
N. Ozkayin ${ }^{1}$, G. Okcu ${ }^{2}$, K. Aktuglu
}

${ }^{1}$ Orthopedics and Traumatology, Ege University, Izmir, Turkey,

${ }^{2}$ Orthopedics and Traumatology, Celel Bayar, Manisa, Turkey

Introduction: The aim of this study was to evaluate the results of surgical treatment of humeral fractures neglected or remained untreated for various reasons.

Materials and methods: The study included 11 of 22 patients underwent surgical treatment because of unacceptable malunions who stayed earlier in the intensive care units of various hospitals from January 2000 to January 2006 and were either neglected or unable to accept surgical treatment because of polytraumas or poor health conditions. Average patient age was $27.2(8-42)$ years. Seven of them were men, and 4 were women. Five of the fractures were localized at the proximal humerus, six were at the $1 / 3$ mid-diaphysis. According to AO classification 7 were Type A, 3 were Type B, and 1 was Type C. None of the patients had any nerve injury before the surgery. Average time interval between the injury and surgical treatment was 7 (5-11) weeks.

Results: Follow-up period was 34.2 (20-77) months. Open reduction was successfully performed in all patients, although osteotomy and shortening of $3 \mathrm{~cm}$ on the average (1-5) were necessary in six patients. Average union time was 13 (10-16) weeks. According to Constant score, $64 \%$ of cases had perfect, $18 \%$ had good, $9 \%$ had poor, and $9 \%$ had worst shoulder functions. As for Mayo Elbow Performance Index, $73 \%$ of cases had perfect, $18 \%$ had good, $9 \%$ had poor, and $0 \%$ had worst elbow functions.

Conclusion: The major problems in such kind of delayed fracture treatment are maintaining reduction and fixation without any harm to surrounding tissues because of the impaired anatomy of the fracture due to callus formation and obtaining stable osteosynthesis permitting early rehabilitation. Stable osteosynthesis may sometimes be difficult to achieve, therefore shortening may present itself as a necessity without any loss of function.

Disclosure: No significant relationships.

\section{$\mathbf{P 1 3 7}$}

\section{SWIMCAST WRIST IMMOBILIZATION (SWIM) TRIAL. COMPARISON OF WATERPROOF VS CONVENTIONAL CAST FOR PEDIATRIC DISTAL RADIUS FRACTURES}

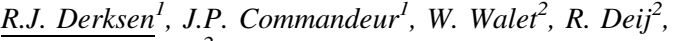 \\ R.S. Breederveld
}

${ }^{1}$ Traumasurgery, VU University Medical Center Amsterdam, Amsterdam, Netherlands, ${ }^{2}$ Red Cross Hospital, Beverwijk, Netherlands

Introduction: Undislocated distal radius fractures in children are traditionally treated in a forearm cast. One disadvantage of a traditional cast is that it does not tolerate getting wet. Skin maceration, rash or penetrant smell usually originate from a cast getting wet. A swim cast is an airy cast without cotton cast liner, that is administered by the MOKcast technique and allows patients to shower and swim. We hypothesize that swimcasts are better tolerated than traditional casts without adverse effects on fracture healing or skincondition.
Materials and methods: A prospective, randomized, single blinded study was conducted comparing traditional to swim cast treatment in children age 5-15 with distal radius fractures type greenstick or torus. Primary endpoint was fracture healing assessed both radiographically by a radiologist and clinically by a surgeon. Both were unaware of the type of cast used. Secondary endpoints consisted of cast-related skinconditions (urticaria, erythema, desquamation and pressure soars) as well as patient and parent satisfaction scores determined by a questionnaire.

Results: In total 68 patients were included (34 per group). Baseline characteristics were similar between groups. There was no difference in radiographic or clinical healing between groups. Skin conditions did not differ significantly between groups. The average comfort score $(0-10)$ judged by the patients was 8.6 in the swimcast group versus 7.5 in the traditional cast group ( $\mathrm{p} 0.002$ ). The comfort score judged by the parents was 8.3 and 7.8 respectively ( $\mathrm{p} 0.02$ ).

Conclusion: Treatment of undislocated, pediatric, distal radius fractures by swimcast yields better patient satisfaction results compared to traditional, cotton lined cast without adverse effects on fracture healing or skincondition.

Disclosure: No significant relationships.

\section{P138}

\section{TENSION BAND WIRING OF FRACTURED OLECRANON: STEEL WIRE OR SUTURES?}

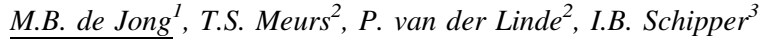

${ }^{1}$ Surgery, Leiden university medical center, Leiden, Netherlands, ${ }^{2}$ Surgery, Haga Hospital, The Hague, Netherlands, ${ }^{3}$ Trauma Surgery, Leiden University Medical Center, Leiden, Netherlands

Introduction: Although tension band wiring is the usual approach in displaced olecranon fractures, it is associated with complications such as irritation of the soft tissue, protrusion of K-wires and of the metal knots, and infections. Frequently (33-67\%), the material has to be removed after fracture healing in a second operation. The aim of this study was to investigate whether sutures can serve as an alternative for conventional steel wire cerclage.

Materials and methods: In a retrospective study, in two hospitals, all patients that received a tension band wiring for an olecranon fracture between 2006 and 2010 were included.

Results: One hundred and eight patients were analysed: 92 patients with steel wire cerclages (group A) and 17 patients received a suture (group B). Postoperatively, five group A patients were treated with a cast for 1-2 weeks, 103 patients were treated with active motion exercises during 6 weeks. In 24 patients complications occurred. Seventeen 'A' and 1 'B' patients developed wound infections. Four re-fractures occurred in group $\mathrm{A}$, three needed a second operation. $\mathrm{K}$-wire failure happened twice in group $\mathrm{A}$ and once in group $\mathrm{B}$. There was no delayed consolidation in both groups. Sixty-one patients had complaints of the metalware (group A 55, group B 6) and 58 had their metalware removed. This was done under local anaesthesia respectively in 9 ' $\mathrm{A}$ ' and 4 ' $\mathrm{B}$ ' patients and regional anaesthesia in respectively 44 ' $A$ ' and 2 ' $B$ ' patients. In 6 ' $A$ ' patients a wound infection occurred after removal of the metalware.

Conclusion: In conclusion, using a suture instead of a metal wire as a cerclage results in less wound infections and less removal operations. Suture cerclage might be an reliable and cheaper alternative for the conventional steel wire in olecranon fractures. Results should be objectivated in a randomised controlled trial.

Disclosure: No significant relationships. 


\section{P139}

\section{THE DIAGNOSTIC ACCURACY OF INTRA-OPERATIVE 3D- IMAGING IN FRACTURES OF THE EXTREMITIES}

\author{
M.S.H. Beerekamp ${ }^{1}$, G.S.I. Sulkers ${ }^{1}$, D.T. Ubbink ${ }^{2}$, N.W.L. Schep ${ }^{1}$, \\ J.C. Goslings
}

${ }^{1}$ Trauma Unit, Department of Surgery, Academic Medical Center, Amsterdam, Netherlands, ${ }^{2}$ Department of Quality and Process Innovations, Academic Medical Center, Amsterdam, Netherlands

Introduction: Intra-operative 3D-imaging was introduced to gain more insight during the surgical treatment of fractures of the extremities [1-3]. The aim of this systematic review was to determine the diagnostic accuracy and image quality of intra-operative 3Dimaging in surgically treated fractures of the extremities compared to other modalities such as radiographs, 2D-fluoroscopy and CTscanning.

Materials and methods: A systematic literature search was performed in Medline and Embase. Cadaver or clinical studies were included when evaluation of reduction and fixation of extremity fractures; description of quality of fracture reduction and fixation of both 3D-imaging (C-arm based) and a reference standard (CTscan, 2D-fluoroscopy, radiographs or autopsy) was described. The QUADAS-tool was used for quality assessment of the studies. If possible a $2 \times 2$ table was extracted to calculate sensitivity and specificity.

Results: A total of 7 cadaver- and 3 clinical studies were included in this review. The quality of these studies fulfilled on average $83 \%$ of the QUADAS-criteria. From only 3 cadaver studies it was possible to extract a $2 \times 2$ table to calculate sensitivity and specificity. These data could not be pooled because of diversity of the outcome parameter (e.g. step-off, screw placement etc.), as was also the case for measurements methods for image quality. However, all studies showed that diagnostic accuracy of intra-operative 3D-imaging was comparable to CT-scanning, but scored lower on subjective image quality than the other imaging modalities.

Conclusion: The diagnostic accuracy of intra-operative 3D-imaging is comparable to CT-scanning, but better than conventional radiographs and 2D-fluoroscopy. However on the subjective image quality, intra-operative 3D-imaging scored lower than all other modalities.

References: 1. Kendoff D, Citak M, Gardner M, Stubig T, Kettek C, Huffner T. Intraoperative 3D-imaging: value and consequences in 248 cases. J Trauma. 2009;66:232-8. 2. Carelsen B, Haverlag R, Ubbink DT, Luitse JS, Goslings JC. Does intraoperative fluoroscopic 3D imaging provide extra information for fracture surgery? Arch Orthop Trauma Surg. 2008;128(12):1419-24. 3. Euler E, Wirth S, Linsenmaier U, Mutschler W, Pfeifer KJ, Hebecker A. Comparative study of the quality of C-arm based 3D imaging of the talus. Unfallchirurg. 2001;104(9):839-46.

Disclosure: I am a research fellow employed at the AMC Research BV which received an unrestricted grant from Philips Healthcare, Best, The Netherlands.

\section{P140}

\section{THE ICI CLASSIFICATION FOR CALCANEAL INJURIES, A VALIDATION STUDY}

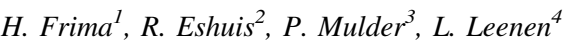

${ }^{1}$ Surgery, Amphia Hospital, Breda, Netherlands, ${ }^{2}$ Diakonessenhuis, Utrecht, Netherlands, ${ }^{3}$ Amphia Hospital, Breda, Netherlands,
${ }^{4}$ Trauma/Surgery, University Medical Center Utrecht, Utrecht, Netherlands

Introduction: The Integral Classification of Injuries (ICI), by Zwipp et al. [1] has been developed as a classification system for injuries of the bones, joints, cartilage and ligaments of the foot. It follows the principles of the Comprehensive Classification of Fractures by Muller et al. [2]. The ICI was developed for everyday use and scientific purposes. Our aim was to perform a validation study for this classification system applied to the calcaneal injuries.

Materials and methods: A panel of five experienced trauma surgeons and orthopaedic surgeons was constituted. This panel used the ICI to score 20 calcaneal injuries based on X-ray and CT scan of the calcis. Two months later a second classification was performed. To determine the inter- and intraobserver variability we used kappa statistics.

Results: All panel members pointed out that based on X-ray and CT scan nothing could be said about capsule and ligamental injuries. Two injuries were excluded as not all panel members scored the same calcis. The intraobserver variability based on 18 injuries of bone and joints was poor, kappa 0.31 (90\% CI $0.22-0.41)$. The interobserver variability was very poor, kappa 0.14 (90\% CI $0.05-0.22)$.

Conclusion: The ICI is a complicated classification system with poor inter- and intraobserver variabilities. It might not be a practical classification system for calcaneal injuries in everyday use or scientific purposes.

References: 1. Zwipp H, Baumgart F, Cronier P, Jorda E, Klaue K, Sands AK, Wai Yung S. Integral classification of injuries (ICI) to the bones, joints, and ligaments-application to injuries of the foot. Injury Int J Care Injured. 2004;35:S-B3-9. 2. Muller ME, Nazarian S, Schatzker J. CCF comprehensive classification of fractures. Pamphlets I and II. ME Muller Foundation. 1996

Disclosure: No significant relationships.

\section{P141}

\section{THE RELIABILITY, VALIDITY AND RESPONSIVENESS OF THE DUTCH VERSION OF THE OXFORD ELBOW SCORE}

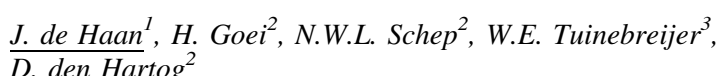

${ }^{1}$ Surgery-Traumatology, Westfriesgasthuis, Hoorn, Netherlands,

${ }^{2}$ Surgery-Traumatolgy, Erasmus MC, University Medical Center Rotterdam, Rotterdam, Netherlands, ${ }^{3}$ Surgey-Traumatology, Erasmus MC, University Medical Center Rotterdam, Rotterdam, Netherlands

Introduction: The Oxford elbow score (OES) is an English questionnaire that measures the patients' subjective experience of elbow surgery. The aim of this study was to develop and evaluate the Dutch version of the translated OES for reliability, validity and responsiveness with respect to patients after elbow trauma and surgery.

Materials and methods: The 12 items of the English-language OES were translated into Dutch. The OES was completed by 69 patients (group A), 60 of whom had an elbow luxation, four an elbow fracture and five an epicondylitis. The QuickDASH, the visual analogue pain scale (VAS) and the Mayo Elbow Performance Index (MEPI) were completed to examine the convergent validity of the OES in group A. To calculate the test-retest reliability and responsiveness of the OES, this questionnaire was completed three times by 43 different patients (group B).

Results: Cronbach's $\alpha$ coefficients for function, pain and socialpsychological domains were $0.90,0.87$ and 0.90 . The intra-class correlation coefficients for the domains were 0.87 for function, 0.89 
for pain, 0.87 for social-psychological. The standardised response means for the domains were $0.69,0.46$ and 0.60 , and the minimal detectable changes were 27.6, 21.7 and 24.0. The convergent validities for the function, pain and social-psychological domains, which were measured as the correlation of the OES domains with the MEPI, were $0.68,0.77$ and 0.77 . The correlation of the OES domains with the QuickDASH were $-0.43,-0.44$ and -0.47 , and the correlations with the VAS were $-0.33,-0.38$ and -0.42 , respectively.

Conclusion: The Dutch OES is a reliable and valid questionnaire.

Disclosure: No significant relationships.

\section{P142}

\section{THE TREATMENT OF PROXIMAL HUMERAL FRACTURES WITH THE USE OF PHN: THE ROLE OF THE ANATOMICAL REDUCTION IN THE POSTOPERATIVE OUTCOME}

\section{S. Tsitsilonis, F. Wichlas}

Centre of Musculosceletal Surgery, Campus Virchow Clinic, Charité University Medicine, Berlin, Germany

Introduction: Proximal humeral nail (PHN) offers an alternative for treatment of proximal humeral fractures (PHF). Protection of the soft tissues and the blood supply is the great advantage. However anatomical reduction is not always achieved. The aim of this study was to examine the importance of anatomical reduction in the postsurgical outcome with the use of PHN.

Materials and methods: 43 patients (mean age 68.56 years) were treated with PHN. 25 healthy subjects were controls. Mean followup was 23.2 months. Function of the shoulder was evaluated with Constant-Murley Score (CMS). Collum-shaft-angle (CSA) in 2 planes and atrophy of the greater tuberosity were radiologically examined. $\mathrm{CSA}>20^{\circ}$ to that of the control group was considered malreduction. CMS of malreduced fractures to reduced fractures, and those with atrophic greater tuberosities to non atrophic was compared.

Results: Control CSA in ap and Y-scapular view were 134.19 (SD 7.11) and 133.73 (SD 12.86). Patient CSA was 137.42 (SD 15.40) in ap and 126.53 (SD 16.99) in scapular-Y view. Mean patient CMS was 74.86 (SD 19.34). Nine patients had atrophy of the greater tuberosity with a CMS of 63.33 (SD 16.88). Malreduction was documented in 13 patients with CMS of 63.85 (SD 21.98). Statistically significant reduction of CMS was provoked by greater tuberosity atrophy and malreduction in ap plane $(\mathrm{p}=0.012)$.

Conclusion: PFN treatment does not always result in anatomical reduction. However functional outcome is not significantly impaired. Malreduction in ap view and greater tuberosity atrophy influence the results. PFN is a good option for PHF treatment.

Disclosure: No significant relationships.

\section{P143}

\section{THE USE OF ENDER NAIL IN INTERTROCHANTERIC FRACTURES SUPPORTING WITH EXTERNAL FIXATION}

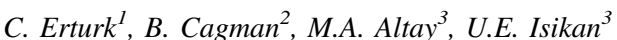

${ }^{1}$ Orthopaedics and Traumatology, Harran University Medical Faculty, Sanliurfa, Turkey, ${ }^{2}$ Orthopaedic Surgeon, Mh Av. Cengiz Gokcek
State Hospital, Gaziantep, Turkey, ${ }^{3}$ Orthopaedics And Traumatology, Harran University Medical Faculty, Sanliurfa, Turkey

Introduction: Treatment of intertrochanteric fractures, therefore, should aim to mobilize the patient quickly, to reduce mortality and hospitalization time, and to restore the patient's functions as much as possible. Intramedullary Ender nailing in intertrochanteric fractures was very popular in the past years. However, this method has fallen in over time, due to complications. We planned to analyze the effectiveness of this combination in cases of intertrochanteric fractures. The purpose of this study was the evaluation results of this method; and possible ways to prevent these complications, including the using of unilateral fixators to supporting the Ender nails.

Materials and methods: This technique was used together in 39 patients $(17 \mathrm{~m} / 22 \mathrm{f}$, mean age 71.4 years). Fractures were classified a according to the AO/OTA. In our surgery, we used the external fixator to support the intramedullary nails. Finally, all patients had been evaluated with Parker-Palmer mobility score, and with the Harris hip score.

Results: The follow-up period was 29.2 months (20-56). Two patients experienced nail migration in the knees, in two patients, varus deformation occurred with a reduction in length $2 \mathrm{~cm}$, and seven patients $(18 \%)$ developed pin-track infection. The average Harris score and Parker-Palmer score of the remaining 14 patients at the last follow up were 64 , and 6.8 , respectively.

Conclusion: This method has demonstrated several advantages, allows the patient to put weight on the extremity after a shorter period of time and enables the fracture to heal rapidly without any serious complications.

References: 1. Eren OT, Küçükkaya M, Tezer M, Yilmaz C, Kuzgun U. Treatment of intertrochanteric fractures of the femur with Ender nails in patients over the age of 65 years. Acta Orthop Traumatol Turc. 2003;37(2):102-6. 2. Kazakos K, Lyras DN, Verettas D, Galanis V, Psillakis I, Xarchas K. External fixation of intertrochanteric fractures in elderly high-risk patients. Acta Orthop Belg. 2007;73(1):44-8.

Disclosure: No significant relationships.

\section{P144}

\section{THREE DIFFERENT TIBIAL FIXATION SYSTEMS IN ACL RECONSTRUCTION WITH STG GRAFT- BIOMECHANICAL STUDY}

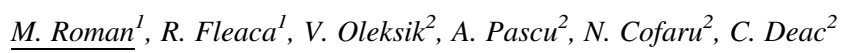

${ }^{1}$ Orthopedic Surgery and Trauma, University of Sibiu, Sibiu, Romania, ${ }^{2}$ Engineering Faculty, University of Sibiu, Sibiu, Romania

Introduction: Primary graft fixation in ACL reconstruction is mechanical. There is no consensus regarding the ideal method of fixation of the STG graft. Tibial fixation is the week link.

Aim: Three systems of tibial fixation were studied in order to prove which is the safest one. Study hypothesis: Hybrid fixation is more efficient then simple fixation.

Materials and methods: The testing protocol consists of 9 bovine knees, 9 human STG tendons (four bundle). Three fixation systems were created: (1) Direct fixation with interference screw, (2) Hybrid fixation (direct fixation with interference screw + indirect, extra cortical fixation over bony bridge), (3) Hybrid fixation (direct fixation with interference screw + indirect, extra cortical fixation over cortical screw). The experiments were performed on the testing machine Instron 5587. Progressive traction of each graft was performed until failure. 
Results: The test showed a different behavior for each system. The maximum load failure (average) was: $430,498 \mathrm{~N}$ for the first system, $941,786 \mathrm{~N}$ for the second and 564,338 $\mathrm{N}$ for the third. Limitations of the study: use of bovine specimens modifies the fixation properties (maximum load failure values greater as those in human specimens). Conclusion: The experiment confirms the initial hypothesis and validates the concept of hybrid tibial fixation. Paper supported by a national investigation grant (CNCSIS nr. 698 19. 01. 2009).

Disclosure: No significant relationships.

\section{P145}

\section{TISSUE ENGINEERED REPLACEMENT OSTEOCHONDRAL} XENOGRAFT FOR TREATMENT OF CARTILAGE INJURY

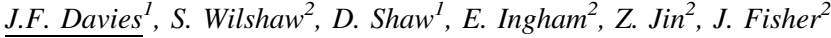 \\ ${ }^{1}$ Orthopaedics, Bradford Teaching Hospitals, Bradford, UK, ${ }^{2}$ Institute \\ Medical Biological Engineering, Leeds, UK
}

Introduction: A porcine derived acellular osteochondral scaffold has been successfully produced in laboratory studies of decellularisation by sodium dodecyl sulphate (SDS) $0.1 \%(\mathrm{v} / \mathrm{v})$ in hypotonic Tris buffer. To determine whether the xenografts were non-cytotoxic and therefore capable of supporting cellular in-growth after decellularisation, studies were undertaken using two cell lines: 3T3 fibroblast (3T3) and Baby hamster kidney (BHK) cells.

Materials and methods: Fresh osteochondral xenografts were tested in comparison to decellularised grafts. For contact inhibition, a cell suspension was added to grafts before incubation, fixation, staining and viewing by light microscopy. Cytotoxicity studies were undertaken to determine whether there were leachable molecules from the scaffolds that affected cell growth, by measuring the relative cellular ATP content of the cells.

Results: Contact studies did not show any inhibition in the growth of cells on fresh or acellular scaffolds. Extract studies showed there was a significant reduction in ATP content of $3 \mathrm{~T} 3$ cells grown with extracts from decellularised grafts that had an extended wash protocol of six washes in SDS compared to one wash, and fresh xenografts compared to one wash $(p<0.05)$. For the BHK cells, a significant reduction was found between extracts from fresh cartilage samples compared to one wash $(\mathrm{p}<0.05)$.

Conclusion: A decellularisation protocol of six SDS washes for the production of an acellular scaffold causes accumulation of SDS which was cytotoxic to $3 \mathrm{~T} 3$ cells. Scaffolds produced by one wash in SDS were biocompatible and therefore suitable for further in vitro cell culture work, to determine whether culturing under appropriate conditions such as cyclic compressive strain can induce chondrogenic differentiation of MSCs.

Disclosure: No significant relationships.

\section{P146}

\section{TREATMENT OF OPEN CRURAL FRACTURES WITH THE MITKOVIĆ EXTERNAL FIXATION SYSTEM}

\author{
I. Lalic, M. Stankovic, V. Kecojevic, Z. Gojkovic, R. Matijevic, \\ B. Vukajlovic
}

Ortopedics, KCV, Novi Sad, Serbia

Introduction: Crural fracture treatment is a great challenge for orthopaedic surgeon due the severity, high frequency as well as uncertain outcome in high percentage.
Objectives: The evaluation of postoperative results in patients with open crural fractures initially treated by the MitkoviÄ $\ddagger$ external fixation system.

Materials and methods: In Clinic for orthopaedic surgery and traumatology in Novi Sad, in period from June 2003 till June 2010, 197 patients with different types of open crural fractures were treated. Sixteen patients $(8 \%)$ had bilateral open crural fractures. Causes of fractures were: traffic $75(38 \%)$ patients, industry and agriculture 52 $(26 \%)$, sports, falls and other $70(36 \%)$ patients. We used Gustilo classification. According to this classification, 34 (17\%) cases were type $1,85(43 \%)$ type $2,48(24 \%)$ type $3 \mathrm{~A}, 20(10 \%)$ type $3 \mathrm{~B}, 10$ (5\%) type 3C.

Results: Complete recovery without major complications was achieved in $120(61 \%)$ cases, additional treatment with intramedullary nail was required in $33(17 \%)$ cases, additional treatment with the Ilizarov device was required in $26(13 \%)$ cases and additional treatment with the Sarmiento cast was required in 18 (9\%) cases. Postoperative infection around the pins of the external fixator developed in $14(7 \%)$ cases and postoperative wound infection developed in $10(5 \%)$ cases. Pseudoarthrosis and absence of bone healing were recorded in $25(13 \%)$ cases, tibial osteitis in $16(8 \%)$ cases and osteitis in the area of pins insertions in $3(1.5 \%)$ cases. Two $(1 \%)$ lower leg amputations were performed due to severe conquasant injury. Average period of the external fixator treatment was 23 (19-33) weeks. Full weightbearing was allowed after nine and a half weeks on average.

Conclusion: The preconditions for quicker healing of open crural fractures with as few complications as possible include correct primary wound management, reasonable antibiotic and antitetanus protection, correct application of the MitkoviÄ $\ddagger$ external fixator as well as constant control and, in some cases, correction of the fixator. Disclosure: No significant relationships.

\section{P147}

\section{TREATMENT OF CRURAL FRACTURE COMPLICATIONS WITH THE ILIZAROV APPARATUS}

\author{
I. Lalic, M. Stankovic, V. Kecojevic, Z. Gojkovic, R. Matijevic, \\ B. Vukajlovic
}

Ortopedics, KCV, Novi Sad, Serbia

Introduction: One of the crural fracture complications is pseudoarthrosis which can be treated using several methods (DCP plates with bone graft, intramedullary nailing, etc.). One of the methods used in our clinic is the Ilizarov method.

Materials and methods: In Clinic for orthopaedic surgery and traumatology in Novi Sad, in period from 2005 to 2010, 39 patients with different types of crural pseudoarthrosis were treated. Thirty four $(87 \%)$ were male patients and five $(13 \%)$ were female patients. We had $3(7 \%)$ patients in the age group 18-30 years old, $9(23 \%)$ in the age group $31-40,10(26 \%)$ in the age group $41-50,8(21 \%)$ in the age group 61 and older. The youngest patient was 21 years old and the oldest was 63 years old. Average age of the patients was 42 . Patients were previously treated by: plates and screws-11 $(28 \%)$ patients, a cast-15 (38\%) patients, external fixator-10 (26\%) patients, intramedullary nail-3 $(8 \%)$ patients. We had $12(31 \%)$ cases of hypotrophic pseudoarthrosis, 10 (26\%) cases of normotrophic pseudoarthrosis, $13(33 \%)$ cases of hypertrophic pseudoarthrosis and $4(10 \%)$ cases of neoarthrosis. We used monolocal closed compression osteosynthesis in 19 (49\%) cases, monolocal open compression osteosynthesis in $6(15 \%)$ cases, monolocal closed compression-distractional osteosynthesis in $8(21 \%)$ cases, monolocal synchronous 
compression-distractional osteosynthesis in $6(15 \%)$ cases. All manipulations with the device were performed in our hospital and patients were later regularly examined in our polyclinic.

Results: Average treatment period was 5 (4-6) months. Complete recovery was recorded in $37(94.8 \%)$ cases whereas in $2(5.2 \%)$ cases the device had to be reapplied due to infection. We analyzed bone parameters (healing, deformation, infection, limb length inequality) and functional parameters (ankle and knee contracture, limping, sympathetic dystrophy, pain, physical inactivity). Bone parameters show that in $23(59 \%)$ cases the results were excellent, in $9(23 \%)$ cases good, in $5(13 \%)$ cases medium, in $2(5 \%)$ cases the results were unsatisfactory. Functional parameters show that in $25(64 \%)$ cases the results were excellent, in $9(23 \%)$ cases good, in $3(8 \%)$ cases medium, in $2(5 \%)$ cases the results were unsatisfactory.

Conclusion: Our experience confirms that treatment by the Ilizarov apparatus for transosseous osteosynthesis is a reliable method for treating crural fracture complications such as pseudoarthrosis.

Disclosure: No significant relationships.

\section{P148}

\section{TREATMENT OF DISTAL CLAVICULAR FRACTURES USING SCORPION PLATE}

\section{Uchino}

\section{Orthopaedic Surgery, Kitasato University, Sagamihara, Japan}

Introduction: Distal clavicular fracture with disruption of the coracoclavicular (CC) ligaments (Neer type 2) is unstable because loss of restraint by the ligaments results in considerable superior and posterior displacement of the proximal fragment. If the distal fragment is small or comminuted, it is more difficult to achieve stable fixation and early mobilisation. We developed a new plate which is named Scopion plate for unstable fracture of the distal clavicle with small or comminuted.

Materials and methods: 16 fractures in 16 patients ( 14 females and 2 males) were treated by using the scorpion plates between 2000 and 2009. The patients averaged 45 years of age at trauma (range 20-74 years) and average of follow-up period was 14 months (range 6-29). We evaluated union rate, union period, complication and outcome (Japanese Orthopaedic Association Score; JOA).

Results: The union rate was $100 \%$ and the average of union period was 5.1 months (range 3-7 months). Two complications were detected, one was the heterotopic ossification and the other was the screw loosening. The average of JOA score was 98.8 points (range 96-100)

Conclusion: The scorpion plate for treatment of unstable fractures of the distal clavicle could achieve good results.

\section{P149}

\section{TREATMENT OF DISTAL RADIUS FRACTURES IN THE NETHERLANDS; RESULTS OF A NATIONWIDE SURVEY}

\section{J. Keizer $^{1}$, R. de Groot $^{2}$, S.J. Rhemrev ${ }^{3}$, S.A.G. Meylaerts ${ }^{3}$}

${ }^{1}$ Surgery and Traumatology, Leiden University Medical Center, Leiden, Netherlands, ${ }^{2}$ Surgery, Leiden University Medical Center,
Leiden, Netherlands, ${ }^{3}$ Surgery, Medical Center Haaglanden, The Hague, Netherlands

Introduction: Fracture management in the Netherlands is provided by both orthopedic surgeons (OS) and trauma surgeons (S). There seems a lack in consensus concerning treatment strategies of distal radius fractures (DRF) and we assessed surgical treatment preferences of both specialties using a questionnaire.

Materials and methods: We used a cross-sectional survey design. 25 questions were sent to one trauma representative of both specialties of each hospital. The questionnaire was based on current literature and consisted of propositions and questions covering current treatment strategies and perioperative imaging.

Results: The response rate was $60 \%$. Surgeons provided $75 \%$ of fracture care in each hospital. $43 \%$ of the $\mathrm{S}$ treated $>25$ patients operatively per year, compared to $7 \%$ of OS. S treated displaced DRF's with plating $(83 \%)$ while OS chose for plating $(50 \%)$ or external fixation (25\%). Preoperative CT-scans were used by $\mathrm{S}$ to choose operative strategy $(60 \%)$; orthopedic surgeons used CT for surgical indications (50\%). For the elderly ( $>70$ years), OS preferred conservative treatment (90\%) while S did not (44\%). Vitamin C as prophylaxis for regional pain syndrome type 1 was uncommon (S43\% and OS19\%). Surgeons acknowledged that DRF's in the elderly population account for a major health problem within the Netherlands more $(75 \%)$ than orthopedic surgeons $(48 \%)$.

Conclusion: There seems no evident consensus regarding the treatment of distal radius fractures in the Netherlands. A difference in preferences between surgeons and orthopedic surgeons appears and is possibly due to a difference in exposure.

Disclosure: No significant relationships.

\section{P150}

\section{TREATMENT OF DISTAL TIBIAL FRACTURES AT OUR DEPARTMENT}

\section{Zagh ${ }^{1}$, G. Merenyi ${ }^{2}$, T. Bogosi ${ }^{2}$}

${ }^{1}$ Traumatology, Karolyi Sandor Hospital, Budapest, Hungary, ${ }^{2}$ Dept. of Traumatology, Karolyi Sandor Hospital, Budapest, Hungary

Introduction: We evaluated the distal tibial fractures treated in the last 5 years at our department retrospectively. We compared the several methods in the treatment of the different types of fractures. We present the methods of fixator interne and the calcaneotibial nailing which were applied at our department in some cases.

Materials and methods: We treated 100 distal tibial fractures in this period. 50 percent of the cases have been caused by high energy trauma. We operated on $85 \%$ of the cases; half of the operation was open surgery. We preferred the stable plate osteosynthesis at the distal tibial fractures. In case of soft tissue problem, mainly at pilon fractures, we applied multistep operative treatment after primary application of external fixator. In selected cases we made primary or secondary calcaneotibial nailing, or so called internal fixator with fibula plate and fibulo-tibial lag screws.

Results: We consider that the soft tissue management and the properly timing stabilisation methods are the most important factors at this type of fractures. Our aim was the early mobilisation of the ankle joint.

Conclusion: By the planning of the treatment of the distal tibial fractures and mainly the pilon fractures caused by high energy trauma the main aspect is the soft tissue management.

Disclosure: No significant relationships. 


\section{$\mathbf{P 1 5 1}$}

\section{TREATMENT OF DISTAL TIBIAL FRACTURES USING LOCKING PLATE}

\author{
A. Khalil-Khan ${ }^{1}$, M. Maru ${ }^{2}$, Z. Khan ${ }^{2}$, S. Scott ${ }^{2}$
}

${ }^{1}$ orthopaedic Surgery, Uhnd, 5tw, United Kingdom, ${ }^{2}$, Uhnd, 5tw, UK

Introduction: Treatment of distal tibial fractures using minimally invasive plate osteosynthesis (MIPO) techniques may minimise damage to soft tissues and the vascular integrity of bony fragments. The use of locking plates is a rigid fixation and proper anatomical reduction is essential in order to avoid non-union.

Materials and methods: Multi-centre retrospective review of 60 patients (41 males and 19 females) treated with MIPO using the AO locking plate for distal tibial fractures. Their mean age was 42 years (range 14-86).

Results: According to $\mathrm{AO}$ fracture classification; there were 32 patients with $43 \mathrm{~A}$ type fractures; twenty $43 \mathrm{~B}$ and eight $43 \mathrm{C}$ type fractures. The commonest cause of injury was low-energy trauma. Fourty six patients had closed injury and 37 patients had closed reduction. Ninety three percent of the patients were non-weight bearing for the first 6 weeks post-op. All patient were fully weight bearing within an average period of 9 weeks (range 0-20) Average time to union was 4 months. Twenty two percent of the patients had further surgery (removal of metalware) due to prominence. Six patients had superficial wound infection successfully treated with antibiotics. There were 2 non-unions; one patient is a chronic heavy smoker and the other patient underwent autologous bone grafting and failed to unite. There were no failures of fixation.

Conclusion: MIPO is an effective method of treatment for distal tibial fractures. The use of indirect reduction techniques and small incisions is technically demanding but decreases surgical trauma to soft tissues. It is a rigid fixation and therefore, proper anatomical reduction is vital.

Disclosure: No significant relationships.

\section{$\mathbf{P 1 5 2}$}

\section{TREATMENT OF INTERTROCHANTERIC AND SUBTROCHANTERIC NONUNION OF THE PROXIMAL FEMUR}

\section{T. Matsumura}

Orthopaedics, Jichi Medical University, Shimotsuke-shi, Tochigi-ken, Japan

Introduction: Nonunion is always a challenging complication for the orthopaedic surgeons, especially in case of intertrochanteric and subtrochanteric nonunion. Nevertheless the treatment of proximal femoral fracture nonunion has been paid little attention in orthopaedic literatures. The purpose of this study was to retrospectively review a consecutive series of patients treated with reoperation for nonunion of fractures of the intertrochanteric and subtrochanteric region of the femur.

Materials and methods: Between 2004 and 2009, 9 patients with a mean age of 48 years (range 20-85 years) with 3 intertrochanteric and 6 subtrochanteric nonunions were treated with additional attempts to achieve union. All patients were followed up for a mean of 22 months (range 12-60 months). Implants used for revision surgery were as follows: four patients were treated with a locking plate, two patients were treated with a 95 degrees dynamic condylar screw, two patients were treated with a compression hip screw and one patient was an intramedullary nail. Eight of 9 patients had autogenous bone grafting. Clinical and radiographic data were reviewed retrospectively and the results and complications were analyzed. All patients were followed up until minimum of 1 year.

Results: All of nonunions healed. At last follow-up, all patients were ambulatory. Eight patients ambulated independently and one patient required a cane. Eight of 9 patients had no pain and one had mild pain in the region of the previous fracture. There were no intraoperative complications. Two patients had a leg length discrepancy, so leg lengthening was performed using Taylor Spatial Frame.

Conclusion: Planed revision internal fixation and autogenous bone grafting for intertrochanteric and subtrochanteric nonunions of the femur led to a high rate union and functional improvement.

References: 1. Antonio B, Gabriel M, Jose F, et al. The treatment of subtrochanteric nonunions with the long Gamma nail: twenty-Six patients with a minimum 2-year follow-up. J Orthop Trauma. 2004;18(6):346-53. 2. Dimon JH, Hughston JC. Unstable intertrochanteric fractures of the hip. JBJS Am. 1967;49:440-50. 3. French BG, Tornetta P 3rd. Use of an interlocked cephalomedullary nail for subtrochanteric fracture stabilization. Clin Orthop. 1998;348:95-100. 4. Haidukewych GJ. Nonunion of fractures of the subtrochanteric region of the femur. Tech Orthop. 2008;23:131-6. 5. Talmo CT, Bono JV. Treatment of intertrochanteric nonunion of the proximal femur using the S-ROM prosthesis. Orthopaedics. 2008;31:1-6.

Disclosure: No significant relationships.

\section{P153}

\section{TREATMENT OF OPEN TIBIAL FRACTURES AMONG RECREATIONAL SKIERS WITH ILIZAROV PROCEDURE}

I. Lalic, M. Stankovic, V. Kecojevic, Z. Gojkovic, R. Matijevic, B. Vukajlovic

Ortopedics, KCV, Novi Sad, Serbia

Introduction: Open tibial fractures among recreational skiers are not as rare as the population engaged in this sport is growing

Materials and methods: In the period since 2005 till 2010. The 31 patients were treated by this method. In relation to the gender structure 22 patients were male and 9 female. The average age of patients was 36 years. All fractures are classified by Gustillo. In 23 patients there was a grade II fracture while 8 patients had grade III fracture. From regional centers are gravitating to the ski slopes, these patients were transported to our clinic with wounds treated by primary surgery and 16 of them with already mounted external fixator and three different types of plaster immobilization. Because of the bad position of the fragments and rigid fixations are averaged after 3 weeks, external fixators were replaced with the Ilizarov apparatus. Results: Complete healing of fractures, was present in 19 patients, prolonged accretion in 8 patients, pseudoarthrosis in two patients and deep infection in two patients.

Conclusion: The application of the Ilizarov apparatus among recreational skiers, provides all the requirements for fast healing of such a serious fracture with minimal risk of entry of infection and other postoperative complications in fractures treated with other operative methods.

Disclosure: No significant relationships. 


\section{$\mathbf{P 1 5 4}$}

\section{TREATMENT OF PERIPROSTHETIC FEMUR FRACTURES}

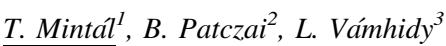

${ }^{1}$ Trauma Department, University of Pécs, Pécs, Hungary, ${ }^{2}$ Trauma Department, University of Pécs, Pécs, Hungary, ${ }^{3}$ Dept. of Traumatology and Hand Surgery, Institut of Musculoskeletal Surgery University Pécs, Pécs, Hungary

Introduction: The authors summarized the increase in the incidence of periprosthetic fractures and their treatment options as the implantation of hip prostheses are becoming more frequent.

Materials and methods: They interpret their own cases between 2003 and 2010. In this 7-year period, 34 patients were examined.

Results: The results were analyzed using the Harris Hip Score. The obtained figures ranged were from 53 to 100 , with a mean value of 82,833 .

Conclusion: The good results depend on the good indication and the high quality implants beside surgical technique.

Disclosure: No significant relationships.

\section{P155}

\section{TREATMENT OF THE FRACTURES OF THE LOWER LEG WITH INTRAMEDULLARY NAILS-AN APPROACH BASED ON 160 CONSECUTIVE CASES}

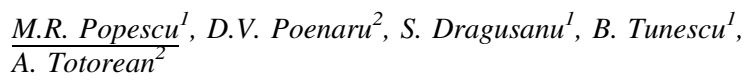

${ }^{1}$ Politraumatology, Casa Austria, County Hospital Timisoara, Timisoara, Romania, ${ }^{2}$ II Ortopaedic Clinic Timisora, Timisoara, Romania

Introduction: Our results regarding the treatment of the tibial and femoral fractures with intramedullary nails.

Materials and methods: Patients admitted with tibial and femoral fractures at Politrauma Casa Austria between 01.08.03 and 14.01.09. We evaluated the following parameters: etiology, ISS, the interval between admission and surgery, type of the fractures, type of osteosynthesis, complications.

Results: 160 patients -119 males (74\%) and 41 females (26\%) with a mean age of $35.43 \pm 14.76$ years, that presented 187 fractures. According to the ISS score: 14 patients with ISS $1-8,33$ patients with ISS $9-15,48$ patients with ISS $16-24,44$ patients with ISS 25-40 and 21 patients with ISS over 40.87 patients had surgery in the first $24 \mathrm{~h}, 61$ patients in the first 2 weeks and 12 patients after 3 weeks. Tibial fractures: 79 diaphyseal, 9 distal and 2 segmental; the femoral fractures: 86 diaphyseal and 11 segmental. We had 38 tibial open fractures and 10 femoral open fractures. Complications: infections (5\%), deep venous thrombosis (3\%), pseudarthrosis $(2 \%)$ breakage of the implant (3\%) and death in 5 cases (3\%).

Conclusion: The severity of the associated lesions (ISS) and the precocity of the surgical treatment are two factors that influence directly the patients' evolution. The intramedullary osteosynthesis is an efficient method of treatment for the tibial and femoral fractures (either opened or closed), with few complications.

References: 1. Babikian MG, White RR. Tibia: shaft. In: Ruedi TP, Murphy WM, editors. AO principles of fracture management, Thieme. New York: Stuttgart. 2000. p. 519-37. 2. Hontzsch D. Femur: shaft (incl. Subtrohanteric). In: Ruedi TP, Murphy WM, editors. AO principles of fracture management, Thieme. New York:
Stuttgart. 2000. p. 457-67. 3. Keel M, Labler L, Trentz O. Damage control in severely injured patients. Eur J Trauma. 2005;31:212-21. Disclosure: No significant relationships.

\section{P156}

\section{TRIAL SETUP AND PRELIMINARY RESULTS OF PROTECT: PROFYLAXIS OF TROMBOEMBOLIC COMPLICATIONS TRIAL}

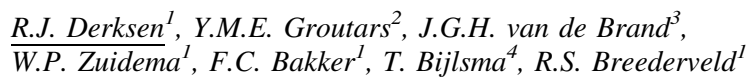

${ }^{1}$ Traumasurgery, VU University Medical Center Amsterdam, Amsterdam, Netherlands, ${ }^{2}$ Emergency Department, Red Cross Hospital, Beverwijk, Netherlands, ${ }^{3}$ Traumasurgery, Medical Center Alkmaar, Alkmaar, Netherlands, ${ }^{4}$ Surgery, Spaarne hospital, Hoofddorp, Netherlands

Introduction: Concerning the need for tromboprofylaxis in ambulatory patients with a nonsurgical fracture of the lower extremity being treated in a below the knee plaster cast, little data have been obtained. Hence, no generally accepted strategy exists in the prevention of tromboembolic complications. The seventh ACCP conference on antitrombotic therapy (2004) does not recommend the routine use of tromboprophylaxis, debating a lack of randomised controlled trials on this subject. The aim of the study is to determine the need for tromboprophylaxis in patients with a fracture of the ankle/foot being treated conservatively in a below-knee plaster cast and to assess if both nadroparin and fondaparinux are effective for this indication.

Materials and methods: The study is designed as a prospective, randomised, controlled, single blinded, multicenter trial. The study groups consist of patients at least 18 years old with an ankle-/footfracture requiring a below-knee plaster cast for at least 4 weeks. Patients are randomly assigned to three groups: Nadroparin, Fondaparinux or no prophylaxis.

Results: At the time of plaster cast removal a colour duplex is performed according to a strict protocol. The technician is blinded to treatment. Safety is assessed as a secondary outcome parameter. Power Analysis. 223 patients are required per group for the study to detect a reduction in the incidence of DVT from $10 \%$ in the control group to $4 \%$ in the intervention groups (power $80 \%$, alfa 0.05 , $\mathrm{NNT}=17)$.

Conclusion: The trial setup and its challenges will be put forward as well as the preliminary data which will comprise about 200 patients by then.

Disclosure: For the study, we received an unrestricted educational grant from GlaxoSmithKline

\section{P157}

\section{VALUE OF INTRAOPERATIVE 3D-IMAGING IN OSTEOSYNTHESIS OF COMPLEX CALCANEUS FRACTURES}

\section{P. Wilde, K. Schmidt-Horlohé, O. Neun, G. Zivko, H.P. Abt, R. Hoffmann}

Unfallchirurgie Und Orthopädische Chirurgie, Berufsgenossenschaftliche Unfallklinik, Frankfurt, Germany

Introduction: Aim of the study was the evaluation of the clinical benefit and the practicability of intraoperative imaging using a 
motorized 3D-C-arm in osteosynthesis of multifragmentary calcaneus fractures.

Materials and methods: We used a 2nd generation 3D-C-arm (Siemens Arcadis Orbic 3D) after conventional open reduction and angular stable plate osteosynthesis of complex calcaneus fractures. Therefore an automated 3D-scan was performed before wound closure. The system software then calculated multiplanar reconstructions which were analyzed by the surgeon with regard to fracture reduction and implant location. Inadequate reduction or implant malposition were corrected immediately. We documented the revision rate and the additional time needed. Clinical benefit and ease of use were rated through the surgeon on a VAS (1-10).

Results: 50 fractures (9 type Sanders 2, 23 type Sanders 3, 18 type Sanders 4 fractures) were included, showing a revision rate of $32 \%$ (16 out of 50 cases with 11 malreductions and 7 implant malpositions). With a steep learning curve the mean additional time was $6.3 \mathrm{~min}(3.5-15)$. Intraoperative 3D imaging was rated very useful by the surgeons with a mean clinical benefit of $8.6(7-10)$ on the VAS. A mean VAS score of 8.8 (7-10) documented the ease of use.

Conclusion: Intraoperative 3D imaging is a very useful tool for the detection of malreduction and implant malposition in calcaneus osteosynthesis. The method allows immediate revisions and is easy to use with only a marginal expenditure of time. In spite of these promising results the effects on the clinical outcome are still open and thus should be analyzed in future studies.

Disclosure: No significant relationships.

\section{P158}

\section{WHAT INFLUENCE HAS A BONY INFECTION (OSTEOMYELITIS) TO THE FUNCTIONAL AND THE BONE HEALING RESULTS AFTER CORRECTION OF LOWER EXTREMITY DEFORMITIES}

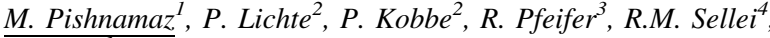 H. Pape}

${ }^{1}$ Department of Trauma Surgery, University of Aachen, Aachen, Germany, ${ }^{2}$ Department of Orthopaedic Trauma, University Hospital of the Rwth Aachen, Aachen, Germany, ${ }^{3}$ Klinik Für Orthopädie Und Unfallchirurgie, Schwerpunkt Unfallchirurgie, Universitaetsklinikum Aachen, Aachen, Germany, ${ }^{4}$ Department of Orthopaedic and Trauma Surgery, University Hospital of the Rwth Aachen, Aachen, Germany

Introduction: Lower extremity deformities are a challenge to both the patient and the treating orthopaedic physician. The management is usually prolonged and has to be performed on a case-adapted basis. We reexamined our patients treated over 2 decades.

Materials and methods: Patients who had previous corrective osteotomies of the lower extremity were considered. Inclusion criteria: surgical correction of femur or tibia, no high tibial osteotomy (HTO), no contralateral amputation, no current malignancy or osteopathy, and less than 65 years old. The clinical reevaluation included range of motion, radiographs, objective and subjective patient complaints (Association for the Study and Application of the Methods of Illizarov (ASAMI) classification).

Results: 48 patients (34 male, 14 female), treatment between 1986 and 2007. Mean age: 30.2 years. $\mathrm{N}=15$ femur, $\mathrm{n}=33$ tibia, $\mathrm{n}=11$ pre-op osteomyelitis. Causes: 27 closed fractures, 16 open fractures, 1 renal osteodystrophy, 2 neoplastic, and 2 congenital malformations. Treatment: 20 patients Ilizarov, 8 nonlocked plates, 5 locked plates, 5 intramedullary (IM) nails, 1 Taylor Spital Frame, 5 unilateral external fixation, and 4 ISKD. Complications: $\mathrm{n}=7$ persistent nonunions, $\mathrm{n}=6$ wound infections. $\mathrm{n}=40$ revisins. ASAMI classification results : $29(60.4 \%)$ excellent bone healing results, 12 (25\%) good, 0 fair, and $7(14.6 \%)$ poor. 31 of $48(64.6 \%)$ excellent functional results, $10(20.8 \%)$ good, $5(10.4 \%)$ fair and $2(4.17 \%)$ poor. Subanalysis osteomyelitis vs. noninfected deformities: Poor and fair bone healing occurred in $27.3 \%$ with pre-operative osteomyelitis versus $10.8 \%$ in uninfected patients. Poor and fair functional outcomes were observed in $18.2 \%$ of patients with osteomyelitis versus $13.5 \%$ in uninfected patients.

Conclusion: In noninfected cases, lower extremity deformity corrections were successful in more than $85 \%$ of patients in this series, thus warranting the high effort. In contrast, given the high failure rate (ongoing nonunion, infection and pain) in cases with preoperative osteomyelitis, it may be wiser to discuss alternative treatments with patients, including amputation.

Disclosure: No significant relationships.

\section{INTERVENTIONAL RADIOLOGY}

\section{P159}

\section{A TWO-PHASE CT PROTOCOL IS A SUFFICIENT PROCEDURE TO DETECT ALL TYPES OF ENDOLEAK ACCURATELY}

\section{B. Walter}

Clinical Radiology, Ludwig-Maximilian Universität Munich, München, Germany

Introduction: The precise detection of endoleaks after aortic aneurysm repair is essential for adequate treatment to prevent the risk of aneurysm rupture. The optimal CT image acquisition protocol, however, is still a subject of debate. The present study intends to show that the combination of arterial and delayed CT phases is a sufficient method to classify all kinds of endoleaks.

Materials and methods: Retrospectively the CT protocols of 400 patients with abdominal and thoracic aortic aneurysms who had undergone stent placements in the last years were reviewed.

Results: 97 patients presented with different types of endoleaks I-V. All kinds of endoleaks could be accurately detected by using the biphasic CT-protocol with an arterial and a delayed venous phase. The non-enhanced imaging phase could be therefore avoided.

Conclusion: Individuals with stent-graft therapy must be followed up lifelong consequently to correct in time endoleaks such as type I and III associated with an increased risk of aneurysmatic rupture or device-related endoleaks correlated with an increased need for secondary interventions. The efficiency of the two phase CT imaging minimizes radiation exposure. But further efforts to reduce the large cumulative doses of ionizing radiation over time are necessary.

References: 1. Cao P, De Rango P, Verzini F, Parlani G. Endoleak after endovascular aortic repair: classification, diagnosis and management following endovascular thoracic and abdominal aortic repair. J Cardiovasc Surg (Torino). 2010;51(1):53-69. 2. Macari M, Chandarana H, Schmidt B et al. Abdominal aortic aneurysm: can the arterial phase at CT evaluation after endovascular repair be eliminated to reduce radiation dose? Radiology. 2006;241(3): 908-14. 3. Buth J, Harris PL, Van Marrewijk C, Fransen G. Endoleaks during follow-up after endovascular repair of abdominal aortic aneurysm. Are they all dangerous? J Cardiovasc Surg (Torino). 2003;44(4):559-66. 4. Heikkinen MA, Arko FR, Zarins CK. What is the significance of endoleaks and endotension. Surg Clin North Am. 2004;84(5):1337-52. 5. Buth J, Harris PL, van Marrewijk C, Fransen G. The significance and management of different types of endoleaks. Semin Vasc Surg. 2003;16(2):95-102. 6. van Marrewijk C, Buth J, Harris PL et al. Significance of endoleaks after 
endovascular repair of abdominal aortic aneurysms: the EUROSTAR experience. J Vasc Surg. 2002;35(3):461-73. 7. Norwood MG, Lloyd GM, Brown MJ, et al. Endovascular abdominal aortic aneurysm repair. Postgrad Med J. 2007;83(975):21-7. 8. Bent CL, Jaskolka JD, Lindsay TF, Tan K. The use of dynamic volumetric CT angiography (DV-CTA) for the characterization of endoleaks following fenestrated endovascular aortic aneurysm repair (f-EVAR). J Vasc Surg. 2010;51(1):203-6. 9. Saba L, Montisci R, Sanfilippo R, Mallarini G. Imaging of the endoleak after endovascular aneurysm repair procedure by using multidetector computer tomography angiography. J Cardiovasc Surg (Torino). 2009;50(4): 515-26. 10. Szucs-Farkas Z, Semadeni M, Bensler S, et al. Endoleak detection with $\mathrm{CT}$ angiography in an abdominal aortic aneurysm phantom: effect of tube energy, simulated patient size, and physical properties of endoleaks. Radiology. 2009;251(2):590-8. 11. Iezzi R, Cotroneo AR, Filippone A, et al. Multidetector-row computed tomography angiography in abdominal aortic aneurysm treated with endovascular repair: evaluation of optimal timing of delayed phase imaging for the detection of low-flow endoleaks. J Comput Assist Tomogr. 2008;32(4):609-15. 12. Kirby JM, Jhaveri KS, Kachura JR. Computed tomography angiography in abdominal aortic endoleaks: what is the optimal protocol? Can Assoc Radiol J. 2007;58(5): 264-71. 13. Lawrence-Brown MM, Sun Z, Semmens JB, Liffman $\mathrm{K}$, et al. Type II endoleaks: when is intervention indicated and what is the index of suspicion for types I or III? Endovasc Ther. 2009;16 Suppl 1:I106-18. 14. Stolzmann P, Frauenfelder T, Pfammatter T, Peter $\mathrm{N}$ et al. Endoleaks after endovascular abdominal aortic aneurysm repair: detection with dual-energy dual-source CT. Radiology. 2008;249(2):682-91. 15. Hong C, Heiken JP, Sicard GA, Pilgram TK, Bae KT. Clinical significance of endoleak detected on follow-up CT after endovascular repair of abdominal aortic aneurysm. AJR Am J Roentgenol. 2008;191(3):808-13. 16. Iezzi R, Cotroneo AR, Filippone A, et al. Multidetector CT in abdominal aortic aneurysm treated with endovascular repair: are unenhanced and delayed phase enhanced images effective for endoleak detection? Radiology. 2006;241(3):915-21. 17. Rozenblit AM, Patlas M, Rosenbaum AT et al. Detection of endoleaks after endovascular repair of abdominal aortic aneurysm: value of unenhanced and delayed helical CT acquisitions. Radiology. 2003;227(2):426-33.

Disclosure: No significant relationships.

\section{P160}

\section{ANGIOGRAPHIC EMBOLIZATION FOR BLUNT RENAL TRAUMA: SINGLE CENTRE RESULTS}

\author{
W. Metsemakers ${ }^{1}$, S. Heye ${ }^{2}$, S. Nijs ${ }^{3}$, A. Sermon ${ }^{4}$, G. Maleux ${ }^{5}$ \\ ${ }^{1}$ Trauma Surgery, University Hospitals Leuven, Leuven, Belgium, \\ ${ }^{2}$ Interventional Radiology, University Hospitals Leuven, Leuven, \\ Belgium, ${ }^{3}$ Department Of Traumatology, University Hospitals \\ Leuven, Leuven, Belgium, ${ }^{4}$ Traumatology, University Hospitals \\ Leuven, Leuven, Belgium, ${ }^{5}$ Interventional Radiology, University \\ Hospitals Leuven, Leuven, Belgium
}

Introduction: Over the last decades the management of high grade, blunt renal injury has shifted from a primarily operative approach to a more conservative treatment protocol. Here angiographic embolization has gained an important role. We performed a retrospective study reviewing patients with blunt renal injury who underwent angiographic embolization.

Material and methods: A retrospective study was performed at the University Hospitals Leuven to determine the outcome of patients who underwent angiographic embolization for the management of blunt renal trauma. Clinical success was defined as the stabilization of vital signs and the absence for further surgical or radiological intervention.

Results: Between January 2003 and October 2010, 10 hemodynamic unstable patients with blunt renal injury were treated with angiographic embolization. The median age of this patient population was 36 years. The median follow up was 2 years. Clinical success was achieved in all patients. One patient died during the months of hospitalization due to severe brain damage. None of the remaining 9 patients developed a new onset of arterial hypertension or procedurerelated impairment of renal function. There were no other complications regarding angiographic embolization or abdominal trauma.

Conclusion: Angiographic embolization in blunt renal injury is a safe and effective procedure. Early identification of patients who would benefit from this procedure is of primordial importance.

Disclosure: No significant relationships.

\section{P161}

\section{EVOLVING NONOPERATIVE MANAGEMENT OF BLUNT SPLENIC INJURY}

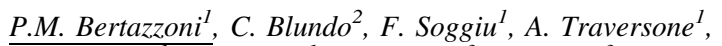
A.M. Marini ${ }^{I}$, P. De Rai ${ }^{1}$, A. Nicolini ${ }^{3}$, S. Crespi ${ }^{3}$, L. Castoldi ${ }^{1}$

${ }^{1}$ Department of Surgery and Emergency Surgery, Fondazione IRCCS Cà Granda-Ospedale Maggiore Policlinico, Milano, Italy,

${ }^{2}$ Department of Surgery and Emergency Surgery, Fondazione IRCCS Cà Granda-Ospedale Maggiore Policlinico, Milano, Italy, ${ }^{3}$ Department Dei Servizi, Fondazione IRCCS Cà Granda-Ospedale Maggiore Policlinico, Milano, Italy

Introduction: The spleen is the most commonly injured organ after blunt trauma. However, the incidence of injury following car accidents is declining, due to obligatory seat belts. In the meantime, nonoperative treatment of splenic lesions, with or without transcatheter embolization of the splenic artery, has gained wide acceptance.

Materials and methods: We conducted a retrospective review of all patients admitted to a Level 1 trauma center with blunt splenic injury. Results: From 1997 to October 2010, 130 patients with blunt splenic injury were admitted. Male to female ratio was 3.8:1 and mean age 36.5 years. Seventy-four patients underwent immediate surgical management and 65 , hemodynamically stable, were managed conservatively, with a $92.3 \%$ salvage rate (failed treatment in 6 patients). In the last 2 years, eight nonoperative treatment were associated to angiography and embolization, in one case of the splenic artery and in seven case distally. All patients had active bleeding at CT scan; five patients had splenic injury grade III and three patients grade IV, according to the OIS of the AAST. Four patients had associated pulmonary, renal or skeletal lesions. One of these eight patients, with multiple fractures, underwent urgent splenectomy for anaemia, but at intervention the spleen was not bleeding. There were no complications, and median hospital stay was 8.5 days. No associated injuries have been missed in the nonoperative treatment group.

Conclusion: In our experience, splenic artery embolization is a valuable adjunct to nonoperative management of blunt splenic injury with bleeding on admission CT scan in hemodynamically stable patients.

Reference: 1. Moore FA. WTA critical decisions in trauma. J Trauma. 2008;65:1007-11.

Disclosure: No significant relationships. 
P162

\section{MANAGEMENT AND PROGNOSIS OF ACUTE MESENTERIC ISCHEMIA: HAS ANYTHING CHANGED?}

C. Camarero $^{1}$, T. Sanchez-Rodriguez ${ }^{2}$, A. Vaquero $^{3}$, L. Alvarez-Llano ${ }^{4}$, A. Fabregues ${ }^{3}$, M. Sanz ${ }^{5}$, F. Turegano 6

${ }^{1}$ Surgery, Gregorio Marañon Hospital, Madrid, Spain, ${ }^{2}$ Surgery, Gregorio Marañon Hospital, Madrid, Spain, ${ }^{3}$ General Surgery, Hospital Gregorio Marañon, Madrid, Spain, ${ }^{4}$ Surgery, Hospital General Universitario Gregorio Marañón, Madrid, Spain, ${ }^{5}$ General Surgery, Gregorio Marañon Hospital, Madrid, Spain, ${ }^{6}$ General And Emergency Surgery, University Hospital Gregorio Maranon, Madrid, Spain

Introduction: Acute mesenteric ischemia (AMI) carries a high mortality, ranging from 40 to $100 \%$. Little progress has been made in the last decades in terms of early diagnosis, management and overall prognosis. Our aim is to assess the main prognostic factors of an adverse outcome in AMI, and the role of interventional radiology.

Materials and methods: Retrospective observational study covering a 20 -year period (January 1990-January 2010). We have assessed the impact of several factors (age, sex, etiology, co-morbidities, hypotension, fever, tachycardia, lactic acidosis, extent of ischemia, time to laparotomy, CPK, LDH, WBC) on mortality. A selected group treated with intra-arterial fibrinolysis was independently assessed.

Results: 183 patients (53\% males) with AMI were treated either surgically or by interventional radiology. The mean age was of 71.4 years, $44.6 \%$ of them had atrial fibrillation, and $32.4 \%$ atherosclerosis. $29.3 \%$ of patients had a massive intestinal ischemia. The mortality rate was of $54 \%$, and the following factors had a significant influence on mortality $(\mathrm{p}<0.05)$ : female sex, age, atrial fibrillation, time to laparotomy, tachycardia, massive ischemia, non-occlusive AMI, and elevated CPK and LDH levels. A selected group with AMI of embolic origin treated by interventional radiology had the best outcome.

Conclusion: AMI still carries a high mortality rate. An early and active surgical implication in the diagnosis of abdominal pain has reduced the time to laparotomy or to interventional radiology, and improved outcome in a selected group of patients

Disclosure: No significant relationships.

\section{$\mathbf{P 1 6 3}$}

WHAT ARE THE SPECIFIC CT SCAN CRITERIA THAT CAN PREDICT OR EXCLUDE THE NEED FOR RENAL ANGIOEMBOLIZATION AFTER HIGH-GRADE RENAL TRAUMA IN A CONSERVATIVE MANAGEMENT STRATEGY?

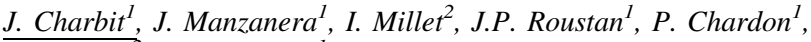 \\ $\overline{P .}$. Taourel $^{2}$, X. Capdevila
}

${ }^{1}$ Département Anesthésie Réanimation Lapeyronie, Réanimation Traumatologique, Hôpital Lapeyronie, CHU Montpellier, Montpellier, France, ${ }^{2}$ Département De Radiologie, Hôpital Lapeyronie, CHU Montpellier, Montpellier, France

Introduction: The indications of renal angioembolization (RAE) highgrade renal trauma (HGRT) are based on angiographic criteria to reduce the failure rate of conservative management $(\mathrm{CM})$. There is no consensus to predict an indication of RAE with a computed tomography $(\mathrm{CT})$ scan. The aim of this study was to evaluate CT specific criteria to predict or exclude the need for RAE.
Materials and methods: All traumatized patients admitted with HGRT (AAST-OIS grade $>2$ ) treated by CM were considered between 2005 and 2009. We collected the demographic, CT criteria, angiographic, management, and outcome data for these patients and they were classified into two groups according to whether they received RAE or not.

Results: Among 101 patients with renal injury, 58 were HGRT, and 53 of these were treated by CM. Ten patients received RAE. There was no significant difference for urologic interventions [2 (20\%) vs. 7 (16\%)] and CM failure rate [1 (10\%) vs. 2 (5\%)] during hospital stay between the RAE group and the no RAE group. Intravascular contrast extravasation (ICE) and perirenal hematoma rim distance (PRD) were independent predictors of the need for RAE on multivariate analysis: OR 24.4 (95\% CI 2.4-248.9, P < 0.01) and OR 31 (95\% CI 3-504.4, $\mathrm{P}<0.005)$, respectively. The absence of ICE associated with a PRD lower than $25 \mathrm{~mm}$ excludes an indication for RAE $(\mathrm{P}<0.001)$.

Conclusion: RAE can be proposed in $20 \%$ of patients with HGRT managed with CM. Specific CT scan criteria can predict with high accuracy and exclude the need for RAE, without reducing the success rate of $\mathrm{CM}$

Disclosure: No significant relationships.

\section{MASS CASUALTIES MANAGEMENT}

\section{P164}

\section{COOPERATION OF FRENCH FORWARD SURGICAL TEAM AND LOCAL HOSPITAL IN BIRAO (NORTH OF CENTRAL AFRICAN REPUBLIC) DURING EUFOR OPERATION IN} 2009

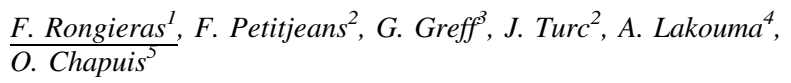

${ }^{1}$ Service De Traumatologie Et Chirurgie Orthopédique, Hopital Militaire Desgenettes, Lyon, France, ${ }^{2}$ Service De Réanimation Et Anesthésie, Hopital Militaire Desgenettes, Lyon, France, ${ }^{3}$ Service De Chirurgie Orthopédique, Hia Desgenettes, Lyon, France, ${ }^{4}$ Hopital Prefectoral De La Vakaga, Birao, Central African Republic, ${ }^{5}$ Service De Chirurgie Viscérale, Hopital D'instruction Des Armées Du Val De Grace, Paris, France

Introduction: During EUFOR military operation in the east of Tchad for 2006-2009, French army had positionned a Forward Surgical Team (FST) in the far north of Central African Republic, just near the frontier of Soudan. This FST was in a very poor region without surgical capabilities.

According to humanitarian rules, in association with local partners of "medecin sans frontieres", this FST worked with the local hospital. Surgical capabilities, X-rays and sonography were used to help civilian population.

Materials and methods: FST has no hospitalisation capacities. For this reason, collaboration with the hospital of Birao was necessary. Consultations were done in Birao hospital. X-rays, sonography and surgical procedures were performed in FST and then patients were evacuated to Birao hospital for further treatment. This was possible because of the good collaboration with local physician.

Results: From February to April 2009, FST performed 168 surgical procedures. Majority of these procedures was general surgery (thyroidectomy, hernia, urology,...) but we performed also war surgery with external fixation device and surgery for old infected wound coming from Soudan. Absence of local dentist or gynecologist requires us to help population by doing teeth treatment and cesarean. $163 \mathrm{X}$-rays were done and 90 sonographies. 320 consultations were performed. 
Conclusion: This experience of collaboration between a local hospital without facilities and a French FST was very interesting. French capabilities had helped local population without substituting to local hospital. This was possible because of proximity of the two structures, local political and military situation.

Disclosure: No significant relationships.

\section{P165}

\section{EARTHQUAKE RELATED INJURIES EXPERIENCES FROM HAITI 2010}

P. Fischer ${ }^{\text {I }}$, M. Rohner ${ }^{2}$, S. Müller ${ }^{2}$, K. Kabir ${ }^{1}$

${ }^{1}$ Orthopaedics and Trauma Surgery, University of Bonn, Bonn, Germany, ${ }^{2}$ University Clinic Bonn, Bonn, Germany

Introduction: Haiti's security, rescue forces and its general population suffer from the Earthquake in 2010 and in the handling of mass casualty incidents.

Materials and methods: The aim of this project was to develop a Disaster Hospital, prepare, doctors, caregivers for medical help of earthquake injuries. And take care of the patients.

Results: Surgical Teams went out for surgery and medical help, and training on the job. In 4 weeks we treated 1,000 patients in a disaster hospital in Port au Prince (Haiti). 70\% were patients with fractures, $40 \%$ of them had lower extremities injuries. $87 \%$ of the treated patients had signs of a PTSD. Most of the severely injured patients were old people and children.

Conclusion: The aim of this project was to develop a Disaster Hospital prepare, doctors, caregivers for medical help of earthquake injuries. And take care of the patients. Trauma Training (like ATLS) and FAST (Sonography in Trauma) for the nurses and doctors were prepared and held. Parallel we took care of the injured patients.

Disclosure: No significant relationships.

\section{P166}

\section{FIRST ITALIAN MRMI: A NEW EDUCATIONAL EXPERIENCE}

\author{
P.C. Bergonzi ${ }^{1}$, R. Faccincani ${ }^{2}$, M. Spessot ${ }^{3}$, M.T. Cibelli ${ }^{1}$, \\ R. Sannicandro ${ }^{3}$, A. Rossodivita ${ }^{4}$
}

${ }^{1}$ Intensive Care Unit, San Raffaele Hospital, Milan, Italy, ${ }^{2}$ Deas, San Raffaele Hospital, Milan, Italy, ${ }^{3}$ Emergency Department, San Raffaele Hospital, Milan, Italy, ${ }^{4}$ Cardiosurgical Sub-intensive Care Unit, San Raffaele Hospital, Milan, Italy

Introduction: Education and training are key elements in increasing the preparedness for major incidents management. We offered to the Italian audience a new training opportunity: the Medical Response to Major Incidents (MRMI) course.

Materials and methods: A tree-days theoretical and practical MRMI course was organized at San Raffaele Hospital in Milan on 5-7 October 2010 in collaboration with ESTES Section of disaster and military surgery. Similarly to the program of the previous MRMI courses organized in other European Countries the trainees followed a full educational day with lectures on major incidents management and training-in-group on simulation method. In the last 2 days the trainees were engaged in real-time macro-simulations of major incidents with hundreds of people involved. We used the MACSIM simulation model created and developed by Prof Sten Lennquist. 52 people attended the course, within these a great variety of professions was represented: healthcare personnel (both doctors and nurses), Hospitals and Public Institution's directors and administrative staff, Civil Protection geologists, etc... The course was conducted by an international faculty of experienced MRMI instructors with the cooperation of an Italian instructors team that had passed the first MRMI instructor course organized in Split (Croatia) on November 2009.

Results: We received extremely positive reviews from all participants regardless of the role they played during the simulations, their usual work and previous experience in major incidents management. The complete evaluation of pre- and post-course tests is in progress.

Conclusion: The MRMI course is an appreciated, valuable and effective training opportunity for staff involved in each role of the Major Incidents management.

Disclosure: No significant relationships.

\section{P167}

\section{FLOODS IN MADEIRA}

\section{P.C. Ramos}

Surgery, Hospital Nelio Mendonça Funchal, Funchal, Portugal

Introduction: we present the 20th February floods in Madeira 2010 and its impact.

Materials and methods: All the logistic of the response of Civil Protection and Health System to the catastrophe.

Results: We present final results of all the injured people.

Conclusion: We present the conclusions of our performance during this event.

Reference: 1. Madeira floods 2010.

Disclosure: No significant relationships.

\section{P168}

\section{INTERNATIONAL ISAF MILITARY HOSPITAL IN KABUL: ONE YEAR OF SURGICAL ACTIVITY}

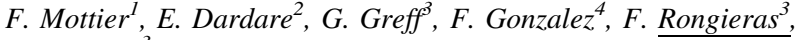 \\ F. Chauvin ${ }^{3}$
}

${ }^{1}$ Service De Traumatologie Et De Chirurgie Orthopédique, Hopital D'instruction Des Armées Desgenettes, Lyon, France, ${ }^{2}$ Service D'anesthesie Et De Reanimation, Hopital Militaire Desgenettes, Lyon, France, ${ }^{3}$ Service De Chirurgie Orthopédique, Hia Desgenettes, Lyon, France, ${ }^{4}$ Service De Chirurgie Viscérale Et Thoracique, Hopital D'instruction Des Armées Percy, Clamart, France

Introduction: International Military hospital of Kabul is based on place of Kabul International Airport. This hospital is a rule 3 NATO hospital constructed 1 year ago. This Hospital replaced the Rule 3 hospital based previously in WAREHOUSE Camp.

Materials and methods: This hospital, commanded by French army, is one of the ISAF Hospital. The mission of this hospital is to treat ISAF soldiers, Afghan soldier of afghan national army, civilians' casualties and also international civilians working in Afghanistan. From July 2009 to October 2010, 1047 surgeries were performed and all datas were collected in a surgical registry.

Results: We propose there to make the analysis of these datas and to focuse on the three last months: epidemiology of trauma, description 
of wounded population, surgical procedures used in this conflict situation.

Conclusion: These datas permits to have some reflections on casualties, development of the conflict and perspectives on further evolution of war wounds treatment.

Disclosure: No significant relationships.

\section{P169}

\section{PATIENT DISTRIBUTION IN A MASS CASUALTY EVENT: LESSONS TO BE LEARNED FROM THE FEBRUARY 2009 TURKISH AIRLINES CRASH}

I.L.E. Postma ${ }^{1}$, H. Weel ${ }^{2}$, M.J. Heetveld ${ }^{3}$, T.S. Bijlsma ${ }^{4}$, I. vd Zande ${ }^{5}$, $\overline{\text { F.W. Bloemers }}^{6}$, J.C. Goslings ${ }^{7}$

${ }^{1}$ Trauma Unit, Department of Surgery, Academic medical Centre, Amsterdam, Netherlands, ${ }^{2}$ VU Medical Center, Amsterdam, Netherlands, ${ }^{3}$ Kennemergasthuis, Haarlem, Netherlands, ${ }^{4}$ Spaarne ziekenhuis, Hoofddorp, Netherlands, ${ }^{5}$ GGD Kennemerland, Haarlem, Netherlands, ${ }^{6}$ Trauma Surgery, VU medica centre, Amsterdam, Netherlands, ${ }^{7}$ Trauma Unit Department of Surgery, Academic Medical Center, Amsterdam, Netherlands

Introduction: Difficulties have been reported in patient distribution, an important part of management of disaster and Mass Casualty Events (MCE). In this study we analysed the protocols and the management of the patient distribution after the Turkish Airlines crash on February 25th 2009 near Amsterdam Schiphol Airport.

Materials and methods: Retrospective analysis of the Patient Distribution Plan (PDP) and executed patient distribution of the 126 casualties of the crash. Data collected included medical treatment capacity (MTC), number of patients per hospital, triage classification, Injury Severity Score (ISS), secondary transfers, distance from the crash site, and critical mortality rate.

Results: The regional PDP contains two ambiguous definitions of MTC. The executed patient distribution was not in compliance with the PDP, with four hospitals receiving $133-213 \%$ of their MTC, and five hospitals receiving 4-20\% MTC. There were 14 receiving hospitals (distance from crash: $5.8-53.5 \mathrm{~km}$ ); three hospitals within $20 \mathrm{~km}$ of the crash did not receive any casualties. Major trauma centres received $89 \%$ of the 'immediate' casualties and $92 \%$ of the casualties with ISS $\geq 16$. They also received $10 \%$ of the 'delayed' casualties and $29 \%$ of the casualties with ISS $\leq 8$. Three patients were secondarily transferred. No casualties died on the way to, or in the hospital (critical mortality rate $=0 \%$ ).

Conclusion: The regional PDP is ambiguous and therefore not practical. The PDP was not followed in this MCE and this has led to uneven casualty load at the hospitals. With respect to secondary transfers and critical mortality rate, patient distribution was performed well.

Disclosure: No significant relationships.

\section{P170}

\section{TRAUMA SURGERY CATASTROPHE AID FOLLOWING THE EARTHQUAKE IN HAITI 2010—SPECIAL CHALLENGES, PROSPECTS}

\section{Winter, I. Osmers, S. Krieger}

Germany-berlin, Ärzte ohne Grenzen/Doctors without borders, Berlin, Germany
Introduction: The 2010 earthquake in Haiti with 300,000 injured has been one of the greatest mass casualties in recent history. Doctorswithout-borders (MSF) started their relief response immediately after the earthquake. While the initial phase was focusing on life saving surgery, the second phase was concentrating on reconstructive surgery of extremities. The magnitude of the interventions and the challenges faced on ground led us to an intense lesson learned process. Herewith we would like to contribute to improve surgical care following disaster.

Materials and methods: Personal experience/internal MSF review Results: MSF performed roughly 173,000 medical consultations and 11,700 surgical interventions in 26 medical facilities during the first 4 months, being one of the main providers of surgery after the earthquake. During the first $72 \mathrm{~h}$ care was provided ad hoc and resources were extremely limited. Triage couldn't be done systematically due to the overload of patients. After $24 \mathrm{~h}$ basic major surgery was possible, provided by Level 2 care-"field-hospitals". These facilities were focusing on saving lives and limbs. Level 3 care was established after 14 days. Further specialized surgery treating osteitis and non-/malunion of primary insufficient treated fractures is still needed.

Conclusion: Crucial for effective patient care is an ability to act as early as possible. Protocols and surgical techniques have to be adapted to the overall situation (triage, damage-control, referral system). Further specially trained and fully equipped rapid-responseteams incl. experienced coordinators should be prepared for future disasters. A MSF-Surgical-Disaster-Preparation-Course is under discussion. In the 1st phase well adapted Level 2 service provides sufficient basic surgery that is cost-effective for a majority of the cases using damage-control concepts. Accurately timed planning for the second and third phase incl. reconstructive surgery, post-op-care and physiotherapy is important. There are poor data records on emergency aid. For a better evidence-based relief action more relevant statistical evaluations are needed.

Disclosure: No significant relationships.

\section{P171}

\section{TRIAGE AND MASS CASUALTIES: THE DJIBOUTIAN EXPERIENCE}

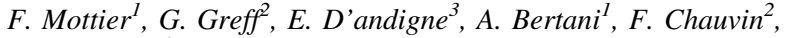 F. Rongieras ${ }^{2}$}

${ }^{1}$ Service De Traumatologie Et De Chirurgie Orthopédique, Hopital D'instruction Des Armées Desgenettes, Lyon, France, ${ }^{2}$ Service De Chirurgie Orthopédique, Hia Desgenettes, Lyon, France, ${ }^{3}$ Hopital Bouffard, Service D'urgence, Djibouti, Djibouti

Introduction: French army has a military hospital located in Republic of Djibouti. This hospital is a NATO rule 3 hospital with an orthopaedic and a visceral surgeon.

During 2008, a conflict between Erythrea and Djibouti was responsible of many wounded soldiers.

Materials and methods: During this conflict, we observed mass casualties which was evacuated on French military hospital BOUFFARD of Djibouti. Wounded and injured Djiboutian soldiers were sorted out and treated.

Results: We reported the datas of 90 war-injured soldiers from sorting to emergency treatment and to definitive care.

Conclusion: Mass casualties is an uncommon situation for a orthopaedic or visceral surgeon in France. This exceptional situation requires surgeon to be very adaptable. By our experience, we attempt to relate how to do to manage this situation.

Disclosure: No significant relationships. 


\section{MASSIVE HEMORRHAGE AND COAGULOPATHY}

\section{$\mathbf{P 1 7 2}$}

\section{ADRENAL GLAND AS A MARKER OF MAJOR TRAUMA}

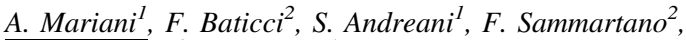
S. Cimbanassi ${ }^{3}$ O. Chiara $^{4}$

${ }^{1}$ Trauma Team, Ospedale Niguarda Ca' Granda, Milano, Italy, ${ }^{2}$ Chirurgia Generale E Mininvasiva, Istituto Clinico Humanitas, Rozzano, Italy, ${ }^{3}$ Emergency, Ospedale Niguarda Ca'granda, Milano, Italy, ${ }^{4}$ Emergency, Ospedale Niguarda Ca' Granda, Milano, Italy

Introduction: To review our experience with adrenal gland injury (AGI) in terms of incidence, demographics, mechanism of injury, associated injuries, mortality, Injury Severity Score (ISS), Revised Trauma Score (RTS), Glasgow Coma Scale (GCS), total length of hospital stay (LOS) and intensive care unit (ICU) LOS.

Materials and methods: We conducted a retrospective analysis of 2,834 trauma patients observed and treated by the Trauma Team of Niguarda Hospital of Milan from October 2002 to June 2010. All patients were studied according to a standard protocol and for each patient we calculated ISS, RTS and probability of Survival (Ps).

Results: Sixty-two patients had AGI (2.18\%) ISS of the AGI + group was significantly higher than the AGI-group (93.4 vs. 53,7\%; $\mathrm{p}<0,001)$, as Ps (0.61 vs. $0.79 ; \mathrm{p}<0,001)$, LOS (length of stay) (24.46 vs. $16.36 ; \mathrm{p}<0,001)$, AGI + patients who needed ICU admission were more numerous then AGI-group (46.7 vs. $12.52 \%$; $\mathrm{p}<0,001$ ), associated injuries were more frequent in AGI + group then in AGI- group (97.9 vs. $21.9 \%$; p < 0,001). In AGI + group mortality was higher then AGI-group but without statistic significance (8 vs. $11.8 \%)$.

Conclusion: AGI is a rare injury, most frequently observed in motor vehicle crash; the right adrenal gland is most frequently involved. It is associated with high morbidity, increased total and ICU LOS, due to associated injuries; rarely it requires surgical or radiological hemostasis. The real meaning of AGI seems to be as a marker of major trauma.

References: 1. Trans Pathol Soc Lond. 1863;14:257. 2. J Trauma. 1993;35:870-874. 3. 3. South Med J. 1994;87: 1269-1271. 4. J Natl Med Assoc. 1995;87:569-571. 5. Urology. 2007; 70:851-855.

Disclosure: No significant relationships.

\section{P173}

Moved to Oral Presentations

\section{$\mathbf{P 1 7 4}$}

\section{EFFECTS OF LARGE VERSUS SMALL VOLUME RESUSCITATION WITH ISOTONIC CRYSTALLOID IN BLUNT TRAUMA PATIENTS}

\section{K. Maekawa, S. Uemura, K. Tanno, K. Mori, Y. Asai}

Trauma \& Critical Care, Sapporo Medical University, Sapporo, Japan

Introduction: Aggressive fluid resuscitation has been the standard of care in bleeding trauma patients. However, animal studies suggested that this approach could produce dilutional coagulopathy and problematic inflammation, increasing the risk of poor outcomes. The objective was to evaluate the effects of small volume resuscitation (SVR) versus large volume resuscitation (LVR) in blunt trauma patients.

Materials and methods: We conducted an observational cohort study over 2 years. Adult blunt trauma patients were eligible for inclusion if they presented directly from the scene to our tertiary care center and survived $24 \mathrm{~h}$ or longer. The fluid resuscitation strategy was decided by attending physicians. We defined LVR as more than $2 \mathrm{~L}$ of isotonic crystalloid administration during bleeding. We used propensity-score matching to adjust for differences between both groups. Outcome measures were hospital mortality, morbidity and length of ICU/hospital stay.

Results: Of 116 eligible patients, 66 were enrolled in the SVR group and 50 were enrolled in the LVR group. The propensity-score matching process selected 21 patients each from both groups. SVR and LVR-matched group had similar clinical profiles except for average volume of crystalloid administration $(1.4$ vs. $2.8 \mathrm{~L}$; $\mathrm{p}<0.0001)$. We did not detect any significant differences between SVR and LVR-matched groups in hospital mortality (14.3 vs. $14.3 \%$; $\mathrm{p}=1.0)$, or occurrence of ARDS (0\% vs. $4.8 \% ; \mathrm{p}=0.99)$ and AKI ( 0 vs. $4.8 \% ; p=0.99)$, length of ICU stay ( 2.5 vs. 6.2 days; $\mathrm{p}=0.14$ ) and hospital stay (12.6 vs. 19.9 days; $\mathrm{p}=0.19$ ).

Conclusion: Large volume resuscitation with about $2.8 \mathrm{~L}$ of isotonic crystalloid was not necessarily associated with poor outcomes. Disclosure: No significant relationships.

\section{P175}

\section{EVALUATION OF PARASPINAL LINE WIDENING ON CHEST X-RAY FILMS FOR IMMEDIATE DIAGNOSIS OF BLUNT THORACIC AORTIC INJURY}

\author{
M. Ueno ${ }^{1}$, N. Kaneko ${ }^{2}$
}

${ }^{1}$ Department of Emergency And Critical Care Medicine, Tokyo Medical University, Shinjuku-ku, Japan, ${ }^{2}$ Department of Emergency And Critical Care Medicine, Tokyo Medical University, Shinjuku, Japan

Introduction: Blunt thoracic aortic injury (BTAI) is associated with a very high mortality rate, and it must be immediately diagnosed on initial management of trauma patients to save their lives. Chest X-ray (CX) is routinely used to enable rapid access to thoracic injuries. Widening of the left paraspinal line (PSL) on CX films has been described as an important but not a chief finding for screening BTAI. However, the significance of PSL widening has not yet been elucidated. The purpose of this study was to determine how PSL appears on CX films in patients without thoracic trauma, and to perform statistical evaluation.

Materials and methods: This was a retrospective study. During the previous 9 months (January-September 2010) both CX and computed tomography (CT) were performed in 110 patients (64 men, 46 women, average age 60.8 years) without thoracic trauma who had been transferred to our emergency department. PSL width was measured on CX films and, in cases of PSL widening, possible causes of those findings were investigated on CT scans. A PSL width (from the left edge of the spine to the line) of over $15 \mathrm{~mm}$ and a non-visible PSL were defined as positive for BTAI.

Results: A total of 31 patients were classified as positive, that is, pseudo-positive for a diagnosis of BTAI. Factors for pseudo-positive findings included a tortuous aorta, arterial calcification, pleural effusion, lung atelectasis, a large thoracic duct, and vertebral fracture. 
Conclusion: The specificity of PSL widening on CX films for a diagnosis of BTAI was $71.8 \%$ in our study. If patients with suspected BTAI are elderly, we may expect the pseudo-positive rate to increase. Disclosure: No significant relationships.

\section{P176}

\section{HAEMOSTATIC AGENTS AND TRAUMATIC LIVER INJURY}

\section{M.J.Mullan ${ }^{1}$, M. Midwinter ${ }^{2}$}

${ }^{1}$ General Surgery, Altnagelvin Area Hospital, Londonderry, United Kingdom, ${ }^{2}$ Royal Centre for Defence Medicine, Birmingham, United Kingdom

Introduction: Haemostatic agents have been the focus of much interest in recent times and have successfully been used in liver trauma patients. The aim of this research was to identify which haemostatic agents may be of use to a surgeon operating on a coagulopathic patient with a severe liver injury without access to angioembolisation.

Materials and methods: An electronic search identified current haemostatic agents. Inclusion and exclusion criteria were drawn up. The agents identified were then searched using MeSH terms on the Pubmed, Cochrane, HONNI and Embase databases. In addition, several eminent surgeons were contacted via email to enquire about 'grey literature'. Returned evidence was appraised with the help of appraisal tools developed by the Critical Appraisal Skills Programme. Results: 96 potential studies for inclusion in the review were identified. When inclusion and exclusion criteria were applied, only 34 studies remained -18 case series or papers relating to guidelines, 7 cohort studies and 9 randomised control trials. Of the ten eminent surgeons contacted, seven replied-five reported on the use of haemostatic agents in patients with liver trauma, no further 'grey literature' studies were identified. Only limited literature relating to emergency surgery and human patients exists.

Conclusion: Much of the published literature concerning haemostatic agents relates to animal based research. Some evidence does exist to support the use of recombinant factor VIIa in such circumstances were a surgeon has to operate on a patient with a severe liver injury. However, current haemostatic agents are not a substitute for surgical technique.

Disclosure: No significant relationships.

\section{$\mathbf{P 1 7 7}$}

\section{HOW WELL DO OUR SURGICAL TRAINEES RECOGNIZE SHOCK IN TRAUMA PATIENTS?}

\section{T.L. Zakrison, N. Ahmed}

Division of General Surgery, St. Michael's Hospital, Toronto, ON, Canada

Introduction: The early recognition of shock in trauma patients is important for expeditious management and improved outcomes. This depends on the recognition of shock by trauma attendings and also by more junior surgical trainees [1]. It is unclear how comfortable or competent these trainees feel about recognizing shock. Our objective was to test how well surgical trainees are able to identify and classify shock in trauma patients at our Level I trauma centre.
Materials and methods: We performed an intervention-based, pilot study of surgical trainees of various disciplines and levels of training. Each participant completed a multiple choice exam with questions exploring the recognition of state and class of shock. Participants were then asked about their comfort in identifying the patient in shock and extent of teaching about shock. The intervention included a didactic and practical module to identify patients in shock using a brief simulated scenario. Trainees were then tested again post-intervention. Pre and post-intervention scores were compared using the paired student's $t$ test.

Results: Ten surgical trainees, from the medical student to 4th year resident level were included for study. Pre-intervention scores for the accurate recognition of shock were low, averaging only $31 \%$. All but one trainee scored $<50 \%$ correct responses. This is in contrast with our post-intervention average score of $70 \%(\mathrm{p}<0.05)$. Most trainees felt that they lacked comfort in recognizing shock and that teaching about shock was inadequate during their training. All trainees felt that the use of a "shock card" for identification would be beneficial.

Conclusion: Shock in the trauma patient is a very poorly recognized phenomenon by surgical trainees of all levels but well recognized after basic teaching. Formal education about shock in trauma patients may be lacking. Long term recognition of shock with or without a "shock card" needs to be explored prospectively.

Reference: 1. Diprose P 1993.

Disclosure: No significant relationships.

P178

\section{INCIDENCE AND DIAGNOSIS OF HEPARIN-INDUCED THROMBOCYTOPENIA (HIT) IN PATIENTS WITH TRAUMATIC INJURIES TREATED WITH UNFRACTIONED OR LOW-MOLECULAR-WEIGHT HEPARIN: A LITERATURE REVIEW}

A. Bloemen $^{1}$, M. Testroote ${ }^{2}$, M.L. Janssen-Heijnen ${ }^{1}$, H.M.J. Janzing ${ }^{2}$

${ }^{1}$ General Surgery, VieCuri Medical Centre, Venlo, Netherlands,

${ }^{2}$ Surgery, VieCuri Medical Centre, Venlo, Netherlands

Introduction: The incidence of thromboembolic complications after major traumatic injuries is high $(>50 \%)$. Thromboprophylaxis, often by low-molecular-weight heparin (LMWH) is routinely administered in major trauma patients and in immobilization for isolated lower leg injuries. Heparin induced thrombocytopenia (HIT) is a rare, potentially fatal immune mediated complication of treatment with LMWH. In the general medical and surgical population the incidence of HIT is $0.2 \%$. Little is known about the incidence of HIT and value of screening in trauma patients and in isolated lower extremity injuries. Therefore, we performed a systematic literature review.

Materials and methods: Medline and EMBASE were searched independently by two authors. Manuscripts were selected for analysis by quantitative and qualitative selection. After eliminating duplicate articles and irrelevant studies, seven relevant papers reporting on the incidence of HIT in trauma patients were identified. No studies specifically reporting on the incidence of HIT in patients with lower leg injuries were found.

Results: The papers varied in study design: three randomized-controlled-trials, three cohort studies and one case-report. Methodological quality also varied. In a total population of 1,920 hospitalized trauma patients, HIT was identified in seven patients $(0.36 \%)$. Pooling of data 
was impossible due to heterogeneity in study design and populations. Clinical guidelines for prevention of HIT of the American College of Chest Physicians advise monitoring of platelet count for early detection of HIT every 2-3 days in patients with an estimated risk of $0.1-1 \%$ for HIT.

Conclusion: Few studies have reported on the incidence of HIT in trauma patients who receive prophylactic LMWH. The incidence of HIT in these patients appears to be low $(0.36 \%)$ and comparable to other patient groups. There is no literature on the incidence of HIT in patients with isolated lower leg injuries receiving LMWH. Regular monitoring of platelet count every $2-3$ days in hospitalized trauma patients can be considered.

Disclosure: No significant relationships.

\section{P179}

\section{MANAGEMENT AND OUTCOME OF 308 CASES OF LIVER TRAUMA IN BOLOGNA TRAUMA CENTER IN 10 YEARS}

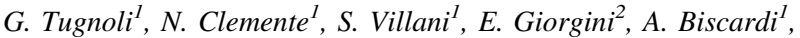

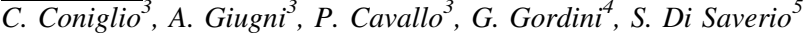

${ }^{1}$ Emergency And Trauma Surgery Unit, Maggiore Hospital Trauma Center, Bologna Local Health District, Bologna, Italy, ${ }^{2}$ Emergency And Trauma Surgery Unit (head Dr. G. Tugnoli), Maggiore Hospital Trauma Center, Bologna Local Health District, Bologna, Italy, ${ }^{3}$ Trauma Icu, Department of Emergency, Maggiore Hospital Trauma Center, Bologna Local Health District, Bologna, Italy, ${ }^{4}$ Trauma Icu, Department of Emergency (head Dr. G. Gordini), Maggiore Hospital Trauma Center, Bologna Local Health District, Bologna, Italy,

${ }^{5}$ Surgery, Emergency and Trauma Surgery Unit, Maggiore Hospital Trauma Center, Bologna, Italy

Introduction: Management of Liver Trauma may vary widely from $\mathrm{NOM} \pm$ angioembolization to Damage Control Surgery. Multidisciplinary management is essential for achieving better outcomes.

Materials and methods: All patients were initially assessed according to ATLS guidelines. In case of haemodynamic instability and FAST evidence of intra-abdominal free fluid, the patients underwent immediate laparotomy. Hemodynamically stable patients, underwent CT scan and admitted in ICU for NOM. During 2000-2009 period 308 patients with liver injury were admitted to level 1 trauma center and recorded in Trauma Registry. Collected data are demographics, AAST grade, initial treatment (operative or non-operative treatment) and outcome (failure of NOM), death.

Results: 94 patients (30.5\%) underwent immediate surgery: 60 patients $(63.8 \%)$ were hemodynamically unstable. In the remaining 34 patients the indications for immediate surgery were clinical peritonitis or CT findings of abdominal organ injury requiring surgical repair. The other 214 patients $(69.5 \%)$ were initially managed with NOM. In 185 patients this was successful. Within the other 29 patients, failure of NOM was due to liver-related causes in 12 patients and non-liver-related causes in 17. Greater the grade of liver injury, fewer patients could be enrolled for NOM $(85.8 \%$ in I-II and $83.3 \%$ in III against $39.8 \%$ in IV-V). Of those initially treated non-operatively, the likelihood of failure was greater in more severely injured patients (24.4\% liver-related failure rate in IV-V against the 1.3 and $1.0 \%$ in I-II and III, respectively).

Conclusion: $\mathrm{NOM} \pm$ angioembolization is safe and effective in any grade of liver injury provided hemodynamic stability. DCS is Gold Standard for hemodynamically unstable patients.

Disclosure: No significant relationships.

\section{P180}

\section{OPTIMAL TREATMENT OF RETAINED HEMOTHORAX IN TRAUMA PATIENTS}

J. van Dijk, M. Bemelman, L.P.H. Leenen

\section{Traumatology, UMC Utrecht, Utrecht, Netherlands}

Introduction: We investigated the best treatment for patients with traumatic retained hemothorax in the past 9 years. Treatment options reviewed are: secondary tube thoracostomy, Video assisted thoracoscopy (VATS), thoracotomy, use of antibiotics or intrapleural streptokinase.

Materials and methods: A structured search in the prospective electronic hospital database was performed using codes for pneumothorax and hemothorax. All patients older than 16 years without former thoracic surgery and discharged between January 2001 and December 2009 were reviewed. Patients with hemothorax were compared to patients with retained hemothorax to find options to prevent a retained hemothorax and outcomes of patients with retained hemothorax were compared between treatment options to find the best treatment option.

Results: 137 patients had a hemothorax; 22 patients developed a retained hemothorax. Even though the retained group had a lower ISS 19 versus 25 ( $\mathrm{p}=0,036)$, they had a significant eventful course. In the retained group: the number of drains used was: 3 versus 1, $\mathrm{p}=0.000$. Presence of empyema: 9 versus $0, \mathrm{p}=0.000$. Median hospital stay: 39 versus 14 days, $\mathrm{p}=0.000$. Mortality 1 versus 31 patient, $\mathrm{p}=0,026$. Intrapleural streptokinase $(\mathrm{n}=2)$ had the lowest mean hospital stay 15.5 days vs. tube thoracostomy 42 days, thoracotomy 31 days and for VATS 43 days. All streptokinase interventions were successful without re-intervention in contrast to the other interventions: $2 / 2$ versus $8 / 9,0 / 2,3 / 9$, respectively.

Conclusion: Retained hemothorax is a serious complication with a significant morbidity and mortality rate. It deserves an adequate therapy, of all therapy modalities intrapleural streptokinase seems to be the most effective treatment with non invasive advantages.

Disclosure: No significant relationships.

\section{P181}

\section{PACKING FOR DAMAGE CONTROL OF NONTRAUMATIC INTRA-ABDOMINAL MASSIVE HEMORRHAGES}
S. Di Saverio ${ }^{1}$, E. Giorgini ${ }^{2}$, F. Filicori ${ }^{3}$, S. Villani $^{3}$, A. Biscardi $^{2}$, G. Tugnoli ${ }^{2}$

${ }^{1}$ Surgery, Emergency and Trauma Surgery Unit, Maggiore Hospital Trauma Center, Bologna, Italy, ${ }^{2}$ Emergency And Trauma Surgery Unit (head Dr. G. Tugnoli), Maggiore Hospital Trauma Center, Bologna Local Health District, Bologna, Italy, ${ }^{3}$ Emergency And Trauma Surgery Unit, Maggiore Hospital Trauma Center, Bologna Local Health District, Bologna, Italy

Introduction: Damage Control Surgery (DCS) for massive intraperitoneal hemorrhage has been shown to significantly reduce the morbidity and mortality in severely injured trauma patients. We applied the principles derived from the traditional traumatic DCS to patients who developed a massive hemorrhage and the "lethal triad" (acidosis, hypothermia, coagulopathy) during a surgical procedure in order to assess feasibility and efficacy of DCS, evaluate the prognostic factors versus mortality prediction scores and compare the data of trauma patients from our trauma registry. 
Materials and methods: We carried out a retrospective analysis of eight consecutive non-traumatic patients with massive intra-operative hemorrhage. Laboratory parameters, fluids requirements, operative times, APACHE II score were analysed, surgical procedure, angioembolization, morbidity, mortality and need for repacking. We compared the results with trauma patients, requiring peri-hepatic packing $(n=35)$ or pelvic packing $(n=9)$.

Results: Average APACHE II score of non traumatic DCS patients was 25.5 (predicted mortality rate $54 \%$ ). Mean ISS in traumatic groups was $36.7 \pm 13.8$ for Hepatic trauma patients and $38.4 \pm 11.2$ for Pelvic Trauma patients. Overall and early mortality in non-traumatic group were $0 \%$, whilst for peri-hepatic Trauma patients was, respectively, 54.3 and $37 \%$. Overall and early mortality for pelvic Trauma patients was 33.4 and $11.1 \%$. Intra-abdominal septic (packing-related) complication rate was $12.5 \%$ for non traumatic patients, $26.1 \%$ for hepatic trauma patients and $33.4 \%$ in pelvic trauma patients.

Conclusion: Intra-abdominal packing was shown to be feasible, safe, and effective for patients with intra-abdominal nontraumatic massive hemorrhage; the application of the principles of DCS may improve survival in cases of surgical hemorrhage with development of lethal triad.

Disclosure: No significant relationships.

\section{P182}

\section{PROGNOSTIC FACTORS FOR TREATMENT OF SPLEEN TRAUMA: NIGUARDA EXPERIENCE}

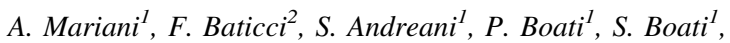
S. Cimbanassi ${ }^{3}$ O. Chiara $^{4}$

${ }^{1}$ Trauma Team, Ospedale Niguarda Ca' Granda, Milano, Italy, ${ }^{2}$ Chirurgia Generale E Mininvasiva, Istituto Clinico Humanitas, Rozzano, Italy, ${ }^{3}$ Emergency, Ospedale Niguarda Ca'granda, Milano, Italy, ${ }^{4}$ emergency, Ospedale Niguarda $\mathrm{Ca}$ ' Granda, Milano, Italy

Introduction: to review our experience with spleen injuries and their treatment.

Materials and methods: we conducted a retrospective analysis of 2,834 trauma patients observed and treated by the Trauma Team of Niguarda Hospital of Milan from October 2002 to June 2010. All patients were studied according to a standard protocol and for each patient we calculated demographic data, Injury Severity Score (ISS), Abbreviated Injury Scale AIS for the spleen injury and associated injuries and were correlated to treatment (splenectomy vs. non operative treatment-NOM).

Results: Of 2,834 trauma patients admitted in the emergency room from October 2002 to June 2010. One hundred and eighty five patients had a spleen injury. Splenectomy was performed 74 patients, a repair of the spleen in 31 patients, NOM was performed in 74 patients and 6 patients was treated with embolization. Groups were compared for age, AIS of the spleen injury, number of organs involved in trauma. Significant differences were found for AIS (NOM vs. operative management $2.75 \pm 0.9$ vs. $3.75 \pm 0.9, p<0.05)$, for organs involved (operative treatment with only spleen injury versus multiorgan involvement 57 versus $61.4 \%, \mathrm{p}<0.04$ ), for ISS (NOM versus operative management $29.6 \pm 0.9$ vs. $37.7 \pm 0.9, \mathrm{p}<0.03)$. No significant evidence was found for age (NOM vs. operative management $32.9 \pm 15.7$ vs. $35.65 \pm 17.3)$.

Conclusion: the mean prognostic factor of the treatment of spleen injury were AIS, ISS and the number of organ involved in trauma are predictor, while age seem to be less important. In stable patients with evidence of free fluid at the E-Fast, CT scan has to be performed for optimal treatment.

References:1. J Surg. 2010;97(11):1696. 2. ANZ J Surg. 2010;80(3): 157.3. 3. J Intensive Care Med. 2006;21:296.

Disclosure: No significant relationships.

\section{P183}

\section{REDUCING ALLOGENIC BLOOD DEMAND PERFORMING POSTOPERATIVE TRANSFUSION OF UNWASHED FILTERED SHED BLOOD IN HEMI-HIP ARTHROPLASTY AFTER TRAUMATIC FEMORAL NECK FRACTURE}

\author{
J. Köttstorfer, R. Schmidt, W. Machold, V. Vecsei
}

Trauma Surgery, General hospital Vienna, Wien, Austria

Introduction: Transfusion of allogenic blood in trauma surgery cause high costs and expose the patient to numerous risks. We performed a prospective randomized trial evaluating the impact of transfusing postoperatively unwashed shed blood on allogenic blood demand. The Bellovac ABT devise was used to salvage drained blood within the first $6 \mathrm{~h}$ after operation. We aimed to assess the changes this system had on patient's allogenic blood requirement, problems in wound healing and duration of hospital stay.

Materials and methods: 162 patients suffering from acute traumatic femoral neck fracture were treated with hip-hemi prosthesis. Pre-operative blood donation was not available for our patients due to the unexpected traumatic circumstances; intraoperative cell salvage was not performed. The patients were randomized to one of the two following groups: 89 patients using standard suction drainage and 73 patients using autologous drains where shed blood was collected within $6 \mathrm{~h}$ postoperatively; A maximum of $500 \mathrm{ml}$ of salvaged blood was reinfused.

Results: The two groups were similar in all aspects. $197.24 \mathrm{ml}$ of autologous shed blood was transfused in 30 patients who were treated with the blood salvage device. Within the study population $43.84 \%$ of the patients received banked blood ( 0.96 units on average). $48.31 \%$ of patients treated with standard Redon drain needed one to four allogenic blood units (1.11 on average).

Conclusion: Evidence showing that retransfusion of shed blood is able to reduce the number of allogenic blood units needed after hemi hip arthroplasty could not be found. However, patients with high postoperative blood loss received significantly more blood units emphasising the importance of intraoperative haemostasis. Taken together standard use of a retransfusion device can not be recommended, but it seems to be effective in patients with high postoperative blood loss.

Disclosure: No significant relationships.

\section{P184}

\section{RUPTURED HEPATIC HEMATOMA IN HELLP SYNDROME CONTEXT-A DIFFERENT APPROACH}

M.S. Ferreira ${ }^{1}$, L.F. Silveira ${ }^{2}$

${ }^{1}$ Cirurgia, Centro Hospitalar Cova da Beira, Covilhã, Portugal, ${ }^{2}$ Cirurgia, Hospital Amato Lusitano, Castelo Branco, Portugal

Introduction: Liver rupture as a complication of HELLP syndrome is rare $(1.8 \%)$ and life-threatening, usually manifesting as an acute 
abdomen and hypotension. In addition to correcting hemodynamic and coagulation disorders, several surgical approaches are described such as perihepatic packing, hepatic lobectomy, ligation or embolization of hepatic artery, and liver transplantation. We report a case in which bleeding control is achieved through intermittent hepatic artery ligation with external control.

Materials and methods: A 37-year-old multiparous woman (G2P1) with a 28 weeks gestation was admitted to the emergency room due to epigastric pain. HELLP syndrome was diagnosed and urgent caesarean section performed. In the intensive care unit (ICU), abdominal pain and hypovolemic shock developed. The abdominal ultrasound revealed hemoperitoneum. An exploratory laparotomy was performed and an extensive ruptured liver hematoma detected. Bleeding was controlled by an intermittent clamping system of the hepatic artery with extra-abdominal control and coagulation factors were administered.

Results: The patient was discharged following 22 days of hospitalization (13 days in ICU). A 6 month follow-up revealed no complications.

Conclusion: Intermittent hepatic artery ligation it's an easily reproducible technique and, when indicated, may be an important therapeutic tool. It resolves the bleeding in just one surgery, don't need intervention radiology and preserves the hepatic artery.

References: 1. Ana C.P.F. Araujo et al. Characteristics and treatment of hepatic rupture caused by HELLP syndrome. Am J Obstet Gynecol. 2006;195:129-33. 2. Miguelote, Rui Filipe, et al. Postpartum spontaneous rupture of a liver hematoma associated with preeclampsia and HELLP syndrome. Arch Gynecol Obstet. 2009;279:923-926.

Disclosure: No significant relationships.

\section{MINIMALLY INVASIVE SURGERY IN NON TRAUMATIC EMERGENCIES}

\section{P185}

COMPUTED ANALYSIS FROM DIGITIZED COLOR TONES IN VIDEOIMAGED INTESTINAL SURFACE COULD BE AN ULTIMATELY NONINVASIVE METHOD TO EVALUATE COMPROMISED TISSUE BLOOD FLOW AND OXYGEN SATURATION OF INTESTINE WITH ISCHEMIAREPERFUSION INJURY

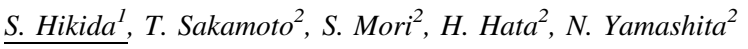

${ }^{1}$ Life Saving Emergency \& Critical Care, Kurume University, Hikida Clinic, Kurume City, Japan, ${ }^{2}$ Life Saving Emergency \& Critical Care, Kurume University Hospital, Kurume City, Japan

Introduction: We aimed to investigate whether the numerical color tones could reflect the amount of tissue blood flow or oxygen saturation in the intestine with compromised blood supply.

Materials and methods: In 12 dogs, the intestinal segment $(20 \mathrm{~cm})$ with $4 \mathrm{~h}$ of ischemia and subsequent $2 \mathrm{~h}$ of reperfusion stress was created by clamping and declamping the marginal vessels and segmental vascular arcade. The intestinal segment and the gauze as an indicator of white balance in the operating field were videotaped under stable brightness every 20 min during the reperfusion period.
Simultaneously, the intestinal tissue blood flow (TBF) and the oxygen saturation $\left(\mathrm{SaO}_{2}\right)$ of the intestinal segment were measured. The three colors of red, green, and blue obtained from video-captured picture were expressed as numerical values and these values were corrected by transforming the color tones of gauze to those of pure white color. Multiple regression analysis was used to compare the corrected values of three colors of the intestinal segment and TBF or $\mathrm{SaO}_{2}$. The linear regression analyses were also used to compare the each color value and $\mathrm{TBF}$ or $\mathrm{SaO}_{2}$.

Results: The TBF or the $\mathrm{SaO}_{2}$ was strictly correlated with corrected values of three color tones. In the three color values, red color tone was the most strictly correlated with $\mathrm{TBF}$ and $\mathrm{SaO}_{2}$.

Conclusion: The results indicate that the color tone value of intestine obtained by non-invasive method could be useful to evaluate intestinal viability as indicated tissue blood flow or oxygen saturation.

Disclosure: No significant relationships.

\section{P186}

\section{CURRENT TRENDS IN THE MANAGEMENT OF ESOPHAGEAL PERFORATION}

T. Sanchez-Rodriguez ${ }^{1}$, C. Camarero $^{2}$, J. martin gil $^{l}$,

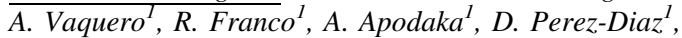
F. Turegano ${ }^{3}$, C. Rey ${ }^{l}$

${ }^{1}$ Surgery, Gregorio Marañon Hospital, Madrid, Spain, ${ }^{2}$ Surgery, Gregorio Marañon Hospital, Madrid, Spain, ${ }^{3}$ General And Emergency Surgery, University Hospital Gregorio Maranon, Madrid, Spain

Introduction: Esophageal perforations are surgical emergencies associated with a high morbidity and mortality rate. Our objective was to review the incidence, etiology of perforation, management, morbidity and mortality of our patient population with an esophageal perforation.

Materials and methods: Observational, descriptive, retrospective analysis of patient with a diagnosis of esophageal perforation treated in our center from 2000 to 2010 . We reviewed demographics, etiology of perforation, diagnosis, location, management, type of surgery, mortality and hospital length of stay.

Results: We report on 24 patients admitted to hospital over that time period. The female/male ratio was of $8 / 16$. The mean age of the patients was of 65 years $( \pm 21) .37 .5 \%$ of patients had associated gastro-esophageal co-morbidities. The causes of perforation were: spontaneous (Boerhaave' syndrome) in $54 \%$, foreign bodies $25 \%$, iatrogenic $16.7 \%$ and caustic $4.2 \%$. Perforation occurred in the cervical esophagus in 3 patients $(12.5 \%)$, in the thorax in $5(20.8 \%)$, and abdominal esophagus in $16(62.5 \%)$. Management of the perforation included primary closure in 7 patients, esophageal exclusion in 1, laparoscopy in 1, and non-surgical therapy in 13 patients (54\%). In $54 \%$ of the patients surgery was performed in the first $24 \mathrm{~h} .19$ CT scans, 7 UGI endoscopies, and 7 esophagograms were done. The mean hospital stay was of 40 days. Five patients died (20\%), two of them treated conservatively, and three of them with surgery performed more than $24 \mathrm{~h}$ after the start of symptoms.

Conclusion: Esophageal perforations are uncommon. In our series non-surgical therapy was done in more than $50 \%$ of cases, in selected patients, with a good final outcome.

Disclosure: No significant relationships. 
P187

Moved to Oral Presentations

\section{P188 \\ EFFICACY OF LAPAROSCOPIC SURGERY FOR STRANGULATED SMALL BOWEL OBSTRUCTION}

\author{
T. Shibusawa ${ }^{I}$, N. Hiroe ${ }^{2}$, M. Murakawa $^{l}$, S. Matsumoto ${ }^{I}$, \\ M. Shimizu ${ }^{I}$, T. Funabiki ${ }^{1}$, T. Egawa ${ }^{I}$, M. Yamazaki ${ }^{1}$, A. Nagashima ${ }^{l}$, \\ M. Kitano ${ }^{l}$
}

${ }^{1}$ Emergency And Critical Care Medicine, Saiseikai Yokohama-shi Tobu Hospital, Yokohama, Japan, ${ }^{2}$ Emergency Surgery, Saiseikai Yokohama-city Tobu Hospital, Yokohama, Japan

Introduction: Strangulated small bowel obstruction is a serious disease causing intestinal necrosis and sepsis. Correct diagnosis of the presence of stragulation and selection of appropriate treatment are required. Recently, efficacy of laparoscopic operations as minimally invasive surgery is reported. The purpose of this study is to consider the efficacy of laparoscopic surgery for strangulated small bowel obstruction.

Materials and methods: 82 patients with small bowel obstruction who operated emergency surgery between April 2007 and September 2010 were selected for this study. Patients who had clear evidence of strangulation, intestinal necrosis, shock, organ failure were performed laparotomy. Laparoscopic operations were performed to the patients who had uncertain evidence of strangulation or had strangulation but didn't have intestinal necrosis.

Results: 59 patients underwent laparotomy and 23 laparoscopic surgery. Among the 23 patients, 12 of them were converted to open surgery ( 5 with small bowel resection, 7 with adhesiolysis only), 2 patients underwent small laparotomy with adhesiolysis (laparoscopic assisted surgery), and in 9 patients laparoscopic operation was completed. Complications of the laparoscopic surgery were small bowel injury (1 patient), massive bleeding (1 patient), and postoperative paralytic ileus (2 patients).

Conclusion: Laparoscopic surgery may be possibility a minimally invasive and safe treatment for strangulated small bowel obstruction. Disclosure: No significant relationships.

\section{P189}

\section{IATROGENIC SPLENIC INJURY: THE BENEFITS OF RADIOFREQUENCY}

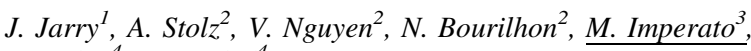 F. Milou ${ }^{4}$ P. Michel ${ }^{4}$}

${ }^{1}$ Surgery, military Hospital Desgenettes, Lyon, France, ${ }^{2}$ Surgery, military hospital Desgenettes, lyon, France, ${ }^{3}$ Chirurgie Viscérale (visceral Surgery), Hôpital d'Instruction des Armées Desgenettes (Military Hospital), LYON CEDEX 03, France, ${ }^{4}$ Military hospital Desgenettes, lyon, France

Introduction: Iatrogenic splenic injury is a well-recognized and potentially serious complication of abdominal surgery. It inevitably results in prolonged operative time, increased blood loss, and extended hospital stay. It is also associated with significant post-operative morbidity and mortality, including the risk of acute, post-splenectomy infection. Splenic salvage is the ultimate goal of iatrogenic splenic injury management, and various surgical techniques have been developed to achieve the hemostasis of the spleen. In animal trauma models, radiofrequency has been reported as a safe method to achieve spleen hemostasis [1]. Herein, we present our experience regarding control of iatrogenic spleen injuries using radiofrequency in human patients.

1. Felekouras E et al. J Trauma. 2004;57(6):1225-9.

Introduction: Iatrogenic splenic injury is a serious complication of abdominal surgery. It inevitably results in prolonged operative time, increased blood loss, extended hospital stay, and is associated with significant morbidity and mortality. Splenic salvage is the ultimate goal of splenic injury management, and various surgical techniques have been developed to achieve the hemostasis.

Materials and methods: Bicentric, retrospective study. From January 2009 to September 2010, all iatrogenic splenic hemorrhages uncontrolled by conventional hemostasis (monopolar or bipolar coagulation, hemostatic meshes) techniques were treated using radiofrequency. Radiofrequency causes coagulative necrosis and desiccation of tissue parenchyma, which can fully obstruct vessels, leading to little or no blood loss from coagulated tissue. The radiofrequency was performed using an internally cooled electrode. The needle tip was continuously perfused with $4^{\circ} \mathrm{C}$ cold water a rate of $100 \mathrm{ml} / \mathrm{min}$ via a channel inside the needle. The needle was straight and was introduced percutaneously into the spleen in order to complete a laparoscopic-controlled hemostasis using radiofrequency.

Materials and methods: Bicentric, retrospective study. From January 2009 to September 2010, all iatrogenic splenic hemorrhages uncontrolled by conventional hemostasis techniques were treated using RF. The RF was performed using a straight needle, which was introduced percutaneously into the spleen.

Results: A total of three patients underwent a splenic hemostasis using RF. They consisted of two men and one woman with a median age of 58 y.o. The splenic injuries occured during a laparoscopic proctectomy in two cases and during open gastrectomy in one case. They were classified as grade III. Complete hemostasis could be achieved in the three patients with a median time of $10 \mathrm{~min}$. No splenectomy was necessary. No conversion was performed. There was no post-operative morbidity and mortality. No recurrent splenic hemorrhage occured during the follow-up period. The financial cost was $350 €$ per RF.

Conclusion: RF is a safe, quick, and effective spleen-preserving technique to stop iatrogenic splenic hemorrhage. One main advantage of the technique is the possibility to treat the laparoscopic iatrogenic injuries of the spleen without conversion and without additional ports. $\mathrm{RF}$ therefore appears to be an ideal device for iatrogenic splenic injuries, and may prove to be an indispensable tool in modern, minimally surgery.

Disclosure: No significant relationships.

\section{P190}

\section{LAPAROSCOPIC APPENDECTOMY WITH LIGASURE IN COMPLICATED AND TECHNICALLY DIFFICULT APPENDICITIS}

\section{G.O. Kucuk}

General Surgery, Samsun Vezirkopru Public Hospital, Samsun, Turkey

Introduction: Laparoscopic appendectomy becomes more difficult when the mesoappendix is bulky and inflamed. Various techniques were described for division of the mesoappendix [1]. In this study laparoscopic appendectomy with Ligasure in complicated and technically difficult appendicitis at a rural hospital was evaluated. 
Materials and methods: This study included patients who underwent laparoscopic appendectomy with Ligasure for acute appendicitis at Vezirkopru Public Hospital between November 2009 and December 2010. The conversion rate, the operative time, the mean hospital stay, the postoperative complications were analyzed.

Results: Laparoscopic appendectomy was performed in 43 patients with Ligasure. 23 of the patients were male and 20 of the patients were female. Four patients were in pediatric age group $(<16$ years). The median age was 27.5 years (range 13-62). The mean operative time was 40.1 min (range 20-75). All operations were performed by the same surgeon. There was no need for conversion to open procedure even if in perforated or technically difficult cases. The postoperative hospital stay ranged from 1 to 5 days with a median of 2.4 days. In this study, perforated appendicitis with typical coexisting complications such as periappendicular inflammatory masses were recognized in 19 patients. In six patients periappendicular abscess drainage was performed intraoperatively. In two female patients, simultaneous laparoscopic removal of the ovarian cysts was performed. There was trocar site infection in two patients. The pathologic examination of the specimen demonstrated carcinoid tumor and appendix diverticula perforation in two patients.

Conclusion: Laparoscopic appendectomy performed by experienced laparoscopic surgeon with Ligasure is a feasible and effective treatment option, independently of the stage or etiology of the appendicitis. In this study, the use of Ligasure in laparoscopic appendectomy at a rural hospital was demonstrated.

Reference: 1. Yauger BJ, Dunlow SG, Lockrow EG. Laparoscopic appendectomy: a series of cases utilizing laparosonic coagulating shears as compared to endo-GIA and endoshears. J Reprod Med. 2005;50:231-234.

Disclosure: No significant relationships.

\section{P191}

\section{LAPAROSCOPIC APPROACH FOR DIFFICULT CHOLECYSTECTOMIES}

I. Lical, M. Lica ${ }^{2}$, D.A. Kinn ${ }^{2}$, C. Stanciu
, A.I. Florea

${ }^{1}$ General Surgery, Clinical Emergency Hospital, Bucharest, Romania, ${ }^{2}$ General Surgery, Clinical Emergency Hospital Bucharest, Bucharest, Romania

Introduction: Laparoscopic cholecystectomy (LC) allows shorter hospital stay, more rapid postoperative rehabilitation and reduced complications and become the "gold standard" for benign gallblader diseases. Nevertheless, conversion to open procedures still occurs in cases of acute or difficult cholecystectomies. Laparoscopic subtotal cholecystectomy (LSC) could be a feasible solution.

Materials and methods: We describe our experience of 54 cases of LSC out of a total of 1,243 LC performed over the last 3 years in our clinic. LSC involved mostly two aspects: difficulty in dissecting the gallblader wall from its bed when we incised the gallblader leaving the posterior wall attached to the liver and cauterizing the mucosa or difficulty in dissecting the gallbladder neck and Calot's triangle when we incised the gallbladder neck, identified the cystic duct from the inside, gradually excised the gallbladder walls and sutured the gallbladder neck. The medical records for those patients were reviewed and analyzed for demographics, diagnosis, operative findings, surgical procedure and postoperative complications and outcomes.
Results: LSC indications were gangrenous gallbladder, empyema or severe inflammatory mass, severe fibrosis or atypical Calot's triangle. The median operative time was approximately $2 \mathrm{~h}$ and only senior, experienced surgeons performed LSC. The median postoperative stay was 6 days. In $20 \%$ of cases complications occurred: bile leak, postoperative subhepatic abscess (requiring relaparoscopy) and portsite superficial infection. No perioperative deaths were recorded.

Conclusion: Laparoscopic subtotal cholecystectomy is a safe and effective treatment for acute or anatomically difficult cholecystitis thus decreasing rate of conversion to open surgery.

References: 1. Ji W, Li LT, Li JS. Role of laparoscopic subtotal cholecystectomy in the treatment of complicated cholecystitis. Hepatobiliary Pancreat Dis Int. 2006;5(4):584-9. 2. Atsushi Horiuchi et al. Delayed laparoscopic subtotal cholecystectomy in acute cholecystitis with severe fibrotic adhesions. Surg Endosc. 2008;22:2720-2723. 3. Philips JAE et al. The use of laparoscopic subtotal cholecystectomy for complicated cholelithiasis. Surg Endosc. 2008;22:1697-1700.

Disclosure: No significant relationships.

\section{P192}

\section{LAPAROSCOPIC MANAGEMENT OF NON-TRAUMATIC HOLLOW VISCUS PERFORATIO: A SERIES OF 100 PATIENTS}

\section{T. Sanchez-Rodriguez ${ }^{1}$, C. Camarero ${ }^{2}$, J. MARTIN-GIL $^{1}$,

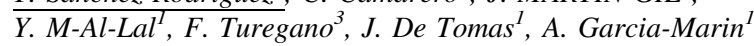

${ }^{1}$ Surgery, Gregorio Marañon Hospital, Madrid, Spain, ${ }^{2}$ surgery, Gregorio Marañon Hospital, Madrid, Spain, ${ }^{3}$ General And Emergency Surgery, University Hospital Gregorio Maranon, Madrid, Spain

Introduction: Perforation of a hollow viscus is an emergency which should prompt a rapid and effective treatment and the laparoscopy appears to be a safe alternative. Our objective was to review the laparoscopic management, complications, and mortality of our patient population with a perforated viscus.

Materials and methods: Observational, descriptive, retrospective analysis of patient with a diagnosis of perforated abdominal viscera in our hospital from 2000 to 2010. Non-traumatic (other than iatrogenic) perforations were excluded from the analysis. We reviewed demographic characteristics, cause of perforation, localization, complications, mortality and hospital length of stay

Results: We report on 588 patients admitted to hospital with a diagnosis of perforation of abdominal viscera over a period of 10 -years. A hundred patients $(17 \%)$ were submitted to emergency laparoscopic surgery, $81 \%$ of them during the last 5 years; 86 patients had a gastroduodenal perforation, 10 small bowel and 4 colorectal. The causes were: gastrointestinal ulcer in $78 \%$, iatrogenic perforation $3 \%$ and malignancy $2 \%$. The most frequently used technique was simple closure $(89 \%)$, and only $6 \%$ of patients required a resection. The most frequent complication was an intra-abdominal fluid collection, followed by suture leaks. The mean hospital stay was of 8.9 days. There was no mortality in the group treated laparoscopically.

Conclusion: Our results add evidence to the feasibility and safety of the laparoscopic management of selected cases of gastrointestinal perforations. A gastroduodenal ulcer perforation is not the only indication for the procedure. Our ELS treated group has increased in the last 5 years, with a low morbidity rate and no mortality.

Disclosure: No significant relationships. 
P193

\section{MASTER OF MINIMALLY INVASIVE SURGERY IN URGENCY AND MODERN TECHNOLOGICAL APPLICATIONS}

\section{Catani}

Dea-emergency Department, Policlinico Umberto I, Rome, Italy

Introduction: The present Master is a postgraduate course dedicated to specialists in general surgery who wish to deepen their knowledge and skills in minimally invasive general emergency surgery. This necessity stems from the evidence that in the last decade the rapid technological progress applied to surgery has played a key role in the development and dissemination of mini invasive surgery that after an initial phase of experimentation, have shown excellent clinical results, justifying an increasingly expansion, even in emergency surgery. And, in fact, it is in the emergency itself, either in abdominal or chest presentation that the minimally invasive surgery is demonstrating to be the best surgical technique, with a diagnostic and operative value at the same time, able to offer those post-operative benefits that are now widely recognized in literature. It must be stressed that the minimally invasive surgery is a specialised surgical action that has to find a specific role only in the hands of a team that has perfected its experience in standard endoscopic surgery procedures and has adequate experience in traditional general surgery.

Materials and methods: The goal of the course stands in providing adequate educational background in minimally invasive surgery, its indications in the urgency and its current limitations, the principles underlying the different surgical procedures, general problems and pitfalls in anesthesiologic management of the patient; to facilitate the acquisition of specific expertise in equipment and technological support, giving to surgeon the mastery of specific equipment; to ensure a broad scientific background with regard to the emergency surgery; to provide the hands-on training, offering the surgeon the opportunity to get their own experience and handicraft.

Results: The professional who has obtained the Master degree will be able to perform the minimally invasive exploration of the abdominal cavity and chest in urgency, the major laparoscopic and thoracoscopic surgery, and will also be able to transfer the knowledge to other colleagues in own workplace.

Conclusion: Advances in minimally invasive surgery are offering us the opportunity to span with this technique in other fields such as the emergency, overwhelming what once were contraindications. The issue is debated and, in this uncertainty, specific institutional educational programs begin to be more and more necessary.

Disclosure: No significant relationships.

P194

Moved to Oral Presentations

\section{P195}

\section{THE ACTUAL CONCEPTS IN DIAGNOSIS AND TREATMENT OF THE ACUTE BILIARY PANCREATITIS}

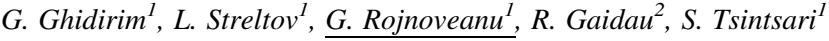 \\ ${ }^{1}$ Surgery No. 1, SMPhU, Chisinau, Moldova, ${ }^{2}$ Interventional \\ Radiology, Municipal Hospital No. 2, Chisinau, Moldova
}

Introduction: A problem for discussion is biliary stones associated with acute pancreatitis.
Materials and methods: Our study includes 217 cases of acute pancreatitis (AP), with biliary origin, which were treated during 2001-2009 years. The diagnosis was established: in virtue of biliary colic in the past and abundant vomiting after taking fatty food, characteristic to AP, the clinical symptoms dependent on variety of lesion of the organs and paraclinical examination: biochemical tests, imagistic examination, ERCP and CT in nine difficult cases.

Results: The forms of the disease were: in 114 cases chronic calculous cholecystitis $(\mathrm{CC})+$ pancreatic edema $(\mathrm{PE})$, predominant in the head and the body of the pancreas, in 69 cases acute $\mathrm{CC}+\mathrm{PE}$, in 6 cases acute $\mathrm{CC}+$ destructive pancreatitis (DP), predominant in the body and tail of the pancreas, in 25 cases chronic $\mathrm{CC}+$ cholelithiasis $+\mathrm{PE}$, in 3 cases acute $\mathrm{CC}+$ cholelithiasis + DP. Jaundice was present in 78 cases. A delay of surgical procedure on biliary lithiasic pathology until the amelioration of AP and the operation has been done from the 4 to 6 th day in acute $\mathrm{CC}$ to the 8 to 12 nd day in chronic CC. In cases with jaundice ERCP has been done in the first 24-48 h and was associated with papilosphincterotomy in 68 cases, later cholecystectomy as described above. In DP the surgical treatment was based on the degree of destruction, has been done " 2 cholecystectomy + drainage of abscessing cyst of the pancreas on term "26th and 43rd days. In the other 7 cases the complex treatment associated with endoscopic papillosphincterotomy resulted in a decrease of pancreatitis and the cholecystectomy has been done on term 34th "42nd days.

Conclusion: Using of modern imagistic techniques increase the accuracy of diagnosis and results of surgical treatment of biliary lithiasis associated with acute pancreatitis. The delay of surgical procedures on biliary lithiasic pathology until the amelioration of AP improved the results of morbidity-seven cases (3.22\%) and mortality-one case $(0.46 \%)$ in these patients.

Disclosure: No significant relationships.

\section{P196}

\section{TWO INCISION LAPAROSCOPIC APPENDICECTOMY AS} A "STEPPING STONE" TO SINGLE INCISION SURGERY

\section{Evans, Y. Haqzad, M. Sinclair}

General Surgery, Ipswich Hospital, Ipswich, United Kingdom

Introduction: Single incision laparoscopic surgery via the "natural scar" of the umbilicus is gaining popularity, mainly driven by the manufacturers and patients' desire for "scarless" surgery. There are some theoretical benefits and many surgeons are keen to develop the technique. As the view and instrument handling is different from traditional laparoscopic surgery it is imperative that there is a safe way to learn. [1].

Materials and methods: We propose a technique of laparoscopic appendicectomy via two incisions (one umbilical incision containing two ports plus one suprapubic incision) as a way of acclimatising surgeons already familiar with standard laparoscopic surgery to the restrictions imposed by single incision surgery without any specialised equipment. All such cases by a single surgeon (LE) were reviewed and compared to standard three incision procedures during the same time period.

Results: During the period 01/09/09 and 31/08/10, 20 two incision and 13 three incision laparoscopic appendectomies were performed. One patient from each group was returned as having normal histology, there were no significant complications. One patient from the two incision group was readmitted due to pain but no abnormality was found on bloods or CT. There was no difference in operating time or median length of stay. 
Conclusion: Two incision laparoscopic appendicectomy is a safe and cost effective way of introducing surgeons to the unique difficulties of single incision surgery.

Reference: 1. Connor S. Single-port-access cholecystectomy: history should not be allowed to repeat. World J Surg. 2009.

Disclosure: No significant relationships.

\section{MINIMALLY INVASIVE SURGERY IN SKELETAL TRAUMA}

\section{P197}

\section{D NAVIGATION OF TRANSILIOSCRAL SCREWS LOWERS RADIATION EXPOSURE FOR THE OPERATION TEAM ONLY}

\section{Behrendt, J. Böhme, M. Mütze, C. Josten}

Traumatology, University of Leipzig, Leipzig, Germany

Introduction: Throughout this study the influence of digital flat panel technology and 3D navigation on the intraoperative radiation exposure for the patient and the operation team as well as the accuracy, and time consumption in the placement of transiliosacral screws was examined.

Materials and methods: A digital mobile X-ray unit (Ziehm Vision FD Vario 3D) with navigation software (Spine \& Trauma Fluoro 3D 1.6, Branlab) was available. 102 cannulated screws were inserted into 15 semipelvic preparations and 9 plastic pelvises not supported by navigation (group I), 2D- (group II) and 3D-navigated (group III).

Results: Radiation exposure to the OP team was significantly lower $(\mathrm{p}<0.0001)$ for group III. Radiation exposure for the patient rose significantly from group I to group III $(\mathrm{p}<0.01)$. Accuracy of $3 \mathrm{D}$ navigated screws was significantly higher $(\mathrm{p}<0.05)$. Time used for navigated screw placement was significantly higher for navigated procedures $(\mathrm{p}<0.01)$.

Conclusion: The lower radiation exposure for the operation team and the higher accuracy represent the main advantages of the flat panel 3D navigation. The higher radiation exposure for the patient is an unfavourable aspect.

Disclosure: The institution of the authors (D.B., J.B., M.M., C.J) has received funding from Brain LAB AG, Feldkirchen, Germany and Ziehm Imaging, Nürnberg, Germany

\section{P198}

\section{ACHILLES TENDON RUPTURE. OPEN PROCEDURE WITH FIBRIN GLUE OR MINIMAL INVASIVE BY PERCUTANEOUS REPAIR?}

\author{
M. Knobe ${ }^{1}$, J. Corsten ${ }^{1}$, R.M. Sellei ${ }^{1}$, P. Kobbe ${ }^{1}$, P. Lichte $^{1}$ \\ R. Pfeifer ${ }^{\prime}$, C. Hoeckle ${ }^{2}$, H. Pape
}

${ }^{1}$ Department of Orthopaedic Trauma, University Hospital of the RWTH Aachen, Aachen, Germany, ${ }^{2}$ Department of Orthopedic Trauma, Marien Hospital Aachen, Aachen, Germany

Introduction: In achilles tendon injuries open surgical repair entails a risk of skin necrosis or infection. Percutaneous methods have lower complication rates, but have a certain amount of risk to compromise the sural nerve. The aim of the present work was to compare the functional long-term outcome of open repair (fibrin glue) versus percutaneous repair.

Materials and methods: 72 patients who had undergone repair with either percutaneous suture (PG, $\mathrm{n}=35,49.2$ years) or open fibrin glue ( $\mathrm{FG}, \mathrm{n}=37,50.7$ years) took part in a follow-up examination (ultrasound, Visual Analog Scale, Thermann + AOFAS score) after an average of 3.7 years (PG) or 5.1 years (FG). Ankle range of motion; thigh, calf and ankle circumferences of the injured leg and the contralateral side, return to work and sports activities time were evaluated. Isokinetic evaluation results were retrieved.

Results: There were no reruptures. Four patients (PG $\mathrm{n}=1(3 \%)$ vs. FG $\mathrm{n}=3(8 \%) ; \mathrm{p}=0.331)$ had superficial wound infections. Lesions of the sural nerve occurred in three patients $(\mathrm{PG} n=2(6 \%) \mathrm{vs}$. FG $\mathrm{n}=1(3 \%) ; \mathrm{p}=0.523)$. The mean American Orthopaedic Foot and Ankle Society score was 92.9 (PG) and 90.4 (FG; p = 0.359), the Thermann Score was $70.7(\mathrm{PG})$ and $71.0(\mathrm{FG} ; \mathrm{p}=0.939)$ and the VAS $8.1(\mathrm{PG})$ versus $8.4(\mathrm{FG} ; \mathrm{p}=0.656)$. Patients returned to work at $9.3(\mathrm{PG})$ and $8.0(\mathrm{FG} ; \mathrm{p}=0.593)$ weeks and to preinjury sportive activities at $16.9(\mathrm{PG})$ and 19.1 (FG; $\mathrm{p}=0.667$ ) weeks. Ankle ROM, ultrasound, circumference and isokinetic measurements did not reveal a significant difference between the two methods. Because of taking fright at possible rerupture 27 patients [PG $\mathrm{N}=18(51 \%)$ vs. FG $9(24 \%) ; \mathrm{p}=0.018]$ changed their sport

Conclusion: These results suggest that minimally invasive and fibrin glue achilles tendon repair provides satisfactory results with early return to previous functional status with low complication rates and without reruptures. Patients after minimal invasive suture changed often their sports activities for fear of rerupture.

Disclosure: No significant relationships.

P199

\section{AN ASSESSMENT OF FUNCTIONAL OUTCOMES AND COMPLICATIONS OF PALMAR VERSUS DORSAL MINIINVASIVE APPROACHES FOR THE SCAPHOID WAIST FRACTURES TREATMENT}

P. Drác $^{1}$, P. Mañák $^{1}$, I. Cizmar $^{2}$

${ }^{1}$ Department of Traumatology, Faculty Hospital Olomouc, Olomouc, Czech Republic, ${ }^{2}$ Traumatology, University Hospital Olomouc, Olomouc, Czech Republic

Introduction: Miniinvasive approaches for the non-displaced or minimally displaced scaphoid waist fractures provide a possibility of early wrist physiotherapy and a shorter sick leave duration. Because of the relatively low complication rate cited in many reports we proceeded this study to compare the complications and the functional outcomes of palmar percutaneous and dorsal limited approaches.

Materials and methods: We compared 51 patients treated by palmar percutaneous osteosynthesis and 58 patients using a limited dorsal approach. Only patients with type A2 and B2 according to Herbert's classification and a minimum follow-up of 12 months were included. We statistically analysed any resting pain, ROM, grip strength, the DASH results and per- and post-operative complications.

Results: The rate of complications was $11.8 \%$ for the percutaneous approach and $12.1 \%$ for the dorsal approach. Most of the observed complications were caused by the surgeon. The differences in the functional outcomes and in the complication rates were statistically insignificant.

Conclusion: We found the two techniques fully comparable based on the statistical analysis of the results of our clinical data. 
References: 1. Adamani DC, Mikola EA, Fraser BJ. Percutaneous fixation of the scaphoid through a dorsal approach: an anatomic study. J Hand Surg. 2008;33A:327-331. 2. Bedi A, Jebson PJL, Jacobson JA, Martus JE. Internal fixation of acute, nondisplaced scaphoid waist fractures via a limited dorsal approach: and assessment of radiographic and functional outcomes. J Hand Surg. 2007;32A: 326-333. 3. Bushnell BD, McWilliams AD, Messer TM. Complications in dorsal percutaneous cannulated screw fixation of nondisplaced scaphoid waist fracture. J Hand Surg. 2007;32A: 827-833.

Disclosure: No significant relationships.

\section{$\mathbf{P 2 0 0}$}

\section{ARTHROSCOPICALLY ASSISTED OSTEOSYNTHESIS OF INTRA-ARTICULAR PROXIMAL TIBIAL FRACTURE}

\section{J. Konecny ${ }^{l}$, M. Reska ${ }^{I}$, J. Ciernik ${ }^{2}$, L. Veverkova ${ }^{1}$}

\author{
${ }^{1} 1$ st Surgical Department, St Ann's Masaryk University Hospital, \\ Brno, Brno, Czech Republic, ${ }^{2}$ Ist Surgical Departmen, St. Ann's \\ University Hospital, Brno, Czech Republic
}

Introduction: Intra-articular proximal tibial fractures are some of the most challenging periarticular injuries to treat. Anatomic restoration of the articular surface with rigid internal fixation, allowing immediate range of motion, is critical to a successful, functional outcome. Arthroscopic techniques possess several advantages over conventional open reduction and internal fixation performed through an arthrotomy. Usually is arthroscopically assisted osteosynthesis recommended for fractures classified $\mathrm{B}$ according to the $\mathrm{AO}$ system.

Materials and methods: In the period from 2005 to 2010, 54 patients with proximal tibial fractures were operated in our department. Fractures AO 42 B1-C3 were diagnosed by CT before operation. In all these cases we used arthroscopically assisted osteosynthesis. Minimally invasive fixation with cancellous screws was used in 9, buttress plate in 24 and LCP in 21 patients. Spongioplasty was carried out in 27 patients and 11 meniscal ruptures were repaired.

Results: 52 patients were healed without complication. Complication was in 2 cases of B type fractures: 1 failure of osteosynthesis and 1 flebothrombosis.

Conclusion: Results and number of complication of arthroscopically assisted osteosynthesis of intra-articular fractures of proximal tibia classified according to the $\mathrm{AO}$ system $\mathrm{B}$ and $\mathrm{C}$ are comparable. That is why arthroscopic techniques could be used for both groups of fractures.

Reference: 1. Mark D. Miller, Brian J. Cole et al. Textbook of Arthroscopy. 2004, Elsevier, Philadelphia.

Disclosure: No significant relationships.

\section{P201}

\section{ATROPHIE OF THE INFRASPINATUS MUSCLE IN TOP LEVEL BEACH VOLLEYBALL PLAYERS}

\section{G. Oberleitner ${ }^{1}$, K. Pieber ${ }^{2}$, T. Paternostro ${ }^{3}$, C. Fialka ${ }^{4}$}

${ }^{1}$ Department of Trauma Surgery, Medical University of Vienna, Vienna, Austria, ${ }^{2}$ Physical Medicine and Rehabilitation, Medical University of Vienna, Vienna, Austria, ${ }^{3}$ Physical Medicin And
Rehabilitation, Medical University of Vienna, Vienna, Austria, ${ }^{4}$ Department of Traumatology, Medical University of Vienna, Vienna, Austria

Introduction: Koppel and Thomson have been the first who described suprascapular nerve entrapment in 1959. One of the most associated clinical findings are the atrophy of the infraspinatus muscle and pain. It is a frequently observed injury in volleyball players.

Materials and methods: We investigated 27 top level beach volleyball players from Austria-12 female players and 15 male players. All of them had been playing at this time at a competitive level. To all of them a Constant score and a SF 36 has been performed. 18 of them, 11 men and 7 women, between 17 and 24 years underwent an electrophysiological examination.

Results: Out of the 27 players we found 4 with atrophy of the infraspinatus muscle-three male and one female. From the 18 players who underwent the electrophysiological examination one player showed chronic neurogenic alterations in the infraspinatus muscle but without relevant side to side differences regarding NCV and without pain and atrophy. EMG in all other players was without pathological findings. In NCV, side to side differences in the compound motor action potential amplitude was $49 \%$ in one player and between 20 and $30 \%$ in three other players. Five players reported pain between five and nine regarding VAS for pain. Three players presented an atrophy of the suprascapularis innervated muscles.

Conclusion: In 17 of 18 players no lesion of the suprascapular nerve was found. Electrophysiological examination indicated a neurogenic lesion in one player without clinical correlation.

References: 1. Ferretti A, Cerullo G, Russo G. Suprascapular neuropathy in volleyball players. J Bone Joint Surg Am. 2007;69A:260-3. 2. Holzgraefe M, Kukowski B, Eggert S. Prevalence of latent and manifest suprascapular neuropathy in highperformance volleyball players. Br J Sports Med. 1994;28:177-9. Disclosure: No significant relationships.

\section{P202}

\section{CLINICAL COMPARISON OF LOCKING PLATES AND ANGULAR PLATES FOR TREATMENT OF EXTRAARTICULAR DISTAL FEMUR FRACTURES}

\author{
N. Ozkayin ${ }^{1}$, G. Okcu ${ }^{2}, K$. Aktuglu ${ }^{3}$
}

${ }^{1}$ Orthopedics And Traumatology, Ege University, İzmir, Turkey, ${ }^{2}$ Orthopedics And Traumatology, Celal Bayar, Manisa, Turkey, ${ }^{3}$ Orthopedİcs And Traumatology, Ege University, Izmir, Turkey

Introduction: Purpose of our study was to compare two different implant types in terms of advantages in the treatment of extraarticular distal femur fractures.

Materials and methods: From January 2000 to January 2006, 51 extremities of a total number of 47 patients were included in this study. First group included 27 extremities of 25 patients treated with angular plate 2 of whom suffered from bilateral fracture. All fractures were classified according to AO classification and 6 were 33-A1 (22.2\%), 12 were $33-\mathrm{A} 2(44.5 \%)$ and 9 were $33-\mathrm{A} 3$ (33.3\%) type fractures. Second group included 24 extremities of 22 patients treated with locking plates 2 of whom suffered from bilateral femur fracture. All fractures were classified according $\mathrm{AO}$ classification and 5 were $33-\mathrm{A} 1(21 \%), 11$ were $33-\mathrm{A} 2(46 \%)$ and 8 were $33-\mathrm{A} 3(33 \%)$ type fractures.

Results: Average follow-up period was 45.2 months (range 22-96). AP radiographic views displayed an average angulation of $4(2-6)^{\circ}$ for $4(14.8 \%)$ patients in the first group among which $1(3.7 \%)$ had 
varus, and $3(11.1 \%)$ had valgus deformity. An average angulation of six $(4-10)^{\circ}$ was identified for four $(17 \%)$ patients in the second group among which two $(8 \%)$ had varus, and two $(8 \%)$ had valgus deformity. In the lateral views, the first group showed an anterior angulation of $5-10^{\circ}$ for six $(22.2 \%)$ cases whereas the second group showed an anterior angulation of $5-10^{\circ}$ for $4(17 \%)$ cases, and an anterior angulation of $10-15^{\circ}$ for eight $(33 \%)$ cases.

Conclusion: Locking plating is reported to be more advantageous with easier application. We believe, however, that this superiority is more important in the intraarticular fractures of the distal femur, and angular plating provides more stable fixation for the protection of distal bone reserve in extraarticular distal femur fractures.

Disclosure: No significant relationships.

\section{P203}

\section{EXTERNAL FIXATION AS TEMPORARILY OR DEFINITIVE METHOD IN SKELETAL TRAUMA}

\section{M.B. Mitkovic, P.M. Stojiljkovic, S. Karalejic, M.M. Mitkovic}

Trauma, University Orthopaedic and Trauma clinic, Nis, Serbia

Introduction: External fixation has already became on the end of last century as routine temporarily method of fracture bone fixation, especially in the light of damage control. External bone fixation has also been accepted in many developed countries as routine temporarily method in treatment of complex articular fractures (knee, ankle, elbow). Sometimes, the skin problem can prolong such fixation for three or more weeks. However, external fixation of tibia and distal radius can be used as method of choose for definitive treatment not only in open but in closed fractures as well. It becomes justified when high mobile and relatively simple external fixation devices have been developed allowing addition gradual, noninvasive correction of reduction. In this paper, we present possibility of using of already applied external fixation device as accurate reduction device.

Materials and methods: One week after external fixation by Mitkovic external fixation device, external fixator was used as accurate reduction device. Once, desirable fracture reduction achieved, internal fixation is very easy and we do not need fluoroscopy control for reduction, just for internal device fixation by minimally invasive method. Using this method, we already treated 15 patients with femur fractures. Method of internal fixation was Selfdynamisable Internal Fixator (SIF).

Results: Operation of transforming external into internal fixation was short-average operation time was $37 \mathrm{~min}$ (23-58). Mean fluoroscopy time was $6 \mathrm{~s}(2-18)$. Intraoperative blood loose was $80 \mathrm{ml}$ (40-200).

Conclusion: From results obtained it can be concluded that Mitkovic external fixator is suitable for routine fractures fixation but also to be used as accurate reduction device useful during the transformation of external to internal fixation.

References: 1. Mitkovic $\mathrm{M}$ et al. Newconcept in external fixation. Acta Chir Iugosl. 2005;52(2):107-11. 2. Mitkovic M et al. Reconstructive procedures on lower extremities using Mitkovic external fixation system. Acta Chir Iugosl. 2005;52(2):117-9.

Disclosure: No significant relationships.

\section{P204}

\section{INTRAMEDULLARY NAILING (ESIN) FOR PAEDIATRIC CLAVICLE FRACTURES: INDICATIONS, PROBLEMS AND PITFALLS}

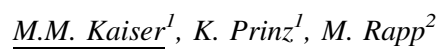

${ }^{1}$ Paediatric Surgery, University Hospital Luebeck, Luebeck, Germany, ${ }^{2}$ Uksh, Klinik für Kinderchirurgie, Lübeck, Germany

Introduction: Paediatric clavicle fractures can often be treated conservatively. In our department ESIN is offered for dislocated fractures in adolescents as a treatment option. Despite some series in adults only little information exists concerning children and adults.

Materials and methods: 20 clavicular osteosynthesis with ESIN were performed: 17 patients with one-sided fracture, one patient with bilateral fracture and one with painful pseudarthrosis. Follow-up was 3 months -3 years and included X-ray before implant removal and clinical (Constand + Murley-Score) and ultrasound examination.

Results: Healing occured in all patients and removal of implants was performed after 2-3 months. Range of motion was unlimited and patients satisfaction was very high. 11 procedures needed open reduction (additional incision $2-3 \mathrm{~cm}$ ) which did not affect the cosmetic result. Three complications occured: one breakage of the nail during kick boxing, two nails had to be exchanged: one due to deformation after a fall on stairs and one while performing icehockey.

Conclusion: ESIN can be offered for dislocated clavicular fractures in adolescents. They benefit from very little pain and early motion. Disadvantage are two operations and anaesthesias. It is alo a minimalinvasive option for rare cases of pseudarthrosis. Nevertheless, sports must be strictly forbidden for 3-4 weeks, contact sports must be avoided for 8 weeks.

References: 1 . Beaty 2006 Bishop 2005. Laer 2007 Trompetter 2008 Kubiak 2002 Dahners 2005 Grassi 2001 Hartmann 2008 Jubel 2003 Kettler 2007 Meier 2006.

Disclosure: No significant relationships.

\section{P205}

\section{MINIMAL INVASIVE TREATMENT OF FRACTURES OF THE PROXIMAL HUMERUS IN ELDERLY PATIENTS}

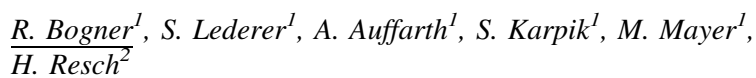

${ }^{1}$ Unfallchirurgie Und Sporttraumatologie, Universitätsklinik Salzburg, Salzburg, Austria, ${ }^{2}$ Traumatology And Sports Injuries, University Hospital Salzburg, Salzburg, Austria

Introduction: Surgical treatment of proximal humeral fractures in osteoporotic bone is still demanding for the surgeon. Until now there is no consensus in which technique treating these fractures leads to best outcomes in elderly patients.

Materials and methods: In the last years a total of 325 proximal humeral fractures were treated at our department by percutaneous 
reduction and internal fixation using the "Humerusblock" (Synthes, Switzerland). Out of this collective we identified 159 fractures in 156 patients aged above 70 . An angulation of more than $30^{\circ}$ of the headfragment and a displacement of the tuberosity of more than $5 \mathrm{~mm}$ were the indication for surgical treatment.

Results: 92 of these patients were accessible for clinical and radiological follow up after a mean of 31.8 months (6-81). The absolute, age-related and side related Constant scores were recorded. 132 fractures $(83 \%)$ healed by first intention. Fragment re-displacement or migration of K-wires was seen in 12 cases (7.5\%). A humeral head necrosis developed in 5 patients $(3.1 \%)$. One deep infection and one delayed healing were recorded. In two-part-fractures we found a mean Constant score of 60.2 points $(83.9 \%$ of the non-injured arm). The Constant score of patients with a three part fracture was 61.2 points (84.9\% of the non-injured arm). In 4-part fractures the mean Constant score was 49.5 points $(68.5 \%$ of the non-injured arm).

Conclusion: Most of the fractures in young patients with a good bony situation can be treated with rigid or semirigid implants in the same way with similar results. In patients aged above 70 years the necessity of a semirigid implant increases to avoid non-unions and cut-out of screws. A minimal invasive treatment can reduce the damage of soft tissue and blood supply at the fracture site. In most of our patients we could achieve good postoperative result with sufficient function for the activities of daily living.

Disclosure: No significant relationships.

P206

\section{MIPO TECHNIQUE OF DISTAL RADIUS FRACTURES WITH A COMMINUTION OF THE METAPHYSIS}

\section{J. Imatani, H. Kondou, Y. Kawakami, M. Hayashi}

Orthopaedic Surgery, Okayama Saiseikai General Hospital, Okayama, Japan

Introduction: Recently minimally invasive plate osteosynthesis (MIPO) for fractures of upper and lower extremities has been described. On the other hand, difficulties in reduction and maintenance of alignment and stability make distal radius fractures with a comminution of the metaphysis a challenge to treat. We have already reported the adaptation of MIPO technique for this complex fracture [1]. This technique used the volar locking plate and left the pronator quadratus intact.

Materials and methods: From 2001 to 2009, thirty one patients who sustained comminuted fractures of the distal metaphysis of radius were treated with MIPO technique. The type of fracture in all cases was AO classification-23-A3-3. The patients were followed for an average follow-up of 16 months.

Results: According to the clinical rating modified from Green and O'Brien, 10 cases were excellent, 16 cases were good and 5 cases were fair. The averaged ranges of motions were almost satisfactory. The alignments of the distal radius were restored without loss of reduction in the twenty five cases.

Conclusion: The current study indicates that adaptation of MIPO may be very efficient to provide good stability and a biological fracture healing for this complex fracture of the radius.

Reference: 1 . J.Imatani et al.: Minimally invasive plate osteosynthesis for comminuted fractures of the metaphysis of the radius.; J. Hand Surg. 30B(2)220-225, 2005.

Disclosure: No significant relationships.
P207

\section{NEW METHOD IN THE THERAPY OF DISTAL BICEPS BRACHII TENDON RUPTURE}

\author{
T. Máthé, L. Novák
}

Department of Traumatology And Hand Surgery, Trauma Centre, University Pécs, Pécs, Hungary

Introduction: The purpose of the present study is to report on a new instrument used to anchor the distal biceps tendon to the tuberosity of the radius.

Materials and methods: We performed reinsertions on seven male patients with rupture of the distal biceps tendon with the help of the thread guide device. The thread guide device is an instrument used to drill two holes in a V shape that meet beneath the cortical layer of the bone. Using a special hook the thread is pulled through the holes. With the help of the thread we reinsert the tendon of the distal biceps to the radial tuberosity.

Results: All seven patients recovered with good results. In one case there were wound healing disturbances, which was controlled with the administration of antibiotics. The range of motion remained within the normal limits. With an exception of a sportsman all patients were able to return to their original profession.

Conclusion: Distal biceps tendon reinsertion using the thread guide device is a solid, simple and low cost method to heal the patient.

References: 1. Bain GI, Prem H, Heptinstall RJ, Verthellen R, Paix D. Repair of Distal Biceps tendon rupture: a new technique using the Endobutton. J Shouldr Elbow Surg 2000;9:120-126. 2. Baker BE, Bierwagen D. Rupture of the distal tendon of the biceps brachii, operative versus non-operative treatment. J Bone Joint Surg Am. 1985;67A:414-21. 3. Barcsa Cs, Mándi A, Gáspár L. The distal tendon injury of biceps brachii. Hungarian Trauma Ortopaedics Hand Plastic Surgery. 1983;26:56-59. 4. Boyd HB, Anderson LD. A method for reinsertion of the distal biceps brachii tendon. J Bone Joint Surg Am. 1961;43A:1041-103. 5. Failla JM, Amadio PC, and Morrey BF. Post-traumatic proximal radio-ulnar synostosis. Results of surgical treatment J Bone Joint Surg Am. 1989 71: 1208-1213. Disclosure: No significant relationships.

\section{P208}

\section{PERCUTANEOUS LOCKING PLATES FOR FRACTURES OF THE DISTAL TIBIA: OUR EXPERIENCE AND A REVIEW OF THE LITERATURE}

\author{
M.A. Ahmad ${ }^{1}$, A. Sivaraman ${ }^{2}$, A. Rai ${ }^{3}$, A.D. Patel $^{3}$
}

${ }^{1}$ Trauma And Orthopaedics, Leeds General Infirmary, Leeds, United Kingdom, ${ }^{2}$ Trauma And Orthopaedics, The Royal London Hospital, London, United Kingdom, ${ }^{3}$ Trauma And Orthopaedics, Norfolk and Norwich University Hospital, Norwich, United Kingdom

Introduction: Distal tibial metaphyseal fractures pose many complexities. This study assessed the outcomes of distal tibial fractures treated with percutaneously inserted medial locking plates.

Materials and methods: 18 patients were selected based on the fracture pattern and classified using the AO classification and stabilised with an AO medial tibial locking plate. Time to fracture union, complications and outcomes were assessed with the American Orthopaedic Foot and Ankle Society ankle score at 12 months. 
Results: $16 / 18$ patients achieved fracture union, with 1 patient lost to follow up. Twelve fractures united within 24 weeks, with average union time 23.1 weeks. Three delayed unions, two at 28 weeks and one at 56 weeks. The average time to union was 32 weeks in the smokers and 15.3 weeks in the non-smokers. Five of the 18 patients (27\%) developed complications; one superficial wound infection and one chronic wound infection, resulting in non-union at 56 weeks, requiring revision. Two patients required plate removal, one after sustaining an open fracture at the proximal end of the plate 6 months after surgery (post fracture union) and the other for painful hardware. One patient had implant failure of three proximal diaphyseal locking screws, but successful fracture union. The average AOFAS ankle score was 88.8 overall, and 92.1 in fractures that united within 24 weeks.

Conclusion: Distal tibial locking plates have high fracture union rates, minimum soft tissue complications, and good functional outcomes. The literature shows similar fracture union and complication rates in locking and non-locking plates.

Disclosure: No significant relationships.

\section{P209}

\section{PSEUDO-ANEURYSM OF THE PERONEAL ARTERY AFTER PERONEAL TENDON REPAIR}

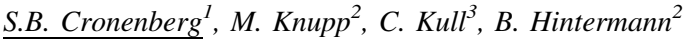 \\ ${ }^{1}$ Orthopedic Surgery, Bürgerspital, Solothurn, Switzerland, \\ ${ }^{2}$ Orthopedic Surgery, Kantonsspital Liestal, Liestal, Switzerland, \\ ${ }^{3}$ Vascular Surgery, Kantonsspital Liestal, Liestal, Switzerland
}

Introduction: Complications in peroneal tendon repair and lateral ligament reconstruction include pseudo-aneurysms of the dorsalis pedis artery, artery of the sinus tarsi and the tib. post. and tib. ant. artery.

We report on a hemodynamic relevant aneurysm of the peroneal artery after peroneal tendon repair.

To our knowledge, this is the first case in literature.

Materials and methods: Case report

In 2008 a 20-year-old patient underwent surgery for a peroneal tendon tear. Surgery included arthroscopy, open reconstruction of the tendon as well as resection of a hypertrophic Bassett ligament.

4 days after surgery the patient discovered a swelling with haematoma.

Surrounding the lateral malleolus with a pulsating swelling with a palpable thrill over the distal fibula.

Doppler sonography revealed an arteriovenous fistula of the fibular artery.

It could show a relevant ateriovenous fistula with increased shunt volumes, being 2-3 times increased compared to the volumes of the right leg.

Revision revealed a small lesion of the peroneal artery and its accompanying veins. Distal to the lesion the artery was damaged, so a ligature of veins and artery was performed.

Results: Discussion

Vascular damages have been previously described in ankle arthroscopy including damages of the artery dorsalis pedis, artery of the sinus tarsi and the tib. post. and tib. ant. artery. However, so far no pseudo-aneurysms of the peroneal artery have been described.
As injury mechanisms we consider over hazardous retractor placement, tangential introduction of the trocar, aggressive mobilisation and excision of scar tissue and shaving.

Conclusion: Insertion of the trocar "straight to the bone".

Careful capsular shaving especially in revisions.

If in doubt (or revision cases) tourniquet should be opened before. wound closure.

References: 1. Karlsson J, Wiger P. Longitudinal Split of the Peroneus Brevis Tendon and Lateral Ankle Instability: Treatment of Concomitant Lesions., J Athl Train. 2002;37(4):463-466. 2. Sadat U, See T, Cousins C, Hayes P, Gaunt M. Peroneal artery pseudoaneurysm-a case report and literature review. BMC Surg. 2007;29:7-4.

Disclosure: No significant relationships.

\section{P210}

\section{PULL-OUT SUTURE FIXATION OF TIBIAL EMINENCE AVULSION FRACTURE USING BIO-ABSORBABLE SUTURE ANCHOR: A CASE REPORT AND TECHNICAL NOTE}

\author{
C.C. Chang
}

\section{Orthopedics, Far Eastern Memorial Hospital, Taipei, Taiwan}

Introduction: Tibial eminence avulsion fracture is not an unusual injury. In displaced fracture, reduction and fixation are required to restore anterior cruciate ligament function and faster recovery. Initially, metal implants such as screw and staple are used intraarticularly for fracture fixation. Since problems in removal implants are frequently encountered, arthroscopic suture repair and pull out fixation with metal post in anterior upper tibia is then developed. With the improvement of bioabsorbable suture anchors, we have the idea of using bioabsorbable suture anchor for treatment of tibial eminence fracture.

Materials and methods: A 19 years old female student who suffered from a traffic accident and displaced tibial eminence fracture ensued. Operation was suggested. We use $5.0 \mathrm{~mm}$ bio-absorbable suture anchor as a post in anterior upper tibia. Then a tunnel was created from above the suture anchor to the intra-articular fracture site using ACL drilling guide. The ethibond suture of the suture anchor was introduced through the tunnel first, then through the distal stump of the anterior cruciate ligament using the suture hook. Finally the ethibond suture was retrieved through the tunnel to the anterior upper tibia and a knot was tied for fixation.

Results: After casting for 2 weeks, function brace was used and protected range of motion exercise was allowed. Three months after operation, $\mathrm{x}$ ray revealed fracture union. The patient has satisfactory recovery of knee range of motion and stability.

Conclusion: Metal post for arthroscopic pull out suture repair of tibial eminence fracture was used for a long time. It provides good fixation and satisfactory result. However, the implant in anterior upper tibia may cause skin irritation and discomfort, and the metal implant must be removed by another operation. With the improvement of the pull-out strength of bioabsorbable suture anchor, it could be a choice for pull out suture repair of tibial eminence fracture. Disclosure: No significant relationships. 
P211

\section{SIMPLE TRANSFORMING OF BRIDGING JOINT FRAME INTO DYNAMIC FRAME DURING INTRAARTICULAR FRACTURE TREATMENT}

\author{
M.B. Mitkovic $^{1}$, S.S. Milenkovic ${ }^{2}$, I. Micic ${ }^{1}$, M.M. Mitkovic ${ }^{1}$
}

${ }^{1}$ Trauma, University Orthopaedic and Trauma clinic, Nis, Serbia, ${ }^{2}$ University Orthopaedic \& traumatology Clinic, Clinical Centre of Nis, Nis, Serbia

Introduction: Bridging external fixation frame leads to joint stiffness and cartilage damage. It is presented one simple method of transforming bridging frame to dynamic frame providing so normal joint motion in the treatment of complex intraarticular fractures.

Materials and methods: As a clinical material we have used 52 patients with rigid or dynamic external fixation of ankle and knee joints. As external fixation device we have used Mitkovic external fixation system which consists of only three components: bar, carrier and clamp. The same system is suitable for axial dynamic fixation of bones, and for dynamic fixation of different joints, providing flexionextension bat preventing varus-valgus. We also used original device for joint axis rotation finder. Minimal internal fixation has been used in 51 fractures.

Results: Average time of healing was 1-4.5 months, depending of joint. End functional results in intraarticular fractures were: very good $20 \%$, good $58 \%$, fair $18 \%$, and poor $4 \%$.

Conclusion: It can be concluded that dynamic external fixation of joint using high mobile Mitkovic external fixation device gives promising functional results in intraarticular fractures treatment

Disclosure: No significant relationships.

\section{$\mathbf{P 2 1 2}$}

THE ANGULAR STABLE LOCKING SYSTEM (ASLS) FOR INTRAMEDULLARY NAILS: PILOT RESULTS FROM THE TREATMENT OF TIBIAL FRACTURES

\section{Hoentzsch ${ }^{1}$, H. Bail ${ }^{2}$, P.M. Rommens ${ }^{3}$}

${ }^{1}$ Abteilung Für Medizin-technische Entwicklung, BG Unfallklinik Tübingen, Tübingen, Germany, ${ }^{2}$, Charité Berlin, Berlin, Germany, ${ }^{3}$ Center of Trauma And Orthopaedic Surgery, Universitätsmedizin Mainz, Mainz, Germany

Introduction: The Angular Stable Locking System (ASLS) using resorbable sleeves was developed to provide angular stability between conventional intramedullary nails and screws, which lack adequate form-locking ability at the nail-screw interface. This pilot investigation was aimed at evaluating the handling and effectiveness of ASLS in a prospective multicentre case series.

Materials and methods: Twenty-eight patients with tibial fractures were treated with an Expert Tibial Nail and ASLS. Patients attended follow-up examinations at 6 weeks, 12 weeks and 6 months. The occurrence of complications was documented and any reported adverse event was evaluated for its relation to the use of ASLS. Functional outcomes of full weight bearing status, pain and walking ability based on the Parker Mobility Score were also determined.

Results: In more than $2 / 3$ of the operations, the surgeon was very to extremely satisfied with the handling of ASLS. None of the postoperative complications were directly related to the use of the new ASLS. Nearly all patients regained full weight bearing (25/26) with minimal pain within the follow-up period. Half of the patients were able to bear full body weight 11.1 weeks after surgery.
Conclusion: This first clinical experience suggests the potential role of the novel screw-sleeve locking system in enhancing fracture stability for optimal bone healing and earlier weight bearing. None of the complications were definitely related to ASLS. The use of ASLS may be associated with a slightly longer duration of surgery. The potential advantages of ASLS with respect to pain progression, weight bearing, gait pattern and fracture healing compared to conventional locking are currently being investigated in a randomised controlled trial.

Disclosure: Support was provided by Synthes $\mathrm{GmbH}$ and the AO Foundation. The authors did not receive any outside funding, grants or additional benefits for this study.

\section{P213}

\section{THE ANTEROMEDIAL MENISCOFEMORAL LIGAMENT: A RARE MRI FINDING NOT TO BE MISTAKEN FOR A BUCKET HANDLE TEAR OF THE MEDIAL MENISCUS AFTER A TORSION TRAUMA OF THE KNEE}

\section{S. Claes, P. Bollars, J. Bellemans}

Orthopedics and Traumatology, KU Leuven, Leuven, Belgium

Introduction: Congenital abnormalities of the medial meniscus are rare and most concern the anterior horn.

Materials and methods: We describe a series of three cases of an additional Anteromedial Meniscofemoral Ligament (AMMFL) in patients with medial knee pain after sustaining a torsional trauma. MRI images demonstrated a suspect image of a bucket handle tear of the medial meniscus. On knee arthroscopy, however, in all cases an additional fibrous band between the anterior horn of the medial meniscus and the femoral intercondylar notch was found, running in between the proximal portions of the ACL and PCL.

Results: In all three cases, there was both clinical and radiological suspicion of a bucket handle tear of the medial meniscus. During the arthroscopy, it turned out to be the AMMFL. In two of these cases, there was a complex tear of the posterior horn of the medial meniscus, whereas in the third there was a flap tear of the anterior horn of lateral meniscus.

Conclusion: In the literature there is no consensus of the clinical impact of the AMMFL. Previous reports suggest a higher risk of future injury and degeneration in the medial meniscus or impingement on the intercondylar notch. The presence of the AMMFL on the MRI of a painful knee after a torsion trauma should not be mistaken for a bucket handle tear of the medial meniscus.

Disclosure: No significant relationships.

\section{P214}

\section{TREATMENT OF FEMUR FRACTURES IN CHILDREN WITH DOUBLE PIN TECHNIQUE: IMMEDIATE INCORPORATED HIP SPICA CASTING BY TWO K-WIRES}

\section{K. Memisoglu, H. Atmaca, C.C. Kesemenli, H. Ceylan}

Orthopaedics And Traumatology, Kocaeli University School of Medicine, Kocaeli, Turkey

Introduction: The immediate hip spica casting is a popular treatment method for the pediatric age group femoral fractures. Femoral shortening is the unacceptable result for the treatment. In this technique we tried to describe immediate spica casting technique pining 
with double K-wire in children and evaluate the results obtained from patients treated with this method.

Materials and methods: Six patients were (4 boys and 2 girls) treated with this technique under general anesthesia in the operating room. Mean age was 6.3 years (range $4-8$ years), 4 fractures were on the right side and two on the left side. First K-wire of $2.5 \mathrm{~mm}$ was introduced in the supracondylar of femur passing from the lateral to the medial side through both cortexes, and protruded through the skin in the medial section. A second $\mathrm{K}$-wire of $2.5 \mathrm{~mm}$ was introduced through the lateral side of proximal fragment and it was allowed to pass only 1-2 mm through the second cortex. While an assistant was applying traction to the pin to maintain the reduction, lower extremity was put in a spica cast on the fractured side, while the knee joint on the other side was excluded from the spica casting process.

Results: The angulation after hip spica cast applied was $9.8^{\circ}$ (range $7^{\circ}-12^{\circ}$ ) on the frontal plane and $10.8^{\circ}$ (range $5^{\circ}-17^{\circ}$ ) on the sagittal plane. After spica cast removal, it was $9.3^{\circ}$ (range $5^{\circ}-13^{\circ}$ ) on the frontal plane and $12^{\circ}\left(\right.$ range $6^{\circ}-17^{\circ}$ ) on the sagittal plane.

Conclusion: Immediate spica casting with double pinning may be an effective method for femoral diaphyseal fractures in children.

References: 1. Kesemenli CC et al. Is external fixation in pediatric femoral fractures a risk factor for refracture. J Pediatr Orthop. 2004;24:17-20. 2. Aksahin E et al. Immediate incorporated hip spica casting in pediatric femoral fractures: comparison of efficacy between normal and high-risk groups. J Pediatr Orthop. 2009;29: 39-43.

Disclosure: No significant relationships.

\section{P215}

\section{TREATMENT OF PERIPROSTHETIC FEMORAL FRACTURES BY RETROGRADE STEM LENGTHENING}

\section{A. Chelnokov, I. Piven}

Orthopaedic Trauma, Ural Scientific Institute of Traumatology and Orthopaedics, Ekaterinburg, Russian Federation

Introduction: Aim of our study was to design a technique of less invasive fixation in periprosthetic fractures of the femoral shaft which can provide primary stability of the stem and the femur.

Materials and methods: Motivated by a case of plate failure in periprosthetic femoral fracture we designed and used a modification of an industrial solid titanium femoral locked nail. Its design provides sliding over the femoral stem with tight fit. Ten patients were treated in 2007-2010 with the technique. There were 7/10 Vancouver B1, 2/10 B2 fractures, and 1/10 B3. The nails were individually custommade to fit the particular stem design and size. The inserted nail was locked distally with two or three $6 \mathrm{~mm}$ screws. Fixator-assisted nailing was used with small wire external distractor for acute or gradual reduction.

Results: Less invasive surgery and stable fixation provided fast recovery. Immediate weight-bearing as tolerated was recommended in most cases. Unassisted walking occurred at 2 month in $4 / 10$ patients, and at 3 month in 9/10. 8 patients were available for followup in 1 year-all fractures healed.

Conclusion: Since 2007 few publications appeared [1, 2] presenting similar approach with good results in series of $18-25$ cases. We conclude that the technique can be an effective solution in problematic cases of failed plating after periprosthetic fractures. For elderly patients with severe comorbidities the technique offers less invasive treatment option with quick recovery. Immediate unrestricted weight-bearing appears safe in majority of cases. Some aspects of the technique concerning indications, possible stressshielding, need of nail dynamization, implant removal require further research.

References: 1. Zuurmond RG, Pilot P, Verburg AD. Retrograde bridging nailing of periprosthetic femoral fractures. Injury. 2007;38(8):958-64. 2. Meyer C et al. Treatment of periprosthetic femoral fractures by effective lengthening of the prosthesis. Clin Orthop Relat Res. 2007;463:120-7.

Disclosure: No significant relationships.

\section{P216}

\section{UNSTABLE TROCHANTERIC FEMORAL FRACTURES. COMPLICATIONS AND OUTCOME AFTER LOCKED MINIMAL INVASIVE PLATING AND FEMORAL NAILING}

\section{Knobe, R.M. Sellei, P. Kobbe, P. Lichte, R. Pfeifer, H.C. Pape}

Department of Orthopaedic Trauma, University Hospital of the RWTH AACHEN, Aachen, Germany

Introduction: Intramedullary fixation has been recommended in unstable intertrochanteric hip fractures (AO 31A2), although screw cut-out has been identified as problematic. This study was performed to ascertain whether treatments with the established nails have advantages over the use of the Percutaneous Compression Plate (PCCP).

Materials and methods: Cohort study. Between 2003 and 2008, 134 patients with unstable fractures were treated with a PCCP, $(n=44$, 78.3 years, ASA 2.8), a PFN $(\mathrm{n}=50,77.2$ years, ASA 2.8), or a PFNA $(\mathrm{n}=40,75.8$ years, ASA 2.6). The patients (31 PCCP, 33 PFN, 30 PFNA) were reexamined clinically and radiologically after approximately 21 months.

Results: The PCCP was found to require less implantation time than the PFN and the PFNA (60 vs. 80 vs. 84 min, p <0.001) and less radiation exposition time (PCCP 139 vs. PFN 283 vs. PFNA $188 \mathrm{~s}$, $\mathrm{p}<0.001)$. The rate of reoperations due to wound infections and hematomas amounted to $2 \%$ for the PCCP, $4 \%$ for the PFN, and $5 \%$ for the PFNA $(p=0.799)$. Due to mechanical complications, $9 \%$ of patients implanted with a PCCP, $13 \%$ of those implanted with a PFN, and $5 \%$ of those implanted with a PFNA had to be reoperated $(\mathrm{p}=0.353)$. The cut-out rate was $2 \%$ after implantation of the PCCP, $4 \%$ after the PFN, and 5\% after implantation of the PFNA $(p=0.799)$. On the basis of their scores according to Merle d'Aubigné and Harris, there was no variation in the results of the follow-up examinations.

Conclusion: The use of the PCCP for the treatment of unstable trochanteric fractures presents a minimally invasive method of implantation, as well as a promising therapy option with regards to operation time, radiologic examination time, and rate of complications. No benefits could be established in an intramedullary treatment with the PFN or the PFNA; thus, it appears that the higher cost of these implants is avoidable.

Disclosure: No significant relationships. 


\section{OPEN FRACTURES}

\section{P217}

\section{HYBRID EXTERNAL FIXATION FOR OPEN SEVERE COMMINUTED FRACTURES OF THE DISTAL FEMUR}

\author{
A. Birjandinejad ${ }^{I}$, E. Ghayem Hassankhani ${ }^{2}$, G. Ghayem \\ Hassankhani $^{I}$
}

${ }^{1}$ Department of Orthopedics Surgery, Mashhad University of Medical Sciences, Mashad, Iran, ${ }^{2}$ Orthopeadic \& Spine, Orthopedic Research Center of Mashad University of Medical Sciences, Mashad, Iran

Introduction: The treatment of open severe comminuted fractures of the distal Femur is still a major problem.

The aim of this study is to evaluate the treatment of these fractures with hybrid external fixator.

Materials and methods: Thirty-four patients with open comminuted distal femur fracture were treated by hybrid external fixator between January 2005 and December 2008.

Results: The bony results were excellent in 24 patients (70.5\%), good in $6(17.7 \%)$, fair in $2(5.9 \%)$, and poor in $2(5.9 \%)$. the functional results were excellent in $10(29.4 \%)$ patients, good in $14(41.2 \%)$, fair in $6(17.6 \%)$, and poor in $4(11.8 \%)$.

Conclusion: Hybrid external fixator is an effective method for treatment of open severe comminuted fractures of the distal Femur References: Kregor PJ. Distal femur fractures with complex articular involvement: management by articular exposure and submuscular fixation. Orthop Clin North Am. 2002;33(1):153-75, ix. Ghayem Hassankhani E, Payvandi Mahamad T, Birjandinejad A. The Ilizarov ring external fixator in complex open fractures of the Tibia. Eur $\mathbf{J}$ Trauma 2006;31:1-7. Kao FC, Tu YK, Su JY. Treatment of distal femoral fracture by minimally invasive percutaneous plate osteosynthesis. J Trauma. 2009; 67(4):719-26.

Disclosure: No significant relationships.

\section{P218}

\section{MULTIPLANAR TUBULAR EXTERNAL FIXATOR APPLICATION FOR DEFECTIVE PEDIATRIC HUMERAL FRACTURE}

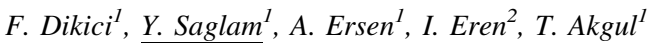

${ }^{1}$ Orthopedics and Traumatology, Istnbul Tip Fakultesi, Istanbul, Turkey, ${ }^{2}$ Orthopadics and Traumatology, Istanbul Tip Fakultesi, Istanbul, Turkey

Introduction: Open fractures of the distal humerus in children with high energy trauma can be associated with considerable bone loss and extensive soft tissue damage. Management of this type of injuries are hard and challenging. The purpose of this case report is to give an opinion for the treatment of open fracture with bone defect in pediatric patients.

Materials and methods: Three years old boy who fallen from a height applied to the emergency. He had Gustilo-Anderson grade IIIA open fracture of the distal humerus with $3.5 \mathrm{~cm}$ of segmental bone loss. After the radical debridement and irrigation the coverage of the remaining part of the humerus was acceptable. The original length of the humerus was restored immediately with AO type tubular external fixator by bridging of elbow.
Results: No complications were encountered during the postoperative period. Bone defect was healed at the end of 5 weeks. The fixator was removed at sixth week. At 20 months follow-up, Mayo Elbow Performance Score was 100 points, which is equivalent to a excellent result.

Conclusion: Comparing with adults, children have high remodeling ability, high healing potential and more resistant to joint stiffness. Periosteum continuity and ligamentotaxis effect provided bone development at the defective zone. The use of external fixators in defective humeral fractures with periosteal continuity is an effective surgical method in pediatric patients.

References: Wiley JJ, McIntyre WM. Fracture patterns in children. In: Current concepts of bone fragility. Berlin: Springer; 1986. p. 159. Fransworth CL, Silva PD, Mubarak SJ. Etiology of supracondylar humerus fractures. J Pediatr Orthop. 1998;18:38. Hanlon CR, Estes WL. Fractures in children: a statical analysis. Am J Surg. 1954;87:312. Dormans JP, Squillante R, Sharf H. Acute neurovascular complications with supracondylar humerus fractures in children. J Hand Surg. 1995;20-A:1. Wilkins KE. Supracondylar fractures: what's new? J Pediatr Ortop B. 1997;6:11. Gustilo RB, Mendoza RM, Williams DN. Problems in the management of type III (severe) open fractures: a new classification of type III open fractures. J Trauma. 1984;24:742. Muller ME, Nazarian S, Koch P, Schaftzken J. Comprehensive classification of fractures of long bones. New York: Springer; 1990. Husain SN, King EC, Young JL, Sarwark JF. Remodeling birth fractures of humeral diaphysis. Pediatr Orthop. 2008 2008;28(1):10-3.

Disclosure: No significant relationships.

\section{P219}

\section{NEW TECHNIQUE FOR INTRAMEDULLARY ANKLE OSTEOSYNTHESIS}

\author{
W. Friedl
}

Orthopädie Unfallchirurgieund Handchirurgie, Klinikum Aschaffenburg, Aschaffenburg, Germany

Introduction: Soft tissue problems in the malleolar area can occur due to trauma damage and arterial and venous disorders. Therefor a implant in the bone and not on the bone surface has several advantages. Additionally intramedullary implants have a higher weight bearing capacity and less risk of implant loosening in osteoporotic bones.

Materials and methods: From 05/2000 to 03/2002 214 ankle fractures were stabilised with a Xs or XXS nail (locked straight nail of 4.5 and $3.5 \mathrm{~mm}$ ). The mean age was 51 year, $59 \%$ were woman. $35 \%$ were Weber $\mathrm{B}$ and $25 \%$ type $\mathrm{C}$ fractures. The re-examination after 6 months could be performed in 91 Patients and was evaluated according to the Ovadia score (clinical and radiological).

Results: At re-examination $71 \%$ showed an excellent and $25 \%$ a good result. Only three Patients were classified as fair (1) or unsatisfactory; in one due to algodystrophia. In two patients haematoma revisions and in two patients with the primary used oblique insertion of the nail showed a proximal fibula fracture. In one case a spilt skin graft was needed. In no case infection of the implant or bone was seen, in no case fracture $r$ implant dislocation occured.

Conclusion: The Xs nail is a new option for ankle stabilisation. It improves the stability and reduces the complication of ankle osteosynthesis due to the lack of implant under the skin on the bone surface 
end due to the higher biomechanical stability of intramedullary implants.

Disclosure: No significant relationships.

\section{PELVIC TRAUMA}

\section{$\mathbf{P 2 2 0}$}

\section{AGE OR ASSOCIATED INJURIES AS A CAUSE OF MORTALITY IN PELVIC FRACTURES?}

\author{
C. Frizis ${ }^{1}$, G. Komninos ${ }^{l}$, N. Vasios $^{l}$, R.-H. Frizis $^{2}$
}

${ }^{1}$ 1st Surgical Department, Ippokratio Hospital of Thessaloniki, Thessaloniki, Greece, ${ }^{2}$ Ippokratio Hospital of Thessaloniki, Thessaloniki, Greece

Introduction: Pelvic fractures, both simple and complex, are still a contradictory surgical entity under continuous study. Based on literature, in the absence of concomitant diseases in elderly patients the same criteria and management guidelines apply as in younger patients. The purpose of the present study is to observe the effect of the factors of age and associated injuries on the mortality in patients with pelvic fractures.

Materials and methods: In the present study, all patients with pelvic fractures and signs of hemodynamic instability, not due to extrapelvic injuries, admitted in a 5-year period were included. Among the 56 patients in total, 43 were under 65 years of age and 13 were more than 65 years old. 45 patients had simple pelvic fractures, whereas, 11 suffered from complex pelvic fractures.

Results: The overall mortality in our study was 5/56 (8.9\%). Among the elderly patients the mortality was $1 / 13(7.7 \%)$ and concerned a patient with a simple pelvic fracture who did not survive due to concomitant diseases. The mortality among the patients under 65 years of age was $4 / 43(9.3 \%)$ due to more severe associated injuries with greater blood loss.

Among the patients with simple pelvic fractures the mortality was $1 / 45(2.2 \%)$, whereas, among the patients with complex fractures the mortality was $4 / 11(36.4 \%)$.

Conclusion: Due to the limited number of patients, the evidence and results of this study could not be fully statistically processed and analysed. However, based on the observations derived from the patients included, we conclude that the severity of associated injuries is a key risk factor for the mortality in patients with pelvic fractures.

In elderly patients, the presence of comorbidities seem to play a significant role even in the presence of simple pelvic fractures.

Disclosure: No significant relationships.

\section{$\mathbf{P 2 2 1}$}

\section{DEEP INFECTIONS AFTER SURGICALLY TREATED PELVIC/ACETABULAR FRACTURES}

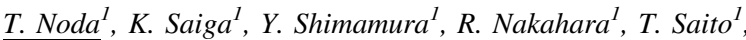 \\ T. Kanazawa ${ }^{1}$, T. Ozaki ${ }^{1}, K$. Ogawa $^{2}$
}

${ }^{1}$ Department of Orthopaedic Surgery, Okayama University Hospital, Okayama City, Japan, ${ }^{2}$ Orthopaedic Trauma, Fukuyama City Hospital Emergency Medical Center, Fukuyama, Japan

Introduction: The results of surgical treatment of pelvic/acetabular fractures have been improved. But treatment of the deep infections after surgery has been very difficult and challenging.
Materials and methods: Retrospective data on surgically treated pelvic/acetabular fractures from 93 patients from 2003 to 2010 was analyzed to investigate the risk factors associated with deep infections after surgery. The data was compared between infected cases and non-infected cases.

Results: Deep infections were occurred in 6 patients. The average age was 48.3 years old. Transcatheter arterial embolization (TAE) was performed in five of six patients. Injury Severity Score (ISS) was ranged $25-57$ (mean 33.3). Total operation time was ranged 4.5-15 h (mean $7.5 \mathrm{~h}$ ). Total blood loss was ranged $1,000-3,000 \mathrm{ml}$ (mean $1,850 \mathrm{ml}$ ). No statistical significance was found in patient age. However, TAE, ISS, total operation time, and total blood loss were associated with deep infections $(\mathrm{p}<0.01)$.

Conclusion: Discussion: In high risk cases such as polytrauma patients with high ISS, we should reduce the total operation time and blood loss in the surgery using a staged operation or minimally invasive surgery. Although TAE is necessary to control retroperitoneal bleeding. Careful attention should be taken to limit excessive TAE.

Disclosure: No significant relationships.

\section{P222}

\section{EMERGENCY STABILIZATION OF PELVIC RING FRACTURES WITH ILIOSACRAL SCREWS}

\author{
G. Merenyi, P. Gergely, I. Zagh
}

\section{Traumatology, Karolyi Sandor Hospital, Budapest, Hungary}

Introduction: Hemorrhage control of the disrupted pelvic fractures requires an urgent reduction of the intrapelvic volume and immediate mechanical stabilization. There are some opportunities: pelvic binder, C-clamp with or without pelvic packing, external fixator. Most of trauma centers applies internal fixations (screws, plates) after a few days, when the patient has already been in haemodynamic stable condition.

Materials and methods: Our treatment protocol is: immediate pelvic sheeting, CT scan, and emergency stabilization of pelvic ring with iliosacral screws and if it is necessary with plating of the symphysis. Advantages of this method: It can be applied even at polytraumatized patients in supine position. Immediate reconstruction of the pelvic ring reduces the bleeding in the same way as the C-clamp or external fixator does, but it gives definitive care. Iliosacral screw fixation is a fast and safe method, its duration is 15-20 min by a skilful surgeon. In the first hours meteorism does not disturb the radiological visualization during the operation. Over a period of 3 years, 15 patients with unstable pelvic ring disruptions were treated by closed reduction and posterior fixation with percutaneous SI screwing. Plating of symphysiolysis was applied at four patients. According to Tile classification, three cases $(20 \%)$ were grouped as type $\mathrm{C} 1$, nine cases $(60 \%)$ as $\mathrm{C} 2$ and three cases $(20 \%)$ as $\mathrm{C} 3$. The follow up period ranged from 8 to 18 months (mean 10 months).

Results: The mortality rate was $13.3 \%$. All the fractures and dislocations healed well. According to the pelvic outcome score, the final results were excellent in five cases $(38.5 \%)$, good in seven cases $(53.8 \%)$, fair in one cases $(7.6 \%)$ There was no neurological deficit or wound infection.

Conclusion: Emergency stabilization of pelvic ring fractures with SI screws results in decreasing of bleeding, possibility of early mobilisation and good functional outcome.

Disclosure: No significant relationships. 
$\mathbf{P 2 2 3}$

\section{EXTRAPERITONEAL PELVIC PACKING TO TREAT SEVERE PELVIC FRACTURE: PRELIMINARY EXPERIENCE}

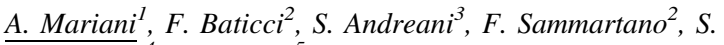 Cimbanassi $^{4}$, O. Chiara ${ }^{5}$}

${ }^{1}$ Trauma Team, Ospedale Niguarda Ca' Granda, Milan, Italy, ${ }^{2}$ Chirurgia Generale E Mininvasiva, Istituto Clinico Humanitas, Rozzano, Italy, ${ }^{3}$ Trauma Team, General Surgery, Niguarda Hospital Milan, Milan, Italy, ${ }^{4}$ Emergency, Ospedale Niguarda Ca' Granda, Milan, Italy, ${ }^{5}$ Emergency, Ospedale Niguarda Ca' Granda, Milan, Italy

Introduction: Mortality from pelvic fractures associated with severe bleeding is still between 40 and $60 \%$ despite several approaches proposed in the last years; pelvic binder, angio with embolization, external fixation.

Materials and methods: Patients arriving at ED with hemodynamic instability unresponsive to temporary stabilization, may be treated in ER with extraperitoneal pelvic packing before angio. This technique is to increase retroperitoneal compartment pressure, through placement three or four tools sideways the bladder, and trying to stop or reduce the bleeding, allowing time for fixation and angio-embolization. Niguarda Hospital Trauma Team managed 2,256 injured patients between October 2002 and December 2008. Among 361 patients with pelvic fracture, 69 arrived in ER with systolic pressure $<60 \mathrm{mmHg}$ and an AIS score of 4 or 5 .

Results: 52 patients had pelvic fracture associated with brain or torso trauma while 17 had isolated complex pelvic fracture. Nine of former group and two of latter were treated with EPP. Five died: four had temporary hemodynamic response after EPP but died for the severity of associated lesions, one died in ICU for a severe associated brain injury. Six survived.

Conclusion: We believe that EPP should be introduced in the surgical skill of Trauma Surgeon as damage control procedure in severe hemorrhage from pelvic fracture.

References:

Totterman A. Extraperitoneal pelvic packing: a salvage procedure to control massive traumatic pelvic hemorrhage. J Trauma. 2007.

Smith WR. Retroperitoneal packing as a resuscitation technique for hemodynamically unstable patients with pelvic fractures: report of two representative cases and a description of technique. J Trauma. 2005. Disclosure: No significant relationships.

\section{$\mathbf{P 2 2 4}$}

\section{HOSPITALIZED PREGNANT PATIENTS DUE TO TRAUMA}

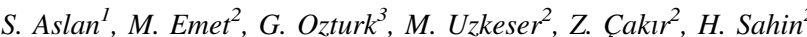

${ }^{1}$ Emergency Department, Ataturk University Aziziye Hospital, Erzurum, Turkey, ${ }^{2}$ Emergency Department, Ataturk University, Erzurum, Turkey, ${ }^{3}$ General Surgery, Ataturk University, Erzurum, Turkey

Introduction: Pregnancy brings a unique set of challenges to trauma patient presentation because care must be provided for two patients: the mother and the fetus. Anatomic and physiologic changes in pregnancy can mask or mimic injury, making diagnosis of traumarelated problems difficult. In this study, we aimed to understand demographic and trauma related differences between women trauma victims with and without pregnancy.
Materials and methods: This is a prospective cross-sectional study. Inclusion criteria were women trauma victims older than 16 years old. Patients were divided into two groups: pregnant and nonpregnant. Difference of means and categorical variables were analyzed using Wilcoxon-Mann-Whitney test and Fisher's exact test, respectively.

Results: Between 01 May 2008 and 29 January 2010, 224 women were hospitalized due to trauma in our hospital. $3.6 \%(\mathrm{n}=8)$ were pregnant. Mean age $(24.7 \pm 3.1$ vs $44.1 \pm 19.8, \mathrm{p}=0.0002)$, and mean Glasgow Coma Scale $(13.5 \pm 4.2$ vs $14.3 \pm 2.1, \mathrm{p}=0.031)$ of pregnant women were significantly lower compared to nonpregnant trauma patients. Mean hospitalization day ( $6.2 \pm 4.6$ vs. $10.5 \pm 10.1$, $\mathrm{p}=0.16)$, mean AIS scores, ISS $(8.9 \pm 6.9$ vs. $12.4 \pm 8.3$, $\mathrm{p}=0.648)$ and NISS $(11.1 \pm 9.7$ vs. $16.2 \pm 11.4, \mathrm{p}=0.691)$ scores did not differ between groups. Mortality rate (12.5 vs. $6.5 \%$, $\mathrm{p}=0.431)$ and operation rate $(37.5$ vs. $50.5 \%, \mathrm{p}=0.722)$ did not differ significantly between pregnant and nonpregnant patients. Characteristics of pregnant trauma victims are listed in Table 1.

Conclusion: Discussed with nonpregnant women, pregnant trauma victims are younger and they have lower GCS sores. Most common trauma mechanism is motor vehicle accident.

Disclosure: No significant relationships.

\section{P225}

\section{ILIOSACRAL SCREW FIXATION FOR AO/OTA CLASSIFICATION 61-B TYPE}

\section{N. Shiota ${ }^{1}$, T. Sato ${ }^{2}$}

${ }^{1}$ Orthopaedic Surgery, Okayama Medical Center, Okayama, Japan, ${ }^{2}$ Orthopaedic, Okayama Medical Center, Okayama, Japan

Introduction: The operation for the pelvic ring fracture of the $\mathrm{AO} /$ OTA classification 61-B type is adapted when the completely disruption of posterior part was occurred, but we cannot distinguish it definitely. We tried to mobilize at once performed minimal invasive surgery to the case which considered that was not operative adaptation once and was being passed a few weeks in beds.

Materials and methods: 15 cases: conservative group (group C) 7 and navigated operation group (group $\mathrm{N}$ ) 8, were performed historical cohort study. It was group $\mathrm{C}$ average age 56.2 years old (23-82), male four cases, woman three, group N 61.3 years old (17-95), male five, woman three. The injury cause was crash two cases, four cases; traffic accident five, four in group $\mathrm{C} /$ group $\mathrm{N}$. Fracture type was B1 two cases, three cases, B2 three, three, B3 two, two. There was not the statistical significance difference in each item. We performed the Ilio-sacral screw fixation using navigation system (Vector vision trauma navigation system, Brain LAB), and the operation inserted two cannulated cancellous screwsdiameter $8.0 \mathrm{~mm}$ in S1, $6.5 \mathrm{~mm}$ in S2. Every cases trained mobilization and standing at parallel bars depending on pain. I examined standing position possibility day in parallel bars/straight leg rising possibility day.

Results: It was average 15.8 days (12-27) in group C and 6.5 (2-12) in group $\mathrm{N}$ that standing position in parallel bars having become possible. SLR became possible on the 17.4 days (12-35) on group C and the $8.3(1-18)$ on group C. The significant difference was accepted among both groups.

Conclusion: Early ambulation was able to be achieved certainly as a result of conducting minimal invasive operation positively also to the case which had been performed conservative treatment. It seemed that it was necessary to reconsider it about a future treatment policy. Disclosure: No significant relationships. 
P226

\section{INCARCERATED DIAPHRAGMATIC HERNIA: CASE REPORT}

T.C. Santos ${ }^{l}$, H.M. Fernandes ${ }^{1}$, J.C. Pereira ${ }^{2}$, A.L. Silva,

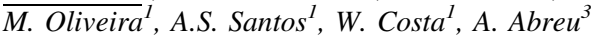

${ }^{1}$ Surgery, Centro Hospitalar do Alto ave, Guimaraes, Portugal, ${ }^{2}$ Surgery, IPO, Porto, Portugal, ${ }^{3}$, Centro Hospitalar do Alto Ave, Guimaraes, Portugal

Introduction: Incarcerated diaphragmatic hernia it's an uncommon situation and might occur after blunt thoraco-abdominal trauma. Its premature detection, although frequently a difficult task, it's essential, since the diaphragmatic rupture is associated with a high mortality and morbidity rate, if not treated.

Materials and methods: The authors present the clinical case study of a 73 years old male patient, with a history of blunt thoracoabdominal trauma, nearly 5 years ago.

Results: The patient presented to the emergency room with history of progressive left thoracic pain, associated with dyspnoea, vomit and food intolerance. He had a chest $\mathrm{x}$-ray that revealed a hydro-aerial level on the left hemithorax. While having a thoracic TC, requested by the doctor, the patient suffered a cardiorespiratory arrest from which he recovered after two cycles of advanced life support. His thoracic CT presented a left hydropneumothorax. The patient was submitted to surgery through abdominal approach, in which a left diaphragmatic rupture with gastric, splenic and colic herniation was detected. The diaphragmatic hernia was then reduced and the diaphragmatic defect repaired using a Polypropylene/Vicryl prosthesis. The patient died in the 21 st day after surgery, in the sequence of a stroke.

Conclusion: Diaphragmatic hernias are well-known sequelae of abdominal and chest wall trauma. However, they may go undiagnosed in the acute setting but present later due to gastrointestinal or respiratory complications. The authors present a distinctive presentation of a diaphragmatic hernia enhancing the principal form of presentation: cardiorespiratory arrest.

Disclosure: No significant relationships.

$\mathbf{P 2 2 7}$

\section{INTRODUCTION OF CT AS THE STANDARD FOR PELVIC RING FRACTURES OVER THE AGE OF 65: WHAT IS THE THERAPEUTIC CONSEQUENCES?}

\section{A. Höch, A. Boldt, J. Böhme, C. Josten}

Trauma Surgery, University of Leipzig, Leipzig, Germany

Introduction: It is known that elderly patients often have an involvement of the posterior pelvic ring after so-called trivial trauma. The literature talks about the "A-B-problem", because often only the CT shows a B-component. Our aim was to evaluate results of the radiological diagnostics and the resulting treatment decision and outcome.

Materials and methods: A total of 196 patients with pelvic ring fractures ( $160 \mathrm{f}, 36 \mathrm{~m}$ ) with an age above 65 years (mean: 80.7 years) treated in the years 2006-2009 were included. From 2007, an initial CT was the standard in this age group with an anterior pelvic ring fracture. We evaluated the trauma mechanism, type of pelvic ring fracture according to the $\mathrm{AO}$, implementation of a $\mathrm{CT}$, type of treatment.
Results: There were 76 pelvic ring fractures of type A, 101 of type B (including 89 type B2) and 19 of type C according to AO. In 151 cases $(77 \%)$ was the accident mechanism a simple fall, only $29(15 \%)$ patients had a high energy trauma, $16(8 \%)$ had no trauma in history. 183 Patients received a diagnostic CT, only 13 patients with A-fractures did not. 159 CT scans were performed initially. In 2006, 15 times the CT scan was done after persisting pain symptoms. In ten cases this was leading to an upgrade from an A- to B-fracture. 143 patients were treated conservatively. 11 of the patients with $\mathrm{C}$-fractures were treated using transiliosacral screws, four using triangular osteosynthesis and four were treated conservative due to the general condition. The B-fractures were treated 38 times by transiliosacral screws. In addition it was done a sacroplasty in ten cases. Eight patients died before surgical treatment. Overall, an increase of surgical treated patients was observed after the indroduction of standard CT. At the same time, we observed a high rate of surgery-related complications.

Conclusion: Even after trivial accident mechanism a participation of the posterior pelvic ring has to be excluded using CT in this age group. The therapeutic consequences must be thought through carefully, because of an increased appearance of surgery-related complications in this age group.

Disclosure: No significant relationships.

P228

\section{MANAGEMENT OF BLUNT ABDOMINAL INJURES IN THE DISTRICT HOSPITAL.}

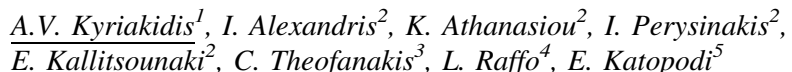

${ }^{1}$ Department of General, General Hospital of Amfissa, Amfissa, Greece, ${ }^{2}$ Department of General Surgery, General Hospital of Amfissa, Amfissa, Greece, ${ }^{3}$ General Surgery, General Hospital of Amfissa, Amfissa, Greece, ${ }^{4}$ Department of Radiology, General Hospital of Amfissa, Amfissa, Greece, ${ }^{5}$ Radiology, General Hospital of Amfissa, Amfissa, Greece

Introduction: The aim of our study is to examine the effectiveness of conservative treatment of blunt abdominal injuries with the use of modern diagnostic approaches which allow early and accurate diagnosis in the Emergency Department

Materials and methods: Between 9/2006 and 9/2010, 56 patients were admitted in our hospital suffering from blunt abdominal injuries. Eighteen patients had liver injuries, 12 patients had unilateral kidney injuries, 10 patients had spleen injuries, 8 patients had injuries in the retroperitoneal space and 8 patients had injuries in the hypogastrium. Results: 14 out of 18 patients with liver injuries had Grade 1-3 injuries and were treated conservatively. Nine patients had Grade 4 injuries and were operated. One patient who was initially managed conservatively was operated due to inability to control hemorrhage. Four out of ten patients with spleen injuries had Grade 4 and Grade 5 injuries and were successfully operated and six had Grade 1 and 2 injuries and were treated conservatively. Patients with injuries of the retroperitoneal space, unilateral kidney injuries were managed conservatively

Conclusion: Blunt abdominal injuries can be successfully and safely managed with conservative measures. CT scan is a very sensitive diagnostic tool, capable of diagnosing intraabdominal hemorrhages, thus being a necessary tool for the physician in order to diagnose accurately any abdominal injury.

Disclosure: No significant relationships. 
P229

\section{PELVIC AND FEMORAL TRAUMA PATIENT TRANSPORT:} VITAL IMPORTANCE

\author{
C. Costa Almeida, E. Afonso, B. Henriques, G. Martinez, L. Reis, \\ C.M. Costa Almeida
}

Surgery, Centro Hospitalar Coimbra, S. Martinho do Bispo, Coimbra, Portugal

Introduction: Male patient that suffered a work accident with pelvic and femur crushing by a concrete block. In addiction to the multiple pelvic bone and femur fractures, he had major vessels injury involving left iliac artery and vein, and left femoral artery and vein. He was transferred from another hospital to our ER $50 \mathrm{~km}$ away, without airway protection, with no pelvic stabilization, and no vessels control with major arterial bleeding.

Materials and methods: He reached the ER without heart beatings and no respiratory movements. He was made reanimation manoeuvres for $30 \mathrm{~min}$ until recovery, iliac and femoral vessels were also controlled in the ER, and was transfused with $12 \mathrm{U}$ of blood and $3 \mathrm{U}$ of plasma. In the end went to the operation room.

Results: In the operation room was applied an external pelvic stabilization by the orthopedics surgeon, and two shunts were made to control haemorrhage and try to save the left lower limb. We used PVC nasogastric tubes to make an arterial and a venous shunt with the main objective to reconstruct the vessels in a second operation after patient recovery. Damage control principles were applied. During this procedures he was transfused again with $40 \mathrm{U}$ of blood, $8 \mathrm{U}$ of plasma, plaquetas and factor VIIa. He was finally transferred for the ICU, however, he had the mortal triade and could not resist.

Conclusion: This is a case of a severe pelvic trauma with major vessels injury that was transported to the ER by health care professionals as he would be by any other person. The fact that he was transported for an ER $50 \mathrm{~km}$ away with major arterial bleeding that was not controlled, without pelvic stabilization, and without airway protection made this severe case an extremely severe one. This bad transport was vital for the final outcome, his death. The patient transport is so important that a good one can save a live.

Disclosure: No significant relationships.

\section{P230}

\section{PELVIC FRACTURE AND HEPATIC INJURY DEADLY ASSOCIATION?}

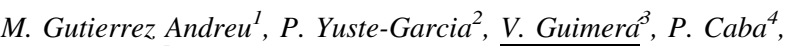 F. Cruz-Vigo}

${ }^{1}$ General Surgery, 12 de Octubre hospital, Madrid, Spain, ${ }^{2}$ General Surgery, 12 de Octubre Hospital, Madrid, Spain, ${ }^{3}$ Orthopaedics and Trauma Surgery, Hospital 12 de Octubre, Madrid, Spain, ${ }^{4}$ Servicio de Cirugia Ortopedica Y Traumatologia Ii, Hospital 12 de Octubre, Madrid, Spain, ${ }^{5}$ General Surgery, Hospital 12 de Octubre, Madrid, Spain

Introduction: The combination of pelvic fracture and hepatic injury is associated with a high mortality rate $(24-51 \%)$. The aim of this study was to determine the epidemiology and type of treatment in this group of patients treated in our hospital.

Materials and methods: Patient and Methods: We retrospectively analyzed a group of 21 patients with fractured pelvis and liver injury between 2001 and 2009. We evaluated the following variables: age, gender, injury mechanism, severity scale (ISS), associated injuries, type of treatment (pelvic fixation, laparotomy, and embolization), and mortality.

Results: 13 men and 8 women. Mean age of 31 . The most common injury mechanism was precipitation (45.4\%). Mean ISS was 38.3, mean NISS of 40.6 and mean PS of 0.61 . Most pelvic fractures were type C (14). Hepatic injuries were 11 grade 1l, 7 grade 111, 2 grade IV and 1 grade V.11 laparotomies and 8 angiographies were performed. Pelvic fractures were treated by angio-embolization in 6 cases and external fixation in 13. The most common complications were hemorrhagic shock in six patients. The mortality rate was $33.3 \%$

Conclusion: A high rate of abdominal injuries is associated with severe pelvic trauma. Patients with pelvic fracture and liver damage have a higher rate of mortality. The treatment of liver injury was a conservative with the exception of those who had associated abdominal injuries that needed a laparotomy. A multidisciplinary approach is essential in order to stablish the correct sequence in diagnostic and therapeutic methods at our disposal.

Disclosure: No significant relationships.

\section{P231}

\section{RESIDUAL PAIN AFTER FRACTURES OF THE PELVIS AND LOWER EXTREMITY: A LONG-TERM ANALYSIS}

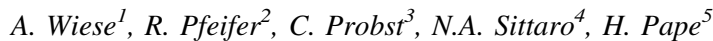

${ }^{1}$ Unfallchirurgie, Universitätsklinikum Aachen, Aachen, Germany, ${ }^{2}$ Klinik Für Orthopädie Und Unfallchirurgie, Schwerpunkt Unfallchirurgie, Universitaetsklinikum Aachen, Aachen, Germany, ${ }^{3}$ Department of Trauma and Orthopaedics, Medizinische Hochschule Hannover, Hannover, Germany, ${ }^{4}$ Hannover Reinsurance, Hannover ReInsurance, Hannover, Germany, ${ }^{5}$ Department of Trauma Surgery, University of Aachen Medical Center, Aachen, Germany

Introduction: Pain after severe trauma of the lower extremity contributes to prolonged rehabilitation, social and professional reintegration. In this study, we examined patients according to their fracture site and residual pain levels more than 10 years after trauma of the pelvis, acetabulum or femur.

Materials and methods: Out of 637 traumatized patients, 49 patients sustained fractures of the pelvis and/or femur who complained about residual pain were re-examined between 2002 and 2003 at the Hanover Medical School. They were interviewed and examined for functional recovery and pain at the respective fracture site. Patients data was analyzed for type of fracture, ISS-score, SF-12-score, HASPOC-score, and Merle d' Aubigné-Postel scores, respectively.

Results: 49 patients complained about residual pain at the pelvis or upper lower extremity. Median age was 32 years ( \pm 11.5 years). The average ISS-score was 21 , SF-12 averaged at $36.8( \pm 9.9)$, HASPOCscore average was $101.95( \pm 54.7)$ and average score of Merle d' Aubigné-Postel-score was $15.1( \pm 4.3)$. 42 of these patients had sustained pelvic fractures (80\%), 11 acetabular fractures $(22 \%), 5$ fractures of the proximal femur (10\%), 13 femoral shaft fractures $(26 \%)$ and 6 fractures of the distal femur (12\%). 39 patients had combined fractures (79\%) and 10 single fractures (21\%).

Conclusion: If combined fractures were present, patients were subjected to residual pain more often compared to single fractures. The data provided indicates that pelvic fracture are most likely to cause long-term pain than other fracture types included in this study. These results are in line with previous findings who also concluded that pelvic trauma is very likely to cause residual pain. Additionally, we found more patients with residual pain who had sustained a femoral 
shaft fractures. This might be due to the fact that femoral shaft fractures were present more often in combined injury patterns.

Disclosure: No significant relationships.

$\mathbf{P 2 3 2}$

\section{SECONDARY PELVIC STABILIZATION AFTER FAILED PRIMARY TREATMENT OF PELVIC FRACTURES}

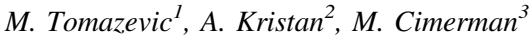

${ }^{1}$ Department of Traumatology, University Medical Centre Ljubljana, Ljubljana, Slovenia, ${ }^{2}$ Department of Traumatology, UMC Ljubljana, Ljubljana, Slovenia, ${ }^{3}$ Traumatology, Univ. Clinical Centre Ljubljana, Ljubljana, Slovenia

Introduction: The function of the pelvis is to transmit forces from axial skeleton to the hip joint. Fractures of the pelvic ring result from high energy trauma. Primary treatment is sometimes insufficient, then secondary stabilization must be done (1).

Materials and methods: 15 consecutive cases of secondary stabilization due to failed primary treatment of pelvic fractures were studied. Secondary stabilization was done at least 2 months after injury and fracture of the pelvis. For evaluation of the stabilization we have used Hannover pelvis scale (HPS). HPS was evaluated after primary treatment and after stabilization was done.

Results: From 15 patients that were treated $8(53 \%)$ patients were primary treated conservatively and $7(47 \%)$ operatively. In eight patients $(53 \%)$ the anterior and posterior pelvis was stabilized in five patients (33\%) the anterior pelvis and in $13 \%$ just posterior pelvis was stabilized. After stabilization average HPS improved by $1.6 / 7$ $(\mathrm{p}<0.000)$ and clinical score by $1.5 / 4(\mathrm{p}<0.000)$, radiologic score improved by $0.1 / 3(\mathrm{p}=0.314)$. We had one complication where symphysiodesis was unsuccessful due to early mobilization and weight bearing. Despite this, osteosynthesis was sufficient and the patient could perform every day activities, but he could not do heavy lifting.

Conclusion: Although there was no significant improvement in radiological outcome after stabilization, the stability of the pelvis and healing brought significant improvement in clinical outcome. The concomitant injuries to the internal organs and neurological deficits could not be improved by stabilization and are still the goal to cure in the future and the burden to carry for the patients.

References: 1. Schatzker J, Tile M. The rationale of operative fracture care. 3rd ed. Berlin: Springer; 2005. . 2. Siegmeth A, Muellner T, Kukla C, Vecsei V. Begleitverletzungen beim schweren Beckentrauma. Der Unfallchirurg 2000;103(7):572-81.

Disclosure: No significant relationships.

\section{P233}

\section{SECONDARY TREATMENT OF SEVERE PELVIC FRACTURES IN POLYTRAUMATIZED PATIENTS CONCERNING PULMONARY COMPLICATIONS}

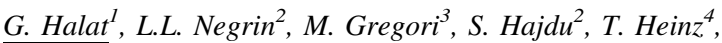 \\ H.K. Widhalm ${ }^{2}$ \\ ${ }^{1}$ Trauma Surgery, Medical University Vienna, Vienna, Austria, \\ ${ }^{2}$ Trauma Surgery, Medical University of Vienna, Vienna, Austria, \\ ${ }^{3}$ Traumatology, Medical University Vienna, Austria, Vienna, Austria, \\ ${ }^{4}$ Trauma Surgery, Med University Vienna, Vienna, Austria
}

Introduction: The development of pulmonary complications in polytraumatized patients plays an important role in the optimal polytrauma treatment. Recent reports showed that acquisition of pulmonary complications is associated with elevated morbidity and mortality rates. The aim of our study was to investigate the causes and the effects of pulmonary complications concerning the date of operative treatment of severe pelvic injuries in polytraumatized patients. Materials and methods: Inclusion criteria for our study were a multiple injured patient with ISS $\geq 16$ and ORIF of the pelvic trauma. Data of 186 polytraumatized patients with severe pelvic trauma were retrospectively collected. 52 patients were selected and separated into 3 groups: Group 1: immediate ORIF Group 2: ORIF within 7 days after admission Group 3: ORIF, later than 7 days after admission We evaluated the cause of injury, as well as the date of operative treatment. Duration of ICU necessity, general stay in hospital and number of ventilator days were assessed. Special attention was paid to the kind of pulmonary complication, amount of complications with cardiac or abdominal origin, thoracic trauma and its AIS code.

Results: Among the 52 operatively treated patients, $21(40 \%)$ underwent secondary ORIF. In $60 \%$ the instable respiratory situation was the cause. Pneumonia was the prevalent complication $(69 \%)$. The most common associated thorax traumatisation was the appearance of rib fractures, with a prevalence of almost $85 \%$ an important contributory factor, followed by lung contusion and pneumothorax. After stabilisation of the respiratory situation these patients underwent ORIF of pelvic fracture at an average of 17 days after trauma. The length of the ICU duration, as well as of ventilator days was increased in secondary treated patients, especially in patients with pulmonary complications.

Conclusion: Pulmonary complications seem to be the most relevant cause of delayed treatment of pelvic fractures in polytraumatized patients.

Disclosure: No significant relationships.

\section{$\mathbf{P 2 3 4}$}

\section{SPHERIC BALL: A NEW 2D NAVIGATION TOOL COMPARED TO 3D NAVIGATION FOR PERIACETABULAR SCREW PLACEMENT}

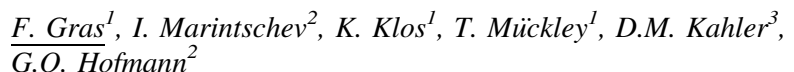

${ }^{1}$ Trauma-, Hand- and Reconstructive Surgery, Friedrich-SchillerUniversity, Jena, Germany, ${ }^{2}$ Department of Trauma-, Hand- and Reconstructive Surgery, Friedrich-Schiller-University Jena, Jena, Germany, ${ }^{3}$ Department of Orthopaedic Surgery, University of Virginia Health System, Charlottesville, USA

Introduction: A new 2D navigation system with a spheric ball tool representing the acetabular joint cavity as "no go zone" was compared to a 3D navigation system for the screw placement in acetabular fracture surgery. We hypothezised that the new 2D navigation procedure is equivalent precise, as the 3D-navigation procedure in preventing intraarticular placement of screws.

Materials and methods: The best $2 \mathrm{D}$ and $3 \mathrm{D}$ navigation images were analyzed for the different osseous corridors: supra-acetabular, anterior column, posterior column; infra-acetabular. Forty guide wires per group were placed in synthetic pelves with a prefabricated soft tissue envelope (10 per group) using a $2 \mathrm{D}$ - or $3 \mathrm{D}$-fluoroscopic navigation procedure. The intraarticular placement rates were evaluated macroscopically and in postoperative 3D scans. Times for different steps of procedure and radiation exposure were measured. 
Results: No intra-articular screw misplacement was observed in both groups, but an increased precision could be achieved using the 3Dbased navigation procedure (perfect placement: 37 vs. 29; secure placement: 2 vs. 7; misplacement: 1 vs. 4). Overall procedure time per pelvis was significantly reduced in the $3 \mathrm{D}$ group compared to the $2 \mathrm{D}$ group $[$ mean $\pm \mathrm{SE} \quad(\mathrm{min}): \quad 50.11 \pm 1.38 \quad$ vs. $\quad 63.42 \pm 2.32$; $\mathrm{p}<0.0001]$. A trend of decrease in image acquisition time $[$ mean $\pm \mathrm{SE}(\min ) 12.37 \pm 1.34$ vs. $15.43 \pm 1.03$, ns), but significant increase in the cumulative fluoroscopy time [mean $\pm \mathrm{SE}$ (s) $64 \pm 9$ vs. $13 \pm 1.3, \mathrm{p}<0.0001$ ) was measured in the $3 \mathrm{D}$ compared to the 2D group, caused by the 3D scan.

Conclusion: Use of both navigation procedures prevents intraarticular screw misplacement, but the determination of the precise entryand exit-points for screw placements was less precise for the $2 \mathrm{D}$ navigation technique (based on summation images) compared to the 3D navigation technique (based on slice images). Furthermore the intraoperative 3D scan, performed for the evaluation of fracture reduction, can be used for the navigation procedure as well, without additional radiation exposure.

Disclosure: No significant relationships.

\section{P234A}

\section{SMALL BOWEL OBSTRUCTION IN PERCUTANEOUS FIXATION OF TRAUMATIC PELVIC FRACTURES.}

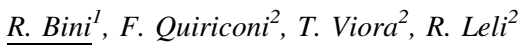

${ }^{1}$ Surgery, Ospedale SG Bosco, ASLTO2, Torino, Turin, Italy, ${ }^{2}$ Surgery, Ospedale SG Bosco ASLTO2, Turin, Italy

Introduction: The use of the external fixator for the initial treatment of unstable and complex pelvic injuries with hemodynamic instability are still a successful treatment of multiply injured patients [1].The authors present a documented case of small bowel entrapment in a sacral fracture stabilized with external fixator.

Materials and methods: A victim of motorcycle accident was admitted to our emergency room with hemodynamic instability. The TC total body showed pelvic fractures of ischiopubic and ileopubic associated with symphyseal disruption. Patient underwent to pelvic fixation with external screw. For abdominal distension we performed a TC scan that showed ileal segment entrapment in the sacral fracture Results: Bowel entrapment in pelvic fractures is rare because, generally, the iliacus and psoas muscles serve as a buffer between the abdominal contents and the posterior pelvic ring injury

Conclusion: Bowel entrapment within a pelvic fracture is a rarely reported but potentially fatal complication [2]. This important diagnosis is rare and difficult to make even with current imaging methods and must be suspected in the presence of postoperative abdominal symptoms especially in patients treated with a percutaneous technique [3].

References: 1. Vilmos V et al. Today's role of external fixation in unstable and complex pelvic fractures. Eur J Trauma Emerg Surg. 2010;36:100-6. 2. Stubbart JR et al. Entrapment within pelvic fractures: a case report and review of the literature. J Orthop Trauma. 1999;13:145-8. 3. Bushnell BD et al. Small bowel obstruction from entrapment in a Sacral fracture stabilized with iliosacral screws: case report and review of the literature. J Trauma. 2008;65:933-7.

Disclosure: No significant relationships.
EMERGENCY SURGERY/VISCERAL TRAUMA

\section{P235}

\section{A STUDY ON DIAPHRAGMATIC TRAUMATIC INJURIES IN IRAN, 2003-2007}

\section{Hosseinpour}

Trauma Research Center, Kashan University of Medical Science, 87196-74591, Iran

Introduction: Diaphragmatic hernias are relatively common after blunt and penetrating traumas. The difficulties in diagnosing traumatic diaphragmatic rupture (TDR) due to coexisting problems and the silent nature of these injuries at the time of initial admission are the common causes of morbidity and mortality.

Materials and methods: Medical records of 34 patients treated for post traumatic diaphragmatic hernias between August 2003 and June 2007 in Al-Zahra Hospital, were studied and data including age, sex, kind and site of trauma, timing and accompanying injuries were recorded and analyzed retrospectively.

Results: There were $28(82.4 \%)$ men and $6(17.6 \%)$ women, with age range of 1-68 years and mean of $32.2 \pm 9.1$. Rupture of diaphragm was left-sided in $22(64.7 \%)$ and right-sided in $11(32.4 \%)$ and both in $1(2.9 \%)$. Blunt trauma accounted for $22(64.7 \%)$ of patients. Elective operation was done in seven $(20.6 \%)$ cases. Diagnosis was established preoperatively in 15 patients $(44.1 \%)$, and intraoperatively in 13 $(38.2 \%)$. The diagnosis was missed in six $(17.7 \%)$ patients in first operation. Strangulation of viscera was found in four patients. The longest interval between trauma and diagnosis was about 3 years in one case. Mean duration of hospital stay was 12.3 days. Multiple associated injuries were observed in 22 patients $(66.7 \%)$. Postoperative complications were encountered in nine (26.5\%) patients, and the overall mortality was $14.7 \%$.

Conclusion: Diaphragmatic hernia should be suspected in all kinds of trauma to thorax and abdomen, and the presence of such an injury should be excluded before terminating the exploratory procedures.

References: Scharff JR. Naunheim KS. Traumatic diaphragmatic injuries. Thorac Surg Clin. 2007;17:81-5. Voeller GR. Reisser JR. Fabian TC, Kudsk K. Mangiante EC. Blunt diaphragm injuries: a fiveyear experience. Am Surg. 1990;56:28-32.

Disclosure: No significant relationships.

\section{P236}

ACUTE ABDOMEN DUE TO INTRALUMINAL TRANSLOCATION OF GASTRIC BAND

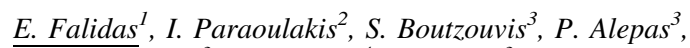
S. Mathioulakis ${ }^{3}$, K. Vlachos ${ }^{4}$, C. Villias ${ }^{3}$

${ }^{1} 1$ st Department of Surgery, 417 Nimts, Veterans Hospital of Athens, Athens, Greece, ${ }^{2}$ Department of General Surgery, Nimts Hospital, Athens, Greece, ${ }^{3} 1$ st Department of General Surgery, Nimts 
Hospital, Athens, Greece, ${ }^{4}$ Department of Surgery, University of Ioannina, Athens, Greece

Introduction: Laparoscopic gastric banding is the most popular surgical procedure for morbid obesity. Long term complication such as slippage, infection and gastric erosion may occur. We report a case of acute abdomen due to intraluminal migration of gastric band.

Materials and methods: A 38 year-old woman arrived to the ED referring acute abdominal pain and projectile vomiting $24 \mathrm{~h}$ lasting. She had a history of laparoscopic gastric banding 3 years ago, while 4 months ago she underwent gastroscopy demonstrating a partial erosion of the gastric wall caused by the band although she refused any further treatment. Ten days ago, she presented projectile vomiting particular evident in upright position. Physical examination revealed diffuse abdominal pain mainly located to the epigastium. Laboratory findings, $\mathrm{x}$-series and ultrasound of the abdomen revealed gastric perforation.

Results: Upon surgical exploration, the gastric band was identified into the gastric lumen, easily movable. Gastrotomy was performed and the band was removed. Omentum was posted on the gastric wall in order to tamponate the point of gastric perforation. Post operative course was uneventful

Conclusion: Erosion of the stomach caused by a gastric band is uncommon but not rare and it usually happens after a mean of 2 years. Total migration of the gastric band into the lumen is a rare event and should be kept in mind in cases of acute abdomen and projectile vomiting in patients with a previous gastric banding.

References:

Suter $\mathrm{M}$ et al. Band erosion after laparoscopic banding: occurrence and results after conversion to Roux-en-Y gastric bypass. Obes Surg. 2004;14(3):381-6.

Disclosure: No significant relationships.

\section{$\mathbf{P 2 3 7}$}

\section{ACUTE PANCREATITIS AND ABDOMINAL COMPARTMENT SYNDROME}

\author{
A.C. Ezanno $^{1}$, M.P. Massoure ${ }^{2}$, C. de Saint Roman ${ }^{1}$, E. Schaeffer ${ }^{3}$, \\ K. Fixot $^{I}, P$. Sockeel ${ }^{1}$
}

${ }^{1}$ General Surgery, HIA Legouest, Metz Cedex 3, France, ${ }^{2}$ Digestive Pathology, HIA Legouest, Metz Cedex 3, France, ${ }^{3}$ Réanimation, HIA Legouest, Metz, France

Introduction: The abdominal compartment syndrome (ACS) is initially described in severe abdominal trauma. But now it is also described in Medical conditions such as severe acute pancreatitis (SAP).

Materials and methods: Six patients were included. Diagnostic was made using intra-vesical pressure, occurring of an organ failure.

Results: Six males were included with an average age of 53.6 years (40-74) who presented a ACS during a SAP. Four patients had a discharge aponeurotomy by laparotomy. Only one was radiologically drained and the last one died before any intervention. The laparostomy was closed down after patient stabilisation, and average of 6 days after initial surgery. After a minimum of 6 months no patients presented an eventration.

Conclusion: The ACS had recently got a lot of attention in the world of critical care. Unfortunately treatment of SAP is based on large fluid resuscitation, which is also a major cause of ACS. ACS which has both local and systemic consequences is often misregarded, because not diagnose and the symptoms can be thought as the consequence of another condition. ACS management often call for a surgical procedure even if various non surgical procedure are described. Many questions still arise in the absence of a consensus: what patient shall have his intra-vesical pressure measured? When to perform aponeurotomy and what procedure shall be used?

Disclosure: No significant relationships.

\section{P238}

\section{ATTITUDE IN RETROPERITONEAL HAEMATOMA FOLLOWING ABDOMINAL TRAUMA}

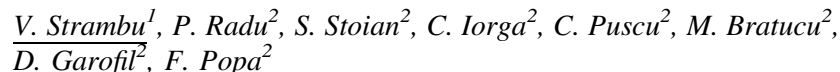

${ }^{1}$ General Surgery, St Pantelimon Hospital, Bucharest, Romania, ${ }^{2}$ General Surgery, St Pantelimon Hospital, Bucharest, Romania

Introduction: Retroperitoneal haematoma is relatively frequent following abdominal trauma and very ofen is difficult to manage.

Materials and methods: 98 consecutive patients found to have a retroperitoneal haematoma $(\mathrm{RH})$ at laparotomy for abdominal trauma over a 5 -year period is reviewed.

Results: Three types of retroperitoneal haematoma are recognized: Type I (central), Type II (flank) and Type III (pelvic).Type I RH was present in 20 patients as a result of injuries to the inferior vena cava, the aorta, the pancreas and duodenum, the portal vein, and to both aorta and inferior vena cava. Fourteen patients died (63.8\%) representing $70 \%$ of all deaths in the series. Type II RH was seen in 69 patients. Thirty-two were stable and the perinephric fascia was not opened with no morbidity or mortality. 39 patients was explored: 14 on pre-operative evidence of renal injury which was confirmed at laparotomy. In the remaining 25 a stable haematoma was explored and no significant lesion found. It is considered that in 45 of 69 Type II RH $(61.0 \%)$ exploration was unnecessary. Five patients died (6.8\%). Type III RH was found in nine patients and was due to iliac vessel injuries and pelvic fractures. Two patients died.

Conclusion: Type I and III RH should be routinely explored. Stable Type II RH discovered at laparotomy should be explored selectively based on pre-operative clinical and radiological assessment of renal injury.

Disclosure: No significant relationships.

\section{P239}

\section{BIOLOGIC MESH IMPLANTS IN COMPLICATED HERNIA AND LAPAROCELIC EMERGENCY SURGERY}

\author{
D. Venditti ${ }^{l}$, V. Balassone ${ }^{l}$, D. D'Andria ${ }^{1}$, G. Sena ${ }^{2}$, C.H. Mazzetti ${ }^{l}$, \\ O.C. Buonomo ${ }^{I}$, G. Petrella ${ }^{2}$
}

${ }^{1}$ Emergency Surgery, Breast Surgery and Transplants, Fondazione Universitaria Policlinico Tor Vergata, Rome, Italy, ${ }^{2}$ Emergency Surgery, Breast Surgery and Transplants, Fondazione Universitaria Policlinico Tor Vergata, Rome, Italy

Introduction: The purpose of this work is to report our experience with Permacol in emergency surgery of abdominal hernia.

Materials and methods: Patients: We reviewed retrospectively data about our patients from 2006 to 2009: 12 patients affected by strangulated inguinal hernia. Mean age was $63.9 \pm 14.24$ SD. Five were females and seven were males. One umbilical strangulated hernia in a female of 73 years old. Five strangulated laparoceles four female and one males. Mean age was $71.16 \pm 2.78$ SD. All patients underwent bowel resection for ischemia or necrosis. 
Inguinal hernias were treated by a Lichtenstein's inguinal mesh repair with Permacol. Laparocele and umbilical hernia were treated with laparotomic access and stapled resection-anastomosis. A mesh of Permacol was used too (2 retro-muscular and 4 subcutaneous). Drains were apposed.

Results: Two wound's infections successfully treated via 3 weeks of VAC therapy. One subcutaneous seroma treated by aspiration. No deaths. No mesh removal. Absence of recurrence at 18 months of follow up. Mean hospitalization of $6.33 \pm 2.3$ SD.

Conclusion: We underline the safety of bio-compatible implants in emergency hernia and laparocele repair.

References: Shaikh FM, Giri SK, Durrani S, Waldron D et al. Experience with porcine acellular dermal collagen implant in onestage tension-free reconstruction of acute and chronic abdominal wall defects. World J Surg. 2007. Liyanage SH, Purohit GS, Frye JNR et al. Anterior abdominal wall reconstruction with a Permacol implant. J Plastic. 2006.

Disclosure: No significant relationships.

\section{P240}

\section{CONSERVATIVE SURGERY OF ESOPHAGEAL PERFORATION: 2 CASE REPORT}

A. Paz-Yánez, M.J. Alvarez-Martin, N. Muñoz-Pérez, T. Torres, J. Martin, J.D. Turiñ luque, S. Gil, J.A. Ferrón

General and Digestive Surgery, Virgen de las Nieves University Hospital, Granada, Spain

Introduction: Report two cases of esophageal perforation managed with conservative surgery

Materials and methods: CASE 1: 64-years-old man with medical history of dilatation of postchemotherapy cicatricial esophageal estenosis 6 days ago, due to a epidermoid carcinoma stage IV. Sudden thoracic pain and fever. BloodTest: WCC 14,230/ $\mu \mathrm{L}$ PMN 89.3\%; $\mathrm{Hb}: 8.7 \mathrm{~g} / \mathrm{dL}$. VBG: $\mathrm{pH} 7.37 ; \mathrm{HCO}_{3}{ }^{-} 22.1 \mathrm{mmol} / \mathrm{L}$ (GAP $4.3 \mathrm{mmol} /$ L); lact. $3.5 \mathrm{meq} / \mathrm{L}$. Thoracoabdominal-CT: widened mediastinum with gas and material inside without leakage of oral contrast. Emergency surgery by midline laparotomy with Pinotti access. Important mediastinitis in mid and inferior esophagus. Abundant clean and mediastinum and left hemitorax drainage, plus feeding jejunostomy and intraoperative endoscopic coated prosthesis. CASE 2: 45-yearsold man with sudden severe epigastric abdominal pain alter an episode of nausea and vomiting. No fever. Diffuse peritonism, taquicardia and hypoventilation of left lung base. BloodTest: creatinine $2.11 \mathrm{mg} / \mathrm{dL}$; urea $151 \mathrm{mg} / \mathrm{dL}$; LDH $645 \mathrm{IU} / \mathrm{L}$. VBG: pH 7.24; $\mathrm{HCO}_{3}{ }^{-} 22.1 \mathrm{mmol} / \mathrm{L}$ (GAP $6.8 \mathrm{mmol} / \mathrm{L}$ ) lact. $5 \mathrm{meq} / \mathrm{L}$. Thoracoabdominal-CT: Distal esophagus perforation with nutritional content outside esophageal lumen. Emergency surgery with Boerhaaves's syndrome by midline laparotomy. After Pinotti access, $7 \mathrm{~cm}$ longitudinal posterolateral perforation $5 \mathrm{~cm}$ above the gastroesophageal junction. Abundant clean, primary suture and gastric fundus plasty (Thal's plasty), with mediastinum and both hemithorax drainage and feeding.

Results: Case 1: 15 days stay ICU due to severe sepsis. Discharge after other 15 days in hospitalization with normal blood-test and radiologic exams and oral feeding restoration. Case 2: Discharge after 10 days: normalization of blood and radiologic test and oral feeding restoration.

Conclusion: Early diagnosis and treatment (within the first 12-24 h) of esophageal perforations allows a conservative surgery, if edges of lesion are viable and no underlying tumor pathology (irresectable tumor, coated endoprotesis is the most suitable option) Distal esophageal perforation requires a wide Pinotti access to exposure the break line and correct clean, debridement and drainage of the mediastinum.

Disclosure: No significant relationships.

P241

\section{DELAYED DIAGNOSIS OF TRAUMATIC DIAPHRAGMATIC HERNIA: OCCULT MARKER OF SERIOUS MORBIDITY}

M. Anastasiu, R. Popescu, N. Micu, C. Popa, A. Ivan, R. Dedu

Emergency and General Surgery, Emergency County Hospital of Buzau, Buzau, Romania

Introduction: Acute traumatic rupture of the diaphragm, usually associated with abdominal injuries, may go unnoticed and there is often a delay between the initial trauma and the diagnosis.

Materials and methods: A review of our past 10 years of all posttraumatic diaphragmatic hernias is analyzed. Our study intends to emphasize the incidence of missed diaphragmatic injuries on initial evaluation, to identify factors contributing to delay the diagnosis and to discuss the surgical procedures performed.

Results: During the specified period, 22 patients with diaphragmatic rupture were admitted in our service. Fourteen patients (64\%) required urgent intervention and five patients were operated within $24 \mathrm{~h}$. Three of $22(13.6 \%)$ diaphragmatic injuries were missed on initial evaluation. Recognition followed 4-10 days later. In our series, the blunt trauma is noted in $86 \%$ cases and $82 \%$ of the ruptures were on the left side. Two diaphragmatic injuries initially presented a normal chest X-ray. Associated injuries were noted in 19 cases $(86 \%)$. Twenty patients underwent repair by laparotomy and two required thoracotomy. The repair with polypropylene mesh was performed in $18 \%$ of cases. In one case with delayed diagnosis a tension fecopneumothorax following diaphragmatic hernia perforation in pleural cavity is a particularly and severe complication. There are three deaths $(13 \%)$ caused by hemorrhagic shock and multisystem organ failure.

Conclusion: Incorrect interpretation of imagistic findings and misinterpretation of clinical features are frequent reasons for a delayed diagnosis in traumatic diaphragmatic hernias. High morbidity and mortality were related primarily to associated injuries.

References: Sirbu $\mathrm{H}$ et al. Late bilateral diaphragmatic rupture: challenging diagnostic and surgical repair. Hernia 2005;9(1):90-2. Sangster G et al. Diaphragmatic rupture: a frequently missed injury in blunt thoracoabdominal patients. Emerg Radiol. 2007;13(5):225-30. Disclosure: No significant relationships.

\section{P242}

\section{DIAGNOSTIC AND ATTITUDE IN SMALL BOWELL CANCER}

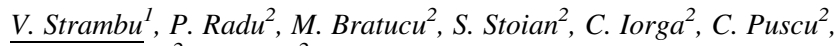
V. Georgescu ${ }^{2}, F$. Popa ${ }^{2}$

${ }^{1}$ General Surgery, St Pantelimon Hospital, Bucharest, Romania, ${ }^{2}$ General Surgery, St Pantelimon Hospital, Bucharest, Romania

Introduction: Primary small bowel cancer is rare. In many cases the diagnosis is difficult, especially in duodenum and jejunum, confirmed in advanced stages with poor prognosis. This paper is intended to clarify the clinical characteristics of primary small bowel cancer. 
Materials and methods: We have reviewed a number of 34 patients with small bowel cancer admitted and operated in our department in the last 6 years. The clinical features, diagnostic method, location, histologic findings of tumour and prognosis were analysed.

Results: The ratio male/female was 1:2.1. The average age was 55 years and in our group the most prevalent age was in sixth decades. The most common site was jejunum with 25 cases, followed by duodenum in 5 cases and ileum in 4 cases. Leyomiosarcoma occurred most frequent in jejunum, adenocarcinoma in duodenum and lymphoma in ileum. The most common symptom was abdominal pain in $70 \%$ of cases. Followed by anemia in $51.5 \%$ cases, and palpable abdominal mass in $43 \%$ of cases. The accuracy rate of preoperative diagnosis or suspicion was about $40 \%$. Diagnosis features used was duodenal endoscopy, abdominal CT for jejunum and ileum. Curative resection was performed in 21 cases, in cases with leiomyoma curative surgery being the most used procedure.

Conclusion: Frequently, small bowel cancer diagnosis was difficult, confirmed in advanced stages. High degree of suspicion, and more endeavor to discover is important in prognosis of these patients. Disclosure: No significant relationships.

\section{P243}

\section{ERRORS CONTRIBUTING TO TRAUMA MORTALITY IN A RUMANIAN LEVEL I TRAUMA CENTER}

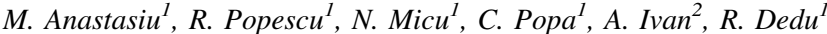

${ }^{1}$ Emergency And General Surgery, Emergency County Hospital of Buzau, Buzau, Romania, ${ }^{2}$ Department of General Surgery, Emergency Departmental Hospital of Buzau, Buzau, Romania

Introduction: The management of acute trauma is complex and dynamic. This study was conducted to identify the incidence, type and setting of errors leading to mortality in trauma patients and to purpose an error-reduction strategies.

Materials and methods: All trauma patients that died during their hospital admission in a period of 5 years (2005-2009) were included in study and analyzed. Data were collected with weekly mortality reports and all deaths were reviewed (by type of errors and phase of care) and were classified into nonpreventable, potentially preventable and preventable deaths. Each case reported was tabulated into next categories: failure to intubate, delay in diagnoses, missed injuries, over resuscitation with fluids and catheters complications.

Results: Between 2005 and 2009, were registered 12,260 trauma patients and resulted 637 deaths (5.2\% of admissions). Twenty-two patients $(0.2 \%$ admissions, $3.5 \%$ deaths $)$ had recognized errors in diagnoses and treatment that contributed to their death. Twelve of them $(54.5 \%$ of deaths) were classified as preventable or potentially preventable deaths. Mean age was 42 years and $82 \%$ were blunt injuries. The major clinical errors included: failure in hemorrhage control (25\%), inappropriate airway management (16.7\%), missed or delayed diagnoses and treatment (33.3\%), complications of procedures $(8.3 \%)$ and over-resuscitation with fluids (16.7\%).

Conclusion: Despite the dramatically changes in trauma therapy, more than half of all deaths are preventable or potentially preventable. Delay in treatment, missed injuries and clinical judgment error are the leading causes of this reality.

Reference: Gedeborg R. The impact of clinically undiagnosed injuries on survival estimates. Crit Care Med. 2009;37:449.

Disclosure: No significant relationships.

\section{P244}

\section{FACTORS ASSOCIATED TO SURGICAL SITE INFECTION IN SECONDARY PERITONITIS: SEVERITY SCORES AND ADEQUATE ANTIBIOTIC TREATMENT}

\author{
E. Membrilla Fernández ${ }^{1}$, J. Sancho ${ }^{2}$, M. Girvent Montllor ${ }^{3}$, \\ G. Adrio ${ }^{4}$, D. Fraccalvieri ${ }^{5}$, J.J. Iniggo Noain ${ }^{3}$, A. Monzón ${ }^{3}$, \\ A. Luna Aufroy ${ }^{3}$, A. Sitges Serra ${ }^{3}$
}

${ }^{1}$ General Surgery and Digestive, Hospital Universitario del Mar, Barcelona, Spain, ${ }^{2}$ Servicio de Cirugía General y Digestiva, Hospital del Mar, Barcelona, Spain, ${ }^{3}$ Hospital del Mar, Barcelona, Spain, ${ }^{4}$ Hospital Arquictecto Manrique, El Ferrol, Spain, ${ }^{5}$ General Surgery, University of Barcelona, Barcelona, Spain

Introduction: Surgical site infections (SSI) are an important reason of morbimortality in secondary peritonitis. The following study analyses its relation to adequate empirical antibiotic treatment.

Materials and methods: An open observational prospective multicentric study at 24 Spanish Hospitals (04/2005-08/2007). There were 362 patients with secondary peritonitis; $262(72.4 \%)$ with communityacquired peritonitis and $100(27.6 \%)$ with postoperative peritonitis (POP). Patients were only included if at least two quadrants of the abdomen were affected and with a positive peritoneal culture. Severity scores, origin of peritonitis, surgical technique, genus, species and microbial sensitivity to antibiotics, (SSI) and mortality were recorded. Results: 164 women and 198 men were included. SSI was the commonest septic complication $(45 \% ; \mathrm{n}=163)$ and more prevalent in POP (64 vs. $38 \%$; $\mathrm{P}=0.0001)$. SSI was superficial in 125 patients (35\%), deep in $25(75 \%)$ and organ/space in $52(14 \%)$. POP suffered more superficial SSI (48 vs. $29 \% ; \mathrm{P}=0.001$ ) and organ/space SSI ( 24 vs. $11 \% ; \mathrm{P}=0.002$ ). Patients with SSI had higher ASA III/IV (48.5 vs. $31.6 \% ; \mathrm{P}=0.0012$ ), worst McCabe\&Jackson score (30 vs. $19 \% ; \mathrm{P}=0.026)$ and higher Mannheim Peritonitis Index (27 \pm 7 vs. $23 \pm 7 ; \mathrm{P}=0.009$ ). Insufficient source control was related to more SSI (69 vs. $43 \%$; $\mathrm{P}=0.006$ ). Deep SSI and organ/space SSI had more mortality ( 28 vs. $6.6 \% ; \mathrm{P}=0.002$ and 17.6 vs. $6.5 ; \mathrm{P}=0.012$ respectively). Empiric antibiotic treatment (EAT) was adequate in $61 \%$ of patients. Inadequate EAT was more prevalent in POP (35 vs. $49 \% ; \mathrm{P}=0.011$ ). Adequate EAT was associated to lower SSI (40 vs. $53 \% ; \mathrm{P}=0,015)$ both in superficial $(\mathrm{P}=0.038)$ and organ-space $(\mathrm{P}=0.040)$. In patients with effective source control $(\mathrm{n}=333$; 92\%), the relationship between SSI and inadequate EAT becomes more significant $(\mathrm{P}=0.011)$. The various antibiotic regimes had a measurable effect on SSI.

Conclusion: The SSI after secondary peritonitis is related to higher mortality levels. The factors associated to SSI were the severity scores and the inadequate empiric antibiotic therapy.

Disclosure: No significant relationships.

\section{P245}

\section{INTRA-ABDOMINAL SOLID VISCERAL WOUNDS IN PATIENTS WITH MULTIPLE INJURIES IN A TERTIARY HOSPITAL OVER 11 YEARS: COMPARISON OF TWO TIME PERIODS}

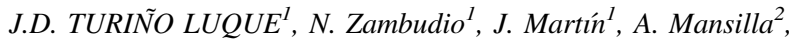 T. TORRES ${ }^{3}$, J.A. FERRÓN ${ }^{3}$}

${ }^{1}$ General And Digestive Surgery, "Virgen de las Nieves" University Hospital, Granada, Spain, ${ }^{2}$ General And Digestive Surgery, Granada, 
Spain, ${ }^{3}$ General And Digestive Surgery, Virgen de las Nieves University Hospital, Granada, Spain

Introduction: Abdominal pathology is one of the most frequent reasons why people attend to the Emergency Room. A small percentage of them are due to trauma causes. Objectives. To assess the incidence of multiple trauma patients admitted in our hospital during an 11-year period by analyzing the etiology of solid organ injuries and the evolution of treatment.

Materials and methods: We reviewed the diagnosis of multiple trauma patients admitted to our emergency room by conducting a search on records from the period between January 1999 to December 2009. The information collected from the hospital database included: age, sex, etiology, abdominal injury, treatment, hospital stay, diagnostic tests, morbidity and mortality.

Results: A total of 790 patients were analyzed. The male to female ratio was 4:1. The mean age was $34.1(0-93)$. The leading causes of injury were: car accident $33.2 \%(\mathrm{n}=262)$, accidental fall $16.8 \%$ $(\mathrm{n}=133)$ and $15.3 \%$ motorcycle accident $(\mathrm{n}=121)$. Mortality amounted to $13.5 \%(\mathrm{n}=107) .611$ patients had abdominal injuries $(77.3 \%)$ that affected: spleen in $42.2 \%(\mathrm{n}=258)$, liver in $29.3 \%$ $(\mathrm{n}=179)$ and pancreas in $2.9 \%(\mathrm{n}=18) .57 .4 \%$ of patients underwent surgery $(\mathrm{n}=351)$ and of these $84.3 \%$ were emergency surgeries $(\mathrm{n}=296) .77$ patients were splenectomized, in 48 patients surgery was conservative and the remaining 133 patients received medical treatment. If we compare the 1999-2004 period with the 2005-2009 period we can observe a decreasing number of splenectomies (36 vs. $21.3 \%, \mathrm{p}=0.016)$. A total of 93 patients died $(15.2 \%)$, without differences between two periods $(\mathrm{p}=0.735)$.The cause of death included: neurological complications (23.7\%), lung disease $(15 \%)$ and abdominal injuries (11.8\%).

Conclusion: Car accidents are the main cause of abdominal injury in trauma patients. The spleen is the most common solid organ injured. There was a statistically significant decrease in the number of splenectomies comparing the two periods.

Disclosure: No significant relationships.

\section{P246}

\section{OUR EXPERIENCE WITH PURULENT PERITONITIS DUE TO PERFORATED APPENDICITIS}

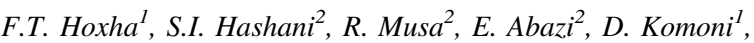

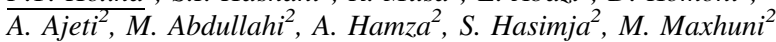

${ }^{1}$ Surgery Department, UCCK, Prishtina, Albania, ${ }^{2}$ UCCK, Prishtina, Albania

Introduction: Despite the improvements in the diagnosis and treatment, perforated rates with purulent peritonitis are still high following urgent operation for appendicitis.

Materials and methods: A retrospective study of a 2-year period, 2008-2009, identified 1,719 patients who underwent conventional emergency appendectomy. Patients were examined for age, sex, histopathological diagnosis, intra operative findings and postoperative complications.

Results: Male gender was presented with $57.53 \%$, mean age of whole group was 59 (range 2-84) years. Generalized purulent peritonitis was recorded in $18.25 \%$ of operated patients suspicious for appendicitis. At operation, perforation was detected in $29.18 \%$. Re intervention due to perforated appendicitis we had in $2.8 \%$ during follow-up, caused mainly cause by abscesses and adhesions (ileus). The overall negative appendectomy rate was $10.43 \%$, at female patients was $17.49 \%$, with a gynecological pathology, including ovarian cysts, pelvic inflammatory diseases.

Conclusion: Delay seeking physicians and delay referral from pre hospital care, a low operative threshold and prolonged observation may delay diagnosis and proper surgical treatment with high rate of complicated perforated appendicitis with purulent peritonitis.

References: Körner H, Söreide JA, Pedersen EJ, Bru T, Söndenaa K, Vatten L. Stability in incidence of acute appendicitis. A population based longitudinal study. Dig Surg 2001; 18:61-6. Humes DJ, Simpson J. Acute appendicitis. BMJ 2006;333:530-4. Ma KW, Chia $\mathrm{NH}$, Yeung HW, Cheung MT. If not appendicitis, then what else can it be? A retrospective review of 1492 appendectomies Hong Kong Med J. 2010;16:12-7.

Disclosure: No significant relationships.

\section{P247}

\section{PENETRATING ABDOMINAL TRAUMA. OUR EXPERIENCE}

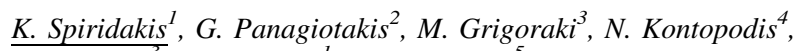
F. Psarakis ${ }^{3}$, I. Volakakis $^{1}$, T. Kokkinakis ${ }^{5}$

${ }^{1} 1$ st Surgical, Venizelion Hospital, Heraklion, Greece, ${ }^{2} 1$ st Surgical Department, Venizelio Hospital, Heraklion Crete, Greece, ${ }^{3} 1 \mathrm{st}$ Surgical, Venizeleion Hospital, Heraklion, Greece, ${ }^{4} 1$ st Surgical, Venizeleion Hoaspital, Heraklion Crete, Greece, ${ }^{5} 1$ st Surgical, Venizeleion Hospital, Heraklion Crete, Greece

Introduction: About $8 \%$ of trauma deaths annually, result from penetrating mechanism. Such injuries may be caused by gunshots, stub wounds and road traffic accidents. The aim of this study is to analyze and evaluate the nature of injuries, the appropriate surgical management and the prognosis of penetrating abdominal trauma based on our experience.

Materials and methods: In the period 2007-2009, 25 patients with penetrating abdominal trauma were admitted to the surgical emergency department of our hospital. 15 patients were male and 10 were female, with average age of 44 years. 14 patients reached the hospital within the first $2 \mathrm{~h}$ after the injury and 11 patients reached the hospital within 2-4 h after the injury. The causes of penetrating abdominal trauma were: gunshots in 4 patients $(16 \%)$, stab wounds in 7 patients $(28 \%)$, motor vehicle collisions in 14 patients $(56 \%)$. After initial resuscitation, laparotomy was performed in 23 patients. In 2 patients with stab wounds in the abdominal region, the treatment was conservative.

Results: In 15 patients there were associated injuries of two or more organs. We analyzed the surgical management and the results were: right hemicolectomy, hepatorrhaphy and packing, splenectomy, nephrectomy, and Hartmann's procedure. Postoperative complications included wound infection in five patients and fecal fistula in three patients. Three patients $(12 \%)$ died during operation or early postoperatively due to severe multiple organ failure. The average hospital stay duration was 36 days.

Conclusion: The care of the trauma patient demands and requires speed and efficiency. Explorative laparotomy for patients with penetrating abdominal trauma proves to be secure and can diminish mortality.

Disclosure: No significant relationships. 


\section{P248}

\section{PMN MOBILISATION FROM THE MARGINATED POOL DURING THE STRESS RESPONSE INDUCED BY TRAUMA}

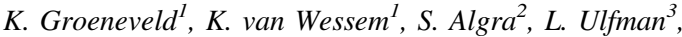
B. Prakken ${ }^{2}$, K. Jansen ${ }^{2}$, L. Leenen ${ }^{4}$, L. Koenderman ${ }^{3}$

${ }^{1}$ Surgery, UMC Utrecht, Utrecht, Netherlands, ${ }^{2}$ Pediatrics, UMC Utrecht, Utrecht, Netherlands, ${ }^{3}$ Respiratory Medicine, UMC Utrecht, Utrecht, Netherlands, ${ }^{4}$ Trauma/Surgery, University Medical Center Utrecht, Utrecht, Netherlands

Introduction: Trauma patients develop an acute (temporary) leukocytosis and neutrophilia, which is likely mediated by stress hormones, such as (plasma) cortisol and epinephrine. Hypothermia on the other hand, can cause a reduction in numbers of circulating leukocytes. We hypothesized that the initial stress response after trauma is the main initiator of release of leukocytes and neutrophils from the marginating pool and hypothermia will shift this process towards more retention in this pool.

Materials and methods: We tested the hypothesis in infants ( $<1$ month of age) undergoing cardiac surgery during deep hypothermia (DHCA) as a trauma model. The results were compared to a rodent trauma model. The leukocyte and neutrophil counts and the presence of functional neutrophil phenotypes were the primary and secondary outcomes, respectively.

Results: The hypothermic phase during DHCA was characterized by a marked decrease in neutrophil counts. These peripheral neutrophils had a decrease in L-selectin and increase in MAC-1 expression. Rewarming lead to an opposite neutrophil phenotype (L-selectin ${ }^{\text {bright/ }}$ $\mathrm{CD} 11 \mathrm{~b}^{\mathrm{dim}}$ ) that was shared by neutrophils mobilized from the marginated pool by treatment with high doses of steroids. Similar data were found in the rodent model.

Conclusion: Hypothermia and stress hormones have opposing effects on the release of PMNs from and sequestration to the marginated pool.

Disclosure: No significant relationships.

\section{P249}

\section{PROGNOSIS AND TREATMENT OF PANCREATICODUODENAL TRAUMATIC INJURIES: WHICH FACTORS ARE PREDICTORS OF OUTCOME?}

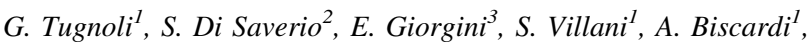

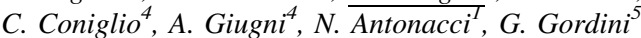

${ }^{1}$ Emergency and Trauma Surgery Unit, Maggiore Hospital Trauma Center, Bologna Local Health District, Bologna, Italy, ${ }^{2}$ Surgery, Emergency and Trauma Surgery Unit, Maggiore Hospital Trauma Center, Bologna, Italy, ${ }^{3}$ Emergency and Trauma Surgery Unit (Head Dr. G. Tugnoli), Maggiore Hospital Trauma Center, Bologna Local Health District, Bologna, Italy, ${ }^{4}$ Trauma ICU, Department of Emergency, Maggiore Hospital Trauma Center, Bologna Local Health District, Bologna, Italy, ${ }^{5}$ Trauma ICU, Department of Emergency (Head Dr. G. Gordini), Maggiore Hospital Trauma Center, Bologna Local Health District, Bologna, Italy

Introduction: Abdominal trauma rarely causes injuries involving the duodenum and pancreas. Associated injuries occur in $46 \%$ of all pancreatic injuries. The morbidity and mortality of pancreaticoduodenal injuries remain high.

Materials and methods: The present study is retrospective review of our experience from 1989 to 2008 in the surgical treatment of traumatic pancreaticoduodenal injuries. Mortality, morbidity, prognostic factors, and the value of surgical techniques were analyzed.

Results: In our level I Trauma Center, between 1989 and 2008, 55 patients had a pancreaticoduodenal injury. In $68.5 \%$ of cases pancreatic injuries were found, $20.4 \%$ had duodenal injury, and $11.1 \%$ suffered combined pancreaticoduodenal injuries; $85.3 \%$ of the patients had blunt abdominal trauma, while $14.9 \%$ had penetrating injuries. We treated $78.1 \%$ of the patients with external drainage and/ or simple suture; distal pancreatectomy was performed in $9 \%$ of cases and duodenal resection with anastomosis $(3.7 \%)$ and diversion procedures $(3.7 \%)$ were performed in an equal number of patients. Age, American Association for the Surgery of Trauma (AAST) grade, organ involved, hemodynamic status, intraoperative cardiac arrest, and operative time remained strongly predictive of mortality on multivariate analysis. The AAST grade represented, on multivariate analysis, the only independent prognostic factor predictive of overall morbidity. In the past decade we have used feeding jejunostomy more frequently, with a reduction of mortality and operating time, due also to better approach from a dedicated trauma team.

Conclusion: Optimal management and better outcome of pancreaticoduodenal injuries seem to be associated with shorter operative time, and with simple and fast damage control surgery (DCS), in contrast to definitive surgical procedures.

Disclosure: No significant relationships.

\section{P250}

\section{SOME DATA ABOUT THE EPIDEMIOLOGY OF COLORECTAL TRAUMA IN ALBANIA}

\author{
M. Torba ${ }^{1}$, A. Gjata ${ }^{2}$, S. Buci ${ }^{2}$, S. Koceku ${ }^{2}$, R. Madani ${ }^{2}$, \\ A. LiLa $^{2}$, E. Faber ${ }^{2}$, K. Subashi ${ }^{2}$
}

${ }^{1}$ Kirurgji, Spitali Ushtarak Qendror universitar, Tirane, Albania, ${ }^{2}$ Kirurgji, Spitali Ushtarak Qendror Universitar, Tirane, Albania

Introduction: The aim of our study was to give some data about the epidemiology of colorectal trauma in our country.

Materials and methods: We studied 138 patients which were present at the National Trauma Center with colorectal trauma during January 2008-October 2010. The data are taken from clinical documentation. We have analyzed the numerical and categorical variable, their relation of dependence.

Results: The patients' age was from 13 to 80 years. Average age was: $32.2 \pm 11.7$ years, males were $117(84.7 \%)$ cases; female 21 (15.2\%); ratio $\mathrm{M} / \mathrm{F}$ 5.5:1. Colorectal trauma according to the anatomic section; right colon is found injured in $25.3 \%$ of cases, transverse colon in $26 \%$ of cases, the left colon $8.6 \%$, sigma to $12.3 \%$ of cases, rectum in $15.9 \%$ of cases and injury of two or three different part of colon together in $11.9 \%$ of cases. The only damage of colony was encountered in $27.8 \%$ of cases and combined with other organs in $72.2 \%$ of cases. We have used primary repair in $26.4 \%$ of cases; hemicolectomy to $5 \%$ of cases; exteriorization of the injury of colon in $3.6 \%$ of cases, Hartman procedure in $57.9 \%$ of cases; primary repair with ileostomy in $14 \%$ of cases; colectomy with ileostomy in $5.7 \%$ of cases. Morbidity: incisional wound infection $11.5 \%$, dehiscence $2.1 \%$; intestinal occlusion $3.5 \%$; intra-abdominal abscess $2.8 \%$; recto-vaginal fistula $1.4 \%$; vesico-rectal fistula $2.1 \%$; parastomal hernia $2.1 \%$; fecal incontinence $1.4 \%$. Mortality $7.59 \%$, because of complications such as: hemorrhagic shock, coagulopatia, toxico-septic status and multi system organ failure.

Conclusion: Colorectal trauma in Albania is increasing. Colorectal traumas associated with other injuries have a high mortality. The time and the rate of contamination of abdominal cavity play an important 
role in prognosis. Colostomia protection should be considered in surgical treatment of rectum and anal canal.

Disclosure: No significant relationships.

\section{$\mathbf{P 2 5 1}$}

\section{SURGICAL TREATMENT PERFORATION AND OBSTRUCTION CARCINOMA OF THE COLON}

\author{
S.D. Sekulic $^{1}$, A.S. Sekulic-Frkovic ${ }^{2}$ \\ ${ }^{1}$ Surgical Clinic, C.H.C.Pristina-Gracanica, Gracanica, Serbia, \\ ${ }^{2}$ Pediatric Clinic, C.H.C.Pristina-Gracanica, Gracanica, Serbia
}

Introduction: Colonic perforation is an abdominal emergency stable with a high morbidity and mortality. In carcinoma of the colon and rectum, obstruction and perforation may occur either alone or together at the site of the neoplasm, proximally. The aim of the surgical intervention must be prevention of this complications, removing the septic focus.

Materials and methods: We present 258 patients with malignant obstruction of the colon and rectum, operated at the Surgical Clinic, Clinical Center Pristina-Gracanica, Medical Faculty PristinaKos.Mitrovica, during the period between 1995 and 2009. Perforation carcinoma of the colon was found in $29(11.2 \%)$ cases.

Results: Patients were operated after a short intensive preparation. Radical procedures were performed in $22(75.8 \%)$ and palliative in 7 $(24.2 \%)$ patients. There were $16(55.2 \%)$ males and $13(44.8 \%)$ females patients. In $15(51.7 \%)$ of them perforation has found to be covered, and in $14(48.3 \%)$ cases perforation has been free. Nine $(31.03 \%)$ patients had diffuse peritonitis. Resection with primary anastomosis was performed in $12(41.3 \%)$ cases. Resection without restoration of the intestinal continuity-Hartamann's-in ten $(34.5 \%)$ and colostomy and drainage in seven $(24.1 \%)$ patients. Postoperative complications occurred in nine $(31.03 \%)$ and mortality in five $(17,24 \%)$ patients.

Conclusion: Primary resection with anastomosis is the procedure of choice in obstructing lesions of the right colon. This has a lower operative mortality and morbidity than a staged procedure. This primary resection with anastomosis is certainly safe as an ileotransverse colostomy. Prevention through screening is an established method for haiting the progress in from malignant disease.

Reference: Chen HS, Sheen-Chen SM. Obstructing and perforation in colorectal adenocarcinoma: an analysis of prognosis and current trends. Surgery 2000;127:370-6.

Disclosure: No significant relationships.

\section{$\mathbf{P 2 5 2}$}

\section{SYSTEMIC NEUTROPHIL RESPONSES TO ISCHEMIA REPERFUSION, HYPOTHERMIA AND TISSUE DAMAGE IN A HUMANE CARDIOPULMONARY MODEL}

\author{
K. Groeneveld ${ }^{1}$, S. Algra ${ }^{2}$, L. Ulfman ${ }^{3}$, B. Prakken ${ }^{2}$, K. Jansen ${ }^{2}$, \\ L. Koenderman ${ }^{3}$, L. Leenen ${ }^{4}$
}

${ }^{1}$ Surgery, UMC Utrecht, Utrecht, The Netherlands, ${ }^{2}$ Pediatrics, UMC Utrecht, Utrecht, The Netherlands, ${ }^{3}$ Respiratory Medicine, UMC Utrecht, Utrecht, The Netherlands, ${ }^{4}$ Trauma/Surgery, University Medical Center Utrecht, Utrecht, The Netherlands

Introduction: Tissue damage, ischemia-reperfusion and hypothermia, which are associated with trauma, can lead to several levels of systemic inflammation. Literature shows that reaching a threshold of inflammation is associated with multi-organ dysfunction syndrome. Neutrophil phenotypes can be used as a read-out of the inflammatory status. Goal of the current study is to gain insight into kinetics of systemic neutrophil activation in response to the above mentioned 'trauma traid'. A humane model of cardiac surgery with cardiopulmonary bypass machine and deep hypothermic circulatory arrest was used as a trauma model. We tested the hypothesis that recirculation and ventilation after aortic arch reconstruction surgery cause immune activation, which leads to a decease in neutrophils with a functional phenotype.

Materials and methods: Infants undergoing aortic arch reconstruction were included in this prospective study. Primary outcome were the kinetics of neutrophil phenotypes measured by flow cytometry. Secondary outcomes were the functional capacities of these neutrophils (reactive oxygen species production, phagocytosis, adhesion).

Results: Results showed a low grade activation (priming) of neutrophils during the surgical procedure, measured as a mild increase in classical activation marker MAC-1 and priming marker fMLPinduced active $\mathrm{Fc} \gamma \mathrm{RII}$. After rewarming and reperfusion were sustained, the expression of MAC-1 returned to baseline. The expression of fMLP-induced active Fc $\gamma$ RII showed a marked decrease, which is a sensitive marker of severe systemic activation of the neutrophil compartment.

Conclusion: Aortic arch reconstruction during deep hypothermic circulatory arrest mildly primed systemic neutrophils. Subsequent recirculation, rewarming and reperfusion caused a marked activation of systemic neutrophils, which is a possible risk factor for inflammatory complications.

Disclosure: No significant relationships.

\section{P253}

\section{TREATING THE TRAUMA OF THE DIGESTIVE TUBE OF THE NATIONAL TRAUMA CENTER, ALBANIA}

\author{
S. Buci, A. Gjata, M. Torba, A. Lila, S. Koceku, E. Faber
}

\section{Kirurgji, Spitali Ushtarak Qendror universitar, Tirane, Albania}

Introduction: Aim: Evaluation of complex trauma of the digestive tube at the National Trauma Center in the period January 2007-May 2009.

Materials and methods: In this study analyzed 204 patients with digestive tube trauma treated at the National Trauma Albania in January 2007-May 2009. This study is performed retrospectively. Information is taken from clinical files with categorical and numerical variable. For data analysis was used SPSS 10.0 statistical package and Microsoft Exel. Significance is accepted $\mathrm{P}<0.05(5 \%)$. The relation of dependence is realized with method of linear regression and the correlation coefficient.

Results: All patients with trauma digestive tube are submitted to surgical intervention. In the first $6 \mathrm{~h}$ are operated $156(76.5 \%)$ patients. In the second $6 \mathrm{~h}$ are operated $30(14.7 \%)$ patients. In the third $6 \mathrm{~h}$ are operated $14(7 \%)$ patients. In the forth $6 \mathrm{~h}$ are operated three $(1.5 \%)$ patients. Intestinum crassum was found injured in $39.2 \%$ of cases; intestinum tenue $47 \%$, stomach $17.6 \%$, esophagus $5.8 \%$, duodenum $3.9 \%$. In 204 patients were injured 324 organs. The distribution by age groups has been: $0-14$ years age group $10 \%$, $14-40$ years $57 \%, 33 \%$ over 40 years. Distribution according to social categories: employees $50.9 \%, 31 \%$ of unemployed, children $10 \%, 6 \%$ retired, $2 \%$ intellectual. Causes of trauma of digestive tube have been: firearm wound $31.3 \%, 25.4 \%$ sharp tools, automobile accidents $17.6 \%, 17.6 \%$ direct collisions, $7.8 \%$ decrease from the height, open trauma $56.8 \%, 43.2 \%$ closed trauma. 
Conclusion: Trauma of the digestive tube was $5.2 \%$ of patients treated at National Trauma Center. A part of the digestive tube damages are identified during the operation. Delayed laparotomy increased mortality. For every damaged organ, stay on the hospital has increased over 4 days. Performance of the trauma of the digestive tube depends on the number of organs damaged, the grade of injury, time of the operation and age.

Disclosure: No significant relationships.

\section{SPINAL AND BRAIN INJURY IN MULTIPLE TRAUMA}

\section{P254}

CT RESULTS AFTER HEAD INJURY IN PATIENTS AFTER ALCOHOL INTAKE COMPARED TO SOBER PATIENTS: RETROSPECTIVE ANALYSIS OF FACTORS AFFECTING USEFULNESS OF COMPUTED TOMOGRAPHY

D. Timler $^{\text {I }}$, S. Niedzwiecki ${ }^{2}$

${ }^{1}$ Emergency Department, Medical University in Lodz, Lodz, Poland, ${ }^{2}$ Memorial Copernicus Hospital in Lodz, Lodz, Poland

Introduction: The diagnosis of the brain concussion may be difficult and uncertain in patients after alcohol intake. The CT is routinely performed in Poland among the patients with head injury after alcohol consumption. The purpose of the study was to compare retrospectively the CT results and other clinical factors after head injury in patients after alcohol intake to sober patients in order to statement of usefulness of CT in alcohol intoxicated patients with head injury.

Materials and methods: The retrospective study took place at the Emergency Department of Copernicus Memorial Hospital in Lodz. The analyses including period between January and December 2009 comprised a group of 306 patients after head injury: 270 sober individuals (group 1) and 36 patients after alcohol intake (group 2). The analysed factors were as follows: age, gender, GCS, clinical symptoms, mechanism of injury and CT abnormalities.

Results: The study revealed no significant difference in age, gender, GCS, clinical symptoms, mechanism of injury between both groups. The total CT abnormalities were found in $21(7.77 \%)$ patients from group 1 and 4 (11.11\%) patients from group 2 (nonsignificant). There was a significant correlation between CT abnormality and age $>62$ years in group 2 . The analyses showed no significant correlation between intracranial and craniofacial soft tissue injury.

Conclusion: CT is an useful diagnostic method in sober as well as in patients after alcohol consumption, particularly in elderly

Reference: Lange RT, Iverson GL, Brubacher JR, Franzen MD. Effect of blood alcohol level on Glasgow Coma Scale scores following traumatic brain injury. Brain Inj. 2010;24(7-8):919-27.

Disclosure: No significant relationships.

\section{P255}

\section{INFECTION RATE IN PATIENTS WITH COMPOUND DEPRESSED SKULL FRACTURES AND PRIMARY BONE FRAGMENT REPLACEMENT, IRAN, 1997-2005}

\section{E. Fakharian}

Trauma Research Center, Kashan University of Medical Science, Kashan, 87196-74591 Iran
Introduction: Compound depressed skull fracture (CDF) is a complication of trauma to the head. In spite of many decades of studies on the management of CDF there is still disagreements regarding primary repair or discarding the bone fragments and late repair. This study has been conducted to evaluate infection rate in primary repair of CDF.

Materials and methods: This study included 60 patients with blunt trauma to head and CDF admitted to Naghavi hospital of Kashan University of Medical Sciences (KAUMS), from 1997 to 2005. Age, sex, the interval between admission and operation, severity of trauma, duration of hospital stay, and infections were recorded and analyzed with descriptive statistics.

Results: Male to female ratio was 7.6:1, and mean age $19 \pm 12.4$ years. It was most common in the age range of 11-20 (19 patients, $31.7 \%)$. Motorcycle was the most common cause of the problem (45 patients, $75 \%$ ). Thirty nine patients $(65 \%)$ had dural laceration. The most common site of fracture was frontal bone (37 and $61.7 \%$ ). Twenty eight cases (46.6\%) had mild, $13(21.7 \%)$ moderate and $19(31.7 \%)$ severe head injury. Fifty four patients (90\%) underwent operation in less than $24 \mathrm{~h}$ of their arrival to hospital and had primary bone replacement. In 6 months follow up there was no infection in either the wound or bone.

Conclusion: In the absence of heavy contamination, and by carrying debridement as soon as possible there will be excellent outcome with primary replacement of bone fragments in CDF patients, excluding the need for another operation and the apprehension about infection. Reference: Francel PC, Honeycutt J. Mild brain injury in children, including skull fractures and growing fractures. In: Richard WH (ed) Youmans neurological surgery, 5th ed. Philadelphia: W.B. Saunders Company; 2005. p. 3461-72.

Disclosure: No significant relationships.

\section{P256}

\section{MOTORCYCLE CRASHES WITH FATAL CASUALTIES: A SERIOUS PUBLIC HEALTH PROBLEM}

\section{C.E. Carrasco, R.B. Carvalho, G.P. Fraga}

Division of Trauma Surgery, State University of Campinas, Campinas, SP, Brazil

Introduction: Motorcyclists are one of the most vulnerable groups in traffic crashes and the increasing incidence of fatal events has become an important concern. The objective was to identify the trauma lesions responsible for deaths.

Materials and methods: All fatal motorcycle crashes $(n=405)$ occurred from January 2001 to December 2008 in Campinas, Brazil, were considered. Official data have been collected from police incident reports, hospitals' charts, and registers of necropsies. The proportion of drunk victims and surgical procedures were analyzed. The moment and causes of death were reviewed. Trauma scores AIS and ISS were calculated.

Results: Fatal accidents increased 124\% from 2001 to 2008. Main accident causes: collisions against cars $(22 \%)$, trucks or buses $(21 \%)$, and falls (13\%). As for the victims: $90 \%$ were male; mean age was 27 (range 1-73); drivers were $85 \%$. Alcoholaemia was positive in $43 \%$ of victims. Forty-two percent died at the scene or during the transport to hospital. Mean AIS for head/neck was 3.39; for thorax, 2,3 and for abdomen, 1.75. Mean ISS was 38.5 (ranged 9-75). Head trauma (69\%), hypovolemic shock (40\%) and thoracic trauma (39\%) were the most relevant events that caused death. Surgical procedures were realized in 85 patients and thoracic drainage, laparotomy and craniotomy were the most frequent procedures. 
Conclusion: Collisions were the main mechanism of accident and alcoholaemia had relevant presence. Head trauma was the most frequent and severe injury evolving motorcycle crashes.

Reference: Ankarath S, Giannoudis PV, Barlow I, Bellamy MC, Matthews SJ, Smith RM. Injury patterns associated with mortality following motorcycle crashes. Injury 2002;33:473-7.

Disclosure: No significant relationships.

\section{$\mathbf{P 2 5 7}$}

\section{THE EPIDEMIOLOGY OF BRAIN INJURY: OUR EXPERIENCE AND REVIEW IN INTERNATIONAL LITERATURE}

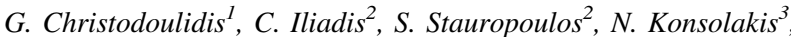
V. Moraitis ${ }^{2}$, D. Dimas ${ }^{4}$, K. Tepetes ${ }^{1}$

${ }^{1}$ General Surgery, University hospital of Larissa, Larissa, Greece, ${ }^{2}$ Neurosurgery, General Hospital Venizelion Krete, Irakeion, Greece, ${ }^{3}$ Neurosurgery, General Hospital Venizelion, Heraklion, Greece,

${ }^{4}$ General Surgery, Elpis, Athens, Greece

Introduction: Traumatic brain injury (TBI) not only has considerable morbidity and mortality, but it is a major cause of epilepsy. We determine the frequency of TBI, special groups at risk, and mortality. Materials and methods: We report epidemiologic characteristics of TBI, including incidence, differences by age, gender, race and ethnic group, and geographic variation, and mortality from our hospital. We reviewed studies of TBI that are either population based or derived from definable catchment areas that allow determination of incidence, identification of risk groups, and mortality.

Results: Population-based studies in the United States suggest that the incidence TBI is between 200 and 250 per 100,000 population per year. Incidence is higher in Europe and especially in South Europe. In our area there are groups at high risk of TBI. This includes males and individuals living in regions characterized by socioeconomic deprivation. Mortality varies by severity but is high in those with severe injury and in the elderly.

Conclusion: TBI is a major public health problem. If primary prevention is to be undertaken, we have to understand the epidemiology of the condition. The primary causes of TBI vary by age, socioeconomic factors, and geographic region, so any planned interventions must tailored accordingly.

Disclosure: No significant relationships.

\section{EMERGENCY SURGERY/VISCERAL TRAUMA/ TRAINING}

\section{P258}

\section{COMPARTMENT PRESSURES WITHIN THE NORTH-WEST OF ENGLAND}

\section{B. Liu, P. Maguire, J. Doyle}

\section{Trauma and Orthopaedics, Fairfield Hospital, Manchester, UK}

Introduction: Diagnosing compartment syndrome in the alert patient from history and examination alone can be challenging, in an obtunded, anaesthetised or paediatric patient diagnosis via history and examination alone can be impossible. The only objective means of assessing compartment pressure is with a hand held device. The aim of this study was to assess how units across the North West of England currently assess patients with suspected compartment syndrome and prepare their junior doctors to approach compartment syndrome.

Materials and methods: 17 orthopaedic departments in the north west. A short five question survey was given to junior surgical trainees at weekly regional teaching sessions regarding their Orthopaedic Unit. Orthopaedic juniors in trusts without a trainee were contacted via telephone.

Results: Of the 17 trusts only 9 units (53\%) had a compartment pressure probe and only $1(11 \%)$ of these taught their junior doctors how to use the probe. Of the remaining trusts seven relied solely on clinical judgement and the remaining trust one used a CVP probe attached to an intravenous cannula. Seven trusts (41\%) arranged teaching for their junior doctors on commencement of their post and five trusts $(29 \%)$ were asked to assess for compartment syndrome more often than fortnightly.

Conclusion: Of the orthopaedic units in the north west, $41 \%$ of trusts are inadequately equipped to objectively assess compartment pressures and to assess patients where the history and examination is impaired (obtunded, anaesthetised or paediatric patients). 59\% of trusts have no formal teaching to prepare their juniors for dealing with patients with suspected compartment syndrome.

Disclosure: No significant relationships.

\section{P259}

\section{EMERGENCY MEDICAL CARE IN JORDAN: IS IT WORTHWHILE?}

\section{A.H. Al Issa}

\section{Ed, Royal Medical Services, Amman, Jordan}

Introduction: This study will determine the current status of EM in Jordan and how to establish consensus on key areas of EM development settings, with respect to scope of EM, residency programs, staffing needs, training requirements, and research priorities.

Materials and methods: This study was conducted in three pilot hospitals (private, public and university sector). These hospitals have EM residency programs for the last decades. Interview with EM specialists or equivalent, with experience in or interest in EM in these hospitals, data were analyzed to provide data on EM scope, staffing, training, and research priorities and challenges for EM in their hospitals.

Results: A total of 268 of 308 subjects ( $81 \%$ ) had responded to the study. There is a consensus that was reached included EM being a specialist-driven service. International training courses should be adapted to local needs. EM research in developing countries should be clinically driven and focused on local issues of importance.

Conclusion: The scope and function of EM and relationships with other specialties are defined. Unambiguous principles are laid out for the development of the specialty in developing world environments. The next step required in this process is translation into practical guidelines for the development of EM in developing world settings where they may be used to drive policy, protocols, and research. References: Bulletin of the World Health Organization. 2002;80(11). Macfarlane S, Racelis M, Muli-Musiime F. Public health in developing countries. Lancet 2000;356:841-6.

Disclosure: No significant relationships. 
$\mathbf{P 2 6 0}$

\section{MANAGEMENT OF DELAYED PRESENTATION OF PANCREATIC TRAUMA AT A TERTIARY CARE HOSPITAL IN KARACHI, PAKISTAN}

\author{
H. Zafar, M.A. Moosa, S.N. Zafar
}

Surgery, Aga Khan University Hospital, Karachi, Pakistan

Introduction: To review the management of pancreatic trauma cases with delayed presentation to a tertiary care hospital.

Materials and methods: Retrospective review of all patients with pancreatic trauma admitted at our center after being transferred from another hospital between January 1990 and December 2009. Data was collected on mode and time of presentation since injury, investigations and treatment received. Outcome measures included pancreatictrauma related complications, length of hospital stay and mortality. Results: A total of 19 patients were included with a mean age of 25.4 $( \pm 7)$ years of which $17(90 \%)$ were male. The mean time from injury to presentation was $112( \pm 175) \mathrm{h}$. Road traffic accidents and firearms accounted for most of the injuries ( 8 cases each). Only 3 patients had been diagnosed with pancreatic trauma at the previous facility and 5 had undergone exploratory laparotomies before presenting at our hospital. At presentation 13 (68\%) were in shock and 18 (95\%) had signs of peritonitis. Ten patients $(53 \%)$ had multiple solid organ injuries. Eight (42\%) patients were diagnosed upon CT scan while 9 $(47 \%)$ were diagnosed upon laparotomy. Surgery was performed in 16 $(84 \%)$ patients, $7(44 \%)$ underwent a drainage procedure, $3(19 \%)$ had damage control surgery, $2(13 \%)$ had a salvage procedure while definitive treatment was performed in $4(25 \%)$ patients. Mean time to surgery from injury was $104( \pm 147)$ hours. Complications occurred in $10(53 \%)$ patients and $4(21 \%)$ patients died.

Conclusion: Presentation of pancreatic injury varies widely and it is rarely found in isolation. Delay in diagnosis due to hospital transfers significantly effects morbidity and mortality.

Disclosure: No significant relationships.

\section{P261}

\section{NONOPERATIVE MANAGEMENT OF PENETRATING ABDOMINAL INJURIES}

A.N. Turhan, M. Gönenç, S. Kapan, H. Alış, I. Başoğlu, I. Gök

General Surgery, Bakirkoy Dr. Sadi Konuk EAH, İstanbul, Turkey

Introduction: Penetrating trauma of the abdomen continues to be a major cause of trauma admission worldwide. After the introduction of sophisticated diagnostic tools within the last 30 years, the pendulum has shifted towards selective conservative treatment of such cases.

Materials and methods: Medical data of the patients who have admitted to Dr. Sadi Konuk Training and Research Hospital, Istanbul, Turkey, with penetrating abdominal injuries between January 2009 and June 2010 were reviewed. The patients who had superficial abdominal injuries were excluded from the study.

Results: Total number of patients was 271. The mean age and male:female ration was 33.7 (14-78) and 252:19, respectively. The mechanism of trauma was gun shot wound in 15 and stab wound in 256. Of 271 patients, 45 (17\%) have had laparotomy, and the rest $(83 \%)$ have been managed by selective non-operative treatment. The mean duration of observation for selective conservative treatment was $36(24-48)$ h. The rate of negative laparotomy was $20 \%(n=9)$. The mortality rate for non-operative treatment group and operative treatment group was 0 and $13 \%(n=6)$, respectively.
Conclusion: Selective conservative treatment is the treatment of choice in patients with penetrating abdominal injury who do not have hemodynamic instability or any sign of peritonitis.

Disclosure: No significant relationships.

P262

\section{NONOPERATIVE MANAGEMENT OF PENETRATING THORACOABDOMINAL GUN SHOT INJURIES}

S. Kapan, A.N. Turhan, H. Alıs, F. Yanar, H. Yırgın, N. Şahin

General Surgery, Bakirkoy Dr. Sadi Konuk EAH, İstanbul, Turkey

Introduction: Abdominal gun shot injuries are one of the most common indications for explorative laparotomy in trauma centers. In this study nonoperative treatment of penetrating abdominal gun shot injuries are evaluated.

Materials and methods: 47 patients, who admitted to our clinic with thoracoabdominal penetrating gun shot injuries between February 2002 and November 2010, managed nonoperatively were included in this study. All of the patients were hemodynamically stable and close physical examinations were done regarding the alterations in the hemodynamic situation and presence of acute abdomen.

Results: Only two patients were female and the others were male with a mean age of $32 \pm 2.04$ (14-75). Bullet wound was in the right upper quadrant of the abdomen in 9 of the patients, in the suprapubic region in 4 of the patients, in the left thoracoabdominal region in 13 of the patients, in the right thoracoabdominal region in 5 of the patients, in the left lower quadrant in 13 of the patients and right lower quadrant in 3 patients. Mean hospital stay was $3.5 \pm 0.4$ days (1-12). One patient with concurrent cranial injury died because of the cranial injury. Other patients were discharged from the hospital with no morbidity and mortality.

Conclusion: Thoracoabdominal gun shot injuries with peritoneal penetration can be managed nonoperatively with close follow up in hemodynamically stable patients preventing unnecessary negative and nontherapeutic laparotomies.

Disclosure: No significant relationships.

\section{P263}

\section{PEDIATRIC REALISTIC SIMULATION: EXPERIENCE IN A THIRD WORLD COUNTRY MEDICAL SCHOOL}

M.C. Reis, F. Belluomini, A.M.A. Fraga, M.B. Brandao, M.P. Zambon, E.C.E. Baracat

Pediatrics, University of Campinas, Valinhos SP, Brazil

Introduction: Pediatric-emergency principles are undervalued/absent in most Medical Schools curricula. Despite low prevalence of critical situations in Pediatric-Emergency, skills are necessary in managing children life-threatening events. Recently, new strategies were implemented in University of Campinas (FCM-UNICAMP), namely Skills LAB during the Pediatric-Emergency module, to contribute for medical formation in recognizing and acting at Pediatric-Emergency situations using realistic simulation.

Materials and methods: Ministered since July/2009, in medical sixth year, using 14-students grouping during 4-h-class in 3 stages: (1) TESTscenario: realistic-simulation using mannequins/equipment pediatric cardiorespiratory emergency situation. (2) Specific-Training: theory training (Pediatric Advanced Life Support (Ped-Support) videos) and 
practice (mannequins/equipment at ER). (a) Airway Evaluation/Management: desobstruction, orotracheal intubation, ventilation in tracheal tube, manual resuscitator and laryngeal mask. (b) Cardiocirculatory Evaluation/Management: cardiocirculatory collapse ER recognition, cardiac reanimation maneuvers and vascular access. (3) POST-TEST scenario: new realistic-simulation in emergency using the procedures/ knowledge recently acquired. Feedback after. Collected data to measure the differences between TEST and POST-TEST skills. PedSupport class is ministered, either before or after the simulation-class (SC). We evaluate the TEST and POST-TEST results comparing if the resuscitation class (RC) has been held or not.

Results: In RC-before-SC group average TEST/POST-TEST results was 6.8/9.25 (max: 10) and in Not-RC group, average TEST/POSTTEST were 6.6/7.7.

Conclusion: Ped-Support class before Skills-Module improved in handling scenarios, although does not prepare for theory in TESTscenario, after the Skills-Module, seems that student who had RC before has achieved better goals compared to group that did not had class. Data are still being collected so further analysis will be possible References: Eppich-WJ, Adler-MD, McGaghie-WC. Emergency critical-care pediatrics: use medical-simulation for training acute pediatric emergencies. Curr Opin Pediatr. 2006;18:266-71.

Disclosure: No significant relationships.

\section{$\mathbf{P 2 6 4}$}

\section{PEDIATRIC TRAUMA RESEARCH IN THE GULF COOPERATION COUNCIL COUNTRIES}

\author{
A.F. Hefny ${ }^{1}$, M. Grivna ${ }^{2}$, A.K. Abbas ${ }^{3}$, F. Branicki ${ }^{4}$, F.M. Abu-Zidan ${ }^{4}$
}

${ }^{1}$ General Surgery, Faculty of Medicine \& Health Sciences, UAE University, Al-Ain, United Arab Emirates, Al-Ain, United Arab Emirates, ${ }^{2}$ Community Medicine, Faculty of Medicine, UAE University, Al-Ain, United Arab Emirates, ${ }^{3}$ Trauma Group, Faculty of Medicine, Al-AIn, United Arab Emirates, ${ }^{4}$ Surgery, Department of Surgery, UAE University, Al-Ain, United Arab Emirates

Introduction: Pediatric trauma is a major health problem worldwide. Most Gulf Cooperation Council (GCC) countries are-high income with developing economies. Pediatric trauma and its effects at national level have not been well studied in this region. Our aim was to review published pediatric trauma research from the (GCC) countries so as to identify research fields that need to be enhanced.

Materials and methods: A MEDLINE search for articles on pediatric trauma from GCC countries during 1960-2010 was performed. The content of articles was analyzed, classified and summarized.

Results: 53 articles were found and retrieved of which 18 (34\%) were published in the last 5 years, $42(79 \%)$ were original articles. The first author was affiliated to a university in 29 reports $(55 \%)$, to a community hospital in $13(25 \%)$ and to a military hospital in 10 $(19 \%)$. All articles were observational studies that included 18 (34\%) case-control studies, $18(34 \%)$ case reports/case series studies, $8(15 \%)$ prospective studies, and $7(13 \%)$ cross sectional studies. The median (range) impact factor of the journals was 1.3 (0.5-3.72). No randomized controlled trials or meta-analysis studies were found.

Conclusion: A strategic plan is required to support pediatric trauma research in GCC countries so as to address unmet needs. Areas of deficiency include prehospital care, post traumatic psychological effects and post traumatic rehabilitation, interventional studies focused on a safe children environment and attitudinal changes, and the socioeconomic impact of pediatric trauma.

Disclosure: No significant relationships.
P265

\section{PREVENTION OF ACUTE MUSCULOSKELETAL INJURY AT COMMUNITY-LEVEL CRICKET THROUGH AN INTEGRATED MUSCULOSKELETAL HEALTH SCREENING SYSTEM WITH COACHING}

\section{Bhattacharyya}

Orthopaedics, LEWISHAM HOSPITAL LONDON, LONDON, United Kingdom

Introduction: There are very few descriptive epidemiologic studies of injury in non elite sport men community-level players. Sports injury surveillance systems have been described in the scientific literature may be implemented in community-level game. Sports specific injury on immature skeleton may restrict mobility in later life. The objective of this study was to implement a method for undertaking routine musculoskeletal health screening and selective sports training in community-level soccer to prevent injuries on skeletally immature young cricketers.

Materials and methods: A cohort of 380 community-level players median aged 13 years were studied and was followed over 1 winter competitive season. Before tournament participation, each player was thoroughly examined and an interviewed to adopt person specific injury prevention program. We have also collected data on participation in matches and training sessions, injuries, and adherence to injury prevention measures.

Results: The incidence rate for match injury events was not observed. A total of four training injury events were reported, giving incidence rates of 1.3 injury events per 100 player-training sessions. The most common injuries were soft tissue injury due to hard ball, followed by sprains and strains of the lower limb.

Conclusion: This study has shown that well designed musculoskeletal health screening and person specific sports training, can be successfully prevent acute injury on the immature skeleton in community-level cricket.

Disclosure: No significant relationships.

\section{P266}

\section{SEATBELT COMPLIANCE AND MORTALITY IN THE GULF COOPERATION COUNCIL COUNTRIES IN COMPARISON WITH OTHER HIGH-INCOME COUNTRIES}

A.K. Abbas ${ }^{1}$, A.F. Hefny ${ }^{2}, \underline{\text { F.M. Abu-Zidan }}{ }^{3}$

${ }^{1}$ Trauma Group, Faculty of Medicine, Al-AIn, United Arab Emirates,

${ }^{2}$ Trauma Group, Faculty of Medicine, Al Ain, United Arab Emirates, ${ }^{3}$ Surgery, Department of Surgery, UAE University, Al-Ain, United Arab Emirates

Introduction: Low compliance of seatbelt usage can be a contributing factor for increased mortality of road traffic collisions. The present study aims to ascertain the presence of a relationship between seatbelt non-compliance of vehicle occupants and vehicle occupants' mortality rates in the Gulf Cooperation Council countries and other high-income countries.

Materials and methods: Data for all Gulf Cooperation Council countries $(n=6)$ and other high-income countries $(n=37)$ were retrieved and compared with regard to population, gross national income, number of vehicles, seatbelt non-compliance and road traffic death rates. Mann-Whitney $U$ test and Spearman's rank correlation test were used for univariate analysis. Multiple linear regression was used to define factors affecting the mortality rates. 
Results: The median road traffic death rates, occupants' death rates, and the percentage of seatbelt non-compliance were significantly higher in the Gulf Cooperation Council countries $(\mathrm{p}<0.0001$, $\mathrm{p}=0.02, \mathrm{p}<0.001$ respectively). There was a strong correlation between occupants' death rates and seatbelt non-compliance $(\mathrm{R}=0.52, \mathrm{p}=0.008)$. Seatbelt non-compliance percentage was the only significant factor predicting mortality in the multiple linear regression model $(\mathrm{p}=0.015)$.

Conclusion: Seatbelt non-compliance percentages in the Gulf Cooperation Council countries are significantly higher than other high-income countries. This is a contributing factor in the increased road traffic collision mortality rate in these countries. Enforcement of seatbelt usage by law is mandatory so as to reduce the toll of death of road traffic collisions in the Gulf Cooperation Council countries.

Disclosure: No significant relationships.

\section{$\mathbf{P 2 6 7}$}

\section{STUDENT CONFIDENCE IN PREHOSPITAL TRAUMA SITUATIONS: QUESTIONNAIRE RESULTS}

\author{
C.B. Frank ${ }^{1}$, T. Gühring ${ }^{1}$, B. Gliwitzky ${ }^{2}$, A. Moghaddam ${ }^{1}$, C. Wölfl ${ }^{1}$
}

${ }^{1}$ Trauma And Orthopedic Surgery, BG Trauma Center Ludwigshafen, Ludwigshafen, Germany, ${ }^{2}$, PHTLS Germany, Aachen, Germany

Introduction: The goal of this study was to assess student's ${ }^{\mathrm{TM}}$ opinions of their own competence in prehospital trauma settings, and to determine if students desire more comprehensive prehospital trauma training.

Materials and methods: Through interviews, standardized questionnaires and self-reflection, we evaluated students' opinions of their university education of prehospital trauma preparation. Additionally, we assessed students' self-confidence in their ability to function as a first responder on a trauma scene.

Results: 111 students $(51$ males $/ 60$ females) were included in the study; $68 \%$ had no experience in prehospital trauma settings. Except 6 , all students said that neither senior students nor new doctors are prepared for prehospital trauma settings. The majority of students did not feel competent, prepared and/or self-confident enough to work as a qualified team member at a trauma. The majority of students expressed interest in a course to improve their theoretical and practical knowledge of prehospital trauma care. $90 \%$ feel that such a course would positively impact their future professional work. The most desired course design is a $10 \mathrm{~h}$ emergency trauma training, with $50 \%$ hands-on practical skills training. $90 \%$ of the students would volunteer to take part in such a course, if provided.

Conclusion: BG Trauma Center Ludwigshafen is inaugurating an emergency trauma training course with practical skill stations to improve the knowledge and self-confidence of final year students. The pilot course will be supported by PHTLS Germany; and, if successful, will be continually offered to all final year students.

References: 1. Rubiano AM, Sanchez AI, Guyette F, Puyana JC. Trauma care training for National Police Nurses in Columbia. Prehosp Emerg Care 2010;14(1):124-30. 2. Esteri RM Jr, Henzi DL, Cohn SM. Senior medical student Boot Campâ: can result in increased self-confidence before starting surgery internships. Curr Surg 2006;63(4): 264-8. 3. Brunt LM, Halpin VJ, Klingensmith ME, Tiemann D, Matthews BD, Spitler JA, Pierce RA. Accelerated skills preparation and assessment for senior medical students entering surgical internship. J Am Coll Surg. 2008;206(5): 897-904.

Disclosure: No significant relationships.

\section{P268}

\section{THE RECORD KEEPING QUALITY OF THE ORTHOPAEDIC TRAUMA OPERATION NOTE}

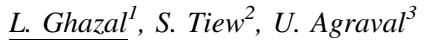

${ }^{1}$ Trauma And Orthopaedics, Central Manchester Foundation Trust, Manchester, United Kingdom, ${ }^{2}$ Postgraduate Education, Central Manchester Foundation Trust, Manchester, United Kingdom,

${ }^{3}$ Trauma And Orthopaedics, Manchester Royal Infirmary, Manchester, United Kingdom

Introduction: It is known that poor quality operation notes can have medicolegal and financial implications for the trauma surgeon. In orthopaedic trauma surgery, poor quality operation notes can have serious consequences on patient safety. As a result, medical and nursing teams lack clear information and postoperative instructions following trauma surgery to deliver good continuity of care. In view of patient safety, we assessed the record keeping quality of the trauma orthopaedic operation note in accordance with "Good Surgical Practice" guidelines [1] published by Royal College of Surgeons, UK. Materials and methods: Two prospective studies of all orthopaedic trauma operation notes at a university affiliated teaching hospital $(n=45)$. A unique aide-memoire prompting tool was developed and attached to blank operation note sheets as the main intervention to improve the quality of orthopaedic trauma operation notes. Aidememoire displayed next to computer screens for electronically typed operation notes.

Results: Initial study showed that poorly recorded data were: correct rectification of mistakes $(0 \%)$, diagnosis $(27 \%)$, details of suture $(58 \%)$ and description of tissue details (42\%). Following implementation of the aide-memoire prompting tool, our follow-up study demonstrated significant improvement: correct rectification of mistakes $(100 \%)$, diagnosis $(45 \%)$, details of suture $(78 \%)$ and description of tissue details ( $78 \%$ ).

Conclusion: An aide-memoire prompting tool is an effective intervention to improve the record keeping quality of the orthopaedic trauma operation note. We advocate the use of this tool in all orthopaedic trauma centers and recommend integration of the "Good Surgical Practice" standards in electronic operation notes.

References: 1. RCSENG. Good surgical practice [online]. http:// www.rcseng.ac.uk/rcseng/content/publications/docs/good-surgicalpractice-1. 2008. Accessed 20/02/2010.

Disclosure: No significant relationships.

\section{P269}

\section{THE VALUE OF WHOLE-BODY COMPUTED TOMOGRAPHY FOR BLUNT TRAUMA PATIENTS IN JAPAN}

T. Fujita, Y. Uchida, K. Nakazawa, R. Yamaguchi, M. Kitamura, H. Ikeda, T. Sakamoto

Trauma And Resuscitation Center, Teikyo University, Tokyo, Japan

Introduction: The use of whole-body computed tomography (WBCT) for trauma has increased rapidly. No data has been available on the frequency of WBCT during trauma evaluation in Japan. The purpose of this study was to determine whether the present use of WBCT is acceptable. 
Materials and methods: We used the data recorded in the Japan Trauma Data Bank (JTDB) 2004-2008 to calculate the probability of survival (Ps) by the trauma registry injury severity score (TRISS) method for 11,056 patients who were transported directly with blunt trauma who were evaluated by either WBCT or non-WBCT methods. Results: One thousand four hundred and ninety-four $(24 \%)$ of the 11,056 patients were evaluated by WBCT. The mean age of the entire population was 45.8 (SD 22.5) and 7917 (72\%) of the patients were male. The mean injury severity score was 16.5 (SD 11.5) for NonWBCT versus 19.0(12.7) for WBCT $(\mathrm{p}<0.001)$ patients. The Ps as calculated by the TRISS method was $0.893(0.2)$ for Non-WBCT versus $0.869(0.23)$ for WBCT $(\mathrm{p}<0.001)$ patients. The survival rate was $0.899(0.302)$ for non-WBCT patients versus $0.899(0.301)$ for those who received WBCT $(p=0.901)$. The unexpected trauma death rate, which was defined as the mortality $>50 \%$ of the Ps was $0.54 \%(0.23)$ for non-WBCT versus $0.50 \%(0.22)$ for WBCT $(\mathrm{p}=0.017)$ patients.

Conclusion: The use of WBCT does not significantly contribute to an increase in the survival rate, although it reduces the unexpected trauma death. This is the first report that has evaluated the use of WBCT examination with the national representative trauma data base in Japan

Disclosure: No significant relationships.

\section{$\mathbf{P 2 7 0}$}

\section{TRAINING PEDIATRIC EMERGENCY ABILITIES AMONG MEDICAL STUDENTS: A NEW APPROACH IN A THIRD WORLD SCHOOL OF MEDICINE}

\author{
M.C. Reis, F. Belluomini, A.M.A. Fraga, M.B. Brandao, \\ M.P. Zambon, E.C.E. Baracat
}

Pediatrics, University of Campinas, Valinhos SP, Brazil

Introduction: Worldwide Pediatric-Emergency concepts are undervalued/absent in Medical Schools curricula. To be in accordance to curricular directive, the emergency modules should be eminently practical, ministered to small groups in distinctive medical assistance scenarios, focusing abilities. Despite low prevalence of critical-situations in Pediatric Emergency-Units, skills are necessary in managing children in life-threatening events. Using mannequins in emergency situations are mandatory. In Brazil, these initiatives are incipient. University of Campinas School of Medicine implemented these strategies, named Modulo de Habilidades (Skills-Module-SM) to contribute in recognizing/acting at Pediatric-Emergency situations for general medical formation.

Materials and methods: Ministered bi-monthly for medical 6th-year, using 14-students group during 3 stages 4-h-class: (1) TEST-scenario: realistic-simulation with mannequins/equipment pediatric cardiorespiratory emergency. (2) Specific-Training: theory training (Pediatric Advanced Life Support (Ped-Support) videos) and practice (mannequins/equipments at ER). Done in rounds with 7 students group in 2 stations: (a) Airway evaluation/management: perform desobstruction, orotracheal intubation, ventilation in tracheal tube, manual resuscitator, and laryngeal mask. (b) Cardiocirculatory Evaluation/ management: cardiocirculatory collapse recognition in ER, perform cardiac reanimation maneuvers and vascular access. (3)Post-TESTscenario: new realistic simulation in emergency using the procedures/ knowledge recently acquired, feed-back after.

Results: Implemented since July, 2009, bi-monthly, for medical students in last year of medical graduation. We are collecting data to measure the differences between TEST and POST-TEST skills.
Conclusion: There has been a positive reaction to the use of Realistic Simulation in Pediatric-Emergency Scenarios.

References: Issenberg-SB, McGaghie-WC, Petrusa-ER, Gordon-DL, Scalese-RJ. Features and use of high-fidelity medical simulations that leads to effective learning: a BEME systematic review. Med Teach. 2005;27(1):10-28. Eppich-WJ, Adler-MD, McGaghie-WC. Emergency and critical-care pediatrics: use of medical simulation for training in acute pediatric emergencies. Curr Opin Pediatr 2006;18: 266-71.

Disclosure: No significant relationships.

\section{P271}

\section{ACUTE TRAUMATIC AORTIC INSUFFICIENCY}

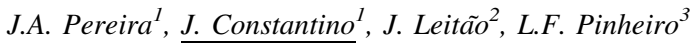

${ }^{1}$ Cirurgia 1, Hospital São Teotónio, Viseu, Portugal, ${ }^{2}$, Hospital São Teotónio, Viseu, Portugal, ${ }^{3}$ Cirurgia Geral, Hospital de São Teotónio, Viseu, Portugal

Introduction: Acute aortic insufficiency of traumatic origin is a rare complication of torso blunt trauma, cited for the first time by in 1830 by Penderleath. Known for more than a century, this entity's pathogeny is still debated, recognizing the importance of a rising pressure gradient between the aortic root and the left ventricle. The low suspicion index and the gravity of the associated lesions are to blame for the lateness of the diagnosis, albeit its importance. It can be the cause of immediate death, but undiagnosed, it can evolve through months before it is identified. The clinical suspicion, by the auscultation of a cardiac sound resembling a seagull's cry, corresponding to an acute diastolic murmur, mandates the observation under echocardiogram or aortography.

Materials and methods: The authors present a case of acute aortic insufficiency after a car crash, with blunt torso trauma. The severity of the associated injuries, namely the pulmonary contusion, did not allowed for a prompt surgical treatment of the situation. So, he was care in a ICU setting and operated latter with valvular substitution.

Results: As a rare case and often missed, the authors discuss its clinical presentation, pathogeny and diagnostic workout.

Conclusion: Despite its low incidence and rareness, the acute aortic insufficiency must be included in the diagnostic workout of torso blunt trauma.

Disclosure: No significant relationships.

\section{P272}

\section{PENETRATING ABDOMINAL TRAUMA: 20 YEARS EXPERIENCE IN A WESTERN EUROPEAN TRAUMA CENTER}

\author{
G. Tugnoli ${ }^{1}$, S. Di Saverio ${ }^{2}$, E. Giorgini ${ }^{3}$, S. Villani ${ }^{1}$, A. Biscardi ${ }^{1}$,

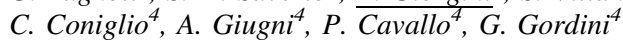

${ }^{1}$ Emergency And Trauma Surgery Unit, Maggiore Hospital Trauma Center, Bologna Local Health District, Bologna, Italy, ${ }^{2}$ Surgery, Emergency and Trauma Surgery Unit, Maggiore Hospital Trauma Center, Bologna, Italy, ${ }^{3}$ Emergency and Trauma Surgery Unit (Head Dr. G. Tugnoli), Maggiore Hospital Trauma Center, Bologna Local Health District, Bologna, Italy, ${ }^{4}$ Trauma ICU, Department of 
Emergency, Maggiore Hospital Trauma Center, Bologna Local Health District, Bologna, Italy

Introduction: Incidence of penetrating abdominal trauma in western Europe is low. While non-operative management (NOM) of blunt trauma has become the gold standard, the management of penetrating trauma is still controversial. NOM and laparoscopy are currently used in selected patients, reducing unnecessary laparotomy.

Materials and methods: We retrospectively reviewed 6,523 patients admitted for thoraco-abdominal trauma $(5,861$ blunt vs. 662 penetrating). We sorted 114 patients with penetrating abdominal trauma in 2 groups (1989-2000 vs. 2001-2008, before and after the establishment of dedicated trauma unit) analyzing characteristics and outcome in comparison.

Results: In the latest period the incidence of penetrating trauma significantly increased (from 4.17/year up to 8.53/year, being now $13.95 \%$ of all trauma laparotomies vs. $7.8 \%$ in the past decade). GSW incidence decreased ( 30 vs. $12.5 \%, \mathrm{p}=\mathrm{ns}$ ) compared to stab; no differences have been recorded in sex, age, GCS (11.8 vs 13.2), ISS ( 22 vs. 18$), \mathrm{pH}, \mathrm{BE}$ and blood transfusion ( 6.4 vs. $4.3 \mathrm{U}$ ) requirement. No changes in major vascular injuries incidence (3.5\% overall) have been observed. Interestingly a marked significant change occurred in demographics of victims $(67.2 \%$ extra-EU origin vs. $8 \%$ in the previous decade, $\mathrm{p}<0.01)$. Recently NOM spread widely in selected stable patients $(21.9 \%)$. Failure rate of NOM was $14.3 \%$. Unnecessary laparotomy decreased from 36 to $21.1 \%(\mathrm{p}=\mathrm{ns})$. Introduction of laparoscopy was helpful for reliable, less invasive exploration, allowing detection of peritoneal penetration and visceral exploration. Two GSW (4\%) versus $3(5.8 \%)$ cases of the latest years required damage control surgery. A recent significant reduction in mortality and morbidity has been reported (3.85 vs. $18 \%$, p $<0.05 ; 20$ vs. $39 \%$, $\mathrm{p}=\mathrm{ns})$.

Conclusion: The recent immigration phenomenon and social changes contributed to significant increase in the incidence of penetrating trauma in Italy. Increased use of NOM and laparoscopy contributed in decreasing incidence of unnecessary laparotomies as well as morbidity and mortality.

Disclosure: No significant relationships.

$$
\text { P273 }
$$

\section{PENETRATING TRAUMA: A COMPLEX REALITY IN A NON VIOLENT SETTING}

\section{H. Santos Sousa, T. Bouça Machado, J. Preto, H. Cristino, R. Bessa de Melo, J. Costa-Maia}

General Surgery Department, São João Hospital, Porto Medical Faculty, Porto, Portugal

Introduction: Penetrating trauma is less common than blunt trauma in our country, however its incidence has been increasing in recent years. In urban centers, the mechanisms of injury may be variable, making their approach a challenge for trauma teams.

Materials and methods: Review of medical records of patients with penetrating trauma treated at the Emergency/Trauma Room of Hospital São João in Porto, Portugal, between January and December 2008.

Results: In this period, 51 patients $(44 \mathrm{M}, 7 \mathrm{~F})$ with a median age of 39 years (16-87 years) were admitted by penetrating trauma. The mechanism of injury was gunshot in 23 cases, 16 stab wounds, work accident in 11 and one iatrogenic lesion. The Revised Trauma Score (RTS) was $<4$ in 5 patients $(9.8 \%)$ and $>4$ in $46(90.2 \%)$. Of the patients with RTS $<4,2$ died in the trauma room, one was submitted to surgery and 2 were admitted in intensive care unit (ICU). In patients with $\mathrm{RTS}>4,22$ were submitted to surgery, 10 were admitted to a lower level of care and the remaining 14 to an upper level of care (ICU). The overall mortality was $15.7 \%(\mathrm{n}=8,4$ with RTS $<4$ and 4 with RTS $>4$ ). All patients who died had head trauma, which was responsible for early mortality of patients (0-6 days after trauma) and in most cases the trauma mechanism was gunshot wounds $(87.5 \%)$.

Conclusion: The penetrating injuries by gunshot are increasing in frequency and usually are associated with a worse prognosis. These injuries are often complex and difficult to approach. The implementation of management protocols may contribute to a better approach and prognosis of these patients.

Disclosure: No significant relationships.

\section{P274}

\section{SURGERY FOR VASCULAR INJURY DUE TO PENETRATING TRAUMA IN SOUTHERN AUSTRIA}

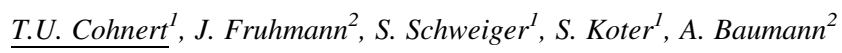

${ }^{1}$ Graz Medical University, Dept. of Vascular Surgery, Graz, Austria, ${ }^{2}$ Dept. Of Vascular Surgery, Graz Medical University, Graz, Austria

Introduction: In vascular surgical practice operations for traumatic lesions are rare with $0.3-4 \%$. Nevertheless there is a considerable risk death in trauma including vessel injuries. Aim of this study was to analyze the necessity for vascular surgery in surgery for penetrating trauma.

Materials and methods: Between 1/1998 and 10/2010 167 trauma patients (pts) underwent vascular surgical operations. Prospectively collected data were analyzed retrospectively.

Results: In 80 pts surgery was necessary for penetrating trauma (72 male and 8 female pts, mean age of $37.3+17.6$ years). Injured was body trunk in 6 pts $(7 / 80=7.5 \%)$, neck in 17 pts $(17 / 80=21.3 \%)$, upper extremity in 37 pts $(37 / 80=46.3 \%)$ and lower extremity in 20 pts $(20 / 80=25.0 \%)$. Five pts died $0-7$ days postoperatively (mortality $5 / 80=6.3 \%)$. Surgery was required for bleeding ( $65 \mathrm{pts}$ ), ischemia ( 8 pts) or a combination ( 7 pts). Trauma mechanism was stab injury in 77 pts and gunshot in 3 pts. In 10 pts the lesion was self inflicted, and in 9 pts a second person had injured the patient intentionally. There were arterial (50), venous (18) and combined (12) lesions. Vascular surgical reconstruction was necessary in 51.7\%: 25 arteries and 5 veins interposition underwent venous bypass. In 8 pts an arterial anastomosis and in 7 vessels a venous patch was performed.

Conclusion: Emergency surgery including an experienced vascular surgeon can help to improve survival in penetrating trauma. The complete extent of vascular injury needs to be determined and treated intraoperatively. A majority of patients will benefit from reconstruction involving vascular surgical techniques.

Disclosure: No significant relationships.

P275

\section{TEVAR OF TRAUMATIC THORACIC AORTIC INJURIES}

\section{Hoermann, M. Gawenda}

Department of Vascular Surgery, University Hospital of Cologne, Cologne, Germany 
Introduction: Approximately 15\% of automobile accident fatalities are associated with thoracic aortic injury, and thoracic aortic transection is the second leading cause of death in fatal accidents. The mortality rates reported for the open repair of traumatic rupture of the thoracic aorta are still high. The aim of this present study was to report the experience with endovascular management of 20 patients with acute traumatic thoracic rupture and to evaluate whether endovascular treatment is valuable in the emergency setting.

Materials and methods: We prospectively between 01/2000 and 10/2010 collected the following parameters: age, sex, Injury Severity Score, endovascular operation time, length of stay in the hospital, length of stay in the ICU and mortality, and follow-up data including computed tomographic angiography.

Results: In a cohort of 205 endovascular thoracic aortic procedures, we treated 21 patients ( $\mathrm{m}: \mathrm{f}=15: 6$; median age 48 [IQR 29-68.5] years) with an acute traumatic thoracic aortic lesion endovascularly. All endovascular procedures were technically successful, and the median operating time was $90 \mathrm{~min}$ (IQR $65-120 \mathrm{~min}$ ). The overall hospital mortality was $19 \%(\mathrm{n}=4)$, and all deaths were unrelated to the aortic rupture or stent placement. No intervention-related mortality occurred during a follow-up of 18 months in median (range 3-72 months; mean, 28.25 months).

Conclusion: On the basis of the experience in elective endovascular procedures, this minimal invasive approach might substantially reduce the mortality and morbidity compared to open surgery in traumatic thoracic aortic lesions. We conclude that immediate endovascular repair is a safe and feasible procedure for such repairs. However, close and long-term follow-up of the relatively young patients is mandatory.

Disclosure: No significant relationships.

\section{Case Reports}

\section{ACUTE CARE SURGERY}

\section{CR1}

\section{A RARE CAUSE OF BOWEL ISCHAEMIA}

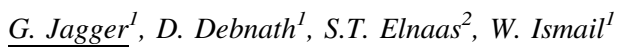

${ }^{1}$ Surgery, Queens Hospital, Romford, United Kingdom, ${ }^{2}$ Radiology, Queens Hospital, Romford, United Kingdom

Introduction: Spontaneous acute portal venous thrombosis is rare. Resulting secondary venous hypertension leading to bowel ischaemia is even rarer.

Case Report: We report a-49-year old man who presented with colicky lower abdominal pain and vomiting for 2 weeks. He was otherwise healthy and not on any regular medication. There was no significant family history. On admission the vital signs were stable. Abdominal examination revealed lower abdominal tenderness. Laboratory tests showed-Hb $13.6 \mathrm{~g} / \mathrm{dL}$, WCC $15.610^{9} / \mathrm{L}$, CRP $104 \mathrm{mg} /$ dL. Coagulation screen, liver function tests and levels of sodium, potassium, urea, creatinine and amylase were within normal limits. Erect chest and supine abdominal X-rays were unremarkable. However $10 \mathrm{~h}$ later he developed lower abdominal peritonitis. An abdominal CT-scan showed small bowel ischaemia and portal vein thrombosis. He underwent emergency laparotomy and resectionanastomosis of $60 \mathrm{~cm}$ of distal ileum. Rest of the bowel and solid viscera were normal. He had an uneventful recovery. Histology confirmed bowel ischaemia with no features of malignancy. He was anticoagulated for 6 months. Follow-ups at three and 6 months were satisfactory. Further investigations did not reveal any definite cause of portal vein thrombosis. Although initial thrombophilia screen was positive for lupus anticoagulant, subsequent screening was equivocal. Discussion: Causes of portal vein thrombosis include thrombophilia, pancreatitis, cirrhosis, hepatobiliary malignancy, intra-abdominal inflammation, sepsis and trauma. Treatments include anticoagulants, shunts, bypass surgery, and liver transplant. The patient in the case had no such cause, and responded to surgery and subsequent anticoagulation. We have not found any such case being reported in the literature.

Disclosure: No significant relationships.

\section{CR2}

\section{A RARE CAUSE OF BOWEL OBSTRUCTION}

\section{Debnath $^{1}$, R. Le Fur ${ }^{2}$}

${ }^{1}$ Surgery, Broomfield Hospital, Chelmsford, United Kingdom,

${ }^{2}$ Surgery, Newham General Hospital, London, United Kingdom

Introduction: Dermoid cyst causing bowel obstruction is rare. Occurrence of such an obstructing dermoid cyst exclusively in the terminal ileum is exceptional.

Case Report: A-65-year old woman presented with abdominal pain, vomiting and constipation of 6 days' duration. Her past medical history included hypertension. Her regular medications included Ramipril and Lacidipine. Her vital signs were normal. Abdominal examination showed distended abdomen and tenderness in lower abdomen. Laboratory tests showed-Hb $15 \mathrm{~g} / \mathrm{dL}$, WBC $7.8 \times 10^{9} / \mathrm{L}$, platelet $300 \times 10^{9} / \mathrm{L}$, normal levels of sodium, potassium, urea, creatinine and amylase. An erect chest X-ray was unremarkable, abdominal X-ray showed small bowel dilatation. An abdominal CT-scan confirmed small bowl obstruction due to a large soft tissue mass in the right iliac fossa, which appeared to be originating in the terminal ileum. The mass was partly calcified. The large bowel was not dilated. She underwent urgent laparotomy which showed an obstructing mass in the right lateral wall of pelvis, involving bladder, loops of ileum and lower pole of caecum, separate from uterus and its adnexae. A limited ileo-caecal resection was performed. She had a slow but uncomplicated recovery. Histology showed the mass to be a calcified dermoid (mature cystic teratomatous) ovarian cyst.

Discussion: Dermoid cysts arising from terminal ileum causing bowel obstruction has rarely been reported. Such an origin may be due to sequestration of ectopic tissue during embryologic development.

Disclosure: No significant relationships.

\section{CR3}

\section{A WOMAN WITH A DISCHARGING SWELLING IN THE GROIN}

D. Debnath, W. Ismail

\section{Surgery, QUEENS HOSPITAL, ROMFORD, United Kingdom}

Introduction: Infected arterial pseudoaneurysm amongst non- intravenous substance users can be misdiagnosed as an abscess.

Case Report: A 57-year-old woman presented with a painful discharging swelling in the right groin of 36 h' duration. She was referred as superficial abscess for incision-drainage. Her past medical history included non-insulin dependent diabetic mellitus and controlled hypertension. Her temperature was $37.4^{\circ} \mathrm{C}$. Local 
examination showed a non-pulsatile tender $7 \times 3 \mathrm{~cm}^{2}$ mass in the right groin just below the mid-inguinal point. There was no cough impulse. All the distal pulses were palpable. Laboratory evaluation showed leucocytosis $\left(16.2 \times 10^{9} / \mathrm{L}\right)$ and raised CRP $(59 \mathrm{mg} / \mathrm{L})$. The alarm bell rang when a thorough history-taking revealed a history of blunt trauma 8 months ago at the same site. Duplex scan clinched the diagnosis of pseudoaneurysm with thrombus formation. She underwent urgent excision of the pseudoaneurysm and repair with a venous patch.

Discussion: Medline search showed 22 articles citing one hundred patients, who presented with arterial pseudoaneurysm. Amongst them, predominantly septic presentation was significantly associated with intravenous substance abusers $(\mathrm{n}=78 ; 91.8 \%$ of 85$)$, when compared with non- substance abusers $(n=9 ; 60 \%$ of 15$)\left[\chi^{2}=11.38\right.$; $\mathrm{p}=0.001]$. The case reported should be a cautionary tale for the unwary while dealing with the rare situation of an exclusively infective presentation of an arterial pseudoaneurysm, particularly amongst non- intravenous substance users without any history of vascular intervention. Lack of pulsatility (due to underlying thrombosis) does not rule out a pseudoaneurysm completely. The case also illustrates the importance of the 'lost art' of skilled history-taking in clinical assessment.

Disclosure: No significant relationships.

\section{CR4}

\section{GASTROINTESTINAL CARCINOID TUMOR PRESENTING WITH ACUTE PAIN IN RIGHT LOWER ABDOMINAL QUADRANT: REPORT OF THREE RARE CASES}

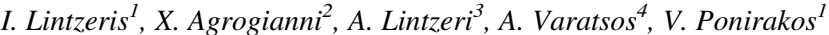

${ }^{1}$ Surgery, General Hospital of Tripolis, Tripolis, Greece,

${ }^{2}$ Postgraduate Student Of Medical School, University of Athens, Athens, Greece, ${ }^{3}$ rrd Hospital of IKA, Athens, Greece, ${ }^{4}$ Pathology, General Hospital of Tripolis, Tripolis, Greece

Introduction: Gastrointestinal carcinoid tumors are rather rare clinical entities. Among them, appendicular carcinoids represent the vast majority. However, the latter are diagnosed in surgical specimen. The aim of this study is to present our experience in defining, in our series, the incidence of gastrointestinal carcinoid tumors that cause acute abdominal pain localized in lower right quadrant.

Materials and methods: We conducted a retrospective review, searching hospital surgical data, medical histories and registries. In a 6 year period time, 625 appendicectomies were applied, in our surgery department, to patients that presented with clinical manifestations of acute appendicitis.

Case Report: We conducted a retrospective review, searching hospital surgical data, medical histories and registries. In a 6 year period time, 625 appendicectomies were applied, in our surgery department, to patients that presented with clinical manifestations of acute appendicitis.

In two cases-involved two males aged 29 and 15 years old-the pathologic and immunohistological examination of the surgical specimens revealed appendicular carcinoid tumor. A third case, where there were no pathognomonic findings in appendix, exposed a carcinoid mass in Meckel's diverticula of 5 year old boy who also appeared with clinical features of acute appendicitis. As a result, all patients were re-evaluated and received the appropriate oncologic care and treatment.

Results: In two cases-involved two males aged 29 and 15 years oldthe pathologic and immunohistological examination of the surgical specimens revealed appendicular carcinoid tumor. A third case, where there were no pathognomonic findings in appendix, exposed a carcinoid mass in Meckel's diverticula of 5 year old boy who also appeared with clinical features of acute appendicitis. As a result, all patients were re-evaluated and received the appropriate oncologic care and treatment.

Discussion: Appendicular and Meckel diverticula's carcinoid tumors are found incidentally in appendectomy and resection specimens after acute appendicitis. The former are extremely rare in young individuals and usually occur in women of fifth decade of life according to current literature data. As for the latter, it certainly consists a very uncommon finding.

Conclusion: Appendicular and Meckel diverticula's carcinoid tumors are found incidentally in appendectomy and resection specimens after acute appendicitis. The former are extremely rare in young individuals and usually occur in women of fifth decade of life according to current literature data. As for the latter, it certainly consists a very uncommon finding.

References: 1. Varas-Lorenzo MJ , Munoz-Agel F, Espinos-Perez JC, Bardaji-Bofil M. Gastrointestinal carcinoid tumors. Rev Esp Enferm Dig. (Madrid) 2010;102(9):533-7. 2. Pelizzo G, La Riccia A, Bouvier R, Chappuis JP, Franchella A. Carcinoid tumors of the appendix in children. Pediatr Surg Int. 2001;17(5-6):399-402. 3. Carpenter SS, Grillis MF. Meckel diverticulitis secondary to carcinoid tumor: an unusual presentation of the acute abdomen in an adult. Curr Surg. 2003;60(3):301-3.

Disclosure: No significant relationships.

\section{CR60}

\section{MANAGEMENT OF A SERIOUS BLUNT ABDOMINAL TRAUMA}

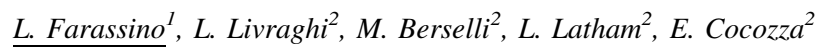

${ }^{1}$ Chirurgia Generale Ii, Ospedale di Circolo e Fondazione Macchi, Varese, Italy, ${ }^{2}$ Chirurgia Ii, Ospedale di Circolo e Fondazione Macchi, Varese, Italy

Introduction: Delay in diagnosis and treatment of abdominal injuries is one of the most common causes of preventable death from blunt or penetrating trauma. Diagnosis of injury after blunt trauma can be difficult and knowledge of appropriate management can be useful.

Case Report: An 18 years old male motorcyclist was involved in a road accident. He suffered of a blunt abdominal trauma. At hospital admission the patient was hemodynamically stable; computed tomography of chest and abdomen was performed with detection of a grade five hepatorenal lesion. After radiological examination impairment of hemodynamics happened. Patient was immediately subjected to laparotomy: Pringle manoeuvre, perihepatic packing and right nephrectomy were carried out. After removal of packing persistent bleeding was detected. Mobilization of right liver, clamping and suture of a little (about $1 \mathrm{~cm}$ ) lesion of inferior vena cava and hemostasis of liver parenchyma were performed; exploration of abdomen looking for further bleeding was negative. After washing laparotomy was effected. The patient was hospitalized in intensive care unit for 21 days. In the postoperative course infection and biliary fistula occurred; conservative management was performed by antibiotics and ERCP-PTC. Periodic washing of abdominal cavity was carried out and laparotomy was closed little by little. Patient was discharged in good general conditions in thirty-second postoperative day. 
Discussion: This report shows as DSTC guidelines can be very helpful in pre and postoperative treatment of a severe blunt abdominal trauma. Careful management of patients and efficient surgical approach are needed to minimize short and long term complications. Disclosure: No significant relationships.

\section{CR31}

\section{EMERGENCY ULTRASONOGRAPHY IN THE EVALUATION OF PATIENT WITH AN ACUTE ABDOMEN: A CASE REPORT}

\section{K.P. Chan}

Emergency Medicine, Singapore General Hospital, Singapore, Singapore

Introduction: I present a case where the application of bedside ultrasonography as a point-of-care investigation in the Emergency Department expedited the definitive treatment for a patient with an acute abdomen.

Case Report: A 68 year-old Chinese lady with no significant past medical history, presented to the Emergency Department with an acute onset of right-sided abdominal pain with nausea and vomiting. She was found to be hypotensive with blood pressure at $90 / 60 \mathrm{mmHg}$ on arrival. A right flank mass with tenderness on palpation was found on physical examination. Bedside ultrasonography via Sonosite M-Turbo was performed and the findings of a right perinephric hyperechoic mass $(8 \times 6 \mathrm{~cm})$ with central hypoechogenicity and negative Doppler flow led to an urgent CT scan of the abdomen and pelvis. The diagnosis of a actively bleeding right lower pole angiomyolipoma $(9.2 \times 7.8 \times 6.5 \mathrm{~cm})$ with large retroperitoneal haematoma was made and an urgent embolisation was performed. The patient recovered uneventfully post procedure and was discharged well 1 week later.

Discussion: Renal angiomyolipomas are intensely echogenic and may cause acoustic shadowing on ultrasonography. Less echogenic areas within the tumor are related to hemorrhage, necrosis, or dilated calyces. Doppler ultrasonography may be used to confirm the rare complication of extension into the renal vein and the IVC. This case illustrates the safe application of emergency bedside ultrasonography as a point-of-care tool on a patient with an acute abdomen which shortens the decision making process and expedites the definitive treatment for the patient.

Disclosure: No significant relationships.

\section{CR33}

\section{SURGICAL MANAGEMENT OF PANCREATIC, BILIARY AND SPLENIC TRAUMA. CASE REPORT}

\section{N. Romano, D. Pietrasanta, V. Prosperi, G. Basili, O. Goletti}

General Surgery Unit, Health Unit 5 Pisa, Pontedera Hospital, Pontedera, Italy

Introduction: Pancreatic trauma is frequently associated with organ injury, which may cause the death of the patient. Severe pancreatic injury presents a post-operative morbidity ranges from 42 to $62 \%$ and its mortality is high (18.1\%). Pancreatic injuries are often associated with complicated treatment strategy. We report our experience in the management of a pancreatic and biliary injuries.

Case Report: A 29 years old man patient (PT) reached to our observation for abdominal trauma with a diagnosis of pancreatic and biliary injury for a traffic accident. The PT was submitted to an abdominal CT scan that confirm the presence of a pancreatic, biliary and splenic injury with massive hemoperitoneum. We have performed in urgency a spleno-pancreasectomy, cholecystectomy and biliary reconstruction with T-Tube. The pt was discharged after 15 days.

Discussion: The pancreatic trauma is associated often with other organ injuries (1). Optima management and better outcome of pts with pancreatic and biliary trauma seem to be associated with shorter operative time and with simple and fast damage control surgery. In our experience the prompt diagnosis, a rapid and effectiveness surgical controls of the injury and intensive care unit stay could allow to improve the survival in this pt.

Disclosure: No significant relationships.

\section{CR34}

\section{THE ROLE OF LAPAROSCOPY IN DIAGNOSING INTESTINAL PERFORATIONS AFTER BLUNT ABDOMINAL TRAUMA}

\section{A. Nicolau}

General Surgery, Emergency Clinical Hospital, Bucharest, Romania

Introduction: Hollow viscus injuries following blunt abdominal trauma are much less common then solid organ injuries. An accurate diagnosis may be difficult within the first few hours after the accident, especially in multiple trauma patients. The clinical examination may be irrelevant in as much as half the patients, while the CT scan only shows indirect signs in most of them (peritoneal fluid in the absence of a solid organ injury). In selected cases, with equivocal clinical and imagistic examinations, diagnostic laparoscopy could be an appropriate diagnostic tool.

Case Report: We present five cases of intestinal perforation after blunt abdominal trauma diagnosed by laparoscopy: four male patients and a female patient, with an average age of 29.6 years. Four patients were involved in motor vehicle accidents, all presenting with multiple injuries and another patient was assaulted, presenting with an abdominal contusion. An ultrasound was performed in all patients and four of them were CT scanned. Three of the patients had indirect CT signs and one patient had free peritoneal air. The patients underwent laparoscopic surgery with a thorough exploration of the entire abdominal cavity, identification of the intestinal injury, lavage and multiple peritoneal draining. We converted all cases to open surgery, performing three intestinal resections and two enterorrhaphies through supra-umbilical mini-laparotomies, three longitudinal and two transverse. There was only one patient with postop complications infected surgical wound. The average length of stay in the surgical ward has been 8.6 days (5-22 days). There were no missed injuries. Discussion: Laparoscopy is an effective diagnostic tool in certain patients with blunt abdominal trauma and suspected intestinal injuries, in which the clinical exam, CT and US examinations are irrelevant. It can avoid an unnecessary as well as a delayed laparotomy, both situations being associated with increased morbidity. Disclosure: No significant relationships. 


\section{CENTRALIZATION CRITERIA AND PRE-HOSPITAL CARE}

\section{CR5}

\section{INTERHOSPITAL TRANSPORT OF AN ARDS PATIENT AFTER MULTIPLE TRAUMA. A CASE REPORT}

\author{
M. Weuster ${ }^{1}$, S. Oestern ${ }^{1}$, S. Lippross ${ }^{1}$, A. Thiem $^{2}$, A. Seekamp ${ }^{3}$, \\ J. Cremer $^{2}$, N. Haake
}

${ }^{1}$ Trauma Surgery, University hospital, Kiel, Germany, ${ }^{2}$ Cardiac Surgery, University hospital, Kiel, Germany, ${ }^{3}$ Unfallchirurgie, Universität Kiel, Kiel, Germany

Introduction: After being hit by a huge tree trunk a 36 year young man was treated as a severely injured patient and transported to a hospital of regional care. Besides severely fractures he had primarily to be operated on a fracture of the upper ankle joint right side. During his stay on the intensive care unit he developed an acute respiratory failure with evidence of pneumonia in X-ray and microbiology sputum testings. Invasive and artificial respiration could not sustain adequate ventilation and oxygenation of the patient. Up to this date the patient had been intubated for about 1 week $(200 \mathrm{~h})$ since trauma.

Case Report: Thus the medical team initially decided to implant an interventional lung assist device (iLA) a pumpless system via femoral vessels requiring sufficient cardiac output. The patient manifested rapidly a septic circulation becoming increasingly cardiopulmonary instable with an increasing need of vasopressors as medicinal support. The effect of the iLA as a pumpless system could be neglected. Measurements of mean $p \mathrm{O}_{2}$ and mean $p \mathrm{CO}_{2}$ hardly differed from each other with counts of $70 \pm 10$ and $60 \pm 10 \mathrm{mmHg}$. Unfortunately conventional methods of artificial respiration had been exhausted. Immediate extracorporeal membrane oxygenation was needed. Transferring the patient to the next trauma level at this stage was unfeasible due to his weak clinical condition. In collaboration with experienced colleagues from a center of maximal care it was possible to conduct an interhospital transport. Therefore analyzing anticoagulation, hemodynamics and respiratory parameters as well as the general history were necessary to prepare the patient's rescue. Supported by the regional fire department the kind of transfer was planned as a ground rescue. Having checked the conditions the patient was handed over on-site and the iLA could be switched to a venoarterial ECMO via already existing femoral vessel cannulation. The operation was uneventful and the patient could be primarily stabilized. Afterwards using an extra large fully equipped intensive care ambulance vehicle including staff the patient could be transferred to the maximum level of trauma care. The whole procedure took place within a few hours and the patient reached the hospital safely. Then we switched the original cannulation of femoral vessels in Seldinger technique to bigger sized cannulas.

Discussion: Critical ill patients with lung failure need to be put to highly specialized units providing extracorporeal cardiopulmonary support. Establishing quickly a sufficient ventilation and oxygenation is essential for the patient's survival preventing multi organ failure. It is the last chance of rapid stabilization.

Disclosure: No significant relationships.

\section{CLINICAL ULTRASOUND IN ACUTE CARE SURGERY}

\section{CR6}

\section{CONSERVATIVE MANAGEMENT OF A IV GRADE BLUNT HEPATIC TRAUMA IN A YOUNG FEMALE PATIENT}

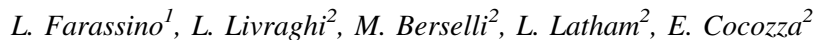

${ }^{1}$ Chirurgia Generale Ii, Ospedale di Circolo e Fondazione Macchi, Varese, Italy, ${ }^{2}$ Chirurgia Ii, Ospedale di Circolo e Fondazione Macchi, Varese, Italy

Introduction: Conservative treatment of blunt hepatic injuries has been extended to a large number of patients in the last years thank to availability of rapid ultrasonography, helical CT and interventional radiology.

Case Report: A 17 years old female had a road incident (fall down from scooter running). At hospital admission patient was hemodynamically stable. Eco-FAST showed free fluid in the abdomen; total body CT scan detected a wide, lacerated and contused wound of the right hepatic lobe (sixth, seventh and eighth segment); a suprahepatic vein was present inside the lesion (IV grade liver injury scale). Active leakage of contrast was not documented. Patient was admitted in Intensive Care Unit and conservative management was started. Continuous monitoring of cardiovascular parameters and of hematocrit values were performed; ultrasonography of the abdomen was performed every hour. Conditions of patient kept stable in successive hours. During hospitalization a right chest drain was positioned after the appearance of reactive pleural effusion conditioning dyspnea. After transfer of patient in General Surgery Unit chest drain was removed and execution of a control CT was performed: a little bile collection of about $4 \mathrm{~cm}$ of diameter was detected and managed without evidence of dimensional increasing. Twenty-two days after incident the patient was discharged in good condition.

Discussion: In hemodynamically stable patients without signs of active bleeding on CT conservative management of serious blunt hepatic trauma is possible. Monitoring of hematocrit values, vital signs, and evolution of hepatic injury by ultrasonography and/or CT is necessary and can avoid surgery.

Disclosure: No significant relationships.

\section{CR7}

\section{SURGEON-PERFORMED CONTRAST ENHANCED US- GUIDED PERCUTANEOUS SEALING OF POSTRAUMATIC SPLENIC PSEUDOANEVRYSM}

\author{
M. Zago, D. Mariani, H. Kurihara, A. Casamassima, F. Luzzana, \\ F. Butti, R.A. Foà
}

General And Emergency Surgery, Istituto Clinico Città Studi, Milano, Italy

Introduction: Non-operative management (NOM) of splenic trauma requires a clear follow-up protocol. CT is widely used to detect the 
AV fistula or pseudoanevrysm. Contrast-enhanced US (CEUS) is a novel tool for detecting solid organ injuries. We describe a case of splenic pseudoanevrysm detected and treated under CEUS follow-up. Case Report: Surgeon-performed CEUS with Sonovue ${ }^{\circledR}$ (Bracco, Italy) is routinely performed by our Team for monitoring NOM of splenic injuries. CEUS is scheduled on day 2 and day 5; B-mode US is performed at day 10 and 30 , then according to the morphologic evolution. A 42 y.o. pt with a grade 3 splenic injury was admitted for NOM. Day2 CEUS showed a typically enhanced $15 \mathrm{~mm}$ pseudoanevrysm at the lower pole, w/o contast extravasation. Due to the clear visibility, a percutaneous US-guided sealing was planned instead of angioembolisation. An informed consent was obtained. During a second CEUS exam, under local anestesia, a 20G needle was inserted into the spleen and the pseudoanevrysm. Two $\mathrm{ml}$ of Trombin were injected under US control. No complications occurred. CEUS performed at day 5 (three after sealing) and day 10, showed complete disappearance of the pseudoanevrysm. Pt was discharged on day 12 . Long term follow-up was uneventful.

Discussion: CEUS is a novel tool for monitoring NOM of splenic injuries. CEUS allows to detect psudoanevrysm and can be used to support interventional percutaneous sealing of this fearful condition. References: 1. Zago M, Mariani, D, Kurihara $\mathrm{H}$, et al. EJTES 2010;36:218-19. 2. Valentino M, Serra C, Pavlica P, Barozzi L. Semin Ultrasound CT MR. 2007;28(2):130-40.

Disclosure: No significant relationships.

\section{CR8 \\ UNUSUAL CASE OF SUPERIOR MESENTERIC VEIN THROMBOSIS AS A COMPLICATION OF CROHN'S DISEASE:}

\section{O. Yener}

Göztepe Training And Research Hospital/surgery, istanbul, Istanbul, Turkey

Introduction: Acute mesenteric venous ischemia is a serious acute abdominal serious condition requiring early diagnosis and intervention to improve to outcome. Surgical resection is still the main curative approach. Superior mesenteric vein stenosis as a consequence of mesenteric fibrosis, causing the development of small bowel varices, is an unrecognised association of Crohn's disease [1-2].

Materials and methods: We report here a case of superior mesenteric vein thrombosis in a 21 year old male patient with no history of crohn's disease and other risk factor of thrombosis.

Case report: We report here a case of superior mesenteric vein thrombosis in a 21 year old male patient with no history of crohn's disease and other risk factor of thrombosis. The patient developed a small bowel obstruction that required a urgent laparotomy, which extreme venous congestion of the small bowel proximal to $30 \mathrm{~cm}$ from the ileoceacal valve, generalized ascites, and creeping fat in the small bowel serosa. $90 \mathrm{~cm}$ of necrotic small intestine was resected (Fig. 1).

Results: The patient developed a small bowel obstruction that required a urgent laparotomy, which extreme venous congestion of the small bowel proximal to $30 \mathrm{~cm}$ from the ileoceacal valve, generalized ascites, and creeping fat in the small bowel serosa. $90 \mathrm{~cm}$ of necrotic small intestine was resected (Fig. 1).

Discussion: Histo-pathologic examination showed underlying mesenteric venous thrombosis with evidence of Crohn $\mathrm{s}$ disease. Hematology workup showed no evidence of a congenitally inherent hypercoagulable state. The patient followed-up by gastroenterology clinic. Sulfasalasine therapy was given to the patient.
Conclusion: Histo-Pathologic examination showed underlying mesenteric venous thrombosis with evidence of Crohn $\mathrm{s}$ disease. Hematology workup showed no evidence of a congenitally inherent hypercoagulable state. The patient followed-up by gastroenterology clinic. Sulfasalasine therapy was given to the patient.

References: 1. Solem CA. Venous thromboembolism in inflammatory bowel disease. Am J Gastroenterol 2004;99: 97-101. 2. Fichera A. Superior mesenteric vein thrombosis after colectomy for inflammatory bowel disease: a not uncommon cause of postoperative acute abdominal pain. Dis Colon Rectum 2003;46: 643-648. 3. Rhee RY and Gloviczki P. Mesenteric venous thrombosis. Surg Clin North Am 1997;77:327-338. 4. Kim AY, Ha HK. Evaluation of suspected mesenteric ischemia: efficacy of radiologic studies. Radiol Clin North Am. 2003;41:327-342.

Disclosure: No significant relationships.

\section{DIFFICULT WOUND MANAGEMENT}

\section{CR9}

\section{"AWAKE" LAPAROSCOPY—SCREENING METHOD IN ASYMPTOMATIC PATIENTS WITH PENETRATING ABDOMINAL TRAUMA?}

\section{S. Tsintsari, G. Rojnoveanu, G. Ghidirim, R. Gurghis, V. Gafton, V. Spataru, V. Bunescu}

Surgery No.1 "n. Anestiadi”, SMPhU "N. Testemitanu”, Chisinau, Moldova

Introduction: The role of laparoscopy in trauma surgery is controversial. The weak points of the method are: the invasive character, the need for general anesthesia and low informativity on some abdominal injuries. The goal of this study was to evaluate the informativity of "awake" laparoscopy (AwL) for the management of asymptomatic patients with penetrating abdominal trauma.

Materials and methods: A retrospective study of 77 patients (2007-2010). Inclusion criteria: abdominal/thoracoabdominal wounds, which penetration cannot be ruled out clinically, absence of indications for exploratory laparotomy. All laparoscopic procedures were performed in the operating room under local anesthesia. Evaluation criteria: penetration confirmation, data suggestive for therapeutic laparotomy.

Case Report: A retrospective study of 77 patients (2007-2010). Inclusion criteria: abdominal/thoracoabdominal wounds, which penetration cannot be ruled out clinically, absence of indications for exploratory laparotomy. All laparoscopic procedures were performed in the operating room under local anesthesia. Evaluation criteria: penetration confirmation, data suggestive for therapeutic laparotomy. There were 68 abdominal wounds: penetration was ruled out in 25 cases (with no false results) and confirmed in 43 patients. For parietal peritoneum lesion the method had: sensitivity $97.67 \%$, specificity $100 \%$, PPV-100\%. For thoracoabdominal wounds $(\mathrm{n}=21)$ : diaphragm injury was ruled out in 11 cases (one false result), direct visualization of the diaphragm injury â€" in 5 and indirect data â€" in 5. In patients with penetrating wounds $(\mathrm{n}=54,56$ procedures), methodâ $€^{\mathrm{TM}_{\mathrm{S}}}$ informativity in decision for therapeutic laparotomy: sensitivity $50 \%$, specificity $78.57 \%$, PPV $43.75 \%$, NPV $82.50 \%$.

Results: There were 68 abdominal wounds: penetration was ruled out in 25 cases (with no false results) and confirmed in 43 patients. For parietal peritoneum lesion the method had: sensitivity $97.67 \%$, specificity $100 \%$, PPV $100 \%$. For thoracoabdominal wounds $(\mathrm{n}=21)$ : diaphragm injury was ruled out in 11 cases (one false result), direct visualization of the diaphragm injury â€" in 5 and 
indirect data â€” in 5 . In patients with penetrating wounds $(\mathrm{n}=54$, 56 procedures), methodâ $€^{\mathrm{TM}_{\mathrm{S}}}$ informativity in decision for therapeutic laparotomy: sensitivity $50 \%$, specificity $78.57 \%$, PPV $43.75 \%$, NPV $82.50 \%$.

Discussion: AwL is a sensitive method for wound penetration confirmation. AwL offers an important diagnostic tool in excluding diaphragm injury. As a part of diagnostic algorithm, AwL would rather provide data for excluding an unnecessary laparotomy than for indicating a therapeutic intervention.

Conclusion: AwL is a sensitive method for wound penetration confirmation. AwL offers an important diagnostic tool in excluding diaphragm injury. As a part of diagnostic algorithm, AwL would rather provide data for excluding an unnecessary laparotomy than for indicating a therapeutic intervention.

Disclosure: No significant relationships.

\section{CR10}

\section{PENETRATING THORACIC INJURY AN INTERESTING CASE REPORT}

\section{Grigoraki $^{1}$, T. Kokkinakis ${ }^{2}$, G. Panagiotakis ${ }^{3}$, A. Moniakis $^{4}$, C. Stroumbos $^{4}$, K. Spiridakis ${ }^{5}$}

${ }^{1} 1$ st Surgical, Venizeleion Hospital, Heraklion, Greece, ${ }^{2}$ 1st Surgical, Venizeleion Hospital, Heraklion Crete, Greece, ${ }^{3} 1$ st Surgical Department, Venizelio Hospital, Heraklion Crete, Greece, ${ }^{4}$ thoracic Surgery, Univercity Hospital, Heraklion, Heraklion, Greece, ${ }^{5} 1$ st Surgical, Venizelion Hospital, Heraklion, Greece

Introduction: Thoracic injuries account for $20-25 \%$ of deaths due to trauma and contribute to $25-50 \%$ of the remaining deaths. Rarely, chest wall hemorrhage can result in exsanguination and may require operative control. This is a case report of a penetrating chest injury. Case Report: A 43 year old male, after a motor vehicle collision on a metallic bar on the highway, is admitted to the ER department, with a penetrating chest trauma-about $90 \%$ skin deficiency of the right chest surface-GCS $6 / 15$, hemodynamically unstable, and with multiple face, neck and upper extremities injuries. Right thoracic drainage tube and tracheal intubation follows, peritoneal drainage tube and urethral catheterization performed by the general surgeons are negative for hemorrhage and the patient is admitted to the operating theatre. Thoracic surgeons performed a frontal thoracotomy through the open trauma, controlled the vascular hemorrhage of the thoracic and neck region, closed the surgical trauma, and placed 2 thoracic drainage tubes. The patient is admitted to the ICU department. CT control revealed multiple trauma. During ICU stay, chest trauma had a surgical site purulent infection. After 24 days, the patient was managed by plastic surgeons.

Discussion: In the western population, it appears that chest wall injury accounts for over $50 \%$ of all thoracic injuries. Associated extra thoracic injuries have been reported in the literature to be $50-75 \%$. Basic education in chest trauma management should be provided to all physicians involved in emergency treatment. Tertiary care of these patients is multidisciplinary and essential to minimize mortality and long-term morbidity.

Disclosure: No significant relationships.

\section{CR11}

\section{PENETRATING TRACHEAL INJURY CAUSED BY A FOREIGN BODY: A CASE REPORT}

\author{
H. Dogan ${ }^{1}$, D.N. Ozucelik ${ }^{2}$, A. avci ${ }^{I}$, M. Kıctr ${ }^{1}$, K. Aciksari $^{1}$, \\ B.M. Ayvacl
}

${ }^{1}$ Emergency Medicine, bakırköy sadi konuk training and research Hospital, Istanbul, Turkey, ${ }^{2}$ Emergency Medicine, Bakırkoy Sadi Konuk Training and Research Hospital, Istanbul, Turkey

Introduction: The posterior membranous part of the trachea is the commonest site of rupture. Anterior rupture of the trachea near the carina is not only rare but can be catastrophic. Here we describe an interesting case of anterior tracheal rupture

Case Report: A 12-years-old girl was admitted to the emergency room after having tripped while running, striking her neck on the balcony railing. The patient was conscious and distressed but hemodynamically stable. Local examination revealed a penetrating injury to the anterior neck. Pulse rate was $106 / \mathrm{min}$, systolic blood pressure was $110 / 80 \mathrm{mmHg}, \mathrm{SaO}_{2}$ was 96 . Clinical examination showed subcutaneous emphysema of the thorax and neck extending to the face. Chest X-ray revealed infiltrates in both lungs, a pneumomediastinum and subcutaneous thoraco cervical emphysema. Cervical spine X-rays confirmed the presence of subcutaneous air without evidence of bony irregularities. She was transferred to the operating room for bronchoscopy.

Discussion: Only a minority of laryngotracheal injuries are caused by penetrating trauma. Penetrating injuries of the neck involve the larynx in $5-15 \%$ of patients, associated carotid artery or digestive track injuries being twice as likely to have airway injuries. The prevailing site of tracheal transection is the junction of the cricoid with the trachea, because the connective tissues in this area are weak.

Disclosure: No significant relationships.

\section{CR12}

\section{UNIQUE APPROACH TO WOUND VACUUM THERAPY IN COMBAT CASUALTIES}

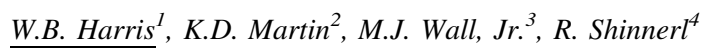

${ }^{1}$ General Surgery, Keesler Medical Center, Biloxi, MS, USA, ${ }^{2}$ Trauma Program, Landstuhl Regional Medical Center, APO AE, USA, ${ }^{3}$ Surgery, Baylor College of Medicine, Houston, TX, USA, ${ }^{4}$, Evansville Surgical Associates, Evansville, IN, USA

Introduction: The introduction of negative-pressure wound therapy devices in 1997 was a landmark breakthrough in acute and chronic wound management. The use of these devices has become particularly high in the management of battlefield trauma, specifically that which results from casualties sustained in Operation Iraqi Freedom (now Operation New Dawn) and Operation Enduring Freedom (OEF). Surge operations in Afghanistan in recent months resulted in a large number of casualties presenting with large open tissue wounds, be it mangled or amputated extremities or flank and perineal wounds. A large portion of these 
injuries are managed with negative-pressure wound therapy with excellent results.

Case Report: Due to surge combat actions in Afghanistan and the use of Improvised Explosive Devices (IED) that are undetectable by conventional mine-sweeping devices, many soldiers and Marines are presenting with high bilateral above knee amputations and complex perineal injuries. Advances in body armor as well as tourniquet technology increase the survival rate of what classically would be mortal injuries. These wounds by their very nature are at high risk for infection and progression of necrosis due to the blast nature of the mechanism of injury. Soldiers and Marines thus benefit greatly from negative-pressure wound therapy for assistance in healing. At US Army Landstuhl Regional Medical Center, unique methods of sponge fixation and troubleshooting of leaks has increased the successful placements of these sponges on these devastating wounds. The military experience using these sponges and description of techniques used for these complicated wounds are discussed from experience of 342 cases over a 9 month period.

Discussion: At US Army Landstuhl Regional Medical Center, our innovative techniques of sponge fixation has increased the successful placement of wound vacuum sponges on these devastating wounds. The military experience of over 300 cases in a 9 month period with descriptions of the techniques used for these complicated wounds are discussed. These techniques limit operative time, improve wound healing, and reduce the number trips to the operating room. Moreover, they enable the successful long-range aeromedical transport of critically ill patients from Germany to the continental United States for further definitive management.

Disclosure: No significant relationships.

\section{CR13}

\section{VACCUM ASSISTED WOUND CLOSURE AND AMPHOTHERICIN B IN DIALYSIS-RELATED CANDIDA ENCAPSULATING PERITONITIS. A CHANCE AFTER THE FAILURE OF A TRADITIONAL THERAPY}

\section{Balassone, O.C. Buonomo, A.V. Granai, D. Venditti, G. Sena, B. Ielpo, G. Petrella}

Emergency Surgery, Breast Surgery And Transplants, Fondazione Universitaria Policlinico Tor Vergata, Rome, Italy

Introduction: Fungal peritonitis related to peritoneal dialysis contributes significantly to morbidity and to an high level of mortality. Up to now, there have not been any studies clearly demonstrating which treatment regimen has the best outcome in this kind of patient. Case Report: A 33 year old man, under continuous ambulatory peritoneal dialysis, was admitted to our department with an infective peritonitis suspect. Results of peritoneal lavage's culture showed a growing of Enterococcus faecalis and colonies of Candida albicans. We decided to immediately explore the abdomen, to remove the peritoneal dialysis catheter and to administer intravenous fluconazole and amphotericin B. Despite 30 days of intravenous combined-antibiotic therapy, cultures were still positives for Candida Albicans. Peritoneal washing with amphotericin B and vacuum assisted abdominal system were started and after 12 cycles we achieved the eradication of the Candida.

Discussion: Up to $80 \%$ of fungal peritonitis in patients on peritoneal dialysis are caused by candida. Catheter removement and prompt initiation of antifungal therapy are indicated, subsequent therapy is based upon culture results, sensitivity, and patient response. Vacuum assisted wound closure, has become the preferred treatment modality for many complex/chronic wounds and the topical negative pressure demonstrated a positive contribution also in managing severe peritonitis. Conclusions: peritoneal sclerosis, can negatively affect results of an intravenous antifungal therapy. Amphotericin B intraperitoneal lavages associated with topical negative abdominal pressure, can represent an effective option for common therapy-resistant peritonitis. Disclosure: No significant relationships.

\section{CR14}

VAC. THERAPY, AS THE LAST CHANCE IN THE MANAGEMENT OF EXTENSIVE SOFT TISSUE ENVELOPE DAMAGE - DEMONSTRATION UNDER TWO CASES

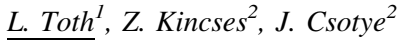 \\ ${ }^{1}$ Traumatology, Pandy Kalman Hospital, Gyula, Hungary, \\ ${ }^{2}$ Traumatology, Pándy Kálmán Hospital, Gyula, Hungary
}

Introduction: The topical negative pressure therapy is a world wide accepted treatment of a variety of wound types including open fractures, extensive degloving injuries, infected wounds, etc. The negative pressure has been shown to accelerate debridement, decreases tissue bacterial levels and increases the rate of cell proliferation. Although its costly, we found it vital in special cases.

Materials and methods: First case: 37 year old female patient was rejected from a car, and sustained an open (Gustilo IIIB) Monteggia fracture. The comminuted ulna fracture was combined with an extended soft tissue, and tip of olecranon bony loss caused by the friction forces. Series of VAC. therapy was used following the debridement and plating, and the soft tissue consolidation was finished with a local rotation flap plus skin graft procedure, in conjunction with the VAC therapy again. Second case: 43 year old diabetic male patient underwent several debridements and necrectomies of his right ankle septic arthritis, resulted a huge soft tissue loss, including the lateral wall of the joint. The continuous VAC therapy was supplemented with joint irrigation, and the articulation was closed with a rotation muscular flap. When the septic arthritis has settled, skin grafting was made.

Case report: First case: 37 year old female patient was rejected from a car, and sustained an open (Gustilo IIIB) Monteggia fracture. The comminuted ulna fracture was combined with an extended soft tissue, and tip of olecranon bony loss caused by the friction forces. Series of VAC. therapy was used following the debridement and plating, and the soft tissue consolidation was finished with a local rotation flap plus skin graft procedure, in conjunction with the VAC. therapy again. Second case: 43 year old diabetic male patient underwent several debridements and necrectomies of his right ankle septic arthritis, resulted a huge soft tissue loss, including the lateral wall of the joint. The continuous VAC. therapy was supplemented with joint irrigation, and the articulation was closed with a rotation muscular flap. When the septic arthritis has settled, skin grafting was made.

With the series of surgeries and the VAC therapy, the patients have recovered with excellent functional and cosmetic outcome, and they turned back to their everyday life and profession. The cases were documented from the initial conditions, thru the intra-operative steps, to the final results, and will be demonstrated, including constant scores.

Results: With the series of surgeries and the VAC therapy, the patients have recovered with excellent functional and cosmetic outcome, and they turned back to their everyday life and profession. The cases were documented from the initial conditions, thru the intraoperative steps, to the final results, and will be demonstrated, including constant scores. 
Discussion: VAC therapy can be regarded as a favorable method of wound care in difficult cases. However its rather used for temporary wound coverage before definitive wound closure. We would like to demonstrate that in almost hopeless cases the longer use of VAC in conjunction with split thickness skin grafting can lead to good result. Conclusion: VAC therapy can be regarded as a favorable method of wound care in difficult cases. However its rather used for temporary wound coverage before definitive wound closure. We would like to demonstrate that in almost hopeless cases the longer use of VAC in conjunction with split thickness skin grafting can lead to good result. Disclosure: No significant relationships.

\section{FRACTURES/DISLOCATIONS}

\section{CR15}

\section{ACUTE PERSISTENT TRAUMATIC ANTERIOR DISLOCATION OF THE FRACTURED RADIAL HEAD: CASE REPORT AND NOVEL SURGICAL TECHNIQUE}

\section{N. Baraza, M. Krkovic}

Trauma and Orthopaedics, University Hospital Coventry and Warwickshire, Coventry, UK

Introduction: Anterior dislocation of the fractured radial head is a rare injury and reduction is challenging. We highlight a case report of the above injury and detail a novel method of maintaining reduction. Case Report: A young male sustained a fracture and anterior dislocation of his left radial head when a fork lift truck landed on his left arm. Initially, he had forearm fasciotomies because of the swelling and an external fixator was applied, but this could only hold the radial head in place with the elbow flexed to $90^{\circ}$ and the forearm in maximum pronation. This position was not acceptable due to the forearm swelling. Attempted repair of the lateral ligamentous complex of the elbow did not reduce the dislocation. Three screws, one each in the capitellum, radial head and radial aspect of the ulna were inserted and tension band wires fashioned between these screws. The tension band wires were then tightened and the lateral collateral ligamentous complex repaired. 18 weeks post operatively the metal work was removed and aggressive physiotherapy instituted. At 6 months, his range of movement was $50^{\circ}$ to $100^{\circ}$ in the flexion-extension arc and supination from neutral to $45^{\circ}$.

Discussion: In the current literature, apart from the use of an external fixator and direct repair of the soft tissues, there is no published surgical method of reducing radial head dislocations. This novel method of radial head reduction should form part of the trauma surgeon's armament when considering surgical treatment of these infrequently encountered injuries.

Disclosure: No significant relationships.

\section{CR16}

\section{CLOSED TOTAL TALAR DISLOCATION}

J. Quintana Plaza ${ }^{1}$, A.A. Jorge Mora ${ }^{2}$, M.R. García-Rayo Rodríguez-

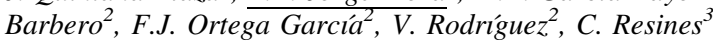

${ }^{1}$ Cot Ii, Hospital 12 de Octubre, Madrid, Spain, ${ }^{2}$ Cot Ii, Hospital 12 de Octubre, Madrid, Spain, ${ }^{3}$ Servicio De Cirugia Ortopedica Y Traumatologia Ii, HOSPITAL 12 DE OCTUBRE, Madrid, Spain
Introduction: Total talar dislocation without fracture associated is a very uncommon injury in adults, with only a few cases having been reported on the literature. It is usually consequence of a severe trauma to the foot because of a motorcycle accident or a fall from height.

Case Report: We introduce a 30 year oldpatient of 30, male, without relevant medical history, who felt from a small wall onto his foot with an inversion mechanism. Physical examination showed a deformation on the lateral of the ankle and midfoot, with an important induration. After radiologic assessment, it showed a total talar dislocation without associated fracture. The patient was brought to the operating theatre to perform a closed reduction under general anesthesia, which was unsuccessful, so an open reduction was realized with later fixation with a Steinmann pin inserted through the heel. After an unremarkable postoperative course, the patient was maintained without load in his damaged foot. After 6 weeks the Steinman pin was removed. At this moment the patient has started to load partially, without pain or signs of avascular necrosis or infection. RNM 6 months form the surgery will probably show avascular necrosis of the talus.

Discussion: Open dislocation is more common in adults, however closed dislocation is common in pediatric patients. Treatment of closed dislocation usually involves closed or open reduction, and internal fixation, with high possibilities of avascular necrosis and variable clinical outcomes.

Disclosure: No significant relationships.

\section{CR17}

\section{COMPLEX SACRAL FRACTURE-CASE REPORT}

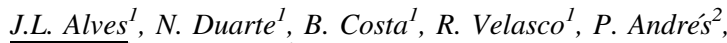 \\ A. Rocha , M.J. Frade
}

${ }^{1}$ Neurosurgery, Centro de Neurocirurgia de Coimbra, Coimbra, Portugal, ${ }^{2}$ Orthopedics, Centro Hospitalar de Coimbra, Coimbra, Portugal

Introduction: Sacral fractures, with a wide range of injury mechanisms, represent less than $2 \%$ of spinal fractures, which can be in part explained as they can go unrecognized in major pelvic fractures. Neurological injury varies from incomplete injury of a single nerve root to involvement of the entire cauda equina. Conservative treatment is an valid option.

Case report: 56-year-old male suffered a fall, with impact in the left buttock. The patient had no other relevant contributory history, injury or illness. Symptoms were pain in left sacro-iliac joint and perineum + paresthesia in left leg and perineum. On examination, tenderness in lumbar-sacral region. There was no motor deficit, hypoesthesia or pelvic injury. The rest of the physical exam was unremarkable. Urinary retention ensued, for what he was catheterized. A CT-scan was obtained, showing fractures in L1-L2 left transverse processes + sacral complex fractures (vertical, transverse and oblique components-reaching the three zones, Denis Classification) + subluxation S1-S2 + fracture of coccyx. The treatment option was bed rest for several weeks, with pain management and progressive mobilization. Discussion: Sacral fractures are commonly associated with major pelvic fracture. In this case, there was no pelvic injury, instead two minor lumbar fractures, a rare association. Another remarkable aspect is the major sacral trauma, unexpected for the severity of the fall, stressing the importance of high suspicion. Comparing operative decompression versus conservative treatment in stable fractures remains a debatable subject, but, as reports show significant improvement with non-operative measures, this approach seems appropriate in a majority of cases.

Disclosure: No significant relationships. 


\section{CR18}

\section{DISTAL RADIUS FRACTURE AND SCAPHOLUNATE DISSOCIATION-CASE REPORT}

\author{
S.I.V.S. Fernandes ${ }^{1}$, J.E. Fraga Ferreira ${ }^{2}$, A.T. Barbosa ${ }^{2}$, \\ J. Oliveira ${ }^{2}$, R. Ferrero ${ }^{2}, V$. Caetano $^{2}$, P. Mendes $^{2}$, M. Loureiro $^{2}$
}

${ }^{1}$ Orthopedics And Traumatology, Centro Hospitalar Alto Ave Guimarães, Guimarães, Portugal, ${ }^{2}$ Orthopaedics And Traumatology, Centro Hospitalar do Alto Ave, Guimarães, EPE, Guimarães, Portugal

Introduction: Management of intra-articular fractures is directed toward restoring the normal anatomy of the distal radius and ulna to maintain the adequacy of reduction of the articular surface. This fracture can be associated with scapholunate dissociation. In scapholunate dissociation, the abnormal kinematics leads to a decrease in surface area contact at the radioscaphoid joint, which causes an increase of the concentration of load, leading to the development of degenerative arthritis. The goals of the surgical reconstruction are to relieve the symptoms associated with the instability, maintain motion, and prevent the development of degenerative arthritis.

Case report: It concerns a 32-year-old that had a fall on an outstretched hand and attended the emergency room of our hospital in $13 / 10 / 08$. At the admission he had deformity, swelling and pain of the wrist.

Radiographs showed intra-articular fracture of the distal radius type 23.B2 AO classification and scapholunate dissociation.

The patient was submitted to open reduction of the fracture and osteosyntesis with volar plate and was orientated to physical rehabilitation.

1 month after surgery the fracture was healing and the scapholunate dissociation was correct, 2 months later the patient had complete mobility and returned to active life and work.

Discussion: The importance of this case is to obtain the notion that after obtaining a correct reduction of the intra-articular distal radius fracture with the osteosyntesis with volar plate we could obtain also a correction of the scapholunate dissociation, prevent the carpal instability and degenerative arthritis and maintain correct carpal alignment.

Disclosure: No significant relationships.

\section{CR19}

FEMORAL CONDYLE FRACTURE AFTER LATERAL PATELLA DISLOCATION. FIXATION TECHNIQUE WITH SUBCONDRAL K WIRES

\section{Roman, R. Fleaca}

\section{Orthopedic Surgery and Trauma, University of Sibiu, Sibiu, Romania}

Introduction: Lateral patellar dislocation can produce a osteochondral fracture of the weight-bearing portion of the lateral femoral condyle which is a less frequent complication. Its diagnosis and treatment can be challenging. The goal of all treatment methods is to provide a stable, congruent joint surface, restore function, and prevent the evolution of osteoarthritis in the injured joint. In the absence of resorbable pins fixation or fibrin glue, fixation with subchondral deepened $\mathrm{K}$ wires, represents a valuable alternative.
Introduction: Lateral patellar dislocation can produce osteochondral fractures of the lateral femoral condyle. Optimal treatment should provide anatomic reduction, stable fixation, restore function.

Materials and methods: We present a case of 26 years old male who suffered a lateral patellar dislocation (single episode-forced knee extension and external rotation) - spontaneously reduced. At admission he presented massive effusion of the knee joint, painful knee, limited ROM. X-Rays (Knee AP, LL, patella axial) revealed a loose fragment in the suprapatellar recess. Surgery was performed within the first $24 \mathrm{~h}$. A $2 / 3 \mathrm{~cm}$ osteochondral fragment was identified. Evaluation of the articular surfaces was made and a defect of the lateral femoral condyle was revealed. Fracture surface preparation (soft curettage) was performed. Microfractures into the remaining subchondral bone were performed and anatomical reduction was obtained. Fixation was performed with $3 \mathrm{~K}$ wires: insertion from lateral to medial and caudal to cranial. The distal end was deepened $1 \mathrm{~mm}$ under the cartilage surface of the lateral condyle. The caudal end was bound and left under the skin on the medial side of the thigh. Postoperative the patient was immobilized in extension for 3 weeks in a fixed knee orthesis and then started full mobilization with the interdiction of weight bearing for 8 weeks.

Case Report: 26 years male suffered a lateral patellar dislocation. At admission he presented massive, painful effusion. XRays: loose fragment in the suprapatellar recess. At surgery a $2 / 3 \mathrm{~cm}$ osteochondral fragment was identified. A lateral femoral condyle defect was identified. Fixation was performed with three Kwires (distal end deepened $1 \mathrm{~mm}$ under the cartilage surface, caudal end bound and left subcutaneously on the medial side of the thigh). Postoperative the patient was immobilized in extension ( 2 weeks), then started full mobilization without weightbearing. At 11 weeks the Kwires were extracted (local anesthesia). Progressive full weightbearing was allowed. Follow up at 6 month, 1, 4 and 7 years showed good integration, no avascular necrosis or arthrosis, no pain and full ROM.

Results: X- Rays were performed at 3, 5 and 8 weeks. Good positioning of the fragment and no migration of the $\mathrm{K}$ wires were noted. At 11 weeks the $\mathrm{K}$ wires were extracted through a minimal medial incision in local anesthesia and progressive full weight bearing was allowed. Full ROM was possible. Radiological and clinical follow up at 6 month, 1, 3, 4 and 7 years showed good integration of the fragment with no signs of avascular necrosis or arthrosis, no pain and full ROM

Arthroscopic assessment of osteochondral fractures of the lateral condyle is the standard procedure but mini arthrotomy should be considered for the reduction and fixation of larger fragments. In case of lack of resorbable pins or fibrin glue, fixation with subchondral deepened $\mathrm{K}$ wires is a good alternative in order to start quick rehabilitation and obtain full recovery.

Discussion: Mini arthrotomy should be considered for reduction/ fixation of larger fragments. In case of lack of resorbable pins, fixation with subchondral deepened $\mathrm{K}$ wires is a good alternative. It is simple, cheap, little cartilage damage done during insertion and stable fixation to allow early movement. Key points in $\mathrm{K}$ wire fixation are: direction of insertion, deepening under the cartilage surface and subcutaneous positioning of the proximal end for an easy extraction. Paper supported by a national investigation grant (CNCSIS nr 698 19.01.2009)

Conclusion: Key points in $\mathrm{K}$ wire fixation are: direction of insertion, deepening under the cartilage surface and subcutaneous positioning of the proximal end for an easy extraction. The advantages of this technique are that it is simple and cheap, there is little cartilage 
damage done during insertion and the fixation is stable enough to allow continuous passive motion. This paper is supported by a national investigation grant (CNCSIS nr 698 19.01.2009).

Disclosure: No significant relationships.

\section{CR20}

\section{FIXATION OF A BIMALLEOLAR ANKLE FRACTURE AND SUBSEQUENT COMPARTMENT SYNDROME}

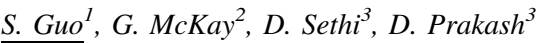

${ }^{1}$ Department Of Trauma \& Orthopaedic Surgery, Weston General Hospital, Weston Super Mare, UK, ${ }^{2}$ Trauma \& Orthopaedic Surgery, Weston General Hospital, Weston Super Mare, UK, ${ }^{3}$ Trauma \& Orthopaedic Surgery, Sandwell General Hospital, West Bromwich, UK

Introduction: Compartment syndrome in the foot after ORIF of ankle fractures is rarely reported. The resultant morbidity associated with compartment syndrome can be severe. We report a case in which standard fixation of a bimalleolar fracture of the left ankle using two medial malleolar screws, lateral neutralisation plate and diastasis screw resulted in compartment pressures consistent with post-operative compartment syndrome of all six compartments in the foot.

Case report: A 28-year-old woman sustained a external rotation and supination injury to her left ankle resulting a Lauge-Hansen Type 4 ankle fracture with a medial and lateral involvement. Neurovascular examination was unremarkable pre-operatively. Fixation was performed the following day with two medial screws, a neutralisation plate and a lag screw, combined with a diastasis screw. $5 \mathrm{~h}$ postoperatively, the patient's neurological function deteriorated in the left foot with severe pain. Clinical features were consistent with compartment syndrome. She was taken back to theatre with the intent of performing fasciotomy of the foot, however compartment pressures fell dramatically following release of the medial and lateral sutures of the ankle wound.

Discussion: Although extremely rare, the morbidity of compartment syndrome in the foot is severe, and the index of clinical suspicion should remain high postoperatively. This is true for ankle fractures as well as foot injuries. We advocate timely operative intervention, but our case highlights the usefulness of removing sutures placed at the time of surgery and subsequent measurement of compartment pressures prior to formal fasciotomy.

Disclosure: No significant relationships.

\section{CR21}

\section{FIXATION OF AN UNUSUAL TYPE OF HAMATE FRACTURE}

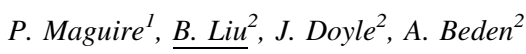

${ }^{1}$ Orthopaedics, Fairfield Hospital, Bury, UK, ${ }^{2}$ Orthopaedics, Fairfield Hospital, Bury, UK

Introduction: The Hamate carpus is an unusual bone to fracture. Many studies have described Hook of Hamate fractures (1). But a fracture of the body is even less common (2). We would like to present a coronal fracture of the body of the hamate with an associated fourth metacarpal subluxation. Initial radiographs were difficult to interpret. A high suspicion of injury is needed following a high energy fall on a hyperextended hand. A CT was obtained prior to surgery which described this unusual injury. The coronal split was displaced $6 \mathrm{~mm}$ with an associated fourth metacarpal drift. No other carpus were affected. The fracture was fixed with a variable pitch cannulated screw under radiographic control.

Case Report: This is a case study of an unusual frature. The patient agreed to publication of the details of the case. This case was recorded prospectively. With follow up of 6 months. Repeat CT images showed the screw to be in a satisfactory position. Patient satisfaction was high. Range of movement of the fingers were comparable to the opposite hand and grip strength was normal.

Results: This case was recorded prospectively. With follow up of 6 months. Repeat CT images showed the screw to be in a satisfactory position. Patient satisfaction was high. Range of movement of the fingers were comparable to the opposite hand and grip strength was normal.

Discussion: We would recommend this type of fixation in this type of fracture. This case gives us insight to treatment of this fracture but also emphasises the need for vigilance in hand injuries to rule out carpus fractures. It is important to realise these injuries are associated with compartment syndrome.

Conclusion: We would recommend this type of fixation in this type of fracture. This case gives us insight to treatment of this fracture but also emphasises the need for vigilence in hand injuries to rule out carpus fractures. It is important to realise these injuries are associated with compartment syndrome.

References: 1. Stark HH. Fracture of the hook of hamate. J Bone Joint Surg AM.1989;71:1202-1207. 2. Milch H. Fracture of the hamate bone. J Bone J Surg AM. 1934;16:459-462.

Disclosure: No significant relationships.

\section{CR22}

\section{FUNCTIONAL FOLLOW-UP POST CLAVICULAR HOOKPLATE USING THE IPHONE 4: A CASE REPORT}

D.W. Good, D. Lui, M. Leonard, S. Morris, M. Neligan, P. Nicholson, J.P. McElwain

The Department Of Trauma Orthopaedics, Adelaide and Meath Incorporating the National Childrens Hospital, Dublin, Ireland

Introduction: Research evaluating procedures relies on post-operative functional assessment. Traditionally this involved follow-up in an outpatient clinic. Retrospective reviews where this was not possible would usually use posted questionnaires/telephone interviews allowing only subjective assessment. For accurate meaningful outcomes to be drawn from studies it requires objective functional assessment.

Case Report: From July 2005 to October 2010 we carried out 36 clavicular hookplates for displaced lateral third clavicle fractures. Oxford and constant scores were used to determine functional outcomes. A functional assessment using Skype was performed, then validated by assessment in the outpatient clinic. Our aim was to determine if video call technology (Iphone4) could be used to accurately assess functional outcome. Only 1 out of our 36 patients had the iphone 4. This patient was happy to be assessed using the iphone 4 . Discussion: Both oxford and constant shoulder scores were not statistically significantly different when assessed with the iphone 4 , Skype and in the outpatient clinic. Patient satisfaction was higher with video technology because it saved time and money on transport into hospital. In recessionary times, research budgets are declining and outpatient clinic waiting lists getting longer, the use of video-technology in the form of both Skype and the iphone 4 enables us to carry out accurate functional assessments. This technology will be most useful in follow-up of large numbers of patients. 
The Iphone 4 is as accurate in assessing the functional outcome of a clavicular hookplate as follow-up in the outpatient department. This provides another novel tool for orthopaedic research.

Disclosure: No significant relationships.

\section{CR23}

\section{INTERNAL FIXATORS}

\section{E. Castaman}

Ortopedia, Ospedale Civile-Ulss5ovestvcientino, Montecchio Maggiore, Italy

Introduction: After the recent introduction of internal fixators, the tipical osteoreperative potentials of elastic synthesis in scheletic trauma are very expanded because of both callogenic validity, of their particular flectent moment, and perfect tolerance.

Case report: Thus, in our hospital, after a careful initial approach of the method with satisfying results, have permitted an extended progressive use of these implants. Internal fixators have been applied on different levels with wide indications, for both complex recent fractures and/or articular fractures even in strongly osteodistrofics sectors and retard consolidation fractures, particularly in hypotrophics pseudoarthrosis or failure of other synthesis devices.

Discussion: We report some interesting cases of particular consolidations and some nonconvenients of the improvable instruments used. Disclosure: After the recent introduction of internal fixators, the tipical osteoreperative potentials of elastic synthesis in scheletic trauma are very expanded because of both callogenic validity, of their particular flectent moment, and perfect tolerance. Thus, $\mathrm{i}$

\section{CR24}

\section{ISOLATED ANTERIOR ELBOW DISLOCATION BY AIRBAG INFLATION. A CASE REPORT}

\author{
E. Fotiadis, E. Iliopoulos, F. Vittis, A. Kravas, D. Tsitouras, T. Ntovas
}

\section{Orthopaedic, General Hospital of Veria, Veria, Greece}

Introduction: Anterior elbow dislocation is usually encountered in patients suffering from Ehlers-Danlos syndrome or accompanied with olecranon and distal humerus fractures. Isolated traumatic anterior dislocation is very uncommon. We present a case with a traumatic anterior elbow dislocation caused by an unusual mechanism of injury.

Case report: We present a case of traumatic isolated anterior elbow dislocation in a 47-year-old manual laborer man. The olecranon was hit and forced forward from the opened air-bag as the elbow was flexed over $90^{\circ}$. Apart from pain due to severe elbow deformity, numbness of the 4th and 5th finger was also reported. Anteroposterior and lateral elbow radiographs revealed anterior elbow dislocation without fracture. The dislocation was successfully reduced by closed means but the elbow was grossly unstable in valgus stress. Repair of the torn MCL was enhanced with a palmaris longus graft using the "Docking technique". The patient returned to his pre-injury occupation and level of activity 10 months after the surgery. Flexion was $120^{\circ}$, extension was $50^{\circ}$, pronation was $80^{\circ}$ and supination was $75^{\circ}$.

Discussion: Therefore, acute repair and reconstruction of the ligament may be considered as the preferred treatment option in young and manual laborer patients.
Results: The patient returned to his pre-injury occupation and level of activity 10 months after the surgery. Flexion was $120^{\circ}$, extension was $50^{\circ}$, pronation was $80^{\circ}$ and supination was $75^{\circ}$.

Conclusion: Therefore, acute repair and reconstruction of the ligament may be considered as the preferred treatment option in young and manual laborer patients .

References: Symeonides PP, Grigoriadis NC, Hatzokos IG. Anterior dislocation of the elbow. J Shoulder Elbow Surg. 2006;15(2):249-51. Torchia ME, DiGiovine NM. Anterior dislocation of the elbow in an arm wrestler. J Shoulder Elbow Surg. 1998;7(5):539-41.

Disclosure: No significant relationships.

\section{CR25}

\section{LENGTHENING Z-OSTEOTOMY OF THE FIBULA TO CORRECT PERSISTENT TALAR SHIFT FOLLOWING OPEN REDUCTION INTERNAL FIXATION OF ANKLE FRACTURES}

\author{
T. Thangarajah, A. Lakdawala, E. Battaloglu, M. Edwards, A. Malik, \\ A. Tillu
}

Trauma and Orthopaedic Surgery, Sandwell and West Birmingham Hospitals NHS Trust, 4HJ, United Kingdom

Introduction: The outcome following open reduction internal fixation of ankle fractures depends upon several factors. From a technical perspective, reduction of the lateral malleolus to restore normal length and produce correct alignment of the talus within the mortice is imperative. As little as $1 \mathrm{~mm}$ of talar shift confers a $42 \%$ reduction in tibiotalar congruity placing increased stress on the articular cartilage. This may eventually result in persistent pain, instability and arthritis. This study describes the technique of lengthening z-osteotomy of the fibula to correct persistent talar shift and achieve internal rotation of the fibula following ankle fracture fixation. The short-term results of the procedure are also discussed.

Case report: Retrospective analysis of three patients who underwent lengthening z-osteotomy of the fibula to correct persistent talar shift following ankle fracture fixation.

The study group consisted of two males and one female with a mean age of 45 (range 38-50) years. The three injuries comprised of a posterior malleolar fracture, a Maisonneuve fracture and an isolated medial malleolar fracture. All patients had talar shift on their initial radiograph which remained despite internal fixation. Lengthening $\mathrm{z}$-osteotomy of the fibula was therefore performed. At 1 year followup all patients were noted to have both a satisfactory clinical and radiological outcome.

Results: The study group consisted of two males and one female with a mean age of 45 (range 38-50) years. The three injuries comprised of a posterior malleolar fracture, a Maisonneuve fracture and an isolated medial malleolar fracture. All patients had talar shift on their initial radiograph which remained despite internal fixation. Lengthening $\mathrm{z}$-osteotomy of the fibula was therefore performed. At 1 year followup all patients were noted to have both a satisfactory clinical and radiological outcome.

Discussion: Lengthening z-osteotomy of the fibula produces satisfactory results when used to correct persistent talar shift following ankle fracture fixation.

Lloyd J, Elsayed S, Hariharan K, Tanaka H. Revisiting the concept of talar shift in ankle fractures. Foot Ankle Int. 2006;27:793-6.

Conclusion: Lengthening z-osteotomy of the fibula produces satisfactory results when used to correct persistent talar shift following ankle fracture fixation.

Disclosure: No significant relationships. 


\section{CR26}

\section{NON-OPERATIVE MANAGEMENT OF A FEMORAL NECK FRACTURE OCCURRING AFTER INTRA-MEDULLARY NAIL REMOVAL. A CASE REPORT}

\section{Pietrogrande ${ }^{1}$, C. Legnani ${ }^{2}, \underline{A . ~ D o n d i ~}^{1}$}

${ }^{1}$ Divisione Di Ortopedia E Traumatologia, Università degli Studi di Milano, Azienda Ospedaliera S. Paolo, Milan, Italy, Milano, Italy, ${ }^{2}$, Scuola di Specializzazione in Ortopedia e Traumatologia, Università degli Studi di Milano, Milan, Italy.

Introduction: Our purpose is to discuss the decision of removing hardware in the elderly osteoporotic patient and to analyze the possibility to conservatively treat an impacted minimally-displaced subcapital fracture occurring after the removal of an intra-medullary nail (IMN) previously inserted for the treatment of a trochanteric fracture. The occurrence of iatrogenic fracture following implants removal is uncommon, but there are reports documenting complications in the outcome of the removal of metal devices. Two recent studies have shown that conservative treatment of impacted fractures avoids surgical intervention and leads to satisfying functional results in $48-54 \%$ of the cases.

Case report: A 67 year-old woman underwent IMN removal inserted for a pertrochanteric fracture occurred 20 months earlier due to continuous discomfort related to the protruding apex of the implant over the great trochanter. Due to pain persistence 2 days after surgery, a CT scan was performed documenting a minimally displaced impacted subcapital femoral neck fracture. Conservative management with close radiographic follow-up was conducted. After 6 months patient had returned to previous daily activities and a satisfactory range of motion was achieved without pain on walking.

Discussion: Conservative treatment should be considered for the management of lesser symptomatic minimally displaced impacted fractures where the intrinsic stability of the fracture allows rapid healing without further surgical operations. In the elderly population with decreased bone quality, the removal of hardware should be carefully evaluated and antiresorptive therapy (bisphosphonate, teriparatide) should be started in order to reduce the risk of further bone loss.

Disclosure: No significant relationships.

\section{CR27}

\section{PIPKIN II FRACTURE OF THE FEMORAL HEAD-CASE PRESENTATION}

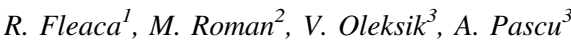

${ }^{1}$ Orthopaedics And Taruma Surgery, University of Medicine Sibiu, Sibiu, Romania, ${ }^{2}$ Orthopedic Surgery And Trauma, University of Sibiu, Sibiu, Romania, ${ }^{3}$ Engineering Faculty, University of Sibiu, Sibiu, Romania

Introduction: The fracture-dislocation of the femoral head is a rare condition, difficult to treat and is frequent associated with a poor functional outcome.

Case Report: This paper present the case of a 31 years old politraumatised patient, treated in Emergency Sibiu Hospital in June 2006, diagnosed with Craniocerebral trauma, multiple wounds, Thoracic trauma with right $\mathrm{C} 4-\mathrm{C} 7$ costal fractures, Lumbar trauma with lumbar hematoma, Posterosuperior right coxofemoral dislocation, right femoral head fracture Pipkin II. In emergency we have proceeded for the reduction of the hip dislocation and immobilization of the right pelvic limb by transscheletal continuous traction. The roentgenograms and control CT scan showed reduction of the hip dislocation, but with a fragment of the femoral head rotated intracotiloidian with $180^{\circ}$. Five days after we have performed an open reduction of the fragment through an anterior Smith Peterson approach, and fixation with 4 RigidFix resorbables pins. Postoperative continuous traction up to 3 weeks, then waking with crouches up to 10 weeks postoperative with partial weight bearing, with roentgenogram control postoperative, then clinical and IRM exam at 6 month, 1 and 2 years, which showed a good reduction, healing of the fracture and no sign of avascular necrosis of the femoral head.

Discussion: There was no sign of avascular necrosis at 2 years and the Harris Hip Score was 100 points. The fractures of the femoral head are difficult to treat and manage, with a high rate of complications.

Disclosure: No significant relationships.

\section{CR28}

\section{SEQUEL OF RADIAL HEAD FRACTURE/DISLOCATION IN A 14 YEARS-OLD CHILD}

S.I.V.S. Fernandes
J. Jiveira
J. J. Je. Reis

${ }^{1}$ Orthopedics And Traumatology, Centro Hospitalar Alto Ave Guimarães, Guimarães, Portugal, ${ }^{2}$ Orthopaedics And Traumatology, Centro Hospitalar do Alto Ave, Guimarães, EPE, Guimarães, Portugal

Introduction: Radial head fractures and dislocations are traumatic lesions which require adequate treatment to prevent functional impairment, deformity, stiffness, pos-traumatic arthritis and nervous lesion.

Case Report: Fourteen year-old child entered the emergency room with deformity and pain in the left elbow without recent traumatic event but with history of a fall with trauma to the left elbow when he was 10 years. Since then he had deformity of the left elbow. He had limitation of the elbow range of motion $40^{\circ}-130^{\circ}$ and impairment of pronosupination.

Radiographs showed sequels of radial head fracture with posterior dislocation. The patient was submitted to surgery with posterolateral approach with radial head excision and after the patient underwent physical therapy.

At 2 months he showed correction of the elbow deformity, restoration of pronosupination $\left(5^{\circ}-70^{\circ}\right.$ of pronation and $10^{\circ}-75^{\circ}$ of supination) and 10 a $135^{\circ}$ of elbow range of motion.

In terms of satisfaction he was much satisfied without elbow deformity, without functional impairment and he regained the capacity of pronosupination. In the evaluation of the Elbow Functional Assessment scale he improved from a preoperative score of 48 to a postoperative of 92 .

Discussion: Radial head fractures can pass unnoticed and many times we came across the need to treat its complications. This represents a rare case of complex radial fracture with posterior dislocation with deformity, pain, reduced elbow range of motion and limitation of the accomplishment of the normal daily activities in a very young person of 14-years-old, that if not treated would had further evolved to more functional impairment and rigidity.

Disclosure: No significant relationships. 


\section{CR29}

THE CONSERVATIVE TREATMENT OF ANTERIOR KNEE DISLOCATION WITH IPSILATERAL OPEN TIBIA SHAFT FRACTURE; A CASE REPORT OF A PROFESSIONAL ATHLETE

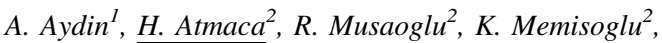 \\ S.U. Muezzinoglu
}

${ }^{1}$ Orthopaedics And Traumatology, Igdir State Hospital, Igdir, Turkey, ${ }^{2}$ Orthopaedics And Traumatology, Kocaeli University School of Medicine, Kocaeli, Turkey

Introduction: Traumatic dislocation of the knee joint is an uncommon complex, multiple ligamentous injury often resulting from a high-energy trauma. Because of both early and late complications of these injuries, significant disability can follow traumatic knee dislocations. Early complications are popliteal artery disruption and peroneal nerve injury. Late complications are related to persistent instability and posttraumatic arthritis. Hence the emergency surgery is necessary due with potential for neurovascular compromise and limb loss. Controversies over operative versus closed immobilization of traumatic complex multiple ligamentous knee injury are still debated. Introduction: Acute dislocation of the knee is a limb threatening injury that often results in extensive soft-tissue damage and disruption of the popliteal blood vessels. While the incidence of knee dislocations is low, this injury is associated with a high rate of complications including amputation.

Case Report: A forty-year-old male professional athlete (Karate) was brought to emergency medical service due to traffic accident. He had severe deformity on his right knee, crepitating and open tibia shaft fracture with $7 \times 4 \mathrm{~cm}$ skin injury. The right ankle pulses were not palpable. In addition, there was pain and swelling on left knee. There was no neurological disorder. Direct radiographs confirmed a short spiral-oblique fracture through the $1 / 3$ mid-distal shaft of the tibia and an anteriorly dislocated right knee and transverse fracture of left patella. Closed reduction of the right knee performed under general anesthesia. After reduction a vascular surgeon examined the distal perfusion. Vascular dissection was not required through tri-phasic distal flow that obtained by doppler device. External fixation performed and gastrocnemius muscle flap used to close open fracture of right tibia. Left patella fracture was treated by open reduction and tension band technique. Physicaltherapy for knee dislocation was started in the third week after closed reduction and continued for 3 months. As the final examination at 24th months he returned his high level sports activity.

Discussion: Although the surgical treatment is the current concept of the traumatic knee dislocation with multiple ligaments injury, It can be achieved the satisfactory results by well-organized conservative treatment by physicaltherapy with the help of patient compliance. Disclosure: No significant relationships.

\section{CR30}

\section{UNEXPECTED NECK OF FEMUR FRACTURE IN A 29 YEAR OLD MAN AFTER A FALL}

\section{N. Baraza, T. Parwez}

Trauma, Bimringham Heatlands Hospital, Birmingham, United Kingdom
Introduction: In this unusual case, a fractured hip was sustained by a young man after a seemingly innocuous fall from a standing height. The aim of this report is to alert clinicians to the fact that a fall from standing height on to the hip is not a trivial form of trauma as is commonly perceived by many orthopaedic surgeons.

Case Report: A healthy 29 year old fell from a standing height. The resulting pain and inability to weight bear brought him to hospital. $\mathrm{He}$ had no past medical history. Examination his left hip revealed tenderness of the greater trochanter and a global reduction of hip movement. A pelvic X-ray demonstrated an undisplaced basal neck of femur fracture. A CT scan of the hip was performed to confirm the diagnosis. Routine bloods including liver function tests were normal and myeloma screen was negative. The fracture was internally fixed with a dynamic hip compression screw, and samples of reamed bone and muscle taken for histology. They returned normal. Six months later his fracture had healed.

Discussion: Patients below the age of 50 who sustain hip fractures often have co-morbidities predisposing to falls or decreased bone strength, or are military recruits and athletes with stress fractures due to the rigors of their training. Our patient did not fall into any of these two groups. This peculiar case highlights the point that a fall on to the hip from a standing height even in a healthy young man possesses sufficient force to fracture the neck of femur.

Disclosure: No significant relationships.

\section{CR30A}

\section{AN EXTENSOR INDICIS-TRANSFER FOR THE TREATMENT OF A SPONTANEOUS EXTENSOR POLLICIS LONGUS RUPTURE}

\section{H.M. Teijgeler, F.H.W.M. van der Heijden}

Surgery, St. Elisabeth Hospital, Tilburg, Netherlands

Introduction: A spontaneous rupture of the extensor pollicis longus (EPL) is relatively rare. It has been described in inflammatory decease as rheumatoid arthritis as well as in chronic tenosynovitis. A fracture of the distal radius is known as a predisposing factor. A rupture of the EPL is occasionally seen as a late complication after treatment of a distal radius fracture with a volair plate osteosythesis. There are several options to repair a rupture of the EPL. Most commonly an extensor indicis-transfer or a palmaris longus transplantation is preformed.

Case Report: Between 2009 and 2010 we have treated five patients with a spontaneous rupture of the EPL. Two patients had prior to the rupture a distal radius fracture which was corrected with a volair plate, 6 and 4 months before the EPL rupture. One patient had a distal radius fracture which had a conservative treatment with a cast, the rupture was a month after the fracture. Two patients had a spontaneous rupture of the EPL without a trauma and had both no predisposing diseases. All the patients were operated and function was restored with an extensor indices-transfer. There were no complications. Post operatively all patients had an immobilisation for 3 weeks. After this period intensive physiotherapy was started. The function of the EPL was restored in all cases.

Discussion: We believe that an extensor indices transfer is a good and safe treatment for correcting a spontaneous rupture of the EPL. The surgically technique is relatively simple with good results. Disclosure: No significant relationships. 


\section{CR32}

\section{IDENTICAL FRACTURE PATTERNS IN COMBAT VEHICLE BLAST INJURIES DUE TO IMPROVISED EXPLOSIVE DEVICES}

\section{J.P. Commandeur $^{1}$, R.J. Derksen ${ }^{1}$, D. MacDonald ${ }^{2}$, R.S. Breederveld ${ }^{3}$}

${ }^{1}$ Traumasurgery, VU University Medical Center Amsterdam, Amsterdam, Netherlands, ${ }^{2}$, Northern Ontario School of Medicine, Sudbury, Canada, ${ }^{3}$ Surgery, Red Cross Hospital/Burn Centre, Beverwijk, Netherlands

Introduction: In November 2008, a surgical team from the Red Cross Hospital Beverwijk, The Netherlands, went to Afghanistan for 3 months, to attend in the army hospital of Kandahar. During their stay, four armoured personnel carriers encountered IEDs and were brought in. The victims were assessed and had sustained strikingly similar injuries.

Case Report: The described paired cases comprise of identical thoracic vertebrae fractures, radial neck fractures, calcaneal fractures and talar fractures in both the driver and co-driver of each of the combat vehicles. The paired injuries were exactly the same in location, extent and subtype of fractures. This is explained by the different mechanisms of blast trauma. We elaborate on the different blast mechanisms and its consequences on injury pattern.

Discussion: From this case series, it seems appropriate to pay extra attention to the injuries other occupants of the same vehicle have sustained. Obviously, this additional attention for specific injuries should be complementary to the regular trauma work-up (e.g. ATLS). The acquired experience from the army Hospital in Kandahar could also be an advisable adjunct to the assessment of other than specific blast injuries, for example the assessment of high energy civilian trauma victims. Moreover, in this case series the importance of determining the type of blast injury is emphasized. Acknowledging the different blast mechanisms is essential for understanding the possible injuries. Blast mechanisms are divided in primary (direct effect of overpressure and implosion), secondary (spreading fragments detaching from the bomb), tertiary (effect of blast wind) and quaternary (e.g. toxic inhalation, burn trauma).

Disclosure: No significant relationships.

\section{MASSIVE HEMORRHAGE AND COAGULOPATHY}

\section{CR35}

\section{COMPLEX POLYTRAUMATISM AFTER CAR CRASH}

\section{Munteanu, G. Molnar}

Surgical Clinic Iii, University of Medicine and Pharmacy, ClujNapoca, Romania

Introduction: Quite frequent, violent impacts, i.e. car crashes are associated with multivisceral lesions, sometimes with posttraumatic large diaphragmatic ruptures. Open repair of such defects is not necessary difficult, more demanding being repair of associated vascular and visceral lesions.

We hereby present the case of a severe car crash; the aim of our presentation is to show that, in spite of technical feasibility of complex vascular and visceral lesions, pulmonary complications might compromise the outcome.
Case report: Three hours after a car crash, a 56 years old female patient, (frontal collision of two cars, patient on the right front seat) was admitted with a severe state of shock.

Surgical exploration revealed: (1) massive hemoperitoneum (3,600 ml); (2) fecaloid peritonitis; (3) bleeding $5 \mathrm{~cm}$ wound of segment III liver; (4) partial ruptures of superior mesenteric artery and vein; (5) rupture of mesentery and avulsion of first $40 \mathrm{~cm}$ jejunum; (6) avulsion and complete rupture of descending colon; complete rupture of inferior mesenteric vein; (7) severe attrition of left lateral and posterior abdominal wall with voluminous retroperitoneal hematoma; (8) rupture of large omentum and gastrocolic ligament; (9) complete rupture of left hemi diaphragm, intrathoracic herniation of colon, small bowel, ruptured spleen.

Vascular lesions, splenectomy were initially managed. Retraction of the abdominal viscera from left hemithorax, double drainage and suture of diaphragm followed. $40 \mathrm{~cm}$ jejunectomy and left hemicolectomy with T-T anastomosis followed.

Patient died with right bronchopneumonia on postoperative day 12 .

Discussion: Grossly inflated stomach (food/alcohol) and initial posttraumatic loss of conscience after being thrown out through car window, caused aspiration of gastric content and early isolated right bronchopneumonia $24 \mathrm{~h}$ later.

Disclosure: No significant relationships.

\section{CR35A}

\section{SUCCESSFUL NONOPERATIVE MANAGEMENT OF VARICEAL GALLBLADDER BLEEDING AND RUPTURE. CASE REPORT}

\section{A. Traversone, P.M. Bertazzoni, C. Blundo, F. Vitale, A.M. Marini, P. De Rai, L. Castoldi; IRCCS Fondazione Ospedale Ca' Granda Policlinico Milano, Chirurgia Generale E D'urgenza, Via Francesco Sforza 35, 20100, Milano, Italy}

Introduction: Gallbladder wall variceal hemorrhage with rupture is a rare event in the cirrhotic patients. We describe such a case managed nonoperatively.

Case Report: A 64-year-old man with alcoholic Child-Pugh C liver cirrhosis was referred to our department for an incarcerated umbilical hernia. A recent abdominal CT showed a normal gallbladder containing calcific stones and sludge, complete thrombosis of the right portal vein and $50 \%$ thrombosis of the extrahepatic portal vein, and massive ascites. The hernia was surgically repaired, with an abdominal drainage left in site to relieve ascites. In the 8th postoperative day the patient had abrupt abdominal pain, with transient hypotension and fresh blood from the drainage $(500 \mathrm{ml})$. A repeat CT scan revealed a perforated gallbladder with stones free in the Morrison's space and active bleeding inside the gallbladder. On account of hemodynamic stability and the high risk of intervention, a nonoperative treatment was chosen. The patient was transfused with blood (5 units) and fresh frozen plasma $(500 \mathrm{ml})$ and the bleeding stopped. He was discharged 33 days later without signs of infection. He died 6 months later from hepatic insufficiency.

Discussion: Child-Pugh C cirrhotic patients have an expected survival rate of $45 \%$ at 1 year without liver transplantation. For major surgical procedures, the reported mortality ranges from 90 to $95 \%$. Gallbladder varices occur in $12-30 \%$ of patients with portal hypertension and thrombosis. Variceal hemorrhage with subsequent spontaneous gallbladder rupture in the cirrhotic patient is a rare event, reported to date in no more than 30 cases. To date, the patient we described is the only one successfully managed with nonoperative treatment.

References: 1. Eric CC, et al. Dig Dis Sci 2002;47:2682-5. 2. Kevans D, et al. Eur J Gastroenterol Hepatol 2009;21:955-57

Disclosure: No significant relationships. 


\section{MINIMALLY INVASIVE SURGERY IN NON TRAUMATIC EMERGENCIES}

\section{CR36}

SMALL-BOWEL OBSTRUCTION DUE TO TRANSMESOCOLIC INTERNAL HERNIA: VIDEOLAPAROSCOPIC TREATMENT

\section{B. Fiore ${ }^{1}$, S. Bona ${ }^{1}$, S. Badiali ${ }^{1}$, C. Verrusio ${ }^{2}$, M. Marconi $^{1}$, S. Imparato $^{3}$, M. Montorsi ${ }^{1}$}

${ }^{1}$ General Surgery, Istituto Clinico Humanitas, IRCCS, Rozzano (MI), Italy, ${ }^{2}$ Minimally Invasive Surgery, Istituto Clinico Humanitas, IRCCS, Rozzano (MI), Italy, ${ }^{3}$ Radiology, Istituto Clinico Humanitas, IRCCS, Rozzano (MI), Italy

Introduction: Congenital internal hernia is defined as a protrusion of abdominal viscera into a normal or abnormal opening within the abdominal or pelvic cavity. This is a very rare cause of small bowel obstruction. Trans-mesocolic hernias are probably the rarest ones. This kind of hernia always involves the transverse mesocolon, it usually has a large orifice situated on the left of the medium colic artery and it can have or not have a sac.

Case Report: We describe the case of a 34-year-old man admitted at our Emergency Department for abdominal pain and vomiting. He denied any previous surgery or other health problems. During the past week he had been admitted several times at the Emergency Department of other institutions for the same reason. He had always been discharged after administration of analgesic therapy obtaining symptom recovery. Physical examination, laboratory tests, abdominal US and X-rays were not contributing. Analgesic drugs had no effect so we decided to perform an abdominal CT-scan that revealed an internal hernia involving a long small bowel segment and conditioning small-bowel obstruction. The patient underwent explorative laparoscopy, reduction of the hernia and closure of the hiatus. The postoperative course was uneventful and the patient was discharged 3 days postoperatively.

Discussion: Internal hernias are rare and preoperative diagnosis based on clinical and radiological findings is very challenging. Management of internal hernias is usually performed through laparotomy, in this case, laparoscopy revealed to be very useful to confirm the diagnosis and for ultimate treatment.

Disclosure: No significant relationships.

\section{MINIMALLY INVASIVE SURGERY IN SKELETAL TRAUMA}

\section{CR37}

\section{ACHIEVING PRIMARY HEALING OF LIGAMENTOUS STRUCTURES AFTER ACROMIOCLAVICULAR JOINT DISLOCATION}

\section{A. Vasireddy ${ }^{I}$, W. Ogufere ${ }^{2}$}

\footnotetext{
${ }^{1}$ Dept of Orthopaedics, University Hospital Lewisham, London, UK, ${ }^{2}$ Orthopaedics, University Hospital Lewisham, London, UK
}

Introduction: Acromioclavicular joint (ACJ) injuries are common and make up $9 \%$ of all shoulder injuries. Type 1 and 2 injuries are treated with physiotherapy alone. Type 4 to 6 are generally treated surgically. Treatment of type 3 injuries is controversial. Surgical options have been shown to frequently fail. However, end-result studies show decreased strength and residual symptoms in patients treated conservatively. The development of acute anatomical coracoclavicular reconstruction techniques represent a minimally invasive method of achieving surgical fixation of type 3 injuries in the appropriate patient.

Case Report: A 23-year-old male manual labourer sustained a type 3 ACJ injury after falling directly onto his shoulder during a football game. He underwent anatomical reconstruction of the coraco-clavicular ligament complex 8 days after the injury, in view of his occupation and athletic interests. The technique involves a two-incision approach to the coracoid and distal clavicle. Two endobuttons and four rows of ethibond sutures are used to restore ACJ alignment. Following surgery, he underwent a physiotherapy programme and returned to work and sport. Follow-up MRI at 10 months showed primary healing of the conoid, trapezoid and acromioclavicular ligaments.

Discussion: Treatment of type 3 injuries is controversial. Many dated studies suggest conservative treatment is appropriate. However, it is becoming apparent that this may lead to a high incidence of residual symptoms. Anatomical reconstruction techniques provide a method of facilitating ACJ realignment in order to achieve primary ligamentous healing. We believe that this is the first case report to illustrate MRI evidence of primary ligamentous healing after ACJ reconstruction. Disclosure: No significant relationships.

\section{CR38}

\section{TRAUMATIC PNEUMOMEDIASTINUM: CASE REPORT}

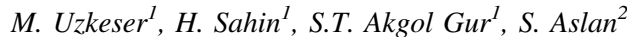

${ }^{1}$ Emergency Department, Ataturk University, Erzurum, Turkey, ${ }^{2}$ Emergency Department, Ataturk University Aziziye Hospital, Erzurum, Turkey

Introduction: Traumatic pneumomediastinum (TPM) is a rare entity. We represent a 43-year-old man with TPM and subcutaneous emphysema occurred after motor vehicle accident (MVA).

Case Report: The patient was brought to our ED approximately $3 \mathrm{~h}$ after MVA with the complaints of angina pectoris and dyspnea. He was cooperated and oriented. His vitals were as follows: TA: 148/86 mmHg, HR: $112 / \mathrm{min}$, RR: $16 / \mathrm{min}$, satO ${ }_{2}: \% 88$, T: $36.5^{\circ} \mathrm{C}$. His positive clinical findings were extensive bilateral subcutaneous emphysema and crepitation on his face, neck and trunk bilaterally. Rales on his right chest and Hamman's sign were obvious with occultation. Unique laboratory anomaly was his high white blood count $\left(20,800 / \mathrm{mm}^{3}\right)$. Electrocardiogram showed first degree AV block. Supine chest X-ray (Fig. 1), lateral neck X-ray (Fig. 2) and thorax CT (Fig. 3) showed subcutaneous and mediastinal air. Thorax CT additionally showed bilateral lung contusion and sternum fracture. After hospitalization, bronchoscopy and endoscopy were performed with normal findings. On the second day, mediastinotomy was performed due to pressure effect of the mediastinal emphysema. On the same day, tube thoracostomy was performed to right chest because of pneumothorax. Complete resolution of the pneumothorax and mediastinal emphysema occurred gradually following days. He was discharged without any complication on the 6th day.

Discussion: TPM may also occur as a result of head, neck, thorax and rarely after oral injury. Hamman's sign is a "crunching" sound timed with the cardiac cycle and heard during cardiac occultation. TPM is an important issue if preexisting trachea or oesophagus injury is present.

Disclosure: No significant relationships. 


\section{OPEN FRACTURES}

\section{CR39}

\section{MEAT GRINDER ACCIDENT RESULTS IN CRITICAL INJURIES FOR WORKER}

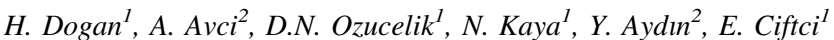

${ }^{1}$ Emergency Medicine, Bakırkoy Sadi Konuk Training and Research Hospital, Istanbul, Turkey, ${ }^{2}$ Emergency Medicine, Bakirköy Sadi Konuk Training and Research Hospital, Istanbul, Turkey

Introduction: Meat Grinder Accident Results in Critical Injuries for Worker. Injury to the upper extremity is common in food preparation workers. If the machinery cannot be safely removed, further tissue loss and higher-level amputation may result.

Case Report: A 21-year-old man's right dominant hand and antebrachium became entrapped in an industrial meat grinder. At the scene, the machine was disconnected from its base. Patient was transported via ambulance to our emergency department with his forearm whilst entrapped in the meat grinder. On presentation, the patient had stable vital signs. His right arm was tightly lodged in the body of the meat grinder, which extended $10-\mathrm{cm}$ proximal to the elbow. His elbow and proximal forearm were intact but within the machine. Mangled digits protruded from the grinding end. No abundant bleeding from the injured extremity was evident. Before proceeding to the operating room, the patient received intravenous cephazolin, gentamicin, and an intramuscular tetanus injection. The operation team used a wrench to turn the shank counterclockwise to remove the grinder. This successfully delivered the remaining tissue from the apparatus without further injury to the forearm or elbow. The patient's distal forearm and hand were mangled; they amputated at the mid-forearm.

Discussion: Occupational injuries represent a significant cause of morbidity and mortality worldwide. Many of these injuries are preventable. Workplace safety advocates have recommended safety measures for meat grinders, using plungers, and placing safety guards to prevent a hand from entering the apparatus.

Disclosure: No significant relationships.

\section{PELVIC TRAUMA}

\section{CR40}

\section{AN USUAL PELVIC RING FRACTURE. CASE REPORT}

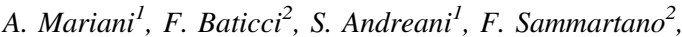 \\ S. Cimbanassi ${ }^{3}$, O. Chiara $^{4}$, R. Squinzi ${ }^{1}$
}

${ }^{1}$ Trauma Team, Ospedale Niguarda Ca' Granda, Milan, Italy, ${ }^{2}$ Emergency Surgery, ICH, Milano, Italy, ${ }^{3}$ Emergency, Ospedale Niguarda Ca' Granda, Milan, Italy, ${ }^{4}$ Emergency, Ospedale Niguarda Ca' Granda, Milan, Italy

Introduction: Vertical shear pelvic ring fracture is an unstable pelvic fracture. Several authors have hypothesized that this mechanism causes less haemorrhage then other types of pelvic ring fracture because the displacement of the emi-pelvis tends to shorter the vascular tree.

Case Report: We report the case of a 24 years old man who was involved in a motorcycle crash. In the prehospital setting the patient was hemodynamically unstable (III class), the clinical exam of the pelvis demonstrated an important caudally dislocation of the left emipelvis with no evidence of open fracture; no other signs of injuries were detected. We performed an extraperitoneal packing in emergency room and then an early fixation of the pelvis. After that a CT scan was performed demonstrating a complete dissection of femoral left artery with an important contrast extravasation. We have called this kind of pelvic fracture a converse vertical shear. This kind of injury is not included in the common classification of pelvic fractures. It is caused by a traction applied in caudal direction on an emipelvis. A caudally displacement of an emipelvis should alert the surgeon to the possibility of massive vascular injury with critical heammorhage.

Discussion: We have called this kind of pelvic fracture a converse vertical shear. This kind of mechanism is not included in the common classification of pelvic fractures. It is caused by a traction applied in caudal direction on an emipelvis. A caudally displacement of an emipelvis should alert the surgeon to the possibility of massive vascular injury with critical hemorrhage.

Disclosure: No significant relationships.

\section{CR41}

\section{EFFECTS OF AN EMERGENCY PELVIC INJURY PROTOCOL IN THE IMPROVEMENT OF THE POLYTRAUMA PATIENT CARE. ABOUT A CASE}

\section{A. Noval, A. Pérez, D. Cárdenes, C.R. Hernández, L. López, M. Sepulveda, C. Araña}

Emergency, Hospital Univeristario Insular de Gran Canaria, Las Palmas de GC, Spain

Introduction: Early activation of the pelvic fracture protocol in polytrauma patients and a skilled team on duty which knows the protocol improves the response time and determines priorities in the treatment of injuries that threatens patients life and therefore reduces the mortality rate.

Case Report: A 53 years old woman fell from a height of $6 \mathrm{~m}$. She had Glasgow 3 and was intubated; the ventilation was correct and she was found to have a pulse of 110 and hypotension (95/70) so she was infused with $4,000 \mathrm{ml}$ saline.

The hospital activated the polytrauma team and the radiologist on call, as an abdominal hemorrhage was highly possible. The first revision was performed, and only found subcutaneous crepitus in the left hemithorax and unstable pelvis immobilized with a sheet.

The eco FAST found free liquid. Portable radiographs were made: thorax (normal) and pelvis (type $\mathrm{C}$ fracture of tile). A blood transfusion was started and the patient was carried urgently to the operating room to fix the cause of the intra-abdominal hemorrhage; the vascular radiologist was then sent to treat the hemorrhage produced by the pelvic fracture.

Discussion: 1. Intra-abdominal hemorrhage is firstly treated in unstable patients with hemoperitoneum and possible pelvic fracture. 2. The arteriography is established as the definitive technique in the treatment of patients with fractured pelvis and hemodynamically unstable when intra-abdominal hemorrhage is disregarded with an eco FAST.

3. As the pelvic portable radiography has poor sensibility and specificity it will only be used when the patient is hemodynamically unstable and pelvic fracture is suspected.

Disclosure: No significant relationships. 


\section{CR42}

\section{EXTRAPERITONEAL PELVIC PACKING IN MULTIPLY INJURIED ELDERLY PATIENT. THE ROLE OF DAMAGE CONTROL SURGERY}

\author{
V. Guimerá Garciá ${ }^{1}$, M. Gutierrez-Andreu ${ }^{2}$, P. Caba Doussoux ${ }^{1}$, \\ P. Yuste-Garcia ${ }^{2}$, J.L. Leon Baltasar ${ }^{1}$, C. Resines Erasun ${ }^{1}$ \\ ${ }^{1}$ Orthopaedic \& Trauma Surgery, Hospital Universitario 12 de \\ Octubre, Madrid, Spain, ${ }^{2}$ General \& Digestive Surgery, Hospital \\ Universitario 12 de Octubre, Madrid, Spain
}

Introduction: Pelvic fractures with hemodynamic instability are challenging for both the orthopaedic and visceral surgeon due to high morbi-mortality rates. No gold standard guidelines to control pelvic hemorrhage have been defined. We would like to outline the importance of a multidisciplinary management and damage control surgery with our case report.

Case Report: 84 year man was crushed by a tree fall. C: chronic obstructive pulmonary disease and diabetes mellitus. Airway was secured by a oro-tracheal tube, a chest tube was inserted and BP was $90 / 60 \mathrm{mmHg}$. Deformity at left thigh and open IIIA ipsilateral tibia fracture were present. FAST excluded intraabdominal bleeding, but pelvic x-ray showed a TileB3 fracture. $\mathrm{Hb}: 7.8 \mathrm{~g} / \mathrm{dl}$, lactate $7 \mathrm{mmol} / \mathrm{l}$, $\mathrm{pH}$ 7.3. Due to hypotension, was transferred to the OR previous pelvic stabilization with a sheet. In the OR, anterior external fixation was performed before an extraperitoneal pelvic packing. SBP raised to 100-110 mmHg. So, was moved to CT that showed active bleeding at ilioisquio rami site. He was still hemodynamically stable and an angiography was performed. It showed no bleeding but also showed a femoral profunda artery obstruction. Afterwards was taken to OR for external fixation of femur and tibia and a popliteal-popliteal by-pass. Five hours later, was transferred to the ICU with lactate $11.2 \mathrm{mmol} / \mathrm{l}$, catecholamine therapy and $<35^{\circ} \mathrm{C} .36 \mathrm{~h}$ after the accident, the patient died.

Discussion: Long surgical interventions must be avoided in multiply injured and unstable patients. In our patient (ISS $=43$ ) the low physiological stock, elderly age and unstable hemodynamical status contraindicated complex and long revascularization techniques.

Disclosure: No significant relationships.

\section{CR43}

\section{REDUCTION TECHNIQUE OF THE OVERLAPPING AND LOCKED PUBIC SYMPHYSIS}

\section{H. Botanlioglu, N. Bilsel, G. Kaynak, M.C. Unlu}

Department of Orthopaedic and Traumatology, Cerrahpaşa Medical Faculty, İstanbul, Turkey

Introduction: These kinds of injuries occur after a lateral compression injury of the pelvic ring and they are classified as type B2 injuries according to Tile classification. Such injuries are rare in literature and first described in 1952. Locked pubic symphysis occurs when one pubic bone becomes entrapped behind the contralateral pubic bone or into the obturator foramen. Closed reduction is not always possible. It is important to know the injury mechanism to choose the treatment modality. In our case we describe an open reduction technique of locked pubic symphysis.

Materials and methods: The patient was a 21-year-old male who was a passenger in a motor vehicle collision. The vehicle crushed to a tree and the passenger squeeze between the left door of the car and the passenger seat. Diagnosis of fracture left ramus pubis inferior, dislocation of the symphysis pubis and locked pubic symphysis was made after the evaluation of the patient. In our case we describe an open reduction technique for a locked pubic symphysis.

Case Report: The patient was a 21-year-old male who was a passenger in a motor vehicle collision. The vehicle crushed to a tree and the passenger squeeze between the left door of the car and the passenger seat. Diagnosis of fracture left ramus pubis inferior, dislocation of the symphysis pubis and locked pubic symphysis was made after the evaluation of the patient. In our case we describe an open reduction technique for a locked pubic symphysis. As a result it is important to know the injury mechanism to plan the treatment of locked pelvis. Knowing the exact injury mechanism facilitates the reduction. By the technique that we described earlier it is possible to succeed in reduction with the help of basic orthopedic devices. Use of reconstruction plates and screws are the best fixations for these cases. Results: As a result it is important to know the injury mechanism to plan the treatment of locked pelvis. Knowing the exact injury mechanism facilitates the reduction. By the technique that we described earlier it is possible to succeed in reduction with the help of basic orthopedic devices. Use of reconstruction plates and screws are the best fixations for these cases.

Discussion: It is important to know the injury mechanism to choose the treatment modality.

Conclusion: It is important to know the injury mechanism to choose the treatment modality.

Reference:

Eggers GW. Dislocations of the os coxae. Am J Surg. 1952;83(3): 300-7.

Disclosure: No significant relationships.

\section{ABDOMINAL EMERGENCIES}

\section{CR44}

\section{ACUTE ABDOMEN: PNEUMATOSIS CYSTOIDS INTESTINALIS COMPLICATED WITH PNEUMOPERITONEUM, CASE REPORT}

H.M. Fernandes, C.S. Costa, A.L. Silva, T.N. Santos, A.S. Santos

Cirurgia Geral, Centro Hospitalar do Alto Ave, Guimarães, Portugal

Introduction: PCI is defined as air filled cysts within the wall of gastro-intestinal tract. The secondary forms of this disease, the most frequent $(85 \%)$, are associated with panoply of gastro-intestinal tract and pulmonary disease. The manifestations of this disease are unspecific. Around 3\% of patients experience complications like pneumoperitoneum, volvulus, intestinal obstruction, intussusceptions. Case Report: A 47-year-old man presented on emergency room with a history of vomiting, abdominal distension and diffuse abdominal pain. He had regular use of methadone and past surgery for peptic ulcer complicated by hemorrhage. On physical examination, generalised palpation tenderness and guarding was present. TC revealed an enormous pneumoperitoneum. At laparotomy was found an uncountable number of cystic lesions in the medium ileum, for a length of 30-40 cm, without signs of perforation; and also a severe stenotic pylorus, associated with a huge stomach. The decision was not to intervene in the affected ileum loops. A Finney pyloroplasty was performed. The post operative course was uneventful.

Results: A 47-year-old man presented on emergency room with a 2 weeks history of vomiting, abdominal distension, and diffuse abdominal pain. He had history of regular methadone use and past surgery for peptic ulcer complicated by hemorrhage. On physical examination, he had generalised palpation tenderness and guarding. 
TC revealed an enormous pneumoperitoneum. At laparotomy, in the medium ileum, and for a length of $30 \mathrm{~cm}$, an uncountable number of cystic lesions were found, without signs of perforation. Also, a severe stenotic pylorus associated with a huge stomach was found. The decision was not to intervene in the alterated ileum loops. A Finney pyloroplasty was performed. The post operative course was uneventful.

Discussion: Pneumoperitoneum is a rare complication of PCI and although surgical approach is not always indicated, it must be considered in case of acute abdomen. The association between pyloric stenosis and PCI, as described in this case, is known and the operative relief of the underlying cause is usually curative of the pneumatosis. So, it is not imperative to remove the segment of intestine involved in pneumatosis, unless there is a clearly evidence of perforation, relevant inflammation, adhesions or risk of obstruction.

Disclosure: No significant relationships.

\section{CR45}

\section{DELAYED PRESENTATION OF DIAPHRAGMATIC RUPTURE}

\author{
E. Falidas $^{\text {I, E. Pavlaki }}{ }^{2}$, G. Christopoulos ${ }^{3}$, S. Mathioulakis ${ }^{4}$, \\ K. Vlachos ${ }^{5}$, C. Villias ${ }^{4}$
}

${ }^{1} 1$ st Department Of Surgery, 417 Nimts, Veterans Hospital Of Athens, Athens, Greece, ${ }^{2}$ Pediatric Hospital "Agia Sofia”, Athens, Greece,

${ }^{3} 1$ st Department Of General Surgery, Nimts Hospital, Athens, Greece, ${ }^{4} 1$ st Department Of General Surgery, Nimts Hospital, Athens, Greece, ${ }^{5}$ Department Of Surgery, University Of Ioannina, Athens, Greece

Introduction: Delayed presentation of diaphragmatic rupture is rare and usually related with signs and symptoms due to hollow viscera herniation through a muscular deficit. We report two cases of delayed presentation of diaphragmatic rupture 1 and 3 months respectively, after the initial blunt trauma.

Materials and methods: CASE A: A 28 year-old man presented to the ED because of acute abdominal pain located to the epigastrium, dyspnea and thoracic pain to the left hemithorax. The patient had a recent history of protracted hospitalization ( 3 months earlier) due to multiple trauma of the chest, spinal cord and multiple bone fractures. On admission, laboratory and radiologic examinations demonstrated a gastric herniation into the chest. CASE B: A 32 year old man arrived to the ED referring dyspnea and acute upper abdominal pain. He had a history of motor accident a month ago, 2 days of hospitalization for blunt trauma while the present symptoms started during the sexual act. X-ray series revealed a gastric herniation to the left thoracic side. Case Report: CASE A: A 28 year-old man presented to the ED because of acute abdominal pain located to the epigastrium, dyspnea and thoracic pain to the left hemithorax. The patient had a recent history of protracted hospitalization (3 months earlier) due to multiple trauma of the chest, spinal cord and multiple bone fractures. On admission, laboratory and radiologic examinations demonstrated a gastric herniation into the chest. CASE B: A 32 year old man arrived to the ED referring dyspnea and acute upper abdominal pain. He had a history of motor accident a month ago, 2 days of hospitalization for blunt trauma while the present symptoms started during the sexual act. X-ray series revealed a gastric herniation to the left thoracic side. Both patients underwent laparotomy. In the first case, the herniated viscera was initially reduced into the abdomen and a like-sleeve gastrectomy was performed. In the second case, the stomach was placed into the abdomen. In both cases the diaphragmatic deficit closed with sutures.
Results: Both patients underwent laparotomy. In the first case, the herniated viscera was initially reduced into the abdomen and a likesleeve gastrectomy was performed. In the second case, the stomach was placed into the abdomen. In both cases the diaphragmatic deficit closed with sutures.

Discussion: Rupture of the diaphragm is uncommon, occurring in $0.8-1.6 \%$ of hospitalized patients with thoracoabdominal blunt trauma and usually on the left side. A high index of suspicion is necessary while treating patients with a positive history of blunt trauma.

Conclusion: Rupture of the diaphragm is uncommon, occurring in $0.8-1.6 \%$ of hospitalized patients with thoracoabdominal blunt trauma and usually on the left side. A high index of suspicion is necessary while treating patients with a positive history of blunt trauma.

Reference: Rashid F, et al. A review on delayed presentation of diaphragmatic rupture. WJ Emerg Surg. 2009;4:32. doi:1D1186/ 1749-7922-4-32.

Disclosure: No significant relationships.

\section{CR46}

\section{DISPROPORTIONAL BILE DUCT INJURY AFTER LAPAROSCOPIC CHOLECYSTECTOMY}

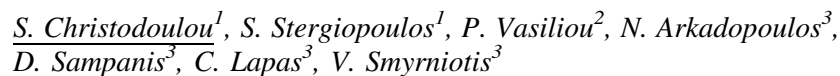

${ }^{1} 4$ th Surgical Clinic, ATTIKON University Hospital, Athens, Greece, ${ }^{2}$ Fourth Surgery Department, University General Hospital 'Attikon', Athens, Greece, ${ }^{3} 4$ th Surgical Clinic, ATTIKON University Hospital, Athens, Greece

Introduction: Laparoscopic cholecystectomy (LC) has become the treatment of choice for patients with symptomatic cholelithiasis, at the cost of slightly higher incidence ( 0.3 vs. $0.1 \%$ for open surgery) of bile duct injuries. Following a benign disease this is an iatrogenic catastrophe associated with significant morbidity, mortality, reduced long-term survival and quality of life, and high rates of subsequent litigation.

Case Report: We report the case of a 34-year-old woman who referred to our academic surgical department for the treatment of a bile duct injury 3 weeks after LC. The patient on 7 th postoperative day developed acute abdomen. On a CT scan large amount of free intraperitoneal fluid was identified. On the emergency laparotomy that followed bile ascites was drained and stitches were placed at the common bile duct. The patient postoperatively developed jaundice, while bile drainage persisted. Magnetic Retrograde Cholangiopancreatography (MRCP) revealed dilation of the left intrahepatic bile ducts, and the Endoscopic RCP that followed failed to resolve the identified obstruction of common hepatic duct. The patient 3 days later became febrile. She underwent laparotomy where occlusion of the left bile duct was identified together with high right sectoral duct transection causing bile and pus leak. A right hepatectomy, with Roux-en-Y hepatojejunostomy was performed. The patient after a prolonged postoperative course, recovered well.

Discussion: Iatrogenic bile duct injuries if not identified intraoperative, should be referred to a tertiary specialized center for optimal outcome. Attempts to repair the damage without the necessary expertise augment the problem and its consequences.

Disclosure: No significant relationships. 


\section{CR47}

\section{HEPATIC TRAUMA}

J.C. Pereira ${ }^{l}$, A.L. Silva ${ }^{l}$, S. Viçoso ${ }^{2}$, T.C. Santos ${ }^{l}$, M. Oliveira $^{l}$,

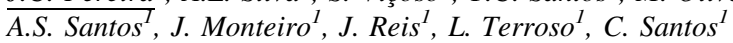

${ }^{1}$ Surgery, Centro Hospitalar do Alto Ave, Unidade de Guimarães, Portugal, Guimarães, Portugal, ${ }^{2}$ Orthopedics, Centro Hospitalar do Alto Ave, Unidade de Guimarães, Guimarães, Portugal

Introduction: Trauma being a major cause of death, the existence of an hospital-wide multidisciplinary team capable of giving an immediate and well organized answer for patients with multiple trauma is essential. Of all the politraumatized patients, about $5 \%$ have an hepatic trauma (HT).

Materials and methods: With this work the authors intend to: Report a case of a patient with 66 years-old with a blunt thoraco-abdominal trauma and a vertebral trauma, after a traffic accident. At the admission in the Emergency Room, she presented an abdominal pain in the right upper quadrant, without signs of peritoneal irritation, associated with hypotension. She also had low-back pain and paraplegia. Initial imaging evaluation (by ultrasound) showed a small amount of free intra-abdominal fluid. The patient responded moderately to the fluids and has done a thoraco-abdomino-pelvic CT and a vertebral CT.

Case report: With this work the authors intend to: Report a case of a patient with 66 years-old with a blunt thoraco-abdominal trauma and a vertebral trauma, after a traffic accident. At the admission in the emergency room, she presented an abdominal pain in the right upper quadrant, without signs of peritoneal irritation, associated with hypotension. She also had low-back pain and paraplegia. Initial imaging evaluation (by ultrasound) showed a small amount of free intra-abdominal fluid. The patient responded moderately to the fluids and has done a thoraco-abdomino-pelvic CT and a vertebral CT.

The evidence of extensive lacerations of the hepatic parenchyma with capsular achievement and intra-peritoneal spillage peri-hepatic, coupled with maintenance of hemodynamic instability, led the surgical team to choose to take an emergency exploratory laparotomy, where several deep lacerations of the hepatic parenchyma associated with a moderate volume of hemoperitoneum were found. Suture of the lacerations and application of a sponge composed of human fibrinogen and thrombin for optimal hemostasis were done. The postoperative period progressed uneventfully and she was then submitted to posterior stabilization of the comminutive fracture with associated dislocation of T11 vertebra shown by the vertebral CT.

Results: The evidence of extensive lacerations of the hepatic parenchyma with capsular achievement and intra-peritoneal spillage perihepatic, coupled with maintenance of hemodynamic instability, led the surgical team to choose to take an emergency exploratory laparotomy, where several deep lacerations of the hepatic parenchyma associated with a moderate volume of hemoperitoneum were found. Suture of the lacerations and application of a sponge composed of human fibrinogen and thrombin for optimal hemostasis were done. The postoperative period progressed uneventfully and she was then submitted to posterior stabilization of the comminutive fracture with associated dislocation of T11 vertebra shown by the vertebral CT.

Discussion: The therapeutic approach of the HT (surgical vs conservative) depends on the extent of initial injury, the patient's hemodynamic stability and signs of peritoneal irritation. The treatment for most patients with HT undergoes a conservative treatment. Hepatic angiography with embolization is a form of conservative treatment, which relieves the surgery, reducing the morbidity of patients. This type of treatment is primarily performed in hemodynamically stable patients. However, as experience increases, even unstable patients may benefit from it.

Conclusion: The therapeutic approach of the HT (surgical vs. conservative) depends on the extent of initial injury, the patient's hemodynamic stability and signs of peritoneal irritation. The treatment for most patients with HT undergoes a conservative treatment. Hepatic angiography with embolization is a form of conservative treatment, which relieves the surgery, reducing the morbidity of patients. This type of treatment is primarily performed in hemodynamically stable patients. However, as experience increases, even unstable patients may benefit from it.

References: "Hepatic trauma", Cothren CC, et al. nÂ ${ }^{\circ} 4$ "Actual-

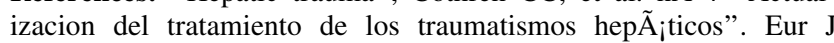
Trauma Emerg Surg 2008. Leonardo Silvio-Estaba, et al. "Angiography and embolisation for solid abdominal organ injury in adults-a current perspective". Circ Esp. 2008. Wallis, et al. World J Emerg Surg 2010;5:18.

Disclosure: No significant relationships.

\section{CR48}

\section{PANCREATIC DUCT REPAIR FOR BLUNT PANCREATIC TRANSECTION}

\author{
M. Shimizu ${ }^{1}$, N. Hiroe ${ }^{2}$, T. Shibusawa ${ }^{3}$, S. Matsumoto ${ }^{3}$, T. Funabiki ${ }^{3}$, \\ M. Yamazaki ${ }^{3}$, M. Kitano ${ }^{2}$
}

${ }^{1}$ Emergency and Surgery, Saiseikai Yokohama-shi Tobu Hospital, Yokohama, Japan, ${ }^{2}$ Emergency Surgery, Saiseikai Yokohama-city Tobu Hospital, Yokohama, Japan, ${ }^{3}$ Emergency and Critical Care Medicine, Saiseikai Yokohama-shi Tobu Hospital, Yokohama, Japan

Introduction: In case of pancreatic duct disruption, the most common operative procedure is distal pancreatectomy (DP). But to preserve the function of the pancreas and the spleen, we select pancreatic duct repair (PDR) for hemodynamically stable patients. The procedure of PDR is as follows, (1) mobilization and triming injured pancreas, (2) cannulating stent into pancreatic duct, (3) performing pancreatic duct anastomsis by 4 stich of 6-0 prolene, (4) suturing pancreas by 4-0 prolene. The aim of this study is to compare PDR to DP.

Materials and methods: We have reviewed retrospectively all our patients received PDR or DP for blunt pancreatic injury between 1985 to 2009. We compared patient demographics, RTS, ISS, intraoperative blood loss, operative time, pancreas related complications and mortality for each procedure.

Case Report: We have reviewed retrospectively all our patients received PDR or DP for blunt pancreatic injury between 1985 to 2009. We compared patient demographics, RTS, ISS, intraoperative blood loss, operative time, pancreas related complications and mortality for each procedure.

Results: Of the total 17 patients, 10 were received PDR and 7 were received DP. There was no significant difference as to demographics, RTS, ISS between both groups. The mean intraoperative blood loss of PDR and DP was similar $(1,542,1685 \mathrm{ml})$, PDR needed significantly longer operative time than DP (200, 139 min). Pancreas related complications of PDR occurred in 4 cases (40\%), 3 pancreatic fistulas and 1 pancreatic cyst, whereas those of DP were 3 pancreatic fistulas $(43 \%)$. In each groups, one patient died because of severe head trauma. 
Discussion: PDR is useful procedure for pancreatic duct injury and can be selected if the patient is hemodynamically stable.

Conclusion: PDR is useful procedure for pancreatic duct injury and can be selected if the patient is hemodynamically stable.

Disclosure: No significant relationships.

\section{CR49}

\section{TREATMENT OF A III GRADES PANCREATIC INJURY. CASE REPORT}

C. Cataldi, T. Mastropietro, S. Manfroni, M. Pistocchi, E. Cingolani, R.L. Meniconi, G. Nardi, D. Antonellis, A. Roveran

Emergency and General Surgery, San Camillo Forlanini, Rome, Italy

Introduction: Pancreatic injury can present a formidable challenge to the surgeon. This injury is present in 5\% of patients with abdominal trauma, is rarely isolated but often associated with other injuries that are directly responsible for most of the morbidity and mortality.

Case Report: A male 34 years, arrived in the A\&E victim of a motorcycle-car crash. Clinical condition: hypovolemic shock, right PNX, facial fracture, evidence of contusion in epigastric region. We placed pleural drainage, performed FAST and transferred the patient to the operative room. The laparotomy showed a significant hemoperitoneum because of the rupture of the left renal vein, the rupture of the inferior splenic pole and the complete rupture of the pancreas isthmus (AAST-OIS Grades III). Surgery treatment: suture of the left renal vein, conservative treatment of the splenic lesion, suturing of the medial and lateral pancreatic border. The patient was sent to the ICU. On the fourth day we observed an increase of the pancreatic enzymes, of the white cells and metabolic acidosis. The angioCT showed a haemorrhagic pancreatitis treated with relaparotomy, epiplon-retrocavity drainage, common bile duct drainage, and bogotà-bag. After $72 \mathrm{~h}$ we proceeded to close the abdomen. The patient was discharged after a long period of hospitalization.

Discussion: The decision of this kind of surgical treatment with a method not recommended in literature was taken for two reasons: to protect the patient from induced diabetes and to avoid the surgical dehiscence due to a predictable pancreatitis. This single clinical case expects to be as a drop in the ocean

Disclosure: No significant relationships.

\section{SPINAL AND BRAIN INJURY IN MULTIPLE TRAUMA}

\section{CR50}

\section{A RARE CASE OF GOOD RECOVERY IN TRAUMATIC OCCIPITAL CONDYLE FRACTURE ASSOCIATED WITH CENTRAL BILATERAL ABDUCENS PALSY}

G. Nardi ${ }^{1}$, A. Cossu ${ }^{2}$, D. Piredda ${ }^{2}$, S. Signoretti ${ }^{3}$, C. Ajmone-Cat ${ }^{2}$, G. Ranaldi ${ }^{2}$

${ }^{1}$ Shock \& Trauma Unit, S. Camillo Hospital, Roma, Italy, ${ }^{2}$ Shock \& Trauma Unit, S. Camillo Hospital, Roma, Italy, ${ }^{3}$ Dept. Of Neurosurgery, S. Camillo Hospital, Rome, Italy

Introduction: Occipital condyle fracture is a rare injury usually associated with a poor outcome. There are limited reports in literature.
We describe a case of unexpected survival with good recovery after a high speed motorbike crash.

Case Report: PA 23, with multiple injuries involving head, chest and abdomen, was transferred to our Trauma Centre from a District Hospital after emergency splenectomy for massive hemoperitoneum. He had a TBI with GCS 7 (E1M5V1), severe thoracic trauma with multiple ribs fractures and pneumothorax and fracture of right leg. Cranio cerebral CT showed a bilateral parieto-occipital subdural hematoma, small left frontotemporal intraparenchymal hemorrhagic foci and cerebral edema. There was also a fracture of skull base causing a dislocation of the left occipital condyle into the foramen magnum and in the spinal canal. The left vertebral artery was dislocated by the bone fragment without evidence of stenosis. Admission MR was negative as for damage of the medulla and of the brain stem and the Pons. The patient was deeply sedated and ICP was normal. On day 8th PA was submitted to occipitocervical stabilization. Sedation was discontinued on day 10. In followings days he improved to a good recovery of the consciousness. PA presented however a tetraparesis with legs hypertonus and upper limbs hypotonus associated with a bilateral abducens nerve palsy. Because of these findings PA was submitted to a new encephalic RM which showed two small symmetric lesions at bulbo-pontine junction detected as hyperintense signals in TR sequences and slightly hypointense in T1. Because of their localization they were interpreted as a damage to the infranuclear 6th nerve area.

Discussion: The abducens nerve is particularly vulnerable to traumatic injury because of its tortuous route from the cranial nerve nucleus located in the pons upward in the subarachnoid space, to the lateral rectus muscle. Abducens nerve damages often occur as the consequence of direct injury to the nerve and are therefore unilateral. The focal lesion of the sixth nerve nucleus are very rare in trauma survivors as the bilateral contusion of the Pons is generally associated with major brain damage and poor outcome. Conservative treatment resulted in residual bilateral abducens nerves palsy after 3 months. Recovery of bilateral traumatic palsy are exceedingly rare according to literature. The only case of complete recovery to our knowledge is reported in a 6 years old Korean child.

Reference: Min-Su Kim, et al. J Korean Neurosurg Soc. 2008;44: 396-8.

Disclosure: No significant relationships.

\section{CR51}

\section{BLUNT TRAUMATIC CARDIAC HERNIA ASSOCIATED WITH TENSION PNEUMOPERICARDIUM: CASE REPORT}

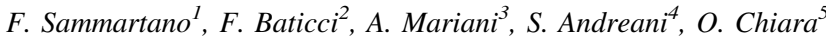

${ }^{1}$ Chirurgia Generale E Mininvasiva, Sezione Di Chirurgia D’urgenza E Trauma, Istituto Clinico Humanitas, Milano, Italy, ${ }^{2}$ Emergency Surgery, ICH, Milano, Italy, ${ }^{3}$ Trauma Team, Ospedale Niguarda Ca' Granda, MILANO, Italy, ${ }^{4}$ Trauma Team, General Surgery, Niguarda Hospital Milan, Milan, Italy, ${ }^{5}$ Emergency, Ospedale Niguarda Ca' Granda, Milan, Italy

Introduction: Tension pneumopericardium and cardiac hernia are rare injuries in trauma with high mortality rate if combined.

Case Report: A 34-years-male was admitted after a fall from $8 \mathrm{~m}$ sustaining a severe blunt head/chest trauma. Patient was unconscious, $\mathrm{SpO}_{2} 60 \%$, BP 140/50, HR 80, GCS3, pupils dilated and fixed. Patient was intubated, evacueed. Prehospital time was $48 \mathrm{~min}$. In ER, HR 90, BP 90/60, $\mathrm{SpO}_{2} 88 \%$, GCS3T. O/E revealed bilateral chest/neck emphysema, absent left and decreased right breath sounds, abdomen unaccessible, pelvis stable with suspected open fracture of right iliac 
spine. GCS3T, pupils unequal. No limbs trauma. Suspected base skull fracture. ET tube was withdrawn with no improvement. Bilateral needle decompression and bilateral chest tube were inserted with transient improvement. FAST, CXR, Pelvis X-ray revealed free-fluid in Morrison space, bilateral pneumothorax mostly right, ribs fractures with unclear mediastinum due to huge emphysema, LC-I pelvis fracture. A second right chest tube was inserted and pelvis contention applied. During CT patient deteriorated, BP 50/30, HR 40. Incomplete scan showed: brain swelling with $\mathrm{SAH}$, penumoencefalum, MXF fractures, bilateral pneumothorax, lung contusions, tension pneumopericardium with left-side cardiac hernia. ED thoracotomy, pericardiotomy, decompression of pericardial sac, cross-clamping was performed and transferred to OR for laparotomy with packing (liver injury OIS II, R-kidney injury OIS I). Despite aggressive fluid resuscitation and active re-warming, patient crashed after $15 \mathrm{~min}$ in OR and coded in 25 min from cardiac arrest.

Discussion: Clark reported 142 cases. Left pleuropericardium (50\%), right pleuropericardium (17\%), diaphragmatic pericardium $(27 \%)$, superior mediastinal pericardium (4\%). Cardiac herniation was found in 31 of left and 5 of right pleuropericardial defects, 4 diaphragmatic. Associated injuries: cardiac (28\%), neurologic (32\%), abdominal (27\%), pelvic/extremity fractures (49\%). Blunt traumatic rupture of pericardium patients should be divided into stable/unstable. Stable: admission in ICU/HDU if not ventilated or without extrathoracic injuries, closed monitoring of vitals/CRX. Simple pneumopericardium: self-limiting. Small, uncomplicated pericardial tears: elective repair. Unstable: emergency surgery. Surgical options are: needle pericardiocentesis, pericardial window (open/VATS),resuscitative thoracotomy.

Disclosure: No significant relationships.

\section{CR52}

\section{POLYTRAUMATIZED PATIENT WITH VERTEBRAL TRAUMA-AN ORTHOPEDIC PERSPECTIVE}

\author{
S.I.V.S. Fernandes ${ }^{1}$, J.E. Fraga Ferreira ${ }^{2}$, A.T. Barbosa ${ }^{2}$, \\ J. Oliveira ${ }^{2}$, J.C. Pereira ${ }^{3}$, M. Oliveira ${ }^{3}$, A.L. Silva ${ }^{3}$, T. Basto ${ }^{4}$

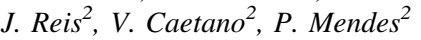

${ }^{1}$ Orthopedics and Traumatology, Centro Hospitalar Alto Ave, Guimarães, Guimarães, Portugal, ${ }^{2}$ Orthopaedics And Traumatology, Centro Hospitalar do Alto Ave, Guimarães, EPE, Guimarães, Portugal, ${ }^{3}$ Surgery, Centro Hospitalar Alto Ave, Guimarães, Guimarães, Portugal, ${ }^{4}$ Orthopaedics and Traumatology, Centro Hospitalar do Alto Ave, Guimarães, EPE, Guimarães, Portugal

Introduction: Polytraumatized patients often have associated abdominal, thoracic and vertebral trauma and life-threatening conditions. Injury to the cord or cauda equina occurs in 10-38\% of thoracolumbar fractures and in approximately $50-60 \%$ of fracture dislocations and nearly $60 \%$ have neurologic deficits.

Case Report: 66 years-old women suffered a traffic accident and at the admission in the emergency room she had abdominal and lowback pain. The patient was hemodynamic unstable. At neurologic examination she had paraplegia with no sensibility below the umbilicus, grade 0 of muscular force of all muscular groups and areflexia of both lower extremities. The lesion was ASIA A with urinary incontinence and no voluntary anal contraction. The mariological study showed extensive hepatic parenchyma lacerations and a T11 comminutive fracture/dislocation. She had a TLCIS (Thoraco-Lumbar Injury Classification and Severity Score) score of 9. The patient underwent laparotomy with hepatic parenchyma laceration suture and after to posterior stabilization with transpedicular fixation T9-T10 and
T12-L1 with PANGEA and initiated physical rehabilitation. At 3 months she is incapable of deambulation, using a wheelchair, but with neurologic function improvement (most of the muscular groups of the both legs have muscular force of 3 and has sensibility in both legs) and is classified in ASIA C.

Discussion: A multidisciplinar approach is needed to improve mortality and morbility in trauma patients. Adequate and precocious treatment of thoracolumbar fractures can prevent neurologic lesions deterioration and functional disability and improve living quality. Unfortunately in vertebral trauma, despite adequate treatment some lesions are so severe that we can't prevent permanent neurologic damage.

Disclosure: No significant relationships.

\section{CR53}

\section{POSTTRAUMATIC CEREBRAL INFARCT}

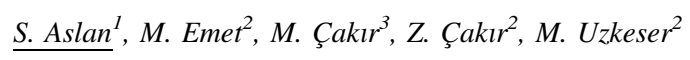

${ }^{1}$ Emergency Department, Ataturk University Aziziye Hospital, Erzurum, Turkey, ${ }^{2}$ Emergency Department, Ataturk University, Erzurum, Turkey, ${ }^{3}$ Neurosurgery, Ataturk University, Erzurum, Turkey

Introduction: Missing a serious traumatic injury is common nightmare of emergency physicians. It is not only crucial for prognosis of the patient, but also for legal and forensic issues. Here, we want to represent a special cerebrovascular event (CVE) that occurs after a prodromal period after mild to moderate brain injury in childhood. It was first described in 1980 s, but many points still need to be illuminated.

Case Report: A 3-year-old girl was brought to our ED due to minor head injury half an hour ago. She was confused with a GCS of 13. No neurologic deficit was observed. Her brain CT was normal. She was observed in ED for $5 \mathrm{~h}$. Seeing that her GCS was improved, she was discharged. Approximately $21 \mathrm{~h}$ after trauma, she readmitted due to difficulty in swallowing and unable to use her right arm. Her parents corrected the trauma mechanism as tumbling of a wardrobe door onto her face. Physical examination revealed aphasia, central facial palsy on the right, and hemiparesis on the same side (upper and lower extremities scores were $1 / 5$ and $2 / 5$, respectively). Her GCS was 14 . Control brain CT showed a hypodense area on left frontoparietal region. Brain magnetic resonance imaging (MRI) obtained half an hour later represented findings compatible with ischemic stroke. She was hospitalized in neurosurgery clinic for 13 days.

Results: A 3-year-old girl was brought to our ED from kindergarten by her teachers due to minor head injury (fallen down on to her head while walking) half an hour ago. She was confused with a Glasgow Coma Scale (GCS) of 13 (EVM). There was a bruise and dermabrasion on her face on the left. No lateralized neurologic deficit was observed. Her laboratory analyses were in normal values except mild leucocytosis $(12550 / \mu \mathrm{L})$, hyperglycemia $(124.4 \mathrm{mg} / \mathrm{dL})$, hypopotassemia $(3.31 \mathrm{meq} / \mathrm{dL})$ and hypocalcemia $(8.33 \mathrm{mg} / \mathrm{dL})$. Her brain computerized tomography (CT) was normal. She was observed in ED for $5 \mathrm{~h}$. Seeing that her GCS was improved and she was able to walk without help, she was discharged. Following day, approximately $21 \mathrm{~h}$ after trauma, she readmitted to ED due to difficulty in swallowing, poor appetite and unable to use her right arm. Her parents corrected the trauma mechanism as tumbling of a wardrobe door onto her face. Physical examination revealed aphasia, central facial palsy on the right, and hemi paresis on the same side (upper and lower extremities scores were $1 / 5$ and $2 / 5$, respectively). Her GCS was 14 . Control brain CT showed a hypodense area on left frontoparietal region (Fig. 1). 
Brain magnetic resonance imaging (MRI) obtained half an hour later represented findings compatible with ischemic stroke (Fig. 2). She was hospitalized in neurosurgery clinic for 13 days. Her B-mode ultrasonography findings of common, external and internal carotid arteries bilaterally were within normal limits. Her echocardiographic evaluation was also normal. The patient was discharged with moderate residual hemiparesis.

Discussion: It is a known fact that mild cranial trauma can cause posttraumatic ischemic infract after a latent period. The pathophysiology of this injury in young children is either thrombus or spasm of end arteries. Other adverse neurological consequences following mild head injury may be migraine, encephalopathy, seizures and focal neurologic deficit

Conclusion: It is a known fact that mild cranial trauma can cause posttraumatic ischemic infract after a latent period. The pathophysiology of this injury in young children is either thrombus or spasm of end arteries. Relative delay of symptoms can be explained on the basis of thrombus generation. Viral infection (especially varicella) may cause vasculopathy and susceptibility to develop arterial thrombosis or spasm following mild head injury. Other adverse neurological consequences following mild head injury may be migraine, encephalopathy, seizures and focal neurologic deficit. Differential diagnoses should include diffuse axonal injury (DAI) (Table 1), hemorrhagic contusion and traumatic subcortical injury.

Disclosure: No significant relationships.

\section{CR54}

\section{RECONSTRUCTION OF A FRONTAL DEPRESSED FRACTURE-CASE REPORT}

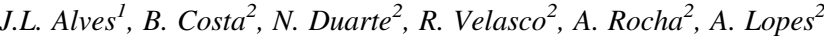

${ }^{1}$ Neurosurgery, Centro de Neurocirurgia de Coimbra, Coimbra, Portugal, ${ }^{2}$, Centro de Neurocirurgia de Coimbra, Coimbra, Portugal

Introduction: Skull fracture is a common phenomenon, due to vehicular accidents. Reconstruction of depressed skull fractures attempts the closure of the defect and cosmetic improvement, restoring anatomical barriers and preventing complications (infections, cerebrospinal fluid leaks). Several techniques/materials are described, and their selection is controversial.

Case Report: 69-year-old male suffered major trauma following motor-vehicle accident. The patient sustained thoracic trauma-rib fractures and pleural effusion, motivating chest tube insertion. Suffered also severe Head Trauma, with frontal impact. CT-scan showed right frontal depressed fracture, with multiple fragments in contact with brain parenchyma, without any other intracranial injury. On examination, GCS -15 , no neurological deficit. Less than 24 h later, after small dural repair, multilayer reconstruction was performed, using aponeurosis, Surgical ${ }^{\circledR}$, fibrin glue and titanium mini-plates/ screws, sustaining multiple reshaped bony fragments. Post-op CTscan shows satisfactory frontal reconstruction, with persistent small median bony fragment + small brain contusions. Treatment concerning thoracic injuries and pneumonia ensued. In follow-up, there were good cosmetic results, with proper contour and stability of the construct.

Discussion: In depressed skull fractures, the outer table of the fracture edge lies below anatomical level of inner table of the intact skull. Depressed bone can produce dural tear and brain damage. This case describes a compound depressed fracture, with frontal location (most frequent) and dural tear. Autogenous tissue and alloplastic material, including cost-effective titanium mini-plates, were used. Bony fragments were retained, eventually preventing a second cranioplasty. There were no complications, and good cosmetic result was obtained (more than $90 \%$ deformity correction comparing to the other side.) Disclosure: No significant relationships.

\section{VASCULAR LESIONS}

\section{CR55}

\section{COMBINED LEFT VENTRICLE AND DESCENDING AORTIC GUN SHOT WOUND}

F. Domaszewski ${ }^{1}$, E. Schwendenwein ${ }^{1}$, J. Dumfarth ${ }^{2}$, M. Greitbauer ${ }^{1}$, M. Gregori ${ }^{1}, F$. Wolf ${ }^{3}$, M. Vögele-Kadletz ${ }^{2}$, G. Wollenek ${ }^{2}$

${ }^{1}$ Traumatology, Medical University Vienna, Austria, Vienna, Austria, ${ }^{2}$ Cardiac Surgery, Medical University Vienna, Austria, Vienna, Austria, ${ }^{3}$ Interventional Radiology, Medical University Vienna, Austria, Vienna, Austria

Introduction: To our knowledge there is no reported combined lesion of the left ventricle and the descending aorta due to a single gunshot bullet in the same patient. Which of these two potentially lethal injuries should be addressed first?

Materials and methods: We report of a 22-year old man who was injured by a small bore gun shot. Computed tomography with contrast medium revealed a penetrating injury to the left ventricle of the heart and the descending aorta. We report on our interdisciplinary diagnostic and therapeutic management regarding these dramatic and potentially lethal injuries.

Case Report: We report of a 22-year old man who was injured by a small bore gun shot. Computed tomography with contrast medium revealed a penetrating injury to the left ventricle of the heart and the descending aorta. We report on our interdisciplinary diagnostic and therapeutic management regarding these dramatic and potentially lethal injuries.

Results: In recent years, thoracic endovascular aortic repair (TEVAR) developed to be the predominant treatment method for blunt aortic injuries. In this case, open surgical approach to one source of bleeding was thought to promote bleeding of the other injury. Therefore endovascular stent grafting under cardiac surgeon's surveillance was considered as the first therapeutic step. However, the patient's cardiac situation worsened, so that the therapy priorities had to be changed immediately. Open cardiac repair at beating heart was performed. Then, with the patient being hemodynamic stable, the stent graft was placed in the same session. After 10 days at the ICU and 10 days at the general ward the patient left the hospital in excellent condition.

Discussion: Although minimal invasive aortic repair is the first treatment option, there is only a very short time frame given by the left ventricular injury. Interdisciplinary collaboration is absolutely necessary to handle such a life-threatening injury combination successfully.

Conclusion: Although minimal invasive aortic repair is the first treatment option, there is only a very short time frame given by the left ventricular injury. Interdisciplinary collaboration is absolutely necessary to handle such a life-threatening injury combination successfully.

Disclosure: No significant relationships. 


\section{CR56}

\section{DELAYED ENDOVASCULAR TREATMENT OF DESCENDING AORTA STENT GRAFT COLLAPSE IN A PATIENT TREATED FOR POST-TRAUMATIC AORTIC RUPTURE}

G. Nano ${ }^{l}$, D. Mazzaccaro ${ }^{2}$, G. Malacrida ${ }^{l}$, M.T. Occhiuto $^{l}$, S. Stegher , D.G. Tealdi ${ }^{T}$

${ }^{1}$ IRCCS POLICLINICO SAN DONATO, San Donato Milanese, Italy, ${ }^{2}$ Chirurgia Vascolare I Divisione, IRCCS POLICLINICO SAN DONATO, San Donato Milanese, Italy

Introduction: We report the case of delayed endovascular correction of graft collapse occurred after emergent Thoracic Endovascular Aortic Repair (TEVAR) for traumatic aortic isthmus rupture.

Case Report: A young man presented a traumatic aortic isthmus rupture after a motocross accident. He had severe hypotension and respiratory impairment so he immediately underwent an endovascular correction (Gore $\mathrm{TAG}^{\circledR} 28-150$ ). In 5th post-operative day, peripheral leg pulses disappeared. A CT scan showed a partial collapse of the endoprosthesis at the descending tract, with no signs of visceral ischemia. Considering patient \& rsquos clinical conditions, the graft collapse wasn $\&$ rsquot treated at that time. Since then, the patient \& rsquos general conditions progressively improved enough to allow reintervention, but the patient refused any new treatment, so he was discharged. Four months later the patient came back complainting severe gluteal and sural intermittent claudication, erectile disfunction and abdominal angina a chest and abdominal radiography and a duplex ultrasound showed subocclusion of the graft. Angiography confirmed the increased collapse of the graft, so we proceeded with a new endovascular correction (Bolton Relay\&trade 28-155 + bare stent). 18 months later a new CT scan showed normal patency of the graft. The patient returned to his previous daily activities.

Discussion: TEVAR represents a significant improvement in the treatment of thoracic aortic rupture. Graft collapse is a rare event, which should be detected and treated as soon as possible. Delayed correction of this complication can be lethal due to the risk of visceral ischemia and limbs loss.

Disclosure: No significant relationships.

\section{CR57}

\section{HUNTING ACCIDENT-SHOT WOUND OF LOWER EXTREMITY}

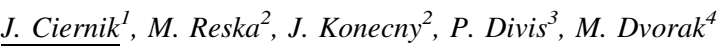

${ }^{1}$ Ist Surgical Department, St. Ann's Univ. Hospital, Brno, Czech Republic, ${ }^{2} 1$ st Surgical Department, St Ann's Masaryk University Hospital, Brno, Brno, Czech Republic, ${ }^{3}$ I.st Surgical Department, St. Ann's Univ. Hospital, Brno, Czech Republic, ${ }^{4}$ Ii.nd Surgical Department, St. Ann's Univ. Hospital, Brno, Czech Republic

Introduction: General overview: Under the term shot wounds are included all the injuries caused by the discharge or bursting of firearms, They are unpredictable puncture wounds that cause major tissue damage. Factors to determine the severity of a gunshot wound are location of the injury, size and speed of the projectile. As a high energy wounds are considered those, when speed of the projectile exceeds $600 \mathrm{~km} / \mathrm{h}$.
Case Report: 29 years old male, hunting accident, rifle shot to the upper part of the right femur. Wound description: Haemoragic shock stage II-III, entry wound $4 \mathrm{~mm}$ to the right dorsal upper femur, exit wound $2 \mathrm{~mm}$ medially. Massive swelling. Periphery pale, pulseless. $\mathrm{X}$-rays-comminutive fracture of the right femur, large number of small metal objects in a soft tissue. Peroperative findings: Primarily secured SFA and PFA (superficial femoral artery a profundic femoral artery) proximally. Multiple laceration to the superficial and deep arteries and veins. Cavity in mid-femur, about $12 \mathrm{~cm}$ in diameter, filled with bone fragments, coagulase and damaged soft tissue. Estimated blood loss about 7 litres at the time. Acute threat of DIC. With clamped major arteries continuing massive diffuse haemorhage. Immediate high femur amputation. Postoperative: ICU care, transfusion, volume restoration a antibiotic therapy. Continues psychological support, rehabilitation. Operation wound healed primarily. Prior to the discharge contacted prosthetic department.

Discussion: Due to the MESS score 7 unsalvageable limb. At young, healthy male, relatively hemodynamically stable patient, would YOU start with primary amputation?

Disclosure: No significant relationships.

\section{CR58}

\section{TRAUMATIC ASPHYXIA: CASE REPORT}

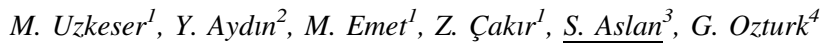

${ }^{1}$ Emergency Department, Ataturk University, Erzurum, Turkey, ${ }^{2}$ Thoracic Surgery, Ataturk University, Erzurum, Turkey,

${ }^{3}$ Emergency Department, Ataturk University Aziziye Hospital, Erzurum, Turkey, ${ }^{4}$ General Surgery, Ataturk University, Erzurum, Turkey

Introduction: Traumatic asphyxia is a rare sequel of thoracic trauma that occurs in children because of the greater flexibility of their thoracic cages. Asphyxia occurs after marked increase in intrathoracic pressure as the result of direct compression of the chest from crushing injury.

Case Report: A 5-year-old boy was found compressed to a part of a tractor while he was playing on it. On arrival, his GCS was 15 . He was oriented and cooperated. Neurological examination was normal. He had got 'mask face' appearance. His face, neck and upper trunk was erythematous and ecchymotic with petechial hemorrhages (photo 1). Bilateral subconjunctival hemorrhages were obvious (photo 2). There was no hearing or visual loss and his tympanic membranes were intact. His initial vitals were as follows: BP 100/60 $\mathrm{mmHg}, \mathrm{HR}$ 90/min, RR 19/min, Temp 36.7 ̈̈̌šC, satO $295 \%$, height $110 \mathrm{~cm}$, weight $19 \mathrm{~kg}$ as a supportive therapy, $\mathrm{O}_{2}$ inhalation was started and the patient was seated upright with a 30Ëš. Jugular vein Doppler ultrasonography, cranial and thoracal computerized tomography were all normal. The patient was hospitalized in thoracic surgery clinic and was discharged without complication on the third day.

Discussion: The clinical manifestations include subconjunctival hemorrhages, facial edema and cyanosis, ecchymotic or petechial hemorrhages on the upper chest, neck and face. Associated neurologic symptoms are altered mental status, brachial plexus injuries, quadriplegia and coma. These findings do occur as a result of cerebral edema and hemorrhage after anoxic brain injury. Morbidity and mortality are generally related to associated injuries.

Disclosure: No significant relationships. 


\section{CR59}

\section{TRAUMATIC TRACHEO-INNOMINATE FISTULA AS A RARE ACUTE COMPLICATION OF PENETRATING NECK INJURY: CASE REPORT}

\author{
A. Kulyapina ${ }^{1}$, T. Sanchez Rodriguez ${ }^{2}$,D. Perez-Diaz, \\ L. Rodriguez Bachiller ${ }^{3}$, J. Rodríguez-Roda Stuart ${ }^{4}$, F. Turegano, \\ D. Sánchez Valenzuela ${ }^{4}$
}

${ }^{1}$ Maxilofacial Surgery, GREGORIO MARAÑON HOSPITAL, Madrid, Spain, ${ }^{2}$ Surgery, GREGORIO MARAÑON UNIVERSITY GENERAL HOSPITAL, Madrid, Spain, ${ }^{3}$ Surgery, GREGORIO MARAÑON HOSPITAL, Madrid, Spain, ${ }^{4}$ Cardiovascular Surgery, GREGORIO MARAÑON HOSPITAL, Madrid, Spain, ${ }^{5}$ General and Emergency Surgery, University Hospital Gregorio Maranon, Madrid, Spain

Introduction: Penetrating injuries in the base of the neck (zone I) are considered to be the most dangerous due to the potential combination of vascular and intrathoracic lesions. The management of these lesions is always challenging as they may not be readily apparent. The case describes an extremely rare combined injury of trachea and innominate artery resulted in formation of traumatic acute tracheo- innominate fistula with delayed massive hemoptysis. Previously these fistulas have only been described as a rare iatrogenic complication of tracheostomy.

Case Report: The patient suffered a penetrating stab neck wound (zone I). Upon his arrival to ED he was stable and almost asymptomatic with a unique finding of blood rests in upper airways. A CT scan was performed which showed widening of mediastinum, elevation of right hemidiaphragm, presence of active arterial bleeding from brachiocephalic trunk with contrast extravasation, formation of pseudoaneurysm and an important left laterocervical and mediastina haematoma. Upon completion of the CT the patient suddenly went into haemodynamic shock from a massive hemoptysis which required urgent tracheal intubation. The patient was operated immediately through a sternotomy which revealed innominate artery disruption and tangential injury to the anterior wall of the trachea. Both lesions were suture -repaired and the patient made an uneventful recovery. Discussion: We hypothesize that the compartment pressure of the expanding neck haematoma prevented early symptoms of tracheal rupture (such as subcutaneous emphysema). The case confirms that penetrating injuries to zone I of the neck can be life threatening in spite of a misleading mild early presentation.

Disclosure: No significant relationships. 